\begin{tabular}{|c|c|}
\hline $\begin{array}{l}\text { FEB } 181998 \quad 4 \text { ENGINEERING DATA TRANSMITTAL } \\
\text { STA } \$ 4\end{array}$ & Pase i of $\frac{1}{1}$ \\
\hline
\end{tabular}

\begin{tabular}{|l|l|}
\hline $\begin{array}{l}\text { 2. To: (Receiving organization) } \\
\text { Distribution }\end{array}$ & $\begin{array}{l}\text { 3. From: Coriginating Organization) } \\
\text { WRAP Engineering }\end{array}$ \\
\hline $\begin{array}{l}\text { 5. Proj./Prog./Dept./Div.: } \\
\text { W-026/WRAP/WMH }\end{array}$ & $\begin{array}{l}\text { 6. Design Authority/ Design Agent/Cog. } \\
\text { Engr:: } \quad \text { K.J. Leist }\end{array}$ \\
\hline $\begin{array}{l}\text { 8. Originator Renarks: } \\
\text { This EDT is to release supporting documentation for the WRAP } \\
\text { Facility to Engineering Files. }\end{array}$
\end{tabular}

11. Receiver Remarks:

11A. Design Basel ine Document?

$[\mathrm{X}]$ Yes [] No

4. Related EDT Ho.:

7. N/A

7. Purchase Order No.:

N/A

9. Equip./Component No.:

$N / A$

10. System/Bldg./Facility: 2336-W

12. Major Assm. Dwg. Ho.: $N / A$

13. Permit/Permit Application Ho.: $N / A$

14. Required Response Date: $N / A$.

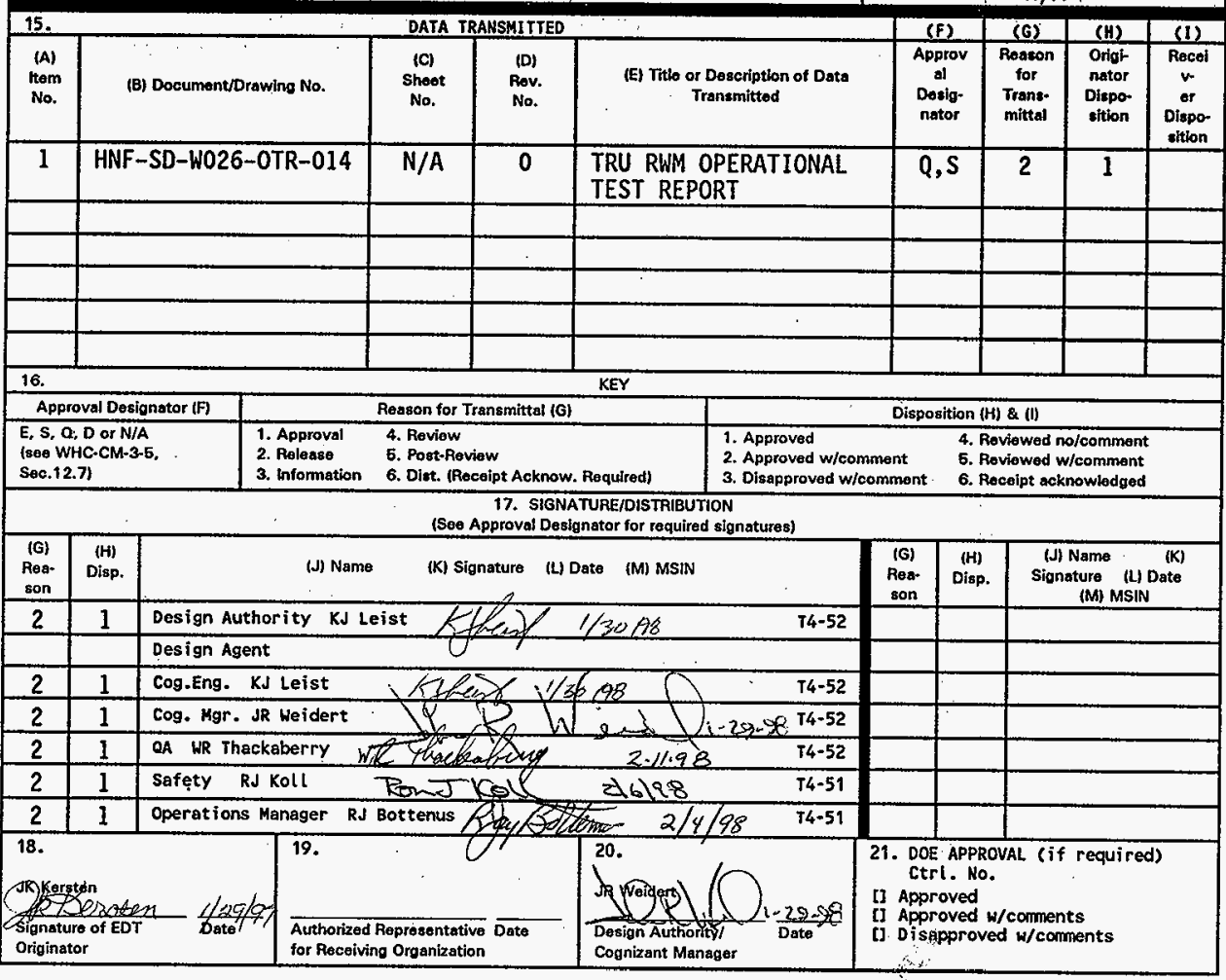




\section{W-026, TRANSURANIC WASTE RESTRICTED WASTE MANAGEMENT (TRU RWM) GLOVEBOX OPERATIONAL TEST REPORT}

KJ Leist

Waste Management Federal Services of Hanford, Richland, WA 99352

U.S. Department of Energy Contract DE-AC06-96RL13200

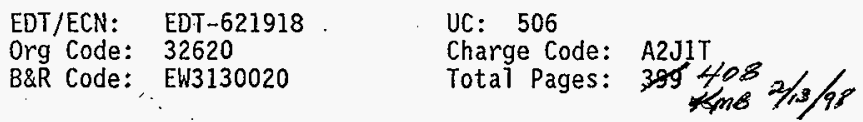

Key Words: WRAP, OTR, Drum, Diversified Metal Products

Abstract: This document verifies the Plant Operating Procedures

related to the TRU RWM GTovebox 401 are accurate so operations can be performed safely: and desired results obtained. It also verifies the Data Management System can interface with the Plant Control System.

* Drath \& Schrader is a registered trademark of Babcock Rohreitungsbau, GMBH, Germany

* Radsorb and Radpad are registered trademarks of Environmental Scientific, Inc., Morrisville, NC.

* ORACLE is a registered trademark of ORACLE Corporation, Redwood Shores, CA.

* Quick Solid is a registered trademark of Chemdal Corporation, Palatine, IL.

* Chemsorb is a registered trademark of Precision Lab Co., North Brook, IL.

* Nylon is a registered trademark of E.I. duPont de Nemours and Co., Wilmington, $D E$.

* Orion is a registered trademakr of Orion Research, Inc., Beverly, MA.

IRADEMARK DISCLAIMER. Reference herein to any specific comerciat product, process, or service by trade name, trademark, manufacturer, or otherwise, does not necessarily constitute or imply its endorsement, recomendation, or favoring by the United States Government or añy agency thereof or its contractors or subcontractors.

Printed in the United States of America. To obtain copies of this document, contact: Document Control Services, P.0. Box 950, Mallstop H6-08, Richland WA 99352, Phone (509) 372-2420; Fax (509) 376-4989.
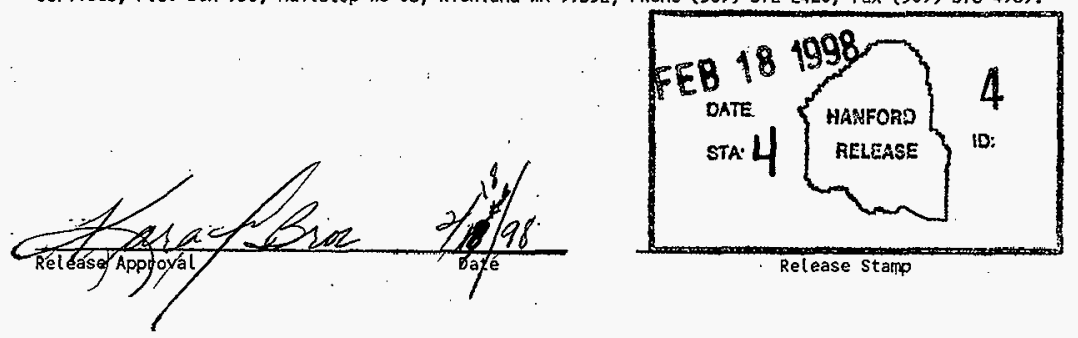

Approved for Public Release 


\title{
WASTE RECEIVING AND PROCESSING FACILITY (WRAP)
}

\section{TRANSURANIC WASTE / RESTRICTED WASTE MANAGEMENT (TRU / RWM)}

\author{
GLOVEBOX 401
}

\section{OPERATIONAL TEST REPORT}

\author{
HNF-SD-W026-OTR-014 Rev. 0 \\ WASTE MANAGEMENT HANFORD COMPANY - PROJECT W-026
}

February 11, 1998 


\section{TRU/RWM GLOVEBOX 401 OPERATIONAL TEST REPORT HNF-SD-W026-OTR-014 Rev. 0}

INTRODUCTION $\ldots \ldots \ldots \ldots \ldots \ldots \ldots \ldots \ldots \ldots \ldots \ldots \ldots \ldots \ldots, \ldots \ldots$

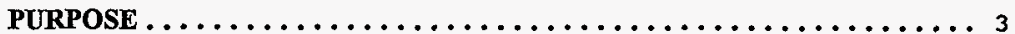

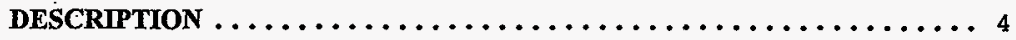

TEST RESULTS $\ldots \ldots \ldots \ldots \ldots \ldots \ldots \ldots \ldots \ldots \ldots \ldots \ldots \ldots \ldots$

Exception Test Reports (ETR's) Summary $\ldots \ldots \ldots \ldots \ldots \ldots \ldots \ldots 5$

Additional Items. . . . . . . . . . . . . . . . . . . . 6

CONCLUSIONS AND RECOMMENDATIONS $\ldots \ldots \ldots \ldots \ldots \ldots \ldots \ldots 6$

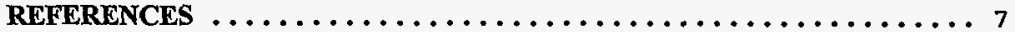

APPENDICES $\ldots \ldots \ldots \ldots \ldots \ldots \ldots \ldots \ldots \ldots \ldots \ldots \ldots \ldots \ldots \ldots$

Appendix A - Exception Test Reports $\ldots \ldots \ldots \ldots \ldots \ldots \ldots \ldots \ldots \ldots 7$

Appendix B - DMS Database Printouts . . . . . . . . . . . . 7

Appendix C - Plant Operating Procedures . . . . . . . . 


\section{TRU/RWM GLOVEBOX 401 OPERATIONAL TEST REPORT HNF-SD-W026-OTR-014 Rev. 0}

\section{INTRODUCTION}

The Transuranic Waste / Restricted Waste Management (LLW/RWM) Glovebox 401 is designed to accept and process waste from the Transuranic Process Glovebox 302 . Waste is transferred to the glovebox via the Drath \& Schraeder Bagless Transfer Port (DO-07-401) on a transfer stand. The stand is removed from the transfer drum with a hoist and the operator inspects the waste (with the aid of the Sampling \& Treatment Director) to determine a course of action for each item.

The waste is separated into compliant and non compliant types. One Trip Port DO-07-402A is designated as "Compliant" and One Trip Port DO-07-402B is designated as "Non Compliant". As the processing (inspection, bar coding, sampling \& treatment) of the transferred items takes place, residue is placed in the appropriate One Trip drum.

The status of the waste items is tracked by the Data Management System (DMS) via the Plant Control System (PCS) barcode interface. As an item is moved for sampling or storage or it's state altered by treatment, the Operator will track an items location using a portable barcode reader and entry any required data on the DMS console.

The Operational Test Procedure (OTP) will perform evolution's (described below) using the Plant Operating Procedures (POP) in order to verify that they are sufficient and accurate for controlled glovebox operation.

\section{PURPOSE}

The intent of this test was to satisfy two main objectives:

a. Verify that the Plant Operating Procedures related to TRU/RWM Glovebox 401 are accurate so that operations within the glovebox can be performed safely and desired results obtained.

During the performance of the OTP, the POP's were redlined to correct errors found during testing. These corrections were incorporated into updated revisions of the POP's. The original POP's from which this OTP was conducted are included with this (Operational Test Report) OTR so the evolution to the present state of the POP's can be traced.

b. Verify that the Data Management System (DMS) could properly interface with the Plant Control System (PCS) to control and display the data obtained during sample/packet movement and treatment.

When both of the above objectives are met, the TRU/RWM Glovebox would be considered ready for operation.

\section{NOTE:}

Verification of glovebox equipment operation was already performed in the TRU/RWM Glovebox Acceptance Test Procedure. 


\section{TRU/RWM GLOVEBOX 401 OPERATIONAL TEST REPORT HNF-SD-W026-OTR-014, Rev. 0}

\section{DESCRIPTION}

The Operational Test Procedure (HNF-SD-W026-OTP-014) was started on July 15, 1997 and consisted of six main sections as described below. A complete description is included at the beginning of each Test Case under General Description.

\section{a. Glovebox Manual Operation}

All three ports (DO-401, DO-402A \& DO-402B) are operated in manual mode. The respective lift table is placed in manual mode and the drum is mated to the port. Then by using manual mode for the Bagless Transfer port or the maintenance menus for the One Trip (Compliant \& None-Compliant) ports, a manual open/closed cycle is completed.

\section{b. Glovebox Aufomatic Test Case 1}

Transfer Drum 1 packets will be sorted and sampled, sample bottles are placed in STC 1 (Sample Transport Container).

c. Glovebox Automatic Test Case 2

Transfer Drum 2 packets will be sorted and sampled, sample bottles are placed in STC 2 (Sample Transport Container).

d. Glovebox Automatic Test Case 3

Transfer Drum 1 Collection Containers will be treated.

e. Glovebox Automatic Test Case 4

Transfer Drum 2 Collection Containers and STC-2 samples will be treated.

\section{f. Glovebox Restart}

The Glovebox Emergency Stop button is pushed during a port opening sequence.

Equipment status is recorded for recovery. 


\section{TRU/RWM GLOVEBOX 401 OPERATIONAL TEST REPORT HNF-SD-W026-OTR-014 Rev. 0}

\section{TEST RESULTS}

\section{Exception Test Reports (ETR's) Summary}

35 ETR's were written during test performance, of which 24 are DMS related. When the test was run, version 2.0 of the DMS software was installed. As of December 17, 1997, all ETR's have been cleared. 


\title{
TRU/RWM GLOVEBOX 401 OPERATIONAL TEST REPORT HNF-SD-W026-OTR-014 Rev. 0
}

\begin{abstract}
Additional Items
The Glovebox Manual Operation portion referenced WRP1-OP-0721 (RWM Gloveboxes Manual Operation) which included operation of lift tables manually.

WRP1-OP-0703 (Glovebox Lift Table Operation) was not referenced and therefore not verified in this OTP.

The "Glovebox Restart" portion of the OTP lacked enough depth to really verify that the glovebox can be successfully restarted for a selection of scenarios. Only one Emergency Stop pushbutton was checked. The balance of the section was merely a status check of the equipment in the glovebox. Operation Procedure OP-0722 should be enhanced to included flow charts or the like to demonstrate a logical course of action after the status of the systems are known.
\end{abstract}

\section{CONCLUSIONS AND RECOMMENDATIONS}

a. The STC Port can be open when the STC has been removed; no mechanical interlock exists. This can result in a loss of glovebox differential pressure and possible contamination of the STC port seal.

b. The Bagless Transfer Port DO-07-401 operation should be incorporated into the existing PLC interface instead of through a separate controller for the following reasons:

-The operator can only perform open and close functions when in automatic mode. Manual operation and recovery must be performed from the D\&S controller.

-There is no feed back to the operator in maintenance mode as to the position of the port components as there is for the entry/exit ports in this glovebox.

-The controller is programmed in German, requiring outside support for program changes.

-By eliminating the controller, the system is made simpler and therefore more reliable.

c. Permanent bar code charts should be added to the glovebox. During the OTP barcodes were taped to a sheet of paper which in time will deteriorate and require replacement.

d.

Since all ETR's have been cleared the Operational Test Procedure can be considered passed. However, modifications to the ports have been made since the completion of the OTP and will require verification prior to operation along with any other items that have been changed or reworked. 


\section{TRU/RWM GLOVEBOX 401 OPERATIONAL TEST REPORT HNF-SD-W026-OTR-014 Rev. 0}

\section{REFERENCES}

Plant Operating Procedures

WRP1-OP-0708 Puncture/Drain Aerosol Cans

WRP1-OP-0710 Glovebox Waste Sampling

WRP1-OP-0711 Waste Treatment

WRP1-OP-0717 LLW/RWM Glovebox Operation

WRP1-OP-0721 RWM Gloveboxes Manual Operation

WRP1-OP-0722 Restart RWM Glovebox Operation

\section{APPENDICES}

Appendix A - Exception Test Reports

Appendix B - DMS Database Printouts

Appendix C - Plant Operating Procedures

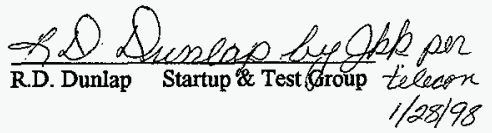


RUST FEDERAL SERVICES HANFORD

HNF-SD-W026-OTR-014

Project W-026, WRAP 1

Operational Test - TRU RWM Glovebox

Rev. 0

Page 1 of 79

ORGANIZATIONAL APPROVAL THAT ENGINEERING OPERATIONAL TEST PROCEDURE HAS BEEN COMPLETED SATISFACTORILY

$\frac{\text { RJ Bottenus } f(3)}{\text { WRAP } 1 \text { Operaterens Manager }} \frac{2 / 4 / 88}{\text { Date }}$

$\frac{\text { JR Weidert } \bigcirc R}{\text { WRAP 1 Engiñeering Manager }}$

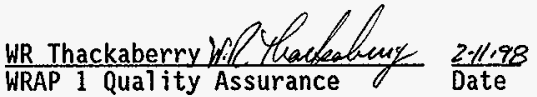

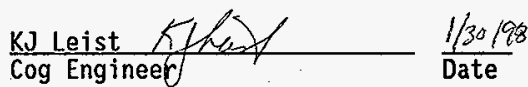

RJ Koll EJKdh WRAP 1 Safety $\frac{216198}{\text { Date }}$

HC Boynton 2/C Boup dulzs WRAP 1 Environmental 1 Compliance officer 
RUST FEDERAL SERVICES HANFORD Project $W-026$. WRAP I

Operational Test - TRU/RWM Glovebox.

HNF-SD-W026-0TR-014- - .......

Rev: 0

Page 2 of 79

\section{TABLE OF CONTENTS}

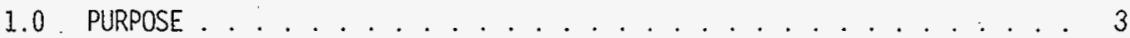

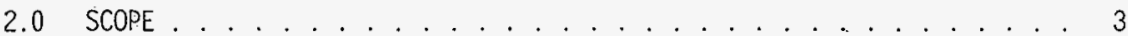

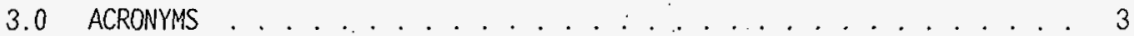

4.0 PRECAUTIONS AND LIMITATIONS . . . . . . . . . . . . . . . . . . . 4

5.0 RESPONSIBILITIES . . . . . . . . . . . . . . 5

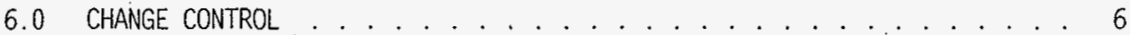

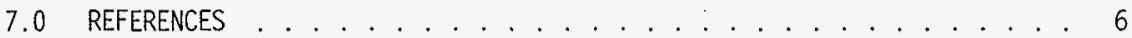

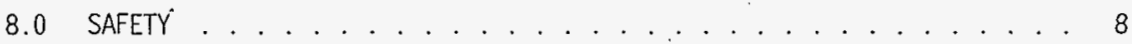

9.0. QualitY ASSURANCE ...................... . . 8

10.0 RECORDS . . . . . . . . . . . . . . . . . . . 8

11.0 PREREQUISITES . . . . . . . . . . . . . . . . . 9

12.0 PROCEDURE . . . . . . . . . . . . . . . . . . 12

13.0 ACCEPTANCE CRITERIA . . . . . . . . . . . . . . . . 13

ATTACHMENTS

ATTACHMENT 1 - TEST EXCEPTION LOG FORM . . . . . . . . . . . . . 14

ATTACHMENT 2 - TEST EXCEPTION REPORT . . . . . . . . . . . . . . . 15

ATTACHMENT 3 - SIGNATURE RECORD _ . . . . . . 16

ATTACHMENT 4 - TEST PLAN, TRU/RWM GLOVEBOX MANUAL OPERATION . . . . . 17

ATTACHMENT 5 - TEST PLAN. TRU/RWM GLOVEBOX AUTOMATIC TEST CASE $1 \ldots 22$

ATTACHMENT 6 - TEST PLAN. TRU/RWM GLOVEBOX AUTOMATIC TEST CASE $2 \ldots \ldots$

ATTACHMENT 7 - TEST PLAN, TRU/RWM GLOVEBOX AUTOMATIC TEST CASE $3 \ldots \ldots$

ATTACHMENT 8 - TEST PLAN, TRU/RWM GLOVEBDX AUTOMATIC TEST CASE $4 \ldots \ldots$

ATTACHMENT 9 - TEST PLAN, TRU/RWM GLOVEBOX RESTART $\ldots \ldots$. . . . . 74

ATTACHMENT 10 - TEST ACCEPTANCE SIGNATURE RECORD . . . . . . . 79 


\subsection{PURPOSE}

1.1 This procedure has been prepared to verify that the TRU/RWM Process GTovebox operates in accordance with system design and specifications. This procedure will also validate the WRAP-1 plant operating procedures 7isted in Section 7.2.

\subsection{SCOPE}

2.1 This Operating Test wi11 demonstrate the waste processing functions for the TRU/RWM Glovebox (107-GB-07-401).

2.2 Other interfacing systems outside the TRU/RWM Glovebox include the Internal Transport System, the Data Management System, the Plant Control System, and the Glovebox Barcode System.

2.3 When possible. this test will demonstrate al1 mechanical and control operations in manual and automatic modes. Restart of the glovebox from an emergency shutdown wi 17 also be demonstrated.

\subsection{ACRONYMS}

3.1 AGV - Automatic Guided Vehicle

3.2 DMS - Data Management System

3.3 HS - Hand Switch

3.4 OCS - Operating Control Station

3.5 OIU - Operator Interface Unit

3.6 OP - Operating Procedure

3.7 OTP - Operational Test Procedure

3.8 PCS - Plant Control System

3.9 PLC - Programmable Logic Controller

3.10 RCT - Radiological Control Technician

3.11 TD - Test Director

3.12 TE - Test Engineer

3.13 MC - Maintenance Craft 
RUST FEDERAL SERVICES HANFORD Project $W-026$. WRAP I -

HNF -SD-W026-OTR-014-2 - ....

Operationa? Test - TRU/RWM Glovebox

Rev: 0

Page 4 of 79

\subsection{PRECAUTIONS AND LIMITATIONS}

4.1 IF during performance of this procedure, any of the following conditions are found:-

- $\quad$ Any equipment malfunction which could prevent fulfillment of functional requirements.

- $\quad$ Personnel error or procedural inadequacy which could prevent fulfillment of OTP procedural requirements.

THEN. IMMEDIATELY NOTIFY the TD and TE.

4.2 The TD and $T E$ shall choose to stop work and place equipment in a safe condition based on the significance of a malfunction, error, or inadequacy.

4.3 Contact the TD and TE for additional instructions if changing plant conditions affect work or delays in work extending beyond (testing) shift.

4.4 IF any waste is generated during performance of this OTP. THEN, consult Environmental Compliance officer for specific instructions to ensure compliance with HNF and DOE environmental standards, as applicable, for correct disposal.

4.5 Comply with HNF and plant/facility specific lock and tag and over-tagging procedures, as applicable.

4.6 Measuring and Test Equipment (M\&TE), except timing devices used in the performance of this OTP, shall meet the following requirements:

- Be within its current calibration cycle as evidenced by an affixed calibration label.

- Be capable of the desired range.

- Have an accuracy (consistent with state-of-the-art limitations) equal to or greater than the accuracy specified in the OTP.

4.7 The equipment number and calibration information of $M$ \& TE used to perform this test sha71.recorded on the data sheets. 
4.8 Timing measurements shaTl be made with commercially available timing devices.

4.9 Al1 test data readings are to be taken and recorded for each location where the capability exists (i.e. local instrument; OIU, DMS terminal).

4.10 All test data readings are to be taken and recorded for each location where the capability exists (i.e. local observable instrument (LOI). operators screen (OS)).

\subsection{RESPONSIBILITIES}

5.1 Test Director:

- $\quad$ Schedules and reschedules operating tests as required.

- Notifies test participants before testing begins.

- Notifies personnel prior to testing of special safety precautions and conditions during testing.

- $\quad$ Schedules and conducts a daj7y pretest meeting with test participants.

- Record Data Exceptions and support OTP notes as required on the Attachment 1, TEST EXCEPTION LOG.

- Controls overall testing process and. change record authorization for this OTP.

- $\quad$ Responsible for conducting the test, collecting data, and ensuring compliance with all OTP requirements.

- Ensures required changes to supporting operating procedures (OPS) are initiated upon completion of OTP testing.

5.2 Test Engineer:

- $\quad$ Provides technical support during testing.

- Provides programming support during testing.

- Participates in pre-test meetings, as necessary.

- Conducts a pre-test system walkdown, as necessary.

- Verifies that mechanical, electrical, and control systems are functioning correctly and can support testing.

5.3 Maintenance Craft:

- $\quad$ Provide assistance during OTP activities. 
RUST FEDERAL SERVICES HANFORD Project W-026. WRAP I

HNF-SD-W026-OTR-01:4- - . Rev: 0

Operational Test - TRU/RWM Glovebox.

Page 6 of 79

5.4 Operations Personnel:

- Perform manipulations on Systems and Test Equipment.

- $\quad$ Start, stop and operate equipment related to OTP.

- Control and monitor parameters from Control Panels and

Control Room.

- Inform Duty Operations Supervisor of conditions affecting

plant stability or safety.

\subsection{CHANGE CONTROL}

6.1 OTP and supporting Operating Procedure (OP) administrative or editorial changes required during testing may be accommodated as redline exceptions in the test report. if these changes do not affect operating facility function. perfornance or safety that could compromise or influence data results. Changes to acceptance criteria, requirement changes, or changes to Cautions, Warnings, or any other safety items, and environmental instructions in the OTP or OP shall be approved by the signatories initially approving the OTP.

\subsection{REFERENCES}

7.1 The following documents were used in the preparation of or are referenced in this procedure:

HSRCM-1, Hanford Site Radiological Control Manual

HNF-CM-5-34. Solid Waste Disposal Operations Administration

HNF-CM-5-36. SWD Internal Requirements

HNF-IP-0263-WRP1, Building Emergency Plan

HNF-CM-5-36. Chapter 1.10. Safety Manual

HNF-CM-5-36. Chapter 1.11. Industrial Hygiene Manual

HNF-CM-5-36. Chapter 3-5. Section 12.7

HNF-CM-5-36. Chapter 7.5. Environmental Comp 7 iance Manual

HNF-CM-5-34. Section 3.1.4. Lock-out, Tag-out 
7.2 The following operating procedures (OPS) will be used to support test activities and objectives and will be validated during this 0TP:

Procedure \# Procedure Title

WRP1-OP-0728, TRU/RWM Glovebox Operation

WRP1-OP-0721. RWM Gloveboxes Manual Operation

WRP1-OP-0722, Restart RWM Glovebox Operation

WRP1-OP-0701, GTovebox Housekeeping

WRP1-OP-0703. Glovebox Lift TabTe Operation

WRP1-0P-0704, Glovebox Manipulator Operation

WRPI-0P-0705. Bagless Transfer Operation

WRP1-0P-0708. Puncture/Drain Aerosol Cans

WRP1-OP-0710, Glovebox Waste Sampling

WRP1-0P-0711, Waste Treatment

A17 copies of the OPs used for testing shall be hand marked with the OTP number at the top of the first page and attached to the completed OTP test package.

Use of operating procedures to support the OTP wi 11 provide validation of the procedures and minimize the amount of test paperwork generated.

7.3 The following procedures will be used for alarm response during OTP activities, as required:

WRP1-AR-0700. Process Area A7arm Response

WRP1-AR-1106. Process Area (Zone II) HVAC A7arm Response

WRP1-AR-1107, Glovebox HVAC Alarm Response

7.4 The following administrative procedures control work performed in this OTP:

HSRCM-1, Hanford Site Radiological Control Manual

HNF-CM-5-34. Section 1.9. Quality Assurance Program Plan

HNF-CM-5-34. Section 3.1.4, Lock-out. Taq-out

HNF-CM-5-36, Chapter 1.10, Safety Manual

HNF-CM-5-36. Chapter 1.11, Industrial Hyqiene Manual

HNF-CM-5-36. Chapter 4-2, Qua lity Assurance Manual

HNF-CM-5-36. Chapter 7-5, Environmental Compliance Manual

HNF-IP-0263-WRP1, Building Emerqency Plan 
RUST FEDERAL SERVICES HANFORD

Project $W-026$. WRAP I

Operational Test - TRU/RWM Glovebox.

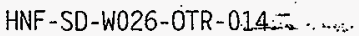

Rev: 0

Page 8 of 79

\subsection{SAFETY}

8.1 WARNING - In addition to construction and/or chemical, electrical. and oil contamination hazards, operators should be aware of the possibility of coming into contact with biological hazards.

8.2 The gloveboxes are equipped with dry chemical fire suppression systems which will discharge into the glovebox if the interior temperature at the top of the gloveboxes rises to $\sim 190^{\circ} \mathrm{F}$.

8.3 Use care to minimize injury. Possible safety hazards include:

- $\quad$ Tripping / Slipping

- Injuries to the hands/head

- $\quad$ Automatic Guided Vehicle traffic.

8.4 RADIATION - CONTAMINATION CONTROL - WRAP 1 is a new facility, no radiation contamination concerns are expected during OTP activities.

\subsection{QUALITY ASSURANCE}

9.1 No Quality Assurance witness, hold points or verifications are required in this OTP.

\subsection{RECORDS}

10.1 This TRU/RWM Glovebox OTP complete with attachments shall be filed as a permanent test record. 
RUST FEDERAL SERVICES HANFORD Project W-026. WRAP I

OperationaT Test - TRU/RWM Giovebox.
HNF-SD-W026-0TR-014- $\cdots$ Rev: 0

Page 9 of 79

\subsection{PREREQUISITES}

11.1 VERIFY barcode Tabels attached to glovebox:

LOCATION

TRURWENTRY

TRURWCMPLT

TRURWEXIT

TRURW PP

TRURWSAMPL

TRURWTREAT
COMMAND LABELS

END. CNCL PREV READ, CNCL OPERATION, YES, NO

END, CNCL PREV READ, CNCL OPERATION, YES, NO

END, CNCL PREV READ, CNCL OPERATION, YES, NO

END, CNCL PREV READ. CNCL OPERATION, YES, NO

END, CNCL PREV READ, CNCL OPERATION, YES, NO

END, CNCL PREV READ, CNCL OPERATION, YES, NO

11.2 VERIFY TRU/RWM process glovebox Acceptance Test Procedure is complete and al1 deficiencies or exceptions arising from the ATP have been resolved and closed out, or will not affect testing.

11.3 VERIFY a1] open (ATP/FTP) items wi 11 not effect OTP performance:

- Quality Assurance Nonconformance: Reports (NCRs)

- Construction Punch Lists

- $\quad$ Outstanding Engineering Change Notices (ECNs)

- $\quad$ Startup-originated Design Change Requests (CRs)

- $\quad$ Test Deficiency Reports

- Master System Punch List items 
RUST FEDERAL SERVICES HANFORD

Project W-026. WRAP I

Operational Test - TRU/RWM Glovebox
HNF-SD-W026-OTR-014:-

Rev: 0

Page 10 of 79

11.4 VERIFY materials are available as follows:

55 ONE TRIP SPA zlulas

Empty 85 gallon Entry/Exit. drum with 7ids and barcode label OTP-TRUR-PD-01

Empty 55 gallon

55 galion D\&S transfer drum with barcode label OTP-TRUR-TD-01 attached, lid, and transfer stand inside containing the following packets:

0TP6-97-000001: unwrapped aerosol can of saline solution.

0TP6-97-000002: aerosol can of saline solution in bubble wrap \& tape.

0TP6-97-000003: aerosol can with no label. wrapped in cloth \& tape.

55 gallon D\&S transfer drum with barcode 7abel OTP-TRUR-TD-02 attached, lid, and transfer stand inside containing the following packets:

0TP6-97-000004: lead brick wrapped in two plastic bags \& tape

0TP6-97-000005: 1L bottle of unknown solvent \& IL bottle of oil. taped together and wrapped in cloth \& tape:

0TP6-97-000006: unwrapped 1L bottle partially fu11 of acetic acid

5 Empty Sample Bottles $(250 \mathrm{ml})$

2 Empty, unlabeled Collection Containers (1L)

2 Empty. unlabeled Treatment Containers (1L)

Sample Transport Container with barcode label 0TP6-97-300001

Sample Transport Container with barcode 1abel 0TP6-97-300002

One Transfer Pig with barcode label 0TP6-97-400001 PIG UNAVAILABLE SPA =18f 8 2/6/98

Dispenser with the following packet and collection container barcode labels, at glovebox sample location:

0TP6-97-000007, 0TP6-97-000008,

OTP6-97-000009, 0TP6-97-000010.

0TP6-97-000011

Dispenser with the following treatment container barcode labels, at glovebox treatment location:

0TP6-97-900001, 0TP6-97-900002,

0TP6-97-900003, OTP6-97-900004,

ОТР6-97-900005

250m7 SODIUM BICARBONATE, QUICK SOLID OR RADSORB. CHEMSORB and VOID SPACE

FILLER as required

11.5 PREREQUISITES 11.1 through 11.4 complete:

TE Initiais/Date: SPA / / /15/97 


\section{NOTE}

Steps 11.6 through 11.15 may be performed in any order.

11.6 PERFORM a pretest briefing for a11 personnel involved in the OTP.

11.7 VERIFY two way portable radio communication (when necessary) between the testing and equipment locations.

11.8 VERIFY the official OTP document and al1 photocopies that will be used during testing are the latest approved revision.

11.9 DETERMINE appropriate interface support (personnel/equipment) from other departments and organizations and that support is available.

11:10 VERIFY personne] performing this procedure are qualified in accordance with HNF-CM-5-34. Section 1.8. Training Plan.

HNF-IP-1242, WRAP 1 RGD Radiation Protection Program and on-the-job training.

11.11 VERIFY all personnel to be involved with this OTP have completed Attachment 3, SIGNATURE RECORD.

11.12 VERIFY a11 required TRU/RWM process glovebox components are insta7led and operational.

11.13 VERIFY a11 permanently installed and portable instrumentation listed in the applicable operating procedures and required for testing, is within it's current calibration cycle.

11.14 VERIFY the DMS, PCS and OIUS for the TRU/RWM process glovebox are available and operational.

11.15 VERIFY process area HVAC and glovebox alarms are activated.

11.16 PREREQUISITES 11.6 through 11.15 complete:

TD Initials/Date: SPA / i/15/97 
RUST FEDERAL SERVICES HANFORD

Project $W-026$. WRAP I

Operational Test - TRU/RWM Glovebox.
HNF-SD-W026-0TR-014- ….

Rev: 0

Page 12 of 79

\subsection{PROCEDURE}

12.1 PERFORM Manual Operation test of the TRU/RWM Glovebox per Attachment 4, TEST PLAN. TRU/RWM GLOVEBOX MANUAL OPERATION.

12.2 PERFORM Automatic Operation test of the TRU/RWM Glovebox for Test Case 1 per Attachment 5, TEST PLAN, TRU/RWM GLOVEBOX AUTOMATIC TEST CASE 1.

12.3 PERFORM Automatic Operation test of the TRU/RWM Glovebox for Test Case 2 per Attachment 6. TEST PLAN, TRU/RWM GLOVEBOX AUTOMATIC TEST CASE 2.

12.4 PERFORM Automatic Operation test of the TRU/RWM Glovebox for Test Case 3 per Attachment 7. TEST PLAN, TRU/RWM GLOVEBOX AUTOMATIC TEST CASE 3.

12.5. PERFORM Automatic Operation test of the TRU/RWM Glovebox for Test Case 4 per Attachment 8. TEST PLAN, TRU/RWM GLOVEBOX AUTOMATIC TEST CASE 4.

12.6 PERFORM Restart test of the TRU/RWM Glovebox per Attachment 9. TEST PLAN, TRU/RWM GLOVEBOX RESTART. 


\subsection{ACCEPTANCE CRITERIA}

13.1 Test results of each section will be considered acceptable if the following conditions are met:

13.1.1 The procedure test section was performed as written with expected results $\underline{\mathrm{OR}}$ :

13.1.1.1 For editorial changes:

- $\quad$ The procedure was redlined to reflect field conditions and the section in question performed as written with expected results.

- Redline and changes shall be in accordance with Section 6 , Change Control.

13.1.1.2 For changes other than editorial:

- Exceptions are documented on Attachment 2, Test Exception Report, and the Disposition Section is approved by the Test Engineer.

- $\quad$ Each Test Exception Report is logged in Attachment 1. Test Exception Log.

- $\quad$ The Test Director shal1 review Attachment 1. Test Exception Log, and ensure timely completion of all Disposition Actions and Retest Requirements. 
RUST FEDERAL SERVICES HANFORD Project W-026. WRAP I

operationaT Test - TRU/RWM GTovebox.
HNF-SD-W026-OTR-014- …4.

Rev: 0

Page 14 of 79

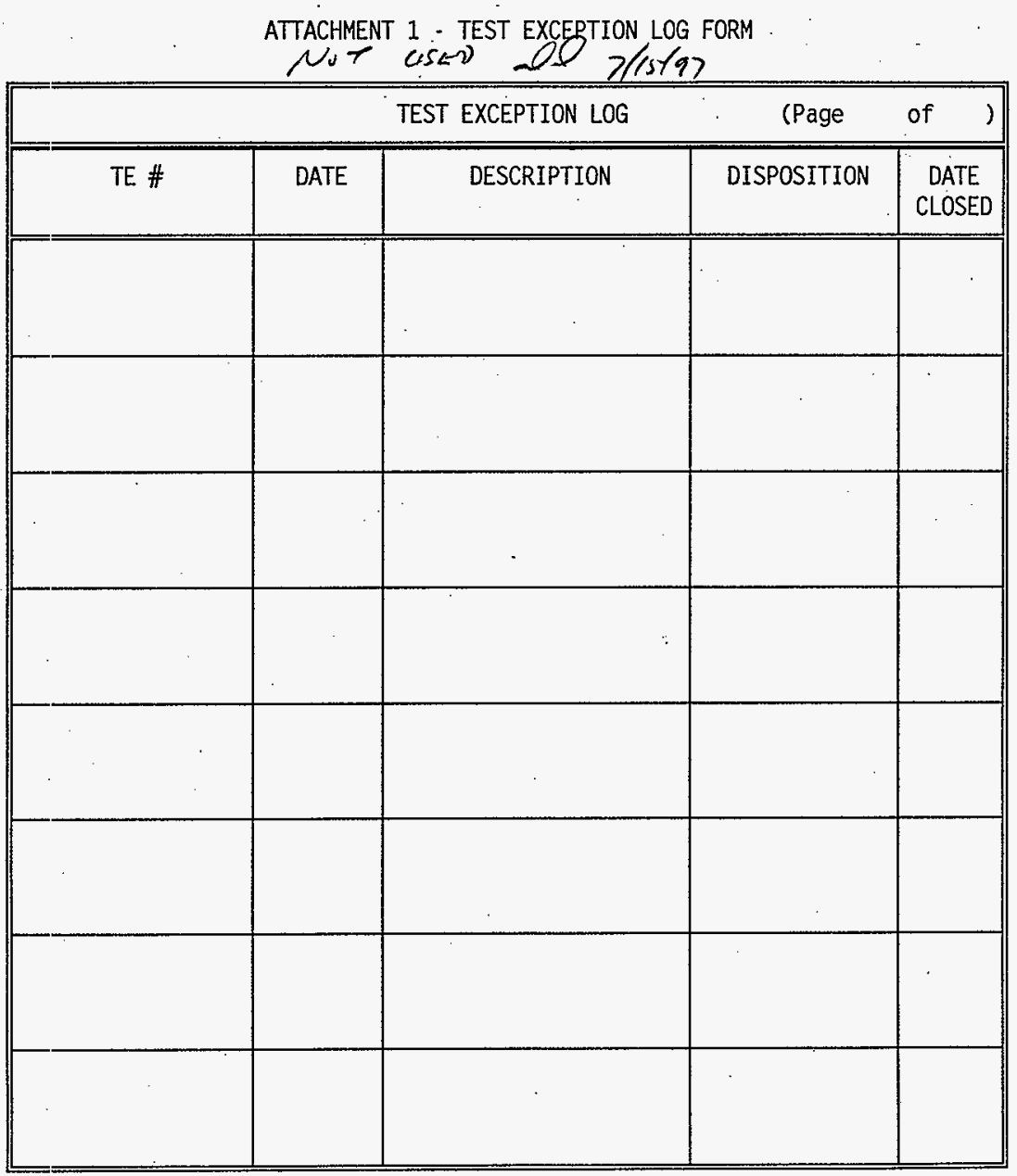




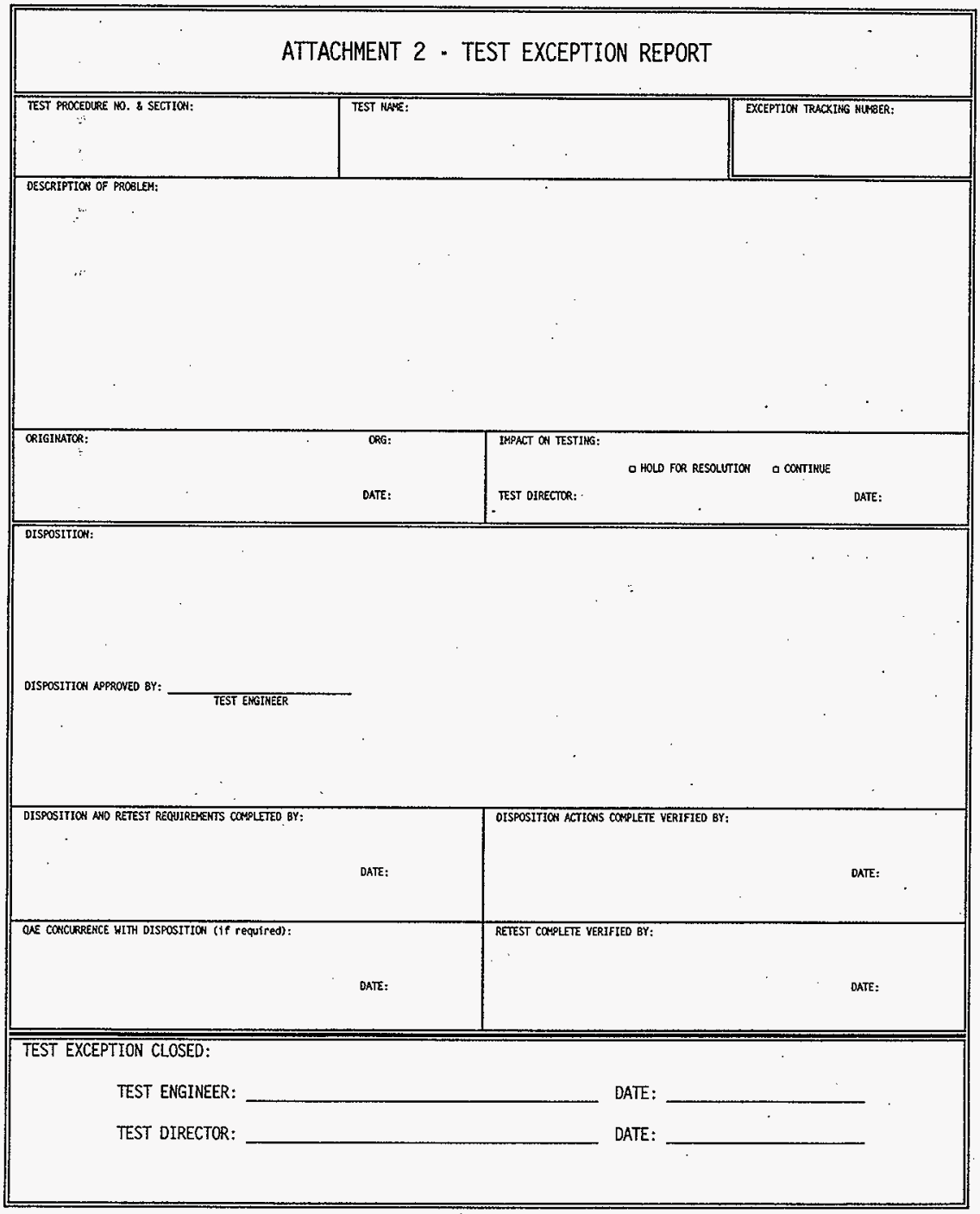


LOW LEVEL. WASTE/RADWASTE MANAGEMENT GLOVEBOX OPERATIONAL TEST INITIAL PRE-JOB PERFORMED 7/15/97 SPA 216/98

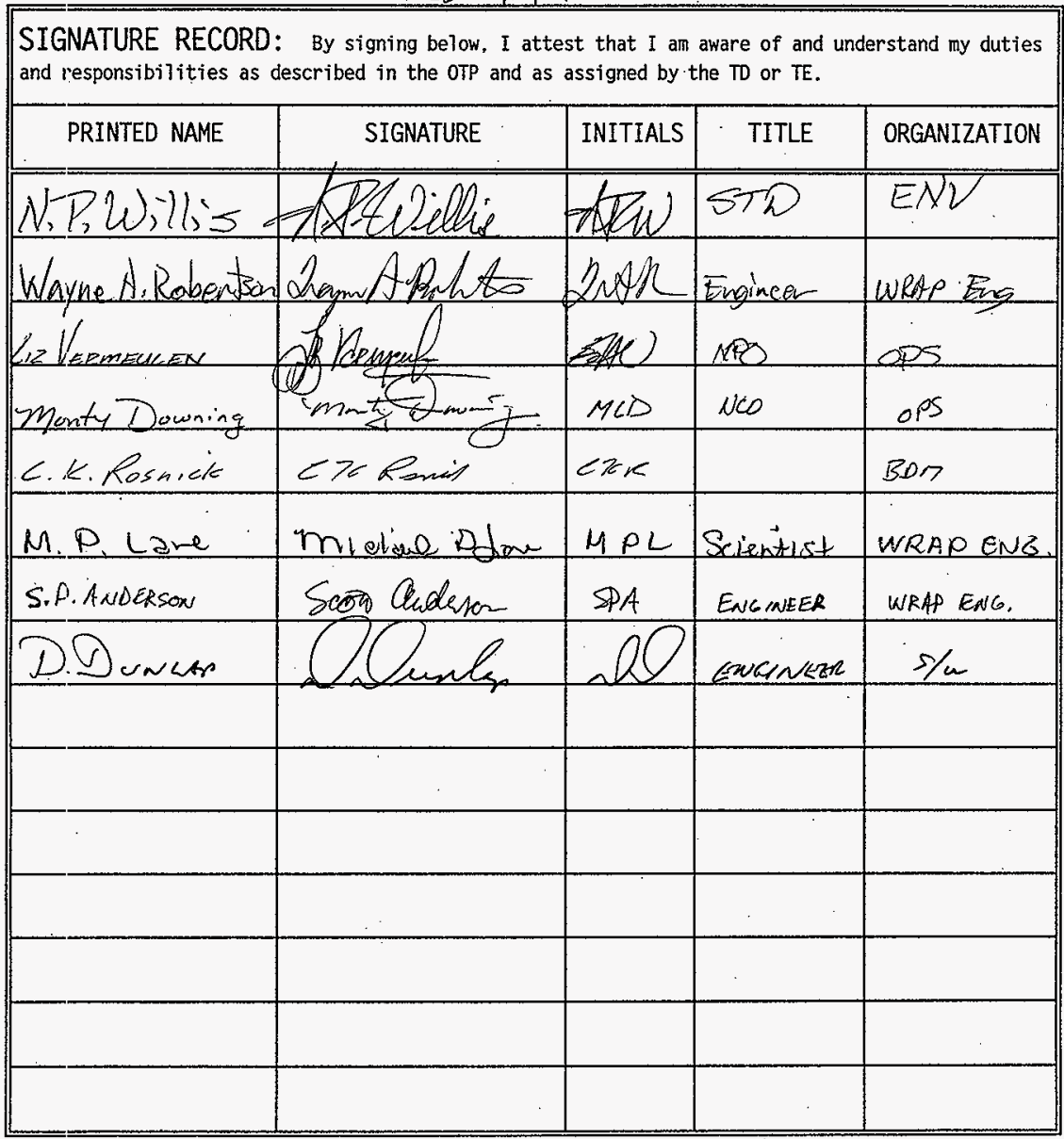


RUST FEDERAL SERVICES HANFORD

ATTACHMENT 4 - TEST PLAN, TRU/RWM GLOVEBOX MANUAL OPERATION

\subsection{GENERAL DESCRIPTION}

1.1 This test plan will verify that each manual function of the glovebox components may be individually operated. These operations will be tested using procedure WRP1-OP-0721. RWM Gloveboxes Manual Operation, thereby also performing the required procedure validation.

\subsection{SAFETY}

2.1 Reference OTP Section 8, SAFETY.

3.0 TOOLS, EQUIPMENT AND SUPPLIES

- $\quad$ Drum handling (manual) equipment or Pallet fork truck

- Spill Kit/Decontamination Equipment (oils/chemicals)

- Tape and rags

4.0 TEST PREREQUISITES

4.1 VERIFY OTP Section 11.0. PREREQUISITES are complete.

4.2 VERIFY PCS and DMS are ready to support testing.

4.3 TEST PREREQUISITES are complete and acceptable.

TD Initials/Date: SA, 2holst 
RUST FEDERAL SERVICES HANFORD Project W-026. WRAP I Operational Test - TRU/RWM Glovebox.
HNF-SD-W026-OTR-014- - . Rev: 0

Page 18 of 79

\section{ATTACHMENT 4 - TEST PLAN, TRU/RWM GLOVEBOX MANUAL OPERATION}

\subsection{PROCEDURE}

5.1 REFERENCE procedure WRP1-OP-0721, RWM GLOVEBOXES MANUAL OPERATION:

5.1.1 PERFORM Section VIII.A. "RESET DIAGNOSTIC FLAGS ON TRU/RWM GLOVEBOX (107-GB-07-401)", of operating procedure as follows:

5.1.1.1 REDLINE procedure as needed during performance.

5.1.1.2 VERIFY results of performance are acceptable.

TD Initials/Date: SPA / T/15/97

5.1.2 PERFORM Section VIII.B. "ACCESS TRU/RWM GLOVEBOX MAINTENANCE MENUS", of operating procedure as follows:

5.1.2.1 REDLINE procedure as needed during performance.

5.1.2.2 VERIFY results of performance are acceptable.

TD Initials/Date: SPA, $/ 7 / 15 / 97$

5.1.3 PERFORM Section VIII.C, "TRU/RWM GLOVEBOX HOIST (107-CR-07-401) MANUAL OPERATIONS", of operating procedure as follows:

5.1.3.1 REDLINE procedure as needed during performance.

5:1.3.2 VERIFY results of performance are acceptable.

TD Initials/Date: SPA, /7/15/97

5.1.4 PERFORM Section VIII.D.1, "MATE DRUM TO BAGLESS TRANSFER PORT (107-00-07-401)", of operating procedure as follows:

5.1.4.1 REDLINE procedure as needed during performance.

5.1.4.2 VERIFY results of performance are acceptable.

TD Initials/Date: SPA / //15/97 
RUST FEDERAL SERVICES HANFORD Project $W-026$, WRAP I

\section{ATTACHMENT 4 - TEST PLAN, TRU/RWM GLOVEBOX MANUAL OPERATION}

5.1.5 PERFORM Section VIII.D.2, "OPEN/CLOSE BAGLESS TRANSFER PORT (107-00-07-401)", of operating procedure as follows:

5.1:5.1 REDLINE procedure as needed during performance.

5.1.5.2 VERIFY results of performance are acceptable.

$$
\text { TD Initials/Date: SPA / I/15/97 }
$$

5.1.6 PERFORM Section VIII.D.3, "REMOVE DRUM FROM BAGLESS TRANSFER PORT (107-D0-07-401)", of operating procedure as follows:

5.1.6.1 REDLINE procedure as needed during performance.

5.1.6.2 VERIFY results of performance are acceptable.

$$
\text { To Initials/Date: SPA / 17/15/97 }
$$

5.1.7 PERFORM Section VIII.E.1, "MATE DRUM TO COMPLIANT WASTE PORT (107-GB-07-402A)", of operating procedure as follows:

5.1.7.1 REDLINE procedure as needed during performance.

5.1.7.2 VERIFY results of performance are acceptable.

TD Initials/Date: SPA / 1/19/97

5.1.8 PERFORM Section VIII.E.2. "OPEN COMPLIANT WASTE PORT (107-GB-07-402A)", of operating procedure as follows:

5.1.8.1 REDLINE procedure as needed during performance.

5.1.8.2 VERIFY results of performance are acceptable.

TD Initials/Date: $\$ A$ / $1 / 15 / 97$ 
RUST FEDERAL SERVICES HANFORD Project $W-026$. WRAP I Operational Test - TRU/RWM Giovebox.

ATTACHMENT 4 - TEST PLAN, TRU/RWM GLOVEBOX MANUAL OPERATION

5.1.9 PERFORM Section VIII.E.3, "CLOSE COMPLIANT WASTE PORT (107-GB-07-402A)" , of operating procedure as follows:

5.1.9.1 REDLINE procedure as needed during performance.

5.1.9.2 VERIFY results of performance are acceptable.

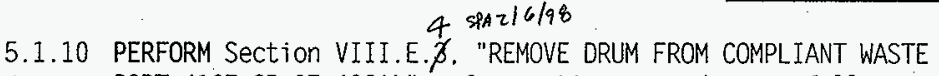
TO Initials/Date: SPA / 7/15/97
SPA z/6/98
"REMOVE DRUM FROM COMPLIANT WASTE
operating procedure as follows:

5.1.10.1 REDLINE procedure as needed during performance.

5.1.10.2 VERIFY results of performance are acceptable.

TO Initials/Date: SPA / 7/15/97

5.1.11 PERFORM Section VIII.F.1, "MATE DRUM TO NON-COMPLIANT WASTE PORT (107-GB-07-402B)", of operating procedure as follows:

5.1.11.1 REDLINE procedure as needed during performance.

5.1.11.2 VERIFY results of performance are acceptable.

TD Initials/Date: $S P A \quad / 1 / 15 / 97$

5.1.12 PERFORM Section VIII.F.2, "OPEN NON-COMPLIANT WASTE PORT

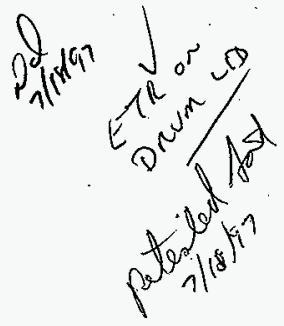
(107-GB-07-402B)", of operating procedure as follows:

5.1.12.1 REDLINE procedure as needed during performance. 5:1.12.2 VERIFY results of performance are acceptable.

TD Initials/Date:

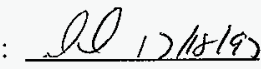


ATTACHMENT 4 - TEST PLAN, TRU/RWM GLOVEBOX MANUAL OPERATION

5.1.13 PERFORM Section VIII.F.3, "CLOSE NON-COMPLIANT WASTE PORT (107-GB-07-402B)", of operating procedure as follows:

5.1.13.1 REDLINE procedure as needed during performance.

5.1.13.2 VERIFY results of performance are acceptable. To Initials/Date: ahe, $7 / 1 \mathrm{~s} / 9 \mathrm{~T}$

5.1.14 PERFORM Section VIII.F.4, "REMOVE DRUM FROM NON-COMPLIANT WASTE PORT (107-GB-07-402B)", of operating procedure aS follows:

5.1.14.1 REDLINE procedure as needed during performance.

5.1.14.2 VERIFY results of performance are acceptable. TD Initials/Date: $\mathrm{hl} \ell_{1}>/ 1 \mathrm{~s} / 9$ 
RUST FEDERAL SERVICES HANFORD

Project W-026. WRAP I -

Operational Test - TRU/RWM GTovebox
HNF-SD-W026-0TR-014:- $\cdots \ldots$.

Rev: 0

Page 22 of 79

\section{ATTACHMENT 5 - TEST PLAN, TRU/RWM GLOVEBOX AUTOMATIC TEST CASE 1}

\subsection{GENERAL DESCRIPTION}

1.1 Test case one will sort and sample Test Drum 1 packets. These operations will be tested using procedures WRP1-OP-0710. Glovebox Waste Sampling, WRP1-OP-0728, TRU/RWM Glovebox Operation, and WRP1-OP-0708. Puncture/Drain Aerosol Cans thereby also performing the required procedure validation for sections performed. The sequence of events is as follows:

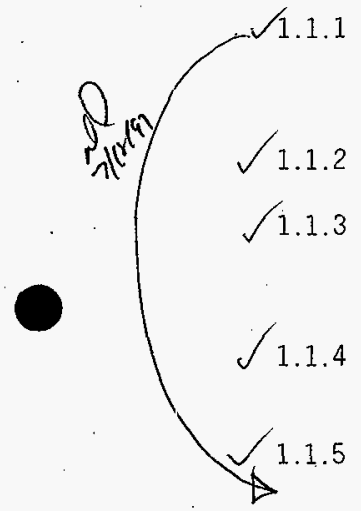

Empty 55 gallon Entry/exit drum OTP-TRUR-PD-11 is connected to the TRU/RWM glovebox compliant loadout port and the lid is removed.

STC OTP6-97-300001 is connected to the STC port.

Transfer drum OTP-TRUR-TD-01 is connected to the TRU/RWM entry port, the lid is removed, and the transfer stand is moved to the sample station.

1.1.6 Packet barcode label 0TP6-97-000007 is attached to a new collection container and the aerosol cans in packets 0TP6-97-000001 and 0TP6-97-000002 are drained in the collection container. The empty aerosol cans and the packet material are placed in the compliant loadout drum.

1.1.7 Packet barcode label 0TP6-97-000008 is attached to a new collection container and the aerosol can in packet 0TP6-97-000003 is drained in the collection container. The empty aerosol can and packet material are placed in the compliant Toadout drum.

1.1.8 Physical component data for the waste in compliant waste loadout drum is entered into DMS.

1.1.9 Field screening is performed on the liquid in collection container OTP6-97-000008. 


\section{ATTACHMENT 5 - TEST PLAN, TRU/RWM GLOVEBOX AUTOMATIC TEST CASE 1}

1.1.10 A sample analysis request is prepared for collection container 0TP6-97-000008. Sample 97-00001 is requested with analyses "AQLIQ" and "COND". One bottle is required for each analysis.

1.1.11 Sample bottle barcode labels 97-00001-01L and 97-00001-02L are printed in the sample management office and the labels are placed on empty sample bottles.

1.1.12 Empty sample bottles 97-00001-01L and 97-00001-02L are loaded into the TRU/RWM glovebox through :the Consumable Materials port.

1.1.13 Liquid from collection container 0TP6-97-000008 is collected in sample bott7es 97-00001-01L and 97-00001-02L.

1.1:14 Sample bottles 97-00001-01L and 97-00001-02L are placed in STC 0TP6-97-300001.

1.1.15 STC 0TP6-97-300001 is removed from TRU/RWM sample port.

1.1.16 A transport pig is not used.

1.1.17 Collection containers 0TP6-97-000007 and 0TP6-97-000008 are placed on the transfer stand and the transfer stand is returned to transfer drum OTP-TRUR-TD-01.

1.1.18 Transfer drum OTP-TRUR-TD-01 is closed and removed from the transfer drum port and from lift table LT-09-201C.

\subsection{SAFETY}

\subsection{Reference OTP Section 8. SAFETY.}

\subsection{TOOLS, EQUIPMENT AND SUPPLIES}

- Drum handing (manual) equipment or Pal iet fork truck

- $\quad$ Spil1 Kit/Decontamination Equipment (oils/chemicals)

- Tape and rags

- Other equipment, material or protective clothing (as required) 
ATTACHMENT 5 - TEST PLAN, TRU/RWM GLOVEBOX AUTOMATIC TEST CASE 1

\subsection{TEST PREREQUISITES}

4.1 VERIFY OTP Section 11.0. PREREQUISITES are complete.

4.2 SET initial conditions as follows:

\section{NOTE}

Supplies required during the test which cannot be loaded through the consumable materials port, will be loaded in the compliant waste loadout drum.

$\checkmark$ 4.2.1 Empty 55 galion. compliant waste loadout drum OTP-TRUR-PD-11 is on lift table LT-09-201B.

$\sqrt{4.2 .2}$ Transfer drum 0TP-TRUR-TD-01 is on lift table LT-09-201C and contains the following packets from the TRU glovebox:

$\mathcal{V}$ 4.2.2.1 Packet 0TP6-97-000001 contains an unwrapped aerosol can of saline solution:

$J_{\text {4.2.2.2 Packet 0TP6-97-000002 contains an aerosol can of }}$ saline solution wrapped in bubble wrap and tape.

4.2.2.3 Packet 0TP6-97-000003 contains an aerosol can with no label, wrapped in cloth and tape.

$\sqrt{4.2 .3 \text { Empty sample transport container 0TP6-97-300001 (STC-1) is at }}$ glovebox ready to connect to the STC port.

4.2.4 Two empty, unlabeled, 1 liter collection containers are available for loading into glovebox through Consumable Materials port.

4.2.5 Two sequential packet barcode labels are available in the glovebox.

$\sqrt{4.2 .6}$ Required sampling supplies are available.

$\sqrt{4.2 .7}$ Two empty, unlabeled, $250 \mathrm{ml}$ sample bottles are in the Sample Management office. 


\section{ATTACHMENT 5 - TEST PLAN, TRU/RWM GLOVEBOX AUTOMATIC TEST CASE 1}

$\sqrt{4.2 .8}$ User with general operator (GENOP) priyjleges is logged on to DMS TRU Terminal (107-TE-12-104). o $7 / 8$ - 197

4.2.9 User with chemist privileges is logged on to Sample Management Office OMS TerminaT (109-TE-12-107).

$\sqrt{4.2 .10}$ Sample-label barcode printer in Sample Management office is loaded with blank labels and ready to print.

4.2.11 Test scripts have been run on the DMS server to:

4.2.11.1 Initialize the DMS database.

4.2.11.2 Load initial data for PINs:

OTP-TRUR-PO-01, OTP-TRUR-PD-11,

OTP-TRUR-TD-01, OTP-TRUR-TD-02,

OTP6-97-000001, OTP6-97-000002.

OTP6-97-000003. OTP6-97-000004,

0TP6-97-000005. 0TP6-97-000006,

0TP6-97-300001, OTP6-97-300002,

OTP6-97-400001.

4.2.11.3 Print report of DMS database.

\subsubsection{VERIFY conditions:}

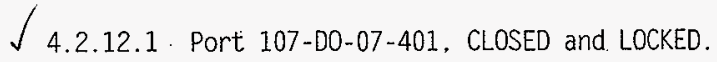

$\sqrt{ }$ 4.2.12.2. Port 107-D0-07-402A, CLOSED and LOCKED.

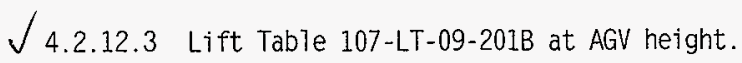

$\sqrt{4.2 .12 .4}$ Lift Table 107-LT-09-201B, MAINTENANCE/OFF/NORMAL $(\mathrm{M} / 0 / \mathrm{N})$ switch in NORMAL.

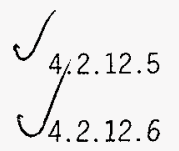

Lift Table 107-LT-09-201C at AGV height.

Lift Tab7e 107-LT-09-201C. MAINTENANCE/OFF/NORMAL $(M / O / N)$ switch in NORMAL. 
RUST FEDERAL SERVICES HANFORD

Project W-026. WRAP I

Operational Test - TRU/RWM Glovebox.
HNF-SD-W026-OTR-0.14:- …s. Rev: 0

Page 26 of 79

ATTACHMENT 5 - TEST PLAN, TRU/RWM GLOVEBOX AUTOMATIC TEST. CASE 1

$\checkmark$ 4.2.12.7 Manipulator 107-EM-07-401 PARKED.

4.2.13 UPDATE PCS Tracking Table as follows:

$\sqrt{ } \quad$ 4.2.13.1 Drum OTP-TRUR-PD-11. at LT-09-201B.

$\sqrt{ }$ 4.2.13.2 Drum OTP-TRUR-TD-01 at LT-09-201C.

$\mathcal{J}$ 4.2.13.3 Drum 0TP-TRUR-PD-01 at LT-09-201A.

\begin{tabular}{||c|c|c|}
\hline \multirow{3}{*}{ PCS TRACKING TABLE FOR TRU/RWM LIFT TABLES } \\
\hline \multirow{2}{*}{ LIFT TABLE } & PIN & Registers \\
\cline { 2 - 3 } & N32 110-116 & N32 121 bit 12 \\
\hline LT-09-201A & N32 122-128 & N32 133 bit 12 \\
\hline$\sqrt{|c|}$ LT-09-201B & N32 134-140 & N32 145 bit 12 \\
\hline LT-09-201C & \\
\hline
\end{tabular}

4.3 TEST PREREQUISITES are complete and acceptable.

TD Initials/Date:

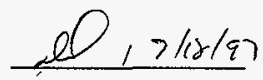


RUST FEDERAL SERVICES HANFORD

Project $W-026$. WRAP I

HNF-SD-W026-OTR-014-- - . -

Rev: 0

Operational Test - TRU/RWM Glovebox.

Page 27 of 79

ATTACHMENT 5 - TEST PLAN, TRU/RWM GLOVEBOX AUTOMATIC TEST CASE 1

\subsection{PROCEDURE}

5.1 REFERENCE procedure WRPI-OP-0710. GLOVEBOX WASTE SAMPLING:

5.1.1 PERFORM Section VII.D.1 "ATTACH STC TO GLOVEBOX", of operating procedure as required to attach empty STC-1 to glovebox.

5.1.1.1 REDLINE procedure as needed during performance.

5.1.1.2 VERIFY results of performance are acceptable.

TD Initials/Date:

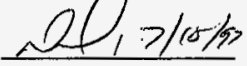

5.2 REFERENCE procedure WRP1-OP-0728. TRU/RWM GLOVEBOX OPERATION:

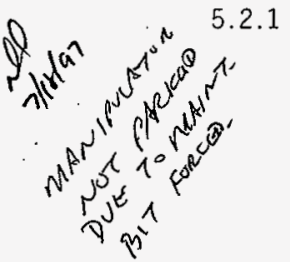

PERFORM Section VII.A, "INITIALIZE SYSTEM", of operating procedure as follows:

5.2.1.1 REDLINE procedure as needed during performance.

5.2.1.2 VERIFY results of performance are acceptable.

TD Initials/Date: $010,7 / 1 / 27$

5.2.2 PERFORM Section VII.B, "OPEN BAGLESS TRANSFER PORT (107-D0-07-401)", of operating procedure as fol lows:

5.2.2.1 REDLINE procedure as needed during performance.

5.2.2.2 VERIFY drum 0TP-TRUR-TD-01 connected to port 107-D0-07-401 and port OPEN.

5.2.2.3 VERIFY results of performance are acceptable.

TD Initials/Date:

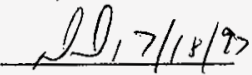


RUST FEDERAL SERVICES HANFORD

Project W-026. WRAP I

HNF-SD-W026-OTR-014- …...

Operationa? Test - TRU/RWM Glovebox.

Rev: 0

Page 28 of 79

ATTACHMENT 5 - TEST PLAN, TRU/RWM GLOVEBOX AUTOMATIC TEST CASE I

5.2.3 PERFORM Section VII.C, "REMOVE TRANSFER STAND FROM DRUM", of operating procedure as follows:

5.2.3.1 REDLINE procedure as needed during performance.

5.2.3.2 VERIFY transfer stand at glovebox sample station.

5.2.3.3 VERIFY results of performance are acceptable.

TO Initials/Date:

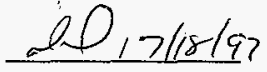

5.2.4 PERFORM Section VII.D, "REMOVE WASTE ITEMS FROM TRANSFER STAND FOR SORTING/SAMPLING", of operating procedure as follows:

5.2.4.1 REDLINE procedure as needed during performance.

5.2.4.2 VERIFY DMSS0341 indicates packets 0TP6-97-000001, OTP6-97-000002, and 0TP6-97-000003 in transfer drum prior to removal from transfer stand.

5.2:4.3 VERIFY DMS indicates packets OTP6-97-000001, 0TP6-97-000002, and 0TP6-97-000003 at TRU/RWM sample station after removal from transfer stand.

5.2.4.4 VERIFY results of performance are acceptable.

TD Initials/Date:

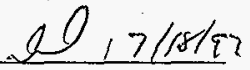

5.2.5 PERFORM Section VII.G, "OPEN COMPLIANT WASTE PORT (107-D0-07-402A)", of operating procedure as follows:

5.2.5.1 REDLINE procedure as needed during performance.

5.2.5.2 VERIFY drum OTP-TRUR-PD-11 connected to port 107-D0-07-402A and port OPEN.

5.2.5.3 VERIFY results of performance are acceptable.

To Initials/Date:

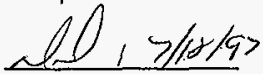


RUST FEDERAL SERVICES HANFORD

Project W-026. WRAP I -

HNF-SD-W026-0TR-014- - . ....

Operational Test - TRU/RWM Glovebox

Rev: 0

Page 29 of 79

\section{ATTACHMENT 5 - TEST PLAN, TRU/RWM GLOVEBOX AUTOMATIC TEST CASE 1}

5.2.6 PERFORM Section VII.I, "SORT WASTE ITEMS", of operating procedure as follows:

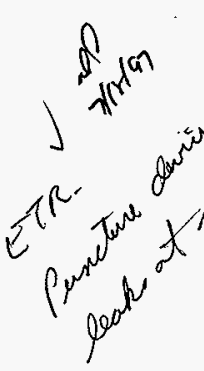

5.2.6.1 REDLINE procedure as needed during performance.

5.2.6.2 VERIFY barcode label 0TP6-97-000007 is attached to a new collection container and aerosol cans in packets 0TP6-97-000001 and 0TP6-97-000002 are drained into collection container.

$\sqrt{5.2 .6 .3}$ SCAN barcodes as directed by operating procedure.

5.2.6.4 ENTER packet information on DMSS0342 when directed

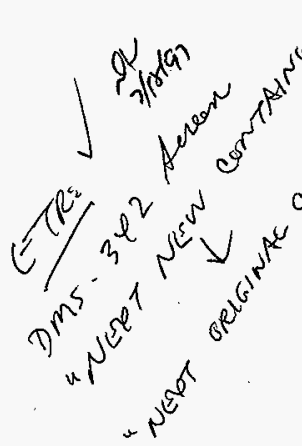
by operating procedure:

0TP6-97-000007

Container type: $C C$ Material Group: LQ Waste Description:

OTP-TRUR-PD-11 Compliant Waste: checked Waste Description: "Two empty saline solution aerosol cans".

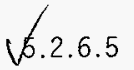

5.2.6.6 SCAN barcodes as directed by operating procedure.

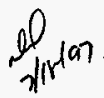

AB sore

"Bottle of Saline solution"

VERIFY barcode label 0TP6-97-000008 is attached to a new collection container and the aerosol can in packet 0TP6-97-000003 is drained into collection container. 
ATTACHMENT 5 - TEST PLAN, TRU/RWM GLOVEBOX AUTOMATIC TEST CASE 1.

$\sqrt{\text { 5.2.6.7 ENTER packet information on DMSSO342 when directed }}$ by operating procedure:

0TP6-97-000008

Container type: CC

Material Group: LQ

Waste Description: "Bottle of unknown 1 1iquid."

OTP-TRUR-PD-11

Compliant Waste: checked

Waste Description: add "Empty unmarked aerosol can".

$\sqrt{5.2 .6 .8}$ VERIFY a11 empty aerosol cans and packet material are placed in compliant loadout.drum.

$\sqrt{5.2 .6 .9}$ VERIFY OTP-TRUR-PD-1I packet information updated in DMS when directed by operating procedure.

5.2.6.10 ENTER compliant waste loadout drum information on DMSS0343 when directed by operating procedure:

- Description: PLASTIC/POLYURATHANE

- Volume Percent: 30

- Weight: 2

- Description: METAL/IRON/GALVANIZED/SHEET

- Volume Percent: 20

- Weight: 1

- Description: CLOTH/RAGS/NYLON/TAPE/ROPE

- Volume Percent: 50

- Weight: 2

5.2.6.11 VERIFY results of performance are acceptable.

TD Initials/Date:

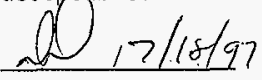

5.2.7 PERFORM Section VII.J, "SCREEN WASTE ITEMS", of operating procedure as follows: 
RUST FEDERAL SERVICES HANFORD

Project W-026. WRAP I

Operational Test - TRU/RWM Glovebox

HNF-SD-W026-0TR-014- - . .

Rev: 0

Page 31 of 79

ATTACHMENT 5 - TEST PLAN, TRU/RWM GLOVEBOX AUTOMATIC TEST CASE 1

5.2.7.1 REDLINE procedure as needed during performance.

$\checkmark$ 5.2.7.2 VERIFY field screening was performed on liquid in collection container 0TP6-97-000008.

$\checkmark$ 5.2.7.3 ENTER data for Screening ID 97-00001-01F on

DMSS0503 when directed by operating procedures:

- Sampling Method: "INS"

- SELECT Analysis "PHDP" from list.

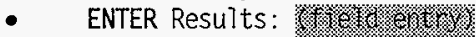

5.2.7.4 VERIFY sample analysis request was prepared in DMS for collection container 0TP6-97-000008. Sample 97-00001 is requested with ana7yses "AQLIQ". and "COND". One bottle is required for each analysis.

$\checkmark$ 5.2.7.5 VERIFY sample bottle barcode labels 97-00001-01L and 97-00001-02L are printed in the sample management office (DMSS0508) and the labels are placed on empty sample bottles.

$\sqrt{\text { 5.2.7.6. VERIFY empty sample bottles 97-00001-01L and }}$ 97-00001-02L are loaded into glovebox through the Consumable Materials port.

$\sqrt{5.2 .7 .7}$ VERIFY liquid from collection container 0TP6-97-000008 is collected in sample bottles 97-00001-01L and 97-00001-02L. Penjigr

$J$

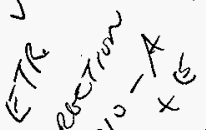

5.2.7.8 ENTER data for Sereening ID 97-00001-01f on 9019 DMSS0501 when directed by operating procedures:

$d \wedge_{x}^{0}$ lis $S_{2}$ Room Temperature: current temp $\int_{0} \int_{0} N_{0}$

$N^{2}+4$ $\bullet$ Comple Matrix:

Comments: "Unknown Tiquid"

TD Initials/Date:
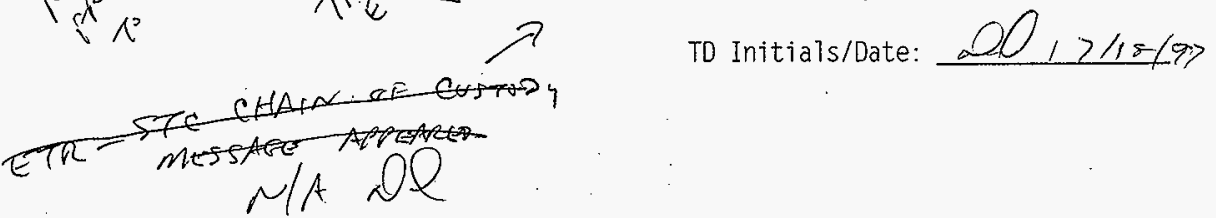
RUST FEDERAL SERVICES HANFORD

Project W-026. WRAP I

operationaT Test - TRU/RWM Glovebox.
HNF-SD-W026-0TR-014- - ...

Rev: 0

Page 32 of 79

\section{ATTACHMENT 5 - TEST PLAN. TRU/RWM GLOVEBOX AUTOMATIC TEST CASE 1}

5.3 REFERENCE procedure WRP1-OP-0710, GLOVEBOX WASTE SAMPLING:

5.3.1 PERFORM Section VII.G. "FINAL SAMPLE PREPARATION AND LOADOUT", of operating procedure.

5.3.1.1 REDLINE procedure as needed during performance.

5.3.1.2 VERIFY sample bottles 97-00001-01L, 97-00001-02L are placed in STC-1 and STC-1 removed from glovebox.

5.3.1.3 VERIFY results of performance are acceptable.

TD Initials/Date:

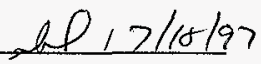

5.4 REFERENCE procedure WRP1-OP-0728. TRU/RWM GLOVEBOX OPERATION:

5.4.1 PERFORM Section VII.M. "ADD WASTE ITEMS TO TRANSFER STAND", of operating procedure as follows:

5.4.1.1 REDLINE procedure as needed during performance.

5.4.1.2 VERIFY DMSS0341 shows collection containers 0TP6-97-000007. 0TP6-97-000008 on transfer stand.

5.4.1.3 . VERIFY after "Refresh" screen DMSS0341 shows all items removed from glovebox sample location.

5.4.1.4 VERIFY results of performance are acceptable.

TO Initials/Date:

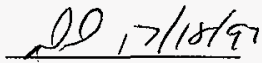


RUST FEDERAL SERVICES HANFORD

Project $W-026$. WRAP I

HNF-SD-W026-0TR-014- $\cdots$

Operational Test - TRU/RWM Glovebox.

Rev: 0

Page 33 of 79

ATTACHMENT 5 - TEST PLAN, TRU/RWM GLOVEBOX AUTOMATIC TEST CASE 1

5.4.2 PERFORM Section VII.N, "LOAD TRANSFER STAND INTO RWM DRUM", of operating procedure as follows:

5.4.2.1 REDLINE procedure as needed during performance.

5.4.2.2 VERIFY transfer stand is returned to transfer drum OTP-TRUR-TD-01.

5.4.2.3 VERIFY results of performance are acceptable.

To Initials/Date:

5.4.3 PERFORM Section VII.0, "CLOSE BAGLESS TRANSFER PORT

(107-D0-07-401)", of operating procedure as follows:

5.4.3.1 REDLINE procedure as needed during performance.

5.4.3.2 VERIFY resuits of performance are acceptable.

TD Initials/Date:

\section{NOTE}

Transfer drum 0TP-TRUR-TD-01 with collection containers 0TP6-97-000007 and OTP6-97-000008 will be used in Test Case 3.0. Storage should be nearby but out of work path.

5.4.4 REMOVE transfer drum OTP-TRUR-TD-01 with contents, from lift table.

5.4.4.1 STORE transfer drum OTP-TRUR-TD-01 unti1 needed.

5.5 RUN DMS Report Script.

To Initials/Date: $d \in, 7 / 18 / 9 ?$ 


\section{ATTACHMENT 6 - TEST PLAN, TRU/RWM GLOVEBOX AUTOMATIC TEST CASE 2}

\subsection{GENERAL DESCRIPTION}

1.1 Test case two will sort and sample Test Drum 2 packets. These operations wi17 be tested using procedures WRP1-OP-0710, Glovebox Waste Sampling and WRP1-OP-0728. TRU/RWM Glovebox Operation, thereby also performing the required procedure validation for sections performed. The sequence of events is as follows:

\subsubsection{STC OTP6-97-300002 is connected to the STC port.}

1.1.2 Transfer drum OTP-TRUR-TD-02 is moved to the TRU/RWM glovebox entry port, the lid is removed, and the transfer stand is moved to the sample station.

1.1.3 Packets 0TP6-97-000004, 0TP6-97-000005, and 0TP6-97-000006 are removed from the transfer stand.

1.1.4 Packet 0TP6-97-000004 is opened and barcodes are scanned to associate the original packet with new non-compliant item 0TP6-97-000009. The wrapping and compliant waste is placed in the compliant loadout drum.

1.1.5 Field screening is performed on non-compliant item 0TP6-97-000009.

1.1.6 Packet 0TP6-97-000005 is opened and separated into two "* non-compliant components. Bar codes are scanned to associate the original packet with new non-compliant items 0TP6-97-000010 and OTP6-97-000011. The wrapping and compliant waste from 0TP6-97-000005 are placed in the compliant loadout drum.

1.1.7 Physical component data for waste in the compliant waste loadout drum is updated in DMS.

1.1.8 Field screening is performed on non-compliant item 0TP6-97-000010.

1.1.9 Two sample analysis requests are prepared for packet OTP6-97-000010. Samples 97-00002 and 97-00003 are requested. both with analysis "SOLVENTS". One bottle is required for each sample. 


\section{ATTACHMENT 6 - TEST PLAN, TRU/RWM GLOVEBOX AUTOMATIC TEST CASE 2}

1.1.10 Field screening is performed on non-compliant item 0TP6-97-000011.

1.1.11 A sample analysis request is prepared for packet OTP6-97-000011. Sample 97-00004 is requested with analyses "OIL". One bottle is required for the analysis.

1.1.12 Field screening is performed on the liquid in packet 0TP6-97-000006.

1.1.13 Barcode labels 97-00002-01L, 97-00003-01L, and 97-00004-01L are printed and the labels are placed on empty sample bott?es.

1.1.14 Empty sample bott7es 97-00002-01L, 97-00003-01L, and 97-00004-01L are loaded into the TRU RWM glovebox through the Consumable Materials port.

1.1.15 Sample material from new packet OTP6-97-000010 is collected in sample bottle 97-00002-01L.

1.1.16 Sample material from new packet 0TP6-97-000010 is collected in sample bottie 97-00003-01L. The packet is then sealed.

1.1.17 Sample material from new packet 0TP6-97-000011 is collected in sample bottle 97-00004-01L. The packet is then sealed.

1.1.18 Sample bott7es 97-00002-01L, 97-00003-01L, and 97-00004-01L are placed in STC 0TP6-97-300002.

1.1.19 STC OTP6-97-300002 is removed from the TRU/RWM STC port and placed in transfer pig 0TP6-97-400001.

1.1.20 Packets 0TP6-97-000006, 0TP6-97-000009, 0TP6-97-000010, and 0TP6-97-000011 are placed on transfer stand and the transfer stand is returned to transfer drum TRU-OTP-TD-02.

1.1.21 Transfer drum TRU-0TP-TD-02 is closed and removed from the transfer drum port and from lift table LT-09-201C.

1.1.22 Run DMS report script. 


\section{ATTACHMENT 6 - TEST PLAN, TRU/RWM GLOVEBOX AUTOMATIC TEST CASE 2}

\subsection{SAFETY}

\subsection{Reference OTP Section 8, SAFETY.}

3.0 TOOLS, EQUIPMENT AND SUPPLIES

- Drum handling (manual) equipment or Pallet fork truck

- Spil1 Kit/Decontamination Equipment (oils/chemicals)

- Tape and rags

- Other equipment, material or protective clothing (as required)

\subsection{TEST PREREQUISITES}

4.1 VERIFY OTP Section 11.0. PREREQUISITES are complete.

4.2 SET initial conditions as follows:

$\checkmark 4.2 .155$ gallon. compliant waste loadout drum OTP-TRUR-PD-11 is connected to port 107-DO-07-402A and OPEN.

$\checkmark$ 4.2.2 Transfer drum OTP-TRUR-TD-02 is on lift table LT-09-201C and contains the following packets from the TRU glovebox:

$\checkmark$ 4.2.2.1 Packet 0TP6-97-000004 contains a lead brick (simulated), wrapped with two plastic bags and tape.

4.2.2.2 Packet 0TP6-97-000005 contains a 1 Titer bottle of unknown solvent and a 1 liter bottle of oil, taped together and wrapped with cloth and tape.

$\sqrt{4}$

4.2.2.3 Packet OTP6-97-000006 contains a partially full 1 liter bottle of acid (simulated).

$\checkmark$ 4:2.3 Empty sample transport container 0TP6-97-300002 (STC-2) is at glovebox ready to connect to STC port.

ro 4.2.4 Transfer pig 0TP6-97-400001 is located near TRU/RWM STC port.

$\checkmark$ 4.2.5 Three sequential packet barcode labels are available in glovebox. 


\section{ATTACHMENT 6 - TEST PLAN, TRU/RWM GLOVEBOX AUTOMATIC TEST CASE 2}

$\sqrt{ }$ 4.2.6 Required sampling supplies are available.

$\checkmark$ 4.2.7 Three empty, unlabeled, $250 \mathrm{ml}$ sample bottles are in the Sample Management office.

$\sqrt{4.2 .8}$ User with general operator (GENOP) privileges is logged on to DMS TRU Terminal (107-TE-12-104).

$\checkmark$ 4.2.9 User with chemist privileges is logged on to Sample Management Office DMS Terminal (109-TE-12-107).

$\sqrt{ }$ 4.2.10 Sample-label barcode printer in Sample Management office is loaded with blank labels and ready to print.

$\sqrt{4.2 .11}$ DMS database is unchanged from Test Case 1.0 completion.

$\sqrt{4.2 .12}$ UPDATE PCS Tracking Table for OTP-TRUR-TD- $0 / 2$ at $1 T-099-201 C$ as
follows: $7 / 2,197$

\begin{tabular}{|c|c|c|}
\hline \multicolumn{3}{|c|}{ PCS TRACKING TABLE FOR TRU/RWM LIFT TABLES } \\
\hline \multirow{2}{*}{ LIFT TABLE } & PIN $:$ & Registers \\
\cline { 2 - 3 } & N32 134-140 & N32 145 bit 12 \\
\hline \hline
\end{tabular}

4.3 TEST PREREQUISITES are complete and acceptabTe.

TD Initials/Date: 20,7 lol $19 ?$ 
RUST FEDERAL SERVICES HANFORD

Project W-026, WRAP I

Operational Test - TRU/RWM GTovebox.

HNF-SD-W026-OTR-014:- $\cdots$....

Rev: 0

Page 38 of 79

ATTACHMENT 6 - TEST PLAN, TRU/RWM GLOVEBOX AUTOMATIC TEST CASE 2

\subsection{PROCEDURE}

5.1 REFERENCE procedure WRP1-OP-0710. GLOVEBOX WASTE SAMPLING:

5.1.1 PERFORM Section VII.C. "LOAD EMPTY SAMPLE BOTTLES INTO . GLOVEBOX", of operating procedure as required to attach empty STC-2 to glovebox.

5.1.1.1 REDLINE procedure as needed during performance.

5.1.1.2 VERIFY resuits of performance are acceptable $7 / 21 / 97$

TD Initials/Date:

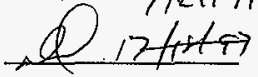

5.2 REFERENCE procedure WRP1-OP-0728, TRU/RWM GLOVEBOX OPERATION:

5.2.1 PERFORM Section VII.A. "INITIALIZE SYSTEM". of operating procedure as follows:

5.2.1.1 REDLINE procedure as needed during performance.

5.2.1.2 VERIFY results of performance are acceptable.

TD Initials/Date:

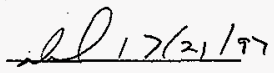

5.2.2 PERFORM Section VII.B, "OPEN BAGLESS TRANSFER PORT (107-D0-07-401)", of operating procedure as follows:

5.2.2.1 REDLINE procedure as needed during performance.

5.2.2.2 VERIFY drum OTP-TRUR-TD-02 connected to port 107-D0-07-401 and port OPEN.

5.2.2.3 VERIFY results of performance are acceptable.

TD Initials/Date:

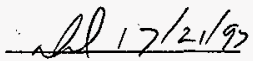




\section{ATTACHMENT 6 - TEST PLAN, TRU/RWM GLOVEBOX AUTOMATIC TEST CASE 2}

5.2.3 PERFORM Section VII.C, "REMOVE TRANSFER STAND FROM DRUM", of operating procedure as follows:

5.2.3.1 REDLINE procedure as needed during performance.

5.2.3.2 VERIFY transfer stand at glovebox sample station.

5.2.3.3 VERIFY results of performance are acceptable.

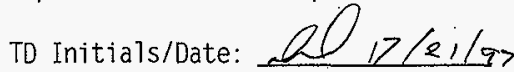

5.2.4 PERFORM Section VII.D. "REMOVE WASTE ITEMS FROM TRANSFER

STAND FOR SORTING/SAMPLING", of operating procedure aS follows:

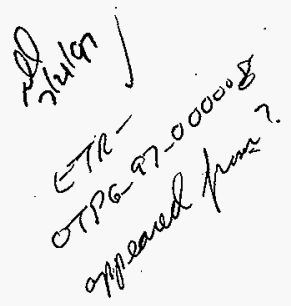

- 5.2.4.1 REDLINE procedure as needed during performance.

$\sqrt{5.2 .4 .2}$ VERIFY DMSS0341 indicates packets OTP6-97-000004, 0TP6-97-000005, and 0TP6-97-000006 in transfer drum prior to removal from transfer stand.

\section{5:2.4.3 VERIFY DMSS0341 indicates packets 0TP6-97-000004,} 0TP6-97-000005, and 0TP6-97-000006 at TRU/RWM sample station after removal from transfer stand.

5.2.4.4 VERIFY results of performance are acceptable.

To Initials/Date:

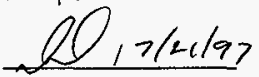

5.2.5 PERFORM Section VII.I. "SORT WASTE ITEMS", of operating procedure as follows:

5.2.5.1 REDLINE procedure as needed during performance.

$\sqrt{\text { 5.2.5.2 VERIFY packet OTP6-97-000004 is opened and barcodes }}$ are scanned to associate the original packet with new non-compliant item OTP6-97-000009. 


\section{ATTACHMENT 6 - TEST PLAN, TRU/RWM GLOVEBOX AUTOMATIC TEST CASE 2}

5.2.5.3 ENTER packet information on DMSS0342 when directed by operating procedure:

OTP6-97-000009

- Container type: PK

- Material Group: PB

- Waste Description: "Lead brick"

OTP-TRUR -PD-11

- Compliant Waste: checked

- Waste Description: add "Plastic sheet".

5.2.5.4 VERIFY packet OTP6-97-000005 is opened and separated into two non-compliant items. Barcodes are scanned to associate original packet with new non-compliant items 0TP6-97-000010 \&

OTP6-97-000011.

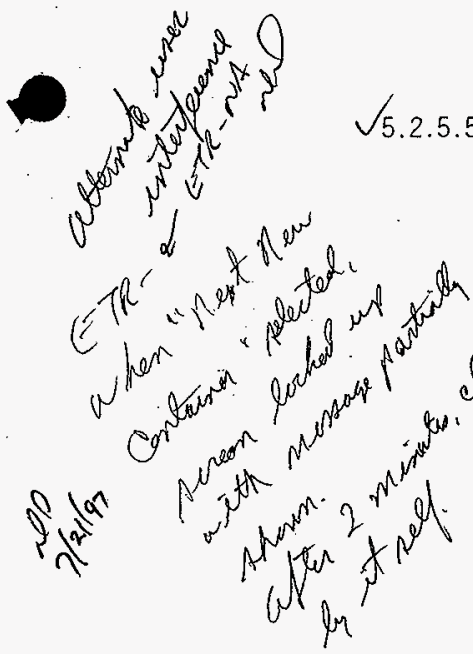

ENTER packet information on DMSS0342 when directed by operating procedure:

$\checkmark$ OTP6-97-000010

- Container type: PK

- Material Group: LQ

(.) Waste Description: "Unknown solvent"

6tp6-97-000011

- Container type: PK

- Material Group: OL

- Waste Description: "0i1"

OTP-TRUR-PD-11

- Comp liant Waste: checked

- Waste Description: add "cloth \& tape".

5.2.5.6 VERIFY wrapping and compliant waste from OTP6-97-000005 are placed in compliant loadout drum. 


\section{ATTACHMENT 6 - TEST PLAN, TRU/RWM GLOVEBOX AUTOMATIC TEST CASE 2}

5.2.5.7 UPDATE compliant waste loadout drum information on DMSS0343 when directed by operating procedure:

- Description: PLASTIC/POLYURATHANE

- Volume Percent: 30

- Weight: 3

- Description: METAL/IRON/GALVANIZED/ SHEET

- Volume Percent: 10

- Weight: 1

$$
\begin{aligned}
& \checkmark \quad \text { Description: CLOTH/RAGS/NYLON/TAPE/ ROPE } \\
& \text { Volume Percent: } 60 \\
& \text { Weight: } 4
\end{aligned}
$$

5.2.5.8 VERIFY results of performance are acceptable.

TD Initials/Date:

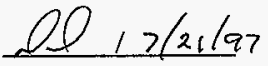

5.2.6 PERFORM Section VII.J, "SCREEN WASTE ITEMS", of operating procedure as follows:

5.2.6.1 REDLINE procedure as needed during performance.

$\checkmark$ 5.2.6.2 VERIFY field screening is performed on non-compliant itern 0TP6-97-000009.

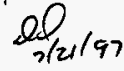

5.2.6.3 ENTER data for Screening ID 97-00002-01F on DMSS0503 when directed by operating procedures:

- Sampling Method: "INS"

- $\quad$ SELECT Analysis "LEAD" from list

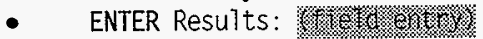
$\int \begin{aligned} & \text { 5.2.6.4 VERIFY field screening is performed on } \\ & \text { non-compliant item 0TP6-97-000010. }\end{aligned}$ 
ATTACHMENT 6 - TEST PLAN, TRU/RWM GLOVEBOX AUTOMATIC TEST CASE 2

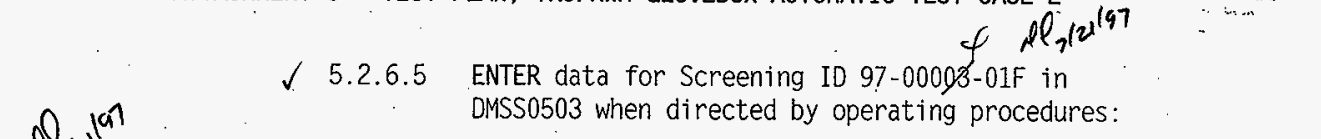

- Sampling Method: "INS"

- SELECT Analysis "CLOR" from list.

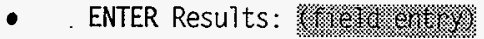

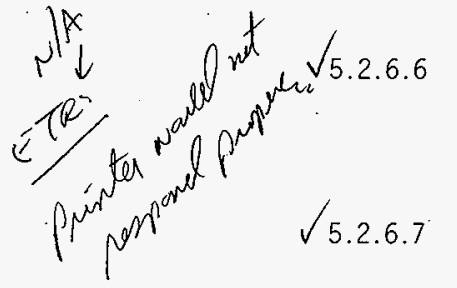

5.2 .6 .8

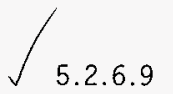

$\sqrt{5}$ 2.6.10 VERIFY field screening is performed on 1iquid in

packet OTP6-97-000006.
$\sqrt{5.2 .6 .11}$ ENTER data for Screening ID 97-00005-01F on packet OTP6-97-000006.

- Sampling Method: "INS"

- $\quad$ SELECT Analysis "PCBO" from list.

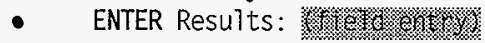

VERIFY two sample analysis requests are prepared in DMS for packet OTP6-97-000010. Sample 97-00002 and 97-00003 requested, both with analysis "SOLVENTS". One bottle is required for each sample.

VERIFY field screening is performed on non-compliant item OTP6-97-000011.

ENTER data for Screening I0 97-0000\%-01F on DMSS0503 when directed by operating procedures:

$$
5 \operatorname{lol}_{10} 197
$$

VERIFY sample analysis request is prepared in DMS for packet 0TP6-97-000011. SampTe 97-00004 is requested with analyses "OIL". One bott7e is required for the analysis. DMSS0503 when directed by operating procedures:

- Sampling Method: "INS."

- SELECT Anatysis "PHDP" from list.

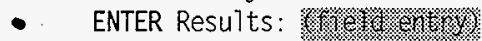

$\sqrt{5.2 .6 .12}$ VERIFY barcode 1abels 97-00002-01L, 97-00003-01L: and 97-00004-01L are printed and placed on empty sample bottles. 
ATTACHMENT 6 - TEST PLAN, TRU/RWM GLOVEBOX AUTOMATIC TEST CASE 2

5.2.6.13 VERIFY empty sample bottles 97-00002-01L. 97-00003-01L, and 97-00004-01L are loaded into glovebox through Consumable Materials port.

$\checkmark$ 5.2.6.14 VERIFY sample material from new packet 0TP6-97-000010 is collected in sample bottle 97-00002-01L.

$\sqrt{\text { 5.2.6.15 ENTER data for sample 97-00002-01L on DMSS0501 when }}$ directed by operating procedures:

- Room Temperature: (current temp)

- Sample Matrix: "L"

- Comments: "Unknown solvent"

$\int$ 5.2.6.16 VERIFY sample material from new packet 0TP6-97-000010 is collected in sample bottle 97-00003-01L. The packet is then sealed.

$\int$ 5.2.6.17 ENTER data for sample 97-00003-01L on DMSS0501 when directed by operating procedures:

- Room Temperature: (current temp).

- Sample Matrix: "L"

- Comments: "Unknown solvent"

$\sqrt{5.2 .6 .18}$

VERIFY sample material from new packet 0TP6-97-000011 is collected in sample bottle 97-00004-01L. The packet is then sealed.

$\sqrt{5.2 .6 .19}$ ENTER data for sample 97-00004-01L on DMSS0501 when directed by operating procedures:

- Room Temperature: (current temp)

- Sample Matrix: "L"

- Comments: "0il"

1 5.2.6.20 VERIFY results of performance are acceptable.

TD Initials/Date: 
RUST FEDERAL SERVICES HANFORD

Project W-026, WRAP I

Operational Test - TRU/RWM Glovebox
HNF-SD-W026-OTR-014-- - ....

Rev: 0

Page 44 of 79

ATTACHMENT 6 - TEST PLAN, TRU/RWM GLOVEBOX AUTOMATIC TEST CASE 2

5.3 REFERENCE procedure WRP1-OP-0710, GLOVEBOX WASTE SAMPLING:

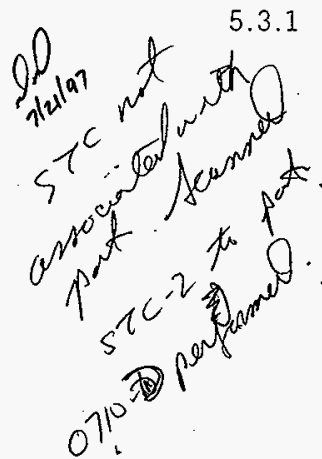

PERFORM Section VII.G. "FINAL SAMPLE PREPARATION AND LOADOUT", of operating procedure.

5.3.1.1 REDLINE procedure as needed during performance.

5.3.1.2 VERIFY sample bottles 97-00002-01L, 97-00002-02L, 97-00002-0,3L. 97-00002-0,4L, are placed in STC-2.

5.3 .1 .3 VERIFY screen DMSS0501 shows sample bottles in STC-2 after refresh.

5.3.1.4 VERIFY STC-2 removed from glovebox teansfer pIig OTP6-97-400001.

5.3.1.5 VERIFY resu7ts of performance are acceptable.

TD Initials/Date: $17 / 21 / 97$

5.4 REFERENCE procedure WRP1-OP-0728. TRU/RWWM GLOVEBOX OPERATION:

5.4.1 PERFORM Section VII.J. "TREATED WASTE ITEM LOADOUT", of $\oint_{1201}$ operating procedure as follows:

5.4.1.1

REDLINE procedure as needed during performance.

Nk 5.4.1.2 VERIFY results of performance are acceptable.

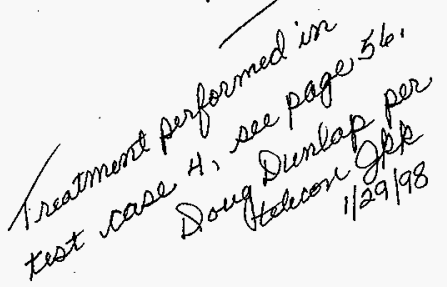

TO Initials/Date: 


\section{ATTACHMENT 6 - TEST PLAN, TRU/RWM GLOVEBOX AUTOMATIC TEST CASE 2}

5.4.2 PERFORM Section VII.M. "ADD WASTE ITEMS TO TRANSFER STAND", of operating procedure as follows:

5.4.2.1 REDLINE procedure as needed during performance.

5.4.2.2 VERIFY DMSS0341 shows packets 0TP6-97-000006, 0TP6-97-000009, OTP6-97-000010, and 0TP6-97-000011 on transfer stand.

5.4.2.3 VERIFY results of performance are acceptable.

TO Initials/Date:

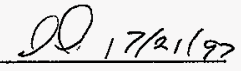

5.4.3 PERFORM Section VII.N, "LOAD TRANSFER STAND INTO RWM DRUM", of operating procedure as follows:

5.4.3.1 REDLINE procedure as needed during performance.

$\int$ 5.4.3.2 VERIFY transfer stand is returned to transfer drum transfer drum OTP-TRUR-TD-02.

5.4.3.3 VERIFY results of performance are acceptable.

TD Initials/Date:

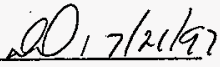

5.4.4 PERFORM Section VII.0, "CLOSE BAGLESS TRANSFER PORT (107-D0-07-401)". of operating procedure as follows:

5.4.4.1 REDLINE procedure as needed during performance.

5.4.4.2 Transfer drum OTP-TRUR-TD-02 is closed and removed from the transfer drum port.

5.4.4.3 VERIFY results of performance are acceptable.

To Initials/Date:

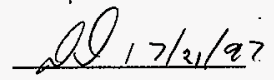


RUST FEDERAL SERVICES HANFORD

Project W-026. WRAP I

Operational Test - TRU/RWM Glovebox.

HNF-SD-W026-0TR-014- - .....

Rev: 0

Page 46 of 79

ATTACHMENT 6 - TEST PLAN, TRU/RWM GLOVEBOX AUTOMATIC TEST CASE 2

\section{NOTE}

Transfer drum OTP-TRUR-TD-02 with packets 0TP6-97-000006, OTP6-97-000009. 0TP6-97-000010, and 0TP6-97-000011 wi 11 be used in Test Case 4. Storage should be nearby but out of work path.

5.4.5 REMOVE transfer drum OTP-TRUR-TD-02 with contents, from lift table.

$\checkmark \quad$ 5.4.5.1 STORE transfer drum OTP-TRUR-TD-02 until needed.

5.4.6 RUN DMS Report Script.

To Initials/Date: a $1 / 2 / 219)$ 
RUST FEDERAL SERVICES HANFORD Project $W-026$, WRAP I

ATTACHMENT 7 - TEST PLAN, TRU/RWM GLOVEBOX AUTOMATIC TEST CASE 3.

\subsection{GENERAL DESCRIPTION}

1.1 Test Case three wi11 treat items in Transfer Drum 1 Collection Containers. These operations wil1 be tested using procedures WRP1-OP-0728, TRU/RWM Glovebox Operation and WRP1-0P-0711, Waste Treatment, thereby also performing the required procedure validation for sections performed. The sequence of events is as follows:

$$
55 \text { hilzalan one TRis }
$$

1.1.1 Empty 85-galion Entry/exit-drum OTP-TRUR-PD-01 is connected to TRU/RWM giovebox treated waste loadout port and lid is removed.

1.1.2 Transfer drum OTP-TRUR-TD-0I is connected to the TRU/RWM

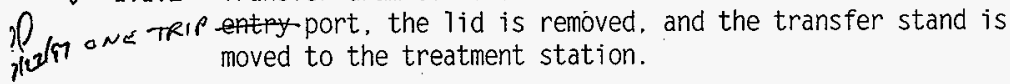

/ 1.1.3 Collection containers 0TP6-97-000007 and 0TP6-97-000008 are removed from the transfer stand.

1.1.4 Treatment container barcode label 0TP6-97-900001 is applied to collection container 0TP6-97-000007. The barcode labels are scanned to associate the treatment container with the collection container.

1.1.5 Contents of treatment container 0TP6-97-900001 are treated.

1.1.6 Treatment container 0TP6-97-900001 and contents are placed in treated waste loadout drum 0TP-TRUR-PD-01.

1.1.7 Treatment container barcode Tabel 0TP6-97-900002 is applied to collection container 0TP6-97-000008. The barcode labels are scanned to associate the treatment container with the collection container:

1.1.8 Contents of treatment container 0TP6-97-900002 are treated.

1.1.9 Treatment Container 0TP6-97-900002 and contents are placed in treated waste loadout drum OTP-TRUR-PD-01.

1.1.10 Physical component data for waste in the treated waste loadout drum is entered into DMS. 
RUST FEDERAL SERVICES HANFORD

Project $W-026$, WRAP I

operationa? Test - TRU/RWM Glovebox.

HNF-SD-W026-OTR-014.- - ......

Rev: 0

Page 48 of 79

ATTACHMENT 7 - TEST PLAN, TRU/RWM GLOVEBOX AUTOMATIC TEST CASE 3

1.1.11 Empty transfer stand is returned to transfer drum OTP-TRUR-TD-01. Transfer drum is closed and removed from transfer drum port and from 1ift table LT-09-201C.

\subsection{SAFETY}

\subsection{Reference OTP Section 8, SAFETY.}

3.0 TOOLS, EQUIPMENT AND SUPPLIES

- Drum handling (manual) equipment or Pallet fork truck

- Spill Kit/Decontamination Equipment (oils/chemicals)

- Tape and rags

- Other equipment, material or protective clothing (as required)

\subsection{TEST PREREQUISITES}

4.1 VERIFY OTP Section 11.0. PREREQUISITES are complete.

4.2 SET initial conditions as follows:

$\checkmark$ 4.2.1 55 gallon, compliant waste loadout drum OTP-TRUR-PD-11 is connected to port 107-D0-07-402A and OPEN.

4.2.2 Transfer drum OTP-TRUR-TD-01 is on lift table LT-09-201C and contains the following collection containers from Test Case 1.0:

- $0 T P 6-97-000007$

- 0 0TP6-97-800008

$$
5 \text { ed } 12 \times 197
$$

$\sqrt{4.2 .3}$ Empty 85 gallon drum OTP-TRUR-PD-01 is at lift table LT-09-201A.

$\sqrt{4.2 .4}$ Two sequential treatment container barcode labels are available in the glovebox.

$\checkmark$ 4.2.5 Required treatment supplies are available.

$\checkmark$ 4.2.6 User with general operator (GENOP) privileges is logged on to DMS TRU Terminal (107-TE-12-104) 
RUST FEDERAL: SERVICES HANFORD

Project W-026. WRAP I

Operational Test - TRU/RWM Glovebox.
HNF-SD-W026-0TR-014-

Rev: 0

Page 49 of 79

ATTACHMENT 7 - TEST PLAN, TRU/RWM GLOVEBOX AUTOMATIC TEST CASE 3

$\checkmark$ 4.2.7 Test scripts have been run to update DMS database as follows:

4.2.7.1 Load data for Treatment Worksheets OTPWRK01. OTPWRK02, OTPWRK03, OTPWRK05 and OTPWRK05.

4.2.7.2 Change Tocation of transfer drums OTP-TRUR-TD-01 and OTP-TRUR-TD-02 to RWM-CRSL.

4.2.7.3 Change location of STC OTP6-97-300001 and transfer pig 0TP6-97-400001 to SAMPLE_MGT.

4.2.7.4 Set BOT_RETURN_DT for sample bottles 97-00002-01L. 97-00003-01L, and 97-00004-01L to the system date/time.

4.2.7.5 Run DMS report script.

$\checkmark$ 4.2.8 PCS database unchanged form Test Case 2.0.

4.2.9 UPDATE PCS Tracking Table for Drum OTP-TRUR-TD-01 at LT-09-201C as follows:

\begin{tabular}{||c|c|c|}
\hline \multicolumn{3}{|c|}{ PCS TRACKING TABLE FOR TRU/RWM LIFT TABLES } \\
\hline \multirow{2}{*}{ LIFT TABLE } & \multicolumn{2}{|c|}{ Registers } \\
\cline { 2 - 3 } & PIN & Drum Present $(=1)$ \\
\hline LT-09-201C & N32 134-140 & N32 145 bit 12 \\
\hline
\end{tabular}

4.3 TEST PREREQUISITES are complete and acceptable.

To Initials/Date: $2(1 / 22 / 97$ 
RUST FEDERAL SERVICES HANFORD

Project W-026. WRAP I

Operational Test - TRU/RWM Glovebox.

HNF-SD-W026-0TR-014-_ - .....

Rev: 0

Page 50 of 79

ATTACHMENT 7 - TEST PLAN, TRU/RWM GLOVEBOX AUTOMATIC TEST CASE 3

\subsection{PROCEDURE}

5.1 REFERENCE procedure WRP1-OP-0728, TRU/RWM GLOVEBOX OPERATION:

5.1.1 PERFORM Section VII.A. "INITIALIZE SYSTEM", of operating procedure as follows:

5.1.1.1 REDLINE procedure as needed during performance.

5.1.1.2 VERIFY results of performance are acceptable.

TD Initials/Date:

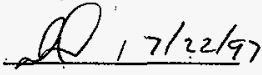

5.1.2 PERFORM Section VII.B, "OPEN BAGLESS TRANSFER PORT (107-D0-07-401)", of operating procedure as follows:

5.1.2.1 REDLINE procedure as needed during performance.

5.1.2.2 VERIFY drum OTP-TRUR-TO-01 connected to port 107-00-07-401 and port OPEN.

5.1.2.3 VERIFY results of performance are acceptable.

TD Initials/Date: $20,7 / 22 / 97$

5.1.3 PERFORM Section VII.C, "REMOVE TRANSFER STAND FROM DRUM", of operating procedure as follows:

5.1.3.1 REDLINE procedure as needed during performance.

$\sqrt{5.1 .3 .2}$ VERIFY transfer stand at glovebox treatment station.

5.1.3.3 VERIFY results of performance are acceptable.

TD Initials/Date:

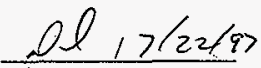


ATTACHMENT 7 - TEST PLAN, TRU/RWM GLOVEBOX AUTOMATIC TEST CASE 3

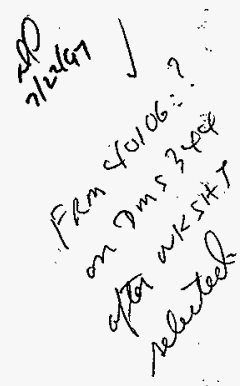

5.1.4 PERFORM Section VII.E. "REMOVE WASTE ITEMS FROM TRANSFER

STAND FOR TREATMENT", of operating procedure as follows:

5.1.4.1 REDLINE procedure as needed during performance.

5.1.4.2 REMOVE items specified on worksheet OTPWRK01.

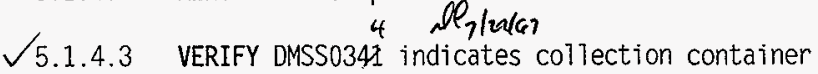
0TP6-97-000007 in transfer drum prior to removal from transfer stand.

5.1.4.4 VERIFY DMSSO34t indicates collection container 0TP6-97-000007 at TRU/RWM treatment station after removal from transfer stand.

$\sqrt{5.1 .4 .5}$ VERIFY results of performance are acceptable.

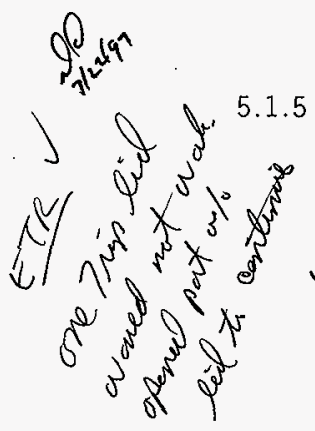

TD Initials/Date:

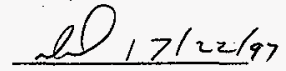

5.1.5 PERFORM Section VII.H, "OPEN NON-COMPLIANT WASTE PORT

(107-D0-07-402B)". of operating procedure as follows:

5.1.5.1 REDLINE procedure as needed during performance.

$\sqrt{5.1 .5 .2}$ VERIFY drum OTP-TRUR-PD-01 connected to port 107-DO-07-402B and port OPEN.

5.1.5.3 VERIFY results of performance are acceptable.

TD Initials/Date: $2, x_{24}+97$ 
RUST FEDERAL SERVICES HANFORD

Project $W-026$. WRAP I

Operational Test - TRU/RWM Glovebox.
HNF-SD-W026-0TR-014-2 ….

Rev: 0

Page 52 of 79

ATTACHMENT 7 - TEST PLAN, TRU/RWM GLOVEBOX AUTOMATIC TEST CASE 3

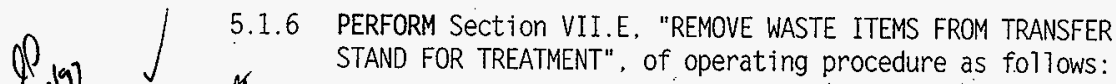

5.1.6.1 REOLINE procedure as needed during performance.

5.1.6.2 REMOVE items specifjed on worksheet OTPWRK02.

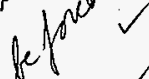<smiles>C1CC1CN1CC1</smiles>

5.1.6.3 * let/r2ta?

VERIFY DMSS0341 indicates collection container 0TP6-97-000008 in transfer drum prior to removal from transfer stand.

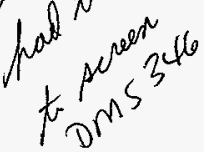

5.1.6.4

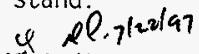

VERIFY DMSS0341 indicates collection container 0TP6-97-000008 at TRU/RWM treatment station after removal from transfer stand.

5.1.6.5 VERIFY resuits of performance are acceptable.

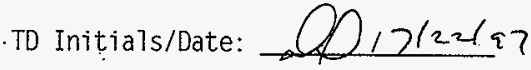

5.1.7 PERFORM Section VII.K, "TREAT NON-COMPLIANT WASTE ITEMS", of operating procedure as follows:

5.1.7.1 REDLINE procedure as needed during performance.

5.1.7.2 TREAT items specified on worksheet OTPWRK01. 
ATTACHMENT 7 - TEST PLAN, TRU/RWM GLOVEBOX AUTOMATIC TEST CASE 3

NOTE

Section VII.K. "TREAT NON-COMPLIANT WASTE ITEMS" of WRP1-OP-0728 directs performance of WRP1-OP-0711, Waste Treatment. Completion of this step wil1 validate WRP1-OP-0711.

5.1.7.3 PERFORM procedure WRP1-OP-0711. Waste Treatment when directed by glovebox operating procedure.

$\sqrt{5.1 .7 .4}$ VERIFY treatment container barcode label -OTP6-97-900001 is applied to collection container 0TP6-97-000007. The barcode labels are scanned to associate the treatment container with the collection container.

$\sqrt{5.1 .7 .5}$ VERIFY contents of treatment container 0TP6-97-900001 are treated.

$\checkmark$ 5.1.7.6 ENTER data for Treatment Container 0TP6-97-900001 on DMSS0346 when directed by operating procedures:

- Waste Description: $1 L$ bottle of saline

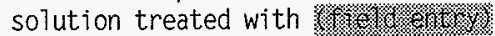

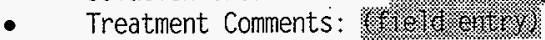

- $\quad$ "Compliant" box for 0TP6-97-000007 not checked

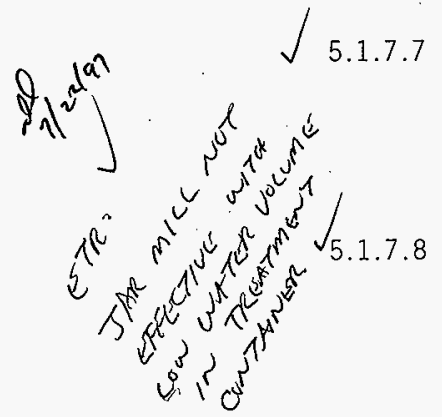

VERIFY treatment container barcode label 0TP6-97-900002 is applied to collection container 0TP6-97-000008 and barcode labels are scanned to associate the treatment container with the collection container.

VERIFY contents of treatment container 0TP6-97-900002 are treated. 
RUST FEDERAL SERVICES HANFORD Project. W-026. WRAP I .

HNF-SD-W026-OTR-014- $\cdots$ Operationa? Test - TRU/RWM GTovebox.

Rev: 0

Page 54 of 79

ATTACHMENT 7 - TEST PLAN, TRU/RWM GLOVEBOX AUTOMATIC TEST CASE 3

$\sqrt{5.1 .7 .9}$ ENTER data for Treatment Container 0TP6-97-900002 on DMSS0346 when directed by operating procedures:

- Waste Description: IL bottle of acetic acid treated with 1 (1)

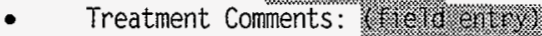

- "Compliant" box for 0TP6-97-000008 not checked

5.1.7.10 VERIFY results of performance are acceptable.

TD Initials/Date:

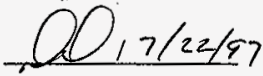

5.1.8 PERFORM Section VII.L. "TREATEO WASTE ITEM LOADOUT", of operating procedure as follows:

5.1.8.1 REDLINE procedure as needed during performance.

5.1.8.2 VERIFY Treatment container 0TP6-97-900001 and contents are placed in non-compliant waste loadout drum OTP-TRUR-PD-01.

5.1.8.3 VERIFY Treatment container OTP6-97-900002 and contents are placed in non-compliant waste loadout drum OTP-TRUR-PD-01.

5.1.8.4 ENTER treated waste drum information on DMSS0348 PHYSCOMP screen when directed by operating procedure:

- Description: ABSORBED AQUUEOUS

- Volume Percent: 95

- Weight: 4

- Description: PLASTIC/POLYURATHANE

- Volume Percent: 5

- Weight: 1

5.1.8.5 VERIFY results of performance are acceptable.

TO Initials/Date: SPA / $/ 2 / \tau_{2} / 97$ 


\section{ATTACHMENT 7 - TEST PLAN, TRU/RWM GLOVEBOX AUTOMATIC TEST CASE 3}

5.1.9 PERFORM Section VII.N, "LOAD TRANSFER STAND INTO RWM ORUM", of operating procedure as follows:

5.1.9.1 REDLINE procedure as needed during performance.

5.1.9.2 VERIFY empty transfer stand is returned to transfer drum OTP-TRUR-TD-01.

5.1.9.3. VERIFY results of performance are acceptable.

TD Initials/Date: SpA $1 / 7 / 22 / 92$

5.1.10 PERFORM Section VII.O. "CLOSE BAGLESS TRANSFER PORT

(107-D0-07-401)", of operating procedure as follows:

5.1.10.1 REDLINE procedure as needed during performance.

5.1.10.2 VERIFY transfer drum is closed and lowered from transfer port.

5.1.10.3 VERIFY results of performance are acceptable.

TD Initials/Date: SPA / $/ 2 z / 97$

\section{NOTE}

Transfer drum 0TP-TRUR-TD-01 wi11 not be used for duration of test.

5.2 REMOVE transfer drum OTP-TRUR-TD-01 from lift table LT-09-201C and STORE for disposal at end of testing.

TD Initials/Date: SPA / 7/2z/97

- TEST SCRIPT RUN 


\section{ATTACHMENT 8 - TEST PLAN, TRU/RWM GLOVEBOX AUTOMATIC TEST CASE 4}

\subsection{GENERAL DESCRIPTION}

1.1 Test Case four will treat items in Transfer Drum 2 packets and samples in STC-2 which were taken from these items. These operations wi 71 be tested using procedures WRP1-OP-0710, Glovebox Waste Sampling, WRP1-OP-0728, TRU/RWM Glovebox Operation and WRP1-OP-0711, Waste Treatment, thereby also performing the required procedure validation for sections performed. The sequence of events is as follows:

1.1.1 STC OTP6-97-300002 is connected to the TRU/RWM glovebox STC port

1.1.2 Transfer drum OTP-TRUR-TD-02 is connected to the TRU/RWM entry port, the lid is removed, and the transfer stand is moved to the treatment station.

1.1.3 The two treatment containers are loaded into the glovebox through the Consumable Materials:port.

1.1.4 Worksheet OTPWRK03 is selected and Packet 0TP6-97-000010 is removed from the transfer stand.

1.1.5 Sample bott7es 97-00002-01L and 97-00003-01L are removed from STC 0TP6-97-300002.

1.1.6 Treatment container barcode label 0TP6-97-900003 is. applied to an empty treatment container. The contents of packet 0TP6-97-000010 and sample bottles 97-00002-01L and 97-00003-01L are added to the treatment container. The packet bottle and sample bottles are added to compliant waste loadout drum OTP-TRUR-PD-11.

1.1.7 The contents of treatment container 0TP6-97-900003 are treated.

1.1.8 Treatment Container OTP6-97-900003 and contents are placed in the treated waste Toadout drum OTP-TRUR-PD-01.

1.1.9 Worksheet OTPWRK04 is selected and Packet 0TP6-97-000011 is removed from the transfer stand. 


\section{ATTACHMENT 8 - TEST PLAN, TRU/RWM GLOVEBOX AUTOMATIC TEST CASE 4}

1.1.10 Sample bottle 97-00004-01L is removed from STC 0TP6-97-300002.

1.1.11 Treatment container barcode label 0TP6-97-900004 is applied to an empty treatment container. The contents of packet 0TP6-97-000011 and sample bottle 97-00004-01L are added to the treatment container. The packet bottle and sample bottle are added to compliant waste loadout drum OTP-TRUR-PD-11.

1.1.12 The contents of treatment container 0TP6-97-900004 are treated.

1.1.13 Treatment container 0TP6-97-900004 and contents are placed in the treated waste loadout drum OTP-TRUR-PD-01.

1.1.14 Worksheet OTPWRK05 is selected and Packet 0TP6-97-000006 is removed from the transfer stand.

1.1.15 Treatment container barcode labei 0TP6-97-900005 is applied to collection container 0TP6-97-000006. The barcode labels are scanned to associate the treatment container with the collection container.

1.1.16 The contents of treatment container 0TP6-97-900005 are treated.

1.1.17 Treatment Container 0TP6-97-900005 and contents are placed in treated waste loadout drum 0TP-TRUR-PD-01.

1.1.18 The transfer stand with packet 0TP6-97-000009 is returned to transfer drum OTP-TRUR-TD-02. The transfer drum is closed and removed from the transfer drum port and from lift table LT-09-201C.

1.1.19 Empty STC 0TP6-97-300002 is removed from TRU/RWM. glovebox STC port.

1.1.20 Physical component data for waste in the treated waste drum is updated in DMS. 
RUST FEDERAL SERVICES HANFORD Project W-026, WRAP. I Operational Test - TRU/RWM Glovebox.
HNF-SD-W026-OTR-014--

Rev: 0

Page 58 of 79

\section{ATTACHMENT 8 - TEST PLAN, TRU/RWM GLOVEBOX AUTOMATIC TEST CASE 4}

1.1.21 Hazardous chemical component data for waste in the treated waste drum is entered into DMS.

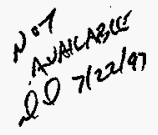

1.1.22 Filler is added to Treated waste drum OTP-FRUR-PQ-01 and the drum is prepared for toadout.

1.1.23 Treated waste drum OTP-TRUR-PO-01 is closed and removed from the treated waste loadout port. The drum is surveyed and a seal attached, then removed from lift table LT-09-201A.

1.1.24 Physical component data for waste in the compliant waste loadout drum is updated in DMS.

1.1.25 Compliant waste loadout drum OTP-TRUR-PD-11 is prepared for loadout.

1.1.26 Compliant waste loadout drum OTP-TRUR-PD-11 is closed and removed from the compliant waste loadout port. The drum is surveyed and a seal attached, then removed from 7 ift table LT-09-201B.

\subsubsection{Print DMS database.}

\subsection{SAFETY}

\subsection{Reference OTP Section 8. SAFETY.}

\subsection{TOOLS, EQUIPMENT AND SUPPLIES}

- Drum handling (manuai) equipment or Pallet fork truck

- $\quad$ Spil1 Kit/Decontamination Equipment (oils/chemica7s)

- Tape and rags

- Other equipment, material or protective clothing (as required) 
RUST FEDERAL SERVICES HANFORD Project W-026. WRAP I Operational Test - TRU/RWM GTovebox.

\section{ATTACHMENT 8 - TEST PLAN, TRU/RWM GLOVEBOX AUTOMATIC TEST CASE 4}

\subsection{TEST PREREQUISITES}

4.1 VERIFY OTP Section 11.0, PREREQUISITES are complete.

4.2 SET initial conditions as follows:

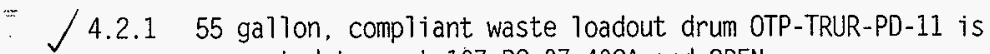
connected to port 107-D0-07-402A and OPEN.

s ale tzala?

4.2.2 85 gallon, non-compliant waste loadout drum 0TP-TRUR-PD-01 connected to port 107-D0-07-402B and OPEN.

$\checkmark$ 4.2.3 Transfer drum OTP-TRUR-TD-02 is on lift table LT-09-201C and contains the following packets from Test Case 2.0:

- 0TP6-97-000006.

- 0 TP6-97-000009

- 0 0TP6-97-000010

- 0TP6-97-000011

$\sqrt{\text { 4.2.4 Sample Transport Container 0TP6-97-300002 (STC-2) is at the }}$ Sample Management office and contains the following samples from Test Case 2.0:

- 97-00002-01L

- 97-00003-01L

- 97-00004-01L

$\sqrt{4.2 .5}$ Two, one liter treatment containers are ready for loading into the glovebox through the Consumable Materials port.

$\sqrt{4.2 .6}$ Three sequential treatment container barcode labels are available in the glovebox.

$\checkmark$ 4.2.7 Required sampling supplies are available.

$\sqrt{4.2 .8}$ User with general operator (GENOP) privileges is logged on to DMS TRU Terminal (107-TE-12-104).

$\sqrt{4.2 .9}$ DMS database is unchanged from Test Case 3.0. 
RUST FEDERAL SERVICES HANFORD Project W-026, WRAP I

Operational Test - TRU/RWM Glovebox.
HNF-SD-W026-0TR-014- - .....

Rev: 0

Page 60 of 79

ATTACHMENT 8 - TEST PLAN, TRU/RWM GLOVEBOX AUTOMATIC TEST CASE 4

4.2.10 PCS database is unchanged from Test Case 3.0.

4.2.11 UPDATE PCS Tracking Table for Drum OTP-TRUR-TD-02 at

LT-09-201C as follows:

\begin{tabular}{|c|c|c|}
\hline \multicolumn{3}{|c|}{ PCS TRACKING TABLE FOR TRU/RWM LIFT TABLES } \\
\hline \multirow{2}{*}{ LIFT TABLE } & \multicolumn{2}{|c|}{ Registers } \\
\cline { 2 - 3 } & PIN & Drum Present $(=1)$ \\
\hline \hline LT.09-201C & N32 134-140 & N32 145 bit 12 \\
\hline
\end{tabular}

4.3 TEST PREREQUISITES are complete and acceptable.

TD Initials/Date: $00,2 / 22 / \Omega 7$ 
RUST FEDERAL SERVICES HANFORD Project W-026, WRAP I a

Operational Test - TRU/RWM GTovebox
HNF-SD-W026-OTR-0.1A= …... Rev: 0

Page 61 of 79

ATTACHMENT 8 - TEST PLAN, TRU/RWM GLOVEBOX AUTOMATIC TEST CASE 4

\subsection{PROCEDURE}

5.1 REFERENCE procedure WRP1-OP-0710, GLOVEBOX WASTE SAMPLING:

5.1.1 PERFORM Section VII.D.I "ATTACH STC TO GLOVEBOX", of operating procedure as required to attach STC-2 to glovebox.

5.1.1.1 REDLINE procedure as needed during performance.

5.1.1.2 VERIFY results of performance are acceptable.

TD Initials/Date:

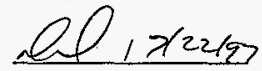

5.1.2 PERFORM Section VII.D.2, "OPEN STC", of operating procedure as required to open STC-2.

5.1.2.1 REDLINE procedure as needed during performance.

5.1.2.2 VERIFY results of performance are acceptable.

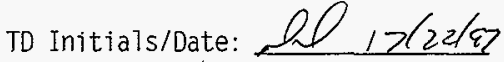

5.2 REFERENCE procedure WRP1-OP-0728, TRU/RWM GLOVEBOX OPERATION:

5.2.1 PERFORM Section VII.A, "INITIALIZE SYSTEM", of operating procedure as follows:

5.2.1.1 REDLINE procedure as needed during performance.

5.2.1.2 VERIFY results of performance are acceptable.

TO Initials/Date:

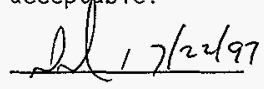


RUST FEDERAL SERVICES HANFORD

Project W-026. WRAP I

Operational Test - TRU/RWM Glovebox.
HNF-SD-W026-0TR-014-- - …2.

Rev: 0

Page 62 of 79

ATTACHMENT 8 - TEST PLAN, TRU/RWM GLOVEBOX AUTOMATIC TEST CASE 4

5.2.2 PERFORM Section VII.B, "OPEN BAGLESS TRANSFER PORT (107-D0-07-401)", of operating procedure as follows:

5.2.2.1 REDLINE procedure as needed during performance.

5.2.2.2 VERIFY drum OTP-TRUR-TD-02 connected to port 107-D0-07-401 and port OPEN.

5.2.2.3 VERIFY results of performance are acceptable.

TD Initials/Date:

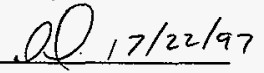

5.2.3 PERFORM Section VII.C. "REMOVE TRANSFER STAND FROM DRUM", of operating procedure as follows:

5.2.3.1 REDLINE procedure as needed during performance.

5.2.3.2 VERIFY transfer stand at glovebox treatment station.

5.2.3.3 VERIFY results of performance are acceptable.

TD Initials/Date:

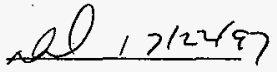

5.2.4 PERFORM Section VII.E, "REMOVE WASTE ITEMS FROM TRANSFER

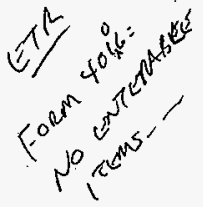
STAND FOR TREATMENT", of operating procedure as follows:

5.2.4.1 REDLINE procedure as needed during performance.

$\checkmark$ 5.2.4.2 REMOVE items specified on worksheet OTPWRK03.

5.2.4.3 VERIFY DMS screen DMSS0344, worksheet OTPWRK03 indicates packet 0TP6-97-000010 in transfer drum prior to removal from transfer stand. 


\section{ATTACHMENT 8 - TEST PLAN, TRU/RWM GLOVEBOX AUTOMATIC TEST CASE 4}

\section{NOTE}

Packet 0TP6-97-000009 is on a different worksheet \& should not show on worksheet OTPWRK02.

5.2.4.4 VERIFY DMS worksheet OTPWRK02 does not indicate packet 0TP6-97-000009.

5.2.4.5 VERIFY packet OTP6-97-000010 removed from transfer stand.

5.2.4.6 VERIFY after "refresh" of DMS screen DMSS0344, Worksheet OTPWRK03 that OMS indicates:

$\checkmark$ - Packet OTP6-97-000010 at glovebox treatment station.

$\checkmark$ - "Itern Containers to be Retrieved" is "0" for packets and "1" for samples.

5.2.4.7 VERIFY results of performance are acceptable.

TD Initials/Date:

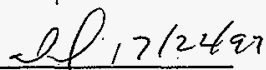

5.2.5 PERFORM Section VII.F, "REMOVE SAMPLE BOTTLES FROM STC FOR TREATMENT", of operating procedure as required to unToad samples from STC, as follows:

5.2.5.1 REDLINE procedure as needed during performance.

$\sqrt{5.2 .5 .2}$ VERIFY DMS screen DMSS0344, worksheet OTPWRK03, indicates sample bottles 97-00002-01L and 97-00003-01L in STC-2 prior to removal from STC.

$\sqrt{5.2 .5 .3}$ VERIFY samples 97-00002-01L and 97-00003-01L, are removed from STC-2. 
RUST FEDERAL SERVICES HANFORD Project W-026, WRAP I

Operational Test - TRU/RWM Glovebox.
HNF-SD-W026-0TR-014- $-\cdots$.

Rev: 0

Page 64 of 79

ATTACHMENT 8 - TEST PLAN, TRU/RWM GLOVEBOX AUTOMATIC TEST CASE 4.

5.2.5.4 VERIFY after "refresh" of DMS screen DMSS0344, Worksheet OTPWRK02 that DMS indicates:

Samples 97-00002-01L and 97-00003-01L at glovebox treatment station.

$\checkmark \quad$ "Item Containers to be Retrieved" is " 0 " for both packets and samples.

5.2.5.5 VERIFY results of performance are acceptable.

TD Initials/Date: $Q Q, 7 / 2 z / 97$

5.2.6. PERFORM Section VII.E. "REMOVE WASTE ITEMS FROM TRANSFER STAND FOR TREATMENT", of operating procedure as follows:

5.2.6.1 REDLINE procedure as needed during performance.

$\sqrt{5.2 .6 .2}$ REMOVE items specified on worksheet OTPWRK04.

5.2.6.3 VERIFY DMS screen DMSS0344, worksheet OTPWRK04 indicates packet 0TP6-97-000011 in transfer drum prior to removal from transfer stand.

$\sqrt{5.2 .6 .4}$ VERIFY packet 0TP6-97-000011 removed from transfer stand.

\$.2.6.5 VERIFY after "refresh" of DMS screen DMSS0344. Worksheet OTPWRKO4 that DMS indicates:

๔. Packet OTP6-97-000011 at glovebox treatment. station.

"Item Containers to be Retrieved" is " 0 " for packets and " 0 " for samples.

5.2.6.6 VERIFY results of performance are acceptable.

TO Initials/Date:

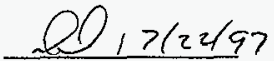


RUST FEDERAL SERVICES HANFORD Project W-026, WRAP I Operational Test - TRU/RWM Glovebox.
HNF-SD-W026-0TR-0.14- - ....

Rev: 0

Page 65 of 79

\section{ATTACHMENT 8 - TEST PLAN, TRU/RWM GLOVEBOX AUTOMATIC TEST CASE 4}

5.2.7 PERFORM Section VII.F, "REMOVE SAMPLE BOTTLES FROM STC FOR TREATMENT", of operating procedure as required to remove sample bottles from STC-2 as follows:

5.2.7.1 REDLINE procedure as needed during performance.

$\sqrt{5.2 .7 .2}$ VERIFY DMS screen DMSS0344; worksheet OTPWRK04. indicates sample bott le 97-00004-01L.

$\checkmark$ 5.2.7.3 VERIFY sample 97-00004-01L removed from STC-2.

$\checkmark$ 5.2.7.4 VERIFY after "refresh" of DMS screen DMSS0344, Worksheet OTPWRKO4 that DMS indicates:

$\checkmark$ Sample 97-00004-01L at glovebox treatment station.

$\checkmark$ "Item Containers to be Retrieved" is "0" for both packets and samples.

5.2.7.5 VERIFY results of performance are acceptable.

TD Initials/Date: $10 / 22(9)$

5.2.8 PERFORM Section VII.K. "TREAT NON-COMPLIANT WASTE ITEMS", of operating procedure as follows:

5.2.8.1 REDLINE procedure as needed during performance.

5.2.8.2 TREAT items specified on worksheet OTPWRK03.

\section{NOTE}

Section VII.K, "TREAT NON-COMPLIANT WASTE ITEMS" of WRP1-OP-0728 directs performance of WRP1-OP-0711. Waste Treatment. Completion of this step wi11 validate WRPI-0P-0711.

5.2.8.3 PERFORM procedure WRP1-OP-0711, Waste Treatment when directed by glovebox operating procedure. 
RUST FEDERAL SERVICES HANFORD

ATTACHMENT 8 - TEST PLAN, TRU/RWM GLOVEBOX AUTOMATIC TEST CASE 4

5.2.8.4 VERIFY empty treatment containers are loaded into glovebox through Consumable Materials port. when required.

5.2.8.5 VERIFY Treatment container barcode label 0TP6-97-900003 is applied to an empty treatment container:

$\sqrt{1}$ ) Contents of packet 0TP6-97-000010 and sample bottles 97-00002-01L and 97-00003-01L are added to the treatment container.

2) Packet bottle and sample bottles are added to compliant waste loadout drum OTP-TRUR-PD-11.

5.2.8.6 VERIFY on DMSSO346 for Treatment Container OTP6-97-900003:

$\checkmark$. "Compliant" boxes "checked" for packet 0TP6-97-000010 and sample bottles 97-00002-01L and 97-00003-01L.

5.2.8.7 UPDATE Compliant Loadout Drum OTP-TRUR-PD-11 information on DMSS0346 when directed by operating procedures:

- Waste Description: add "Empty $1 L$ plastic bottle. Two empty sample bottles."

5.2.8.8 VERIFY contents of treatment container 0TP6-97-900003 are treated.

5.2.8.9 ENTER data for Treatment Container OTP6-97-900003 on DMSS0346 when directed by operating procedures:

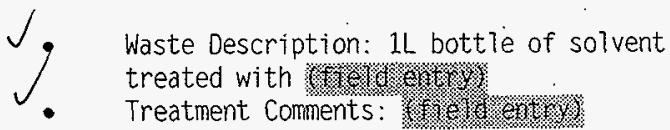




\section{ATTACHMENT 8 - TEST PLAN, TRU/RWM GLOVEBOX AUTOMATIC TEST CASE 4}

5.2.8.10 VERIFY treatment container barcode label 0TP6-97-900004 is applied to empty treatment container:

$\checkmark$ 1) VERIFY contents of packet 0TP6-97-000011 and sample bottle 97-00004-01L are added to the treatment container OTP6-97-900004.

$\sqrt{ }$ 2) VERIFY packet bottle and sample bottle are added to compliant waste loadout drum OTP-TRUR-PD-11.

5.2.8.11 ENTER data for Treatment Container OTP6-97-900004 on DMSS0346 when directed by operating procedures:

$\checkmark \quad$ Check "Compliant" boxes for packet OTP6-97-000011 and sample bottle 97-00004-01L.

5.2.8.12 UPDATE compliant waste loadout drum information on DMSS0346 when directed by operating

\%aste Description. modiph ol 7 (2) (a) Waste Description: getd "Two empty $1 \mathrm{~L}$ plastic bottles. Three empty sample bottles."

$\checkmark / 5.2 .8 .13$ VERIFY contents of treatment container 0TP6-97-900004 are treated.

\$.2.8.14 ENTER data for Treatment Container OTP6-97-900004 on DMSS0346 when directed by operating procedures:

- Waste Description: 1L bottle of oil treated with अ:

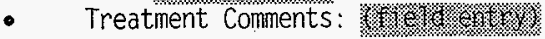


RUST FEDERAL SERVICES HANFORD

Project $W-026$. WRAP I

operational Test - TRU/RWM Glovebox.
HNF-SD-W026-OTR-014- - ....

Rev: 0

Page 68 of 79

ATTACHMENT 8 - TEST PLAN, TRU/RWM GLOVEBOX AUTOMATIC TEST CASE 4

\subsubsection{PERFORM Section VII.E, "REMOVE WASTE ITEMS FROM TRANSFER} STAND FOR TREATMENT", of operating procedure as follows:

5.2.9.1 REDLINE procedure as needed during performance.

5.2.9.2 REMOVE items specified on worksheet OTPWRK05.

5.2.9.3 VERIFY DMS screen OMSS0344, worksheet OTPWRK05 indicates packet 0TP6-97-000006 in transfer drum prior to removal from transfer stand.

$\checkmark$ 5.2.9.4 VERIFY packet 0TP6-97-000006 removed from transfer stand.

$\sqrt{5.2 .9 .5}$ VERIFY after "refresh" of OMS screen DMSS0344, Worksheet OTPWRKO4 that DMS indicates:

- Packet 0TP6-97-000006 at glovebox treatment station.

- $\quad$ "Item Containers to be Retrieved" is " 0 " for packets and "1" for samples.

To Initials/Date:

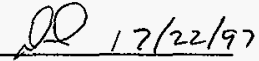

$\checkmark$ 5.2.9.7 VERIFY treatment container barcode label OTP6-97-900005 is applied to collection container OTP6-97-000006 and barcode labels are scanned to associate the treatment container with the collection container.

5.2.9.8 VERIFY contents of treatment container OTP6-97-900005 are treated. 


\section{ATTACHMENT 8 - TEST PLAN. TRU/RWM GLOVEBOX AUTOMATIC TEST CASE 4}

5.2.9.9 ENTER data for Treatment Container 0TP6-97-900005

on DMSS0346 when directed by operating procedures:

$\checkmark$ Waste Description: 1 L bottle of acid treated with

- Treatment comments: 1/ (1)

- "Compliant" box for 0TP6-97-000006 "not checked"

5.2.9.10 VERIFY results of performance are acceptable.

TD Initials/Date:

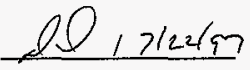

5.2.10 PERFORM Section VII.L, "TREATED WASTE ITEM LOADOUT", of operating procedure as follows:

5.2.10.1 REDLINE procedure as needed during performance.

$\checkmark$ 5.2.10.2 VERIFY Treatment container 0TP6-97-900003 and contents are placed in non-compliant waste loadout drum OTP-TRUR-PD-01.

$\sqrt{5.2 .10 .3}$ VERIFY Treatment container OTP6-97-900004 and contents are placed in non-compliant. waste loadout drum OTP-TRUR-PD-01.

5.2.10.4 VERIFY Treatment container OTP6-97-900005 and contents are placed in non-compliant waste loadout drum OTP-TRUR-PD-01. 
ATTACHMENT 8 - TEST PLAN, TRU/RWM GLOVEBOX AUTOMATIC TEST CASE 4

5.2.10.5 UPDATE treated waste drum information on DMSS0348

PHYSCOMP when directed by operating procedure:

- Description: ABSORBED AQUEOUS

- Volume Percent: 60

- Weight: 6 . 5 2/22/a)

- Description: PLASTIC/POLYURATHANE

- VoTume Percent: 5

- Weight: 2

- Description: haZARDOUS CONSTITUENTS

- Volume Percent: 45

- Weight: 4

5.2.10.6 ENTER treated waste drum information on DMSS0348 CHEMCOMP when directed by operating procedure:
Temp op2a.
- ID: 67-64-I
- Description: ACETONE
- Weight: 2

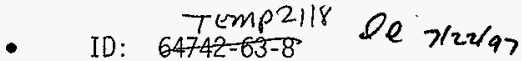
- Description: REFINED PETROLEUMOIL

5.2.10.7 VERIFY results of performance are acceptable.

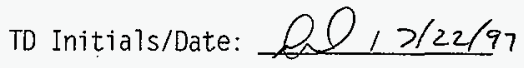

5:2.11 PERFORM Section VII.N, "LOAD TRANSFER STAND INTO RWM DRUM". of operating procedure as follows:

5.2.11.1 REDLINE procedure as needed during performance.

5.2.11.2 VERIFY transfer stand with packet 0TP6-97-000009 is returned to transfer drum OTP-TRUR-TD-02.

5.2.11.3 VERIFY results of performance are acceptable.

To Initials/Date:

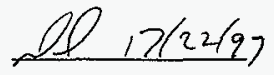


ATTACHMENT 8 - TEST PLAN, TRU/RWM GLOVEBOX AUTOMATIC TEST CASE 4

5.2.12 PERFORM Section VII.0, "CLOSE BAGLESS TRANSFER PORT (107-00-07-401)", of operating procedure as follows:

5.2.12.1 REDLINE procedure as needed during performance.

\section{NOTE}

Transfer drum OTP-TRUR-TD-01 will not be used for duration of test.

5.2.12.2 VERIFY transfer drum is closed and lowered from transfer port.

UPDATE compliant waste loadout drum information DMSS0343 PHYSCOMP when directed by operating procedure:

- Description: PLASTIC/POLYURATHANE

- Volume Percent: 40

- Weight: 4

- Description: METAL/IRON/GALVANIZED/ SHEET

- Volume Percent: 10

- Weight: 1

- Description: CLOTH/RAGS/NYLON/TAPE/ROPE

- Volume Percent: 50

- Weight: 4

5.2.12.4 VERIFY results of performance are acceptable.

TD Initials/Date:

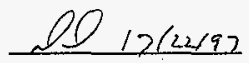

5.2.13 PERFORM Section VII.P, "CLOSE COMPLIANT WASTE PORT (107-D0-07-402A)", of operating procedure as follows:

5.2.13.1 REDLINE procedure as needed during performance.

$\int$ 5.2.13.2 VERIFY compliant waste loadout drum 0TP-TRUR-PD-11 is prepared for loadout. 
PLAN, TRU/RWM GLOVEBOX AUTOMATIC TEST CASE 4

ENTER compliant waste loadout drum data on DMSS0343 when directed by operating procedures:

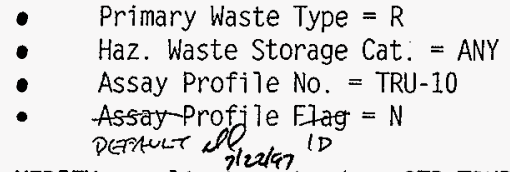
VERIFY compliant waste drum OTP-TRUR-PD-11 is
closed and lowered from port.

VERIFY drum is surveyed by RCT and a seal attached.

ENTER treated waste drum data on DMSS0343 when directed by operating procedures:

\section{- Beta/Gamma Dose Rate $=5 \mathrm{mr}$}

5.2.13.7 VERIFY results of performance are acceptable. $7(22 / 9)$

TD Initials/Date:

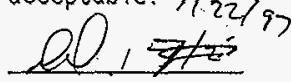

5.2.14 PERFORM Section VII.Q. "CLOSE NON-COMPLIANT WASTE PORT (107-00-07-402B)", of operating procedure as follows:

5.2.14.1 REDLINE procedure as needed during performance.

$\int$ 5.2.14.2 VERIFY Filler is added to non-compliant waste drum OTP-TRUR-PD-01 and the drum is prepared for loadout.

5.2.14.3 ENTER treated waste loadout drum data on DMSS0348 when directed by operating procedures:

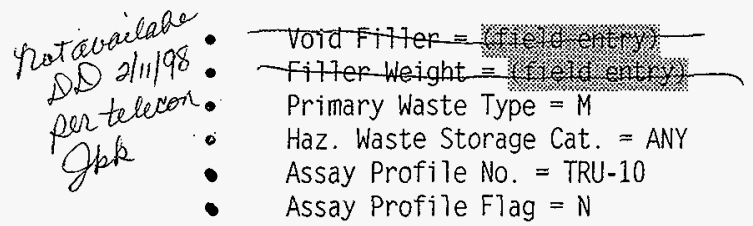




\section{ATTACHMENT 8 - TEST PLAN, TRU/RWM GLOVEBOX AUTOMATIC TEST CASE 4}

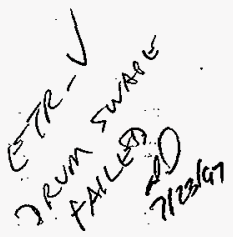
5.2.14.4 VERIFY drum OTP-TRUR-PD-01 is closed and lowered
from port.

5.2.14.5 VERIFY drum is surveyed by RCT and a seal attached.

5.2.14.6 ENTER treated waste loadout drum data on screen DMSS0348 when directed by operating procedures:

- Beta/Gamma Dose Rate $=\underline{5}$

5.2.14.7 VERIFY results of performance are acceptable.

To Initiais/Date: $0,2 / 23 / 9$

5.3 REFERENCE procedure WRP1-OP-0710. GLOVEBOX WASTE SAMPLING:

5.3.1 PERFORM Section VII.G, "FINAL SAMPLE PREPARATION AND

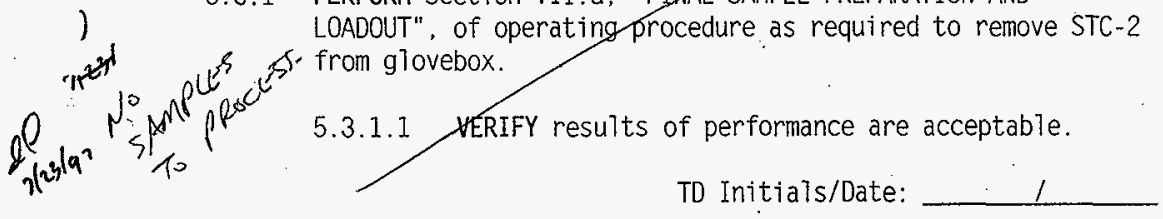

\section{NOTE}

Steps 5.4 through 5.6 may be performed in any order.

5.4 REMOVE transfer drum OTP-TRUR-TD-01 from lift table LT-09-201C.

TD Initiais/Date: el $17 / 23 / 97$

5.5 REMOVE drum OTP-TRUR-PD-01 from lift table LT-09-201A.

TO Initials/Date:

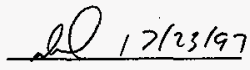

5.6 REMOVE drum OTP-TRUR-PD-1I from lift table LT-09-201B.

TD Initials/Date:

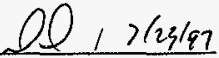

5.7 RUN DMS Report Script.

TD Initials/Date:

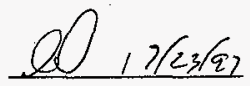


RUST FEDERAL SERVICES HANFORD Project $W-026$, WRAP I

Operational Test - TRU/RWM GTovebox.
HNF-SD-W026-0TR-014- - - . Rev: 0

Page 74 of 79

\section{ATTACHMENT 9 - TEST PLAN, TRU/RWM GLOVEBOX RESTART}

\subsection{GENERAL DESCRIPTION}

1.1 This procedure provides instructions for testing restart of the TRU/RWM Glovebox. These operations will be tested using procedure WRP1-OP-0722, Restart RWM Glovebox Operation, thereby also performing the required procedure validation.

\subsection{SAFETY}

\subsection{Reference OTP Section 8. SAFETY.}

\subsection{TOOLS, EQUIPMENT AND SUPPLIES}

- $\quad$ Drum handling (manual.) equipment or Pallet fork truck

- Spill Kit/Decontamination Equipment (oils/chemicals)

- Tape and rags

- Other equipment, material or protective clothing (as required)

\subsection{TEST PREREQUISITES}

4.1 VERIFY OTP Section 11.0. PREREQUISITES are complete.

4.2 Transfer drum OTP-TRUR-TD-01 on lift table LT-09-201C.

4.3 Drum 0TP-TRUR-PD-01 on lift table LT-09-201A.

4.4 Drum OTP-TRUR-PD-11 on Tift table LT-09-201B.

TD Initials/Date: $5 P A \quad / 7 / 15 / 97$ 
RUST FEDERAL SERVICES HANFORD Project $W-026$. WRAP I Operationa? Test - TRU/RWM Glovebox.
HNF-SD-W026-0TR-014- - .... Rev: 0

Page 75 of 79

ATTACHMENT 9 - TEST PLAN, TRU/RWM GLOVEBOX RESTART

4.5 UPDATE PCS Tracking Table as follows:

4.5.1 Drum OTP-TRUR-PD-11 at LT-09-201B.

4.5.2 Drum OTP-TRUR-TO-01 at LT-09-201C.

4.5.3 Drum OTP-TRUR-PD-01 at LT-09-201A.

\begin{tabular}{|c|c|c|}
\hline \multicolumn{2}{|c|}{ PCS TRACKING TABLE FOR TRU/RWM LIFT TABLES } \\
\hline \multirow{2}{*}{ LIFT TABLE } & RIN & Registers \\
\cline { 2 - 3 } & N32 110-116 & N32 121 bit 12 \\
\hline LT-09-201A & N32 122-128 & N32 133 bit 12 \\
\hline LT-09-201B & N32 134-140 & N32.145 bit 12 \\
\hline LT-09-201C & \\
\hline
\end{tabular}

4.6 TEST PREREQUISITES are complete and acceptable.

TD Initials/Date: SPA / I/15/97 
RUST FEDERAL SERVICES HANFORD Project W-026, WRAP I Operational Test - TRU/RWM Glovebox.

HNF-SD-W026-0TR-014- $-\ldots . .$. Rev: 0

Page 76 of 79

ATTACHMENT 9 - TEST PLAN, TRU/RWM GLOVEBOX RESTART

\subsection{PROCEDURE}

5.1 REFERENCE procedure WRP1-OP-0728. TRU/RWM GLOVEBOX OPERATION:

5.1.1 PERFORM Section VII.A. "INITIALIZE SYSTEM", of operating procedure as follows:

5.1.1.1 VERIFY results of performance are acceptable.

TD Initials/Date: SPA, $/ 1 / 5 / 97$

5.1.2 PERFORM Section VII.B, "OPEN BAGLESS TRANSFER PORT

(107-D0-07-401)", of operating procedure as follows:

5.1.2.1 VERIFY drum OTP-TRUR-TD-01 connected to port 107-D0-07-401 and port OPEN.

5.1.2.2 VERIFY results of performance are acceptable.

TD Initials/Date: SPA, $7 / 15 / 97$ 
RUST FEDERAL SERVICES HANFORD

Project W-026. WRAP I

Operational Test - TRU/RWM Glovebox.
HNF-SD-W026-OTR-014- $=\ldots$

Rev: 0

Page 77 of 79

ATTACHMENT 9 - TEST PLAN, TRU/RWM GLOVEBOX RESTART

5.1.3 PERFORM Section VII.C, "REMOVE TRANSFER STAND FROM DRUM", of operating procedure as follows:

5.1.3.1 VERIFY transfer stand at glovebox sample station.

5.1.3.2 VERIFY results of performance are acceptable.

TO Initials/Date: SPA /7/15/97

\section{NOTE}

The exact point at which EMERGENCY STOP is pressed is not critical, so long as port 107-D0-07-402A has not completed the opening sequence.

5.1.4 PERFORM Section VII.G, "OPEN COMPLIANT WASTE PORT (107-D0-07-402A)", of operating procedure as follows:

5.1.4.1 WHEN port 107-D0-07-402A is approximately half way open.

THEN, PRESS EMERGENCY STOP switch (107-HS-07-315) on DMS console (107-TE-12-103).

TD Initiais/Date: $S P A \quad / 7 / 15 / 97$

5.1.4.2 VERIFY TRU/RWM Glovebox operations STOP.

TO Initials/Date: 4 Al $7 / 15 / 97$

5.2 REFERENCE procedure WRP1-OP-0722, Restart RWM GTovebox Operation:

5.2.1 PERFORM Section VII.A. "DETERMINE GLOVEBOX EQUIPMENT STATUS". of operating procedure as follows:

5.2.1.1 REDLINE procedure as needed during performance.

5.2.1.2 VERIFY results of performance are acceptable.

TD Initials/Date: SPA / / /15/97 
RUST FEDERAL SERVICES HANFORD

Project $W-026$. WRAP I

Operational Test - TRU/RWM Glovebox.
HNF-SD-W026-0TR-014-二 …5.

Rev: 0

Page 78 of 79

ATTACHMENT 9 - TEST PLAN, TRU/RWM GLOVEBOX RESTART

5.2.2 PERFORM Section VII.B. "RETURN RWM GLOVEBOXES TO OPERATION", of operating procedure as follows:

5.2.2.1 REDLINE procedure as needed during performance.

5.2.2.2 VERIFY results of performance are acceptable.

TD Initials/Date: SPA / 7/15/97 


\section{LLW RWM GLOVEBOX OTP WASTE AND SAMPLE TRACKING}

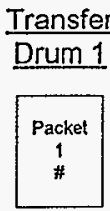

Aerosol Can

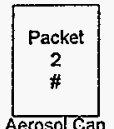

whrapping

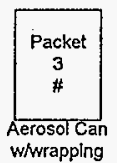

Transfer Drum 2
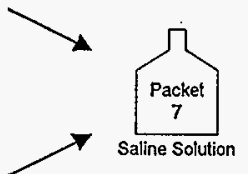

$\frac{\text { Transfer }}{\text { Drum } 1}$

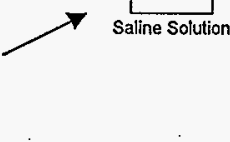

$\underline{\text { STC } 1}$
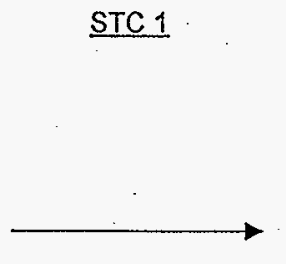

Product Drum PIN Transfeer Drum PIN Packet PIN

STC PIN

Transport Pig PIN

Treatment Container

Sample Bottle ID

Field Screen ID
OTP-LLWR-PD-XX OTP-LLWR-TD-XX OTP6-97-0000XX OTP6-97-3000XX OTP6-97-4000XX OTP6-97-9000XX 97-000XX-XXL 97-000XX-01F

ffreatment

Container: Treated saline Treated Saltin
Solution
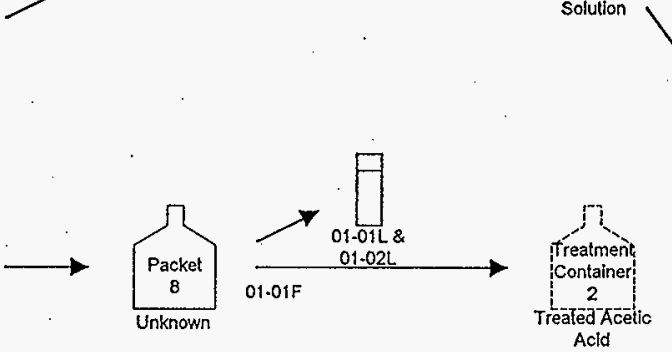

\begin{tabular}{c}
\hline Packet \\
5 \\
$\#$ \\
\hline
\end{tabular}

Two Bottles whrrapping $\frac{\text { Transfer }}{\text { Drum } 2}$

$\underline{\operatorname{STC} 2}$

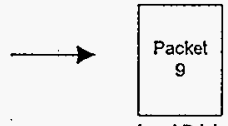

Lead Brick

$02-01 F$

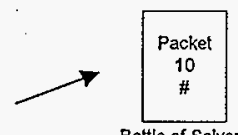

Bottle of Solvent
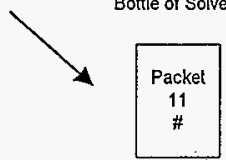

Bottle of Oil

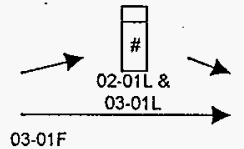

03-01F
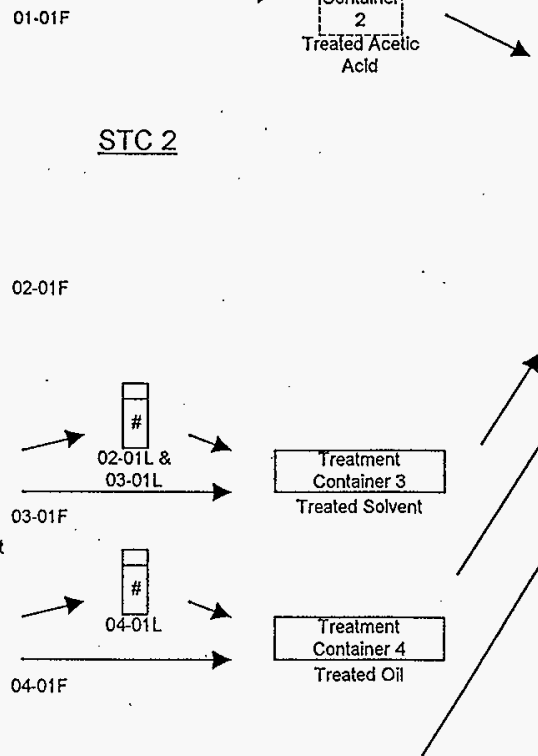

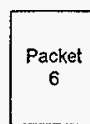

Bottle of Acid

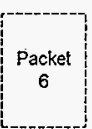

Botle of Acid

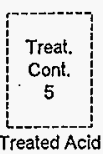

Product Drum 1 Treated Waste

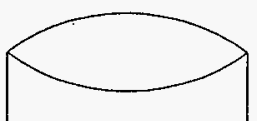

Product Drum 11 Compliant Waste

Receives waste from containers with \# 


$$
\therefore: N
$$

DMS

Step : Att 5, 5.2.4 Equipment Tag \#: DMS Software

Exception : 1

\section{Revision :}

Specification Section: DMS

Test Witness/Author: Rosnick

Test Operator:

Responsible Company: WMH

Responsible Subcontractor: BDM

Responsible Individual: Weldert

Exception Description :

[DMS screen DMSSO341. When there is no transfer drum present and [Exit] button is selected, the pop-up message "FRM-40202 field must be entered [OK]" is displayed. When [OK] is selected screen is exited.

Exception Disposition :

Duplicate of \#162 and \#181.

\section{Exception Status :}

Exception Closed By: Rosnick $\subset \geq R$ Date : 7/24/97

\section{APPROVAL OF RESOLUTION}

Contractor Representative: (Only as neaded)

WMH Representative:

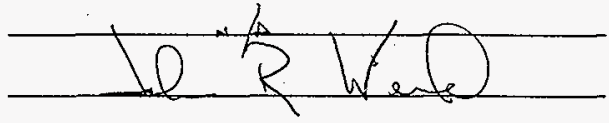

Additional Approval:

(Only as needed)
Date:

Date: $12-10-97$

Date:

FDNW Representative:

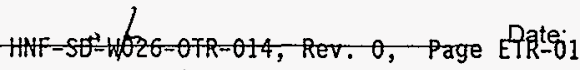


ATP_YN : N

DMS

Test : HNF-SD-W026-OTP-014

Step : ATT. 5, 5.2.4 Equipment Tag \#: DMS Software
Exception :

Revision :

Specification Section: DMS

Test Witness/Author:

Rosnick

Test Operator:

$\begin{array}{ll}\text { Responsible Company: } & \text { WMH } \\ \text { Responsible Subcontractor: } & \text { BDM } \\ \text { Responsible Individual: } & \text { Weidert }\end{array}$

Exception Description :

DMS Screen MMSSO347. When there is no transfer drum present and] [Exit] button is selected, the pop-up message "FRM-40202 Field must be entered [ok]" is displayed. When [ok] is selected screen is exited.

\section{Exception Disposition :}

Duplicate of \#132 and \#181. Sotware corrected.

\section{Exception Status :}

Ready to test.

Exception Closed By : Dunlap

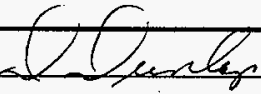

Date : 9/15/97

\section{APPROVAL OF RESOLUTION}

Contractor Representative: (Only as needed)

WMH Representative:

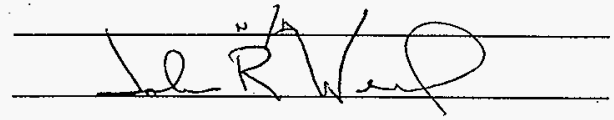

Date:

Date: 12.0.97

Additional Approval:

(Only as needed)

Date:

FDNW Representative:

$N / D$

Date: 


\section{WRAP ACCEPTANCE TEST PROCEDURE EXCEPTION LOG}

ATP YN : N

DMS

Test : HNF-SD-W026-OTP-014

Step : ATT. 5, 5.2.6 Equipment Tag \#: DMS Software
Exception : 2

Revision :

Date:

Specification Section : DMS

Test Witness/Author:

Test Operator:

\begin{tabular}{ll} 
Responsible Company: & WMH \\
\hline Responsible Subcontractor : & BDM \\
\hline Responsible Individual: & Wetden
\end{tabular}

Exception Description :

DMS Screen DMSS0342.

When the screen is accessed, the complaint loadout drum PIN is displayed only if there are NCIP messages to process. The screen should always display the PIN if a drum is present.

\section{Exception Disposition :}

Software corrected.

\section{Exception Status :}

Closed

\section{Exception Closed By: Dunlap}

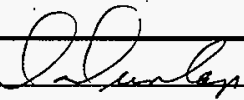

Date : $8 / 6 / 97$

\section{APPROVAL OF RESOLUTION}

Contractor Representative: (Only as needed)

WMH Representative:

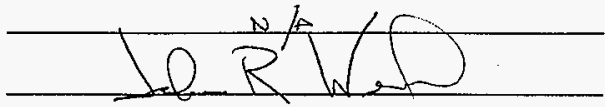

Additional Approval:

(Only as needed)
Date:

Date: $12-10-87$

Date:

Date: 
ATP_YN : N

DMS

Test : HNF-SD-W026-OTP-014

Step : ATT. 5, 5.2.6.4 Equipment Tag \#: DMS Software

Specification Section: DMS

Test Witness/Author:

Test Operator:
Exception : 3

Revision :

Date:

\section{Responsible Company: WMH \\ Responsible Subcontractor: $\mathrm{BDM}$ \\ Responsible Individual: Weldent}

\section{Exception Description :}

DIMS Screen DMSSO342.

The date/time for the packet to complaint - loadout - drum relationship record (CONR_DT) does not include the time. Time is set to 00:00:00.

\section{Exception Disposition:}

Problem fixed and tested in dry run.

\section{Exception Status :}

\section{Exception Closed By : Dunlap

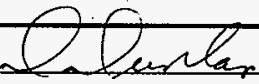 \\ Date : 9/3/97}

\section{APPROVAL OF RESOLUTION}

Contractor Representative: (Only as needed)

WMH Representative:

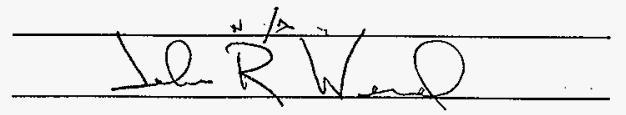

Date:

Date: $12 \cdot 10-97$

Additional Approval:

(Only as needed)

Date:

FDNW Representative:

$\sim /$

Date: 
ATP_YN : N

DMS

Test : HNF-SD-W026-OTP-014

Step : ATT. 5, 5.2.6.6. Equipment Tag \#: DMS Software
Exception : 4

Revision :

Specification Section: DMS

Test Witness/Author :

Test Operator:

\begin{tabular}{ll} 
Responsible Company: & WMH \\
\hline Responsible Subcontractor: & BDM \\
Responsible Individual: & Weldert
\end{tabular}

Exception Description :

DMS screen DMSS0504.

The "Analysis Selected" field is not wide enough. "Solvents" is truncated to "OLVENTS".

\section{Exception Disposition :}

Software corrected.

\section{Exception Status :}

Closed.

Exception Closed By : Dunlap

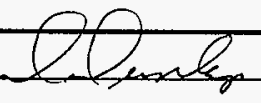

Date : $8 / 6 / 97$

\section{APPROVAL OF RESOLUTION}

Contractor Representative: (Only as needed)

WMH Representative:

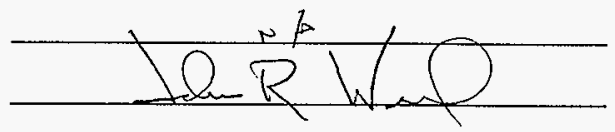

Date:

Date: $12-10.97$

Additional Approval:

(Only as needed)

Date:

FDNW Representative:

$+10$

Date: 
ATP_YN : N

DMS

Exception : 5

Test : HNF-SD-W026-OTP-014

Step : ATT.5, 5.2.6.10 Equipment Tag \#: DMS Software

Revision :

Date:

Specification Section : DMS

Test Witness/Author :

Rosnick

Test Operator:

Responsible Company: WMH

Responsible Subcontractor: BDM

Responsible Individual: Weldert

Exception Description :

DMS Screen DMSS0343.

When entering the screen, the complaint waste loadout drum Proflie ID was automatically set to NULL and the Default Profile to "N". NULL is not a valid Profile ID.

Exception Disposition :

Problem fixed and tested in dry run.

\section{Exception Status :}

Exception Closed By: Dunlap Date: 9/3/97

\section{APPROVAL OF RESOLUTION}

Contractor Representative: (Only as needed)

WMH Representative:

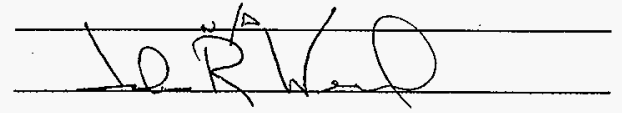

Date:

Date: $12-6-97$

Additional Approval:

(Only as needed)

Date:

FDNW Representative:

$N / \Delta$

Date: 
ATP_YN : N

DMS

Test : HNF-SD-W026-OTP-014

Step : ATT.5, 5.2.6.10 Equipment Tag \#: DMS Software

\section{Exception : 6}

Revision :

Date:

Specification Section: DMS

Test Witness/Author :

Rosnick

Test Operator:

Responsible Company: WMH

Responsible Subcontractor: BDM

Responsible Individual: Wetdent

Exception Description :

DMS Screen DMSS0341. When returning from DMSS0342 to DMSS0347, screen DMSS0341 is not refreshed. See PSDD, Appendix F, 3.1.3.4, UP 5.

Exception Disposition :

Problem fixed in ary run.

\section{Exception Status :}

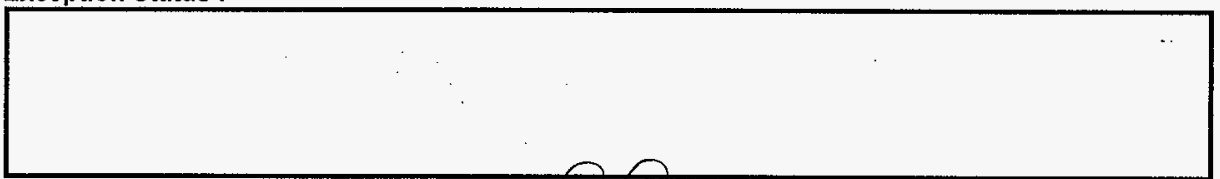

Exception Closed By : Dunlap

to

Date : $9 / 3 / 97$

\section{APPROVAL OF RESOLUTION}

Contractor Representative: (Only as needed)

WMH Representative:

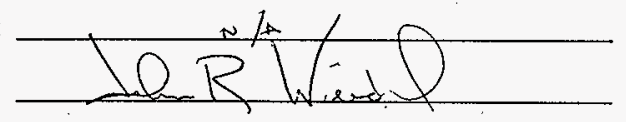

Additional Approval:

(Only as needed)
Date:

Date: $12-10-97$

Date:

Date: 


\section{WRAP ACCEPTANCE TEST PROCEDURE EXCEPTION LOG}

Tracking \#: $\quad 168$

\section{Exception : 7}

Revision :

Date:

Specification Section: DMS

Test Witness/Author : Rosnick

Test Operator:

\begin{tabular}{ll} 
Responsible Company: & WMH \\
\hline Responsible Subcontractor: & BDM \\
Responsible Individual: & Weldert \\
\hline
\end{tabular}

Exception Description :

DTMS Screen DMSS0503.

The PIN List of values is in descending order. The LOV should be in ascending order, like the PIN LOV screen for DMSS0504.

\section{Exception Disposition :}

Software corrected.

\section{Exception Status :}

closed.

Exception Closed By : Dunlap

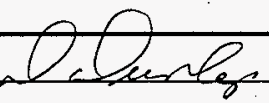

\section{APPROVAL OF RESOLUTION}

Contractor Representative: (Only as needod)

WMH Representative:

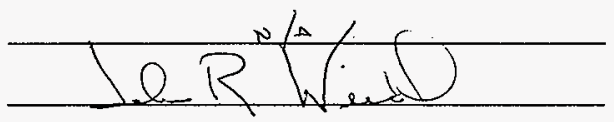

Date:

Date: $12 \cdot 10-94$

Additional Approval:

(Only as needed)

Date:

FDNW Representative:

$N A$

Date: 
Test : HNF-SD-W026-OTP-014

Step : ATT.5,5.2.7.3/4 Equipment Tag \#: DMS Software

Revision :

Date:

\section{Specification Section: DMS}

Test Witness/Author :

Rosnick

Test Operator:

$\begin{array}{ll}\text { Responsible Company: } & \text { WMH } \\ \text { Responsible Subcontractor: } & \text { BDIV } \\ \text { Responsible Individual: } & \text { Weidert }\end{array}$

Exception Description :

When entering screen DMS'50503 (Field screening) there is an automatic LOV for PIN number. Screen DMSSO504 (Analysis Request) does not have an automatic LOV for the PIN. The screens should be consistent.

\section{Exception Disposition :}

Sotware corrected.

\section{Exception Status :}

Closed.

\section{Exception Closed By : Dunlap \\ APPROVAL OF RESOLUTION}

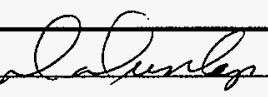

Contractor Representative: (Only as needed)

WMH Representative:

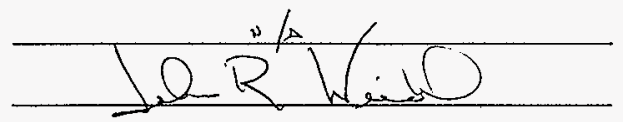

Additional Approval: (Only as needed)
Date:

Date: $12 \cdots 0.97$

Date:

Date: 
ATP YN : N

DMS

Test : HNF-SD-W026-OTP-014

Step : ATT.6, 5.3.1.4 Equipment Tag \#: 'DMS Software

Specification Section : DMS

Test Witness/Author:

Test Operator:
Exception : 9

Revision :

Date:

\begin{tabular}{ll} 
Responsible Company: & WMH \\
\hline Responsible Subcontractor: & BDM \\
Responsible Individual: & Weldert \\
\hline
\end{tabular}

Exception Description :

The transport pig PIN is shown as the STC PIN and no sample bottles are listed. If the most recent $P$ IN is a pig (CONEXT_USE_CD = 'TP') the STC PIN field should be blank.

Exception Disposition :

Problem fixed and tested in dry run.

Exception Status :

Ready for testing

\section{Exception Closed By : Rosnick
APPROVAL OF RESOLUTION}

Contractor Representative: (Only as needed)

WMH Representative:

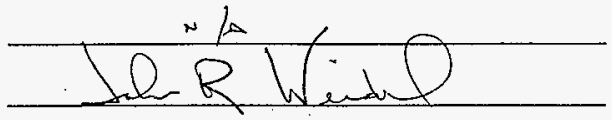

Date:

Date: $12-10-97$

Additional Approval:

(Only as needed)

Date:

N/A

Date: 
ATP_YN : N

DMS

Test : HNF-SD-W026-OTP-014

Step: ATT.7, 5.1.4 Equipment Tag \#: DMS Software
Exception : 10

Revision :

Specification Section : DMS

Test Witness/Author :

Rosnick

\section{Test Operator:}

\begin{tabular}{ll} 
Responsible Company: & WMH \\
\hline Responsible Subcontractor: & .BDIh \\
\hline Responsible Individual: & Weldert. \\
\hline
\end{tabular}

Exception Description :

DMMS Screen DIVSSO344.

The Worksheet LOV truncates the last digit.

See LLW/RWM OTP Exception 10 for screen DMSS0324 exception.

Exception Disposition :

Problem fixed and tested in dry run

\section{Exception Status :}

Exception Closed By : Dunlap

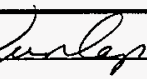

Date : $8 / 6 / 97$

\section{APPROVAL OF RESOLUTION}

Contractor Representative: (Only as needed)

WMH Representative:

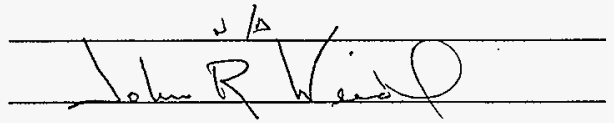

Date:

Date: $12 \cdot 10-97$

Additional Approval:

Date:

(Only as needed)

FDNW Representative:

$n / \infty$

Date: 


\section{WRAP ACCEPTANCE TEST PROCEDURE EXCEPTION LOG}

ATP_YN : N

DMS

Test : HNF-SD-W026-OTP-014

Step : ATT.7, 5.1.7 Equipment Tag \#: DMS Software

Specification Section : DMS

Test Witness/Author :

Test Operator:
Exception : 11

Revision :

\section{Responsible Company: ' WMH \\ Responsible Subcontractor : BDM \\ Responsible Individual: Weidert}

\section{Exception Description :}

DDMS Screen DMSSO346.

When [List of Values] was selected and there were no treatment containers to list, the screen was left in query mode and it was necessary to cancel the query from the menu in order to Exit the screen.

\section{Exception Disposition :}

software corrected.

\section{Exception Status :}

Ready for retest.

Exception Closed By : Dunlap

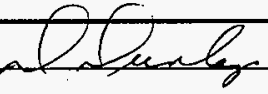

\section{APPROVAL OF RESOLUTION}

Contractor Representative: (Only as needed)

WMH Representative:

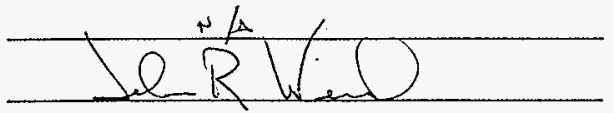

Additional Approval:

(Only as needed)
Date:
Date: $12-10-97$

Date:

Date: 
ATP_YN : N

DMS

Test : HNF-SD-W026-OTP-014

Step : ATT.7, 5.1.7.7 Equipment Tag \#: DMS Software

Specification Section : DMS

Test Witness/Author:

Test Operator:

\begin{tabular}{ll}
\hline Responsible Subcontractor: & BDM \\
Responsible Individual: & Weider
\end{tabular}

Exception Description :

DMSS Screen DMSSO344.

The treatment container was listed with "Packets/Samples not on Worksheet". A treatment container used with the current worksheet should not be listed.

See LLW/RWM OTP Exception 18 for screen DMSS0324 exception.

\section{Exception Disposition :}

Problem fixed and tested in dry run.

\section{Exception Status :}

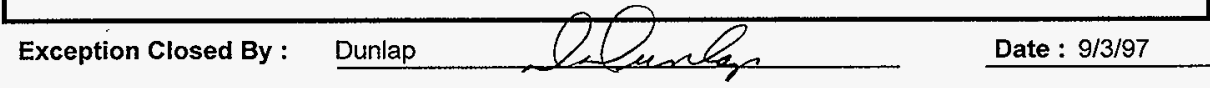

\section{APPROVAL OF RESOLUTION}

Contractor Representative: (Only as needed)

WMH Representative:

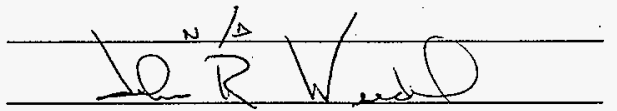

Date:

Date: $12-10-37$

Additional Approval:

Date:

(Only as needed)

FDNW Representative:

$w / D$

Date: 
ATP_YN : N

DMS

Test : HNF-SD-W026-OTP-014

Step : ATT.7, 5.1.7.7 Equipment Tag \#: DMS Software
Exception : 13

Revision :

Date:

Specification Section : DMS

Test Witness/Author:

Rosnick

Test Operator:

\begin{tabular}{ll} 
Responsible Company: & WMH \\
\hline Responsible Subcontractor: & BDIV \\
\hline Responsibie Individual: & Weldert \\
\hline
\end{tabular}

\section{Exception Description :}

DMS screen DMSS0344.

The [OK] button on "Packet/Sample Not on Worksheet" pop-up does not work. The pop-up on DMSS0324 is identical but an exception was not noted in the LLW/RWM OTP.

\section{Exception Disposition :}

Problem fixed and tested in dry run

\section{Exception Status :}

\section{Exception Closed By : Dunlap \\ APPROVAL OF RESOLUTION}

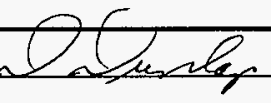

Date : $9 / 3 / 97$

Contractor Representative: (Only as needed)

WMH Representative:

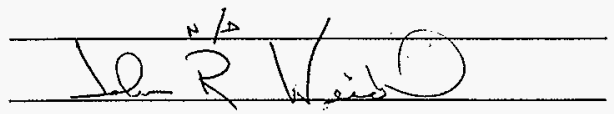

Date:

Date: $12-10-97$

Additional Approval:

Date:

(Only as needed)

FDNW Representative:

$+1$

Date: 


\section{WRAP ACCEPTANCE TEST PROCEDURE EXCEPTION LOG}

ATP_YN : N

DMS

Test: HNF-SD-W026-OTP-014

Step : ATT.7, 5.1.8.4 Equipment Tag \#: DMS Software

Specification Section : DMS

Test Witness/Author :

\section{Test Operator:}

Tracking \#: 175

Exception : 14

Revision :

Date:

\section{Responsible Company: WMH \\ Responsible Subcontractor: BDM \\ Responsible Individual: Weldert}

\section{Exception Description :}

\section{DMMS Screen DMSS0348.}

When entering the screen, the complaint waste loadout drum Porfile ID was automatically set to NULL and the Default Profile to "N". NULL is not a valid Profile ID.

\section{Exception Disposition :}

Problem fixed and tested in dry run.

\section{Exception Status :}

.

Exception Closed By : Dunlap

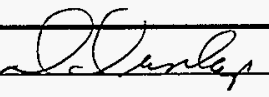

\section{APPROVAL OF RESOLUTION}

Contractor Representative: (Only as needed)

WMH Representative:

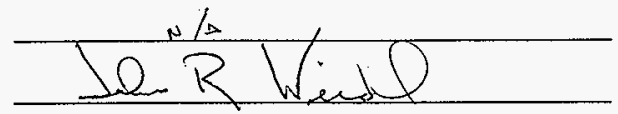

Additional Approval:

(Only as needed)
Date:

Date: $12 \cdot 10-97$

Date:

Date: 
ATP_YN : N

DMS

Test : HNF-SD-W026-OTP-014

Step : ATT:8, 5.2.8.11 Equipment Tag \#: DMS Software

Specification Section : DMS

Test Witness/Author:

Test Operator:

Responsible Company: WMH

\section{Responsible Subcontractor: BDM}

Responsible Individual: . Weidert

Exception Description :

DMS Screen DMSS0346.

When data had been entered but not committed and [Return] was selected, pop-up screen "E617 - Must be entered to send packages to PCS [OK]" was displayed.

\section{Exception Disposition :}

Problem fixed and tested in dry run.

\section{Exception Status :}

Exception Closed By : Dunlap Date: 9/3/97

\section{APPROVAL OF RESOLUTION}

Contractor Representative: (Only as needed)

WMH Representative:

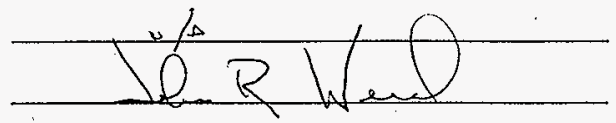

Date:

Date: $12-10-97$

Additional Approval:

(Only as needed)

Date:

FDNW Representative:

$N$.

Date: 
ATP_YN : N

DMS

Test : HNF-SD-W026-OTP-014

Step : ATT.8, 5.2.10.6 Equipment Tag \#: DMS Software
Exception : 16

\section{Revision :}

Specification Section : DMS

Test Witness/Author:

Rosnick

Test Operator:

Responsible Company: WMH

Responsible Subcontractor: BDM

Responsible Individual: Weldert

Exception Description :

DDS Screen DMSS0348.

The hazardous component weight percent is required on this screen. However, this value is not known at this time since the total waste weight is not known. The weight percent is calculated and recorded at the airlock after the drum is weighed.

\section{Exception Disposition :}

Software changed so weight percent is not required. Tested in dry run.

\section{Exception Status :}

Exception Closed By : Dunlap

Date : $9 / 3 / 97$

\section{APPROVAL OF RESOLUTION}

Contractor Representative: (Only as needed)

WMH Representative:

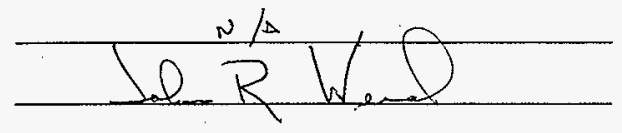

Date:

Date: $12-10-97$

Additional Approval:

Date:

(Only as needed)

$N / \infty$

Date: 


\section{WRAP ACCEPTANCE TEST PROCEDURE EXCEPTION LOG}

ATP_YN : N

DMS

Test : HNF-SD-W026-OTP-014

Step : ATT.8, 5.2.14 Equipment Tag \#: DMS Software
Exception : 17

Revision :

Specification Section: DMS

Test Witness/Author:

Rosnick

\section{Test Operator:}

\begin{tabular}{ll} 
Responsible Company: & WMH \\
\hline Responsible Subcontractor: & BDM \\
Responsible Individual: & Wetder
\end{tabular}

Exception Description :

DMS Screen DMSS0348.

The LOV pop-ups for this screen have mixed fonts.

\section{Exception Disposition :}

Problem fixed and tested in dry run.

\section{Exception Status :}

Exception Closed By : Dunlap

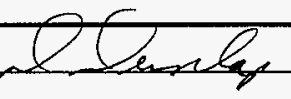

Date : $9 / 3 / 97$

\section{APPROVAL OF RESOLUTION}

Contractor Representative: (Only as needed)

WMH Representative:

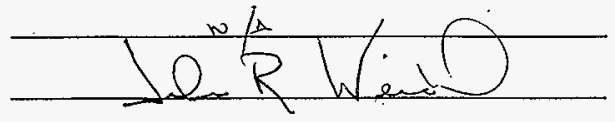

Additional Approval:

(Only as needed)
Date:

Date: $12-10-97$

Date:

Date: 
ATP_YN : N

DMS

Test : HNF-SD-W026-OTP-014

Step : ATT.8, 5.2.14.6 Equipment Tag \#: DMS Software
Exception : 18

\section{Revision :}

Date:

Specification Section: DMS

Test Witness/Author :

\section{Rosnick}

Test Operator:

\begin{tabular}{ll} 
Responsible Company: & WMH \\
\hline Responsible Subcontractor: & BDM \\
Responsible Individual: & WeIdert
\end{tabular}

\section{Exception Description :}

DMS Screen SMSS0348.

"Beta/Gamma Dose Rate" and "Container QC Seal No." are write protected.

Exception Disposition :

Problem fixed and tested in dry run.

\section{Exception Status :}

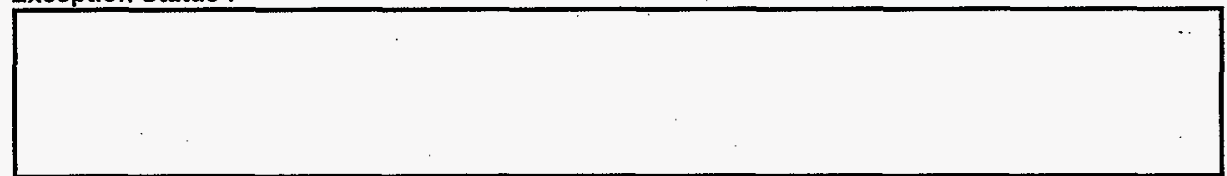

Exception Closed By: Rosnick $2<1<$ Date : 7/24/97

\section{APPROVAL OF RESOLUTION}

Contractor Representative: (Only as needed)

WMH Representative:

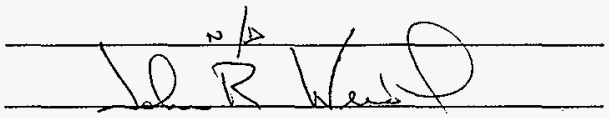

Additional Approval:

(Only as needed)

FDNW Representative:
Date:

Date: $12+10-97$

Date:

Date: 
ATP_YN : N

DMS

Test : HNF-SD-W026-OTP-014

Step : ATT. 5, 5.2.4 Equipment Tag \#: DMS Software
Exception : 1

Revision :

Specification Section : DMS

Test Witness/Author:

Rosnick

Test Operator:

\begin{tabular}{ll} 
Responsible Company: & WMH \\
\hline Responsible Subcontractor: & BDM \\
Responsible Individual: & Weloter
\end{tabular}

\section{Exception Description :}

DMS Screen DMSS0341. When there is no transfer drum present and [Exit]

button is selected, the pop-up message "FRM-40202 Field must be entered [OK]" is displayed. When [OK] is selected screen is exited.

Exception Disposition :

Problem fixed and tested in dry run.

\section{Exception Status :}

Closed.

Exception Closed By: Dunlap Date: 9/3/97

\section{APPROVAL OF RESOLUTION}

Contractor Representative: (Only as needed)

WMH Representative:

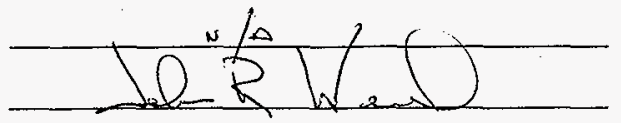

Additional Approval:

(Only as needed)
Date:

Date: $12-10 \cdot 97$

Date:

- Date:

FDNW Representative:

10 
ATP_YN : N

DMS

Test : HNF-SD-W026-OTP-0114

Step : Att. 5, 5.2.5 · Equipment Tag \#: DMS Software
Exception : 19

Revision :

Specification Section : DMS

Test Witness/Author:

Rosnick

Test Operator:

\begin{tabular}{ll} 
Responsible Company: & WMH \\
\hline Responsible Subcontractor: & BDM \\
Responsible Individual: & WeIdert
\end{tabular}

Exception Description :

DMSPSDD, Apendix F, 3.1.8. up 21

DMSCOM The secondary waste type group (RDET_SWTY_GRP) was not set for the product durms.

\section{Exception Disposition :}

Problem Corrected.

\section{Exception Status :}

closed.

Exception Closed By : Dunlap

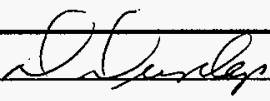

Date : $8 / 5 / 97$

\section{APPROVAL OF RESOLUTION}

Contractor Representative: (Only as needed)

WMH Representative:

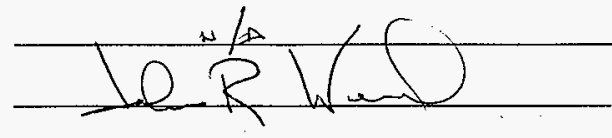

Date:

Date: $12-10-97$

Additional Approval:

Date:

(Only as needed)

FDNW Representative:

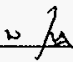

Date: 
ATP_YN : N

DMS

Test : HNF-SD-W026-OTP-014

Step : Att. 5, 5.1.6

Equipment Tag \#: DMS Software
Exception : 20

Revision :

Date:

Specification Section: DMS

Test Witness/Author:

Rosnick

Test Operator:

Responsible Company: $\quad W M H$

Responsible Subcontractor : BDM

Responsible Individual: Wedert

Exception Description :

DDMSCOM The contain status (CONEXT_CNTR_STATUS) for transfer drum 1 was not changed from 'P' to 'E' when all packets been removed for treatment.

Exception Disposition :

Problem corrected.

\section{Exception Status :}

Closed.

\section{Exception Closed By :}

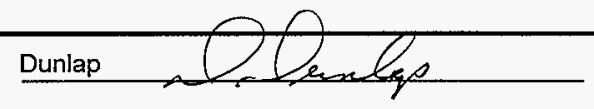

Date : $8 / 6 / 97$

\section{APPROVAL OF RESOLUTION}

Contractor Representative: (Onily as needed)

WMH Representative:

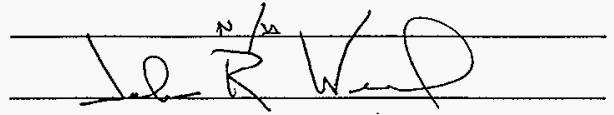

Date:

Date: $12 \cdot 10-97$

Additional Approval:

(Only as needed)

Date:

FDNW Representative:

$\sim 4$

Date: 
ATP_YN : N

Test: HNF-SD-W026-OTP-014

Step : Att.5.2.14.4 A8 Equipment Tag \#: DO-07-402B

Specification Section :

Test Witness/Author:

Test Operator:

13461

Duntap
Exception : 1

Revision :

Date:

\begin{tabular}{ll} 
Responsible Company: & WMH \\
\hline Responsible Subcontractor : & \\
\hline Responsible Individual: & Leist \\
\hline
\end{tabular}

Exception Description :

Drum swage failed 
ATP YN : N

Test : HNF-SD-W026-OTP-014

Step : 5.2.13.4 A8 Equipment Tag \#: DO-07-402A

Specification Section : 13461

Test Witness/Author :

Test Operator:

\begin{tabular}{l|l} 
Responsible Company: & WMH \\
\hline Responsible Subcontractor: & \\
\hline Responsible Individual: & Leist \\
\hline
\end{tabular}

Exception Description :

Drum stubs on port when lowered.

\section{Exception Disposition :}

\section{Exception Status :}

9-3-97 Duplicateof \#49. Closed.

Exception Closed By : Leist $1-2 / 16 / 97$ Date : 9/3/97

\section{APPROVAL OF RESOLUTION}

Contractor Representative: (Only as needed)

WMH Representative:

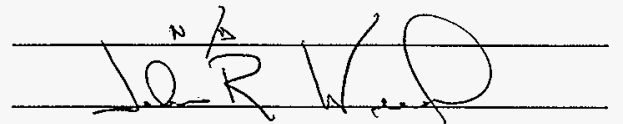

Date:

Date: $12-10-97$

Additional Approval:

(Only as needed)

Date:

FDNW Representative:

N

Date: 


\section{WRAP ACCEPTANCE TEST PROCEDURE}

EXCEPTION LOG

ATP_YN : N

Test : HNF-SD-W026-OTP-014

Step : 5.2.13.4 A8 Equipment Tag \#: DO-07-402A
Exception : 3

Revision :

Specification Section : 13461

Test Witness/Author:

Dunlap

Test Operator:

Responsible Company: WMH

Responsible Subcontractor :

Responsible Individual: _ Leist

Exception Description :

"Sequence fail" alarm in closing port. South side lock sensor misadjusted.

\section{Exception Disposition :}

\section{Exception Status :}

9-3-97-MPLane to retest. 12-12-97. Detested sat is factury. Clorkd

Exception Closed By :

Micieas

PQne

Date : $12-12-9 / 9$

\section{APPROVAL OF RESOLUTION}

Contractor Representative: (Only as needed)

WMH Representative:

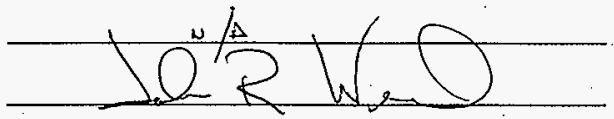

Date:

Date: $12-12-27$

Additional Approval:

(Only as needed)

Date:

FDNW Representative:

$\checkmark / 2$

Date: 
ATP_YN : N

Test : HNF-SD-W026-OTP-014

Step : $5.2 .6 \mathrm{~A} / 6$

Equipment Tag \#: ACP DEVICE-111
Exception : 11

Revision :

Date:

Specification Section : 13462

Test Witness/Author:

Dunlap

Test Operator:

\begin{tabular}{|c|c|}
\hline Responsible Company: & WMH \\
\hline Responsible Subcontractor: & \\
\hline Responsible Individual: & Leist \\
\hline
\end{tabular}

Exception Description :

Aerosol can punture device leaks at handle.

\section{Exception Disposition :}

\section{Exception Status :}

8-3-97 Duplicate of \$34. Closed.

Exception Closed By: Leist

\section{APPROVAL OF RESOLUTION}

Contractor Representative: (Only as needed)

WMH Representative:

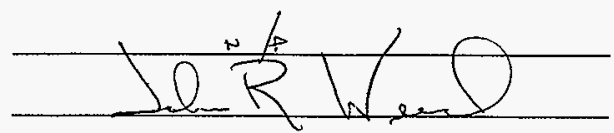

Date:

Date: $12-10-97$

Additional Approval:

(Only as needed)

Date:

FDNW Representative:

$N / A$

Date: 
ATP_YN : N

Test : HNF-SD-W026-OTP-014

Step : 5.1.7.8 A7

Equipment Tag \#: $X X-07-4-2$
Exception : 4

Revision :

Date:

Specification Section : 13462

Test Witness/Author:

Dunlap

Test Operator:

\begin{tabular}{ll} 
Responsible Company: & WMH \\
\hline Responsible Subcontractor: & N/A \\
\hline Responsible Individual: & WIIIS \\
\hline
\end{tabular}

Exception Description :

Jar Mill not effective when low volume of water in treatment container

\section{Exception Disposition :}

\section{Exception Status :}

Sar mil has been remowed fuom the glave liox

Exception Closed By :

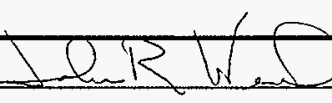

Date: $12-10 \cdot 97$

\section{APPROVAL OF RESOLUTION}

Contractor Representative: (Only as needed)

WMH Representative:

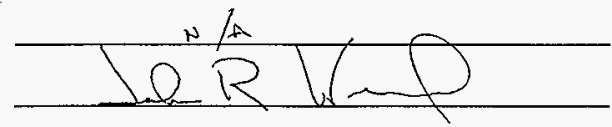

Date:

Date: $12-10-97$

Additional Approval:

(Only as needed)

Date:

FDNW Representative:

$N / A$

Date: 


\section{WRAP ACCEPTANCE TEST PROCEDURE EXCEPTION LOG}

ATP YN : N

DMS

Test : HNF-SD-W026-OTP-014

Step : 5.1.6 Att.7 Equipment Tag \#: DMS Software
Exception : 5

Revision :

Date:

Specification Section: DMS

Test Witness/Author:

Duniap

Test Operator:

Responsible Company: WMH

Responsible Subcontractor: $S I$

Responsible ndividual: —TBD

Exception Description :

Location record for a treatment container was advertently scanned twice while trying to establish a treatment to collection container relation. Had to manually enter Conloc for treatment container.

Exception Disposition :

Add code to PCS to prevent scanning the same PIN twice drums this condition.

Exception Status :

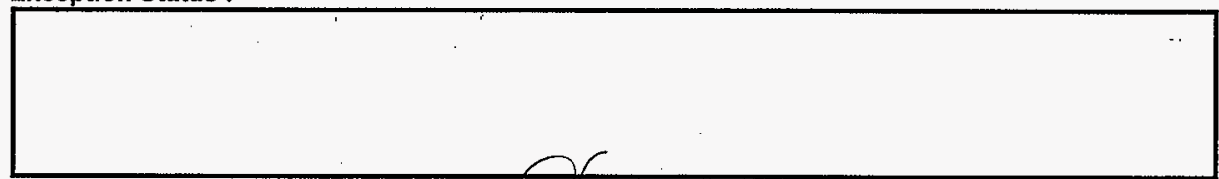

Exception Closed By : Dunlap

Date : $9 / 12 / 97$

\section{APPROVAL OF RESOLUTION}

Contractor Representative: (Only as needed)

WMH Representative:

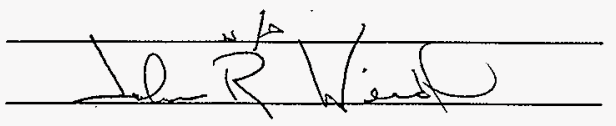

Date:

Date: $\quad 12-10-87$

Additional Approval:

(Only as needed)

Date:

FDNW Representative:

$N / D$

Date: 
ATP_YN : N

Exception : 6

Test : HNF-SD-W026-OTP-014

Step :

Equipment Tag \#: PCS S $W$

Revision :

Date:

Specification Section : 13462

Test Witness/Author :

Dunlap

Test Operator:

\begin{tabular}{ll} 
Responsible Company: & WMH \\
\hline Responsible Subcontractor: & SII \\
\hline Responsible Individual: & TBD \\
\hline
\end{tabular}

Exception Description :

If is possible to scan an item other than a treated or treatment container to a non-compliant drum for loadout. DMS records are being generated inadvertantly.

\section{Exception Disposition :}

\section{Exception Status :}

12-12.97. Coele madified tolimit treated it em loddent valial scans to trestiment contuines.
clorel.

Exception Closed By :

Micue Pdre

Date: $12-12-97$

\section{APPROVAL OF RESOLUTION}

Contractor Representative: (Only as needed)

WMH Representative:

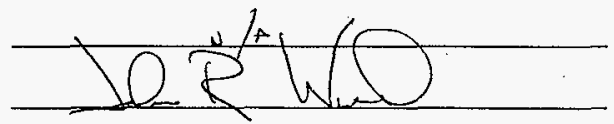

Date:

Date: $\varepsilon^{-2}-97$

Additional Approval:

(Only as needed)

Date:

FDNW Representative:

$\sim \rho$

Date: 
ATP_YN : N

Test : HNF-SD-W026-OTP-014

Step : 5.2.4 Att.6 Equipment Tag \#: PCS SN

Exception : 7

Revision :

Date:

Specification Section : $\quad 13461$

Test Witness/Author :

Duntap

Test Operator:

\begin{tabular}{llll} 
Responsible Company: & WMH \\
\hline Responsible Subcontractor: & ST \\
\hline Responsible Individual: & TBD & & \\
\hline
\end{tabular}

Exception Description :

PCS sends a previously composed message when TRURWSAMPL. Location is scanned, this caused an extra container to appear at that location.

\section{Exception Disposition :}

Caused by a race condition in the ladder logic where a location bit is unlatched before the new message is composed.

\section{Exception Status :}

Removed location bit check to eliminate race condition. The çondition did not occur later in the OTP under the same circumstances.

Exception Closed By :

Thickon POrar

Date: $12 / 2 / 97$

\section{APPROVAL OF RESOLUTION}

Contractor Representative: (Only as needed)

WMH Representative:

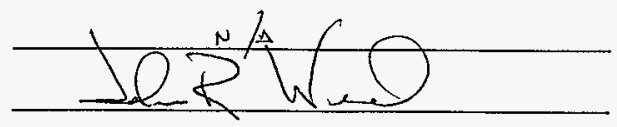

Date:

Date: $[2-12-97$

Additional Approval:

(Only as needed)

Date:

FDNW Representative:

$\checkmark$ pe

Date: 
ATP_YN : N

Test : HNF-SD-W026-OTP-014

Step : 5.2.7/AC

Equipment Tag \#: OP-710
Exception : 9

Revision :

Date:

Specification Section : $\quad 13462$

Test Witness/Author:

Duniap

Test Operator:

\begin{tabular}{ll} 
Responsible Company: & WMH \\
\hline Responsible Subcontractor : & PARSONS \\
\hline Responsible Individual: & Schaefter \\
\hline
\end{tabular}

Exception Description :

OP-710 does not provide direction from section $A, B, C, E$.

\section{Exception Disposition :}

\section{Exception Status :}

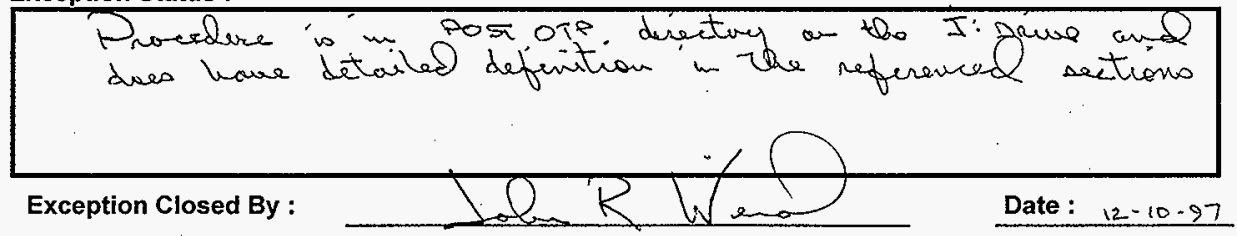

\section{APPROVAL OF RESOLUTION}

Contractor Representative: (Only as needed)

WMH Representative:

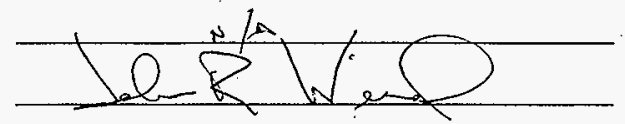

Additional Approval:

(Only as needed)
Date:

Date: $12-10-97$

Date:

Date: 


\section{WRAP ACCEPTANCE TEST PROCEDURE EXCEPTION LOG}

ATP_YN : N

DMS

Test : HNF-SD-W026-OTP-014

Step : 5.2.6/AC

Equipment Tag \#: DMS Software
Exception : 10

Revision :

Date:

Specification Section: DMS

Test Witness/Author:

Dunlap

Test Operator:

Responsible Company: WMH

Responsible Subcontractor: BDM

Responsible Individual: Weldent

Exception Description :

DMMS Screen 342.

Button "Next New Container" needs wording change to include both "orginal" \& "new" container.

Exception Disposition :

Software corrected.

\section{Exception Status :}

Ready to test.

\section{Exception Closed By : Dunlap \\ APPROVAL OF RESOLUTION}

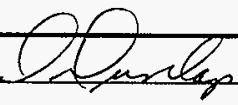

Date : $9 / 12 / 97$

Contractor Representative: (Only as needed)

WMH Representative:

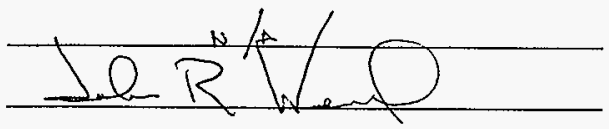

Additional Approval:

(Only as needed)
Date:

Date: $12-$ io -97

Date:

Date: 
ATP_YN : N

Test : HNF-SD-W026-OTP-014

Step : 5.1.12 A/C Equipment Tag \#: DO-07-402B
Exception : 12

\section{Revision :}

Date:

Specification Section : 13462

Test Witness/Author :

Dunlap

Test Operator:

\begin{tabular}{ll} 
Responsible Company: & WMH \\
\hline Responsible Subcontractor : & N/A \\
\hline Responsible Individual: & Lelst \\
\hline
\end{tabular}

Exception Description :

One trip port drum ild would not fit to allow port to open.

Exception Disposition :

Another lid was used and port operated properly.

Exception Status :

closed

Exception Closed By : Dunlap

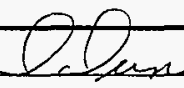

tep

Date : $7 / 18 / 97$

\section{APPROVAL OF RESOLUTION}

Contractor Representative: (Only as needed)

WMH Representative:

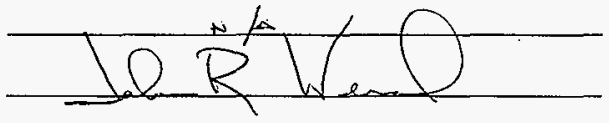

Date:

Date: $12-10-27$

Additional Approval:

(Only as needed)

Date:

FDNW Representative:

$N \Delta$

Date: 
ATP_YN : N

DMS

Test : HNF-SD-W026-OTP-014

Step : 5.14 A7, 5.2.4 Equipment Tag \#: DMS Software

Specification Section : DMS

Test Witness/Author :

Dunlap

Test Operator:

Responsible Company: WMH

Responsible Subcontractor: BDM

Responsible Individual: Weldert

Exception Description :

Message "FRM 40106 no ????? items" would be displayed unexpectedly.

\section{Exception Disposition :}

Software corrected.

\section{Exception Status :}

Ready to test.

\section{APPROVAL OF RESOLUTION}

Contractor Representative: (Only as needed)

WMH Representative:

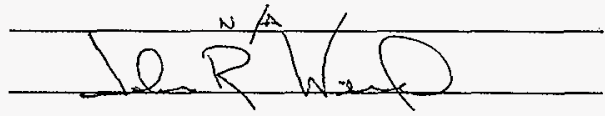

Date:

Date: $12 \cdot 10-97$

Additional Approval:

(Only as needed)

N乎

Date: 


\section{WRAP ACCEPTANCE TEST PROCEDURE}

EXCEPTION LOG

ATP_YN : Y

Test : HNMF-SP-W026-OTP-014

Step : AH.5,5.3

Equipment Tag \#: DMS Software

\section{Exception :}

Revision :

Date:

Specification Section: DMS

Test Witness/Author :

Dunlap

Test Operator:

Responsible Company: $\quad$ WMH
Responsible Subcontractor:
Responsible Individual:

\section{Exception Description :}

Duplicate sample/container (SAMREL) records were recorded in the DMS when the barcodes were scanned multiple times.

Specification is WHC-SD-W026-SDD-001.

\section{Exception Disposition :}

Software corrected.

\section{Exception Status :}

Ready to test.

Exception Closed By :

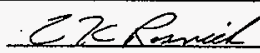

Date: $11 / 12 / 57$

\section{APPROVAL OF RESOLUTION}

Contractor Representative: (Only as needed)

WMH Representative:

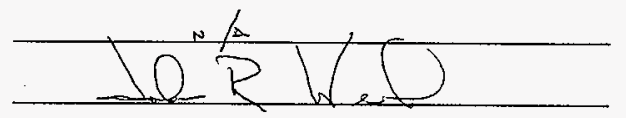

Additional Approval:

(Only as needed)
Date:

Date: $\{z-10-97$

Date:

Date: 
CASE1A.718 DMS DATABASE AT THE END OF TRU/RWM GLOVEBOX OTP CASE $17 / 18 / 97$

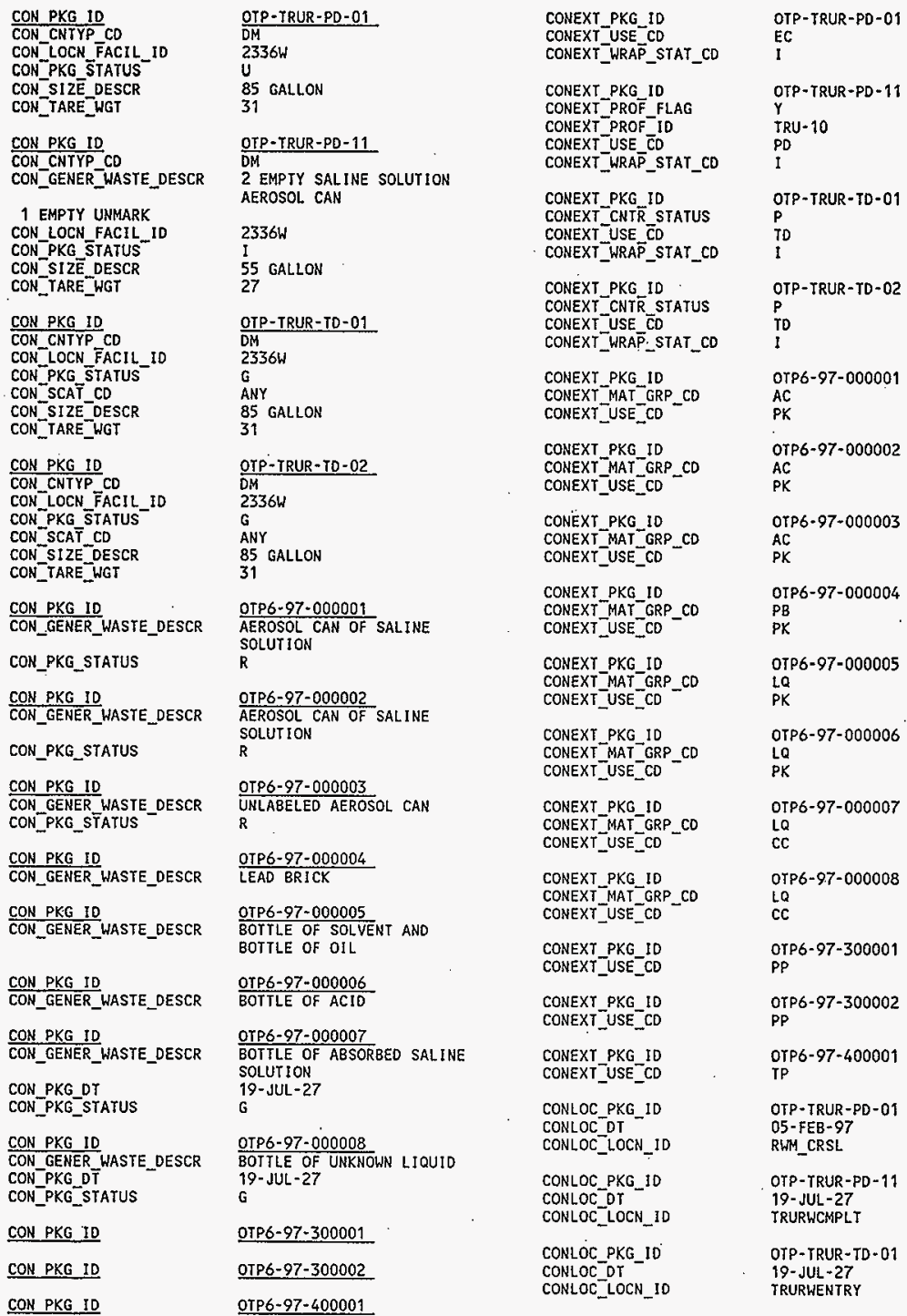




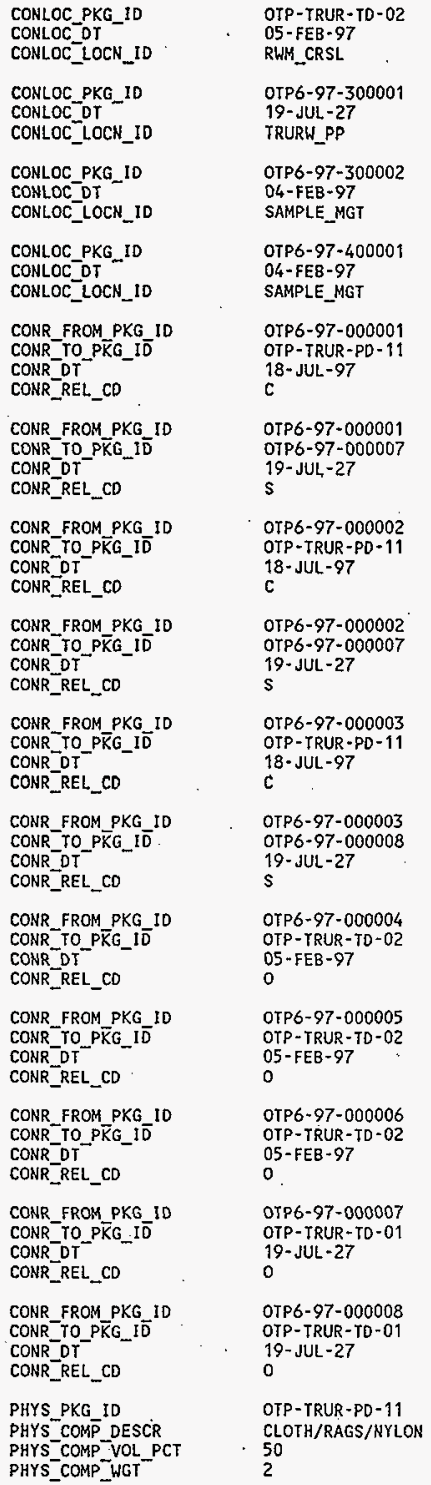

\begin{tabular}{|c|c|}
\hline $\begin{array}{l}\text { PHYS_PKG_ID } \\
\text { PHYS_COMP_DESCR } \\
\text { PHYS_COMP_VOL_PCT } \\
\text { PHYS_COMP_WGI }\end{array}$ & $\begin{array}{l}\text { OIP-TRUR -PD }-11 \\
\text { METAL/IRON/GALVANIZED/SHE } \\
\text { ET } \\
20 \\
1\end{array}$ \\
\hline $\begin{array}{l}\text { PHYS_PKG_ID } \\
\text { PHYS_CONP_DESCR } \\
\text { PHYS_COMP_VOL_PCT } \\
\text { PHYS_COMP_WGT }\end{array}$ & $\begin{array}{l}\text { OTP-TRUR-PD }-11 \\
\text { PLASTIC/POLYURATHANE } \\
30 \\
2\end{array}$ \\
\hline $\begin{array}{l}\text { SAM_SAMPLE_ID } \\
\text { SAM_CLOSED_FLAG } \\
\text { SAM_COMMENTS } \\
\text { SAM_LOCN_ID } \\
\text { SAM_MAIRIX_CD } \\
\text { SAM_IAKEN_ } B Y \\
\text { SAM_TAKEN_DT } \\
\text { SAM_IEMP - } \\
\text { SAM_PKG_IO }\end{array}$ & $\begin{array}{l}97-00001 \\
\text { N } \\
\text { Unknown liquid } \\
\text { TRURWSAMPL } \\
L \\
62264 \\
18-J U L-97 \\
22 \\
\text { OTP6-97-000008 }\end{array}$ \\
\hline $\begin{array}{l}\text { SAR_SAMPLE_ID } \\
\text { SAR_ANAL_CD }\end{array}$ & $\begin{array}{l}97-00001 \\
A Q L I Q\end{array}$ \\
\hline $\begin{array}{l}\text { SAR_SAMPLE_ID } \\
\text { SAR_ANAL_CO }\end{array}$ & $\begin{array}{l}97-00001 \\
\text { COND }\end{array}$ \\
\hline $\begin{array}{l}\text { BOT_BOTTLE_ID } \\
\text { BOT_SAMPLE_ID } \\
\text { BOT_ANAL_CD }\end{array}$ & $\begin{array}{l}97-00001-01 L \\
97-00001 \\
A 0 L 10\end{array}$ \\
\hline $\begin{array}{l}\text { BOr_BOTTLE_ID } \\
\text { BOr_SAMPLE_ID } \\
\text { BOr_ANAL_CD }\end{array}$ & $\begin{array}{l}97-00001-021 \\
97-00001 \\
\text { COND }\end{array}$ \\
\hline $\begin{array}{l}\text { SAMREL_BOTTLE_IO } \\
\text { SAMREL_PKG_ID } \\
\text { SAMREL_DT } \\
\text { SAMREL_USE_CO }\end{array}$ & $\begin{array}{l}97-00001-011 \\
\text { OIPG-97-000008 } \\
18-J U L-97 \\
\text { CC }\end{array}$ \\
\hline $\begin{array}{l}\text { SAMREL_BOTTLE_IO } \\
\text { SAMREL_PKG_ID } \\
\text { SAMREL_OT - } \\
\text { SAMREL_USE_CO }\end{array}$ & $\begin{array}{l}97-00001-01 \mathrm{~L} \\
0 T P 6-97-300001 \\
19-J U L-27 \\
\text { PP }\end{array}$ \\
\hline $\begin{array}{l}\text { SAMREL_BOTTLE_ID } \\
\text { SAMREL_PKG_ID } \\
\text { SAMREL_DT } \\
\text { SAMREL_USE_CO }\end{array}$ & $\begin{array}{l}97-00001-02 L \\
\text { OTP6-97-000008 } \\
18-J U L-97 \\
\text { CC }\end{array}$ \\
\hline $\begin{array}{l}\text { SAMREL_BOTTLE_ID } \\
\text { SAMREL_PKG_ID } \\
\text { SAMREL_DT } \\
\text { SAMREL_USE_CD }\end{array}$ & $\begin{array}{l}97-00001-02 L \\
\text { OTP6-97-300001 } \\
19-J U L-27 \\
\text { PP }\end{array}$ \\
\hline $\begin{array}{l}\text { SAMREL_BOTYLE_ID } \\
\text { SAMREL_PKG_ID- } \\
\text { SAMREL_DT } \\
\text { SAMREL_USE_CD }\end{array}$ & $\begin{array}{l}97-00001-02 L \\
\text { orP }-97-300001 \\
19-J U L-27 \\
\text { PP }\end{array}$ \\
\hline $\begin{array}{l}\text { SAMREL_BOTTLE_ID } \\
\text { SAMREL_PKG_ID- } \\
\text { SAMREL_DT } \\
\text { SAMREL_USE_CO }\end{array}$ & $\begin{array}{l}97-00001-02 L \\
\text { OTP6-97-300001 } \\
19-J U L-27 \\
\text { PP }\end{array}$ \\
\hline $\begin{array}{l}\text { MSGLOG_DT } \\
\text { MSGLOG_ERROR_FLAG } \\
\text { MSGLOG_GEN_TYYPE } \\
\text { MSGLOG_SEQ_NUM } \\
\text { MSGLOG_SPEC TYYPE } \\
\text { MSGLOG_STRINGI }\end{array}$ & 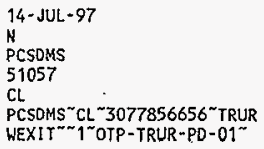 \\
\hline $\begin{array}{l}\text { MSGLOG_OT } \\
\text { MSGLOG_ERROR_FLAG } \\
\text { MSGLOG_GEN_TYYPE }\end{array}$ & $\begin{array}{l}14-\text { JUL- } 97 \\
N \\
\text { PCSDMS }\end{array}$ \\
\hline
\end{tabular}


CASE1A.718 DMS DATABASE AT THE END OF TRU/RWM GLOVEBOX OTP CASE $17 / 18 / 97$

\begin{tabular}{|c|c|c|c|}
\hline $\begin{array}{l}\text { CON PKG ID } \\
\text { CON_CNTYP_CD } \\
\text { CON_LOCN FACIL_IO } \\
\text { CON_PKG_STATUS }\end{array}$ & $\begin{array}{l}\text { OTP-TRUR-PD-01 } \\
\text { DM } \\
2336 \mathrm{~W} \\
\mathrm{U}\end{array}$ & $\begin{array}{l}\text { CONEXT_PXG_ID } \\
\text { CONEXT_USE_CD } \\
\text { CONEXT_WRAP_SIAT_CD }\end{array}$ & $\begin{array}{l}\text { OTP-TRUR-PD-01 } \\
\text { EC } \\
\text { I }\end{array}$ \\
\hline $\begin{array}{l}\text { CON_SIZE_OESCR } \\
\text { CON_TARE_WGT }\end{array}$ & $\begin{array}{l}85 \text { GALLON } \\
31\end{array}$ & $\begin{array}{l}\text { CONEXT_PKG_ID } \\
\text { CONEXT_PROF_FLAG }\end{array}$ & $\begin{array}{l}\text { OTP-TRUR - PD- } 11 \\
Y\end{array}$ \\
\hline $\begin{array}{l}\text { CON PKG 10 } \\
\text { CON_CNYYP_CD } \\
\text { CON_GENER_WASTE_DESCR }\end{array}$ & $\begin{array}{l}\text { OTP-TRUR-PD-11 } \\
\text { DM EMPTY SALINE SOLUTION }\end{array}$ & $\begin{array}{l}\text { CONEXT_PROF_ID } \\
\text { CONEXT_USE_CO } \\
\text { CONEXT_WRAP_STAT_CD }\end{array}$ & $\begin{array}{l}\mathrm{TRU}-10 \\
\mathrm{PD} \\
\mathrm{I}\end{array}$ \\
\hline 1 EMPTY UNMARK & AEROSOL CAN & $\begin{array}{l}\text { CONEXT_PKG_ID } \\
\text { CONEXT_CNTR_STATUS }\end{array}$ & $\begin{array}{l}\text { OTP-TRUR -TD-01 } \\
\text { P }\end{array}$ \\
\hline $\begin{array}{l}\text { CON_LOCN_FACIL_ID } \\
\text { CON_PKG_STATUS } \\
\text { CON_SIZE_DESCR }\end{array}$ & $\begin{array}{l}2336 \mathrm{~W} \\
\text { I } 55 \text { GALLON }\end{array}$ & $\begin{array}{l}\text { CONEXT_USE_CD } \\
\text { CONEXT_WRAP_STAT_CD }\end{array}$ & TO \\
\hline CON_TARE_WGT $^{-}$ & & $\begin{array}{l}\text { CONEXT_PKG_IO } \\
\text { CONEXT_CNTR_STATUS }\end{array}$ & $\begin{array}{l}\text { OTP-TRUR-TD-02 } \\
\text { P }\end{array}$ \\
\hline CON PKG 10 & OTP-TRUR-TD-01 & CONEXI_USE_CD & TO \\
\hline $\begin{array}{l}\text { CON_CNTYP CD } \\
\text { CON_LOCN_FACIL_IO }\end{array}$ & $2336 \mathrm{~W}$ & CONEXI_WRAP_STAI_CD & 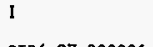 \\
\hline $\begin{array}{l}\text { CONPPKG STIATUS } \\
\text { CON_SCAT_CO }\end{array}$ & & $\begin{array}{l}\text { CONEXI_PKG_ID } \\
\text { CONEXT_MAT_GRP CD }\end{array}$ & $\begin{array}{l}\text { OIPG-97-000001 } \\
A C\end{array}$ \\
\hline $\begin{array}{l}\text { CON_SIZE_DESCR } \\
\text { CON_TARE_WGT }\end{array}$ & $\begin{array}{l}85 \text { GALLON } \\
31\end{array}$ & CONEXI_USE_CD & PK \\
\hline CON PKG ID & OTP-TRUR - TO-02 & $\begin{array}{l}\text { CONEXT_PKG_ID } \\
\text { CONEXT_MAT_GRP_CO }\end{array}$ & $\begin{array}{l}\text { OTP6-97-000002 } \\
\text { AC }\end{array}$ \\
\hline $\begin{array}{l}\text { CON_CNTYP CD } \\
\text { CON_LOCN_FACIL_ID }\end{array}$ & $2336 \mathrm{~W}$ & CONEXT_USE_CD & PK \\
\hline $\begin{array}{l}\text { CON_PKG_STATUS } \\
\text { CON_SCAT_CD }\end{array}$ & G ANY & $\begin{array}{l}\text { CONEXY_PKG_ID } \\
\text { CONEXY_MAT_GRP_CD }\end{array}$ & $\begin{array}{l}\text { OTPG-97-000003 } \\
\text { AC }\end{array}$ \\
\hline $\begin{array}{l}\text { CON_SIZE_DESCR } \\
\text { CON_TARE_WGT }\end{array}$ & $\begin{array}{l}85 \\
31\end{array}$ & CONEXT_USE_CD & PK \\
\hline CON PKG ID & $01 P 6-97-000001$ & $\begin{array}{l}\text { CONEXT_PKG_ID } \\
\text { CONEXT_MAT_GRP_CD }\end{array}$ & $\begin{array}{l}\text { OTP6-97-000004 } \\
\text { PB }\end{array}$ \\
\hline$\overline{C O N}$ COENER_WASTE_DESCR & $\begin{array}{l}\text { AEROSOL CAN OF SALINE } \\
\text { SOLUTION }\end{array}$ & CONEXT_USE_CO - & PK \\
\hline CON_PKG_STATUS & $\mathrm{R}$ & $\begin{array}{l}\text { CONEXT_PKG_ID } \\
\text { CONEXT_MAT_GRP_CD }\end{array}$ & $\begin{array}{l}\text { OTP6-97-000005 } \\
\text { LQ }\end{array}$ \\
\hline$\frac{\text { CON_PKG ID }}{\text { CON_GENER_WASTE_DESCR }}$ & $\frac{\text { OIP6-97-000002 }}{\text { AEROSOL CAN OF SALINE }}$ & CONEXT_USE_CD & PK \\
\hline CON_PKG_STATUS & $\begin{array}{l}\text { SOLUTION } \\
R\end{array}$ & $\begin{array}{l}\text { CONEXT_PKG_ID } \\
\text { CONEXT_MAT_GRP_CD }\end{array}$ & OTP6-97-000006 \\
\hline CON PXG ID & OIP6-97-000003 & CONEXT_USE_CO & PK \\
\hline $\begin{array}{l}\text { CON_GENER_WASTE_DESCR } \\
\text { CON_PKG_STATUS }\end{array}$ & $\begin{array}{l}\text { UNLABELED AEROSOL CAN } \\
R\end{array}$ & $\begin{array}{l}\text { CONEXT_PKG_ID } \\
\text { CONEXT_MAT_GRP_CD } \\
\text { CONEXT_USE_CD - }\end{array}$ & $\begin{array}{l}\text { OTP6-97-000007 } \\
\text { LQ } \\
\text { CC }\end{array}$ \\
\hline$\frac{\text { CON PKG ID }}{\text { CON_GENER_WASTE DESCR }}$ & 0TP6-97-000004 & & \\
\hline CON_GENER_WASTE_DESCR & LEAD BRICK & $\begin{array}{l}\text { CONEXT_PKG_ID } \\
\text { CONEXT_MAT_GRP_CD }\end{array}$ & $\begin{array}{l}\text { OTP6-97-000008 } \\
\text { LQ }\end{array}$ \\
\hline$\frac{\text { CON_PKG ID }}{\text { CON_GENER_WASTE_DESCR }}$ & $\frac{\text { OTP6-97-000005 }}{\text { BOTYLE OF SOLVENT AND }}$ & CONEXT_USE_CO & $\mathrm{CC}$ \\
\hline & BOTTLE OF OIL & $\begin{array}{l}\text { CONEXT_PKG_ID } \\
\text { CONEXT_USE_CD }\end{array}$ & $\begin{array}{l}\text { OTP6-97-300001 } \\
\text { PP }\end{array}$ \\
\hline $\begin{array}{l}\text { CON PKG ID } \\
\text { CON GENER_WASTE DESCR }\end{array}$ & OTP6-97-000006 & & \\
\hline CON_GENER_WASTE_DESCR & BOTTLE OF ACID & $\begin{array}{l}\text { CONEXT_PKG_1D } \\
\text { CONEXT_USE_CD }\end{array}$ & $\begin{array}{l}\text { OTP6-97-300002 } \\
\text { PP }\end{array}$ \\
\hline CON_GENER_WASTE_DESCR & $\begin{array}{l}\text { BOTTLE OF ABSORBED SALINE } \\
\text { SOLUTION } \\
19-J U L-27\end{array}$ & $\begin{array}{l}\text { CONEXT_PKG_ID } \\
\text { CONEXT_USE_CD }\end{array}$ & $\begin{array}{l}\text { OTP6-97-400001 } \\
\text { TP }\end{array}$ \\
\hline CON_PKG_STATUS & $0706=0700000$ & $\begin{array}{l}\text { CONLOC_PKG_ID } \\
\text { CONLOC_DT }\end{array}$ & $\begin{array}{l}\text { OTP-TRUR-PD-01 } \\
05-F E B-97\end{array}$ \\
\hline CON_PKG ID & $\frac{\text { OTP6-97-000008 }}{\text { BOTTLE OF UNKNOWN LIQUID }}$ & CONLOC_LOCN_IO & RWM_CRSL \\
\hline $\begin{array}{l}\text { CON_PKG_D̄ } \\
\text { CON_PKG_STATUS }\end{array}$ & G $19-\mathrm{JUL}-27$ & $\begin{array}{l}\text { CONLOC_PKG_IO } \\
\text { CONLOC_OT } \\
\text { CONLOC_LOCN_10. }\end{array}$ & $\begin{array}{l}\text { OTP-TRUR-PD- } 11 \\
\text { 19-JUL-27 } \\
\text { TRURWCMPLT }\end{array}$ \\
\hline CON PKG 10 & OTP6-97-300001 & & \\
\hline CON PKG 10 & OTP6-97-300002 & $\begin{array}{l}\text { CONLOC_PKG_IO } \\
\text { CONLOC_DT }\end{array}$ & $\begin{array}{l}\text { OTP-TRUR-TD-01 } \\
19-J U L-27 .\end{array}$ \\
\hline CON PKG 10 & OTP6-97-400001 & CONLOC_ & TRURWENTRY \\
\hline
\end{tabular}




\begin{tabular}{|c|c|}
\hline $\begin{array}{l}\text { CONLOC_PKG_ID } \\
\text { CONLOC_DT } \\
\text { CONLOC_LOCN_ID }\end{array}$ & $\begin{array}{l}\text { OTP-TRUR-TD-02 } \\
05-F E B-97 \\
\text { RWM_CRSL }\end{array}$ \\
\hline $\begin{array}{l}\text { CONLOC_PKG_IO } \\
\text { CONLOC_DT } \\
\text { CONLOC_LOCN_ID }\end{array}$ & $\begin{array}{l}\text { OTPG-97-300001 } \\
\text { 19-JUL-27 } \\
\text { TRURW_PP }\end{array}$ \\
\hline $\begin{array}{l}\text { CONLOC_PKG_ID } \\
\text { CONLOC_DT } \\
\text { CONLOC_LOCN_ID }\end{array}$ & $\begin{array}{l}\text { OTPG-97-300002 } \\
\text { O4-FEB-97 } \\
\text { SAMPLE_MGT }\end{array}$ \\
\hline $\begin{array}{l}\text { CONLOC_PKG_ID } \\
\text { CONLOC_DT - } \\
\text { CONLOC_LOCN_ID }\end{array}$ & $\begin{array}{l}\text { OTP6-97-400001 } \\
\text { O4-FEB-97 } \\
\text { SAMPLE_MGT }\end{array}$ \\
\hline $\begin{array}{l}\text { CONR_FROM_PKG_ID } \\
\text { CONR_TO_PKG_ID } \\
\text { CONR_DT- } \\
\text { CONR_REL_CD }\end{array}$ & $\begin{array}{l}\text { OTP6-97-000001 } \\
\text { OTP-YRUR-PD- } 11 \\
18-J U L-97 \\
\text { C }\end{array}$ \\
\hline $\begin{array}{l}\text { CONR_FROM_PKG_ID } \\
\text { CONR_TO_PKG_ID } \\
\text { CONR_DT- } \\
\text { CONR_REL_CD }\end{array}$ & $\begin{array}{l}\text { OTP6-97-000001 } \\
\text { OTP6-97-000007 } \\
19-J U L-27 \\
\text { s }\end{array}$ \\
\hline $\begin{array}{l}\text { CONR_FROM_PKG_ID } \\
\text { CONR_TO_PKG_ID } \\
\text { CONR_DT } \\
\text { CONR_REL_CD }\end{array}$ & $\begin{array}{l}\text { OTP6-97-000002 } \\
\text { OTP-TRUR-PD-11 } \\
18-J U L-97 \\
\text { C }\end{array}$ \\
\hline $\begin{array}{l}\text { CONR_FROM_PKG_ID } \\
\text { CONR_TO_PKG_ID } \\
\text { CONR_OT- } \\
\text { CONR_REL_CD }\end{array}$ & $\begin{array}{l}\text { OTP6-97-000002 } \\
\text { OTP6-97-000007 } \\
19-J U L-27 \\
S\end{array}$ \\
\hline $\begin{array}{l}\text { CONR_FROM_PKG_ID } \\
\text { CONR_TO_PKG_ID } \\
\text { CONR_DT } \\
\text { CONR_REL_CD }\end{array}$ & $\begin{array}{l}\text { OTP6-97-000003 } \\
\text { OTP-TRUR-PD- } 11 \\
18-J U L-97 \\
\text { C }\end{array}$ \\
\hline $\begin{array}{l}\text { CONR_FROM_PKG ID } \\
\text { CONR_YO_PKG_ID } \\
\text { CONR_DT } \\
\text { CONR_REL_CD }\end{array}$ & $\begin{array}{l}\text { OTP6-97-000003 } \\
\text { OTP6-97-000008 } \\
19-\mathrm{JUL}-27 \\
\text { s }\end{array}$ \\
\hline $\begin{array}{l}\text { CONR_FROM_PKG_10 } \\
\text { CONR_TO_PKG_ID } \\
\text { CONR_DT } \\
\text { CONR_REL_CD }\end{array}$ & $\begin{array}{l}\text { OTP6-97-000004 } \\
\text { OTP-TRUR-TD-02 } \\
\text { O5-FEB-97 } \\
0\end{array}$ \\
\hline $\begin{array}{l}\text { CONR_FROM_PKG_ID } \\
\text { CONR_YO_PKG_ID } \\
\text { CONR_OT } \\
\text { CONR_REL_CD }\end{array}$ & $\begin{array}{l}\text { OTP6-97-000005 } \\
\text { OTP-TRUR-TD-02 } \\
\text { 05-FEB-97 } \\
0\end{array}$ \\
\hline $\begin{array}{l}\text { CONR_FROM_PKG ID } \\
\text { CONR_TO_PKG_10 } \\
\text { CONR_DT } \\
\text { CONR_REL_CD }\end{array}$ & $\begin{array}{l}\text { OTPG-97-000006 } \\
\text { OTP-TRUR - TD-02 } \\
\text { O5-FEB-97 } \\
0\end{array}$ \\
\hline $\begin{array}{l}\text { CONR_FROM_PKG_ID } \\
\text { CONR_TO_PKG_I } \overline{0} \\
\text { CONR_DT- } \\
\text { CONR_REL_CD }\end{array}$ & $\begin{array}{l}\text { OTPG-97-000007 } \\
\text { OTP-TRUR-TD-01 } \\
\text { 19-JUL-27 } \\
0\end{array}$ \\
\hline $\begin{array}{l}\text { CONR_FROM_PKG_ID } \\
\text { CONR_TO_PKG_ID } \\
\text { CONR_DT- } \\
\text { CONR_REL_CO }\end{array}$ & $\begin{array}{l}\text { OTPG-97-000008 } \\
\text { OTP-TRUR-TO-01 } \\
19-J U L=27 \\
0\end{array}$ \\
\hline $\begin{array}{l}\text { PHYS_PKG_ID } \\
\text { PHYS_COMP_DESCR } \\
\text { PHYS_COMP_VOL_PCT } \\
\text { PHYS_COMP_WGT- }\end{array}$ & $\begin{array}{l}\text { OTP-TRUR-PD- } 11 \\
\text { CLOTH/RAGS/NYLON } \\
50 \\
2\end{array}$ \\
\hline
\end{tabular}

\begin{tabular}{|c|c|}
\hline $\begin{array}{l}\text { PHYS_PKG_10 } \\
\text { PHYS_COMP_DESCR } \\
\text { PHYS_COMP_VOL_PCT } \\
\text { PHYS_COMP_WGT }\end{array}$ & $\begin{array}{l}\text { OTP-TRUR-PD- } 11 \\
\text { METAL/IRON/GALVANIZED/SHE } \\
\text { ET } \\
20 \\
1\end{array}$ \\
\hline $\begin{array}{l}\text { PHYS_PKG_10 } \\
\text { PHYS_COMP_DESCR } \\
\text { PHYS_COMP_VOL_PCT } \\
\text { PHYS_COMP_WGT }\end{array}$ & $\begin{array}{l}\text { OTP-TRUR-PO- } 11 \\
\text { PLASTIC/POLYURATHANE } \\
30 \\
2\end{array}$ \\
\hline $\begin{array}{l}\text { SAM_SAMPLE_IO } \\
\text { SAM_CLOSED_FLAG } \\
\text { SAM_COMMENTS } \\
\text { SAM_LOCN_ID } \\
\text { SAM_MATRIX_CD } \\
\text { SAM_TAKEN_BY } \\
\text { SAM_TAKEN_DT } \\
\text { SAM_TEMP_ } \\
\text { SAM_PKG_ID }\end{array}$ & $\begin{array}{l}97-00001 \\
N \\
\text { unknown I iquid } \\
\text { TRURWSAMPL } \\
L \\
62264 \\
.18-J U L-97 \\
22 \\
\text { OTP6-97-000008 }\end{array}$ \\
\hline $\begin{array}{l}\text { SAR_SAMPLE_IO } \\
\text { SAR_ANAL_CD }\end{array}$ & $\begin{array}{l}97-00001 \\
\text { AQLIO }\end{array}$ \\
\hline $\begin{array}{l}\text { SAR_SAMPLE_IO } \\
\text { SAR_ANAL_CD }\end{array}$ & $\begin{array}{l}97-00001 \\
\text { COND }\end{array}$ \\
\hline $\begin{array}{l}\text { 8OT_BOTTLE_ID } \\
\text { 8OT_SAMPLE_IO } \\
\text { BOT_ANAL_Cō }\end{array}$ & $\begin{array}{l}97-00001-01 L \\
97-00001 \\
\text { AQLIQ }\end{array}$ \\
\hline $\begin{array}{l}\text { BOT_BOTTLE_IO } \\
\text { BOT_SAMPLE_ID } \\
\text { BOT_ANAL_CD }\end{array}$ & $\begin{array}{l}97-00001-02 L \\
97-00001 \\
\text { COND }\end{array}$ \\
\hline $\begin{array}{l}\text { SAMREL_BOTYLE_ID } \\
\text { SAMREL_PKG_ID } \\
\text { SAMREL_DT - } \\
\text { SAMREL_USE_CD }\end{array}$ & $\begin{array}{l}97-00001-01 L \\
\text { OTP6-97-000008 } \\
18-J U L-97 \\
\text { CC }\end{array}$ \\
\hline $\begin{array}{l}\text { SAMREL_BOTTLE_ID } \\
\text { SAMREL_PKG_ID } \\
\text { SAMREL_DT_ } \\
\text { SAMREL_USE_CO }\end{array}$ & $\begin{array}{l}97-00001-01 L \\
0 T P 6-97-300001 \\
19-J U L-27 \\
\text { PP }\end{array}$ \\
\hline $\begin{array}{l}\text { SAMREL_BOTTLE_ID } \\
\text { SAMREL_PKG_ID- } \\
\text { SAMREL_DT - } \\
\text { SAMREL_USE_CD }\end{array}$ & $\begin{array}{l}97-00001-02 L \\
\text { OTP6-97-000008 } \\
18-J U L-97 \\
\text { CC }\end{array}$ \\
\hline $\begin{array}{l}\text { SAMREL_BOTTLE_ID } \\
\text { SAMREL_PKG_IO } \\
\text { SAMREL_DT } \\
\text { SAMREL_USE_CD }\end{array}$ & $\begin{array}{l}97-00001-02 L \\
0 T P 6-97-300001 \\
19-J U L-27 \\
\text { PP }\end{array}$ \\
\hline $\begin{array}{l}\text { SAMREL_BOTTLE_ID } \\
\text { SAMREL_PKG_ID-- } \\
\text { SAMREL_DT_- } \\
\text { SAMREL_USE_CD }\end{array}$ & $\begin{array}{l}97-00001-02 L \\
\text { OTP6-97-300001 } \\
19-J U L-27 \\
\text { PP }\end{array}$ \\
\hline $\begin{array}{l}\text { SAMREL_BOTTLE_10 } \\
\text { SAMREL_PKG_ID- } \\
\text { SAMREL_DT - } \\
\text { SAMREL_USE_CD }\end{array}$ & $\begin{array}{l}97-00001-02 L \\
\text { OTP6-97-300001 } \\
19-J U L-27 \\
\text { PP }\end{array}$ \\
\hline $\begin{array}{l}\text { RADMAT_ID } \\
\text { RADMAT_DESCR } \\
\text { RADMAT_LIMIT } \\
\text { RADMAT_RAD_TOT } \\
\text { RADMAT_UNITS } \\
\text { RADMAT_ALARM. }\end{array}$ & $\begin{array}{l}\text { F } \\
\text { WRAP } 1 \text { FACILITY } \\
100 \\
10 \\
\text { CI } \\
N\end{array}$ \\
\hline $\begin{array}{l}\text { RADMAT_10 } \\
\text { RADMAT_DESCR } \\
\text { RADMAT_LIMIT }\end{array}$ & $\begin{array}{l}\text { R } \\
\text { TRU RWM GLOVEBOX } \\
200\end{array}$ \\
\hline
\end{tabular}


HNF-SD-W026-OTR-014, Rev. 0, Page DMS-5

RADMAT_RAD_YOT

RADMAT_UNTTS

RADMAT_ALARM

RADMAT_IO

RADMAT DESCR

RADMAT_LIMIT

RADMAT RAD TOT

RADMAT UNITS

RADMAT_ALARM

150
FGE
N
T
TRU GLOVEBOX
200
110
FGE
$\mathrm{N}$


CASE2A.721 DMS DATABASE AT THE END OF TRU/RWM GLOVEBOX OTP CASE 2 7/21/97

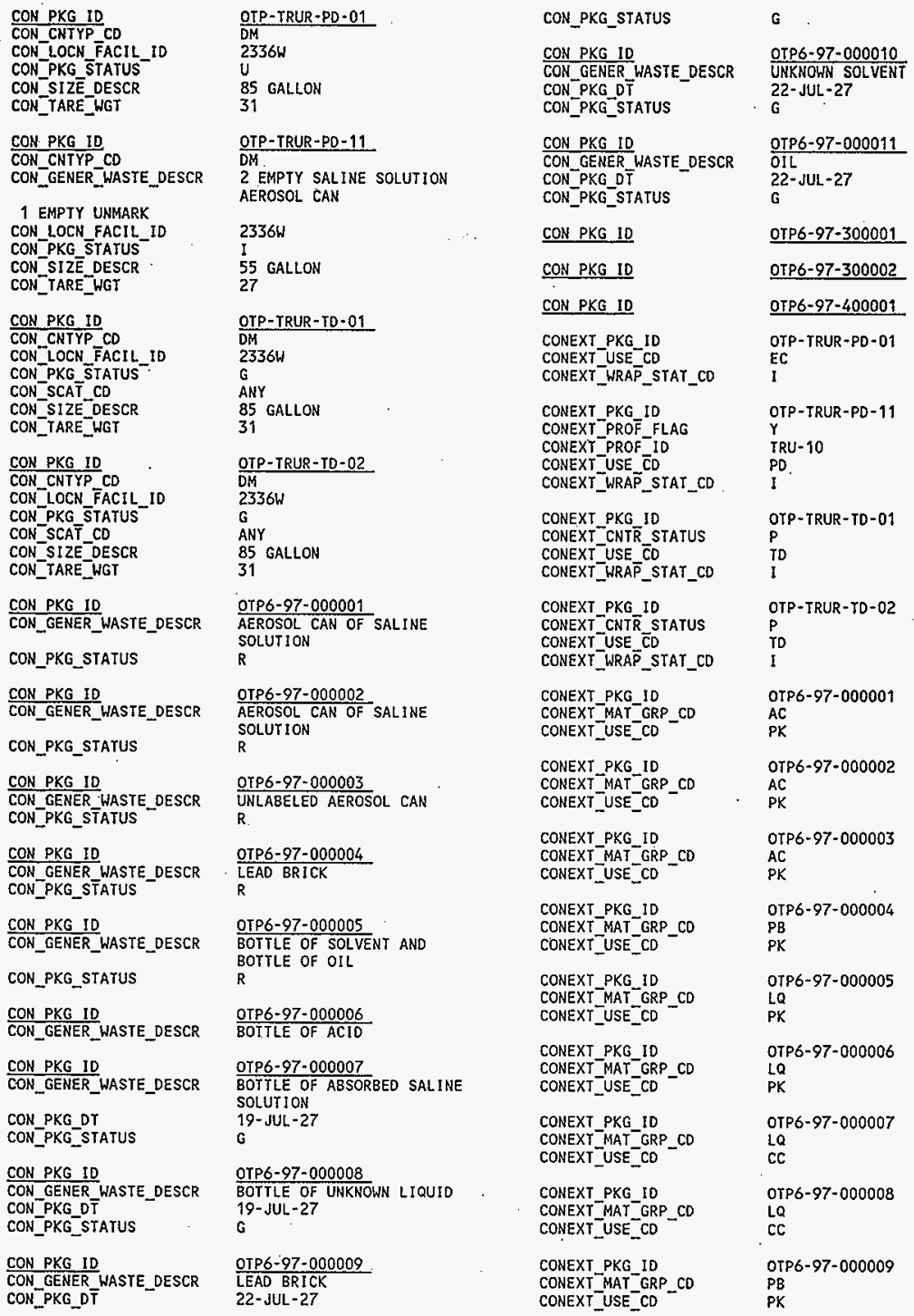




\begin{tabular}{|c|c|}
\hline $\begin{array}{l}\text { CONEXT_PKG_ID } \\
\text { CONEXT_MAT_GRP_CD } \\
\text { CONEXT_USE_CD - }\end{array}$ & $\begin{array}{l}\text { OTPG-97-000010 } \\
\text { LQ } \\
\text { PK }\end{array}$ \\
\hline $\begin{array}{l}\text { CONEXT_PKG_ID } \\
\text { CONEXT_MAT_GRP_CD } \\
\text { CONEXT_USE_CD }\end{array}$ & $\begin{array}{l}\text { OTP6-97-000011 } \\
\text { OL } \\
\text { PK }\end{array}$ \\
\hline $\begin{array}{l}\text { CONEXT_PKG_ID } \\
\text { CONEXT_USE_CD }\end{array}$ & $\begin{array}{l}\text { OIP6-97-300001 } \\
\text { PP }\end{array}$ \\
\hline $\begin{array}{l}\text { CONEXT_PKG_ID } \\
\text { CONEXT_USE_CD }\end{array}$ & $\begin{array}{l}\text { OTP6-97-300002 } \\
\text { PP }\end{array}$ \\
\hline $\begin{array}{l}\text { CONEXT_PKG_ID } \\
\text { CONEXT_USE_CD }\end{array}$ & $\begin{array}{l}\text { OTPG-97-400001 } \\
\text { TP }\end{array}$ \\
\hline $\begin{array}{l}\text { CONLOC_PKG_ID } \\
\text { CONLOC_DT } \\
\text { CONLOC_LOCN_ID }\end{array}$ & $\begin{array}{l}\text { OTP-TRUR-PD-01 } \\
\text { O5-FE8-97 } \\
\text { RWM_CRSL }\end{array}$ \\
\hline $\begin{array}{l}\text { CONLOC_PKG_ID } \\
\text { CONLOC_DY } \\
\text { CONLOC_LOCN_ID }\end{array}$ & $\begin{array}{l}\text { OTP-TRUR-PO-11 } \\
19-J U L-27 \\
\text { TRURWCMPLT }\end{array}$ \\
\hline $\begin{array}{l}\text { CONLOC_PKG_ID } \\
\text { CONLOC_DT } \\
\text { CONLOC_LOCN_ID }\end{array}$ & $\begin{array}{l}\text { OTP-TRUR-TO-01 } \\
19-J U L-27 \\
\text { TRURWENTRY }\end{array}$ \\
\hline $\begin{array}{l}\text { CONLOC_PKG_ID } \\
\text { CONLOC_DI } \\
\text { CONLOC_LOCN_ID }\end{array}$ & $\begin{array}{l}\text { OTP-TRUR-TD-02 } \\
22-J U L-27 \\
\text { TRURWENTRY }\end{array}$ \\
\hline $\begin{array}{l}\text { CONLOC_PKG_10 } \\
\text { CONLOC_DT } \\
\text { CONLOC_LOCN_ID }\end{array}$ & $\begin{array}{l}\text { OTP6-97-000008 } \\
22-J U L-27 \\
\text { TRURWSAMPL }\end{array}$ \\
\hline $\begin{array}{l}\text { CONLOC_PKG_ID } \\
\text { CONLOC_OT } \\
\text { CONLOC_LOCN_ID }\end{array}$ & $\begin{array}{l}\text { OTP6-97-300001 } \\
19-J U L-27 \\
\text { TRURW_PP }\end{array}$ \\
\hline $\begin{array}{l}\text { CONLOC_PKG_10 } \\
\text { CONLOC_DI } \\
\text { CONLOC_LOCN_ID }\end{array}$ & $\begin{array}{l}\text { OTP6- } 97-300002 \\
22-J U L-27 \\
\text { TRURW_PP }\end{array}$ \\
\hline $\begin{array}{l}\text { CONLOC_PKG_IO } \\
\text { CONLOC_DT } \\
\text { CONLOC_LOCN_ID }\end{array}$ & $\begin{array}{l}\text { OTP6-97-400001 } \\
04-F E 8-97 \\
\text { SAMPLE_MGT }\end{array}$ \\
\hline $\begin{array}{l}\text { CONR_FROM_PKG_ID } \\
\text { CONR_TO_PKG_ID } \\
\text { CONR_DT } \\
\text { CONR_REL_CD }\end{array}$ & $\begin{array}{l}\text { OTP6-97-000001 } \\
\text { OTP-TRUR-PD- } 11 \\
\text { 18-JUL-97 } \\
\text { C }\end{array}$ \\
\hline $\begin{array}{l}\text { CONR_FROM_PKG_ID } \\
\text { CONR_TO_PKG_ID } \\
\text { CONR_DT_ } \\
\text { CONR_REL_CD }\end{array}$ & $\begin{array}{l}\text { OTP6-97-000001 } \\
\text { OTP6-97-000007 } \\
19-J U L-27 \\
\text { s }\end{array}$ \\
\hline $\begin{array}{l}\text { CONR_FROM_PKG_ID } \\
\text { CONR_IO_PKGG_ID } \\
\text { CONR_DI } \\
\text { CONR_REL_CD }\end{array}$ & $\begin{array}{l}\text { OTPG-97-000002 } \\
\text { OTP-TRUR-PD- } 11 \\
18-\text { JUL- } 97 \\
\text { C }\end{array}$ \\
\hline $\begin{array}{l}\text { CONR_FROM_PKG_ID } \\
\text { CONR_TO_PKG_ID } \\
\text { CONR_OT } \\
\text { CONR_REL_CD }\end{array}$ & $\begin{array}{l}\text { OTP6-97-000002 } \\
\text { OTP6-97-000007 } \\
19-J U L-27 \\
\text { s }\end{array}$ \\
\hline $\begin{array}{l}\text { CONR_FROM_PKG_ID } \\
\text { CONR_IO_PKG_ID } \\
\text { CONR_DY } \\
\text { CONR_REL_CD }\end{array}$ & $\begin{array}{l}\text { OTP6-97-000003 } \\
\text { OTP-TRUR-PD- } 11 \\
18-J U L-97 \\
\text { C }\end{array}$ \\
\hline $\begin{array}{l}\text { CONR FROM_PKG_ID } \\
\text { CONR_TO_PKG_I }\end{array}$ & $\begin{array}{l}\text { OTP6-97-000003 } \\
\text { 0TP6-97-000008 }\end{array}$ \\
\hline
\end{tabular}

\begin{tabular}{|c|c|}
\hline $\begin{array}{l}\text { CONR_OT } \\
\text { CONR_REL_CD }\end{array}$ & $\begin{array}{l}19-J U L-27 \\
s\end{array}$ \\
\hline $\begin{array}{l}\text { CONR_FROM_PKG_ID } \\
\text { CONR_TO_PKG_IO } \\
\text { CONR_DT } \\
\text { CONR_REL_CD }\end{array}$ & $\begin{array}{l}\text { OTP6-97-000004 } \\
\text { OTP-TRUR -PD } 11 \\
21-J U L-97 \\
C\end{array}$ \\
\hline $\begin{array}{l}\text { CONR_FROM_PKG_ID } \\
\text { CONR_TO_PKG_ID } \\
\text { CONR_DT } \\
\text { CONR_REL_CD }\end{array}$ & $\begin{array}{l}\text { OTP6-97-000004 } \\
\text { OTP6-97-000009 } \\
22-J U L-27 \\
\$\end{array}$ \\
\hline $\begin{array}{l}\text { CONR_FROM PKG ID } \\
\text { CONR_TO_PKG_ID } \\
\text { CONR_DT } \\
\text { CONR_REL_CD }\end{array}$ & $\begin{array}{l}\text { OTP6-97-000005 } \\
\text { OrP-IRUR-PD-11 } \\
21-J U L-97 \\
\text { C }\end{array}$ \\
\hline $\begin{array}{l}\text { CONR_FROM_PKG_ID } \\
\text { CONR_TO_PKG_ID } \\
\text { CONR_DT } \\
\text { CONR_REL_CO }\end{array}$ & $\begin{array}{l}\text { OrP6-97-000005 } \\
\text { OTP6-97-000010 } \\
22-J U L-27 \\
\text { S }\end{array}$ \\
\hline $\begin{array}{l}\text { CONR_FROM_PKG_ID } \\
\text { CONR_TO PKG_ID } \\
\text { CONR_DT } \\
\text { CONR_REL_CD }\end{array}$ & $\begin{array}{l}\text { OTP6-97-000005 } \\
\text { OTP6-97-000011 } \\
22-J U L-27 \\
\text { S }\end{array}$ \\
\hline $\begin{array}{l}\text { CONR_FROM_PKG_ID } \\
\text { CONR_TO_PKG_ID } \\
\text { CONR_DT } \\
\text { CONR_REL_CD }\end{array}$ & $\begin{array}{l}\text { OTP6-97-000006 } \\
\text { OTP-TRUR-TO-02 } \\
22-J U L-27 \\
0\end{array}$ \\
\hline $\begin{array}{l}\text { CONR_FROM_PKG_ID } \\
\text { CONR_YO_PKG_ID } \\
\text { CONR_DT } \\
\text { CONR_REL_CD }\end{array}$ & $\begin{array}{l}\text { OTP6-97-000007 } \\
\text { OTP-TRUR-TD-01 } \\
19-\text { JUL-27 } \\
0\end{array}$ \\
\hline $\begin{array}{l}\text { CONR_FROM_PKG_ID } \\
\text { CONR_TO_PKG_ID } \\
\text { CONR_OT } \\
\text { CONR_REL_CO }\end{array}$ & $\begin{array}{l}\text { OTP6-97-000009 } \\
\text { OTP-TRUR-TD-02 } \\
22-J U L-27 \\
0\end{array}$ \\
\hline $\begin{array}{l}\text { CONR_FROM_PKG_ID } \\
\text { CONR_TO_PKG_ID } \\
\text { CONR_OT } \\
\text { CONR_REL_CD }\end{array}$ & $\begin{array}{l}\text { OTPG-97-000010 } \\
\text { OTP-TRUR-TO-02 } \\
22-J U L-27 \\
0\end{array}$ \\
\hline $\begin{array}{l}\text { CONR_FROM_PKG_ID } \\
\text { CONR_TO_PXG_ID } \\
\text { CONR_DT- } \\
\text { CONR_REL_CD }\end{array}$ & $\begin{array}{l}\text { OTP6-97-000011 } \\
\text { OTP-TRUR -TD-02 } \\
22-J U L-27 \\
0\end{array}$ \\
\hline $\begin{array}{l}\text { PHYS_PKG_ID } \\
\text { PHYS_COMP_DESCR } \\
\text { PHYS_COMP_VOL_PCT } \\
\text { PHYS_COMP_WGT }\end{array}$ & $\begin{array}{l}\text { OTP-TRUR-PD- } 11 \\
\text { CLOTH/RAGS/NYLON } \\
60 \\
4\end{array}$ \\
\hline $\begin{array}{l}\text { PHYS_PKG_ID } \\
\text { PHYS_COMP_DESCR } \\
\text { PHYS_COMP_VOL_PCT } \\
\text { PHYS_COMP_WGT }\end{array}$ & $\begin{array}{l}\text { OTP-TRUR-PD- } 11 \\
\text { METAL/IRON/GALVANIZED/SHE } \\
\text { ET } \\
10 \\
1\end{array}$ \\
\hline $\begin{array}{l}\text { PHYS_PKG_ID } \\
\text { PHYS_COMP_DESCR } \\
\text { PHYS_COMP_VOL_PCT } \\
\text { PHYS_COMP_WGT }\end{array}$ & $\begin{array}{l}\text { OTP-TRUR-PD- } 11 \\
\text { PLASTIC/POLYURATHANE } \\
30 \\
3\end{array}$ \\
\hline $\begin{array}{l}\text { SAM_SAMPLE_ID } \\
\text { SAM_CLOSED_FLAG } \\
\text { SAM_COMMENTS } \\
\text { SAM_LOCN_ID } \\
\text { SAM_MATRIX_CD } \\
\text { SAM_TAKEN_BY } \\
\text { SAM_TAKEN_DT }\end{array}$ & $\begin{array}{l}97-00001 \\
N \\
\text { unknown Iliquid } \\
\text { TRURWSAMPL } \\
\mathrm{L} \\
62264 \\
18+J U L-97\end{array}$ \\
\hline
\end{tabular}




\begin{tabular}{|c|c|}
\hline $\begin{array}{l}\text { SAM_TEMP } \\
\text { SAM_PKG_ID }\end{array}$ & $\begin{array}{l}22 \\
\text { OTP6-97-000008 }\end{array}$ \\
\hline $\begin{array}{l}\text { SAM_SAMPLE_ID } \\
\text { SAM_CLOSED_FLAG } \\
\text { SAM_COMMENTS } \\
\text { SAM_LOCN_IO } \\
\text { SAM_MAIRIX_CD } \\
\text { SAM_TAKEN_BY } \\
\text { SAM_TAKEN_DT } \\
\text { SAM_TEMP - } \\
\text { SAM_PKG_ID }\end{array}$ & $\begin{array}{l}97-00002 \\
\text { N } \\
\text { unkown solvents } \\
\text { TRURWSAMPL } \\
\text { L } \\
62264 \\
21-J U L-97 \\
22 \\
\text { OTP6-97-000010 }\end{array}$ \\
\hline $\begin{array}{l}\text { SAM_SAMPLE_ID } \\
\text { SAM_CLOSED_FLAG } \\
\text { SAM_COMMENTS } \\
\text { SAM_LOCN_ID } \\
\text { SAM_MATRIX_CD } \\
\text { SAM_TAKEN_BY } \\
\text { SAM_TAKEN_DI } \\
\text { SAM_TEMP - } \\
\text { SAM_PKG_ID }\end{array}$ & $\begin{array}{l}97-00003 \\
N \\
\text { Unknown solvent } \\
\text { TRURWSAMPL } \\
\text { 6 } \\
62264 \\
21=\mathrm{JUL}-97 \\
22 \\
\text { OTP6-97-000010 }\end{array}$ \\
\hline $\begin{array}{l}\text { SAM_SAMPLE_IO } \\
\text { SAM_CLOSED_FLAG } \\
\text { SAM_COMMENTS } \\
\text { SAM_LOCN_ID } \\
\text { SAM_MATRTX_CD } \\
\text { SAM_TAKEN_BY } \\
\text { SAM_IAKEN_DT } \\
\text { SAM_TEMP } \\
\text { SAM_PKG_ID }\end{array}$ & $\begin{array}{l}97-00004 \\
N \\
\text { oil } \\
\text { TRURWSAMPL } \\
L \\
62264 \\
21-J U L-97 \\
22 \\
\text { OTP6-97-000011 }\end{array}$ \\
\hline $\begin{array}{l}\text { SAR_SAMPLE_ID } \\
\text { SAR_ANAL_CD }\end{array}$ & $\begin{array}{l}97-00001 \\
\text { AQL1Q }\end{array}$ \\
\hline $\begin{array}{l}\text { SAR_SAMPLE_ID } \\
\text { SAR_ANAL_CD }\end{array}$ & $\begin{array}{l}97-00001 \\
\text { COND }\end{array}$ \\
\hline $\begin{array}{l}\text { SAR_SAMPLE ID } \\
\text { SAR_ANAL_CD }\end{array}$ & $\begin{array}{l}97-00002 \\
\text { SOLVENTS }\end{array}$ \\
\hline $\begin{array}{l}\text { SAR_SAMPLE ID } \\
\text { SAR_ANAL_CD }\end{array}$ & $\begin{array}{l}97-00003 \\
\text { SOLVENTS }\end{array}$ \\
\hline $\begin{array}{l}\text { SAR_SAMPLE_ID } \\
\text { SAR_ANAL_C } \bar{D}\end{array}$ & $\begin{array}{l}97-00004 \\
\text { OILS }\end{array}$ \\
\hline $\begin{array}{l}\text { BOT_BOTTLE_IO } \\
\text { BOT_SAMPLE_IO } \\
\text { BOT_ANAL_CD }\end{array}$ & $\begin{array}{l}97-00001-01 L \\
97-00001 \\
\text { AQL1Q }\end{array}$ \\
\hline $\begin{array}{l}\text { BOT_BOTTLE_ID } \\
\text { BOT_SAMPLE_ID } \\
\text { BOT_ANAL_CD }\end{array}$ & $\begin{array}{l}97-00001-02 L . \\
97-00001 \\
\text { COND }\end{array}$ \\
\hline $\begin{array}{l}\text { BOT_BOTTLE_ID } \\
\text { BOT_SAMPLE_ID } \\
\text { BOT_ANAL_CD }\end{array}$ & $\begin{array}{l}97-00002-01 L \\
97-00002 \\
\text { SOLVENTS }\end{array}$ \\
\hline $\begin{array}{l}\text { BOT_BOTTLE_ID } \\
\text { BOT_SAMPLE_ID } \\
\text { BOT_ANAL_CD }\end{array}$ & $\begin{array}{l}\text { 97-00003-01L } \\
97-00003 \\
\text { SOLVENTS }\end{array}$ \\
\hline $\begin{array}{l}\text { BOT_BOTTLE_ID } \\
\text { BOT_SAMPLE_ID } \\
\text { BOT_ANAL_CD }\end{array}$ & $\begin{array}{l}97-00004-01 L \\
97-00004 \\
\text { OILS }\end{array}$ \\
\hline $\begin{array}{l}\text { SAMREL_BOTTLE_ID } \\
\text { SAMREL_PKG_ID-I } \\
\text { SAMREL_DT - } \\
\text { SAMREL_USE_CD }\end{array}$ & $\begin{array}{l}97-00001-01 L \\
\text { oTP6-97-000008 } \\
18-J U L-97 \\
C C\end{array}$ \\
\hline $\begin{array}{l}\text { SAMREL_BOT TLE_ID } \\
\text { SAMREL_PKG_ID- } \\
\text { SAMREL_DT. }\end{array}$ & $\begin{array}{l}97-00001-01 \mathrm{~L} \\
\text { OTP6-97-300001 } \\
19-\mathrm{JUL}-27\end{array}$ \\
\hline
\end{tabular}

\begin{tabular}{|c|c|}
\hline SAMREL_USE_CD & PP \\
\hline $\begin{array}{l}\text { SAMREL_BOTTLE_ID } \\
\text { SAMREL_PKG_ID-ID } \\
\text { SAMREL_DT } \\
\text { SAMREL_USE_CD }\end{array}$ & $\begin{array}{l}97-00001-02 L \\
\text { OTP6-97-000008 } \\
18-J U L-97 \\
\text { CC }\end{array}$ \\
\hline $\begin{array}{l}\text { SAMREL_BOTTLE_ID } \\
\text { SAMREL_PKG_ID-ID } \\
\text { SAMREL_DT } \\
\text { SAMREL_USE_CD }\end{array}$ & $\begin{array}{l}97-00001-02 L \\
\text { 0TP6-97-300001 } \\
19-J U L-27 \\
\text { PP }\end{array}$ \\
\hline $\begin{array}{l}\text { SAMREL_BOTTLE_ID } \\
\text { SAMREL_PKG_ID } \\
\text { SAMREL_DT - } \\
\text { SAMREL_USE_CD }\end{array}$ & $\begin{array}{l}97-00001-02 L \\
0 T P 6-97-300001 \\
19-J U L-27 \\
\text { PP }\end{array}$ \\
\hline $\begin{array}{l}\text { SAMREL_BOTTLE_ID } \\
\text { SAMREL_PKG_ID- } \\
\text { SAMREL_OT_ } \\
\text { SAMREL_USE_CD }\end{array}$ & $\begin{array}{l}97-00001-02 L \\
\text { OTP6-97-300001 } \\
19-J U L-27 \\
\text { PP }\end{array}$ \\
\hline $\begin{array}{l}\text { SAMREL_BOTTLE_ID } \\
\text { SAMREL_PKG_10- } \\
\text { SAMREL_OY } \\
\text { SAMREL_USE_CD }\end{array}$ & $\begin{array}{l}97-00002-01 \mathrm{~L} \\
07 P 6-97-000010 \\
21-J U L=97 \\
\text { PK }\end{array}$ \\
\hline $\begin{array}{l}\text { SAMREL_BOTTLE_ID } \\
\text { SAMREL_PKG_1D- } \\
\text { SAMREL_OT } \\
\text { SAMREL_USE_CD }\end{array}$ & $\begin{array}{l}97-00002-011 \\
\text { OTP6-97-300002 } \\
22-J U L-27 \\
\text { PP }\end{array}$ \\
\hline $\begin{array}{l}\text { SAMREL_BOTTLE_ID } \\
\text { SAMREL_PKG_ID- } \\
\text { SAMREL_DT } \\
\text { SAMREL_USE_CO }\end{array}$ & $\begin{array}{l}97-00003-01 \mathrm{~L} \\
0 T P 6-97-000010 \\
21-J U L-97 \\
\text { PK }\end{array}$ \\
\hline $\begin{array}{l}\text { SAMREL_BOTTLE_10 } \\
\text { SAMREL_PKG_10-10 } \\
\text { SAMREL_OI } \\
\text { SAMREL_USE_CD }\end{array}$ & $\begin{array}{l}97-00003-01 \mathrm{~L} \\
\text { OTP6-97-300002 } \\
22-\mathrm{JUL}-27 \\
\text { PP }\end{array}$ \\
\hline $\begin{array}{l}\text { SAMREL_BOTTLE_10 } \\
\text { SAMREL_PKG_ID- } \\
\text { SAMREL_OI } \\
\text { SAMREL_USE_CD }\end{array}$ & $\begin{array}{l}97-00004-01 \mathrm{~L} \\
\text { OTP6-97-000011 } \\
21-J U L-97 \\
\text { PK }\end{array}$ \\
\hline $\begin{array}{l}\text { SAMREL_BOTTLE_ID } \\
\text { SAMREL_PKG_ID- } \\
\text { SAMREL_DT } \\
\text { SAMREL_USE_CD }\end{array}$ & $\begin{array}{l}97-00004-01 L \\
\text { OTP6-97-300002 } \\
22-J U L-27 \\
\text { PP }\end{array}$ \\
\hline $\begin{array}{l}\text { SCRN_SCRN_ID } \\
\text { SCRN_PKG_IO } \\
\text { SCRN_DT } \\
\text { SCRN_LOCN_ID } \\
\text { SCRN_PERS_10 } \\
\text { SCRN_SAMPLING_METHOD_CD }\end{array}$ & $\begin{array}{l}97-00003-01 F \\
\text { OTPG-97-000009 } \\
21-J U L-97 \\
\text { TRURWSAMPL } \\
62264 \\
\text { INS }\end{array}$ \\
\hline $\begin{array}{l}\text { SCRN_SCRN_ID } \\
\text { SCRN_PKC_ID } \\
\text { SCRN_DT } \\
\text { SCRN_LOCN_ID } \\
\text { SCRN_PERS-1D } \\
\text { SCRN_SAMPLING_METHOD_CD }\end{array}$ & $\begin{array}{l}97-00004-01 \mathrm{~F} \\
\text { OTP6-97-000010 } \\
21-J U L-97 \\
\text { TRURWSAMPL } \\
\text {-62264 } \\
\text { INS }\end{array}$ \\
\hline $\begin{array}{l}\text { SCRN_SCRN_10 } \\
\text { SCRN_PKG_ID } \\
\text { SCRN_DT } \\
\text { SCRN_LOCN_10 } \\
\text { SCRN_PERS_ID } \\
\text { SCRN_SAMPLING_METHOD_CD }\end{array}$ & $\begin{array}{l}97-00005-01 F \\
\text { OTP6-97-000011 } \\
21-J U L-97 \\
\text { TRURWSAMPL } \\
\text { 62264 } \\
\text { INS }\end{array}$ \\
\hline $\begin{array}{l}\text { SCRN_SCRN_10 } \\
\text { SCRN_PKG_10 } \\
\text { SCRN_DT }\end{array}$ & $\begin{array}{l}97-00006-01 \mathrm{~F} \\
\text { OTP6-97-000006 } \\
21-J U L=97\end{array}$ \\
\hline
\end{tabular}




\begin{tabular}{|c|c|}
\hline $\begin{array}{l}\text { SCRN_LOCN_ID } \\
\text { SCRN_PERS_ID } \\
\text { SCRN_SAMPLING_METHOD_CD }\end{array}$ & $\begin{array}{l}\text { TRURWSAMPL } \\
62264 \\
\text { INS }\end{array}$ \\
\hline $\begin{array}{l}\text { FIELD_SCRN_10 } \\
\text { FIELD_FAT_CD } \\
\text { field_res }\end{array}$ & $\begin{array}{l}\text { 97-00003-01F } \\
\text { LEAD } \\
\text { FIELD ENTRY }\end{array}$ \\
\hline $\begin{array}{l}\text { FIELO_SCRN_10 } \\
\text { FIELD_FAT_CD } \\
\text { field_res }\end{array}$ & $\begin{array}{l}97-00004-01 F \\
\text { CLOR } \\
\text { FIELD ENTRY }\end{array}$ \\
\hline $\begin{array}{l}\text { FIELD_SCRN_ID } \\
\text { FIELD_FAT_CD } \\
\text { field_res }\end{array}$ & $\begin{array}{l}97-00005-01 F \\
\text { PCBO } \\
\text { FIELD ENTRY }\end{array}$ \\
\hline $\begin{array}{l}\text { FIELD_SCRN_ID } \\
\text { FIELD_FAT_CD } \\
\text { field_res }\end{array}$ & $\begin{array}{l}97-00006-01 F \\
\text { PHDP } \\
\text { FIELD ENTRY }\end{array}$ \\
\hline $\begin{array}{l}\text { RADMAT_ID } \\
\text { RADMAT_DESCR } \\
\text { RADMAT_LIMIT } \\
\text { RADMAT_RAD_TOT } \\
\text { RADMAT_UNITS } \\
\text { RAOMAT_ALARM }\end{array}$ & $\begin{array}{l}\text { W } \\
\text { WRAP } 1 \text { FACILITY } \\
100 \\
10 \\
\mathrm{CI} \\
\mathrm{N}\end{array}$ \\
\hline $\begin{array}{l}\text { RAOMAT_ID } \\
\text { RADMAT_DESCR } \\
\text { RADMAT_LIMIT } \\
\text { RADMAT_RAD_TOT } \\
\text { RADMAT_UNITS } \\
\text { RAOMAT_ALARM }\end{array}$ & $\begin{array}{l}\text { R } \\
\text { TRU RWM GLOVEBOX } \\
200 \\
150 \\
\text { FGE } \\
N\end{array}$ \\
\hline $\begin{array}{l}\text { RADMAT_IO } \\
\text { RADMAT_DESCR } \\
\text { RADMAT_LIMIT } \\
\text { RADMAT_RAO TOT } \\
\text { RADMAT_UNITS } \\
\text { RAOMAT_ALARM }\end{array}$ & $\begin{array}{l}T \\
\text { IRU GLOVEBOX } \\
200 \\
110 \\
\text { FGE } \\
\mathrm{N}\end{array}$ \\
\hline
\end{tabular}


otp_tr2.sq] $7 / 15 / 97$

SCRIPT TO UPDATE THE DMS DATABASE PRIOR TO THE START OF THE TRU RHM GLOVEBOX OTP CASE 3

spool otp_tr2.out;

UPDATE CONLOC

SET CONLOC DT' = sysdate, CONLOC_LOCN_ID = 'RWM_CRSL'

WHERE CONLOCC_PKG_ID = 'OTP-TRUR-TD-OT';

UPDATE CONLOC

SET CONLOC DT = sysdate, CONLOC LOCN ID = 'RWM_CRSL'

WHERE CONLOC_ PKG_ID = 'OTP-TRUR-TD-0 $\overline{2}$ !;

UPDATE CONLOC

SET CONLOC DT = sysdate,

CONLOC LOCN ID = 'SAMPLE MGT'

WHERE CONLOC_PKG_ID = 'OTTP6-97-300001';

UPDATE CONLOC

SET CONLOC DT = sysdate,

CONLOC LOCN ID = 'SAMPLE MGT'

WHERE CONLOC_PKG_ID = 'OTP6-97-400001';

UPDATE BOTTLE

SET BOT RETURN DT = sysdate

WHERE BOT_BOTTLE_ID $=$ '97-00002-01L';

UPDATE BOTTLE

SET BOT RETURN DT = sysdate

WHERE BOT_BOTTLE_ID = '97-00003-01L';

UPDATE BOTTLE

SET BOT RETURN DT = sysdate

WHERE BOT_BOTTLE_ID $=$ '97-00004-01L';

INSERT INTO WORKSHEET(WORKSHEET_ID)

VALUES('OTPWRKOI');

INSERT INTO WORKSHEET (WORKSHEET_ID)

VALUES( 'OTPWRKO2');

insert into worksheet(worksheet_id)

values ('OTPWRK03');

insert into worksheet (worksheet_id)

values ('OTPWRKO4');

insert into worksheet(worksheet_id)

values ('OTPWRK05');

update worksheet

set worksheet_name $=^{\prime}$ Packet $7^{\prime}$, 
worksheet_proc_num $=$ 'OP-0711-ABS',

worksheet_proc_rev num $=$ ' $A$ ',

worksheet_proc_instr = 'Treat Saline Solution Items: OTP6-97-000007

Instructions:

Treat in collection container

Add Chemsorb'

where worksheet_id = 'OTPWRK01';

update worksheet

set worksheet_name $={ }^{\prime}$ Packet $8^{\prime}$,

worksheet_proc_num = 'OP-0711-NEUT',

worksheet_proc_rev_num $={ }^{\prime} A$ ',

worksheet proc instr $=$ 'Treat Acetic Acid Solution Items:

0TP6-97-000008

Instructions:

Treat in collection container

Add

Add Bicarbonate of soda

where worksheet_id = 'OTPWRKO2';

update worksheet

set worksheet name $={ }^{\prime}$ Packet $10^{\prime}$,

worksheet proc num = 'OP-0711-ABS',

worksheet proc rev num $={ }^{\prime} A$ ',

worksheet_prō $\bar{c}_{-} i n \bar{s} t r=$ 'Treat Solvent : OTP6-97-000010 OTP00002-01L

Instructions:

OTP00003-01L

Use treatment container

Add Embiber beads'

where worksheet_id = 'OTPWRKO3';

update worksheet

set worksheet name $={ }^{\prime}$ Packet $11^{t}$,

worksheet proc num = 'OP-0711-ABS',

worksheet proc $\bar{r}$ ev num $=$ ' $A$ ',

worksheet_prōc_instr = 'Treat oil Items: 0TP6-97-000011

OTP00004-01L

97-00004-01L

Instructions:

Use treatment container

Add chemsorb'

where worksheet_id $=$ 'OTPWRKO4';

update worksheet

set worksheet_name $=^{\prime}$ Packet $\sigma^{\prime}$, worksheet_proc num $=$ 'OP-071I-NEUT', worksheet_proc-rev_num $={ }^{\prime} A$ ', worksheet_proc instr = 'Treat Acid solution Items: 0 0TP6-97-000006

Instructions:

0TP00004-011

Treat in packet container

Add bicarbonate of soda 
Add chemsorb'

where worksheet_id = 'OTPWRK05';

insert into workitem

values ('OTPWRKO1', 'OTP6-97-000007');

insert into workitem

values ('0TPWRKO2' , '0TP6-97-000008');

insert into workitem

values( 'OTPWRK03', ' OTP6-97-000010');

insert into workitem

values('0TPWRK03', ' 97-00002-01L');

insert into workitem

values('OTPWRK03' , ' 97-00003-01L');

insert into workitem

- values('OTPWRK04', ' 97-00004-01L');

insert into workitem

values('OTPWRK04' , 'ATP6-97-000011');

insert into workitem

values('OTPWRK05' , '0TP6-97-000006!') ; commit;

spool off; 
CASE3B.722 DMS DATABASE AT THE START OF TRU/RWM GLOVEBOX OTP CASE $3 \quad 7 / 22 / 97$

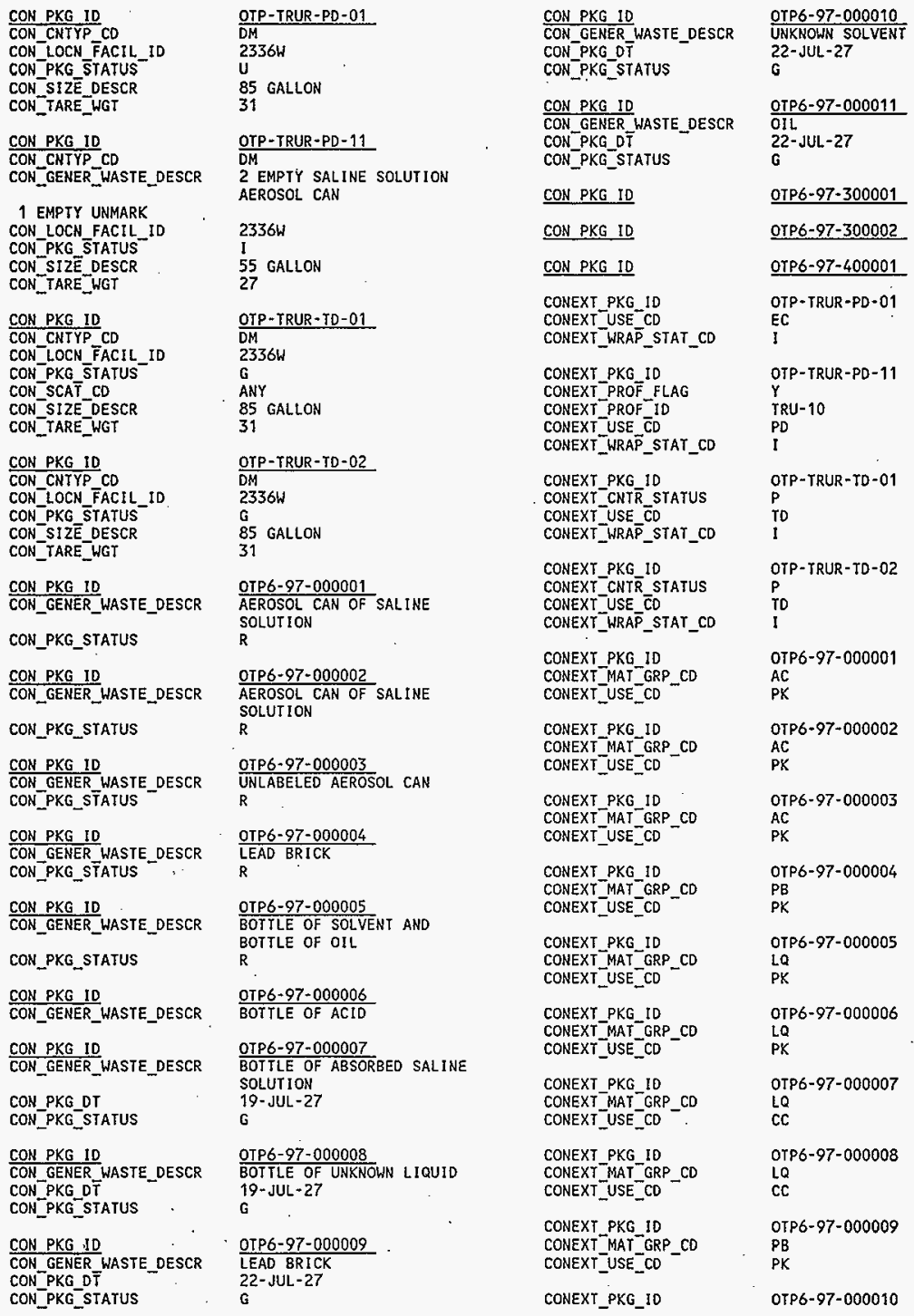




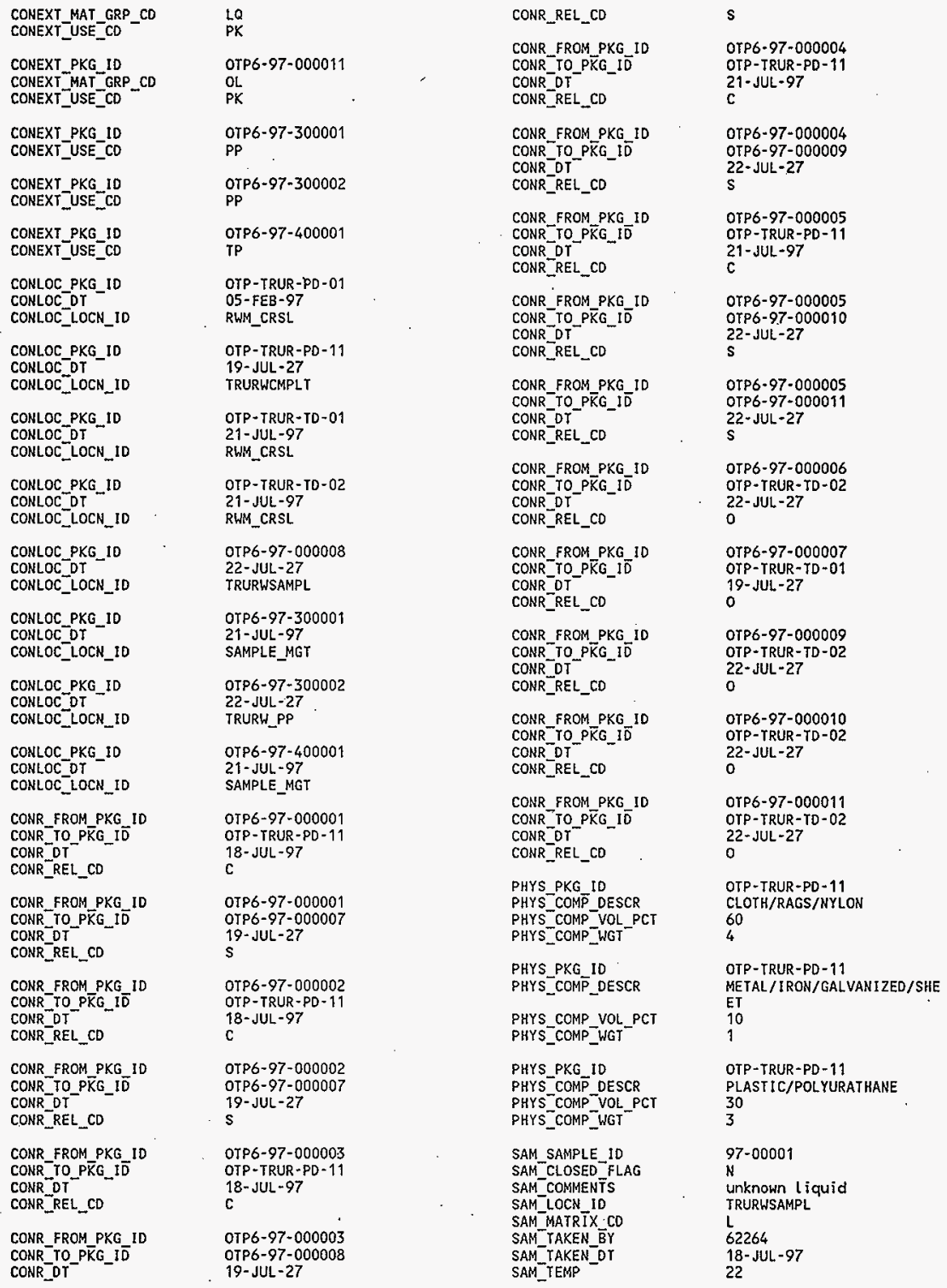




\begin{tabular}{|c|c|}
\hline SAM_PKG_ID & 0rp6-97-000008 \\
\hline $\begin{array}{l}\text { SAM_SAMPLE_ID } \\
\text { SAH_CLOSED_FLAG } \\
\text { SAM_COMMENTS } \\
\text { SAM_LOCN_ID } \\
\text { SAM_MATRIX_CD } \\
\text { SAM_TAKEN_BY } \\
\text { SAM_TAKEN_DT } \\
\text { SAM_TEMP_- } \\
\text { SAM_PKG_IO }\end{array}$ & $\begin{array}{l}97-00002 \\
N \\
\text { unkown solvents } \\
\text { TRURWSAMPL } \\
\text { L } \\
62264 \\
21-J U L-97 \\
22 \\
\text { OTP6-97-000010 }\end{array}$ \\
\hline $\begin{array}{l}\text { SAM_SAMPLE_ID } \\
\text { SAM_CLOSED_FLAG } \\
\text { SAM_COMMENIS } \\
\text { SAM_LOCN_ID } \\
\text { SAM_MATRIX_CD } \\
\text { SAM_TAKEN_BY } \\
\text { SAM_TAKEN_DT } \\
\text { SAM_TEMP - } \\
\text { SAM_PKG_ID }\end{array}$ & $\begin{array}{l}97-00003 \\
N \\
\text { Unknown solvent } \\
\text { TRURWSAMPL } \\
\text { L } \\
62264 \\
21-\text { JUL- } 97 \\
22 \\
\text { OTP6-97-000010 }\end{array}$ \\
\hline $\begin{array}{l}\text { SAM_SAMPLE_ID } \\
\text { SAM_CLOSED_FLAG } \\
\text { SAM_COMMENTS } \\
\text { SAM_LOCN_ID } \\
\text { SAM_MATRIX_CD } \\
\text { SAM_TAKEN_BY } \\
\text { SAM_IAKEN_DT } \\
\text { SAM_TEMP } \\
\text { SAM_PKG_ID }\end{array}$ & $\begin{array}{l}97-00004 \\
N \\
\text { oil } \\
\text { IRURWSAMPL } \\
\text { L } \\
62264 \\
21-J U L-97 \\
22 \\
\text { OTP6-97-000011 }\end{array}$ \\
\hline $\begin{array}{l}\text { SAR_SAMPLE_ID } \\
\text { SAR_ANAL_CD }\end{array}$ & $\begin{array}{l}97-00001 \\
\text { AQLIO }\end{array}$ \\
\hline $\begin{array}{l}\text { SAR_SAMPLE_IO } \\
\text { SAR_ANAL_CD }\end{array}$ & $\begin{array}{l}97-00001 \\
\text { COND }\end{array}$ \\
\hline $\begin{array}{l}\text { SAR_SAMPLE_ID } \\
\text { SAR_ANAL_CD }\end{array}$ & $\begin{array}{l}97-00002 \\
\text { SOLVENTS }\end{array}$ \\
\hline $\begin{array}{l}\text { SAR_SAMPLE_ID } \\
\text { SAR_ANAL_CD }\end{array}$ & $\begin{array}{l}97-00003 \\
\text { SOLVENTS }\end{array}$ \\
\hline $\begin{array}{l}\text { SAR_SAMPLE_ID } \\
\text { SAR_ANAL_CD }\end{array}$ & $\begin{array}{l}97-00004 \\
\text { OILS }\end{array}$ \\
\hline $\begin{array}{l}\text { BOT_BOTTLE_ID } \\
\text { BOT_SAMPLE_IO } \\
\text { BOT_ANAL_C } \bar{D}\end{array}$ & $\begin{array}{l}97-00001-01 L \\
97-00001 \\
\text { AQL } 10\end{array}$ \\
\hline $\begin{array}{l}\text { BOT_BOTYLE_ID } \\
\text { BOT_SAMPLE_ID } \\
\text { BOT_ANAL_CD }\end{array}$ & $\begin{array}{l}97-00001-02 L \\
97-00001 \\
\text { COND }\end{array}$ \\
\hline $\begin{array}{l}\text { BOT_BOTTLE_IO } \\
\text { BOT_SAMPLE_ID } \\
\text { BOT_RETURN_DT } \\
\text { BOT_ANAL_CD }\end{array}$ & $\begin{array}{l}97-00002-01 L \\
97-00002 \\
21-J U L-97 \\
\text { SOLVENTS }\end{array}$ \\
\hline $\begin{array}{l}\text { BOT_BOTTLE_ID } \\
\text { BOT_SAMPLE_ID } \\
\text { BOT_RETURN_DT } \\
\text { BOT_ANAL_CD }\end{array}$ & $\begin{array}{l}97-00003-01 L \\
97-00003 \\
21-J U L-97 \\
\text { SOLVENTS }\end{array}$ \\
\hline $\begin{array}{l}\text { BOT_BOTTLE_ID } \\
\text { BOT_SAMPLE_ID } \\
\text { BOT_RETURN_DT } \\
\text { BOT_ANAL_CD }\end{array}$ & $\begin{array}{l}97-00004-01 L \\
97-00004 \\
21-J U L-97 \\
\text { OILS }\end{array}$ \\
\hline $\begin{array}{l}\text { SAMREL_BOTTLE_ID } \\
\text { SAMREL_PKG_ID } \\
\text { SAMREL_DT } \\
\text { SAMREL_USE_CD }\end{array}$ & $\begin{array}{l}97-00001-01 L \\
\text { 0TP6-97-000008 } \\
18-J U L-97 \\
\text { CC }\end{array}$ \\
\hline SAMREL_BOTTLE_ID & $97-00001-01 L$ \\
\hline
\end{tabular}

\begin{tabular}{|c|c|}
\hline $\begin{array}{l}\text { SAMREL_PKG_10 } \\
\text { SAMREL_DI - } \\
\text { SAMREL_USE_CD }\end{array}$ & $\begin{array}{l}\text { OTP6-97-300001 } \\
19-J U L-27 \\
\text { PP }\end{array}$ \\
\hline $\begin{array}{l}\text { SAMREL_BOTTLE_ID } \\
\text { SAMREL_PKG_IO- } \\
\text { SAMREL_DT } \\
\text { SAMREL_USE_CD }\end{array}$ & $\begin{array}{l}97-00001-02 L \\
\text { OTP6-97-000008 } \\
18-J U L-97 \\
\text { CC }\end{array}$ \\
\hline $\begin{array}{l}\text { SAMREL_BOTTLE_ID } \\
\text { SAMREL_PKG_ID } \\
\text { SAMREL_DI - } \\
\text { SAMREL_USE_CD }\end{array}$ & $\begin{array}{l}97-00001-02 \mathrm{~L} \\
\text { 0IP6-97-300001 } \\
19-\mathrm{JUL}-27 \\
\text { PP }\end{array}$ \\
\hline $\begin{array}{l}\text { SAMREL_BOTTLE_ID } \\
\text { SAMREL_PKG_1D- } \\
\text { SAMREL_DT } \\
\text { SAMREL_USE_CD }\end{array}$ & $\begin{array}{l}97-00001-02 L \\
\text { OTP6-97-300001 } \\
19-J U L-27 \\
\text { PP }\end{array}$ \\
\hline $\begin{array}{l}\text { SAMREL_BOTTLE_ID } \\
\text { SAMREL_PKG_ID } \\
\text { SAMREL_DT } \\
\text { SAMREL_USE_CD }\end{array}$ & $\begin{array}{l}97-00001-02 L \\
\text { OTPG-97-300001 } \\
19-J U L-27 \\
\text { PP }\end{array}$ \\
\hline $\begin{array}{l}\text { SAMREL_BOTTLE_ID } \\
\text { SAMREL_PKG_ID- } \\
\text { SAMREL_DT } \\
\text { SAMREL_USE_CO }\end{array}$ & $\begin{array}{l}97-00002-01 \mathrm{~L} \\
\text { OTP6-97-000010 } \\
21-J U L-97 \\
\text { PK }\end{array}$ \\
\hline $\begin{array}{l}\text { SAMREL_BOTTLE_ID } \\
\text { SAMREL_PKG_ID- } \\
\text { SAMREL_DT - } \\
\text { SAMREL_USE_CD }\end{array}$ & $\begin{array}{l}97-00002-01 \mathrm{~L} \\
\text { OTP6-97-300002 } \\
22-J U L-27 \\
\text { PP }\end{array}$ \\
\hline $\begin{array}{l}\text { SAMREL_BOTTLE_ID } \\
\text { SAMREL_PKG_ID- } \\
\text { SAMREL_DT } \\
\text { SAMREL_USE_CD }\end{array}$ & $\begin{array}{l}97-00003-01 \mathrm{~L} \\
0 T P 6-97-000010 \\
21-\mathrm{JUL}-97 \\
\text { PK }\end{array}$ \\
\hline $\begin{array}{l}\text { SAMREL_BOTTLE_10 } \\
\text { SAMREL_PKG_IO- } \\
\text { SAMREL_OT - } \\
\text { SAMREL_USE_CD }\end{array}$ & $\begin{array}{l}97-00003-01 \mathrm{~L} \\
\text { OTP6-97-300002 } \\
22-\text { JUL }-27 \\
\text { PP }\end{array}$ \\
\hline $\begin{array}{l}\text { SAMREL_BOTTLE_ID } \\
\text { SAMREL_PKG_ID- } \\
\text { SAMREL_DT - } \\
\text { SAMREL_USE_CO }\end{array}$ & $\begin{array}{l}97-00004-01 \mathrm{~L} \\
\text { OTP6-97-000011 } \\
21-J U L-97 \\
\text { PK }\end{array}$ \\
\hline $\begin{array}{l}\text { SAMREL_BOTTLE_ID } \\
\text { SAMREL_PKG_ID- } \\
\text { SAMREL_OT - } \\
\text { SAMREL_USE_CD }\end{array}$ & $\begin{array}{l}97-00004-01.4 \\
\text { OTP6-97-300002 } \\
22-J U L-27 \\
\text { PP }\end{array}$ \\
\hline $\begin{array}{l}\text { SCRN_SCRN_ID } \\
\text { SCRN_PKG_ID } \\
\text { SCRN_DT - . } \\
\text { SCRN_LOCN_ID } \\
\text { SCRN_PERS_ID } \\
\text { SCRN_SAMPLING_METHOD_CD }\end{array}$ & $\begin{array}{l}97-00003-01 F \\
\text { OTP6-97-000009 } \\
21-J U L-97 \\
\text { TRURWSAMPL } \\
62264 \\
\text { INS }\end{array}$ \\
\hline $\begin{array}{l}\text { SCRN_SCRN_ID } \\
\text { SCRN_PKG_ID } \\
\text { SCRN_DT- } \\
\text { SCRN_LOCN_ID } \\
\text { SCRN_PERS_ID } \\
\text { SCRN_SAMPLING_METHOD_CD }\end{array}$ & $\begin{array}{l}97-00004-01 \mathrm{~F} \\
\text { OTP6-97-000010 } \\
21-J U L-97 \\
\text { TRURWSAMPL } \\
\text { 62264 } \\
\text { INS }\end{array}$ \\
\hline $\begin{array}{l}\text { SCRN_SCRN_ID } \\
\text { SCRN_PKG_ID } \\
\text { SCRN_DT } \\
\text { SCRN_LOCN_ID } \\
\text { SCRN_PERS_ID } \\
\text { SCRN_SAMPLENG_METHOD_CD }\end{array}$ & $\begin{array}{l}97-00005-01 \mathrm{~F} \\
\text { OTP6-97-000011 } \\
21-J U L-97 \\
\text { TRURWSAMPL } \\
62264 \\
\text { INS }\end{array}$ \\
\hline SCRN_SCRN_ID & $97-00006-01 F$ \\
\hline
\end{tabular}




\begin{tabular}{|c|c|}
\hline $\begin{array}{l}\text { SCRN_PKG_ID } \\
\text { SCRN_DT } \\
\text { SCRN_LOCN_ID } \\
\text { SCRN_PERS_ID } \\
\text { SCRN_SAMPI.ING_METHOD_CO }\end{array}$ & $\begin{array}{l}\text { OTP6-97-000006 } \\
21-J U L-97 \\
\text { TRURWSAMPL } \\
\text { 62264 } \\
\text { INS }\end{array}$ \\
\hline $\begin{array}{l}\text { FIELD_SCRN_ID } \\
\text { FIELD_FAT_CD } \\
\text { field_res }\end{array}$ & $\begin{array}{l}97-00003-01 F \\
\text { LEAD } \\
\text { FIELD ENTRY }\end{array}$ \\
\hline $\begin{array}{l}\text { FIELD_SCRN_ID } \\
\text { FIELD_FAT_CD } \\
\text { field_res }\end{array}$ & $\begin{array}{l}97-00004-01 F \\
\text { CLOR } \\
\text { FIELD ENTRY }\end{array}$ \\
\hline $\begin{array}{l}\text { FIELD_SCRN_ID } \\
\text { FIELD_FAT_CD } \\
\text { field_res }\end{array}$ & $\begin{array}{l}97-00005-01 \mathrm{~F} \\
\text { PCBO } \\
\text { FIELD ENTRY }\end{array}$ \\
\hline $\begin{array}{l}\text { FIELD_SCRN_IO } \\
\text { FIELD_FAT_CO } \\
\text { field_res }\end{array}$ & $\begin{array}{l}97-00006-01 F \\
\text { PHDP } \\
\text { FIELD ENTRY }\end{array}$ \\
\hline $\begin{array}{l}\text { WORKSHEET_ID } \\
\text { WORKSHEET_NAME } \\
\text { WORKSHEET_PROC_NUM } \\
\text { WORKSHEET_PROC_REV NUM } \\
\text { WORKSHEET_PROC_INSTR } \\
\text { I TEMS }\end{array}$ & $\begin{array}{l}\text { OTPWRKO1 } \\
\text { PACKET } 7 \\
\text { OP-0711-ABS } \\
\text { A } \\
\text { TREAT SALINE SOLUTION } \\
\text { OTP6-97-000007 }\end{array}$ \\
\hline $\begin{array}{l}\text { WORKSHEET_ID } \\
\text { WORKSHEET_NANE } \\
\text { WORKSHEET_PROC_NUM } \\
\text { WORKSHEET_PROC_REV_NUM } \\
\text { WORKSHEET_PROC_INSTR }\end{array}$ & $\begin{array}{l}\text { OTPWRKO2 } \\
\text { PACKET } 8 \\
\text { OP-0711-NEUT } \\
\text { A } \\
\text { TREAT ACETIC ACID } \\
\text { SOLUTION ITEMS } \\
\text { OTP6-97-000008 }\end{array}$ \\
\hline $\begin{array}{l}\text { WORKSHEET_ID } \\
\text { WORKSHEET_NAME } \\
\text { WORKSHEET_PROC_NUM } \\
\text { WORKSHEET_PROC_REV_NUM } \\
\text { WORKSHEET_PROC_INSTR }\end{array}$ & $\begin{array}{l}\text { OTPWRK03 } \\
\text { PACKET } 10 \\
\text { OP-0711-ABS } \\
\text { A } \\
\text { TREAT SOLVENT } \\
\text { OTP6-97-000010 }\end{array}$ \\
\hline $\begin{array}{l}\text { WORKSHEET_ID } \\
\text { WORKSHEET_NAME } \\
\text { WORKSHEET_PROC_NUM } \\
\text { WORKSHEET_PROC_REV_NUM } \\
\text { WORKSHEET_PROC_INSTR }\end{array}$ & $\begin{array}{l}\text { OTPWRKO4 } \\
\text { PACKET 11 } \\
\text { OP-0711-ABS } \\
\text { A } \\
\text { TREAT OIL ITEMS } \\
\text { OTPG-97-000011 }\end{array}$ \\
\hline $\begin{array}{l}\text { WORKSHEET_ID } \\
\text { WORKSHEET_NAME } \\
\text { WORKSHEET_PROC_NUM } \\
\text { WORKSHEET_PROC_REV_NUM } \\
\text { WORKSHEET_PROC_INSTR } \\
\text { ITEMS }\end{array}$ & $\begin{array}{l}\text { OTPWRK0 } \\
\text { PACKET } 6 \\
\text { OP-0711-NEUT } \\
\text { A } \\
\text { TREAT ACID SOLUTION } \\
\text { OTP6-97-000006 }\end{array}$ \\
\hline $\begin{array}{l}\text { WORKITEM_IO } \\
\text { WORKITEM_PKG_ID }\end{array}$ & $\begin{array}{l}\text { OTPWRK01 } \\
\text { OIP6-97-000007 }\end{array}$ \\
\hline $\begin{array}{l}\text { WORKITEM_ID } \\
\text { WORKITEM_PKG_ID }\end{array}$ & $\begin{array}{l}\text { OTPWRK02 } \\
\text { OTP6-97-000008 }\end{array}$ \\
\hline $\begin{array}{l}\text { WORKITEM_ID } \\
\text { WORKITEM_PKG_ID }\end{array}$ & $\begin{array}{l}\text { OTPWRK03 } \\
97-00002-01 L\end{array}$ \\
\hline $\begin{array}{l}\text { WORKITEM_ID } \\
\text { WORKITEM_PKG_ID }\end{array}$ & $\begin{array}{l}\text { OTPWRK03 } \\
97-00003-01 \mathrm{~L}\end{array}$ \\
\hline $\begin{array}{l}\text { WORKITEM_ID } \\
\text { WORKITEM_PKG_ID }\end{array}$ & $\begin{array}{l}\text { OTPWRKO3 } \\
\text { OTP6-97-000010 }\end{array}$ \\
\hline
\end{tabular}

\begin{tabular}{|c|c|}
\hline $\begin{array}{l}\text { WORKIIEM_1D } \\
\text { WORKITEM_PKG_IO }\end{array}$ & $\begin{array}{l}\text { OTPWRK04 } \\
97-00004-01 \mathrm{~L}\end{array}$ \\
\hline $\begin{array}{l}\text { WORKITEM_ID } \\
\text { WORKITEM_PKG_ID }\end{array}$ & $\begin{array}{l}\text { OTPWRK04 } \\
\text { OTP6-97-000011 }\end{array}$ \\
\hline $\begin{array}{l}\text { WORKITEM_IO } \\
\text { WORKITEM_PKG_ID }\end{array}$ & $\begin{array}{l}\text { OTPWRK05 } \\
\text { OTP6-97-000006 }\end{array}$ \\
\hline $\begin{array}{l}\text { RADMAT_ID } \\
\text { RADMAT_DESCR } \\
\text { RADMAT_LIMIT } \\
\text { RADMAT_RAD_TOT } \\
\text { RADMAT_UNITS } \\
\text { RADMAT_ALARM }\end{array}$ & $\begin{array}{l}\text { F } \\
\text { WRAP } 1 \text { FACILITY } \\
100 \\
10 \\
\text { CI } \\
\text { N }\end{array}$ \\
\hline $\begin{array}{l}\text { RADMAT_ID } \\
\text { RADMAT_DESCR } \\
\text { RADMAT_LINIT } \\
\text { RADMAT_RAD_TOT } \\
\text { RADMAT_UN! TS } \\
\text { RADMAT_ALARM }\end{array}$ & $\begin{array}{l}\text { R } \\
\text { TRU RWM GLOVEBOX } \\
200 \\
150 \\
\text { FGE } \\
N\end{array}$ \\
\hline $\begin{array}{l}\text { RADMAT_ID } \\
\text { RADMAT_DESCR } \\
\text { RADMAT_LIMIT } \\
\text { RADMAT_RAD TOT } \\
\text { RADMAT_UNITS } \\
\text { RADMAT-ALARM }\end{array}$ & $\begin{array}{l}T \\
\text { TRU GLOVEBOX } \\
200 \\
110 \\
\text { FGE } \\
\mathrm{N}\end{array}$ \\
\hline
\end{tabular}


CASE3A.722 DMS DATABASE AT THE END OF TRU/RWM GLOVEBOX OTP CASE $3 \quad 7 / 22 / 97$

CON_PKG 10
CON_CNTYP_CO
CON_LOCN_FACIL_ID
CON_PKG_STATUS
CON_PWTYP_CD
CON_SCAT_CD
CON_S1ZE_DESCR
CON_TARE_WGT
CON PKG 10
CON_CNTYP_CD
CON_GENER_WASTE_DESCR

1 EMPTY UNMARK

CON_LOCN_FACIL_ID

CON_PKG STATUS

CON ${ }^{-} I Z \bar{E}$ DESCR

CON_TARE_WGT

CON PKG ID

CON CNTYP CD

CON_LOCN FACIL ID

CONPKG STATUS

CON SCAT_CD

CON SIZEDESCR

CON_TARE_WGT

CON PKG 10

CON CNTYP CD

CONLOCN FACIL ID

CONPKG_STATUS

CON_SIZE_DESCR

CON_TARE_WGT

CON PKG ID

CON PKG ID

CON_GENER_WASTE_DESCR

CON_PKG_STATUS

CON PKG ID

CON_GENER_WASTE_DESCR

CON_PKG_STATUS

CON PKG ID

CON GENER WASTE DESCR

CON_PKG_STATUS

CON PKG ID

CON GENER WASTE DESCR

CONPKG_STTATUS

CON PKG ID

CON_GENER_WASTE_DESCR

CON_PKG_STATUS

CON PKG ID

CON_GENER_WASTE_DESCR

CON PKG ID

CON_GENTER_WASTE_DESCR

CON_PKG_DT

CON_PKG_STATUS

CON PKG ID

CON_GENER_WASTE_DESCR

CON PKG D $\bar{T}$

CON_PKG_STATUS

\begin{tabular}{l} 
OTP-TRUR-PD-01 \\
\hline DM \\
$2336 \mathrm{~W}$ \\
$\mathrm{U}$ \\
$\mathrm{R}$ \\
TRU \\
85 GALLON \\
31
\end{tabular}

OTP-TRUR-PD-11

2 EMPTY SALINE SOLUTION AEROSOL CAN

$2336 \mathrm{~W}$
I 5 GALLON
27

OTP-TRUR-TD-01 CON PKG ID

DM

$2336 \mathrm{~W}$

ANY

85 GALLON

31

OTP-TRUR-TO-02

DM $336 \mathrm{~W}$

85 GALLON

31

OTP1-OTP100001

OTP6-97-000001

AEROSOL CAN OF SALINE

SOLUTION

R

OTP6-97-000002

AEROSOL CAN OF SALINE

SOLUTION

$\mathrm{R}$

0IP6-97-000003

UNLABELED AEROSOL CAN

$R$

OIP6-97-000004

LEAD BRICK

$R$

0TP6-97-000005

BOTILE OF SOLVENT AND BOTTLE OF OIL. $R$

0IP6-97-000006

BOTTLE OF ACID

0TP6-97-000007

BOTTLE OF ABSOR8ED SALINE

SOLUTION

19-JUL-27

G

0IP6-97-000008

BOTTLE OF UNKNOWN LIQUIO

19- JUL-27

$G$

\begin{tabular}{|c|c|}
\hline $\begin{array}{l}\text { CON_PKG_ID } \\
\text { CON_GENER_WASTE_DESCR } \\
\text { CON_PKG_DT } \\
\text { CON_PKG_STATUS }\end{array}$ & $\begin{array}{l}\text { OTP6-97-0000009. } \\
\text { LEAD BRICK } \\
\text { 22-JUL-27 } \\
\text { G }\end{array}$ \\
\hline $\begin{array}{l}\text { CON PKG 1D } \\
\text { CON_GENER_WASTE_DESCR } \\
\text { CON_PKG_DT } \\
\text { CON_PKG_STATUS }\end{array}$ & $\begin{array}{l}\text { OTPG-97-000010 } \\
\text { UNKMOWN SOLVENT } \\
22-\text { JUL-27 } \\
G\end{array}$ \\
\hline $\begin{array}{l}\text { CON_PKG ID } \\
\text { CON_GENER_WASTE_DESCR } \\
\text { CON_PKG_DT } \\
\text { CON_PKG_STATUS }\end{array}$ & $\begin{array}{l}\text { OTP6-97-000011 } \\
\text { OIL } \\
22-\text { JUL-27 } \\
\text { G }\end{array}$ \\
\hline CON PKG ID & OTP6-97-300001 \\
\hline CON PKG ID & OTP6-97-300002 \\
\hline CON PKG ID & OTP6-97-400001 \\
\hline$\frac{\text { CON_PKG ID }}{\text { CON_GENER_WASTE_DESCR }}$ & $\frac{\text { OTP6-97-900001 }}{1 L \text { BOTTLE OF SAL INE }}$ \\
\hline$\frac{\text { CON PKG ID }}{\text { CON_GENER_WASTE_DESCR }}$ & $\frac{\text { OTP6-97-900002 }}{\text { TL BOTTLE OF ACETIC ACID }}$ \\
\hline $\begin{array}{l}\text { CONEXT_PKG_ID } \\
\text { CONEXT_PROF_FIAG } \\
\text { CONEXT_PROF_ID } \\
\text { CONEXT_USE_CD } \\
\text { CONEXT_WRA }{ }_{\text {STAT_CO }}\end{array}$ & $\begin{array}{l}\text { OTP-TRUR-PD-01 } \\
Y \\
\text { TRU-10 } \\
\text { PD } \\
1\end{array}$ \\
\hline $\begin{array}{l}\text { CONEXT_PKG_ID } \\
\text { CONEXT_PROF_FLAG } \\
\text { CONEXT_PROF_ID } \\
\text { CONEXT_USE_CD } \\
\text { CONEXT_WRAP_STAT_CD }\end{array}$ & $\begin{array}{l}\text { OTP-TRUR-PD-11 } \\
Y \\
\text { TRU-10 } \\
\text { PO } \\
1\end{array}$ \\
\hline $\begin{array}{l}\text { CONEXT_PKG_ID } \\
\text { CONEXT_CNTR_STATUS } \\
\text { CONEXT_USE_CD } \\
\text { CONEXT_WRAP_STAT_CD }\end{array}$ & $\begin{array}{l}\text { OTP-TRUR-TO-01 } \\
\text { P } \\
\text { TO } \\
I\end{array}$ \\
\hline $\begin{array}{l}\text { CONEXT_PKG_ID } \\
\text { CONEXT_CNTR_STATUS } \\
\text { CONEXT_USE_CD } \\
\text { CONEXT_WRAP_STAT_CO }\end{array}$ & $\begin{array}{l}\text { OTP-TRUR-TD-02 } \\
P \\
\text { TD } \\
I\end{array}$ \\
\hline $\begin{array}{l}\text { CONEXT_PKG_ID } \\
\text { CONEXT_USE_CD }\end{array}$ & $\begin{array}{l}\text { OIP1-0TP100001 } \\
\text { PP }\end{array}$ \\
\hline $\begin{array}{l}\text { CONEXT_PKG_ID } \\
\text { CONEXT_MAT_GRP_CO } \\
\text { CONEXT_USE_CD }\end{array}$ & $\begin{array}{l}\text { OTPG-97-000001 } \\
A C \\
P K\end{array}$ \\
\hline $\begin{array}{l}\text { CONEXT_PKG_ID } \\
\text { CONEXT_MAT_GRP_CD } \\
\text { CONEXT_USE_CD }\end{array}$ & $\begin{array}{l}\text { OTP6-97-000002 } \\
\text { AC } \\
\text { PK }\end{array}$ \\
\hline $\begin{array}{l}\text { CONEXT_PKG_ID } \\
\text { CONEXT_MAI_GRP_CO } \\
\text { CONEXT_USE_CO }\end{array}$ & $\begin{array}{l}\text { OTP6-97-000003 } \\
\text { AC } \\
\text { PK }\end{array}$ \\
\hline $\begin{array}{l}\text { CONEXT_PKG_IO } \\
\text { CONEXT_MAT_GRP_CO } \\
\text { CONEXT_USE_CO }\end{array}$ & $\begin{array}{l}\text { OTP6-97-000004 } \\
\text { PB } \\
\text { PK. }\end{array}$ \\
\hline $\begin{array}{l}\text { CONEXT_PKG_ID } \\
\text { CONEXT_MAT_GRP_CD } \\
\text { CONEXT_USE_CO }\end{array}$ & $\begin{array}{l}\text { OTPG-97-000005 } \\
\text { LQ } \\
\text { PK }\end{array}$ \\
\hline CONEXT_PKG_IO & OTP6-97-000006 \\
\hline
\end{tabular}




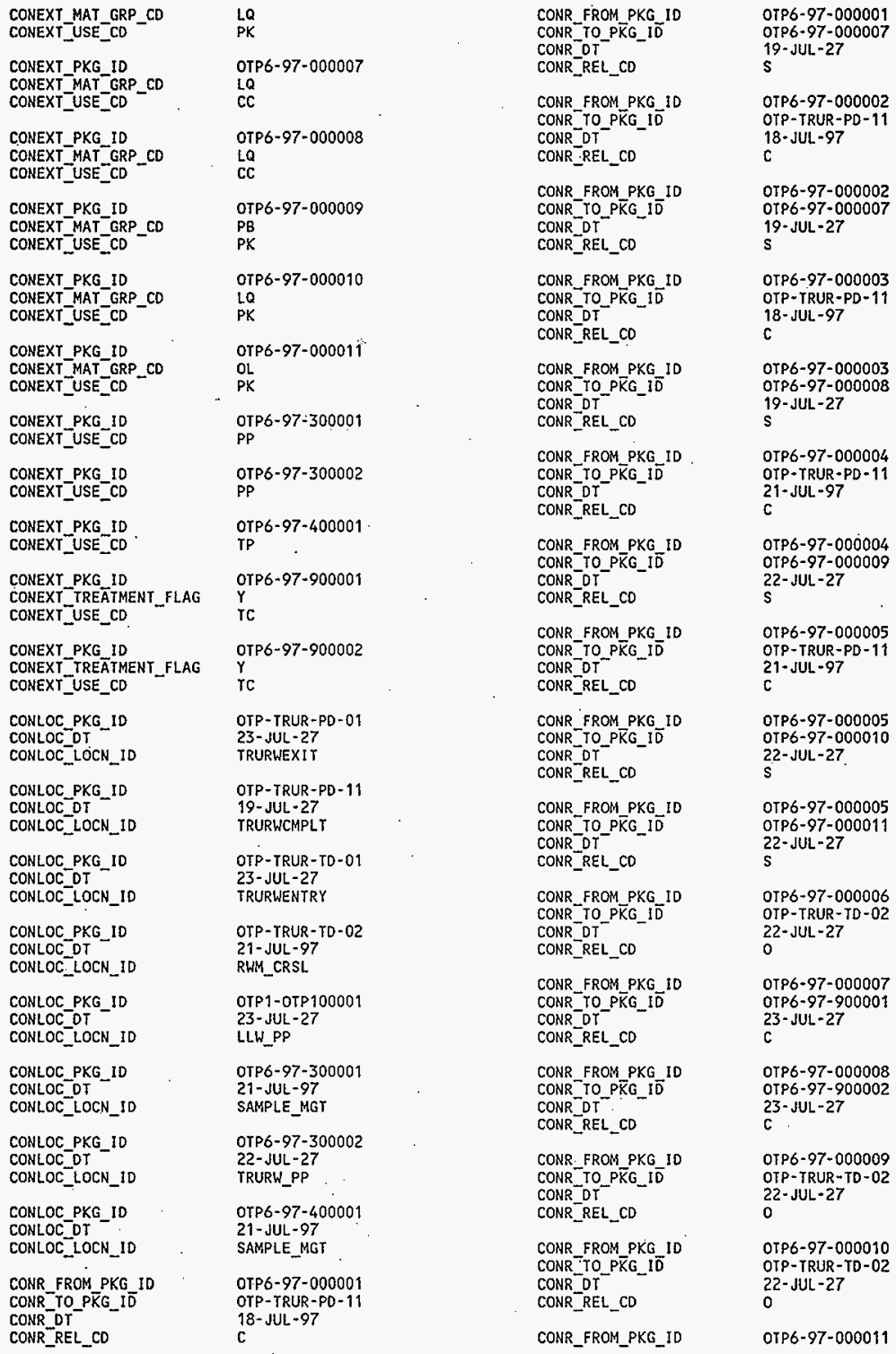




\begin{tabular}{|c|c|c|c|}
\hline $\begin{array}{l}\text { CONR_TO_PKG_IO } \\
\text { CONR_DT } \\
\text { CONR_REL_CD }\end{array}$ & $\begin{array}{l}\text { OTP-TRUR-TD-02 } \\
22-J U L-27 \\
0\end{array}$ & $\begin{array}{l}\text { SAM_MATRIX_CD } \\
\text { SAM_TAKEN_BY } \\
\text { SAM_TAKEN_DT } \\
\text { SAM TEMP - }\end{array}$ & $\begin{array}{l}\mathrm{L} \\
62264 \\
21-\mathrm{JUL}-97 \\
22\end{array}$ \\
\hline $\begin{array}{l}\text { CONR_FROM PKG ID } \\
\text { CONR TO PKG ID }\end{array}$ & $\begin{array}{l}\text { OTPG-97-900001 } \\
\text { OTP-TRUR-PD -01 }\end{array}$ & SAM_PKG_1D & 0TP6-97-000010 \\
\hline CONRDT & $23-J U L-27$ & SAM SAMPLE IO & $97-00004$ \\
\hline CONR_REL_CD & c & $\begin{array}{l}\text { SAM_CLOSED FLAG } \\
\text { SAM_COMMENTS }\end{array}$ & $\begin{array}{l}\text { N } \\
\text { oil }\end{array}$ \\
\hline CONR_FROM_PKG_ID & OTP6-97-900002 & SAM LOCN_ID & TRURWSAMPL \\
\hline $\begin{array}{l}\text { CONRTOOPKG_10 } \\
\text { CONR_DT } \\
\text { CONR_REL_CD }\end{array}$ & $\begin{array}{l}\text { OTP-TRUR-PD-01 } \\
23-J U L-27 \\
\text { C }\end{array}$ & $\begin{array}{l}\text { SAM_MATRTX_CD } \\
\text { SAM_TAKEN_BY } \\
\text { SAM_TAKEN_DT } \\
\text { SAM_TEMP }\end{array}$ & $\begin{array}{l}\mathrm{L} \\
62264 \\
21-\mathrm{JUL}-97 \\
22\end{array}$ \\
\hline $\begin{array}{l}\text { CONTREAT_PKG_ID } \\
\text { CONTREAT_DT }\end{array}$ & $\begin{array}{l}\text { OTP6-97-900001 } \\
22-\mathrm{JUL}-97\end{array}$ & SAM_PKG_ID & 0TP6-97-000011 \\
\hline CONTREAT_COMMENT & $X X X$ & SAR_SAMPLE_ID & $97-00001$ \\
\hline CONTREAT_USER_ID & USER1 & SAR_ANAL_CD & AQL IQ \\
\hline CONIREAT_WS_ID & OTPWRK01 & SAR_SAMPLE_ID & $97-00001$ \\
\hline $\begin{array}{l}\text { CONTREAT_PKG_ID } \\
\text { CONTREAT_DT }\end{array}$ & $\begin{array}{l}\text { OTP6-97-900002 } \\
22-J U L-97\end{array}$ & SAR_ANAL_C $\bar{D}$ & CONO \\
\hline $\begin{array}{l}\text { CONTREAT_COMHENT } \\
\text { CONTREAT_USER_ID } \\
\text { CONTREAT_WS_ } 1 \bar{D}\end{array}$ & $\begin{array}{l}X X X \\
\text { USER1 } \\
\text { OTPWRKO2 }\end{array}$ & $\begin{array}{l}\text { SAR_SAMPLE_ID } \\
\text { SAR_ANAL_CD }\end{array}$ & $\begin{array}{l}97-00002 \\
\text { SOLVENTS }\end{array}$ \\
\hline $\begin{array}{l}\text { PHYS_PKG_ID } \\
\text { PHYS_COMP_DESCR } \\
\text { PHYS_COMP_VOL_PCT } \\
\text { PHYS_COMP_WGT }\end{array}$ & $\begin{array}{l}\text { OTP-TRUR-PD- } 01 \\
\text { ABSORBED AQUEOUS SOLUTION } \\
95 \\
4\end{array}$ & $\begin{array}{l}\text { SAR_SAMPLE_ID } \\
\text { SAR_ANAL_CD } \\
\text { SAR_SAMPLE_ID } \\
\text { SAR_ANAL_CD }\end{array}$ & $\begin{array}{l}97-00003 \\
\text { SOLVENTS } \\
97-00004 \\
\text { OILS }\end{array}$ \\
\hline $\begin{array}{l}\text { PHYS_PKG_ID } \\
\text { PHYS_COMP_DESCR } \\
\text { PHYS_COMP_VOL_PCT } \\
\text { PHYS_COMP_WGT }\end{array}$ & $\begin{array}{l}\text { OTP-TRUR -PD - } 01 \\
\text { PLASTIC/POLYURATHANE } \\
5 \\
1\end{array}$ & $\begin{array}{l}\text { BOT_BOTTLE_IO } \\
\text { BOT_SAMPLE_ID } \\
\text { BOT_ANAL_CD }\end{array}$ & $\begin{array}{l}97-00001-01 L \\
97-00001 \\
\text { AQLIQ }\end{array}$ \\
\hline $\begin{array}{l}\text { PHYS_PKG_ID } \\
\text { PHYS_COMP_DESCR } \\
\text { PHYS_COMP_VOL_PCT }\end{array}$ & $\begin{array}{l}\text { OTP-TRUR-PD-11 } \\
\text { CLOTH/RAGS/NYLON } \\
60\end{array}$ & $\begin{array}{l}\text { BOT_BOTTLE_ID } \\
\text { BOT_SAMPLE_ID } \\
\text { BOT_ANAL_CD }\end{array}$ & $\begin{array}{l}97-00001-02 L \\
97-00001 \\
\text { COND }\end{array}$ \\
\hline PHYS_COMP_WGT & 4 & $\begin{array}{l}\text { BOT_BOTILE_IO } \\
\text { BOT_SAMPLE_ID }\end{array}$ & $\begin{array}{l}97-00002-01 L \\
97-00002\end{array}$ \\
\hline PHYS_PKG_ID & OTP-TRUR - PD- 11 & BOT_RETURN_DT & $21-J U L-97$ \\
\hline PHYS_COMP_DESCR & $\begin{array}{l}\text { METAL/IRON/GALVAN I ZED/SHE } \\
\text { ET }\end{array}$ & BOT_ANAL_CD & SOLVENTS \\
\hline $\begin{array}{l}\text { PHYS_COMP_VOL_PCT } \\
\text { PHYS_COMP_WGT }\end{array}$ & 10 & $\begin{array}{l}\text { BOT_BOTTLE_ID } \\
\text { BOT_SAMPLE_ID } \\
\text { BOT_RETURN_OT }\end{array}$ & $\begin{array}{l}97-00003-01 L \\
97-00003 \\
21-J U L-97\end{array}$ \\
\hline $\begin{array}{l}\text { PHYS_PKG_ID } \\
\text { PHYS_COMP_DESCR }\end{array}$ & $\begin{array}{l}\text { OTP-TRUR-PD- } 11 \\
\text { PLASTIC/POLYYRATHANE }\end{array}$ & BOT_ANAL_CD & SOLVENTS \\
\hline PHYS_COMP_VOL_PCT & 30 & BOT_BOTTLE_ID & $97-00004-01 L$ \\
\hline PHYS_COMP_WGT & 3 & $\begin{array}{l}\text { BOT_SAMPLE_ID } \\
\text { BOT_RETURN_DT }\end{array}$ & $\begin{array}{l}97-00004 \\
21-J U L-97\end{array}$ \\
\hline $\begin{array}{l}\text { SAM_SAMPLE_ID } \\
\text { SAM_CLOSED_FLAG }\end{array}$ & $\begin{array}{l}97-00001 \\
N\end{array}$ & BOT_ANAL_CD & OILS \\
\hline $\begin{array}{l}\text { SAM_COMMENTIS } \\
\text { SAM_LOCN_ID } \\
\text { SAM_MATR IX CO }\end{array}$ & $\begin{array}{l}\text { unknown liquid } \\
\text { TRURWSAMPL } \\
\text { L. }\end{array}$ & $\begin{array}{l}\text { SAMREL_BOTTLE_ID } \\
\text { SAMREL_PKG_ID } \\
\text { SAMREL_DT }\end{array}$ & $\begin{array}{l}97-00001-01 L \\
\text { OTP6-97-000008 } \\
18-J U L-97\end{array}$ \\
\hline $\begin{array}{l}\text { SAM TAKEN } \bar{B} Y \\
\text { SAM TAKEN DT }\end{array}$ & $\begin{array}{l}62264 \\
18-J U L-97\end{array}$ & SAMREL_USE_CD & CC \\
\hline SAM_IEMP - & 22 & SAMREL_BOTTLE_ID & $97-00001-01 \mathrm{~L}$ \\
\hline SAM_PKG_ID & 07P6-97-000008 & $\begin{array}{l}\text { SAMREL_PKG_ID } \\
\text { SAMREL_DT }\end{array}$ & $\begin{array}{l}\text { OTPG-97-300001 } \\
19-\mathrm{JUL}=27\end{array}$ \\
\hline $\begin{array}{l}\text { SAM_SAMPLE_ID } \\
\text { SAM_CLOSED_FLAG }\end{array}$ & $\begin{array}{l}97-00002 \\
N\end{array}$ & SAMREL_USE_CD & $\mathrm{PP}$ \\
\hline $\begin{array}{l}\text { SAM_COMMENTS } \\
\text { SAM_LOCN_ID }\end{array}$ & $\begin{array}{l}\text { Unkown solvents } \\
\text { TRURWSAMPL }\end{array}$ & $\begin{array}{l}\text { SAMREL_BOTTLE_ID } \\
\text { SAMREL_PKG_ID }\end{array}$ & $\begin{array}{l}97-00001-02 \mathrm{~L} \\
\text { 0TP6-97-000008 }\end{array}$ \\
\hline $\begin{array}{l}\text { SAM_NATRIX_CD } \\
\text { SAM_TAKEN_BYY } \\
\text { SAM_TAKEN DT }\end{array}$ & $\begin{array}{l}\mathrm{L} \\
62264 \\
21-\mathrm{JUL}-97\end{array}$ & $\begin{array}{l}\text { SAMREL_DT - } \\
\text { SAMREL_USE_CD }\end{array}$ & $\begin{array}{l}18-J U L-97 \\
C C\end{array}$ \\
\hline SAM_TEMP - - & & SAMREL_BOTTLE_ID & $97-00001-02 \mathrm{~L}$ \\
\hline SAM_PKG_ID & OTP6-97-000010 & $\begin{array}{l}\text { SAMREL_PXG_ID } \\
\text { SAMREL_DT }\end{array}$ & $\begin{array}{l}\text { orp } 6-97-300001 \\
19-\mathrm{JUL}-27\end{array}$ \\
\hline $\begin{array}{l}\text { SAM_SAMPLE_ID } \\
\text { SAM_CLOSED_FLAG }\end{array}$ & $\begin{array}{l}97-00003 \\
N\end{array}$ & SAMREL_USE_CD & $\mathrm{PP}$ \\
\hline $\begin{array}{l}\text { SAM_COMMENTS } \\
\text { SAM LOCN IO }\end{array}$ & $\begin{array}{l}\text { unknown solvent } \\
\text { TRURWSAMPL }\end{array}$ & $\begin{array}{l}\text { SAMREL_BOTTLE_ID } \\
\text { SAMREL_PKG_ID }\end{array}$ & $\begin{array}{l}97-00001-02 L \\
\text { 0TP6-97-300001 }\end{array}$ \\
\hline
\end{tabular}




\begin{tabular}{|c|c|}
\hline $\begin{array}{l}\text { SAMREL_DT } \\
\text { SAMREL_USE_CD }\end{array}$ & $\begin{array}{l}\text { 19- JUL - } 27 \\
\text { PP }\end{array}$ \\
\hline $\begin{array}{l}\text { SAMREL_BOTTLE_ID } \\
\text { SAMREL_PKG_10- } \\
\text { SAMREL_DT - } \\
\text { SAMREL_USE_CD }\end{array}$ & $\begin{array}{l}97-00001-02 L \\
\text { OTP6-97-300001 } \\
19-J U L-27 \\
\text { PP }\end{array}$ \\
\hline $\begin{array}{l}\text { SAMREL_BOTTLE_ID } \\
\text { SAMREL_PKG_10- } \\
\text { SAMREL_DT- } \\
\text { SAMREL_USE_CD }\end{array}$ & $\begin{array}{l}97-00002-01 \mathrm{~L} \\
\text { OTP6-97-000010 } \\
21-J U L-97 \\
\text { PK }\end{array}$ \\
\hline $\begin{array}{l}\text { SAMREL_BOTTLE_ID } \\
\text { SAMREL_PKG_ID- } \\
\text { SAMREL_DT - } \\
\text { SAMREL_USE_CD }\end{array}$ & $\begin{array}{l}97-00002-011 \\
\text { OTP6-97-300002 } \\
22-J U L-27 \\
\text { PP }\end{array}$ \\
\hline $\begin{array}{l}\text { SAMREL_BOTTLE_ID } \\
\text { SAMREL_PKG_ID } \\
\text { SAMREL_DT - } \\
\text { SAMREL_USE_CD }\end{array}$ & $\begin{array}{l}97-00003-01 \mathrm{~L} \\
\text { OTP6-97-000010 } \\
21-J U L-97 \\
\text { PK }\end{array}$ \\
\hline $\begin{array}{l}\text { SAMREL_BOTTLE_ID } \\
\text { SAMREL_PKG_ID- } \\
\text { SAMREL_DT } \\
\text { SAMREL_USE_CD }\end{array}$ & $\begin{array}{l}97-00003-01 \mathrm{~L} \\
\text { OTP6-97-300002 } \\
22-J U L-27 \\
\text { PP }\end{array}$ \\
\hline $\begin{array}{l}\text { SAMREL_BOTYLE_10 } \\
\text { SAMREL_PKG_1D-_ } \\
\text { SAMREL_DT } \\
\text { SAMREL_USE_CD }\end{array}$ & $\begin{array}{l}97-00004-01 L \\
\text { OTP6-97-000011 } \\
21-5 U L-97 \\
\text { PK }\end{array}$ \\
\hline $\begin{array}{l}\text { SAMREL_BOTTLE_ID } \\
\text { SAMREL_PKG_1D- } \\
\text { SAMREL_DT } \\
\text { SAMREL_USE_CD }\end{array}$ & $\begin{array}{l}97-00004-01 L \\
\text { OTP6-97-300002 } \\
22-J U L-27 \\
\text { PP }\end{array}$ \\
\hline $\begin{array}{l}\text { SCRN_SCRN_1D } \\
\text { SCRN_PKG_ID } \\
\text { SCRN_DT } \\
\text { SCRN_LOCN_10 } \\
\text { SCRN_PERS_1D . } \\
\text { SCRN_SAMPLING_METHOD_CD }\end{array}$ & $\begin{array}{l}97-00003-01 \mathrm{~F} \\
\text { OTP6-97-000009 } \\
21-\text { JUL }-97 \\
\text { TRURWSAMPL } \\
62264 \\
\text { INS }\end{array}$ \\
\hline $\begin{array}{l}\text { SCRN_SCRN_ID } \\
\text { SCRN_PKG_ID } \\
\text { SCRN_OT } \\
\text { SCRN_LOCN_ID } \\
\text { SCRN_PERS_1D } \\
\text { SCRN_SAMPLING_METHOD_CD }\end{array}$ & $\begin{array}{l}97-00004-01 \mathrm{~F} \\
\text { OTP6-97-000010 } \\
21-\text { JUL-97 } \\
\text { TRURWSAMPL } \\
\text { 62264 } \\
\text { INS }\end{array}$ \\
\hline $\begin{array}{l}\text { SCRN_SCRN_ID } \\
\text { SCRN_PKG_ID } \\
\text { SCRN_DT } \\
\text { SCRN_LOCN_ID } \\
\text { SCRN_PERS_ID } \\
\text { SCRN_SAMPLING_METHOD_CD }\end{array}$ & $\begin{array}{l}97-00005-01 \mathrm{~F} \\
\text { OTPG-97-000011 } \\
21-\text { JUL-97 } \\
\text { TRURWSAMPL } \\
62264 \\
\text { INS }\end{array}$ \\
\hline $\begin{array}{l}\text { SCRN_SCRN_10 } \\
\text { SCRN_PKG_ID } \\
\text { SCRN_DT } \\
\text { SCRN_LOCN_ID } \\
\text { SCRN_PERS_ID } \\
\text { SCRN_SAMPLIING_METHOD_CD }\end{array}$ & $\begin{array}{l}\text { 97-00006-01F } \\
\text { OTP6-97-000006 } \\
21-J U L-97 \\
\text { TRURWSAMPL } \\
62264 \\
\text { INS }\end{array}$ \\
\hline $\begin{array}{l}\text { FIELD_SCRN_ID } \\
\text { FIELD_FAT_CD } \\
\text { field_res }\end{array}$ & $\begin{array}{l}97-00003-01 F \\
\text { LEAD } \\
\text { FIELD ENTRY }\end{array}$ \\
\hline $\begin{array}{l}\text { FIELD_SCRN_1D } \\
\text { FIELD_FAT_CD } \\
\text { field_res }\end{array}$ & $\begin{array}{l}97-00004-01 F \\
\text { CLOR } \\
\text { FIELD ENTRY }\end{array}$ \\
\hline $\begin{array}{l}\text { FIEL } \\
\text { FIEL }\end{array}$ & $\begin{array}{l}97-00005-01 F \\
\text { РСBO }\end{array}$ \\
\hline
\end{tabular}

$\begin{array}{ll}\text { field_res } & \text { FIELD ENTRY } \\ \text { FIELD_SCRN_ID } & 97-00006-01 \mathrm{~F} \\ \text { FIELO_FAT_CO } & \text { PHDP } \\ \text { field_rES } & \text { FIELD ENTRY } \\ \text { WORKSHEET_ID } & \text { OTPWRKO1 } \\ \text { WORKSHEET_NAME } & \text { PACKET 7 } \\ \text { WORKSHEET_PROC_NUM } & \text { OP-OT11-ABS } \\ \text { WORKSHEET_PROC_REV_NUM } & \text { A } \\ \text { WORKSHEET_PROC_INSTR } & \text { TREAT SALINE SOLUTION } \\ \text { ITEMS } & \text { OTP6-97-000007 }\end{array}$

WORKSHEET_10 OTPWRKO2

WORKSHEET NAME PACKET 8

WORKSHEET PROC NUM OP-0711-NEUT

WORKSHEET PROC REV NUM A

WORKSHEET PROC INSTR TREAT ACEYIC ACID

SOLUT ION ITEMS

OTP6-97-000008

WORKSHEET_IO OTPWRKO3

WORKSHEE T NAME PACKET 10

WORKSHEET PROC_NUM OP-0711-ABS

WORKSHEET_PROC_REV_NUM A

WORKSHEET PROCINSTR TREAT SOLVENT

OIP6-97-000010

WORKSHEET_IO OTPWRKO4

WORKSHEET HAME PACKET 11

WORKSHEET PROC_NUM OP-0711-ABS

WORKSHEET PROC REV NUM A

WORKSHEET PROCINSTR TREAT OIL ITEMS

OIP6-97-000011

WORKSHEET_ID OTPWRKOS

WORKSHEET WAME PACKET 6

WORKSHEET_PROC_MUM OP-0711-NEUT

WORKSHEET PROC REV NUM A

WORKSHEET_PROC_INSTR TREAT ACID SOLUTION

ITEMS - OTP6-97-000006

$\begin{array}{ll}\text { WORKITEM_ID } & \text { OTPWRKO1 } \\ \text { WORKITEM_PKG_IO } & \text { OTP6-97-000007 } \\ \text { WORKITEM_ID } & \text { OTPWRK02 } \\ \text { WORKITEM_PKG_ID } & \text { OTP6-97-000008 } \\ \text { WORKITEM_ID } & \text { OTPWRK03 } \\ \text { WORKITEM_PKG_IO } & 97-00002-011 \\ \text { WORKITEM_ID } & \text { OTPWRK03 } \\ \text { WORKITEM_PKG_ID } & 97-00003-01 \mathrm{~L} \\ \text { WORKITEM_ID } & \text { OTPWRK03 } \\ \text { WORKITEM_PKG_ID } & \text { OTP6-97-000010 } \\ \text { WORKITEM_ID } & \text { OIPWRKO4 } \\ \text { WORKITEM_PKG_ID } & 97-00004-01 \mathrm{~L} \\ \text { WORKITEM_ID } & \\ \text { WORKITEM_PKG_ID } & \text { OTPWRKO4 } \\ \text { WORKITEM_ID } & \text { OTP6-97-000011 } \\ \text { WORKITEM_PKG_ID } & \text { OTPWRK05 } \\ \text { RAOMAT_ID } & \text { OTP6-97-000006 } \\ \text { RAOMAT_DESCR } & \text { F } \\ \text { RADMAT_LIMIT } & \text { WRAP 1 FACILITY } \\ \text { RADMAT_RAD_TOT } & 100 \\ \text { RADMAT_UNITS } & 10 \\ \text { RADMAT_ALARM } & \text { CI } \\ & \text { N }\end{array}$


HNF-SD-W026-0TR-014, Rev. 0, Page DMS-21

$\begin{array}{ll}\text { RADMAT_IO } & \text { R } \\ \text { RADMAT_DESCR } & \text { TRU RWM GLOVEBOX } \\ \text { RADMAT_LIMIT } & 200 \\ \text { RADMAT_RAD_TOT } & 150 \\ \text { RADMAT_UNITS } & \text { FGE } \\ \text { RADMAT_ALARM } & N \\ \text { RADMAT_10 } & \text { T } \\ \text { RADMAT_DESCR } & \text { TRU GLOVEBOX } \\ \text { RADMAT_LIMIT } & 200 \\ \text { RADMAT_RAD_TOT } & 110 \\ \text { RADMAT_UNITS } & \text { FGE } \\ \text { RADMAT_ALARM } & N\end{array}$


CASE4A.723 DMS DATABASE AT THE END OF TRU/RWM GLOVEBOX OTP CASE $4 \quad 7 / 23 / 97$

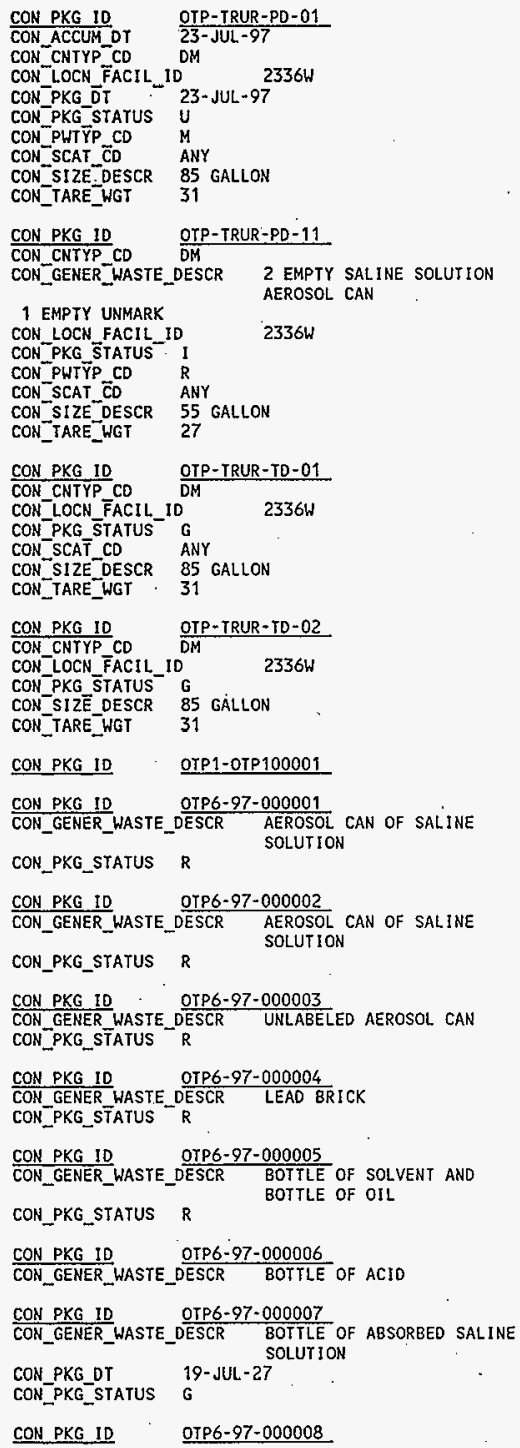

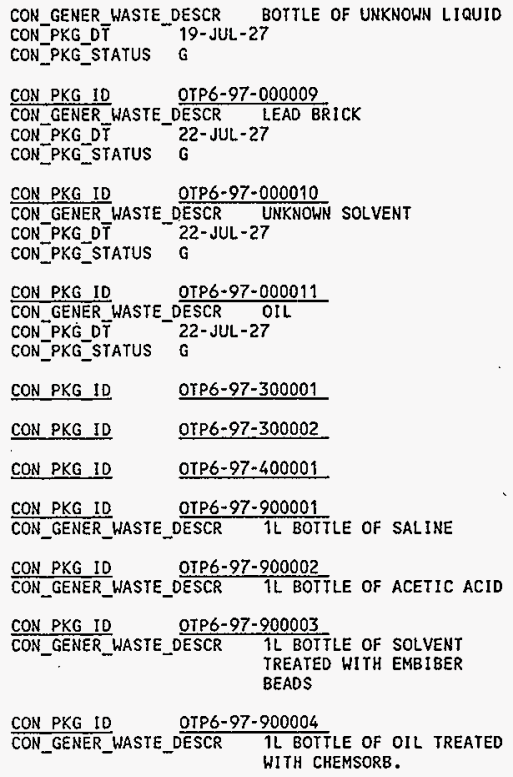




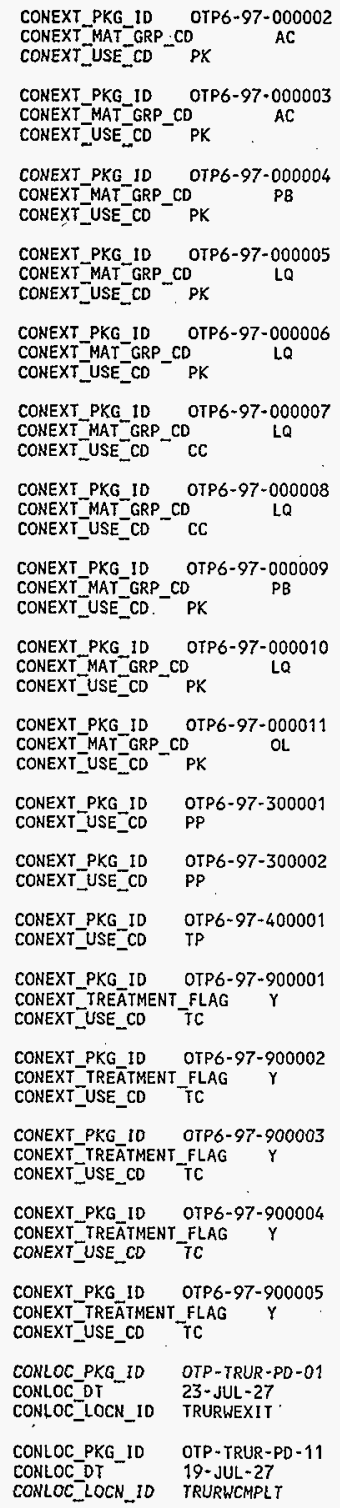

$\begin{array}{ll}\text { CONLOC_PKG_ID } & \text { OTP-TRUR-TO-01 } \\ \text { CONLOC_DT } & 23-J U L-27 \\ \text { CONLOC_LOCN_ID } & \text { IRURLENTRY } \\ \text { CONLOC_PKG_ID } & \text { OTP-TRUR-ID-02 } \\ \text { CONLOC_DT } & 23-J U L-27 \\ \text { CONLOC_LOCN_ID } & \text { TRURWENTRY } \\ \text { CONLOC_PKG_ID } & \text { OTP1-OTP100001 } \\ \text { CONLOC_DT } & \text { 23-JUL-27 } \\ \text { CONLOC_LOCN_ID } & \text { LLW_PP } \\ \text { CONLOC_PKG_ID } & \text { OTP6-97-300001 } \\ \text { CONLOC_DT } & 21-J U L-97 \\ \text { CONLOC_LOCN_ID } & \text { SAMPLE_MGT } \\ \text { CONLOC_PKG_ID } & \text { OTP6-97-300002 } \\ \text { CONLOC_DT } & 22-J U L-27 \\ \text { CONLOC_LOCN_ID } & \text { TRURW_PP } \\ \text { CONLOC_PKG_ID } & \text { OTP6-97-400001 } \\ \text { CONLOC_DT } & 21-J U L-97 \\ \text { CONLOC_LOCN_ID } & \text { SAMPLE_MGT } \\ \text { CONLOC_PKG_10 } & \text { OTP6-97-900005 } \\ \text { CONLOC_DT } & \text { 23-JUL-27 } \\ \text { CONLOC_LOCN_10 } & \text { TRURWTREAT }\end{array}$

CONR FROM PKG I0 . OTPG-97-000001 CONR_TO PKG_ID OTP-TRUR-PD-11 CONR DT $18-\mathrm{JUL}-97$

CONR_REL_CD C

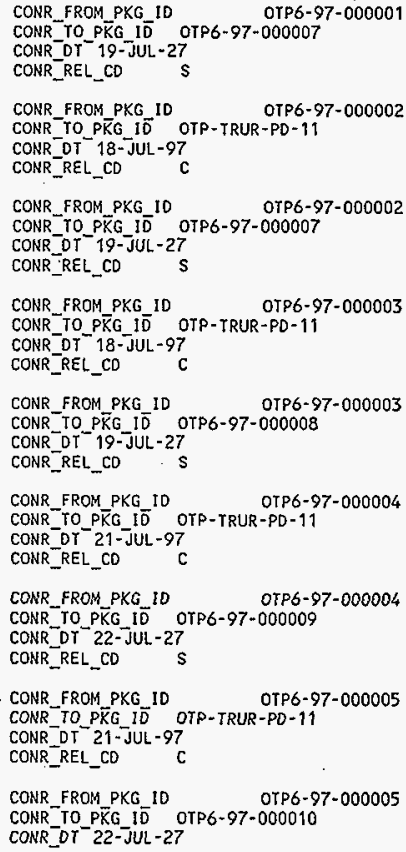


CONR_REL_CD S

CONR_FROH_PKG_ID

CONR TO PKG ID OTP6-97-000019

CONR_DT 22-JUL-27

CONR_REL_CD S

CONR_FROM_PKG_ID

OTP6-97-000006

CONR TO PKG ID OTP-IRUR-PD-01

CONR_DT 23-JUL-27

CONR_REL_CD C

CONR FROM PKG ID OIP6-97-000006

CONR TO PKG JD OTP6-97-900005

CONR_DT $23-J U L-27$

CONR_REL_CD C

CONR_FROMPXG_ID OTP6-97-000007

CONRTOP P $\overrightarrow{K G} I \bar{D}$ OTP6-97-900001

CONR-DT 23-JUL-27

CONR_REL_CO C

CONR FROM PKG 1D OTP6-97-000008

CONR_TO_PKG_ID OTPG-97-900002

CONR_DT $23-\mathrm{JUL}-27$

CONR_REL_CD C

CONR FROM PKG ID

CONR TO PKG ID OTP-TRUR-TO-OD

CONR_OT $22-J U L-27$

CONR_REL_CD 0

CONR_FROM_PKG_ID

CONR TO PKG $1 \bar{D}$ OTP-TRUR-PD-11

CONR_DT $22-J U L-97$

CONR_REL_CD C

CONR_FROM_PKG_ID OTP6-97-000010

CONR TO PBKG ID OTP6-97-900003

CONR OI $23-\mathrm{JUL}-27$

CONR REL CD .C

CONR FROM PKG ID OTP6-97-000011

CONR TO $P \overline{P K}$ ID $\bar{D}$ OTP-TRUR-PD-11

CONRDT 22-JUL-97

CONR_REL_CD C

CONR FROM PKG ID OTPG-97-000011

CONR_TOPFKG $1 \overline{0}$ OTP6-97-900004

CONR_DT $23-J U L-27$

CONR_REL_CO C

CONR FROM PKG 10

CONR TO $P \bar{K} G I \bar{O}$ OTP-TRUR+PD-O

CONR_DT $23-\mathrm{JUL}-27$

CONR_REL CD C

CONR_FROM_PKG_10

CONR TO PEKG I $\overline{0}$ OTP-TRUR-PD-01

CONR DT $23-$ JUL-27

CONR_REL_CO C

CONR_FROM_PKG_10 OTP6-97-900003

CONR TO $P \bar{K} G$ ID $\bar{D}$ OTP-TRUR-PD-O1

CONR DT 23-JUL-27

CONR REL CD C

CONR FROM PKG ID OIPG-97-900004

CONR_TO_PKG_ID OTP-TRUR-PD-01

CONR DT $23-J U L-27$

CONR_REL_CD

CONTREAT PKG ID OTPG-97-900001

CONTREAT-DT - 22-JUL-97

CONTREAT COMMENT XXX

CONTREAT_USER_ID USER1
CONTREAT_WS_ID OTPWRKO1

$\begin{array}{lc}\text { CONTREAT_PKG_ID OTP6-97-900002 } & \text { OT-JUL-97 } \\ \text { CONTREAT_DT_ } & 22-J U L-97 \\ \text { CONTREAT_COMMENT } & \text { XXX } \\ \text { CONTREAT_USER_ID } & \text { USER1 } \\ \text { CONTREAT_WS_ID } & \text { OTPWRKO2 }\end{array}$

CONTREAT PKG ID OTP6-97-900003

CONTREAT_OT 22-JUL-97

CONTREAT COMMENT FIELD ENTRY

CONTREAT USER ID USER1

CONTREAT_WS ID OTPWRK03

CONTREAT_PKG_ID. OTP6-97-900004

CONTREATDT $-22-J U L-97$

CONTREAT USER ID USER1

CONTREAT_WS I $I \vec{D}$ OTPWRKO4

\begin{tabular}{|c|c|}
\hline $\begin{array}{l}\text { CONTREAT_PKG_ID } \\
\text { CONTREAT_DT } \\
\text { CONTREAT_COMMENT } \\
\text { CONTREAT_USER_ID }\end{array}$ & $\begin{array}{c}\text { OTP6-97-900005 } \\
22-J U L-97 \\
\text { XXX } \\
\text { USER! }\end{array}$ \\
\hline CONTREAT_WS_I & OTPWRK05 \\
\hline $\begin{array}{l}\text { RDET_PKG_ID } \\
\text { ROET_BG_DOSE_RATE }\end{array}$ & $\begin{array}{c}\text { OTP-TRUR-PD-01 } \\
E\end{array}$ \\
\hline $\begin{array}{l}\text { PHYS_PKG_ID } \\
\text { PHYS_COMD_DESCR } \\
\text { PHYS_COMP_VOL_PCT } \\
\text { PHYS_COMP_WGT }\end{array}$ & $\begin{array}{l}\text { OTP-TRUR - PD-01 } \\
\text { ABSORBED AQUEOUS } \\
T_{6}^{2} 50 \\
{ }_{6}\end{array}$ \\
\hline
\end{tabular}

PHYS PKG ID OTP-TRUR-PD-01

PHYS COMP DESCR HAZARDOUS CONSTI IUENTS

PHYS COMP VOL PCT 45

PHYS_COMP_WGT 4

PHYS PKG ID OTP-TRUR-PD-01

PHYS COMP_DESCR PLASTIC/POLYURATHANE

PHYS CONP VOL PCT 5

PHYS_CONP_WGT 2

PHYS PKG ID OTP-TRUR-PD-11

PHYS COMP DESCR CLOTH/RAGS/NYLON

PHYS COMP VOL PCT

PHYS COMP WGT 4

PHYS PKG_10 OTP-TRUR-PD-11

PHYS COMP DESCR METAL/IRON/GALVANIZED/SHEET

PHYS_COMP_VOL_PCT 10

PHYS COMP WGT 1

PHYS PKG ID ' DTP-TRUR-PD- 11

PHYS COMP DESCR PLASTIC/POLYURATHANE

PHYS COMP VOL PCT 50

PHYSCOMP_WGT 4.

HAZ PKG ID OTP-TRUR-PD-01

HAZ_COMP_10 TEMP0429

HAZ COMP_TEXT ACETONE

HAZ COMP WGT 2

HAZ_COMP_WGT PCT 50

HAZ_EPCR $\bar{A}$ FL $\overline{A G}$

HAZ PKG_ID OTP-TRUR-PD-O 9

HAZ COMP ID TEMP3526

HAZ_COMP_TEXT SOLVENT REFINED PETROLEUM

DISTILLATES

HAZ COMP WGT PCT 50

HAZ EPCRA FLA $\bar{A}$,

SAM_SAMPLE_10 97-00001

SAM CLOSED FLAG N

SAM_COMMENTS unknown liquid 


\begin{tabular}{|c|c|}
\hline $\begin{array}{l}\text { SAM_LOCN_ID } \\
\text { SAM_MATR } \bar{I} X \text { CD } \\
\text { SAM_TAKEN } \bar{B} Y \\
\text { SAM_TAKEN_OT } \\
\text { SAM_TEMP - } \\
\text { SAM_PKG_ID }\end{array}$ & $\begin{array}{l}\text { TRURWSAMPL } \\
L \\
62264 \\
18-J U L-97 \\
22 \\
\text { OTP6-97-000008 }\end{array}$ \\
\hline $\begin{array}{l}\text { SAM_SAMPLE_1D } \\
\text { SAM_CLOSED_FLAG } \\
\text { SAM_COMMENTS } \\
\text { SAM_LOCN_ID } \\
\text { SAM_MATR IX_CD } \\
\text { SAM_TAKEN_BY } \\
\text { SAM_TAKEN_DT } \\
\text { SAM_TEMP - } \\
\text { SAM_PKG_ID }\end{array}$ & $\begin{array}{l}97-00002 \\
N \\
\text { unkown solvents } \\
\text { TRURWSAMPL } \\
1 \\
62264 \\
21-J U L-97 \\
22 \\
\text { OTP6-97-000010 }\end{array}$ \\
\hline $\begin{array}{l}\text { SAM_SAMPLE_ID } \\
\text { SAM_CLOSED_FLAG } \\
\text { SAM_COMMENTS } \\
\text { SAM_LOCN_ID } \\
\text { SAM_MATRIX_CD } \\
\text { SAM_TAKEN_BY } \\
\text { SAM_TAKEN_DT } \\
\text { SAM_TEMP_- } \\
\text { SAM_PKG_ID }\end{array}$ & $\begin{array}{l}97-00003 \\
N \\
\text { Unknown solvent } \\
\text { TRURWSAMPL } \\
L \\
62264 \\
21-J U L-97 \\
22 \\
\text { OTP6-97-000010 }\end{array}$ \\
\hline $\begin{array}{l}\text { SAM_SAMPLE_ID } \\
\text { SAM_CLOSED_FLAG } \\
\text { SAM_COMMENTS } \\
\text { SAM_LOCN_ID } \\
\text { SAM_MATRIX_CO } \\
\text { SAM_TAKEN_BY } \\
\text { SAM_TAKEN_DT } \\
\text { SAM_TEMP_- } \\
\text { SAM_PKG_ID }\end{array}$ & $\begin{array}{l}97-00004 \\
N \\
\text { OIL } \\
\text { TRURWSAMPL } \\
\text { L } \\
62264 \\
21-J U L-97 \\
22 \\
\text { OTP6- } 97-000011\end{array}$ \\
\hline $\begin{array}{l}\text { SAR_SAMPLE_ID } \\
\text { SAR_ANAL_CD }\end{array}$ & $\begin{array}{l}97-00001 \\
A Q L 10\end{array}$ \\
\hline $\begin{array}{l}\text { SAR_SAMPLE_IO } \\
\text { SAR_ANAL_CD }\end{array}$ & $\begin{array}{l}97-00001 \\
\text { CONO }\end{array}$ \\
\hline $\begin{array}{l}\text { SAR_SAMPLE_10 } \\
\text { SAR_ANAL_CD }\end{array}$ & $\begin{array}{l}97-00002 \\
\text { SOLVENTS }\end{array}$ \\
\hline $\begin{array}{l}\text { SAR_SAMPLE_ID } \\
\text { SAR_ANAL_CD }\end{array}$ & $\begin{array}{l}97-00003 \\
\text { SOLVENTS }\end{array}$ \\
\hline $\begin{array}{l}\text { SAR_SAMPLE_ID } \\
\text { SAR_ANAL_CD }\end{array}$ & $\begin{array}{l}97-00004 \\
\text { OILS }\end{array}$ \\
\hline $\begin{array}{l}\text { BOT_BOTTLE_ID } \\
\text { BOT_SAMPLE_ID } \\
\text { BOT_ANAL_CD }\end{array}$ & $\begin{array}{l}97-00001-01 L \\
97-00001 \\
A Q L I Q\end{array}$ \\
\hline $\begin{array}{l}\text { BOT_BOTTLE_ID } \\
\text { BOT_SAMPLE_ID } \\
\text { BOT_ANAL_CD }\end{array}$ & $\begin{array}{l}97-00001-02 L \\
97-00001 \\
\text { COND }\end{array}$ \\
\hline $\begin{array}{l}\text { BOT_BOTTLE_ID } \\
\text { BOT_SAMPLE_ID } \\
\text { BOT_DISP_BY } \\
\text { BOT_DISP_DT } \\
\text { BOT_RETURN_DT } \\
\text { BOT_ANAL_CD }\end{array}$ & $\begin{array}{l}97-00002-01 L \\
97-00002 \\
\text { USER1 } \\
22-J U L-97 \\
21-J U L-97 \\
\text { SOLVENTS }\end{array}$ \\
\hline $\begin{array}{l}\text { BOT_BOTILE_ID } \\
\text { BOT_SAMPLEIID } \\
\text { BOT_DISP_BY } \\
\text { BOT_DISP_DT } \\
\text { BOT_RETURN_DT } \\
\text { BOT_ANAL_CD }\end{array}$ & $\begin{array}{l}97-00003-01 \mathrm{~L} \\
97-00003 \\
\text { USER1 } \\
22-\text { JUL }-97 \\
21-J U L-97 \\
\text { SOLVENTS }\end{array}$ \\
\hline $\begin{array}{l}\text { BOT_BOTTLE_ID } \\
\text { BOT_SAMPLE_ID }\end{array}$ & $\begin{array}{l}97-00004-01 L \\
97-00004\end{array}$ \\
\hline
\end{tabular}

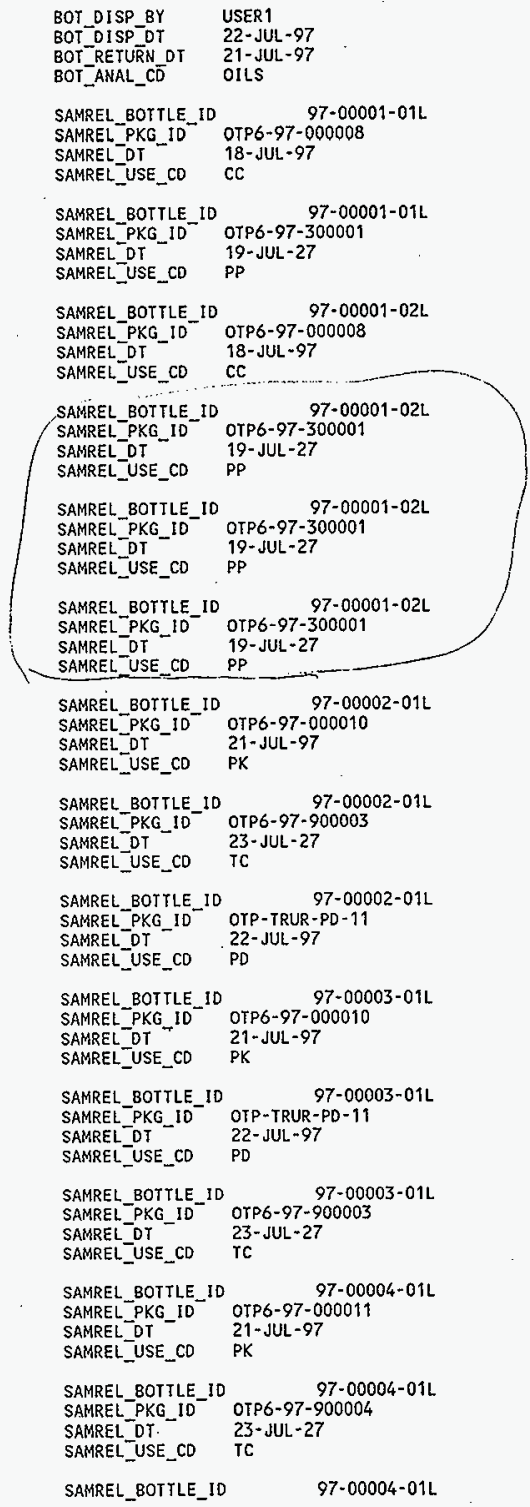




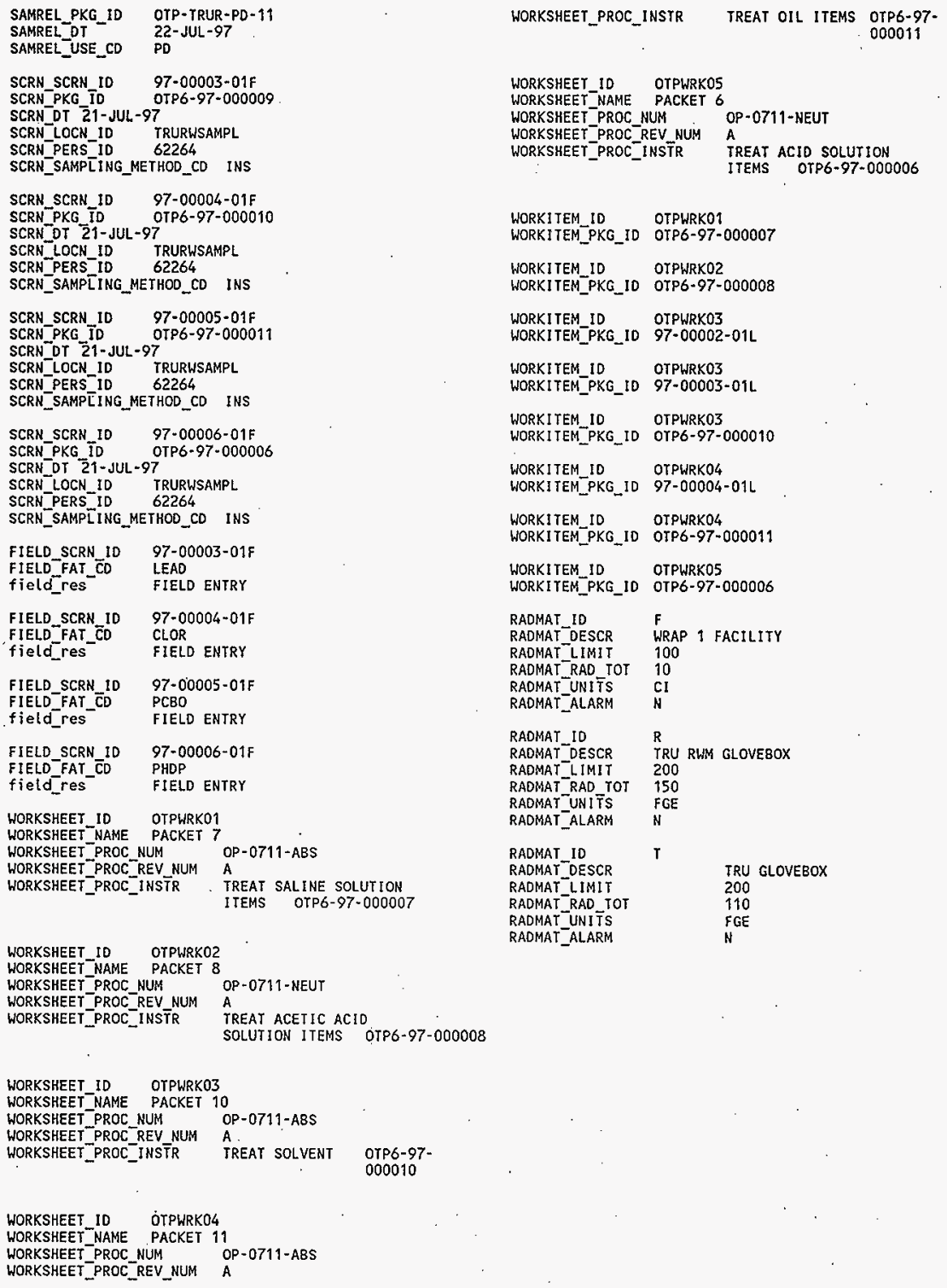


CON PKG 10:2 OIP-TRUR - PD-01

CON_CNTYP_CD DM

CON LOCN FACIL 1D 2336W

CONPKG STATUS

CON_STZE__DESCR $\quad 85$ GALLON

CON_TARE_WGT 31

CON PKG ID:2 OTP-TRUR - PD-11

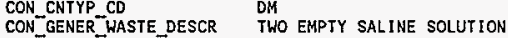

CON_LOCN_FACIL_ID $2336 \mathrm{~W}$

CON-PKG_STATUS ${ }^{-10}$

$\begin{array}{ll}\text { CON_SIZE__DESCR } & 55 \text { GALLON } \\ \text { CON_TARE_WGT } & 27\end{array}$

CON PKG ID:2 OIP-TRUR-TD-01

CON_LOCN_FACIL_ID 2336W

CON-PKG STATUS

CON $S C A \bar{T}$ CD ANY

CON_SIZE_DESCR 85 GALLON

CON_TARE_WGT 31

CON PKG ID:2 OTP-TRUR-TD-02
CON CNTYP CD OM

CON_LOCN FACIL_ID 2336W

CON_PKG STATUS $\mathrm{G}$

CON SCAT CD ANY

$\begin{array}{ll}\text { CON_SIZE_DESCR } & 85 \text { GALLON } \\ \text { CON_TARE_WGT } & 31\end{array}$

CON PKG $10: 2$ OTP6-97-000001

CON_GENER_WASTE_DESCR AEROSOL CAN OF SALINE

CON_PKG_SIATUS SOLUTION

CON PKG ID:2 OIP6- $97-000002$

CON_GENER WASIE DESCR AEROSOL CAN OF SALINE

CON_PKG_STATUS

SOLUTION

CON PKG 1D:2 OTP6-97-000003

CON_GENER_WASTE_DESCR UNLABELED AEROSOL CAN

CON_PKG_STTATUS

R

CON PKG ID:2 OTP6-97-000004

$\begin{array}{ll}\text { CON_GENER_WASTE_DESCR } & \text { LEAD BRICK } \\ \text { CON_PKG_STATUS } & \text { R }\end{array}$

CON_PKG ID:2 OTP6-97-000005

CON_GENER_WASTE_DESCR BOTTLE OF SOLVENT ANO

CON_PKG_STATUS

BOTTLE OF OIL

CON PKG ID:2 OTP6-97-000006

CON_GENER_WASTE_DESCR BOTTLE OF ACIO

CON PKG ID:2 OTP6-97-000007

CON GENER WASTE DESCR BOTILE OF SALINE SOLUTION

CON PKG_DT

CONPKG STATUS

$09-11-97 \quad 14: 25: 20$

G

CON PKG ID $: 2$ OTP6-97-000008

CON GENER WASTE DESCR BOTTLE OF UNKNOWN LIQUIO

CON PKG DT

CONPKG STATUS

$09-11-97 \quad 14: 25: 22$

G

CON PKG ID:2 OTP6-97-000009

CON_GENER WASTE DESCR LEAD BRICK

CON PKG DT

CON_PKG_STATUS

09-11-97 14:33:49

$\mathrm{G}$
CON PKG ID:? 0IP6-97-000010

CON_GENER_WASTE_DESCR UNKNOWN SOLVENT

CON PKG D $\overline{\mathrm{T}} \quad 09-11-97 \quad 14: 33: 50$

CON_PKG_STATUS G

CON PKG ID:2 OIP6-97-000011

CON_GENER_WASIE_DESCR OII

CON_PKG DT

CON_PKG_STATUS

09-11-97 14:33:56

CON PKG 10:2 OTP6-97-300001

CON PKG ID:2 OTPG-97-300002

CON PKG ID:2 OIP6-97-400001

$\begin{array}{ll}\text { CON_PKG_DI } & 09-11.97 \\ \text { CON_PKG_STATUS } & G\end{array}$

CON PKG ID:2 OTP6-97-900002

CONEXT PKG 10:20TP-TRUR - PD-01

CONEXT_USE-CO

CONEXT_PKG_ID:20TP-TRUR -PD - 11

$\begin{array}{ll}\text { CONEXT_USE_CO } & \text { PD } \\ \text { CONEXT_WRAP_STAT_CD } & \text { I }\end{array}$

CONEXT PKG ID:20IP-TRUR - TD - 01

CONEXT_CNTR_SIATUS E

CONEXT_USE CO TO

CONEXT_WRAP_STAT_CO I

CONEXT PKG ID:20TP-TRUR -TD-02

CONEXT_CNTR_STATUS $\quad$ P

CONEXT_WRAPי_STAT_CD I

CONEXT PKG ID:20TP6-97-000001

$\begin{array}{ll}\text { CONEXT_MAT_GRP_CD } & \text { AC } \\ \text { CONEXY_USE_CD } & \text { PK }\end{array}$

CONEXT_PKG_ID:20TP6-97-000002

CONEXT_MAT_GRP_CO AC

CONEXT_USE_CD ${ }^{-} \quad P K$

CONEXT PKG ID:20TP6-97-000003

$\begin{array}{ll}\text { CONEXT_MAT_GRP_CD } & \text { AC } \\ \text { CONEXT_USE-CD } & \end{array}$

CONEXT PKG ID:20TP6-97-000004

CONEXT_MAT_GRP_CD P8

CONEXT_USE_CD

CONEXT PKG ID:20TP6-97-000005

CONEXT_MAT_GRP_CD LQ

CONEXT_USE_CD -

CONEXT_PKG_ID:20TP6-97-000006

CONEXT_MAT_GRP_CD. LQ

CONEXT_USE_CD ${ }_{-}^{-}$PK

CONEXT PKG ID:20TP6-97-000007

CONEXT_MAT_GRP_CD LO

CONEXT_PKG_ID : 20IP6-97-000008

CONEXT_MAT_GRP_CD LQ

CONEXT_USE_CD $-C C$

CONEXT_PKG_ID:20TP6-97-000009

CONEXT_MAT_GRP_CD PB

CONEXT_USE_CD ${ }_{-}^{-}$PK 
CONEXT_PKG_ID:20TP6-97-000010

$\begin{array}{ll}\text { CONEXI_MAT_GRP_CD } & \text { LQ } \\ \text { CONEXI_USE_CO - } & \text { PK }\end{array}$

CONEXI_PKG 10:20TP6-97-000011

CONEXI_MAT_GRP_CD $\quad$ OL

CONEXT PKG 10:20TPG-97-300001

CONEXT_USE_CO PP

CONEXT PKG 10:20TP6-97-300002

CONEXT_USE_CD PP

CONEXT_PKG_10:20TP6-97-400001

CONEXT_USE_CO TP

CONEXT PKG 10:20IP6-97-900002

CONEXT_USE_CD IC

RDET_PKG ID:2OIP-TRUR-PD- 11

RDEI_SWTYTP_GROUP TRU

SAM_SAMPLE_10:297-00001

SAM_CLOSED_FLAG N

SAM LOCN $1 \bar{D}$. TRURWSAMPL

SAM_TAKEÑ_BY 62264

SAM TAKEN DT 09-12-97 10:05:33

SAM_PKG_10 $\quad$ OTPG-97-000008

SAM SAMPLE 10:297-00003

SAM CLOSEO FLAG

SAM LOCN ID

SAM TAKEN BY

SAM TAKEN DT

SAM_PKG_ID

N

TRURWSAMP'L

62264

$09-12-97 \quad 10: 14: 12$

OTP6-97-000007

SAR_SAMPLE_10:297-00001

SAR_ANAL_CD $\bar{O}$ AQLIQ

SAR_SAMPLE_10:297-00001

SAR_ANAL_CD ${ }^{-10}$ COND

SAR SAMPLE ID:297-00003

SAR_ANAL_CD.

80T_BOTTLE_1D:297-00001-01L

BOT_SAMPLE_ID 97-00001

BOT_ANAL_CD AQLIQ

BOT BOTILE ID:297-0000\%-02L

$\begin{array}{ll}\text { BOT-SAMPLE } & 97-00001 \\ \text { BOT ANAL CD } & \text { COND }\end{array}$

BOT BOTTLE ID:297-00003-01L

BOT_SAMPLE_10 97-00003

BOT_ANAL_CD $\bar{D}$ AQL $1 Q$

SAMREL BOITLE 1D:2 97-00001-01L

SAMREL-PKG 10 $\quad$ OTP6-97-300001

SAMREL_DT $09-11-9714: 31: 14$

SAMREL_USE_CD PP

SAMREL BOTTLE ID:2 97-00001-01L

SAMREL PKG ID 0 OTPG-97-000008

SAMREL_DT - 09-12-97 00:00:00

SAMREL_USE_CD

CC

SAMREL_BOTTLE_ID:2 97-00001-02L

SAMREL_PKG ID- OTPG-97-300001

SAMRELDI - $09-11-9714: 31: 18$

SAMRELUSE_CD . PP

SAMREL_BOTTLE_ID:2 97-00001-02L

SAMREL-PKG IO- 0 OTPG-97-000008

SAMREL_DT $09-12-9700: 00: 00$

SAMREL_USE_CD
SAMREL_BOTTLE_10:2 97-00003-01L

SAMREL_PKG_ID $\quad$ OIP6-97-000007

SAMREL DI - 09-12-97 00:00:00

SAMREL_USE_CD · CC

WORKSHEET ID: 2 OIPWRK01

WORKSHEET NAME

WORKSHEET PROC NUM

WORKSHEEI PROC_REV NUM

WORKSHEE T PROC INSTR

I tems

Packet 7

OP-0711-ABS

A

Treat Satine solution

0TP6-97-000007

WORKSHEET_10:2 OTPWRKO2

WORKSHEET NAME

WORKSHEET PROC NUM

WORKSHEET PROC REV NUM

WORKSHEET PROC INSTR

Packet 8

OP-0711-NEUT

A

Ireat Acetic Acid

Solution I tems

0TP6-97-000008

WORKSHEE T_IO:2 OTPWRKO3

WORKSHEET NAME

WORKSHEET PROC NUM

WORKSHEET PROC REV NUM

WORKSHEE T_PROC_INSTRR

Packet 10

OP-0711-ABS

A

Treat solvent

orp6-97-000010

WORKSHEET ID:2 OTPWRKO4

WORKSHEET NAME

WORKSHEET PROC_NUM

WORKSHEET PROC REV NUM

WORKSHEET_PROC_INSTR

Packet 11

OP-0711-ABS

A

Treat oil Items

OTP6-97-000011

WORKSHEET ID:2 OTPWRK05

WORKSHEET NAME

WORKSHEET PROC NUM

WORKSHEET PROC REV NUM

WORKSHEEI_PROC_INST̃R

Packet 6

OP-0711-NEUT

Treat Acid solution Items OTP6-97-000006

WORKI TEM ID:20TPWRK01

WORKITEM_PKG_10

OTP6-97-000007

WORKI TEM 10 : 2OTPWRKO2

WORKITEM_PKG_ID

$97-00001-01 L$

WORKITEM ID: 2OTPWRKO2

WORKITEM_PKG_ID

97-00001-021.

WORKITEM 10:20TPWRK02

WORKITEM_PKG_10

OTPS-97-000008

WORKITEM_10:2OTPWRK03

WORKITEM_PKG_ID

$97-00002-011$

WORKI IEM_ID:20TPWRKO3

WORKITEM_PKG_ID

97-00003-01L

WORKITEM ID:2OTPWRK03

WORKI TEM_PKG_10

0TP6-97-000010

WORKITEM 10:2OTPWRK04

WORKITEM_PKG_10

97-00004-01L

WORKITEM 10 : 20TPWRK04

WORKITEM_PKG_ID

OTP6-97-000011

WORKITEM ID:20TPWRK05

WORKITEM_PKG_10

OTP6-97-000006

CONLOC_PKG_ID:20TP-TRUR - PD - 01

CONLOCDT

02-05-97 13:00:00 
CONLOC_LOCN_ID

RWM_CRSL

CONLOC_PKG_ID:20TP-TRUR-PD- 11

CONLOC OT

09-11-97 14:25:16

CONLOC_LOCN_L 10

TRURWCMPLT

CONLOC PKG IO:2OTP-TRUR-TD-01

CONLOCDT

$09-11-97 \quad 15: 41: 16$

CONLOC_LOCN_ID

TRURWENTRY

CONLOC_PKG_ID:2OTP-TRUR -TD-02

CONLOC OT

09-11-97 15:41:16

CONLOC_LOCN_10

RWM_CRSL

CONLOC PKG 10:20TP6-97-000007

CONLOCDT

$09-13-27 \quad 16: 21: 39$

CONLOC_LOCN ID

TRURWSAMPL

CONLOC_PKG_10:20TP6-97-300001

CONLOC-DT

CONLOC_LOCN_10

$09-11-97 \quad 15: 41: 16$

TRURW_PP

CONLOC PKG_10:20TP6-97-400001

CONLOCDOT

CONLOC_LOCN_ID

09-11-97 15:41:16

SAMPLE MGT

CONLOC_PKG_ID:20TP6-97-900002

CONLOC OT

09-13-27 17:16:22

CONLOC_LOCN_1D TRURWTREAT

CONR FROM PKG 10:2 OTP6-97-000001

CONR_YO_PKG_ID

CONRDT

CONR_REL_CD OTP-TRUR-PD- 11 $09-11-97 \quad 14: 27: 40$

CONR FROM PKG ID:2 OTP6-97-000001

CONR_TO_PKG_ID

CONR OT

CONR_REL_CD 0TP6-97-000007 09-11-97 14:25:20

CONR FROM PKG 10:2 OTP6-97-000002

CONR_TO_PKG_ID

CONRDT

CONR_REL_CD

\section{OTP-TRUR - PD - 11} $09-11-97 \quad 14: 27: 57$

c

CONR FROM PKG ID:2 OTP6-97-000002

$\begin{array}{ll}\text { CONR_IO_PKG_1D } & \text { OTP6-97-000007 } \\ \text { CONR_DY_ } & 09-11-9714: 25: 22 \\ \text { CONR_REL_CD } & \text { S }\end{array}$

CONR FROM PKG 1D:2 0TP6-97-000003

CONR_TO PKG_ID

CONR_REL_CD

OTP-TRUR-PD- 11

$09-11-97 \quad 14: 28: 40$

c

CONR FROM PKG ID:2 OTP6-97-000003

CONR IO PKG ID

CONRDT

CONR_REL_CD $09-11-97 \quad 14: 25: 2$

$\mathrm{s}$

CONR_FROM PKG ID:2 OTP6-97-000004

CONRTTOPKG_ID $\quad$ OTP-TRUR-PD-11

CONR_DT

CONR_REL_CD

09-11-97 $14: 45: 35$

c

CONR_FROM_PKG_ID:2 OTP6-97-000004
CONR TO PKG ID. OTP6-97-000009
CONR_DT
CONR_REL_CD $09-11-97 \quad 14: 33: 49$
$\mathrm{S}$

CONR FROM PKG_10:2 0TP6-97-000005

CONR TO PKG ID $\bar{D}$ OTP-TRUR-PO- 11

CONR_DT

CONR_REL_CD

$09-11-97 \quad 14: 46: 46$

c

CONR_FROM_PKG_ID:2 OTP6-97-000005

CONR_TO PKG ID

OTP6-97-000010
CONR DT

CONR REL CO

$09-11-97$ 14:33:50

CONR_FROM_PKG_10:2 OTP6-97-000005

CONR TO PKG $1 \overrightarrow{0}$ OTP6-97-000011

CONR_DT

CONR REL_CO 09-11-97 14:33:56

CONR_FROM PKG 10:2 OTP6-97-000006

CONRTIO PKG ID

CONRDT

CONR_REL_CD

OTP-TRUR-TD-02

09-11-97 14:48:14

CONR FROM PKG 10:2 OTPG-97-000008

CONR TO P $\overline{K G}_{I} \bar{D}^{-}$

CONR OT

CONR_REL_CD

OTP6-97-900002

$09-13-27 \quad 17: 16: 22$

CONR_FROM_PKG_1D:2 OTP6-97-000009

CONR TO PKG $1 \bar{D}$ OTP-TRUR-TO-02

CONROT

CONR_REL_CD

09-11-97 $14: 48: 22$

CONR_FROM_PKG_10:2 OTP6-97-000010

CONR_TO_P $\overrightarrow{\text { KG_IO }}$

$\begin{array}{ll}\text { CONROT } & 09-11-9714: 48: 23 \\ \text { CONRREL CD } & 0\end{array}$

CONR_FROM_PKG_10:2 OTP6-97+000011

CONR TO PKG IO OTP-TRUR-TD-02

CONRDI $\quad 09-11-97$ 14:48:35

CONR_REL_CD

0

CONR_FROM_PKG_1D:2 OTP6-97-300002

CONR TOP $P \bar{B} G$ ID

CONROT

CONR_REL_CD

OTP6-97-400001

09-11-97 14:48:07

RADMAI_10:2F

RADMAT DESCR

RAOMAT LIMIT

RADMAT_RAD_TOT

RADMAT_UNITS

RAOMAT_ALARM

0

RADMAT ID:2R

RADMAT_DESCR

RADMAT LIMIT

RADMAT_RAD_TOT

RAOMAT_UNITS

RADMAT_ALARM

WRAP 1 FACILITY

100

10

C:

RADMAT 1D:2T

RADNAT DESCR

RADMAT_LIMIT

RADMAI_RAD TOT

RADMAT UNITS

RADMAT_ALARM

IRU RUM GLOVEBOX

200

150

FGE

H

MSGLOG_DT:209-12-97 09:31:54

MSGLOG ERROR FLAG N

MSGLOG GEN TYPPE PCSOMS

MSGLOG_SEQ_NUN 108310

MSGLOG SPEC TYPE REMT

MSGLOG_STRINTG1 PCSDMS"REMT"874081299"TRU

RWSAMPL.OTP-TRUR-TD-01 OT

MSGLOG_DT:209-12-97 09:32:00

MSGLOG ERROR FLAG N

MSGLOG_GEN_TYPE PCSRDMS

MSGLOG SEQ NUM 108311

MSGLOG_SPEC_TYPE RDMS

MSGLOG STRIÑG1 . PCSRDMS RDMS

MSGLOG_DT:209-12-97 09:32:01

MSGLOG_ERROR_FLAG N 
MSGLOG_GEN_TYPE
MSGLOG_SEQ_NUM
MSGLOG_SPEC TYPE

MSGLOG_STRIN̈G1

MSGLOG_OT:209-12-97 09:32;45

MSGLOG ERROR FLAG N

MSGLOG GEN TYPE. PCSRDMS

MSGLOG SEQ NUM 108313

MSGLOG_SPEC TYPE ROMS

MSGLOGSSTRING1 PCSRDMS ${ }^{\sim}$ RDMS $^{\sim}$

MSGLOG DT : 209-12-97 09:32:46

MSGLOG ERROR FLAG N

MSGLOG_GEN_TYPE - DMSPCS

MSGLOG SEQ NUM 108314

MSGLOG SPEC TYPE SDMS

MSGLOG_STRING1 OMSPCS SDMS $00000000^{\sim 1000}$

"FUNCTIONING PROPERLY

MSGLOG_DT:209-12-97 09:33:30

MSGLOG ERROR FLAG

MSGLOG_GEN_TYPE PCSRDMS

MSGLOG SEQ NUM 108315

WSGLOG SPE $\bar{C}$ TYPE ROMS

MSGLOG STRINTGI PCSRMS ROMS

MSGLOG DT:209-12-97 09:33:31

MSGLOG_ERROR_FLAG N

MSGLOG GEN TYYPE DMSPCS

MSGLOG SEQ NUM 108316

MSGLOG SPE $\bar{C}$ TYPE SDMS

MSGLOG_STRINTG1 DMSPCS SDMS $00000000 \% 1000$

"FUNCTIONING PROPERLY

MSGLOG DT:209-12-97 09:34:15

MSGLOG_ERROR_FLAG N

MSGLOG GEN TYYPE PCSRDMS

MSGLOG SEQ-NUM 108317

MSGLOG SPEC TYPE ROMS

MSGLOG_STRINTG1 PCSROMS ${ }^{\sim}$ RDMS

MSGLOG DT:209-12-97 09:34:16

MSGLOG_ERROR_FLAG N

MSGLOG GEN TYYPE DMSPCS

MSGLOG SEQ NUM 108318

MSGLOG SPEC TYPE SDMS

MSGLOG STRING1 DMSPCS ${ }^{\sim}$ SDMS $\approx 00000000^{\sim} 1000$

"FUNCTIONING PROPERLY

MSGLOG DT:209-12-97 09:35:00

MSGLOG ERROR FLAG N

MSGLOG GEN TYYPE PCSROMS

MSGLOG_SEQ_NUM 108319

MSGLOG SPEC TYPE RDMS

MSGLOG STRINTG1 PCSRDMS ${ }^{-R^{-}}$

MSGLOG DT:209-12-97 09:35:01

MSGLOG ERROR FLAG

MSGLOG_GEN_TYPE

MSGLOG SEO NUM 108320

MSGLOG_SPEC TYPE SOMS

NSGLOG STRINGI

FUNCTIONING PROPERLY

MSGLOG DT:209-12-97 09:35:45

MSGLOG ERROR FLAG N

MSGLOG GEN TYYPE PCSRDMS

MSGLOG SEQ NUM 108321

MSGLOG SPE $\bar{C}$ TYPE ROMS

MSGLOG_STRIÑG1 - PCSRDMS ${ }^{-R^{-}}$

MSGLOG DT:209-12-97 09:35:46

MSGLOG ERROR FLAG

MSGLOG_GEN_TYPE DHSPCS

MSGLOG SEQ NUM. 108322 $\begin{array}{ll}\text { MSGLOG_SPEC_TYPE } & \text { SOMS } \\ \text { MSGLOG_STRING } & \text { DMSPCS" SOMS }{ }^{\sim} 00000000^{\sim} 1000 \\ & \text { "FUNCTIONING PROPERLY }\end{array}$

MSGLOG DT:209-12-97 09:36:30

MSGLOG_ERROR_FLAG N

MSGLOG GEN TYPE PCSROMS

MSGLOG_SEQ_NUM 108323

MSGLOG_SPEC TYPE ROMS

MSGLOG_STRIN̄GI PCSROMS ${ }^{-1 D M S^{*}}$

MSGLOG_DT:209-12-97 09:36:31

MSGLOG ERROR FLAG N

MSGLOG_GEN_T YYPE DMSPCS

MSGLOG SEQ NUM 108324

MSGLOG_SPEC

MSGLOG_STRINTE1

"FUNCTIONING PROPERLY"

MSGLOG_DT:209-12-97 09:37:15

MSGLOG_ERROR_FLAG

MSGLOG GEN TYYPE PCSROMS

MSGLOG SEQ NUM 108325

MSGLOG_SPEC_TYPE ROMS

MSGLOG_STRINGI PCSROMS ${ }^{\sim}$ RDMS $^{\sim}$.

MSGLOG DT:209-12-97 09:37:16

MSGLOG ERROR FLAG N

MSGLOG_GEN_TYYPE DMSPCS

MSGLOG SEQ NUM 108326

MSGLOG SPE $\bar{C}$ TYPE SOMS

MSGLOG_STRING1 DMSPCS“SDMS $00000000 \% 1000$

"FUNCTIONING PROPERLY"

MSGLOG DT:209-12-97 09:38:00

MSGLOG ERROR FLAG

MSGLOG_GEN_TYPE PCSRDMS

MSGLOG_SEQ_NUM 108327

MSGLOG ${ }^{-}$SPE $\bar{C}$ TYPE RDMS

MSGLOG_STRENG1 PCSRDMS"ROMS

MSGLOG_DT:209-12-97 09:38:01

MSGLOG ERROR FLAG N

MSGLOG GEN TYPE DMSPCS

MSGLOG_SEO_NUM 108328

MSGLOG SPEC TYPE SDMS

MSGLOG_SIRINGT DMSPCS SDMS 000000001000

FUNCTIONING PROPERLY

MSGLOG_DT:209-12-97 09:38:45

MSGLOG ERROR FLAG N

MSGLOG_GEN_TYPE PCSRDMS

MSGLOG_SEQ_NUM 108329

MSGLOG SPE $\bar{C}$ TYPE RDMS

MSGLOG_STRINTG1 PCSRDMS ${ }^{2}$ RDMS

MSGLOG DT:209-12-97 09:38:46

MSGLOG_ERROR_FLAG N

MSGLOG GEN TYYPE DMSPCS

MSGLOG SEQ NUM 108330

MSGLOG_SPEC TYPE SOMS

MSGLOG STRINีG1 DMSPCS“SDMS $00000000 \approx 1000$

FUNCTIONING PROPERLY

MSGLOG DT:209-12-97 09:39:30

MSGLOG ERROR FLAG N

MSGLOG GEN TYYPE PCSRDMS

MSGLOG_SEQ_NUM 108331

MSGLOG SPE $\bar{C}$ TYPE ROMS

MSGLOG STRINTG1 PCSRDMS"ROMS"

MSGLOG DT:209-12-97 09:39:31

MSGLOG ERROR FLAG

MSGLOG_GEN_TYPE

MSGLOG SEQ NUM 108332

MSGLOG_SPEC_TYPE SOMS 
MSGLOG_STRING1

DMSPCS“SOMS $00000000 \% 1000$ FUNCTIONING PROPERLY

MSGLOS_OT:209-12-97 09:40:15

MSGLOG ERROR FLAG

MSGLOG_GEN_TYPE PCSRDMS

MSGLOG_SEQ NUM 108333

MSGLOG SPE $\bar{C}$ TYPE RDMS

MSGLOG_STRINGG1 PCSRDMS RDMS

MSGLOG_OT:209-12-97 09:40:16

MSGLOG_ERROR_FLAG N

MSGLOG GEN TYPE DMSPCS

MSGLOG_SEQ_NUM 108334

MSGLOG SPE $\bar{C}$ TYPE SDMS

MSGLOG_STRING1 DNSPCS“SDMS"00000000 1000

"FUNCTIONING PROPERLY

MSGLOG DT:209-12-97 09:41:01

MSGLOG ERROR FLAG

MSGLOG GEN TYPE PCSRDMS

MSGLOG SEQ NUM 108335

MSGLOG SPEC TYPE RDMS

MSGLOG_STRIN̄G1 PCSRDMS"RDHS

MSGLOG DT:209-12-97 09:41:02

MSGLOG_ERROR_FLAG N

MSGLOG GEN TYYPE DMSPCS

MSGLOG_SEQ_NUM 108336

MSGLOG SPE C TYPE SOMS

MSGLOG_STRIN̄G1 DMSPCS“SDHS“00000000 1000

FUNCT IONING PROPERLY

MSGLOG_DT:209-12-97 09:41:46

MSGLOG ERROR FLAG N

MSGLOG GEN TŸPE PCSRDMS

MSGLOG_SEQ_NUM 108337

MSGLOG_SPEC TYPE RDMS

MSGLOG_STRINTG1 PCSROMS"RDMS"

MSGLOG OT:209-12-97 09:41:47

MSGLOG ERROR FLAG N

MSGLOG GEN TYPPE DMSPCS

MSGLOG SEQ NUM , 108338

MSGLOG SPEC TYPE SOMS

MSGLOG STRING1 DMSPCS"SDMS $00000000 \approx 1000$

"FUNCTIONING PROPERLY

MSGLOG DT:209-12-97 09:42:31

MSGLOG ERROR FLAG N

MSGLOG GEN TYYPE PCSROMS

MSGLOG SEQ NUM 108339

HSGLOG_SPEC_TYPE RDMS

MSGLOG SIRINNGI PCSROMS"RDMS

MSGLOG DT:209-12-97 09:42:32

MSGLOG ERROR FLAG N

MSGLOG_GEN_TYYPE OMSPCS

MSGLOG SEQ NUM 108340

MSGLOG-SPE $\bar{C}$ TYPE SOMS

MSGLOG_STRING1 DMSPCS-SDMS $00000000^{-1000}$

"FUNCTIONING PROPERLY"

MSGLOG DI:209-12-97 09:43:16

MSGLOG ERROR FLAG

MSGLOG_GEN_TYYPE PCSRDMS

MSGLOG SEQ NUM 108341

MSGLOG SPE $\bar{C}$ TYPE ROMS

MSGLOG STRINGG . PCSROMSNROMS

MSGLOG DT: 209-12-97 09:43:17

MSGLOGERROR_FLAG N N

MSGLOG GEN TYYPE DMSPCS

MSGLOG SEQ NUM 108342

MSGLOG SPEC TYPE, SOMS

MSGLOG_SIRING1 ' DMSPCS SOMS $00000000^{\sim} 1000$

"FUNCTIONING PROPERLY"
MSGLOG_DT:209-12-97 09:44:01

MSGLOG ERROR FLAG

MSGLOG GEN TYPE PCSRDMS

MSGLOG SEQ NUM . 108343

MSGLOG SPEC IYPE RDMS

MSGLOG_STRINTG1 PCSRDMS“RDMS

MSGLOG OT:209-12-97 09:44:02

MSGLOG ERROR FLAG N

MSGLOG GEN TYYPE DMSPCS

MSGLOG_SEQ_NUM 108344

MSGLOG SPE C TYPE SDMS

MSGLOG_STRINGT DMSPCS SDMS 0000000 1000

"FUNCTIONING PROPERLY

MSGLOG_DT:209-12-97 09:44:46

MSGLOG ERROR FLAG

MSGLOG GEN TYPE PCSRDMS

MSGLOG_SEQ_NUM 108345

MSGLOG SPED TYPE RDMS

MSGLOG_STRIN̄GT PCSRDMS"RDMS

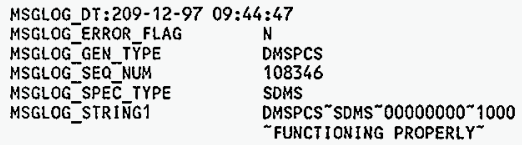

MSGLOG DI:209-12-97 09:45:31

MSGLOG_ERROR_FLAG N

MSGLOG GEN TYPE PCSRDMS

MSGLOG_SEO_NUM 108347

MSGLOG SPE $\bar{C}$ TYPE ROMS

MSGLOG STRINTG1 PCSROMS"RDMS

MSGLOG_OT:209-12-97 09:45:32

MSGLOG ERROR FLAG

MSGLOG_GEN_TYPE. DMSPCS

MSCLOG SEO NUM 108348

MSGLOG SPEC TYPE SOMS

MSGLOG_STRINGT

"FUNCTIONING PROPERLY"

MSGLOG OT:209-12-97 09:46:16

MSGLOG ERROR FLAG

MSGLOG_GEN_TYPE

MSGLOG SEQ NUM 108349

MSGLOG_SPE.

MSGLOG STRINTCT PCSRDMS"ROMS"

MSGLOG_DT: 209-12-97 09:46:17

MSGLOG ERROR FLAG N

MSGLOG_GEN_TYPE DMSPCS

MSGLOG_SEO_NUM 108350

MSGLOG SPEC TYPE SDMS

MSGLOG_STRINTG1 DMSPCS"SDMS $00000000 \% 1000$

FUNCTIONING PROPERLY ${ }^{\sim}$

MSGLOG_DT:209-12-97 09:47:01

MSGLOG ERROR FLAG N

MSGLOG_GEN_TYPE PCSRDMS

MSGLOG SEQ NUM 108351

MSGLOG SPEC TYPE ROMS

MSGLOG STRING1 PCSRDMS“RDMS

MSGLOG_OT:209-12-97 09:47:02

MSGLOG ERROR FLAG N

MSGLOG GEN TYYPE DMSPCS

MSGLOG_SEQ_NUM 108352

MSGLOG SPE $\bar{C}$ TYPE * SOMS

MSGLOG_STRING1 ' DMSPCS“SDMS 00000000 1000

FUNCTIONING PROPERLY

MSGLOG_OT:209-12-97 09:47:46

MSGLOG_ERROR_FLAG N 


\begin{tabular}{|c|c|}
\hline $\begin{array}{l}\text { MSGLOG_GEN_TYPE } \\
\text { MSGLOG_SEQ_NUM } \\
\text { MSGLOG_SPEC TYPE } \\
\text { MSGLOG_STRINGI }\end{array}$ & $\begin{array}{l}\text { PCSRDMS } \\
108353 \\
\text { RDMS } \\
\text { PCSRDMS"RDMS }\end{array}$ \\
\hline $\begin{array}{l}\text { MSGLOG_DT:209-12-9 } \\
\text { MSGLOG_ERROR_FLAG } \\
\text { MSGLOG_GEN_TYPE } \\
\text { HSGLOG_SEQ_NUM } \\
\text { MSGLOG_SPEC_TYPE } \\
\text { MSGLOG_STRINGI }\end{array}$ & $\begin{array}{l}7: 47 \\
N \\
\text { OMSPCS } \\
108354 \\
\text { SDMS } \\
\text { DMSPCS SOMS OOOOOOOO } 1000 \\
\text { "FUNCTIONING PROPERLY }\end{array}$ \\
\hline
\end{tabular}

HSGLOG DT:209-12-97 09:48:31

MSGLOG_ERROR_FLAG N

MSGLOG_GEN TYPE PCSROMS

MSGLOG SEQ NUM 108355

MSGLOG_SPEC $T Y P E$ ROMS

MSGLOG_STRING1 PCSROMS"ROMS"

MSGLOG_DT:209-12-97 09:48:32

MSGLOG ERROR FLAG

MSGLOG_GEN_TYPE DMSPCS

MSGLOG SEQ NUM 108356

MSGLOG SPE $\bar{C}$ TYPE SDMS

MSGLOG_STRIN̄G1 DMSPCS“SDMS $00000000^{\sim} 1000$

"FUNCTIONING PROPERLY"

MSGLOG DT:209-12-97 09:48:58

MSGLOG ERROR FLAG N

MSGLOG_GEN_TYYPE PCSDMS

MSGLOG SEO NUM 108357

MSGLOG SPEC TYPE SPDP

MSGLOG_STRIN̄G1 PCSDMS SPDP $174082323 \sim T R U$

RWSAMPL 97-00003-01L“OTP6

MSGLOG DT:209-12-97 09:49:16

MSGLOG_ERROR FLAG N

MSGLOG GEN TYYPE PCSRDMS

MSGLOG_SEQNNUM 108358

MSGLOG SPEC TYPE RDMS

MSGLOGSTRIÑGI PCSRDMS"RDMS

MSGLOG_DT:209-12-97 09:49:17

MSGLOG ERROR FLAG

MSGLOG_GEN TYPE DMSPCS

MSGLOG SEQ NUM 10835

MSGLOG_SPEC TYPE SDMS

MSGLOG STRING

"FUNCTIONING PROPERLY"

MSGLOG DT:209-12-97 09:50:01

MSGLOG ERROR FLAG N

MSGLOG_GEN_TYPE

MSGLOG SEQ NUM 108360

MSGLOG_SPEC_TYPE ROMS

MSGLOG_STRING1 PCSRDMS RDMS

MSGLOG_DT :209-12-97 09:50:02

MSGLOG ERROR FLAG N

MSGLOG_GEN_TYPE DMSPCS

MSGLOG SEO NUM 108361

MSGLOG SPEC TYPE SDMS

MSGLOG_STRING1 DMSPCS SOMS $00000000^{\sim} 1000$

"FUNCTIONING PROPERLY

MSGLOG DT:209-12-97 09:50:46

MSGLOG ERROR FLAG N

MSGLOG_GEN_TYPE - PCSRDMS

MSGLOG SEQ NUM. 108362

MSGLOG SPEC TYPE RDMS

MSGLOG_STRINTG1 PCSRDMS RDMS

MSGLOG_DT:209-12-97 09:50:47

MSGLOG ERROR FLAG N

MSGLOG_GEN_TYPE DMSPCS
MSGLOG_SEQ_NUM 108363

MSGLOG SPE $\bar{C}$ TYPE , SOMS

MSGLOG_STRINTG1 DNSPCS SOMS 00000000 1000

FUNCTIONING PROPERLY

MSGLOG OT:209-12-97 09:51:31

MSGLOG ERROR FLAG N

MSGLOG_GEN_TYPE PCSROMS

MSGLOG SEQ NUM 108364

MSGLOG SPE $\bar{C}$ TYPE ROMS

MSGLOG_STRINTGT PCSROMSNRDMS

MSGLOG_OT:209-12-97 09:51:32

MSGLOG ERROR FLAG N

MSGLOG GEN TYPE DMSPCS

MSGLOG SEQ MUM 108365

MSGLOG SPEC TYPE SDMS

MSGLOG_STRINTG1 DMSPCS“SDMS $00000000 \% 1000$

"FUNCTIONING PROPERLY

MSGLOG_DT:209-12-97 09:52:16

MSGLOG ERROR FLAG N

HSGLOG_GEN_TYPE PCSRDNS

MSGLOG_SEQ_NUM 108366

MSGLOG SPE $\bar{C}$ TYPE ROMS

MSGLOG_STRIÑG1 PCSROMS“RDMS

MSGLOG_DT:209-12-97 09:52:17

MSGLOG ERROR FLAG N

MSGLOG GEN TYYPE DMSPCS

MSGLOG_SEQ_NUM . 108367

MSGLOG SPEC TYPE SDMS

MSGLOG_STRIN̄G1 DMSPCS SDMS $00000000^{\sim} 1000$

FUNCTIONING PROPERLY

MSGLOG_DT:209-12-97 09:53:01

MSGLOG ERROR FLAG N

MSGLOG GEN TYYPE PCSROMS

MSGLOG_SEQ_NUM 108368

MSGLOG SPE $\vec{C}$ TYPE ROMS

MSGLOG_STRIN̄G1 PCSRDMS ROMS"

MSGLOG DT:209-12-97 09:53:02

MSGLOG_ERROR_FLAG N

MSGLOG GEN TYPE DNSPCS

MSGLOG SEQ NUM 108369

NSGLOG SPE $\bar{C}$ TYPE SDMS

MSGLOG STRI $\overline{M G 1}$ DMSPCS ${ }^{-}$SDMS $00000000 \% 1000$

"FUNCTIONING PROPERLY"

NSGLOG DT:209-12-97 09:53:46

MSGLOG_ERROR_FLAG N

MSGLOG GEN TYPE PCSROMS

MSGLOG SEQ NUM 108370

MSGLOG_SPE $\bar{C}$ TYPE ROMS

MSGLOG STRIN̈G1 PCSRDMS“RDMS

MSGLOG_DT:209-12-97 09:53:47

MSGLOG ERROR FLAG in

MSGLOG_GEN_TYPE DMSPCS

MSGLOG SEO NUM 108371

MSGLOG SPE $\bar{C}$ TYPE SOMS

MSGLOG STRING1 DMSPCS SOMS 00000000 1000

"FUNCTIONING PROPERLY"

MSGLOG DT:209-12-97 09:54:31

MSGLOG ERROR FLAG

MSGLOG GEN TYYPE

MSGLOG SEQ NUM 108372

MSGLOG_SPEC $\bar{C}$ TYPE RDMS

MSGLOG STRINTG1 PCSRDMS RDMS

MSGLOG_DT:209-12-97 09:54:32

MSGLOG ERROR FLAG N

MSGLOG_GEN_TYYPE DMSPCS

MSGLOG SEQ NUM 108373

MSGLOG_SPEC_IYPE' SOMS 


\begin{tabular}{|c|c|}
\hline MSGLOG_STRINGI & $\begin{array}{l}\text { DMSPCS } \text { SONS }^{000000000} 1000 \\
\text { "FUNCTIONING PROPERLY }\end{array}$ \\
\hline $\begin{array}{l}\text { MSGLOG_DT:209-12-9 } \\
\text { MSGLOG_ERROR_FLAG } \\
\text { MSGLOG_GEN_IYPE } \\
\text { MSGLOG_SEQ_NUM } \\
\text { MSGLOG_SPEC_TYPE } \\
\text { MSGLOG_STRINGI }\end{array}$ & $\begin{array}{l}5: 16 \\
N \\
\text { PCSRDMS } \\
108374 \\
\text { RDMS } \\
\text { PCSRDMS ROMS }\end{array}$ \\
\hline $\begin{array}{l}\text { MSGLOG_DT:209-12-9 } \\
\text { MSGLOG_ERROR_FLAG } \\
\text { MSGLOG_GEN_TYYPE } \\
\text { NSGLOG_SEQ_NUM } \\
\text { MSGLOG_SPEC_TYPE } \\
\text { MSGLOG_STRIN̄GI }\end{array}$ & $\begin{array}{l}5: 17 \\
\text { N } \\
\text { DMSPCS } \\
108375 \\
\text { SDMS } \\
\text { DMSPCS SOMS OOOOOOOO } 1000 \\
\text { "FUNCTIONING PROPERLY. }\end{array}$ \\
\hline $\begin{array}{l}\text { HSGLOG_DT:209-12-9 } \\
\text { HSGLOG_ERROR_FLAG } \\
\text { NSGLOG_GEN_TYPE } \\
\text { MSGLOG_SEQ_NUM } \\
\text { MSGLOG_SPEC TYPE } \\
\text { MSGLOG_STRINGI }\end{array}$ & $\begin{array}{l}6: 01 \\
\text { N } \\
\text { PCSROHS } \\
108376 \\
\text { ROMS } \\
\text { PCSROMS ROMS }\end{array}$ \\
\hline $\begin{array}{l}\text { MSGLOG_DT: } 209-12-9 \\
\text { MSGLOG_ERROR_FLAG } \\
\text { MSGLOG_GEN_TYYPE } \\
\text { MSGLOG_SEQ_NUM } \\
\text { MSGLOG_SPECT_TYPE } \\
\text { MSGLOG_STRINTG1 }\end{array}$ & $\begin{array}{l}6: 02 \\
\text { N } \\
\text { DMSPCS } \\
108377 \\
\text { SDMS } \\
\text { OMSPCS SOMS } \sim 0000000{ }^{\sim} 1000 \\
\text { “FUNCIIONING PROPERLY }\end{array}$ \\
\hline $\begin{array}{l}\text { MSGLOG_DT:209-12-9 } \\
\text { MSGLOG_ERROR_FLAG } \\
\text { MSGLOG_GEN_TYYPE } \\
\text { MSGLOG_SEQ_NUM } \\
\text { MSGLOG_SPEC TYYPE } \\
\text { MSGLOG_STRINTG1 }\end{array}$ & $\begin{array}{l}: 29 \\
N \\
\text { PCSOMS } \\
108378 \\
\text { SPOP } \\
\text { PCSDMS } \\
\text { RWSPMPL }-97-00003-01 L^{*} \text { "OTPG }\end{array}$ \\
\hline
\end{tabular}

MSGLOG_DT:209-12-97 09:56:46

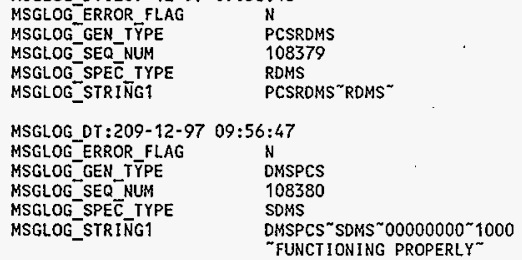

MSGLOG_DT:209-12-97 09:57:31

MSGLOG_ERROR_FLAG N

MSGLOG_GEN TYPE PCSROMS

MSGLOG_SEQ NUM 108381

MSGLOG SPEC TYPE RDMS

MSGLOG_STRINTG1 PCSRDMS RDMS

$\begin{array}{ll}\text { MSGLOG_DT :209-12-97 09:57:32 } \\ \text { MSGLOG_ERROR_FLAG } & \text { N } \\ \text { MSGLOG_GEN_TYPE } & \text { DMSPCS } \\ \text { MSGLOG_SEQ_NUM } & 108382 \\ \text { MSGLOG_SPEC TYYPE } & \text { SDMS } \\ \text { MSGLOG_STRINTG1 } & \text { DMSPCS SDMS 00000000 1000 } \\ & \text { "FUNCTIONING PROPERLY }\end{array}$

MSGLOG DT :209-12-97 09:58:16

MSGLOGERROR_FLAG $N$

MSGLOG GEN TYYPE PCSROMS

MSGLOG_SEQ_NUM 108383

MSGLOG SPE $\bar{C}$ TYPE RDMS
MSGLOG_STRINGI PCSROMS"RDMS

\begin{tabular}{|c|c|c|}
\hline MSGLOG_DT : $209-12-9$ & $8: 17$ & . \\
\hline MSGLOG_ERROR_FLAG & N & \\
\hline MSGLOG_GEN_TYPE & DMSPCS & \\
\hline MSGLOG SEQ NUM & 108384 & \\
\hline MSGLOG_SPEC \IYPE & SDMS & \\
\hline MSGLOG_STRIN̄GI. & $\begin{array}{l}\text { DMSPCS } \\
\text { FUNCT }\end{array}$ & $\begin{array}{l}000000 \% 1000 \\
\text { ROPERLY }\end{array}$ \\
\hline
\end{tabular}

MSGLOG_DT:209-12-97 09:59:01

MSGLOG ERROR FLAG

MSGLOG GEN TYYPE PCSROMS

MSGLOG_SEQ_NUM 108385

MSGLOG SPE $\bar{C}$ TYPE RDMS

MSGLOG_STRINTG1 PCSRDMS"RDHS"

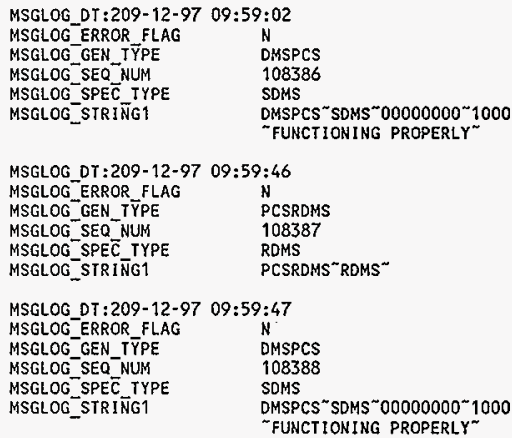

MSGLOG DT:209-12-97 10:00:31

MSGLOG ERROR FLAG . N

ASGLOGGEN TYPE PCSRDMS

MSGLOG SEQ NUM 108389

MSGLOG SPE $\bar{C}$ TYPE RDMS

MSGLOG STRINTG1 PCSROMS"RDMS"

MSGLOGOT:209-12+97 10:00:32

MSGLOG ERROR FLAG N

MSGLOG_GEN_TYPE DMSPCS

MSGLOG SEQ MUM 108390

MSGLOG SPEC TYPE SDMS

MSGLOG_STRINTG1 DMSPCS SOMS $0000000 \% 1000$

"FUNCTIONING PROPERLY"

MSGLOG DT:209-12-97 10:01:16

$\begin{array}{ll}\text { MSGLOG_ERROR FLAG } & \text { N } \\ \text { MSGLOG_GEN_TYPE } & \text { PCSROMS } \\ \text { MSGLOG_SEQ_NUM } & \text { 108391 } \\ \text { MSGLOG_SPEC̄_TYPE } & \text { RDMS } \\ \text { MSGLOG_STRINTG1 } & \text { PCSRDMS“RDMS }\end{array}$

MSGLOG DT:209-12-97 10:01:17

MSGLOG ERROR FLAG N

MSGLOG GEN TYPE DMSPCS

MSGLOG_SEQ NUM 108392

MSGLOG SPE $\bar{C}$ TYPE SDMS

MSGLOG_STRIN̈GI . DMSPCS"SOMS $00000000 \sim 1000$

FUNCT IONING PROPERLY

MSGLOG_DT:209-12-97 10:02:01

$M S G L O G-E R R O R$ FLAG

MSGLOG_GEN_TYPE PCSRDHS

MSGLOG_SEQ_NUM 108393

MSGLOG SPEC TYPE ROMS

MSGLOG_STRINGI PCSROMS ROMS 


$\begin{array}{ll}\text { MSGLOG_DT:209-12-97 } & 10: 02: 02 \\ \text { MSGLOG_ERROR FLAG } & \text { N } \\ \text { MSGLOG_GEN_TYPE } & \text { DMSPCS } \\ \text { MSGLOG_SEO_NUM } & 108394 \\ \text { MSGLOG_SPEC TYPE } & \text { SDHS } \\ \text { MSGLOG_STRING } & \text { DMSPCS SDMS-00000000 } 1000 \\ & \text { FUNCTIONING PROPERLY" }\end{array}$

MSGLOG_DT :209-12-97 10:02:46

MSGLOG ERROR FLAG N

MSGLOG_GEN_TYYPE PCSRDMS

MSGLOG SEQ NUM . 108395

MSGLOG SPEC TYPE RDMS

MSGLOG_STRINTG1 PCSROMS"ROMS"

MSGLOG_DT:209-12-97 10:02:47

MSGLOG_ERROR FLAG N

MSGLOG GEN TYYPE DMSPCS

MSGLOG SEQ NUM 108396

MSGLOG SPEC $T Y P E \quad$ SDMS

MSGLOG_STRINGI OMSPCS"SOMS"00000000 1000

FFUNCTIONING PROPERLY

MSGLOG_DT:209-12-97 10:03:31

MSGLOG_ERROR_FLAG N

MSGLOG_GEN_TYYPE PCSRDMS

MSGLOG_SEQ_NUM 108397

MSGLOG SPEC TYPE - RDMS

MSGLOG_STRINTG1 PCSRDMS"ROMS"

MSGLOG DT:209-12-97 10:03:32

MSGLOG_ERROR_FLAG N

MSGLOG GEN TYYPE DMSPCS

MSGLOG_SEQ_NUM 108398

MSGLOG SPE $\bar{C}$ TYPE SDMS

MSGLOG_STRINAT1 OMSPCS“SOMS“00000000 1000

FEUNCTIONING PROPERLY

MSGLOG DT:209-12-97 10:04:16

MSGLOG_ERROR_FLAG $\mathrm{N}$

MSGLOG GEN TYYPE PCSROMS

MSGLOG_SEQ_NUM 108399

MSGLOG SPE $\bar{C}$ TYPE ROMS

MSGLOG-STRINTG1 PCSRDMS RDMS

MSGLOG OT:209-12-97 10:04:17

MSGLOG_ERROR_FLAG N

MSGLOG_GEN TYPE

MSGLOG SEQ NUM 108400

MSGLOG_SPEC̈_TYPE SDMS

MSGLOGSTRINTG1 DMSPCS ${ }^{*}$ SOMS $00000000^{\sim} .1000$

־ FUNCTIONING PROPERLY

MSGLOG DT:209-12-97 10:05:01

MSGLOG_ERROR FLAG N

MSGLOG_GEN_TYPE

MSGLOG SEQ NUM 108401

MSGLOG_SPEC_TYPE RDMS

MSGLOG STRIN̄G1 PCSRDMS ${ }^{\sim}$ RDMS

MSGLOGDT:209-12-97 10:05:02

MSGLOG ERROR FLAG

MSGLOG_GEN_TYYPE OMSPCS

MSGLOG SEQ NUM 108402

MSGLOG SPEC TYPE SDMS

MSGLOG STRING1 1 DMSPCS SOMS 00000000 1000

FUNCTIONING PROPERLY

MSGLOG DT:209-12-97 10:05:46

MSGLOG ERROR FLAG N

MSGLOG_GEN_TYYPE PCSRDMS

MSGLOG SEQ NUM 108403

MSGLOG_SPECE_TYPE - RDMS

MSGLOG_STRINTG1 PCSROMS ROMS

MSGLOG_DT:209-12-97 10:05:47

MSGLOG_ERROR_FLAG N
MSGLOG_GEN_TYPE

MSGLOG SEQ NUM

MSGLOG SPE $\bar{C}$ TYPE

MSGLOG_STRIN̄GI

DNSPCS

108404

SONS

DMSPCS SOMS $00000000 \% 1000$

"FUNCTIONING PROPERLY"

MSGLOG_DT:209-12-97 10:06:3

MSGLOG ERROR FLAG

MSGLOG_GEN_TYPE PCSROMS

MSGLOG SEQ NUM 108405

MSGLOG_SPEC_TYPE ROMS

MSGLOG SIRING1 PCSROMS"RDMS

MSGLOG_DI:209-12-97 10:06:32

MSGLOG ERROR FLAG

MSGLOG_GEN_TYPE DMSPCS

MSGLOG SEQ NUM 108406

MSGLOG SPE $\bar{C}$ TYPE SOMS

MSGLOG_STRINGI DMSPCS"SOMS $00000000^{\sim 1000}$

"FUNCTIONING PROPERLY"

MSGLOG_DT:209-12*97 10:07:16

MSGLOG ERROR FLAG

MSGLOG GEN IYYPE PCSRDNS

$M S G L O G$ SEQ NUM 108407

MSGLOG SPEC TYPE RDMS

MSGLOG_STRINGI PCSROMS ${ }^{* R D M S}$

MSGLOG_DT:209-12-97 10:07:17

MSGLOC ERROR FLAG

MSGLOG GEN TYYYE DMSPCS

MSGLOG SEQ NUM 108408

MSGLOG SPEC TYPE SDMS

MSGLOG_STRINGI DMSPCS SOMS $00000000 \sim 1000$

FUNCTIONING PROPERLY

MSGLOG_DT:209-12-97 10:08:01

MSGLOG ERROR FLAG

MSGLOG_GEN_TY YPE PCSROMS

MSGLOG SEQ NUM 108409

MSGLOG SPE $\bar{C}$ TYPE ROMS

MSGLOG_STRIN̄G1 PCSROMS ${ }^{*}$ ROMS

MSGLOG DT:209-12-97 10:08:02

MSGLOG ERROR FLAG

MSGLOG GEN TTYPE DMSPCS

MSGLOG-SEO NUM 108410

MSGLOG SPEC TYPE. SOMS

MSGLOG STRINGI DMSPCS SDMS $00000000^{\sim} 1000$

FUNCTIONING PROPERLY

MSGLOG_DT:209-12-97 10:08:46

MSGLOG ERROR FLAG

MSGLOG GEN TYYPE PCSRDMS

MSGLOG_SEQ_NUM 108411

MSGLOG SPE $\bar{C}$ TYPE RDMS

MSGLOG STRIN̄GI PCSRDMS"RDMS

MSGLOG DT:209-12-97 10:08:47

MSGLOG ERROR. FLAG

MSGLOG GEN TYPE DMSPCS

MSGLOG SEQ NUM 108412

MSGLOG_SPEC

MSGLOG STRINTEI OMSPCS ${ }^{\sim}$ SDMS ${ }^{\sim 00000000} 1000$

"FUNCTIONING PROPERLY

MSGLOG DT:209-12-97 10:09:31

MSGLOG ERROR FLAG

MSGLOG GEN $T \overline{Y P E}$ PCSRDMS

MSGLOG ${ }^{-}$SEQ $N$ NUM 108413

MSGLOG SPEC TYPE RDMS

MSGLOG STRINGG1 PCSRDMS ${ }^{\sim}$ RDMS

MSGLOG DIT:209-12-97 10:09:32

MSGLOG ERROR FLAG

MSGLOG_GEN_TYPE DMSPCS

MSGLOG SEQ NUM 108414 
MSGLOG_SPEC_TYPE

MSGLOG_SIRIN̄GI

MSGLOG DT:209-12-97 10:10:16

MSGLOG_ERROR_FLAG N

MSGLOG GEN TYPE PCSROMS

MSGLOG SEQ NUM 10841.5

MSGLOG_SPEC TYPE RDMS

MSGLOG STRIN̈G1 PCSROMS ${ }^{2}$ ROMS

MSGLOG DT:209-12-97 10:10:17

MSGLOG ERROR FLAG

MSGLOG GEN TYYPE DHSPCS

MSGLOG SEQ NUM 108416

HSGLOG SPE $\bar{C}$ TYPE SDMS

MSGLOG_STRING1 $\quad$ DMSPCS“SDMS“00000000 1000

"FUNCTIONING PROPERLY"

MSGLOG DT:209-12-97 10:11:01

MSGLOG ERROR FLAG N

MSGLOG_GEN_TYPE PCSRDMS

MSGLOG SEO NUM 108417

MSGLOG_SPEC TYPE RDMS

MSGLOG STRINTG1 PCSRDMS ROMS

MSGLOG_DT:209-12-97 10:11:02

MSGLOG ERROR FLAG : . N

MSGLOG GEN TYPE DMSPCS

MSGLOG SEQ NUM _. 108418

MSGLOG SPEC TYPE SOMS

MSGLOG_STRINTG1 DMSPCS“SDMS $00000000 \% 1000$

"FUNCTIONING PROPERLY

MSGLOG_DT:209-12-97 10:11:46

MSGLOG ERROR FLAG

MSGLOG_GEN_TYPE PCSRDMS

MSGLOG_SEQ MUM 108419

MSGLOG SPE $\bar{C}$ TYPE RDMS

MSGLOG_STRINTG1 PCSRDMS"ROMS

HSGLOG_DT : 209-12-97 10:11:47

MSGLOG_ERROR_FLAG N

MSGLOG GEN TYYPE DMSPCS

MSGLOG_SEO_NUM 108420

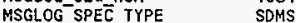

MSGLOG_STRINGG1 DMSPCS ${ }^{-10 M S^{\sim} 00000000} 1000$

FUNCTIONING PROPERLY

MSGLOG_DT:209-12-97 $10: 12: 31$

MSGLOG ERROR FLAG N

MSGLOG GEN TYPE PCSRDMS

MSGLOG_SEQ_NUM 108421

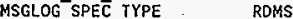

MSGLOG STRINTGI PCSRDMS“RDHS

MSGLOG DT:209-12-97 10:12:32

MSGLOG_ERROR FLAG N

MSGLOG_GEN_TYPE DMSPCS

MSGLOGSEO NUM . 108422

MSGLOG_SPEC_TYPE SDMS

MSGLOG_STRINTGI DMSPCS SDMS $00000000^{\sim} 1000$

"FUNCTIONING PROPERLY

MSGLOG DT:209-12-97 10:13:16

MSGLOG_ERROR_FLAG N

MSGLOG GEN TYPE PCSRDMS

MSGLOG SEQ NUM 108423

MSGLOG SPEC TYPE RDMS

MSGLOGSTRIN̄GI PCSRDMS"RDMS

MSGLOG DT:209-12-97 10:13:17

MSGLOG ERROR FLAG

MSGLOG_GEN_TYPE DHSPCS

MSGLOG SEQ NUM 108424

MSGLOG_SPEC_IYPE SOMS
MSGLOG_STRINGI DMSPCS SDMS"00000000 1000

FUNCTIONING PROPERLY"

MSGLOG DT :209-12-97 10:14:01

MSGLOG ERROR FLAG N

MSGLOG_GEN_TYYPE PCSROMS

MSGLOG SEQ NUM 108425

MSGLOG_SPEC

MSGLOG_SIRINGI PCSROMS“RDMS

MSGLOG_DT :209-12-97 10:14:02

MSGLOG ERROR FLAG

MSGLOG GEN TYTPE DMSPCS

MSGLOG_SEQ_NUM 108426

MSGLOG SPEC TYPE SOMS

MSGLOG_STRINTG

FUNCTIONING PROPERLY

MSGLOG_OT:209-12-97 10:14:46

MSGLOG ERROR FLAG

MSGLOG_GEN TYYPE. PCSROMS

MSGLOG_SEQ_NUM 108427

MSGLOG SPEC TYPE RDMS

MSGLOG_STRING1 PCSRDMS ${ }^{-R D M S}{ }^{*}$

MSGLOG_DT:209-12-97 10:14:47

MSGLOG-ERROR FLAG

MSGLOG GEN TYPPE DMSPCS

MSGLOG_SEQ_NUM 108428

MSGLOG SPE $\bar{C}$ TYPE SOHS

MSGLOG STRINTGI DMSPCS ${ }^{2}$ SDMS $00000000 \% 1000$

"FUNCIIONING PROPERLY"

MSGLOG_DT:209-12-97 10:15:31

MSGLOG ERROR FLAG

MSGLOG GEN TŸPE PCSRDMS

MSGLOG_SEQ_NUM 108429

MSGLOG SPE $\bar{C}$ TYPE RDMS

MSGLOG_STRINTG1 PCSRDMS ROMS"

MSGLOG DT:209-12-97 10:15:32

MSGLOG_ERROR FLAG N

MSGLOG GEN TYYPE - OMSPCS

MSGLOG SEQ MUM 108430

MSGLOG SPEC TYPE SDMS

MSGLOG STRINGG1 DMSPCS ${ }^{2}$ SDMS $00000000 * 1000$

"FUNCT IONING PROPERLY"

MSGLOG DT:209-12-97 10:16:16

ISSGLOG_ERROR FLAG N

MSGLOG GEN TYPE. PCSROMS

MSGLOG SEQ NUM 108431

MSGLOG SPE $\overline{\mathrm{C}}$ TYPE ROMS

MSGLOG STRING1 PCSROMS ${ }^{\sim}$ ROMS

MSGLOG_DT:209-12-97 10:16:17

MSGLOG ERROR FLAG

MSGLOG_GEN_TYPE DMSPCS

MSGLOG SEQ NUM 108432

MSGLOG SPE $\bar{C}$ TYPE SDMS

MSGLOG STRIN̈G1 DMSPCS SOMS 00000000 1000

F FUNCTIONING PROPERLY

MSGLOG OT:209-12-97 10:17:01

MSGLOG ERROR FLAG

MSGLOG_GEN_TYYPE PCSRDMS

MSGLOG SEQ NUM 108433

MSGLOG SPEC TYPE ROMS

MSGLOG STRINTG1 PCSROMS"RDMS

MSGLOG_DT:209-12-97 10:17:02

MSGLOG ERROR FLAG

MSGLOG_GEN_TYPE OMSPCS

MSGLOG SEQ NUM 108434

MSGLOG SPE $\bar{C}$ TYPE SOMS

MSGLOG_STRINTI DHSPCS SDMS $00000000 \sim 1000$

־FUNCTIONING PROPERI $\gamma^{\sim}$ 
MSGLOG DT:209-12-97 10:17:46

$\begin{array}{ll}\text { MSGLOG_ERROR_FLAG } & \text { N } \\ \text { MSGLOG_GEN_IYPE } & \text { PCSRDMS } \\ \text { MSGLOG_SEQ_NUM } & 108435 \\ \text { MSGLOG_SPEC_TYPE } & \text { ROMS } \\ \text { MSGLOG_STRING 1 } & \text { PCSRDMS }{ }^{\sim R D M S ~}\end{array}$

MSGLOG DT:209-12-97 10:17:47

MSGLOG_ERROR FLAG

MSGLOG_GEN TYYPE DMSPCS

MSGLOG SEQ NUM 108436

NSGLOG_SPEC _TYPE SDMS

MSGLOG STRINTG1 DMSPCS“SDMS ${ }^{\sim} 00000000 \% 1000$

"FUNCTIONING PROPERLY"

MSGLOG DT:209-12-97 $10: 18: 31$

MSGLOG_ERROR FLAG N

MSGLOG GEM TYYPE PCSRDMS

MSGLOG_SEQ_NUM 108437

MSGLOG_SPEC TYPE ROMS

MSGLOG_STRIN̄G1 PCSRDMS“RDMS

MSGLOG_DT:209-12-97 10:18:32

MSGLOG ERROR FLAG

MSGLOG_GEN_TYPE DMSPCS

MSGLOG SEQ NUM 108438

MSGLOG SPEC TYPE SDMS

MSGLOG_STRINTG1 DMSPCS“SDMS $00000000^{\sim} 1000$

"FUNCTIONING PROPERLY"

MSGLOG DT:209-12-97 10:19:16

MSGLOG ERROR FLAG

MSGLOG_GEN_TYPE $\quad$ PCSRDMS

MSGLOG SEQ MUM 108439

MSGLOG SPE $\bar{C}$ TYPE ROMS

MSGLOG STRING1 $\quad$ PCSRDMS ROMS

MSGLOG_DT:209-12-97 10:19:17

MSGLOG ERROR FLAG N

MSGLOG_GEN TYYPE DMSPCS

MSGLOG SEQ NUM 108440

MSGLOG SPEC $T Y P E \quad$ SDMS

MSGLOG STRING1 DMSPCS ${ }^{\circ}$ SDMS $00000000^{-1000}$

"FUNCTIONING PROPERLY" .

MSGLOG_DT:209-12-97 10:20:01

MSGLOG ERROR FLAG

MSGLOG_GEN_TYPE PCSRDMS

MSGLOG_SEQ MUM 10844

MSGLOG SPE $\bar{C}$ TYPE ROMS

MSGLOG_STRING1 PCSRDMS"RDMS"

MSGLOG_DT:209-12-97 10:20:02

MSGLOG ERROR FLAG N

MSGLOG GEN TYPE DMSPCS

MSGLOG_SEQ_NUM 108442

MSGLOG SPEC TYPE SDMS

MSGLOG_STRING

-FUNCTIONING PROPERLY

MSGLOG_OT:209-12-97 10:20:46

MSGLOG ERROR FLAG N

MSGLOG GEN TYPE PCSRDMS

MSGLOG_SEQ_NUM 108443

MSGLOG SPEC TYPE ROMS

MSGLOG_STRIN̄GT PCSROMS RDMS

MSGLOG OT:209-12-97 10:20:47

MSGLOG_ERROR FLAG N

MSGLOG GEN TYPE

MSGLOG SEQ NUM 108444

MSGLOG_SPEC TYPE SOMS

MSGLOG_STRINGI DMSPCS SDMS $00000000 \% 1000$

"FUNCTIONING PROPERLY

MSGLOG_DT:209-12-97 10:21:31

MSGLOG ERROR FLAG N

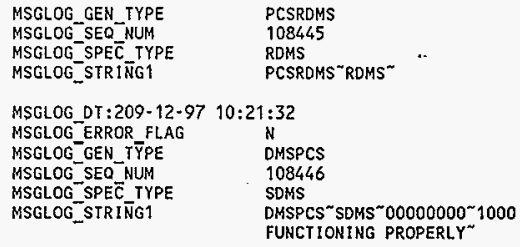

MSGLOG_DT :209-12-97 10:22:16

MSGLOG ERROR FLAG $\mathrm{N}$

MSGLOG GEN TYYPE PCSROMS

MSGLOG_SEQ_NUM . 108447

MSGLOG SPE $\bar{C}$ TYPE ROMS

MSGLOG_STRINNG1 PCSRDMS“RDMS"

MSGLOG DT:209-12-97 10:22:17

MSGLOG_ERROR_FLAG N

MSGLOG GEN TYYPE DMSPCS

MSGLOG SEQ NUM 108448

MSGLOG_SPEC _TYPE SOMS

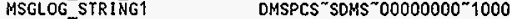

"FUNCTIONING PROPERLY"

MSGLOG DT:209-12-97 10:23:01

MSGLOG ERROR FLAG N

MSGLOG GEN TYYPE PCSRDMS

MSGLOG_SEQ_NUM 108449

MSGLOG_SPE $\overline{\mathrm{C}}$ TYPE ROMS

MSGLOG STRINTG1 PCSRDMS“RDMS

MSGLOG_DT:209-12-97 10:23:02

MSGLOG ERROR FLAG $\mathrm{N}$

MSGLOG_GEN_TYPE DMSPCS

MSGLOG SEQ NUM 108450

MSGLOG SPE $\bar{C}$ TYPE SDMS

MSGLOG STRIN̄GT DMSPCS“SOMS 00000000 1000

"FUNCTIONING PROPERLY ${ }^{\sim}$

MSGLOG DT:209-12-97 10:23:46

MSGLOG_ERROR FLAG N

MSGLOG_GEN_TYPE PCSRDMS

MSGLOG SEQ NUM 108451

MSGLOG SPE C TYPE ROMS

MSGLOG STRING1 PCSRDMS"RDMS

MSGLOG_OT:209-12-97 10:23:4?

MSGLOG ERROR FLAG N

MSGLOG_GEN_TYPPE DMSPCS

MSGLOG SEQ NUM 108452

MSGLOG SPEC TYPE SDMS

MSGLOG_STRIWT1 DHSPCS SDMS $00000000 \% 1000$

-FUNCTIONING PROPERLY

MSGLOG DT:209-12-97 10:24:25

MSGLOG ERROR FLAG N

MSGLOG_GEN_TYPE PCSROMS

MSGLOG SEQ NUM 108453

MSGLOG SPE $\bar{C}$ TYPE NCIT

MSGLOG STRING1 PCSROMS"NCIT"874084450\%TR

URWTREAT"OTP6-97-000008\%

MSGLOG DT : 209-12-97 $10: 24: 31$

MSGLOG ERROR FLAG N

MSGLOG_GEN_TYPE

MSGLOG SEQ $^{-}$NUM 108454

MSGLOG SPE $\bar{C}$ TYPE ROMS

MSGLOG STRIN̄G1 PCSRDMS RDMS

MSGLOG DT:209-12-97 10:24:32

MSGLOG ERROR FLAG N

MSGLOG_GEN_TYPE DMSPCS

MSGLOG_SEQ_NUM 108455 
MSGLOG_SPEC_TYPE MSGLOG_STRINGG

SOMS

DMSPCS NOMS $00000000 \sim 1000$

"FUNCTIONING PROPERLY"

$\begin{array}{ll}\text { MSGLOG_OT:209-12-97 10:25:16 } \\ \text { MSGLOG_ERROR_FLAG } & \text { N } \\ \text { MSGLOG_GEN_TYPE } & \text { PCSRDMS } \\ \text { MSGLOG_SEQ_NUM } & 108456 \\ \text { MSGLOG_SPEC TYPE } & \text { ROMS } \\ \text { MSGLOG_STRING1 } & \text { PCSROMS }\end{array}$

MSGLOG DT :209-12-97 10:25:17

MSGLOG ERROR FLAG N

MSGLOG_GEN_TYPE DMSPCS

MSGLOG SEQ NUM 108457

MSGLOG SPE $\bar{C}$ TYPE SDMS

MSGLOG STRING1 DMSPCS ${ }^{\text {SDMS }}$ 00000000 1000

FUNCTIONING PROPERLY

MSGLOG DT:209-12-97 10:26:01

$\begin{array}{ll}\text { MSGLOG_ERROR_FLAG } & \text { N } \\ \text { MSGLOG_GEN_TYPE } & \text { PCSRDMS } \\ \text { MSGLOG_SEQ_NUM } & 108458 \\ \text { MSGLOG_SPEC TYPE } & \text { RDMS } \\ \text { MSGLOG_STRING1 } & \text { PCSRDMS RDMS }\end{array}$

MSGLOG_DT:209-12-97 10:26:02

MSGLOG ERROR FLAG $N$

MSGLOG_GEN_TYPE DMSPCS

MSGLOG_SEQ NUM 108459

MSGLOG SPEC TYPE SDMS

MSGLOG_STRING1 OMSPCS ${ }^{\sim}$ SDMS ${ }^{\sim} 00000000^{\sim} 1000$

"FUNCTIONING PROPERLY

MSGLOG DT:209-12-97 $10: 26: 37$

MSGLOG ERROR FLAG

MSGLOG_GEN_TYPE PCSDMS

MSGLOG SEQ NUM 108460

MSGLOG SPEC TYPE - TCIC

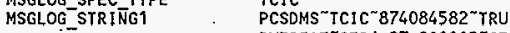

RWTREAT ${ }^{*}$ OTP6-97-900002\%OT

MSGLOG DT:209-12-97 10:26:46

MSGLOG_ERROR_FLAG N

MSGLOG GEN TYYPE PCSRDMS

MSGLOG SEQ NUM 108461

MSGLOG SPE$\overline{\mathrm{C}}$ TYPE RDHS

MSGLOG_STRIN̄G1 PCSRDMS ${ }^{\sim}$ RDMS

MSGLOG DT:209-12-97 $10: 26: 47$

MSGLOG ERROR FLAG

MSGLOG_GEN_TYPE DMSPCS

MSGLOG SEQ NUM 108462

MSGLOGSSPEC TYPE SDMS

MSGLOG_STRINTG1 DMSPCS SDMS $00000000 \sim 1000$

־UNCTIONING PROPERLY

MSGLOG_DT:209-12-97 10:27:31

MSGLOG ERROR FLAG

MSGLOG_GEN_TYPE

MSGLOG SEQ NUM 108463

MSGLOG SPE $\overline{\mathrm{C}}$ TYPE RDMS

MSGLOG_STRINTGI PCSRDMS RDMS

MSGLOG_DT:209-12-97 10:27:32

MSGLOG ERROR FLAG

MSGLOG_GEN_TYPE DMSPCS

MSGLOG SEQ NUM 108464

MSGLOG SPE $\bar{C}$ TYPE SDMS

MSGLOG_STRINTG1 DMSPCS SDKS $00000000 \approx 1000$

"FUNCTIONING PROPERLY"

MSGLOG OT:209-12-97 10:28:16

MSGLOG ERROR FLAG N

MSGLOG_GEN_TYPE PCSRDMS

MSGLOG SEQ NUM 108465
MSGLOG_SPEC_TYPE ROMS

MSGLOG_STRLT̄G1 PCSRDMS ${ }^{\sim R O M S}$

MSGLOG_DT:209-12-97 10:28:17

MSGLOG ERROR FLAG

MSGLOG_GEN_TYYPE DNSPCS

MSGLOG_SEQ_NUM 108466

MSGLOG SPEC TYPE SDMS

MSGLOG_STRIT̄G1 DKSPCS ${ }^{\sim}$ SDMS ${ }^{\sim 00000000} 1000$

"FUNCTIONING PROPERLY

MSGLOG DT:209-12-97 10:28:18

MSGLOG ERROR FLAG

MSGLOG_GEN_TYPE PCSOMS

MSGLOG SEQ NUM 108467

MSGLOG SPE $\bar{C}$ TYPE TCIC

MSGLOG STRINGI

RWTREAT ${ }^{20 T P 6-97-900002 \% O T ~}$

MSGLOG DT:209-12-97 10:29:01

MSGLOG_ERROR_FLAG

MSGLOG GEN TYPE PCSROMS

MSGLOG_SEQ_NUM 108468

MSGLOG SPE $\vec{C}$ TYPE $\quad$ RDMS

MSGLOG STRIÑG1 PCSRDMS ${ }^{\sim}$ RDMS

MSGLOG DT:209-12-97 10:29:02

MSGLOG_ERROR_FLAG N

MSGLOG_GEN_TYPE DMSPCS

MSGLOG SEQ NUM 108469

MSGLOG_SPEC

MSGLOG STRIÑT1 DMSPCS“SOMS 00000000 1000

"FUNCTIONING PROPERLY

MSGLOG DT:209+12+97 10:29:46

MSGLOG_ERROR_FLAG N

MSGLOG GEN TYPE

MSGLOG SEQ NUM 108470

MSGLOG_SPEC TYPE ROMS

MSGLOG STRIN̄G1 PCSRDMS"RDMS"

MSGLOG_DT:209-12-97 10:29:47

MSGLOG ERROR FLAG N

MSGLOG_GEN_TYPE DMSPCS

MSGLOG SEQ NUM 108471

MSGLOG SPEC TYPE

MSGLOG STRINTG1 DMSPCS ${ }^{\sim}$ SDMS $\sim 00000000 \sim 1000$

"FUNCTIONIHG PROPERLY

MSGLOG DT:209-12-97 10:30:31

MSGLOG ERROR FLAG

MSGLOG_GEN_TYPE PCSRDMS

MSGLOG SEO NSUM 108472

MSGLOG SPE $\bar{C}$ TYPE ROMS

MSGLOG STRINTG1 PCSROMS RDMS

MSGLOG DT:209-12-97 10:30:32

MSGLOG ERROR FLAG N

MSGLOG GEN TYPE DMSPCS

MSGLOG SEQ NUM 108473

MSGLOG SPE $\bar{C}$ TYPE SDMS

MSGLOG_STRINTG1 DMSPCS“SDMS ${ }^{\sim} 00000000^{\sim} 1000$

"FUNCTIONING PROPERLY ${ }^{\sim}$

MSGLOG_DT:209-12-97 10:31:16

MSGLOG ERROR FLAG N

MSGLOG_GEN TŸPE PCSRDMS

MSGLOGSSEQ-NUM 108474

MSGLOG SPEC TYPE RDMS

MSGLOG_STRIN̄G1 PCSROMS"RDMS

MSGLOG_OT:209-12-97 10:31:17

MSGLOG ERROR FLAG N

MSGLOG GEN TYYPE DMSPCS

MSGLOG SEQ NUM 108475

MSGLOG_SPE $\bar{C}$ TYPE SOMS 
HNF-SD-H025-0TR-014, Rev. 0, Page DMS-38

MSGLOG_STRING!

DMSPCS $\sim$ SOMS $0000000 \% 1000$

"FUNCTIONING PROPERLY"

MSGLOG_DT:209-12-97 10:32:01

MSGLOG ERROR FLAG N

MSGLOG_GEN TYPE PCSRDMS

MSGLOG SEQ NUM 108476

MSGLOG SPEC TYPE RDMS

MSGLOG_STRING1 , , PCSRDMS"ROMS

MSGLOG_DT:209-12-97 10:32:02

MSGLOG ERROR FLAG N

MSGLOG GEN TY PE

MSGLOG SEQ NUM

MSGLOG SPE $\bar{C}$ TYPE

DMSPCS

108477

SDMS

MSGLOG_STRING

DMSPCS ${ }^{\sim}$ SOMS $0000000 \% 1000$

"FUNCTIONING PROPERLY 
HNF-SD-WO26-OTR-O14 REV.O

\section{WRAP 1 ALARM RESPONSE PROCEDURE}

\section{WRP1-AR-0700}

\section{PROCESS AREA/GLOVEBOXES}

\begin{tabular}{|c|c|c|c|}
\hline $\begin{array}{c}\text { Alarm } \\
\text { Designator }\end{array}$ & $\begin{array}{c}\text { PCS Panel Tag \#s } \\
\text { in Alphanumeric } \\
\text { Order }\end{array}$ & Associated Alarms & $\begin{array}{l}\text { Page } \\
\text { Number }\end{array}$ \\
\hline $0700 / 1$ & 07-HS-305 & $\begin{array}{l}\text { TRU PROCESS GLOVEBOX SUITE } \\
\text { EMERGENCY STOP }\end{array}$ & 3 \\
\hline $0700 / 1$ & O7-HS-315 & $\begin{array}{l}\text { LLW PROCESS GLOVEBOX SUITE } \\
\text { EMERGENCY STOP }\end{array}$ & 3 \\
\hline $0700 / 2$ & $09-X S-215 A / B$ & $\begin{array}{l}\text { DRUM TRANSFER CONVEYOR A } \\
\text { ROPE SWITCH PULLED }\end{array}$ & 4 \\
\hline $0700 / 2$ & $09-X S-216 A / B$ & $\begin{array}{l}\text { DRUM TRANSFER CONVEYOR B } \\
\text { ROPE SWITCH PULLED }\end{array}$ & 4 \\
\hline $0700 / 3$ & $09-X S-221 A / B$ & $\begin{array}{l}\text { DRUM LIFT TABLE LT-09-201A } \\
\text { ROPE SWITCH PULLED }\end{array}$ & 5 \\
\hline $0700 / 2$ & $09-X S-222 A / B$ & $\begin{array}{l}\text { EMPTY DRUM CONVEYOR CV-09-203 } \\
\text { ROPE SWITCH PULLED }\end{array}$ & 4 \\
\hline $0700 / 3$ & $09-X S-223 A / B$ & $\begin{array}{l}\text { DRUM LIFT TABLE LT-09-201B } \\
\text { ROPE SWITCH PULLED }\end{array}$ & 5 \\
\hline $0700 / 2$ & $09-X S-224 A / B$ & $\begin{array}{l}\text { EMPTY DRUM CONVEYOR CV-09-204 } \\
\text { ROPE SWITCH PULLED }\end{array}$ & 4 \\
\hline $0700 / 3$ & $09-x S-225 A / B$ & $\begin{array}{l}\text { DRUM LIFT TABLE LT-09-201C } \\
\text { ROPE SWITCH PULLED }\end{array}$ & 5 \\
\hline $0700 / 3$ & $09-X S-227 A / B$ & $\begin{array}{l}\text { DRUM LIFT TABLE LT-09-201D } \\
\text { ROPE SWITCH PULLED }\end{array}$ & 5 \\
\hline $0700 / 3$ & $09-X S-229 A / B$ & $\begin{array}{l}\text { DRUM LIFT TABLE LT-09-201E } \\
\text { ROPE SWITCH PULLED }\end{array}$ & 5 \\
\hline $0700 / 3$ & $09-X S-231 A / B$ & $\begin{array}{l}\text { DRUM LIFT TABLE LT-09-201F } \\
\text { ROPE SWITCH PULLED }\end{array}$ & 5 \\
\hline
\end{tabular}

\begin{tabular}{|c|c|c|c|c|}
\hline 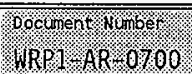 & 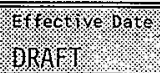 & 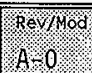 & For & Wov $/ 1 \%$ of 9 \\
\hline
\end{tabular}




\section{WRAP 1 ALARM RESPONSE PROCEDURE}

\begin{tabular}{|c|c|c|c|}
\hline $\begin{array}{l}\text { Alarm } \\
\text { Designator }\end{array}$ & $\begin{array}{c}\text { PCS Pane1 Tag \#s } \\
\text { in ATphanumeric } \\
\text { Order }\end{array}$ & Associated ATarms & $\begin{array}{l}\text { Page } \\
\text { Number }\end{array}$ \\
\hline $0700 / 3$ & $09-X S-233 A / B$ & $\begin{array}{l}\text { DRUM LIFT TABLE LT-09-202A } \\
\text { ROPE SWITCH PULLED }\end{array}$ & 5 \\
\hline $0700 / 3$ & $09-X S-235 A / B$ & $\begin{array}{l}\text { DRUM LIFT TABLE LT-09-202B } \\
\text { ROPE SWITCH PULLED }\end{array}$ & 5 \\
\hline $0700 / 3$ & $09-X S-237 A / B$ & $\begin{array}{l}\text { DRUM LIFT TABLE LT-09-202C } \\
\text { ROPE SWITCH PULLED }\end{array}$ & 5 \\
\hline $0700 / 3$ & $09-X S-239 A / B$ & $\begin{array}{l}\text { DRUM LIFT TABLE LT-09-202D } \\
\text { ROPE SWITCH PULLED }\end{array}$ & 5 \\
\hline $0700 / 3$ & $09-X S-241 A / B$ & $\begin{array}{l}\text { DRUM LIFT TABLE LT-09-203A } \\
\text { ROPE SWITCH PULLED }\end{array}$ & 5 \\
\hline $0700 / 3$ & $09-X S-243 A / B$ & $\begin{array}{l}\text { DRUM LIFT TABLE LT-09-203B } \\
\text { ROPE SWITCH PULLED. }\end{array}$ & 5 \\
\hline $0700 / 3$ & $09-X S-245 A / B$ & $\begin{array}{l}\text { DRUM LIFT TABLE LT-09-203C } \\
\text { ROPE SWITCH PULLED }\end{array}$ & 5 \\
\hline $0700 / 3$ & $09-X S-247 A / B$ & $\begin{array}{l}\text { DRUM LIFT TABLE LT-09-202E } \\
\text { ROPE SWITCH PULLED }\end{array}$ & 5 \\
\hline $0700 / 4$ & D0-07-105 & $\begin{array}{l}\text { LLW SORTING GLOVEBOX BAGLESS TRANSFER } \\
\text { PORT TROUBLE }\end{array}$ & 7 \\
\hline $0700 / 4$ & D0-07-201 & LLW RWM BAGLESS TRANSFER PORT TROUBLE & 7 \\
\hline $0700 / 4$ & D0-07-305 & $\begin{array}{l}\text { TRU SORTING GLOVEBOX BAGLESS TRANSFER } \\
\text { PORT TROUBLE }\end{array}$ & 7 \\
\hline $0700 / 4$ & D0-07-308 & $\begin{array}{l}\text { TRU EMPTY DRUM LOADOUT BAGLESS } \\
\text { TRANSFER PORT TROUBLE }\end{array}$ & 7 \\
\hline $0700 / 4$ & $D 0-07-401$ & $\begin{array}{l}\text { TRU RWM GLOVEBOX BAGLESS TRANSFER } \\
\text { PORT TROUBLE }\end{array}$ & 7 \\
\hline $0700 / 5$ & $\mathrm{HU}-07-304 T / \mathrm{A}$ & COMPACTOR TROUBLE & 9 \\
\hline
\end{tabular}

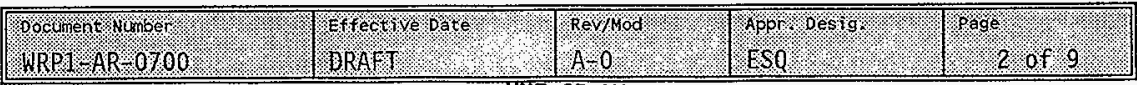


EQUIPMENT STATUS

$0700 / 1$

\begin{tabular}{|c|c|c|}
\hline Alarm Description & PCS Panel Tag \# & Setpoint/Source \\
\hline $\begin{array}{c}\text { TRU PROCESS GLOVEBOX SUITE } \\
\text { EMERGENCY STOP }\end{array}$ & $07-H S-305$ & $\begin{array}{c}\text { Operator at local DHS } \\
\text { Control Pedestal pushes the } \\
\text { Emergency Stop Pushbutton }\end{array}$ \\
$\begin{array}{c}\text { LLW PROCESS GLOVEBOX SUITE } \\
\text { EMERGENCY STOP }\end{array}$ & $07-H S-315$ & \\
\hline
\end{tabular}

\section{ALARM DESCRIPTION}

The Operator at the local DMS Control Pedestal pushes the Emergency Stop Pushbutton if a visually perceived potential problem exists. The associated glovebox equipment stops, and an alarm signal is sent to PCS.

II. ALARM PANEL LOCATION

WRAP 1 Control Room, Central Control Station

\section{ALARM RESPONSES}

1. Automatic Actions:

A. Al1 associated glovebox equipment stops.

2: Operator Actions:

A. ACKNOWLEDGE the alarm.

B. - INFORM the Duty Operations Supervisor.

C. COORDINATE with the Process Area Operator(s) to determine why the Emergency Stop Pushbutton was pushed.

D. WHEN condition requiring the Emergency Stop is rectified, RESET the associated glovebox per WRP1-0P-0702, Restart GTovebox Operation.

3. Indications:

A. Associated glovebox equipment stops.

4. Possible Causes:

A. Operator pushed the Emergency Stop Pushbutton at the local DMS Control Pedestal.

\section{REFERENCES}

1. H-2-131922, Sheet 1 .

2. H-2-131924, Sheet 1

\begin{tabular}{|c|c|c|c|c|}
\hline WRPJ 2 AR 0700 & 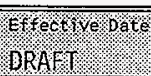 & R/O & (6) & prof $/ 2 . \%$ \\
\hline
\end{tabular}




\section{WRAP 1 ALARIM RESPONSE PROCEDURE}

EQUIPMENT STATUS

0700/2

\begin{tabular}{|c|c|c|}
\hline ATarm Description & PCS Panel Tag \# & Setpoint/Source \\
\hline $\begin{array}{c}\text { DRUM TRANSFER CONVEYOR A } \\
\text { ROPE SWITCH PULLED }\end{array}$ & $09-X S-215 A / B$ & \\
$\begin{array}{c}\text { DRUM TRANSFER CONVEYOR B } \\
\text { ROPE SWITCH PULLED }\end{array}$ & $09-X S-216 \mathrm{~A} / \mathrm{B}$ & $\begin{array}{c}\text { Manual actuation of } \\
\text { Rope Switch }\end{array}$ \\
$\begin{array}{c}\text { EMPTY DRUM CONVEYOR CV-09-203 } \\
\text { ROPE SWITCH PULLED } \\
\text {. }\end{array}$ & $09-X S-222 A / B$ \\
$\begin{array}{c}\text { EMPTY DRUM CONVEYOR CV-09-204 } \\
\text { ROPE SWITCH PULLED }\end{array}$ & $09-X S-224 A / B$ & \\
\hline
\end{tabular}

\section{ALARM DESCRIPTION}

There are pullcords run parallel to each conveyor. When a pullcord is actuated, it opens a switch, instantly deenergizing the Conveyor. An alarm is sent to PCS.

VI. ALARM PANEL LOCATION

WRAP I Control Room, Central Control Station

\section{ALARM RESPONSES}

1. Automatic Actions:

A. Affected Conveyor deenergizes.

2. Operator Actions:

A. ACKNOWLEDGE the alarm.

B. INFORM the Duty Operations Supervisor.

C. DISPATCH an Operator to. DETERMINE the reason the rope switch was pulled.

3. Indications:

A. Affected Conveyor stops.

4. Possible Causes:

A. Operator pulled rope switch.

VIII. REFERENCES

B. Faulty switch.

H-2-131854, Sheet 1

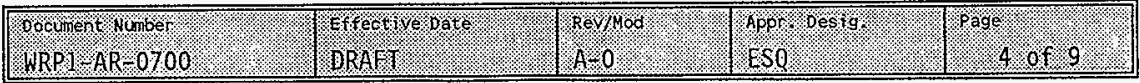




\section{ALARM DESCRIPTION}

There are pullcords associated with each lift table. When a pullcord is actuated, it opens a switch, instantly deenergizing the lift table motor. An 27 arm is sent to PCS.

\section{ALARM PANEL LOCATION}

WRAP 1 Control Room, Central Control Station

\section{ALARM RESPONSES}

1. Automatic Actions:

A. Affected lift table deenergizes.

2. Operator Actions:

A. ACKNOWLEDGE the a $\mathrm{arm}$.

B. INFORM the Duty Operations Supervisor.

C. DISPATCH an Operator to DETERMINE the reason the rope switch was pulted.

3. Indications:

A. Affected lift table stops.

4. Possible Causes:

A. Operator pulled rope switch.

B. Faulty switch.

XII. REFERENCES

1. H-2-131853, Sheets 3 and 4

2. $\mathrm{H}-2-131854$, Sheet 1 


\begin{tabular}{|c|c|c|}
\hline & \multicolumn{2}{|c|}{ EQUIPMENT STATUS } \\
\hline Alarm Description & PCS Panel Tag \# & Setpoint/Source \\
\hline $\begin{array}{l}\text { LLW SORTING GLOVEBOX } \\
\text { BAGLESS TRANSFER PORT } \\
\text { TROUBLE }\end{array}$ & D0-07-105： & \\
\hline $\begin{array}{l}\text { LLW RWH } \\
\text { BAGLESS TRANSFER PORT } \\
\text { TROUBLE }\end{array}$ & D0-07-201 & \\
\hline $\begin{array}{l}\text { TRU SORTING GLOVEBOX } \\
\text { BAGLESS TRANSFER PORT } \\
\text { TROUBLE }\end{array}$ & D0-07-305 & $\begin{array}{l}\text { Bagless Transfer Port Controller } \\
\text { does not return OPEN/CLOSED } \\
\text { signal to PCS within specified }\end{array}$ \\
\hline $\begin{array}{l}\text { TRU EMPTY DRUM LOADOUT } \\
\text { BAGLESS TRANSFER PORT } \\
\text { TROUBLE }\end{array}$ & D0-07-308 & \\
\hline $\begin{array}{l}\text { TRU RWM GLOVEBOX } \\
\text { BAGLESS TRANSFER PORT } \\
\text { TROUBLE }\end{array}$ & D0-07-401 & \\
\hline
\end{tabular}

\section{ALARM DESCRIPTION}

When the Operator selects "OPEN PORT" ("CLOSE PORT") from the Operator Interface Unit (OIU), PCS sends a signal to the associated Bagless Transfer Port Controller commanding the port to open (close). If confirmation that the command has been carried out is not received by PCS, a trouble alarm is generated.

\section{ALARM PANEL LOCATION}

WRAP 1 Control Room, Central Control Station

\section{ALARM RESPONSES}

1. Automatic Actions:

None

2. Operator Actions:
A. ACKNOWLEDGE the al arm.
B. INFORM the Duty Operations Supervisor.
C. COORDINATE with the Process Area Operator(s) to locally
investigate/resolve the alarm at the Bagless Transfer Port Controljer.

3. Indications: 


\section{WRAP 1 ALARM RESPONSE PROCEDURE}

A. Local observation only.

4. Possible Causes:

A. Obstruction in port.

B. Bagless Transfer Port Controller problem.

C. Port position sensor problem.

D. Port mechanically bound.

\section{REFERENCES}

1. H-2-131922, Sheets 2, 5

2. H-2-131926, Sheet 1 


\section{WRAP 1 ALARM RESPONSE PROCEDURE}

EQUIPMENT STATUS

0700/5

\begin{tabular}{|c|c|l|}
\hline Alarm Description & PCS Panel Tag \# & \multicolumn{1}{|c|}{ Setpoint/Source } \\
\hline COMPACTOR TROUBLE & HU-07-304 & $\begin{array}{l}\text { PCS receives HU-07-304/T, } \\
\text { Compaction Trouble, signai from } \\
\text { Hydraulic Power Unit (HPU) } \\
\text { Controller. }\end{array}$ \\
\hline
\end{tabular}

\section{ALARM DESCRIPTION}

PCS receives HU-07-304/T Compaction Trouble signal from Hydraulic Power Unit (HPU) Controller. The cause of the HU-07-304/T Compaction Trouble signal is one of thirty-one fault messages generated by the OIU-12-104C HPU Controller, and the cause must be determined at the local message display.

\section{II. . ALARM PANEL LOCATION}

WRAP 1 Control Room, Central Control Station

\section{ALARM RESPONSES}

1. Automatic Actions:

None

2. Operator Actions:

A. ACKNOWLEDGE the alarm.

B. INFORH the Duty Operations Supervisor.

C. COORDINATE with the Process Area Operator(s) to Tocally

determine the cause of the alarm and resolve any problem that

the HPU Controller message display may indicate per

WRP1-AR-0701, Supercompactor ATarm Response Procedure.

3. Indications:

A. Local observation on ly.

4. Possible Causes:

A. Multiple causes (31 fault messages) as addressed by WRP1-AR-0701, Supercompactor A1 arm Response Procedure.

\section{REFERENCES}

H-2-131922, Sheet 3

\begin{tabular}{|c|c|c|c|c|}
\hline 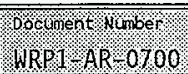 & $\begin{array}{l}\text { Effect } \\
\text { BRAFI }\end{array}$ & H & $\begin{array}{l}\mathrm{por} \\
\mathrm{SO}\end{array}$ & poge \\
\hline
\end{tabular}




\section{WRP1-AR-1106 PROCESS AREA (ZONE II) HVAC}

\begin{tabular}{|c|c|c|c|}
\hline $\begin{array}{c}\text { Alarm } \\
\text { Designator }\end{array}$ & $\begin{array}{c}\text { PCS Panel Tag \#s } \\
\text { in Alphanumeric } \\
\text { Order }\end{array}$ & Associated Alarms & $\begin{array}{c}\text { Page } \\
\text { Number }\end{array}$ \\
\hline $1106 / 1$ & $11-F I-618 \mathrm{~A}$ & $\begin{array}{l}\text { PROCESS EXHAUST FILTER TRAIN A LOW AIRFLOW } \\
425 \mathrm{CMM}(15,000 \mathrm{CFM})\end{array}$ & 3 \\
\hline $1106 / 2$ & $11-F I-618 A$ & $\begin{array}{l}\text { PROCESS EXHAUST FILTER TRAIN A HIGH AIRFLOW } \\
450 \mathrm{CMM}(16,000 \mathrm{CFM})\end{array}$ & 4 \\
\hline $1106 / 1$ & $11-F I-618 B$ & $\begin{array}{l}\text { PROCESS EXHAUST FILTER TRAIN B LOW AIRFLOW } \\
425 \text { CMM }(15,000 \text { CFM })\end{array}$ & 3 \\
\hline $1106 / 2$ & $11-F I-618 B$ & $\begin{array}{l}\text { PROCESS EXHAUST FILTER TRAIN B HIGH AIRFLOW } \\
450 \mathrm{CMM}(16,000 \mathrm{CFM})\end{array}$ & 4 \\
\hline $1106 / 3$ & $11-P D I S H-601 \mathrm{~A}$ & $\begin{array}{l}\text { PROCESS AHU A PRE-FILTER } \\
\text { HIGH DIFF PRESS }\end{array}$ & 5 \\
\hline $1106 / 4$ & $11-\mathrm{PDISH}-602 \mathrm{~A}$ & $\begin{array}{l}\text { PROCESS AHU A FINAL FILTER } \\
\text { HIGH DIFF PRESS }\end{array}$ & 6 \\
\hline $1106 / 3$ & $11-\mathrm{PDISH}-601 \mathrm{~B}$ & $\begin{array}{l}\text { PROCESS AHU B PRE-FILTER } \\
\text { HIGH DIFF PRESS }\end{array}$ & 5 \\
\hline $1106 / 4$ & 11 -PDISH-602B & $\begin{array}{l}\text { PROCESS AHU B FINAL FILTER } \\
\text { HIGH DIFF PRESS }\end{array}$ & 6 \\
\hline $1106 / 5$ & 11-PDISH-612A & $\begin{array}{l}\text { PROCESS EXHAUST A PRE - FILTER } \\
\text { HIGH DIFF PRESS }\end{array}$ & 7 \\
\hline $1106 / 5$ & $11-P D I S H-612 B$ & $\begin{array}{l}\text { PROCESS EXHAUST B PRE-FILTER } \\
\text { HIGH DIFF PRESS }\end{array}$ & 7 \\
\hline $1106 / 6$ & 11-PDISH-614A & $\begin{array}{l}\text { PROCESS EXHAUST A FIRST HEPA FILTER } \\
\text { HIGH DIFF PRESS }\end{array}$ & 8 \\
\hline $1106 / 6$ & 11-PDISH-614B & $\begin{array}{l}\text { PROCESS EXHAUST B FIRST HEPA FILTER } \\
\text { HIGH DIFF PRESS }\end{array}$ & 8 \\
\hline $1106 / 7$ & $11-\mathrm{PDISH}-616 \mathrm{~A}$ & $\begin{array}{l}\text { PROCESS EXHAUST A SECOND KEPA FILTER } \\
\text { HIGH DIFF PRESSURE }\end{array}$ & 9 \\
\hline $1106 / 7$ & 11 -PDISH-6168 & $\begin{array}{l}\text { PROCESS EXHAUST B SECOND HEPA FILTER } \\
\text { HIGH DIFF PRESSURE }\end{array}$ & 9 \\
\hline
\end{tabular}

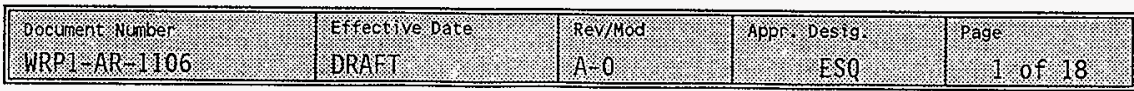




\begin{tabular}{|c|c|c|c|}
\hline $\begin{array}{c}\text { Alarm } \\
\text { Designator }\end{array}$ & $\begin{array}{c}\text { PCS Panel Tag \#s } \\
\text { in Alphanumeric } \\
\text { Order }\end{array}$ & Associated Alarms & $\begin{array}{l}\text { Page } \\
\text { Number }\end{array}$ \\
\hline $1106 / 8$ & $11-T 1-609 \dot{A}$ & $\begin{array}{l}\text { PROGESS AREA HIGH TEMPERATURE } \\
32^{\circ} \mathrm{C}\left(90^{\circ} \mathrm{F}\right)\end{array}$ & 10 \\
\hline $1106 / 9$ & $11-71-609 A$ & $\begin{array}{l}\text { PROCESS AREA LOW TEMPERATURE } \\
16^{\circ} \mathrm{C}\left(60^{\circ} \mathrm{F}\right)\end{array}$ & 11 \\
\hline $1106 / 8$ & $11-T 1-609 B$ & $\begin{array}{l}\text { PROCESS AREA HIGH TEMPERATURE } \\
32^{\circ} \mathrm{C}\left(90^{\circ} \mathrm{F}\right)\end{array}$ & 10 \\
\hline $1106 / 9$ & $11-T I-609 B$ & $\begin{array}{l}\text { PROCESS AREA LOW TEMPERATURE } \\
16^{\circ} \mathrm{C}\left(60^{\circ} \mathrm{F}\right)\end{array}$ & 11 \\
\hline $1106 / 10$ & $11-T I-618 A$ & $\begin{array}{l}\text { PROCESS EXHAUST FILTER TRAIN A } \\
\text { LOW TEMPERATURE }\end{array}$ & 12 \\
\hline $1106 / 10$ & $11-T I-618 B$ & $\begin{array}{l}\text { PROCESS EXHAUST FILTER TRAIN B } \\
\text { LOW TEMPERATURE }\end{array}$ & 12 \\
\hline $1106 / 11$ & $11-X A-601 A$ & $\begin{array}{l}\text { PROCESS SUPPLY AHU CONTROLLER } \\
\text { C-11-201A TROUBLE }\end{array}$ & 13 \\
\hline $1106 / 11$ & $11-X A-601 B$ & $\begin{array}{l}\text { PROCESS SUPPLY AHU CONTROLLER } \\
\text { C-11-201B TROUBLE }\end{array}$ & 13 \\
\hline $1106 / 12$ & $11-X A-612 A$ & $\begin{array}{l}\text { PROCESS EXHAUST SYSTEM CONTROLLER } \\
\text { C-11-202A TROUBLE }\end{array}$ & 14 \\
\hline $1106 / 12$ & $11-X A-612 B$ & $\begin{array}{l}\text { PROCESS EXHAUST SYSTEM CONTROLLER } \\
C-11-202 B \text { TROUBLE }\end{array}$ & 14 \\
\hline $1106 / 13$ & $E F-11-202 A$ & PROCESS EXHAUST FILTER TRAIN A FAN FAILED & 15 \\
\hline $1106 / 13$ & $E F-11-202 B$ & PROCESS EXHAUST FILTER TRAIN B FAN FAILED & 15 \\
\hline $1106 / 14$ & $S F-11-201 A$ & PROCESS AHU A SUPPLY FAN FAILED & 17 \\
\hline $1106 / 14$ & $S F-11-201 B$ & PROCESS AHU B SUPPLY FAN FAILED & 17 \\
\hline
\end{tabular}

\begin{tabular}{|c|c|c|c|c|}
\hline 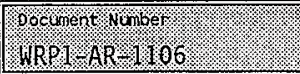 & WRAF & A. 0.2 & 9. & 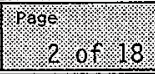 \\
\hline
\end{tabular}




\section{WRAP 1 ALARM RESPONSE PROCEDURE}

EQUIPMENT STATUS

$1106 / 1$

\begin{tabular}{c|c|c|}
\hline Alarm Description & PCS Panel Tag \# & Setpoint/Source \\
\hline $\begin{array}{c}\text { PROCESS EXHAUST FILTER } \\
\text { TRAIN A/B LOW AIRFLOW } \\
425 \text { CMM }(15,000 \text { CFM })\end{array}$ & $11-F I-618 A / B$ & 425 CMM $(15,000 \mathrm{CFM})$ \\
\hline
\end{tabular}

\section{ALARM DESCRIPTION}

Zone II Process Exhaust Filter Train Fan flow is sensed by a flow element at the discharge of the fan. This alarm alerts the operator if the flow from the fan is below the expected range.

\section{ALARM PANEL LOCATION}

WRAP 1 Control Room, Central Control Station

\section{ALARM RESPONSES}

1. Automatic Actions:

None

2. Operator Actions:

A. ACKNOWLEDGE the alarm.

B. INFORM the Duty Operations Supervisor.

C. CHECK other HVAC parameters.

D. SHIFT to the standby Process Exhaust Filter Train as necessary per WRP1-0P-1101, Operation of the HVAC System.

3. Indications:

A. Low airflow in the Process Area.

4. Possible Causes:

A. Faulty control circuit.

B. Instrument error

C. Clogged HEPA Filter.

\section{REFERENCES}

H-2-131893 Sheet 1

\begin{tabular}{|c|c|c|c|c|}
\hline 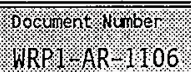 & 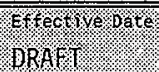 & Revmod & \%pprovero & wav \\
\hline
\end{tabular}


PCS Panel Tag.\#

Setpoint/Source

PROCESS EXHAUST FILTER TRAIN A/B HIGH AIRFLOW

450 CMM $(16,000$ CFM $)$

\section{ALARM DESCRIPTION}

Zone II Process Exhaust Filter Train Fan flow is sensed by a flow element at the discharge of the fan. This alarm alerts the operator if the flow from the fan is above the expected range.

\section{ALARM PANEL LOCATION}

WRAP 1 Control Room, Central Control Station

\section{ALARM RESPONSES}

1. Automatic Actions:

None

2. Operator Actions:

A. ACKNOWLEDGE the alarm.

B. INFORM the Duty Operations Supervisor.

C. CHECK other HVAC parameters.

3. Indications:

A. High airflow in the Process Area.

4. Possible Causes:
A. Faulty control circuit.
B. Instrument error.
C. Punctured HEPA Filter.

\section{REFERENCES}

H-2-131893 Sheet 1

\begin{tabular}{|c|c|c|c|c|}
\hline 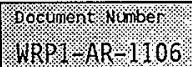 & $\begin{array}{l}\text { Effect } \\
\text { BRARI }\end{array}$ & Rey $10 \%$ & mpropso & page \\
\hline
\end{tabular}




\section{WRAP 1 ALARM RESPONSE PROCEDURE}

EQUIPMENT STATUS

$1106 / 3$

\begin{tabular}{|c|c|c|}
\hline A1arm Description & PCS Panel Tag \# & Setpoint/Source \\
\hline $\begin{array}{l}\text { PROCESS AHU A/B } \\
\text { PRE-FILTER } \\
\text { HIGH DIFF PRESS }\end{array}$ & 1I-PDISH-601A/B & $0.60^{\prime \prime}$ W.C. \\
\hline
\end{tabular}

\section{ALARM DESCRIPTION}

Particulate/moisture has built up on the Process Area Supply AHU Pre-Filter to the point where the differential pressure has exceeded the setpoint. An alarm signal is sent to the PCS.

\section{ALARM PANEL LOCATION}

WRAP 1 Control Room, Central Control Station

\section{ALARM RESPONSES}

1. Automatic Actions:

None

2. Operator Actions:

A. ACKNOWLEDGE the al arm.

B. INFORM the Duty Operations Supervisor.

C. ENSURE Process Room 117 is being maintained at $-0.15^{\text {" W.C. as }}$ indicated on the associated RTAP screen.

D. DISPATCH an operator to locally investigate and observe the local indications of alarming Pre-Filter.

E. IF alarm is due to particulate buildup (clogged filter), SHIFT to the standby Process Area Suppiy AHU per WRP1-OP-1101, Operation of the HVAC System.

3. Indications:

A. Local differentia] pressure indication.

4. Possible Causes:

A. Particuiate or moisture buildup.

B. Instrument error.

\section{REFERENCES}

H-2-131892

\begin{tabular}{|c|c|c|c|c|}
\hline WRPR AR L 106 & $\begin{array}{l}\text { Effect } \\
\text { OAATI }\end{array}$ & $\begin{array}{l}\mathrm{RevNo} \\
\mathrm{A}=0\end{array}$ & Hop $\%$ WESO & $1 \%$ of 18 \\
\hline
\end{tabular}


EQUIPMENT STATUS

\section{Alarm Description}

PROCESS AHU A/B

FINAL FILTER

HIGH DIFF PRESS

\section{EQUIPIMENT STATUS}

PCS Panel Tag \#

11-PDISH-602A/B
$1106 / 4$

Setpoint/Source

1.00 " H.C.

\section{ALARM DESCRIPTION}

Particulate/moisture has built up on the Process Area Supply AHU Final Filter to the point where the differential pressure has exceeded the setpoint. An alarm signal is sent to the PCS.

\section{ALARM PANEL LOCATION}

WRAP 1 Control Room, Central Control Station

\section{ALARM RESPONSES}

\section{Automatic Actions:}

None

2. Operator Actions:
A. ACKNOHLEDGE the al arm.
B. INFORM the Duty Operations Supervisor.
C. ENSURE Process Room 117 is being maintained at - $0.15^{\prime \prime}$ W.C. as
C. indicated on the associated RTAP screen.
D. DISPATCH an operator to locally investigate and observe the local indications of alarming Final Filter.

3. Indications:

A. LocaT differential pressure indication.

4. Possible Causes:

A. Particulate or moisture buildup.

B. Instrument error.

\section{REFERENCES}

H-2-131892

\begin{tabular}{|c|c|c|c|c|c|}
\hline 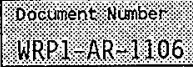 & 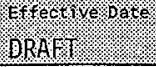 & 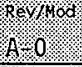 & mporpose & past. & 0678 \\
\hline
\end{tabular}




\section{WRAP 1 ALARIM RESPONSE PROCEDURE}

EQUIPMENT STATUS

$1106 / 5$

\begin{tabular}{|c|c|c|}
\hline A1arm Description & PCS Pane1 Tag \# & Setpoint/Source \\
\hline $\begin{array}{c}\text { PROCESS EXHAUST A/B } \\
\text { PRE-FILTER } \\
\text { HIGH DIFF PRESS }\end{array}$ & 11-PDISH-612A/B & $225 \mathrm{~Pa}\left(0.9^{\prime \prime}\right.$ W.C.) \\
\hline
\end{tabular}

\section{ALARM DESCRIPTION}

Particulate/moisture has built up on the affected Zone II Process Exhaust Pre-Filter to the point where differential pressure has reached the setpoint. An alarm signal is sent to the PCS.

\section{ALARM PANEL LOCATION.}

\section{WRAP 1 Control Room, Central Control Station}

\section{ALARM RESPONSES}

1. Automatic Actions:

None

2. Operator Actions:

A. ACKNOWLEDGE the alarm.

B. INFORM the Duty Operations Supervisor.

C. CHECK related system parameters.

D. DISPATCH an operator to locally investigate and observe the local indications of alarming Pre-Filter.

E. IF alarm is due to particulate buildup (clogged filter), SHIFT to the standby Process Exhaust Filter Train per WRPI-OP-1101, Operation of the HVAC System.

3. Indications:

A. LocaT pressure differential indication.

4. Possible Causes:

A. Particulate/moisture buildup.

B. Instrument error.

\section{REFERENCES}

H-2-131893 Sheet 1

\begin{tabular}{|c|c|c|c|c|}
\hline 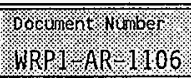 & Effect & 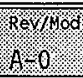 & hopr ops & past 1 of 18 \\
\hline
\end{tabular}




\section{WRAP 1 ALARM RESPONSE PROCEDURE}

EQUIPMENT STATUS

\begin{tabular}{|c|c|c|}
\hline \multicolumn{3}{|c|}{ EQUIPMENT STATUS } \\
\hline Alarm Description & PCS Panel Tag \# & Setpoint/Source \\
\hline $\begin{array}{c}\text { PROCESS EXHAUST A/B } \\
\text { FIRST HEPA FILTER } \\
\text { HIGH DIFF PRESS }\end{array}$ & 11-PDISH-614A/B & $750 \mathrm{~Pa}\left(3.0^{\prime \prime}\right.$ W.C. $)$ \\
\hline
\end{tabular}

\section{ALARM DESCRIPTION}

Particulate/moisture has built up on the affected Zone II Process Exhaust First HEPA Filter to the point where differential pressure has reached the setpoint. An alarm signal is sent to the PCS.

\section{ALARM PANEL LOCATION}

WRAP 1 Control Room, Central Control Station

\section{ALARM RESPONSES}

1. Automatic Actions:

None

2. Operator Actions:

A. ACKNOWLEDGE the alarm.

B. INFORN the Duty Operations Supervisor.

C. CHECK related system parameters.

D. DISPATCH an operator to locally investigate and observe the local indications of alarming First HEPA Filter.

3. Indications:

A. Local pressure differential indication.

4. Possible Causes:

A. Particulate/moisture buildup.

B. Instrument error.

\section{REFERENCES}

H-2-131893 Sheet 1 


\section{WRAP 1 ALARM RESPONSE PROCEDURE}

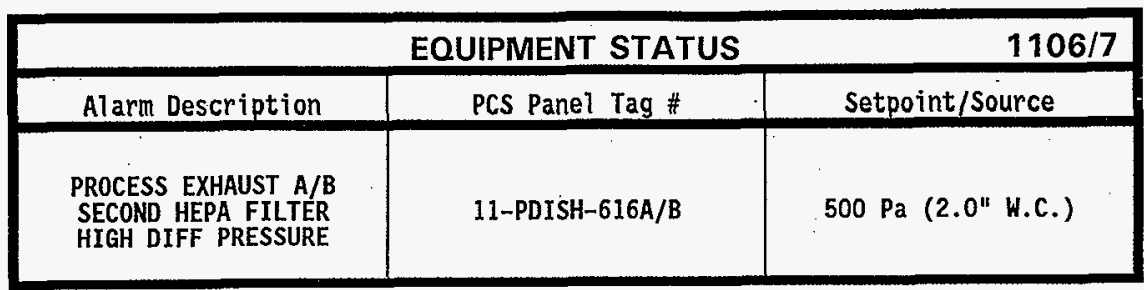

\section{ALARM DESCRIPTION}

Particulate/moisture has built up on the affected Zone II Process Exhaust Second HEPA Filter to the point where differential pressure has reached the setpoint. An alarm signal is sent to the PCS.

\section{ALARM PANEL LOCATION}

WRAP 1 Control Room, Central Control Station

\section{ALARM RESPONSES}

1. Automatic Actions:

None

2. Operator Actions:

A. ACKNOWLEDGE the al arm.

B. INFORM the Duty Operations Supervisor.

C. CHECK related system parameters.

D. DISPATCH an operator to locally investigate and observe the local indications of alarming Second HEPA Filter.

3. Indications:
A. Local pressure differential indication.

4. Possible Causes:

A. Particulate/moisture buildup.

B. Instrument error.

\section{REFERENCES}

\section{H-2-131893 Sheet 1}

\begin{tabular}{|c|c|c|c|c|}
\hline 6ocond & 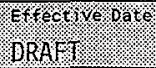 & Key $100 \%$ & Hopros & 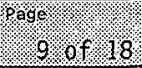 \\
\hline
\end{tabular}


Alarm Description

PROCESS AREA HIGH TEMPERATURE $32^{\circ} \mathrm{C}\left(90^{\circ} \mathrm{F}\right)$
PCS Panel Tag \#

- 11-TI-609A/B
Setpoint/Source

$32^{\circ} \mathrm{C}\left(90^{\circ} \mathrm{F}\right)$

\section{ALARM DESCRIPTION}

When Process Area temperature exceeds the setpoint, an alarm is sent to the PCS.

\section{ALARM PANEL LOCATION}

WRAP 1 Control Room, Central Control Station

\section{ALARM RESPONSES}

1. Automatic Actions:

None

2. Operator Actions:

A. ACKNOWLEDGE the al arm:

B. INFORM the Duty Operations Supervisor.

C. VERIFY proper operation of the in-service Process Area Air

Handling Unit, 203-AH-11-201A/B per WRP1-OP-110I, Operation of the HVAC System.

D. SHIFT to the standby Process Area Air Handling Unit per WRP1-0P-1101, if necessary.

3. Indications:

A. Uncomfortably hot in the Process Area.

4. Possible Causes:

A. Faulty Process Area Air Handling Unit or associated Control Circuit.

B. Faulty sensor/alarm circuit.

\section{REFERENCES}

H-2-131892

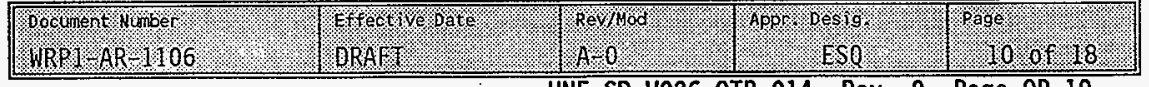




\section{WRAP 1 ALARIM RESPONSE PROCEDURE}

\section{EQUIPMENT STATUS}

$1106 / 9$

Alarm Description

PCS Panel Tag \#

Setpoint/Source

PROCESS AREA

LOW TEMPERATURE

$16^{\circ} \mathrm{C}\left(60^{\circ} \mathrm{F}\right)$

11-TI-609A/B

$16^{\circ} \mathrm{C}\left(60^{\circ} \mathrm{F}\right)$

\section{ALARM DESCRIPTION}

When Process Area temperature falls below the setpoint, an alarm is sent to the PCS.

\section{ALARM PANEL LOCATION}

WRAP 1 Control Room, Central Control Station

\section{ALARM RESPONSES}

1. Automatic Actions:

None

2. Operator Actions:

A. ACKNOWLEDGE the a $7 \mathrm{arm}$.

B. INFORM the Duty Operations Supervisor.

C. VERIFY proper operation of the in-service Process Area Air Handling Unit, 203-AH-11-201A/B per WRP1-0P-1101, Operation of the HVAC System.

D. SHIFT to the standby Process Area Air Handling Unit per WRP1-OP-1101, if PCS control cannot be re-established.

3. Indications:

A. Uncomfortably cold in the Process Area.

4. Possible Causes:

A. Faulty Process Area Air Handling Unit or associated Control Circuit.

B. Faulty sensor/alarm circuit.

C. During extremely cold weather, the heating system may not have sufficient capacity to maintain setpoint.

D. Heat Recovery System failure.

\section{REFERENCES}

H-2-131892

\begin{tabular}{|c|c|c|c|c|}
\hline 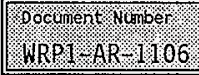 & $\begin{array}{l}\text { Effect verate } \\
\mathrm{PRA}\end{array}$ & A- & 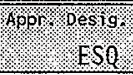 & 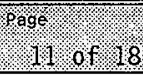 \\
\hline
\end{tabular}




\section{WRAP 1 ALARM RESPONSE PROCEDURE}

\section{EQUIPMENT STATUS}

$1106 / 10$

\begin{tabular}{|c|c|c|}
\hline Alarm Description & PCS Panel Tag \# & Setpoint/Source \\
\hline $\begin{array}{c}\text { PROCESS EXHAUST FILTER } \\
\text { TRAIN A/B } \\
\text { LOH TEMPERATURE }\end{array}$ & $11-\mathrm{TI}-618 \mathrm{~A} / \mathrm{B}$ & $6^{\circ} \mathrm{C}\left(43^{\circ} \mathrm{F}\right)$ \\
\hline
\end{tabular}

\section{ALARM DESCRIPTION}

When the Heat Recovery System has cooled the exhaust air to less than $6^{\circ} \mathrm{C}$ $\left(43^{\circ} \mathrm{F}\right)$, an a] arm is sent to the PCS.

\section{ALARM PANEL LOCATION}

WRAP 1 Control Room, Central Control Station

\section{ALARM RESPONSES}

1. Automatic Actions:

None

2. Operator Actions:

A. ACKNOWLEDGE the a $1 \mathrm{arm}$.

B. INFORM the Duty Operations Supervisor.

C. VERIFY proper operation of the Process HVAC Heating Units/Heat Recovery Contro1 System per WRP1-OP-1101, Operation of the HVAC System.

3. Indications:

None

4. Possible Causes:

A. Failed Heat Recovery Control System.

B. Faulty sensor.

\section{REFERENCES}

H-2-131893 Sheet 1 


\section{WRAP 1 ALARM RESPONSE PROCEDURE}

EQUIPMENT STATUS

$1106 / 11$

Alarm Description
PROCESS SUPPLY AHU
CONTROLLER C-11-2O1A/B

TROUBLE

$11-\mathrm{XA}-601 \mathrm{~A} / \mathrm{B}$

Digital input

from DCS to PCS

\section{ALARM DESCRIPTION}

The Process Supply AHU Controller self monitors for indication of proper operation: When an abnormal condition is detected, it is displayed locally on the controller and an alarm is sent to the PCS. This alarm may also indicate a problem when a communicator 1ink or NCU (Network Control Unit) needs maintenance and digital communication is not possible.

\section{ALARM PANEL LOCATION}

WRAP 1 Control Room, Central Control Station

\section{ALARM RESPONSES}

1. Automatic Actions:

None

2. Operator Actions:

A. ACKNOWLEDGE the alarm.

B. INFORM the Duty Operations Supervisor.

C. CHECK related HVAC parameters.

D. DISPATCH an operator to the alarming Process Supply AHU to investigate the trouble alarm.

3. Indications:

A. Local indication on the Controller.

4. Possible Causes:

A. Internal AHU Controller problem.

B. Internal NCU (Network Control Unit) problem.

C. Circuit communication problem.

\section{REFERENCES}

H-2-131893 Sheet 1 


\begin{tabular}{|c|c|c|}
\hline Alarm Description & PCS Panel Tag \# & Setpoint/Source \\
\hline $\begin{array}{c}\text { PROCESS EXHAUST SYSTEM } \\
\text { CONTROLLER C-11-202A/B } \\
\text { TROUBLE }\end{array}$ & I1-XA-612A/B & $\begin{array}{c}\text { Digital input } \\
\text { from DCS to PCS }\end{array}$ \\
\hline
\end{tabular}

\section{ALARM DESCRIPTION}

The Process Exhaust System Controller self monitors for indication of proper operation. When an abnormal condition is detected, it is displayed locally on the controller and an alarm is sent to the PCS. This alarm may a I so indicate a problem when a communicator 1ink or NCU (Network Control Unit) needs maintenance and digital communication is not possible.

\section{ALARM PANEL LOCATION}

WRAP 1 Control Room, Central Control Station

\section{ALARM RESPONSES}

1. Automatic Actions:

None

2. Operator Actions:

A. ACKNOWLEDGE the alarm.

B. INFORM the Duty Operations Supervisor.

C. CHECK related HVAC parameters.

D. DISPATCH an operator to the ajarming Process Exhaust System Controller to investigate the trouble alarm.

3. Indications:

A. Local indication on the Controller.

4. Possible Causes:

A. Internal Controller problem.

B. Internal NCU (Network Control Unit) problem.

C. Circuit communication probiem.

\section{REFERENCES}

H-2-131893 Sheet 1

\begin{tabular}{|c|c|c|c|c|}
\hline HRO $-\mathrm{AR}-106$ & $\begin{array}{l}\text { Effect vepto } \\
\text { oRAEF }\end{array}$ & ReY $10 \%$ & Hopospos & 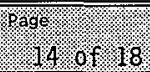 \\
\hline
\end{tabular}




\section{WRAP 1 ALARM RESPONSE PROCEDURE}

\begin{tabular}{|c|c|c|}
\hline & EQUIPMENT STATUS & $1106 / 13$ \\
\hline Alarm Description & PCS Panel Tag \# & Setpoint/Source \\
\hline $\begin{array}{c}\text { PROCESS EXHAUST FILTER } \\
\text { TRAIN A/B } \\
\text { FAN FAILED }\end{array}$ & EF-11-202 A/B & $\begin{array}{c}\text { PDISL-618 A/B senses 1ow } \\
\text { differential pressure } \\
\text { across Zone II Process } \\
\text { Exhaust Filter Train Fan } \\
\text { EF-11-202 A/B }\end{array}$ \\
\hline
\end{tabular}

\section{ALARM DESCRIPTION}

Zone II Process Exhaust Filter Train Fan operation is sensed by a differential pressure sensor. It measures the pressure drop across the fan. When the differential pressure (airflow) between the two points drops below the setpoint (indicating a loss of the fan) an alarm is sent to the PCS. Another signal places the standby unit online.

\section{ALARM PANEL LOCATION}

WRAP 1 Control Room, Central Control Station

\section{ALARM RESPONSES}

1. Automatic Actions:

A. System shifts to the Standby Exhaust Filter Train Fan/AHU when there is a loss of airflow on the running Exhaust Filter Train Fan/AHU.

2. Operator Actions:

A. ACKNOWLEDGE the a $7 \mathrm{arm}$.

B. INFORM the Duty Operations Supervisor.

C. VERIFY the Standby Zone II Process Exhaust Filter Train Fan has started and system parameters have stabilized.

3. Indications:

A. Duct smoke detector alarm (fire dampers shut).

B. Smoke and burning odor at the motor feeder breaker.

C. Low airflow.

D. System has shifted to the Standby Exhaust Finter Train Fan/AfU due to the loss of airflow on the running Exhaust Filter Train Fan/AHU.

E. Fire alarm.

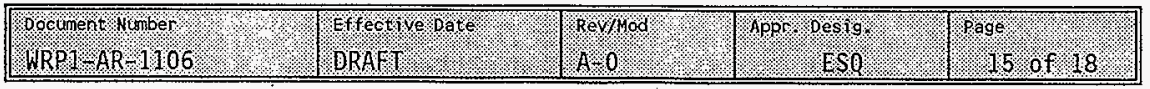




\section{Possible Causes:}
A. Failed motor.
B. Loss of electrical power.
C. Feeder breaker trip:
- MCC-11-102 for EF-11-202A
D. Broken drive belt.
E. Loss of control power.
$F$. Loss of instrument air.

\section{REFERENCES}

H-2-131893 Sheet 1 


\begin{tabular}{|c|c|c|}
\hline Alarm Description & PCS Panel Tag \# & Setpoint/Source \\
\hline $\begin{array}{c}\text { PROCESS AHU A/B } \\
\text { SUPPLY FAN } \\
\text { FAILED }\end{array}$ & SF-11-201A/B & $\begin{array}{c}\text { PDISL-603 A/B senses low } \\
\text { differentia pressure } \\
\text { across Supply Fan }\end{array}$ \\
\hline
\end{tabular}

\section{ALARM DESCRIPTION}

Process Air Handling Unit Supply Fan operation is sensed by a differential pressure sensor across the fan. When the differential pressure (airflow) between the two points drops below the setpoint (indicating a loss of the fan) an alarm is sent to the PCS. Another signal places the standby unit ont ine.

\section{ALARM PANEL LOCATION}

WRAP 1 Control Room, Central Control Station

\section{ALARM RESPONSES}

1. Automatic Actions:

A. System shifts to the Standby Supply Fan/AHU when there is a loss of airflow on the running Supply Fan/AHU.

2. Operator Actions:

A. ACKNOWLEDGE the alarm.

B. INFORM the Duty Operations Supervisor.

C. VERIFY the Standby Process Air Handling Unit Supply Fan has started and system parameters have stabilized.

3. Indications:

A. Duct smoke detector alarm (fire dampers shut).

B. Loss of glovebox HVAC.

C. Smoke and burning odor at the motor feeder breaker.

D. Low Process Area airflow.

E. System has shifted to the standby Supply Fan/AHU due to the

loss of airflow on the running Supply Fan/AHU.

F. Fire alarm.

\begin{tabular}{|c|c|c|c|c|}
\hline 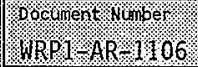 & 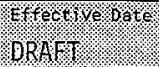 & Revmod & \%pproespg & $\begin{array}{l}\text { page } \\
1 \% 17 \% \text { f } \\
18\end{array}$ \\
\hline
\end{tabular}


4. Possible Causes:
A. Failed motor.
B. Loss of electrical power.
C. Feeder breaker trip:
- MCC-11-102 for SF-11-201A
D. Broken drive be1t.
MCC-11-103 for SF-11-201B
E. Loss of control power.
$F$. Loss of instrument air.

\section{REFERENCES}

H-2-131892 
HNF-SD-WOZ6-OTP-OI4 REV-O

\section{WRAP 1 ALARIM RESPONSE PROCEDURE}

\section{WRP1-AR-1107}

\section{GLOVEBOX (ZONE I) HVAC}

\begin{tabular}{|c|c|c|c|}
\hline $\begin{array}{c}\text { Alarm } \\
\text { Designator }\end{array}$ & $\begin{array}{c}\text { PCS Panel Tag \#s } \\
\text { in Alphanumeric } \\
\text { Order }\end{array}$ & Associated Alarms & $\begin{array}{l}\text { Page } \\
\text { Number }\end{array}$ \\
\hline $1107 / 1$ & $11-F I-314$ & TRU GLOVEBOX LOW AIRFLOW & 2 \\
\hline $1107 / 2$ & $11-\mathrm{FI}-316$ & LLW GLOVEBOX LOW AIRFLOW & 4 \\
\hline $1107 / 3$ & $11-\mathrm{FI}-371$ & TRU RESTRICTED WASTE GLOVEBOX LOW AIRFLOW & 6 \\
\hline $1107 / 4$ & $11-\mathrm{FI}-391$ & LLW RESTRICTED WASTE GLOVEBOX LOW AIRFLOW & 8 \\
\hline $1107 / 5$ & $11-P D I-611 A$ & GLOVEBOX EXHAUST FILTER TRAIN A HIGH DIFF PRESS & 10 \\
\hline $1107 / 5$ & $11-\mathrm{PDI}-611 \mathrm{~A}$ & GLOVEBOX EXHAUST FILTER TRAIN A LOW DIFF PRESS & 10 \\
\hline $1107 / 6$ & $11-P D I-611 B$ & GLOVEBOX EXHAUST FILTER TRAIN B HIGH DIFFF PRESS & 11 \\
\hline $1107 / 6$ & $11-\mathrm{PDI}-611 \mathrm{~B}$ & GLOVEBOX EXHAUST FILTER TRAIN B LOW DIFF PRESS & 11 \\
\hline $1107 / 7$ & $11-P D I S H-613 A$ & $\begin{array}{l}\text { GLOVEBOX EXHAUST A FIRST HEPA FILTER HIGH DIFF } \\
\text { PRESSURE }\end{array}$ & 12 \\
\hline $1107 / 7$ & $11-$ PDISH- 6138 & $\begin{array}{l}\text { GLOVEBOX EXHAUST B FIRST HEPA FILTER HIGH DIFF } \\
\text { PRESSURE }\end{array}$ & 12 \\
\hline $1107 / 8$ & 11 -PDISH-615A & $\begin{array}{l}\text { GLOVEBOX EXHAUST A SECOND HEPA FILTER HIGH DIFF } \\
\text { PRESSURE }\end{array}$ & 13 \\
\hline $1107 / 8$ & 11-POISH-615B & $\begin{array}{l}\text { GLOVEBOX EXHAUST B SECOND HEPA FILTER HIGH DIFF } \\
\text { PRESSURE }\end{array}$ & 13 \\
\hline $1107 / 9$ & $E F-11-201 A$ & GLOVEBOX EXHAUST FILTER TRAIN A FAN FAILED & 14 \\
\hline $1107 / 9$ & $E F-11-201 B$ & GLOVEBOX EXHAUST FILTER TRAIN B FAN FAILED & 14 \\
\hline
\end{tabular}

\begin{tabular}{|c|c|c|c|c|}
\hline \%ocon & $\begin{array}{l}\text { seffect } \\
\text { PRATI }\end{array}$ & Revod & 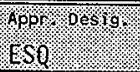 & pag ov. \\
\hline
\end{tabular}


EQUIPMENT STATUS

$1107 / 1$

\begin{tabular}{|c|c|c|}
\hline Alarm Description & PCS Panel Tag \# & Setpoint/Source \\
\hline $\begin{array}{c}\text { TRU GLOVEBOX } \\
\text { LOW AIRFLOW }\end{array}$ & $11-\mathrm{FI}-314$ & 9.8 CMM (345 CFM) \\
\hline
\end{tabular}

\section{ALARM DESCRIPTION}

A single point thermal anemometer (FT-314) Tocated in the TRU Glovebox (107-GB-09-301, 302, 306, 307, 308 and 309) exhaust duct measures glovebox exhaust airflow. If flow from the glovebox is below the alarm setpoint, an a] arm is sent to the PCS.

\section{ALARM PANEL LOCATION}

WRAP 1 Control Room, Central Control Station, 201-CNS-12-105

\section{ALARM RESPONSES}

1. Automatic Actions:

None

2. Operator Actions:

A. ACKNOWLEDGE the alarm.

B. INFORM the Duty Operations Supervisor.

C. SUSPEND TRU G]ovebox (107-GB-09-301, 302, 306, 307, 308, and 309.) operations until TRU Glovebox airflow is returned to normal and this al arm clears.

D. CHECK the TRU Glovebox and associated TRU Glovebox HVAC parameters for off-normal conditions.

E. COORDINATE with the process area operators to investigate and identify corrective action(s) required to remediate the alarm condition.

F. RECORD the following in the Control Room Log:

(I) Corrective action(s) taken.

(2) Proposed corrective action(s) required if initial attempts to rectify the problem are unsuccessfut.

3. Indications:

A. Low airflow through the TRU Glovebox HEPA Filters.

\begin{tabular}{|c|c|c|c|c|}
\hline 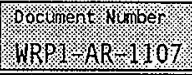 & 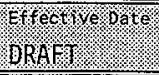 & Rerodo & 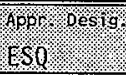 & 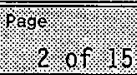 \\
\hline
\end{tabular}


4. Possible Causes:

A. Instrument error.

B. Faulty Glovebox Controller, 107-C-11-200B.

C. Loss of process HVAC.

D: Faulty damper and/or faulty damper actuator (107-DP-11-204B).

E. Loss of instrument air.

\section{REFERENCES}

1. H-2-131893; Sheet 2

2. H-2-131898 


\section{WRAP 1 ALARM RESPONSE PROCEDURE}

\begin{tabular}{|c|c|c|}
\hline & EQUIPMENT STATUS & $1107 / 2$ \\
\hline Alarm Description & PCS Panel Tag \# & Setpoint/Source \\
\hline $\begin{array}{c}\text { LLW GLOVEBOX } \\
\text { LOW AIRFLOW }\end{array}$ & $11-$ FI-316 & 9.2 CMM (325 CFM) \\
\hline
\end{tabular}

\section{ALARM DESCRIPTION}

A single point thermat anemometer (FT-316) located in the LLW Glovebox (107-GB-07-1.01, 102, 104, and 105) exhaust duct measures glovebox exhaust airflow. If flow from the glovebox is below the alarm setpoint, an alarm is sent to the PCS.

\section{ALARIM PANEL LOCATION}

WRAP 1 Control Room, Central Control Station, 201-CNS-12-105

\section{ALARM RESPONSES}

1. Automatic Actions:

None

2. Operator Actions:

A. ACKNOWLEDGE the alarm.

B. INFORM the Duty Operations Supervisor.

C. SUSPEND LLW G7ovebox (107-GB-07-101, 102, 104, and 105)

operations until LLW Glovebox airflow is returned to normal and this al arm clears.

D. CHECK the LLW Glovebox and associated LLW Glovebox HVAC parameters for off-normal conditions.

E. COORDINATE with the process area operators to investigate and identify corrective action(s) required to remediate the alarm condition.

F. RECORD the following in the Control Room Log:

(1) Corrective action(s) taken.

(2) Proposed corrective action(s) required if initial attempts to rectify the problem are unsuccessfut.

3. Indications:

A. Low airflow through the LLW Glovebox HEPA Filters.

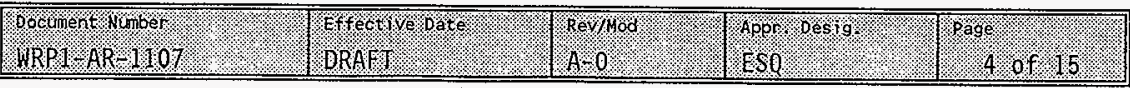


4. Possible Causes:

A. Instrument error.

B. Faulty Glovebox Controller, 107-C-11-200A.

C. Loss of process HVAC.

D. Faulty damper and/or faulty damper actuator (107-DP-11-204A).

E. Loss of instrument air.

\section{REFERENCES}

1. H-2-131893 Sheet 2

2. $\mathrm{H}-2-131898$ 
EQUIPMENT STATUS

\begin{tabular}{|c|c|c|}
\hline Alarm Description & PCS Panel Tag \# & Setpoint/Source \\
\hline $\begin{array}{c}\text { TRU RESTRICTED WASTE } \\
\text { GLOVEBOX } \\
\text { LOW AIRFLOW }\end{array}$ & $1 I-F I-371$ & $2.8 \mathrm{CMH}$ (100 CFM) \\
\hline
\end{tabular}

\section{ALARM DESCRIPTION}

A single point thermal anemometer (FT-371) located in the TRU Restricted Waste Giovebox (107-GB-07-401) exhaust duct measures glovebox exhaust airflow. If flow from the glovebox is outside the expected range, an alarm is sent to the PCS.

\section{ALARM PANEL LOCATION}

WRAP 1 Control Room, Central Control Station, 201-CNS-12-105

ili. ALARM RESPONSES

1. Automatic Actions:

None

2. Operator Actions:

A. ACKNOWLEDGE the alarm.

B. INFORM the Duty Operations Supervisor.

C. SUSPEND TRU Restricted Waste G1ovebox (107-GB-07-401) operations until TRU Restricted Waste Glovebox airflow is returned to normal and this alarm is clear.

D. CHECK the TRU Restricted Waste GTovebox and associated TRU Restricted Waste Glovebox HVAC parameters for off-normal conditions.

E. COORDINATE with the process area operators to investigate and identify corrective action(s) required to remediate the atarm condition.

F. RECORD the following in the Control Room Log:

(1) Corrective action(s) taken.

(2) Proposed corrective action(s) required if initial attempts to rectify the problem are unsuccessful.

3. Indications:

A. Low airflow through the TRU Restricted Waste Glovebox HEPA Fi7ters. 
4. Possible Causes:

A. Instrument error.

B. Faulty Glovebox Controller, 107-C-11-200D.

C. Loss of process HVAC.

D. Faulty damper and/or faulty damper actuator (107-DP-11-203B).

E. Loss of instrument air.

\section{REFERENCES}

1. $\mathrm{H}-2-131893$ Sheet 2

2. $\mathrm{H}-2-131898$ 


\section{WRAP 1 ALARM RESPONSE PROCEDURE}

EQUIPMENT STATUS

$1107 / 4$

Alarm Description

PCS Panel Tag \#

Setpoint/Source

LLW RESTRICTED WASTE

GLOVEBOX

LOW AIRFLOW

11-FI-39I

2.8 CMM (100 CFM)

\section{ALARM DESCRIPTION}

A single point thermal anemometer (FT-391) located in the LLW Restricted Waste-Glovebox (107-GB-07-201) exhaust duct measures glovebox exhaust airflow. If flow from the glovebox is below the alarm setpoint, an alarm is sent to the PCS.

\section{ALARM PANEL LOCATION}

WRAP 1 Control Room, Central Control Station, 201-CNS-12-105

\section{ALARM RESPONSES}

1. Automatic Actions:

None

2. Operator Actions:

A. ACKNOWLEDGE the aTarm.

B. INFORM the Duty Operations Supervisor.

C. SUSPEND LLW Restricted Waste Glovebox (107-GB-07-201) operations until LLW Restricted Waste Glovebox airflow is returned to normal and this alarm is clear.

D. CHECK the LLW Restricted Waste Glovebox and associated LLW Restricted Waste Glovebox HVAC parameters for off-normal conditions.

E. RECORD the following in the Control Room Log:

(1) Corrective action(s) taken.

(2) Proposed corrective action(s) required if initial attempts to rectify the problem are unsuccessful.

3. Indications:

A. Low airflow through the LLW Restricted Waste Glovebox HEPA

Filters.

\begin{tabular}{|c|c|c|c|c|}
\hline ORPA-AR- 107 & 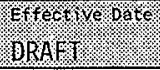 & R\% & $\mathrm{spor}$ & 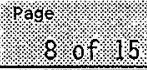 \\
\hline
\end{tabular}




\section{WRAP 1 ALARIM RESPONSE PROCEDURE}

4. Possible Causes:

A. Instrument error.

B. Faulty Glovebox Controller, 107-C-11-200C.

C. Loss of process HVAC.

D. Faulty damper and/or faulty damper actuator (107-DP-11-203A).

E. Loss of instrument air.

\section{REFERENCES}

1. $\mathrm{H}-2-131893$ Sheet 2

2. $\mathrm{H}-2-131898$ 


\section{WRAP 1 ALARM RESPONSE PROCEDURE}

EQUIPMENT STATUS

$1107 / 5$

\begin{tabular}{|c|c|c|}
\hline Alarm Description & PCS Panel Tag\# & Setpoint/Source \\
\hline $\begin{array}{c}\text { GLOVEBOX EXHAUST FILTER } \\
\text { TRAIN A/B } \\
\text { HIGH DIFF PRESS }\end{array}$ & $11-$ PDI-611A/B & $2740 \mathrm{~Pa}$ (11.0" W.C.) \\
\hline
\end{tabular}

\section{ALARM DESCRIPTION}

Differential pressure is outside the expected range for the GTovebox Exhaust Filter Train (113-FT-11-201 A/B). An alarm signal is sent to the PCS.

\section{ALARM PANEL LOCATION}

WRAP 1 Control Room, Central Control Station, 201-CNS-12-105

\section{ALARM RESPONSES}

1. Automatic Actions:

None

2. Operator Actions:

A. ACKNOWLEDGE the alarm.

B. INFORM the Duty Operations Supervisor.

C. DISPATCH an operator to investigate/confirm the alarm condition.

D. SHIFT to the standby Glovebox Exhaust Fitter Train (113-FT-11-201 A/B) per WRPI-OP-1101, Operation of the HVAC System, if the on-line filter train condition cannot be cleared.

3. Indications:

A. Loss of, or reduced, glovebox airfiow:

4. Possible Causes:

A. Faulty instrument.

B. Faulty control circuit.

C. Clogged filters.

\section{REFERENCES}

H-2-131893 Sheet 1

\begin{tabular}{|c|c|c|c|c|}
\hline 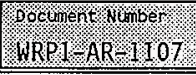 & 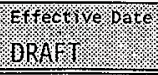 & ReV Mod & 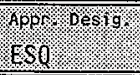 & \%ag $10 \%$ of \\
\hline
\end{tabular}




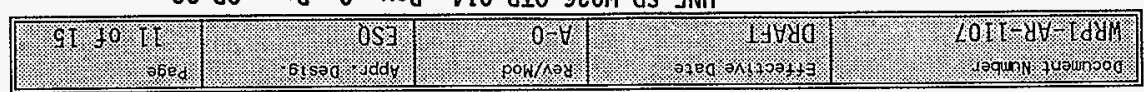

I 7əวบS $\varepsilon 68 I \varepsilon[-Z-H$

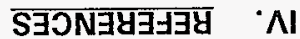

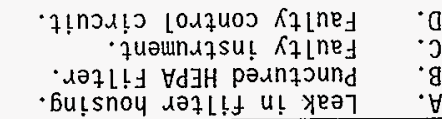

:səsnes

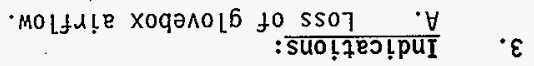

parbajo

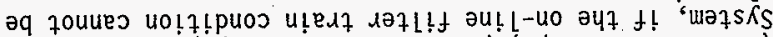

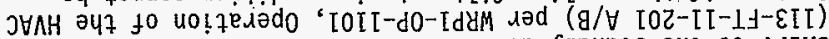

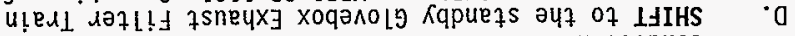

- $00 \div 7 !$ puos

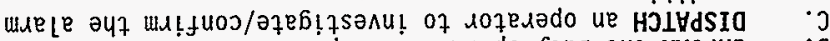

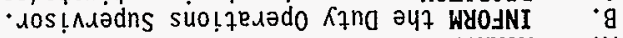

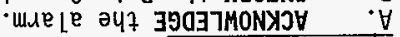

:SU0!7गु do7ededo 2

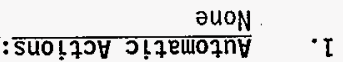

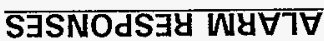

'III

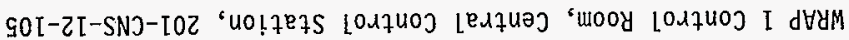

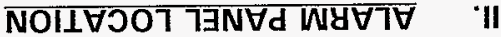

STd

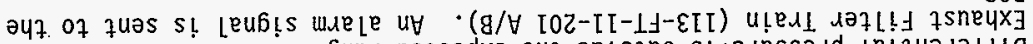

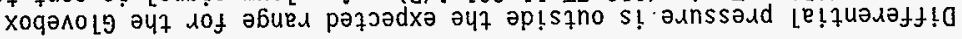

NOIIdIYOS $\exists$ Wप्d

\begin{tabular}{|c|c|c|}
\hline$\left(\circ \cdot M^{n} g^{\circ} L\right)$ ed $0 \angle 8 T$ & g/VII9-IUd-II & 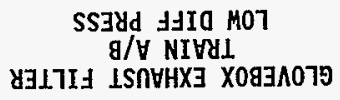 \\
\hline 20.nos/7u!od7as & $\#$ \#E $\perp$ Loued STd & uo!7d!̣usod ude!t \\
\hline 9/LOLL & $\forall \perp S$ LNヨWdI & \\
\hline
\end{tabular}




\section{WRAP 1 ALARM RESPONSE PROCEDURE}

EQUIPMENT STATUS

$1107 / 7$

Alarm Description
GLOVEBOX EXHAUST A/B
IST HEPA FILTER
HIGH DIFF PRESSURE

\section{EQUIPINENT STATUS}

\section{ALARM DESCRIPTION}

Particulate/mojsture has built up on the affected Zone I Process (Glovebox) Exhaust (113-FT-11-201 A/B) Ist HEPA Filter to the point where differentia1 pressure has reached the setpoint. An alarm signal is sent to the PCS.

\section{ALARM PANEL LOCATION}

WRAP 1 Control Room, Central Control Station, 201-CNS-12-105

\section{ALARM RESPONSES}

1. Automatic Actions:

None

2. Operator Actions:

A. ACKNOWLEDGE the alarm.

B. INFORM the Duty Operations Supervisor.

C. CHECK related system parameters.

D. DISPATCH an operator to locally investigate and observe the local indications of alarming 1st HEPA Filter (113-FT-11-201 $A / B)$.

3. Indications:

A. Local pressure differential indication.

4. Possible Causes:

A. Particulate/moisture buildup.

B. Instrument error.

\section{REFERENCES}

H-2-131893 Sheet 1

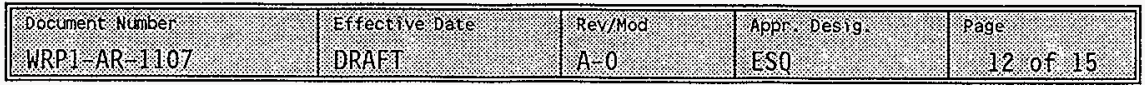




\section{WRAP 1 ALARM RESPONSE PROCEDURE}

\begin{tabular}{|c|c|c|}
\hline & EQUIPMENT STATUS & $1107 / 9$ \\
\hline Alarm Description & PCS Panel Tag \# & Setpoint/Source \\
\hline $\begin{array}{c}\text { GLOVEBOX EXHAUST } \\
\text { FILTER TRAIN A/B } \\
\text { FAN FAILED }\end{array}$ & EF-11-201A/B & $\begin{array}{c}\text { PDISL-617A/B - senses } \\
\text { low dp across Zone I } \\
\text { Process Exhaust Filter } \\
\text { Train Fan EF-1I-201 A/B }\end{array}$ \\
\hline
\end{tabular}

\section{ALARM DESCRIPTION}

Zone I Process (Glovebox) Exhaust Filter Train Fan (113-EF-11-201 A/B) operation is sensed by a differential pressure sensor. It measures the pressure drop across the fan. When the differential pressure between the two points drops below the setpoint (indicating a loss of the fan) an alarm is sent to the PCS. Another signal places the standby unit oni ine.

II. ALARM PANEL LOCATION

WRAP 1 Control Room, Centrai Control Station, 201-CNS-12-105

III. ALARM RESPONSES

1. Automatic Actions:

A. System shifts to the Standby Zone I (GTovebox) Exhaust Filter Train Fan (113-EF-11-201 A/B).

B. MCC-07-101, glovebox equipment circuit trips if glovebox ventilation is lost.

2. Operator Actions:

A. ACKNOWLEDGE the a] arm.

B. INFORM the Duty Operations Supervisor.

C. VERIFY the Standby Zone I Process (Glovebox) Exhaust Filter

Train Fan (113-EF-11-201 A/B) has started and system parameters have stabilized.

\begin{tabular}{|c|c|c|c|c|}
\hline HRPLFAR 1 I07 & $\begin{array}{l}\text { Efer weroth } \\
\text { BRAs }\end{array}$ & $\begin{array}{l}\mathrm{BeV} \\
\mathrm{A}=\mathrm{O}\end{array}$ & $\mathrm{Appor} / \mathrm{pos}$ & 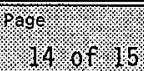 \\
\hline
\end{tabular}




\section{WRAP 1 ALARM RESPONSE PROCEDURE}

NOTE - Upon fai7ure of the operating Zone I Process Exhaust Fi7ter Train Fan, if the Standby Zone I Process Exhaust Fi7ter Train Fan fails to start, the Zone II Process Exhaust Filter Train Fans should stop on interlock.

D. IF the Standby Zone 1 Process Exhaust Filter Train Fan has not started, PERFORM the following:

(1) SUSPEND associated Glovebox operations until Zone I HVAC is returned to operating status.

(2) ENSURE the ZONE II Process Exhaust Filter Train Fans/AHUs have stopped on interlock.

3. Indications:

A. Duct smoke detector alarm (fire dampers shut).

B. Smoke and burning odor at the motor feeder breaker.

C. Low airflow.

D. System has shifted to the Standby Zone I (GTovebox) Exhaust Filter Train Fan/AHU due to the loss of airflow on the running Zone I Exhaust Filter. Train Fan/AHU.

4. Possible Causes:

A. Failed motor.

B. Loss of electrical power.

C. Feeder breaker trip:

- MCC-11-102 for EF-11-201A

D. Broken drive belt.

E. Loss of control power.

F. Loss of instrument air.

\section{REFERENCES}

1. $\mathrm{H}-2-131819$

2. $\mathrm{H}-2-131820$, Sheet 2

3. H-2-131834, Sheet 11

4. H-2-131893, Sheet 1

\begin{tabular}{|c|c|c|c|c|}
\hline 6ocumen & $\begin{array}{l}\text { sffer } \\
\mathrm{HAF}\end{array}$ & 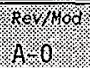 & 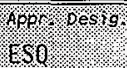 & page \\
\hline
\end{tabular}


HNF-SD-WOZG-OTR-O14 REV. O

\section{WRAP 1 ALARM RESPONSE PROCEDURE}

\section{WRP1-AR-1108}

\section{ZONE DIFFERENTIAL PRESSURE}

\begin{tabular}{|c|c|c|c|}
\hline $\begin{array}{c}\text { Alarm } \\
\text { Designator }\end{array}$ & $\begin{array}{c}\text { PCS Panel Tag \#s } \\
\text { in Alphanumeric } \\
\text { Order }\end{array}$ & Associated Alarms & $\begin{array}{l}\text { Page } \\
\text { Number }\end{array}$ \\
\hline $1108 / 1$ & $11-P D I-315$ & TRU GLOVEBOX LOW DIFF PRESSURE & 2 \\
\hline $1108 / 2$ & $11-\mathrm{PDI}-317$ & LLW GLOVEBOX LOW DIFF PRESSURE & 4 \\
\hline $1108 / 3$ & $11-\mathrm{PDI}-372$ & TRU RESTRICIED WASTE GLOVEBOX LOW DIFF PRESS & 6 \\
\hline $1108 / 4$ & $11-\mathrm{PDI}-392$ & LLW RESTRICTED WASTE GLOVEBOX LOW DIFF PRESS & 8 \\
\hline $1108 / 5$ & $11-$ PDIT-601 & NON-CONFINEMENT/ZONE II PROCESS HVAC LOW DIFF PRESS & 10 \\
\hline $1108 / 6$ & $11-P D I T-602$ & NON-CONFINEMENI/ZONE II PROCESS LOW DIFF PRESS & 12 \\
\hline $.1108 / 7$ & $11-P D I T-603$ & ZONE II PROCESS AVAC/ZONE II PROCESS LOW DIFF PRESS & 14 \\
\hline $1108 / 8$ & $11-P D 1 T-606$ & NON-CONEINEMENT/ATMOSPHERE LOH DIFF PRESS & 16 \\
\hline $1108 / 9$ & $11-P D I T-607$ & ATMOSPHERE/ZONE II PROCESS LOW DIFF PRESS & 18 \\
\hline
\end{tabular}

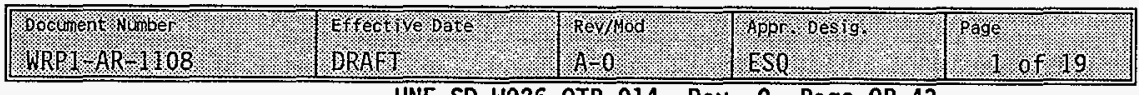




\section{Alarm Description}

TRU GLOVEBOX LOW DIFF PRESSURE
PCS Panel Tag \#

11-PDI-315
Setpoint/Source

$175 \mathrm{~Pa}$ (0.7" W.C.)

\section{ALARM DESCRIPTION}

When a lower than expected differential pressure exists between the TRU Glovebox (107-GB-07-301, 302, 306, 307, 308 and 309) and the Process Area (Rm 107), an alarm is sent to the PCS by PDT-315.

\section{ALARM PANEL LOCATION}

WRAP 1 Control Room, Central Control Station, 201-CNS-12-105

\section{ALARM RESPONSES}

1. Automatic Actions:

None

2. Operator Actions:

A. ACKNOWLEDGE the alarm.

B. INFORM the Duty Operations Supervisor.

C. SUSPEND TRU GIovebox operations until differential pressure between TRU Glovebox and Process Area is restored to normal.

D. CHECK TRU GTovebox and TRU Glovebox HVAC for off-normal conditions.

E. COORDINATE with the process area operators to investigate, identify, and correct the alarm condition.

F. RECORD the following in the Control Room Log:

(1) Corrective action(s) taken.

(2) Proposed corrective action(s) required if initial attempts to rectify the problem are unsuccessful.

3. Indications:

A. Low airflow through TRU Glovebox HEPA filters.

4. Possible Causes:

A. Instrument error/failure.

B. Duct blockage.

C. Glovebox Controller (107-C-11-200B) failure.

D. Loss of process HVAC exhaust fan (13-EF-11-201A/B).

E. Faulty damper/damper actuator (107-DP-11-204B).

F. Loss of Instrument Air.

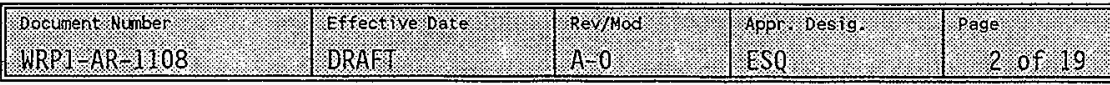




\section{REFERENCES}

1. $\mathrm{H}-2-131893$

2. $\mathrm{H}-2-131898$ 


\section{WRAP 1 ALARM RESPONSE PROCEDURE}

EQUIPMENT STATUS

$1108 / 2$

\section{Alarm Description}

LLW GLOVEBOX

LOW DIFF PRESSURE
PCS Panel Tag \#.

11-PDI-317
Setpoint/Source

$175 \mathrm{~Pa}\left(0.7^{\prime \prime}\right.$ H.C.)

\section{ALARM DESCRIPTION}

When a lower than expected differential pressure exists between the LLW Glovebox (107-GB-07-101, 102, 104, and 105) and the Process Area (Rm 107), an alarm is sent to the PCS by PDT-317.

\section{ALARM PANEL LOCATION}

WRAP 1 Control Room, Central Control Station, 201-CNS-12-105

\section{ALARM RESPONSES}

1. Automatic Actions:

None

2. Operator Actions:

A. ACKNOWLEDGE the alarm.

B. INFORM the Duty Operations Supervisor.

C. SUSPEND LLW Glovebox operations until differential pressure between LLW Glovebox and Process Area is restored to normal.

D. CHECK LLW Glovebox and LLW Glovebox HVAC for off-norma 1 conditions.

E. COORDINATE with the process area operators to investigate, identify, and correct the alarm condition.

F. RECORD the following in the Control Room Log:

(1) Corrective action(s) taken.

(2) Proposed corrective action(s) required if initial attempts to rectify the problem are unsuccessful.

3. Indications:

A. Low airflow through Glovebox HEPA filters.

4. Possible Causes:

A. Instrument error/failure.

B. Duct blockage.

C. Glovebox Controller (107-C-11-200A) failure.

D. Loss of process HVAC exhaust fan (113-EF-11-201A/B)

E. FauTty damper/damper actuator (113-EF-11-204A)

F. Loss of Instrument Air.

\begin{tabular}{|c|c|c|c|c|}
\hline WIRPA-AR 1008 & $\begin{array}{l}\text { Effeculopto } \\
\mathrm{BRAH}\end{array}$ & A $\$ 0 \%$ & 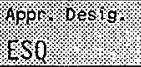 & Mass 4 of 19 \\
\hline
\end{tabular}




\section{REFERENCES}

1. H-2-131893

2. $\mathrm{H}-2-131898$ 


\section{WRAP 1 ALARIM RESPONSE PROCEDURE}

EQUIPMENT STATUS

$1108 / 3$

\begin{tabular}{|c|c|c|}
\hline Alarm Description & PCS Panel Tag \# & Setpoint/Source \\
\hline $\begin{array}{l}\text { TRU RESTRICTED } \\
\text { WASTE GLOVEBOX } \\
\text { LOW DIFF PRESS }\end{array}$ & 11-PDI-372 & $175 \mathrm{~Pa}$ (0.7" W.C.) \\
\hline
\end{tabular}

\section{ALARM DESCRIPTION}

When a lower than expected differential pressure exists between the TRU Restricted Waste Management (RWM) Glovebox (1.07-GB-07-40I) and the Process Area (Rm 107), an alarm is sent to the PCS by PDT-372.

\section{ALARM PANEL LOCATION}

WRAP 1 Control Room, Central Control Station, 201-CNS-12-105

\section{ALARM RESPONSES}

1. Automatic Actions:

None

2. Operator Actions:

A. ACKNOWLEDGE the al arm.

B. INFORM the Duty Operations Supervisor.

C. SUSPEND TRU RWM Glovebox operations until differential pressure between TRU RWM Glovebox and Process Area is restored to normal.

D. CHECK TRU RWM Glovebox and TRU RWM HVAC for off-normat conditions.

E. COORDINATE with process area operators to investigate, identify, and correct the alarm condition.

F. RECORD the following in the Control Room Log:

(1) Corrective action(s) taken.

(2) Proposed corrective action(s) required if initial attempts to rectify the problem are unsuccessful.

3. Indications:

A. LOW airfiow through TRU RWM Glovebox HEPA filters.

4. Possible Causes:
A. Instrument error/failure.
B. Duct blockage.
C. TRU RWM Glovebox Controller (107-C-11-200D) failure.
D. Loss of Zone I HVAC exhaust fan (113-EF-201A/B).
E. Faulty damper/damper actuator (107-DP-11-203B).

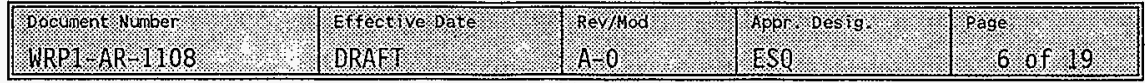


IV. REFERENCES

1. H-2-131893

2. $\mathrm{H}-2-131898$ 
LLW RESTRICTED WASTE GLOVEBOX. LOW DIFF. PRESS
11-PDI-392
$175 \mathrm{~Pa}$ (0.7" W.C.)

\section{ALARM DESCRIPTION}

When a lower than expected differential pressure exists between the LLW Restricted Waste Management (RWM) Glovebox (107-GB-07-201) and the Process Area (Rm 107), an alarm is sent to the PCS by PDT-392.

\section{ALARM PANEL LOCATION}

WRAP 1 Control Room, Central Control Station, 201-CNS-12-105

\section{ALARM RESPONSES}

I. Automatic Actions:

None

2. Operator Actions:

A. ACKNOWLEDGE the alarm.

B. INFORM the Duty Operations Supervisor.

C. SUSPEND LLW RWM Glovebox operations until differential pressure between LLW RWM Glovebox and Process Area is restored to normal.

D. CHECK LLW RWM and LLW RWM HVAC for off-normal conditions.

E. COORDINATE with process area operators to investigate, identify, and correct the alarm condition.

F. RECORD the following in the Control Room Log:

(I) Corrective action(s) taken.

(2) Proposed corrective action(s) required if initial attempts to rectify the problem are unsuccessful.

3. Indications:

A. Low airflow through LLW RWM Glovebox HEPA filters.

4. Possible Causes:

A. Instrument error/failure.

B. Duct blockage.

C. LLW RWM Glovebox Controller (107-C-11-200C) failure.

D. Loss of Zone I HVAC exhaust fan (113-EF-11-201A/B).

E. Faulty damper/damper actuator (107-DP-11-203A, 202-DP-11-201A/B, 203-DP-11-202A/B).

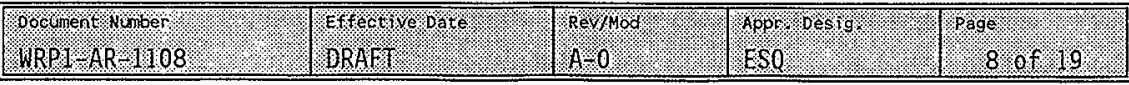




\section{REFERENCES}

1. $\mathrm{H}-2-131893$

2. $\quad \mathrm{H}-2-131898$ 


\begin{tabular}{|c|c|c|}
\hline Alarm Description & PCS Panel Tag \# & Setpoint/Source \\
\hline $\begin{array}{c}\text { NON-CONFINEMENT/ZONE II } \\
\text { PROCESS HVAC } \\
\text { LOW DIFF PRESS }\end{array}$ & 11-PDIT-601 & $25 \mathrm{~Pa}\left(0.10^{\mathrm{n}}\right.$ W.C. $)$ \\
\hline
\end{tabular}

\section{ALARM DESCRIPTION}

When a lower than expected differential pressure exists between the Non-Confinement Area (sensed in Corridor 117) and the Zone II Process HVAC Area (Room 113), an alarm is sent to the PCS by PDIT-601.

\section{ALARM PANEL LOCATION}

WRAP 1 Control Room, Central Control Station, 201-CNS-12-105

\section{ALARM RESPONSES}

1. Automatic Actions:

None

2. Operator Actions:
A. ACKNOWLEDGE the al arm.
B. INFORM the Duty Operations Supervisor.
C. SUSPEND all Glovebox/Process Area operations until differential pressure between Process HVAC Area and Non-Confinement Area is restored to norma 7 .
D. CHECK Zone II Exhaust Fan (113-EF-11-202A/B) for off-normal conditions.
E. CHECK Zone II Air Handling Unit (203-AH-11-201A/B) for off- normat conditions.
F. COORDINATE with process area operators to investigate, identify, and correct alarm condition.
G. RECORD the following in the Control Room Log:
(1) Corrective action(s) taken.
(2) Proposed corrective action(s) required if initial attempts to rectify the problem are unsuccessful.

3. Indications:
A. Low airflow through Zone II Exhaust Fan train (113-AH-11-202A/B).
B. Low airflow through Zone II Air Handling Unit (203-AH-11-201A/B).

\begin{tabular}{|c|c|c|c|c|}
\hline 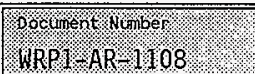 & ERffect ro vate & A+\% & 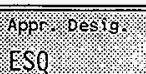 & 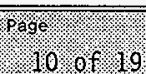 \\
\hline
\end{tabular}




\section{WRAP 1 ALARM RESPONSE PROCEDURE}

4. Possible Causes:

A. Instrument error/failure.

B. Duct blockage.

C. Exhaust System Controller (113-C-11-202A/B) failure.

D. Boundary between zones penetrated.

E. Faulty damper/damper actuator (113-DP-11-206A/B/C/D).

\section{REFERENCES}
1. $\mathrm{H}-2-131892$
2. $\mathrm{H}-2-131898$ 


\section{WRAP 1 ALARM RESPONSE PROCEDURE}

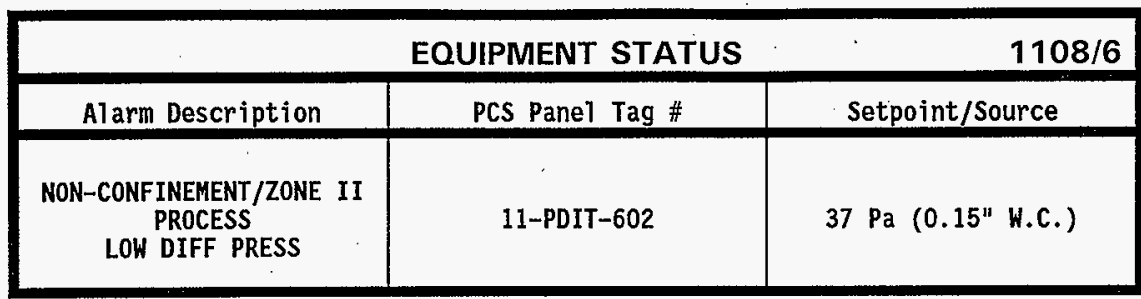

\section{ALARM DESCRIPTION}

When a lower than expected differential pressure exists between the Non-Confinement Area (sensed in Corridor 117) and the Zone II Process Area (Room 107), an alarm is sent to the PCS by PDIT-602.

\section{ALARM PANEL LOCATION}

WRAP 1 Control Room, Central Control Station, 201-CNS-12-105

\section{ALARM RESPONSES}

1. Automatic Actions:

None

2. Operator Actions:

A. ACKNOWLEDGE the alarm.

B. INFORM the Duty Operations Supervisor.

C. SUSPEND a11 Glovebox/Process Area operations until differential pressure between Process Area and Non-Confinement Area is restored to normal.

D. CHECK Zone II Exhaust Fan (113-EF-11-202A/B) for off-normal conditions.

E. CHECK Zone II Air Handling Unit (203-AH-11-201A/B) for offnormal conditions.

F. COORDINATE with process area operators to investigate, identify, and correct alarm condition.

G. RECORD the following in the Control Room Log:

(1) Corrective action(s) taken.

(2) Proposed corrective action(s) required if initial attempts to rectify the problem are unsuccessful.

3. Indications:

A. Low airflow through Zone II Exhaust Fan train

(113-AH-11-202A/B).

B. Low airflow through Zone II Air Hand7ing Unit

(203-AH-11-201A/B).

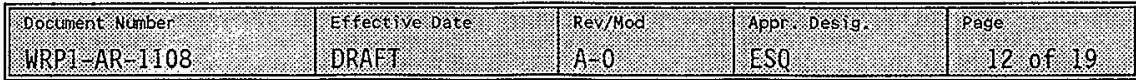


4. Possible Causes:

A. Instrument error/fajlure.

B. Duct blockage.

C. Supply Damper (203-DP-11-203C) failed open or controlling too far in the open position.

D. Boundary between zones penetrated.

E. Fautty damper/damper actuator (113-DP-11-206A/B/C/D).

\section{REFERENCES}

1. $\mathrm{H}-2-131892$

2. $\mathrm{H}-2-131898$ 
EQUIPMENT STATUS

$1108 / 7$

\begin{tabular}{|c|}
\hline Alarm Description \\
ZONE II PROCESS HVAC/ \\
ZONE II PROCESS \\
LOW DIFF PRESS
\end{tabular}

\section{ALARM DESCRIPTION}

When a lower than expected differential pressure exists between the Zone II Process Area (Room 107) and the Zone II Process HVAC Area (Room 113), an alarm is sent to the PCS by PDIT-603.

\section{ALARM PANEL LOCATION}

WRAP 1 Control Room, Central Control Station, 201-CNS-12-105

III. ALARM RESPONSES

1. Automatic Actions:

None

2. Operator Actions:

A. ACKNOWLEDGE the al arm.

B. INFORM the Duty Operations Supervisor.

C. CHECK Zone II Exhaust Fan (113-EF-11-201A/B) for off-norma] conditions.

D. CHECK Zone II Air Handling Unit (203-AH-11-201A/B) for off-normal conditions.

E. COORDINATE with process area operators to investigate, identify, and correct al arm condition.

F. RECORD the following in the Control Room Log:

(1) Corrective action(s) taken.

(2) Proposed corrective action(s) required if initia? attempts to rectify the problem are unsuccessfut.

3. Indications:

A. High airflow from Room 113 to Room 107.

4. Possible Causes:

A. Instrument error/failure.

B. Loss of HVAC.

C. Boundary between zones penetrated.

D. Exhaust system controller (113-C-11-202A/B) failure.

E. Faulty damper/damper actuator (113-DP-11-206A/B/C/D).

\begin{tabular}{|c|c|c|c|c|}
\hline HRP $=\mathrm{AR}-1108$ & 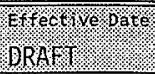 & 4.0 & Hopol & $1 \% 1 \%$ f $/ 19$ \\
\hline
\end{tabular}




\section{REFERENCES}

1. $\quad H-2-131892$
2. $\quad H-2-131898$ 


\begin{tabular}{|c|c|c|}
\hline Alarm Description & PCS Panel Tag \# & Setpoint/Source \\
\hline $\begin{array}{c}\text { NON-CONFINEMENT/ATMOSPHERE } \\
\text { LOW DIFF PRESS }\end{array}$ & I1-PDIT-606 & $5 \mathrm{~Pa}(0.02$ " W.C.) \\
\hline
\end{tabular}

\section{ALARM DESCRIPTION}

When a lower than expected differential pressure exists between the Non-Confinement Area (sensed in Corridor 117) and atmospheric pressure (sensed by a probe (PE-601) mounted on the exhaust stack), an alarm is sent to the PCS by PDAL-606.

\section{ALARM PANEL LOCATION}

WRAP 1 Control Room, Central Control Station, 201-CNS-12-105

III. ALARM RESPONSES

1. Automatic Actions:

None

2. Operator Actions:

A. ACKNOWLEDGE the al arm.

B. INFORM the Duty Operations Supervisor.

C. CHECK Administration Area Air Handling Unit (203-AH-11-401) for off-normal conditions.

D. COORDINATE with the process area operators to investigate, identify, and correct the al arm condition.

E. RECORD the following in the Control Room Log:

(1) Corrective action(s) taken.

(2) Proposed corrective action(s) required if initial attempts to rectify the problem are unsuccessful.

3. Indications:

A. Local pressure differential indication (PDI-606).

4. Possible Causes:

A. Instrument error/failure.

B. Loss of Administration Area Air Handler (203-AH-11-401).

C. Air Handler Controller (203-C-11-401) failure.

\begin{tabular}{|c|c|c|c|c|}
\hline MRPJ AR 1108 & BRAf & 80 & SOSO & $1696.09 \% 19$ \\
\hline
\end{tabular}




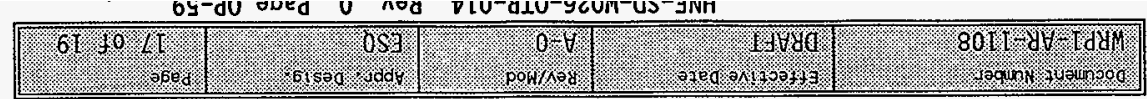

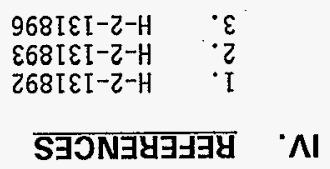




\section{WRAP 1 ALARM RESPONSE PROCEDURE}

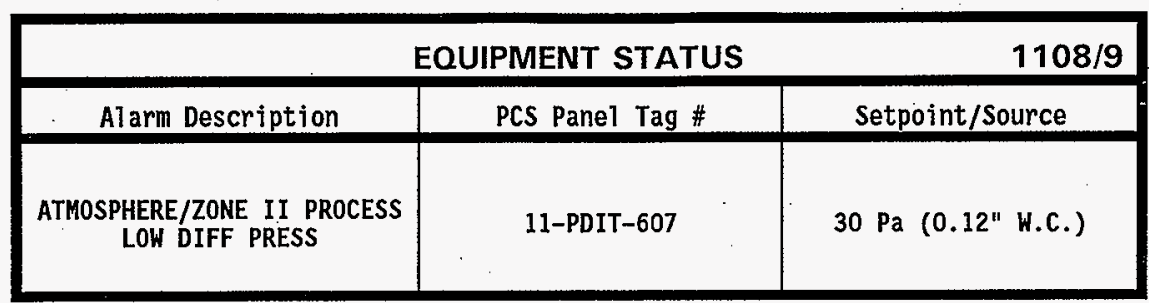

\section{ALARM DESCRIPTION}

When a lower than expected differential pressure exists between the Zone II Process Area ( $\mathrm{Rm}$ 107) and atmospheric pressure (sensed by a probe (PE-601) mounted on the exhaust stack), an alarm is sent to PCS by PDAL-607.

\section{ALARM PANEL LOCATION}

WRAP 1 Control Room, Central Control Station, 201-CNS-12-105

\section{ALARM RESPONSES}

1. Automatic Actions:

None

2. Operator Actions:

A. ACKNOWLEDGE the al arm.

B. INFORM the Duty Operations Supervisor.

C. SUSPEND all G1ovebox/Process Area operations until differential pressure between Process Area and atmospheric pressure is restored to normal.

D. CHECK Zone II Exhaust Train (113-EF-11-202A/B) for off-normal conditions.

E. COORDINATE with process area operators to investigate, identify, and correct alarm condition.

F. RECORD the following in the Control Room Log:

(1) Corrective action(s) taken.

(2) Proposed corrective action(s) required if initial attempts to rectify the problem are unsuccessful.

3. Indications:

A. Local pressure differential indication (PDI-607).

4. Possible Causes:

A. Instrument error/failure.

B. Loss of Zone II exhaust train.

C. Boundary between zones penetrated.

D. Zone II exhaust system controller (113-C-11-202A/B) failure.

E. Faulty damper/damper actuator (113-DP-206A/B/C/D).

\begin{tabular}{|c|c|c|c|c|}
\hline 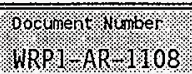 & 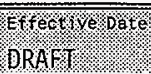 & A & ESOFr & orge 18 of \\
\hline
\end{tabular}


IV. REFERENCES

1. $\mathrm{H}-2-131892$

2. $\mathrm{H}-2-131893$

\begin{tabular}{|c|c|c|c|c|}
\hline Wooding & $\begin{array}{l}\text { Hffect } \\
\text { ARAPl }\end{array}$ & $8 \% 0 \%$ & $\begin{array}{l}\text { Appr } \\
\mathrm{SSO}\end{array}$ & Pro ow \\
\hline
\end{tabular}




\title{
WRAP 1. PLANT OPERATING PROCEDURE
}

\author{
SYSTELT: RROCESS
}

\section{GLOVEBOX HOUSEKEEPING}

\section{SYSTEM DESCRIPTION}

This procedure provides instructions for cleanup of spills, debris and general housekeeping in WRAP-1 gloveboxes.

Glovebox housekeeping shall be performed after processing of each waste stream batch is completed, and as needed during glovebox operations for spills or other debris buildup. This will ensure adequate, current, process knowledge to help determine possible sampling requirements \& environmental compliance when disposing of cleanup residue.

\section{REFERENCE DOCUMENTS}

WRP1-OP-0704, G1ovebox Manipulator Operation.

WRP1-OP-0713, LLW Entry Glovebox Operation.

WRP1-OP-0714, LLW Sorting GTovebox Operation.

WRP1-0P-0716, LLW Exit GTovebox Operation.

WRP1-0P-0717, LLW/RWM Process GIovebox-Operation.

WRP1-0P-0705, Bagless Transfer Manual Operation

WRP1-OP-0710, Waste Sampling

HSRCM-1, Hanford Site Radiological Control Manual.

\section{PRESTART CONDITIONS}

All personnel performing this procedure shall be qualified in accordance with WHC-CM-5-34, Section 1.8, "Training and Qual ification, " and on-the-job training.

\section{SAFETY}

A11 potential hazards, such as lifting heavy containers or handling sharp objects present in waste containers, must be mitigated by protective equipment, procedures, and administrative controls to ensure acceptablerisk operating conditions.

\section{Radiological Safety}

A17 work in gloveports must be performed per applicable Radiation Work Permits (RWPs).

\begin{tabular}{|c|c|c|c|c|c|}
\hline 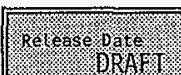 & 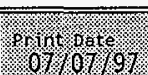 & Wokgesig & 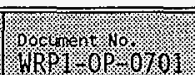 & reyod & 8)ge \\
\hline
\end{tabular}




\section{WRAP 1 PLANT OPERATING PROCEDURE}

\section{TOOLS AND SUPPLIES}

Approved cleaning agents and supplies as required.

\section{TABLE OF CONTENTS}

PAGE

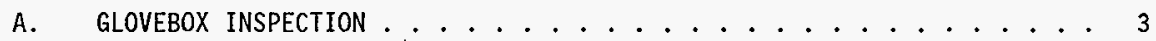

B. CLEANUP SOLID DEBRIS AND MATERIALS $\ldots \ldots \ldots \ldots \ldots$

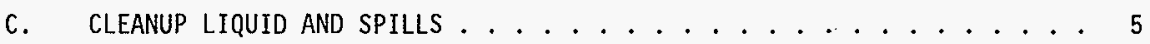

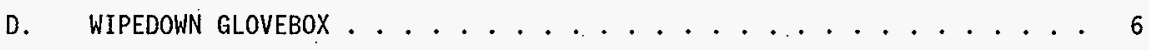

E. SAMPLING AND LOADOUT $\ldots \ldots \ldots \ldots \ldots \ldots \ldots$

\section{ATTACHMENTS}

None

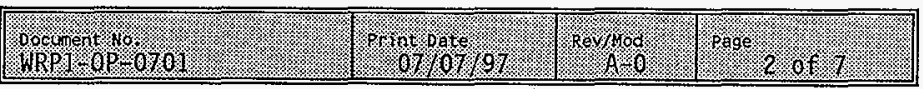




\section{WRAP 1 PLANT OPERATING PROCEDURE}

\section{PROCEDURE}

\section{CAUTION}

INTRODUCTION OF UNAPPROVED CLEANING AGENTS INTO GLOVEBOXES MAY RESULT IN ENVIRONMENTAL NON-COMPLIANCE, UNWANTED CHEMICAL REACTIONS OR OTHER UNDESIRABLE EFFECTS. USE ONLY FACILITY APPROVED CLEANING AGENTS.

Note - Administrative hold steps are identified by letters in parentheses at the left margin of the procedure step. The direction given in the procedure step must be satisfied before work continues.

(M) - Operations management sha77 approve operation.

(R) - Radiological Control (RC) shall complete surveys or agree to permit continued operation.

\section{A. GLOVEBOX INSPECTION}

1. CHECK, visually throughout glovebox for: a. Loose items, spills, droppings or tailings from waste
handiting process.

b. Improperty stored glovebox tools and equipment.

c. Leaks from glovebox hydraulic systems.

d. Left-over agents introduced for cleaning or other purposes.

e. Foreign matter on glovebox walls, windows and/or equipment.

2. CHECK glovebox equipment (manipulators, ports, Tift tables, etc) for damage, leaks, etc. 


\section{WRAP 1 PLANT OPERATING PROCEDURE}

3. PERFORM glovebox cleanup as required, per the following:

a. VERIFY VoTatile Organic Compound (VOC) in glovebox is less than (TBD)\%.

b. CLEANUP solid debris and materials per Section VII.B.

c. CLEANUP Tiquid and spills per Section VII.C.

d. WIPEDOWN glovebox per Section VII.D.

NOTE - Radiological survey is required upon exiting gloveports after each use.

e. UPON exiting gloveports at any point in this procedure, PERFORM a self-survey.

(1) IF self-survey 1 imits are exceeded, THEN, CONTACT Radiological Control Technician.

\section{B. CLEANUP SOLID DEBRIS AND MATERIALS}

NOTE 1 - Glovebox manipulators are operated per WRP1-0P-0704, Glovebox Manipulator Operation.

1. USING manipulators to the extent possible, PERFORM the following:

a. PICKUP waste and debris items and PLACE on glovebox sorting table.

(1) USE (with manipulators) broom, dustpan, and shovel located inside gilovebox, as needed to aid cleanup.

b. MOVE glovebox tools and equipment to proper glovebox storage Tocation.

2. IF any items cannot be accessed with manipulators, THEN :

a. ENSURE alT moving equipment in glovebox is SHUTDOWN.

b. LOCATE gioveport(s) which provide best access to item(s) and PROCESS items per Steps VII.B.1.a and VII.B.1.b.

3. If other cleanup work is to be performed,

THEN, GO TO applicable section(s),

OTHERHISE, GO TO Section VII.E, Sampling and Loadout.

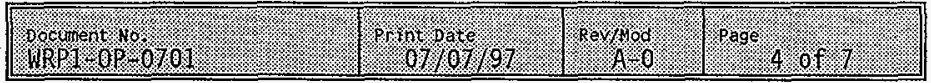




\section{WRAP 1 PLANT OPERATING PROCEDURE}

\section{c. CLEANUP LIQUID AND SPILLS}

NOTE - Spill kits will norma77y be pre-packaged in marked sample transfer canisters and stored in the Warm Maintenance Area, Room 108.

1. TRANSFER required Spill Cleanup Kit into glovebox through bagless transfer port, sample transfer port or consumable entry port, as required, per applicable operating procedure(s):

- WRP1-0P-0705, Bagless Transfer Manual Operation.

- WRPI-0P-0710, Waste Sampling.

2. IF work is to be performed through gloveports, THEN, ENSURE all moving equipment in glovebox is SHUTDOWN.

3. WORKING with manipulators and/or through gloveport(s), as required, PERFORM the following:

NOTE - Cleanup is accomplished by best suited method. The following steps are used as a guideline.

a. PLACE dam, dike, or absorbent containment from spill kit around spit7.

b. ABSORB, using Rad-Pads and absorbent, as required, a11 spilled material.

c. PLACE used absorbent and Rad-Pads into plastic bag(s).

d. PLACE bagged items on glovebox sorting table.

4. IF necessary,

THEN, WIPEDOWN spi11 area per, Section VII.D.

5. IF other cleanup work is to be performed,

THEN, GO TO applicable section(s),

OTHERWISE, GO TO Section VII.E, Sampling and Loadout. 


\section{WRAP 1 PLANT OPERATING PROCEDURE}

D. . WIPEDOWN GLOVEBOX

1. TRANSFER required materials (approved cleaning agents, rags, Rad-Pads) into glovebox through bagless transfer port, sample transfer port or consumable entry port, as required, per applicable operating procedure(s):

- WRP1-0P-0705, Bagless Transfer Manual Operation.

- WRP1-0P-0710, Waste Sampling.

2. If work is to be performed through gloveports, THEN, ENSURE all moving equipment in glovebox is SHUTDOWN.

3. WORKING with manipulators and/or through gloveport(s), as required, PERFORM the following:

NOTE - Cleanup is accomplished by best suited method (i.e, cleaning agents may be poured directly on area or applied to rags or pads, then wiped over area). The following steps are used as a guideline.

a. APPLY cleaning agent to affected area(s).

b. SCRUB or BRUSH affected area(s).

c. WIPE, affected area(s) and equipment with clean, dry, Rad-Pads or rags.

d. PLACE used Rad-Pads, rags and other items to be disposed, into plastic bag(s).

e. PLACE bagged items on glovebox sorting tabie.

4. If other cleanup work is to be performed, THEN, GO TO applicable section(s), OTHERWISE, GO TO Section VII.E, Sampling and Loadout. 


\section{WRAP 1 PLANT OPERATING PROCEDURE}

\section{E. SAMPLING AND LOADOUT}

NOTE 1 - Once cleaned up and placed on sorting table, cleanup items are treated as if processing ordinary waste for applicable glovebox (i.e. passed to RWM. glovebox for sorting, sampling \& disposal per applicable procedures).

NOTE 2 - Sampling requirements are determined by review of process history for waste stream preceding cleanup activities.

(H)

1. DETERMINE sampling requirements for items placed on glovebox sorting table during cleanup activities.

2. PERFORM sampling per WRP1-0P-0710, Glovebox Waste Sampling, as directed.

3. PROCESS remaining cleanup items as directed, per applicable glovebox operating procedures:

- WRP1-0P-0713, LLW Entry Glovebox 0peration.

- WRP1-0P-0714, LLW Sorting Glovebox Operation.

- WRP1-0P-0716, LLW Exit Glovebox Operation.

- WRP1-0P-0717, LLW/RWM Process GTovebox Operation. 


\title{
WRAP 1 PLANT OPERATING PROCEDURE
}

\author{
SYSTIEH: PROCESS
}

\section{GLOVEBOX LIFT TABLE MANUAL OPERATION}

\section{SYSTEM DESCRIPTION}

This procedure provides instructions for manual operation of drum lift tables at various locations throughout the facility. Automatic operation of 1 ift tables is described in applicable glovebox operating procedures.

\section{REFERENCE DOCUMENTS}

WRPI-0P-0704, Glovebox Manipulator Operation. WRP1-0P-0713, LLW Entry GTovebox Operation. WRP1-0P-0714, LLW Sorting Glovebox Operation. WRP1-0P-0716, LLW Exit GTovebox Operation. WRP1-0P-0717, LLW/RWM Process Glovebox Operation.

\section{PRESTART CONDITIONS}

A11 personnel performing this procedure shall be qualified in accordance with WHC-CM-5-34, Section 1.8, "Training and Qualification," and on-the-job training.

\section{SAFETY}

Al1 potential hazards, such as lifting heavy containers or handling sharp objects present in waste containers, must be mitigated by protective equipment, procedures, and administrative controls to ensure acceptablerisk operating conditions.

CAUTION - In manual control (maintenance) mode there is no interlock to prevent drum over-travel. If the reverse switch is held in after the drum reaches the Automatic Guided Vehicle (AGV) end of the table and the AGV is not in place, the drum will fall to the floor. There is also no interlock to stop up-travel based on weight setpoint and no indication that lift table is at drum centering height.

WARNING - Lift tables LT-09-20IC, LT-09-202D, and LT-09-202E, when Towered, interfere with AGV travel near the LLW and TRU RWM giovebox lines. Before lowering these tables below AGV height $(27+1-1 / 8$ inches from floor to top of rollers), the operator must ensure that the AGV will not travel into the area by disabling the AGV.

\section{TOOLS AND SUPPLIES}

None

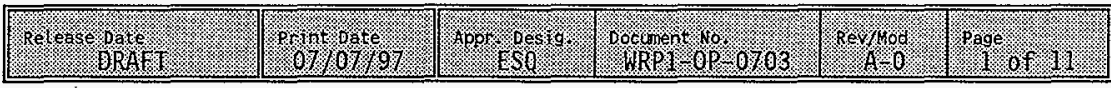


-

-

$\div$ 


\section{WRAP 1 PLANT OPERATING PROCEDURE}

\section{TABLE OF CONTENTS}

PAGE

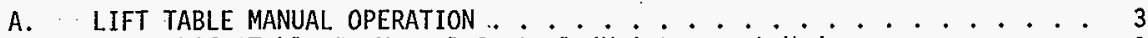

Place Lift Table In Manual Control (Maintenance) Mode . . . . . . . 3

Lower Drum From AGV Load Height . . . . . . . . . . . . . . 3

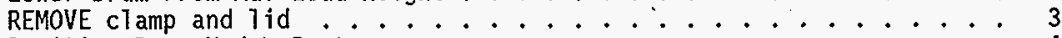

Position Drum Under Port ............... . . 4

Lift Drum To Port.................. . . 4

Lower Drum From Port . . . . . . . . . . . . . . . . 7

MOVE Drum To AGV End of Lift Tabie ............. . 8

B. RETURN LIFT TABLE TO NORMAL OPERATION ............... 8

c. REHOME LIFT TABLE ..................... 9

FIGURE 1 : LifT TABLE LOCAL CONTROL PANEL ............... . . 10

TABLE 1: LIFT TABLE/CONTROL PANEL DESCRIPTIONS . . . . . . . . . . . 10

TABLE 2: DRUM MATING PRESSURE/CENTERING HEIGHT ............ 11

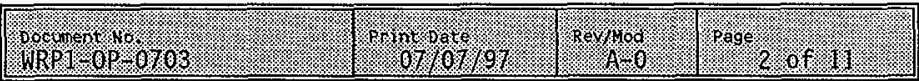

HNF-SD-W026-0TR-014, Rev. 0, Page 0P-70 


\section{WRAP 1 PLANT OPERATING PROCEDURE}

\section{PROCEDURE}

NOTE 1 - This procedure covers manual operation of all WRAP lift tables, therefore equipment tag numbers are not referenced in the individual steps. Tag numbers for referenced equipment are located in Table 1. When equipment is called out by name, reference to applicable equipment number is understood.

NOTE 2 - Un7ess otherwise stated, switches and indicators are located on the applicable Lift Table Control Panel.

\section{A. LIFT TABLE MANUAL OPERATION}

1. Place Lift Table In Manual Control (Maintenance) Mode

a. ENSURE glovebox manipulator is PARKED (Reference WRP1-OP-0704, Glovebox Manipulator Operation).

b. PLACE "MODE" (MAINT/OFF/NORMAL) switch to "MAINT".

2. Lower Drum From AGV Load Height

NOTE - Onily those Lift Tables indicated by (*) in Tab7e 1, need to be lowered from AGV Load Height for drum to clear port. Port clearance height is checked visually or with a tape measure when visual inspection is not possible.

a. ENSURE Lift Table is in manual control mode.

b. PRESS and HOLD "DOWN" switch until drum is at port clearance height. REFERENCE Table 2, Drum Clearance Height for required clearance between port bottom and drum top.

c. IF "DOWN OVERTRAVEL" light comes ON, THEN, PRESS "UP" switch until light goes OFF.

3. REMOVE clamp and Tid, as needed, from drum per applicable procedure:

- WRP1-0P-0713, LLW Entry GTovebox Operation.

- WRP1-0P-0714, LLW Sorting Glovebox Operation.

- WRP1-0P-0716, LLW Exit G1ovebox Operation.

- WRP1-0P-0717, LLW/RWM Process GTovebox 0peration.

- WRPI-OP-OT28, TRU/RLOM GLOVEBOX OPERATION

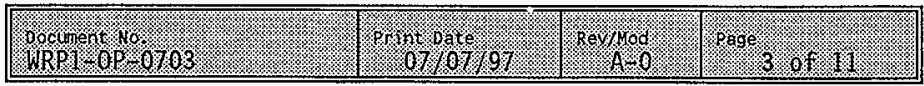




\section{WRAP 1 PLANT OPERATING PROCEDURE}

4. Position Drum Under Port

a. VERIFY the following heights of the 1 isted items as applicablè:

(1) 55 gallon drum with 7 id and clamp band attached: $\quad 345 / 8+/-1 / 8$ in.

(2) 85 gal lon overpack drum with 7 id and clamp band attached:

$391 / 16+/-1 / 8$ in.

b. ENSURE Lift Table is in manual control mode.

NOTE - On7y those Lift Tables indicated by (*) on Attachment 1, Tab7e 1, need 7owered from AGV Load Height for drum to clear port. Port clearance height is checked visually.

c. IF necessary,

THEN, ENSURE Lift Table is lowered to port clearance height.

d. PRESS and HOLD "FORWARD" switch unti 1 drum is centered beneath port and "DRUM UNDER PORT" 1 ight is STEADY ON.

e. IF necessary,

THEN, jog drum into position as follows:

(1) CHECK drum position visually in relation to port.

(2) PRESS "FORWARD" and/or "REVERSE" buttons as necessary to properly position drum under port.

5. Lift Drum To Port

a. ENSURE Lift Table is in manual control mode.

b. ENSURE drum is properTy positioned under port.

c. ENSURE drum port is CLOSED, per applicable procedure.

d. VERIFY the following for Entry/Exit ports:

(1) Centering devices are retracted.

(2) HVAC seal is deflated, if present.

(3) Lid Detach rod is RETRACTED.

\begin{tabular}{|c|c|c|c|}
\hline 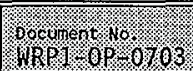 & 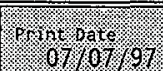 & 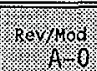 & pags \\
\hline
\end{tabular}




\section{WRAP 1 PLANT OPERATING PROCEDURE}

e. VERIFY correct drum pin, as per applicable procedure:

- WRP1-0P-0713, LLW Entry G1ovebox Operation.

- WRP1-0P-0714, LLW Sorting Glovebox Operation.

- WRP1-0P-0716, LLW Exit Glovebox Operation.

- WRP1-0P-0717, LLW/RWM-Process Glovebox Operation.

NOTE - $\quad$ Proper drum to port mating is determined by drum to port mating pressure or drum height, depending on port type.

f. DETERMINE correct drum to port mating pressure or drum centering height per Table 2.

g. RAISE drum to correct height, non-ENTRY/EXIT Port:

\section{CAUTION}

DO NOT EXCEED LIFT TABLE WEIGHT SETPOINT BY MORE THAN 50 KILOGRAMS.

(1) ENSURE that lift table weight scale (on top of table control pedestal) units, are "kg" for kilograms.

(2) PRESS and HOLD "UP" switch until drum mates to port.

(3) VERIFY correct weight setpoint is s-reached___-

h. RAISE drum to correct height, ENTRY/EXIT Port:

(1) PRESS and HOLD "UP" switch until lift table is at "Drum Centering Height".

(2) VERIFY lift table at "Drum Centering. Height" using measuring tape and values from Table 2.

(3.) EXTEND centering clamps.

(4) VERIFY centering clamps extended.

NOTE: $\quad$ All entry/exit port maintenance menus have a pilot light that indicates when the port "7id in position" sensor is activated by the drum 7id.

(5) PRESS and HOLD "UP" switch until lift table is at "Lid in Position Height".

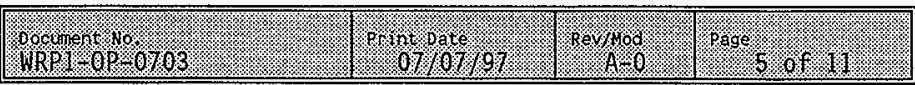




\section{WRAP 1 PLANT OPERATING PROCEDURE}

(6) VERIFY lift table at "Lid in Position Height".

(7) TURN ON port vacuum.

(8) VERIFY vacuum generated.

(9) IF HVAC SeaT Present:

(a) INFLATE HVAC sea?.

(b) VERIFY HVAC seal inflated.

(10) UNLOCK port.

(11) VERIFY port unlocked.

NOTE: $\quad$ All entry/exit port maintenance menus have a pilot light that indicates when the port "DRUM in position" sensor is activated by raising the port door approximately $1 / 2$ inch above the glovebox floor.

(12) PRESS and HOLD "UP" switch until lift table raises to "DRUM IN POSITION" height.

(13) VERIFY Lift Table at "DRUM IN POSITION" height.

\section{CAUTION}

TABLE RESPONDS QUICKLY TO PRESSURE ON CONTROL SWITCHES. A "LIGHT TOUCH" MUST BE USED ON SWITCHES WHEN JOGGING DRUM INTO POSITION. OVER-DRIVING DRUM INTO PORT COULD RESULT IN EQUIPMENT DAMAGE.

i. JOG drum with "UP/DOWN". switches to adjust position/mating pressure as required.

j. OPERATE drum port per applicable procedure.

\begin{tabular}{|c|c|c|}
\hline 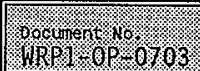 & 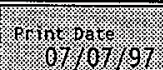 & Re\% \\
\hline
\end{tabular}




\section{WRAP 1 PLANT OPERATING PROCEDURE}

6. Lower Drum From Port

a. ENSURE Lift Table is in manual control mode.

b. ENSURE drum port is CLOSED, per applicable procedure.

c. PERFORM the following steps for Entry/Exit Ports:

(1) PRESS and HOLD "DOWN" switch AS NEEDED until 1 ift table is at "Lid in Position Height".

(2) VERIFY lift table at "Lid in Position Height".

(3) VERIFY Port Door Lowered.

(4) LoCK Port Door.

(5) VERIFY Port Door Locked.

(6) EXTEND 1id detach cylinder

(7) PRESS and HOLD "DOWN" switch until lift table is at "Drum Centering Height".

(8) VERIFY 7 ift table at "Drum Centering Height".

(9) RETRACT lid detach cylinder.

(10) RETRACT centering clamps.

(11) VERIFY centering clamps are retracted.

(12) IF HVAC seal present:

(a) DEFLATE HVAC seaT.

(b) VERIFY HVAC seal is deflated.

NOTE - Only those Lift Tables indicated by (*) in Table 1, need lowered from AGV Load. Height for drum to clear port. Port clearance height is checked visually.

d. PRESS and HOLD "DOWN" switch until drum is at AGV Load Height ("AGV LOAD HEIGHT" light STEADY ON), or port clearance height, as applicable.

e. IF "DOWN OVERTRAVEL" light comes ON, THEN, PRESS "UP" switch until light goes OFF. 


\section{WRAP 1 PLANT OPERATING PROCEDURE}

7. MOVE Drum To AGV End of Lift Table

\section{CAUTION}

IN MANUAL CONTROL (MAINTENANCE) MODE THERE IS NO INTERLOCK TO PREVENT DRUM OVER-TRAVEL. IF THE REVERSE SWITCH IS HELD IN AFTER THE DRUM REACHES THE AUTOMATIC GUIDED VEHICLE (AGV) END OF THE TABLE AND THE AGV IS NOT IN PLACE, THE DRUM WILL FALL TO THE FLOOR. THERE IS ALSO NO INTERLOCK TO STOP UP-TRAVEL BASED ON WEIGHT SETPOINT AND NO INDICATION THAT LIFT TABLE IS AT DRUM CENTERING HEIGHT.

a. ENSURE Lift Table is in manual control mode.

NOTE - On7y those Lift Tables indicated by $(*)$ in Table 1, need lowered from AGV Load Height for drum to clear port. Port clearance height is checked visually.

b. If necessary,

THEN, ENSURE Lift Table is Towered to port clearance height.

\section{WARNING}

LIFT TABLE REVERSE WILL NOT AUTOMATICALLY STOP WHEN DRUM REACHES END OF LIFT TABLE. ENSURE THAT DRUM DOES NOT EXTEND PAST END OF LIFT TABLE BY RELEASING "REVERSE" SWITCH AS NEEDED.

c. PRESS and HOLD "REVERSE" switch until drum is near end of lift table and "DRUM AT AGV END" light is STEADY ON.

d. VERIFY drum not past end of lift table.

e. IF drum moves past desired position,

THEN, PRESS "FORWARD" switch until drum moves back to desired position.

B. RETURN LIFT TABLE TO NORMAL OPERATION

1. PLACE "MODE" (MAINT/OFF/NORMAL) switch to "NORMAL".

NOTE - On7y Lift Tab7es 107-LT-09-201A through 201F are equipped with Table Control switch.

2. IF equipped with "TABLE CONTROL" (AGV/PANEL) switch, THEN, PLACE "TABLE CONTROL" switch to "AGV".

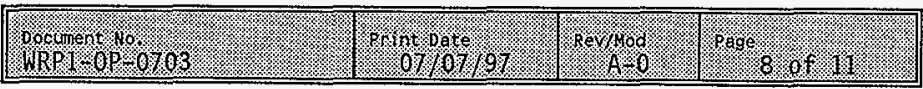




\section{WRAP 1 PLANT OPERATING PROCEDURE}

C. REHOME LIFT TABLE

NOTE - Lift tables must be rehomed:

1. After every power outage.

2. After use in manual mode.

3. After a lift table rope switch is activated.

1. PLACE glovebox in Maintenance Mode.

2. VERIFY drum is NOT present on the lift table.

3. POSITION Lift Tables 201D, 202C, 202D, and 202E below AGV height.

4. POSITION all other Lift Tables ABOVE AGV height.

5. SELECT Misce11 aneous Equipment Maintenance Menu.

6. PRESS "REHOME LIFT TABLE LT-09-20XX" button.

NOTE - $\quad X X$ refers to the last two digits in the lift table tag number.

7. VERIFY Lift Table at AGV height (AGV indicator illuminated; and/or lift table rollers at $27+/ 1 / 8$ inch above floor). 


\section{WRAP 1 PLANT OPERATING PROCEDURE}

FIGURE 1: LIFT TABLE LOCAL CONTROL PANEL

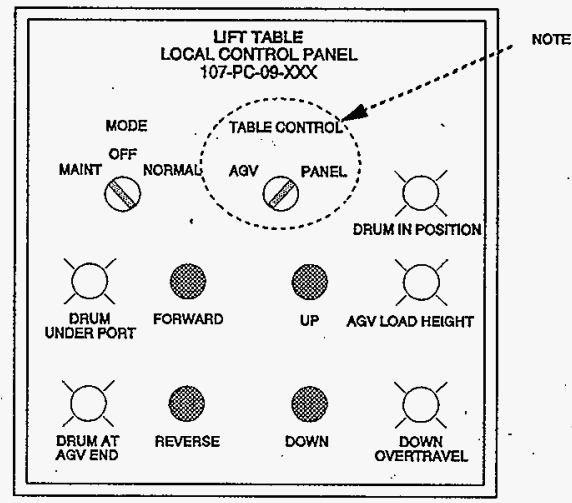

TABLE 1. LITT TABLELCONTROL PANEL DESCR IPTIONS

VOCA. OONTROL PANEL NUGBER

$107.2 \mathrm{pc}=09.2011 \mathrm{~A}$

107- $\mathrm{PC}$ - $09-201 \mathrm{~B}$

$107.1 \mathrm{C}-09.201 \mathrm{C}$ $107.3 \mathrm{c}-09-2010$

$107 \cdot \mathrm{RC}-09-201 \mathrm{~L}$

$107-10-09-2017$

$107 . \mathrm{BC}-0.9-202 \mathrm{~A}$

107 PC-0. $09202 B$

$107 . \mathrm{PC}-09=202 \mathrm{C}$

107. PC. $09-2020$

107. $\mathrm{PC}-09-202 \mathrm{E}$

107. $\mathrm{PC}-09-203 \mathrm{~A}$

$107-\mathrm{PC}-09-203 \mathrm{~B}$

$107.9 \mathrm{CC} 09.203 \mathrm{C}$
LIFT TABLE NUMBER

107. $17.09 .201 \mathrm{~A}$

107. T T-09-201B

107- IT-09-201C

107. 1 T -092010

107.4 . $09.201 \mathrm{E}$

$107-17=09-2015$

107. 1 T. $09=202 \mathrm{~A}$

$107.115-09-202 \mathrm{~B}$

$107.11 .09 .202 \mathrm{C}$

$107.41 .09-2020$

107: 1 - $=09-202 \mathrm{E}$

107-1 T $=09=203 \mathrm{~A}$

$107-17=09-203 B$

107. $11.09=2030$
DESCRIPT TION

TRU BHU TREATED WASTE LOADOUT PORT ILITI TABLE

TRU RWH COMPLIANT WASTE LOADOUT RORL/ITH TABLE

TRU RHM TRANSEER DRUM PORT IIVITI TABLE

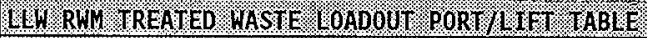

LLW RWM COMPLIANT WASTL LOADOUT RORT LLITT TABLE.

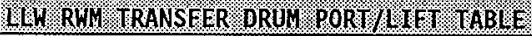

GH. ENTRY GLOVEBOX PORT II IT T TABLE

TRU ENTRY GLOUEBOX PORT/LWIT IIABLE

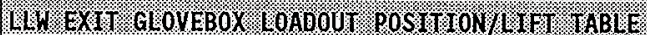

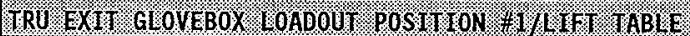

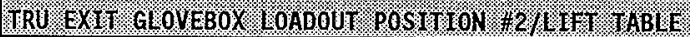

44. TRANSFER PORT/L IIT TABLE EOR RWH TRANSFER ORUNS

TRU TRANSFER PORT LLI TI TABLE FOR RH TRUASERE DRUUS

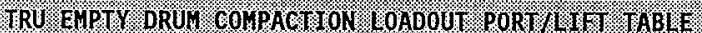

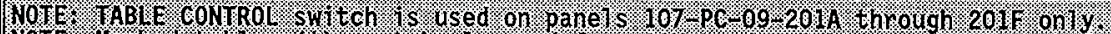

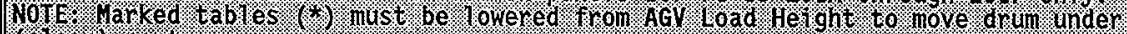
(clear) port.

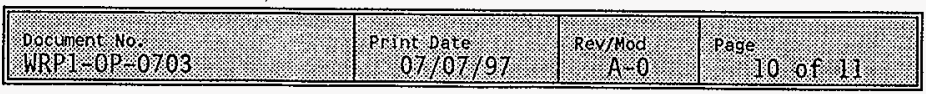




\section{WRAP 1 PLANT OPERATING PROCEDURE}

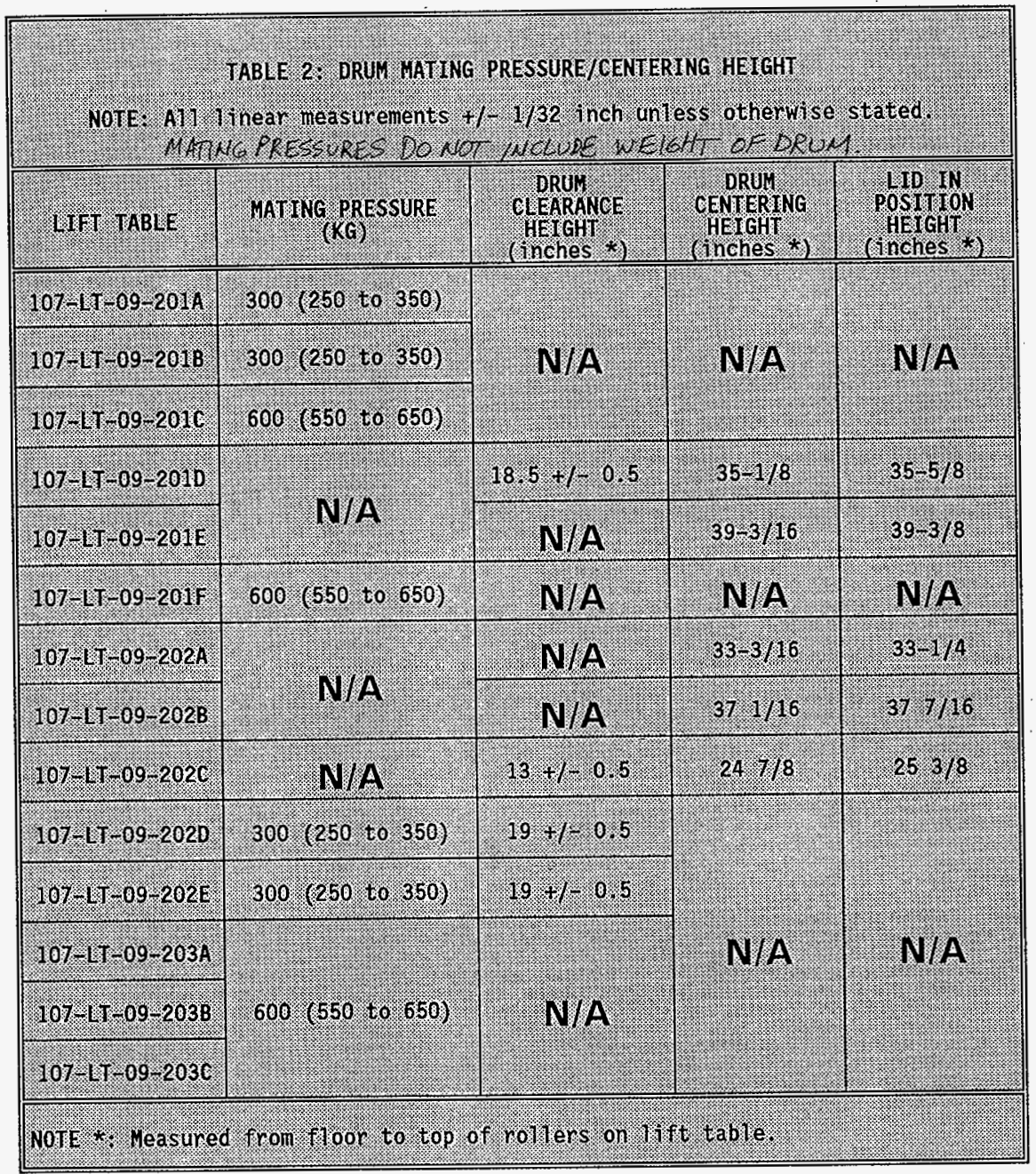

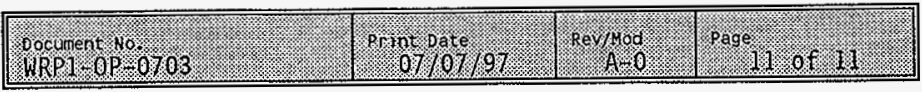


HNF-SD-WO26-OTR-OI4 REV:O

\section{WRAP 1 PLANT OPERATING PROCEDURE}

\section{STSTEH: PROCESS}

\section{GLOVEBOX MANIPULATOR}

\section{SYSTEM DESCRIPTION}

This procedure provides instructions for operation of Schilling Robotic Systems, Glovebox Manipulators.

Giovebox Manipulators are used for moving materials and equipment, and performing tasks inside the gloveboxes.

\section{REFERENCE DOCUMENTS}

None.

\section{PRESTART CONDITIONS}

A11 personnel performing this procedure shall be qualified in accordance with WHC-CM-5-34, Section 1.8, "Training Plan," and on-the-job training.

\section{SAFETY}

Potential hazards, such as lifting heavy containers or handling sharp objects present in waste containers, must be mitigated by protective equipment, procedures, and administrative controls to ensure acceptablerisk operating conditions.

\section{TOOLS AND SUPPLIES}

None. 


\section{TABLE OF CONTENTS}

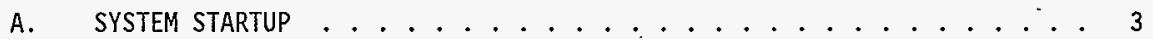

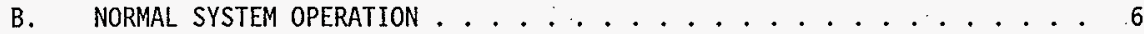

Master Arm 0peration. . . . . . . . . . . . . . . 8

Changing Slave Arm To Master Arm Correspondence . . . . . . . . . 8

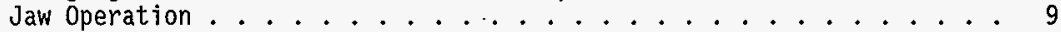

Controlling Wrist Rotation ................ . 10

Moving Carriages ................. 11

Vertical Carriage Obstructions . . . . . . . . . . . . . 11

Horizontal Carriage Obstructions . . . . . . . . . . . 12

Changing Individuai System Operating Characteristics . . . . . . . 13

c. STARTUP WITH SYSTEM ERRORS . . . . . . . . . . . . . . 13

D. SET SLAVE ARM CHARACTERISTICS .............. 16

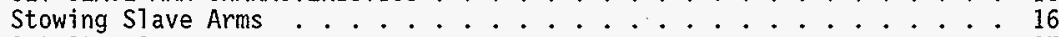

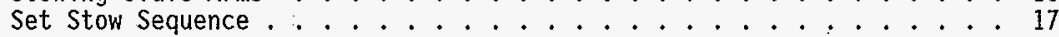

Freezing Entire Slave Arm ................ . . 18

Freezing Individual STave Arm Joints ........... 19

Controlling Slave Arm Speed ................... 20

Enabling And DisabTing S1ave Arm Hydraulics ............ 21

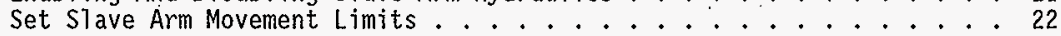

E. SET JAW OPERATING CHARACTERISTICS . . . . . . . . . . . 24

Set Jaw Closing Speed . . . . . . . . . . . . . . . . 24

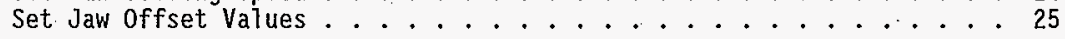

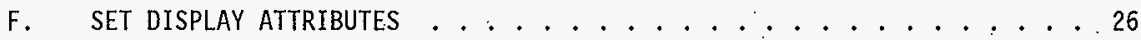

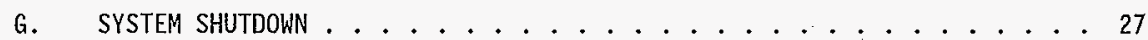

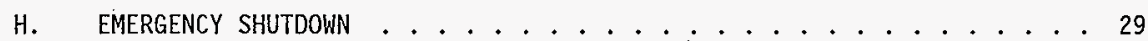

\section{ATTACHMENTS}

FIGURE 1 - CARRIAGE CONTROLLER . . . . . . . ...... 30

FIGURE 2 - MASTER CONTROL PANEL . . . . . . . . . . . . . . . 31

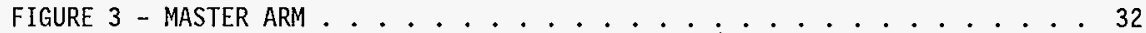




\section{WRAP 1 PLANT OPERATING PROCEDURE}

\section{PROCEDURE}

\section{A. SYSTEM STARTUP}

\section{CAUTION}

ERRATIC, UNCONTROLLED MOVEMENT OF SLAVE ARMS MAY DAMAGE EQUIPMENT.

1. If at any time during startup or operation, slave arms move erratical ly or uncontrolled,

THEN immediately:

a. PRESS EMERGENCY STOP button on either side of carriage controller (Figure 1) IN.

b. TURN master control panel (Figure 2) POWER switch OFF.

NOTE - $\quad$ Prob7em which caused erratic movement must be corrected prior to restart of system.

c. CONTACT Duty Operation Supervisor (DOS).

2. PERFORM pre-start checks:

a. INSPECT, visually, fasteners and fittings are properly connected and in good condition.

b. INSPECT, visually, hoses are free of cuts, nicks, kinks, and excessive sagging.

c. INSPECT, visually, slave arms are free of hydrautic leaks.

3. SET disconnect switch on Control \& Instrumentation (C\&I) enclosure to ONE.

4. ENSURE EMERGENCY STOP buttons on carriage controller are OUT.

5. TURN master control panel POWER switch ON.

NOTE - Screen briefly displays "Establishing communication with s]ave unit" when power is first applied.

6. IF screen displays no readable text,

THEN, TOGGLE master control POWER switch (OFF and back ON).

a. IF screen remains blank,

THEN, NOTIFY DOS. 
7. IF NORMAL STARTUP screen displays, THEN, PROCEED as fOllows:

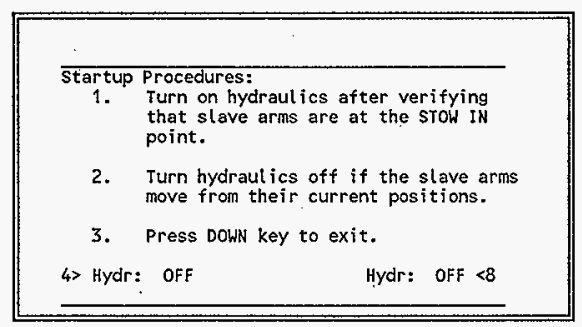

NOTE -

HPU 107-HU-07-302 is common to all manipulators and will be running already if another manipulator is operating HPU Control Panel is located at HPU on overhead walkway.

a. IF Hydraulic Power Unit (HPU), 107-HU-07-302 is OFF,

THEN, PRESS "START" button on HPU Control Panel.

NOTE -

Pressing indicated function keys toggles hydraulic

power to slave arms by opening and closing isolation valves. Corresponding screen flag will change to indicate state OFF/ON.

b. PRESS the following STARTUP screen keys to enable slave arm hydrautic power:

- "4> HYDR" - enable left slave arm

- $\quad$ "8> HYDR" - enable right slave arm

c. CHECK, HYDR indicators on display:

- 4> HYDR: ON

- 8> HYDR: ON

d. PRESS DN (down) function key on master control panel. 
(Step VII.A.7 Cont.)

e. VERIFY STOW OUT menu displayed.

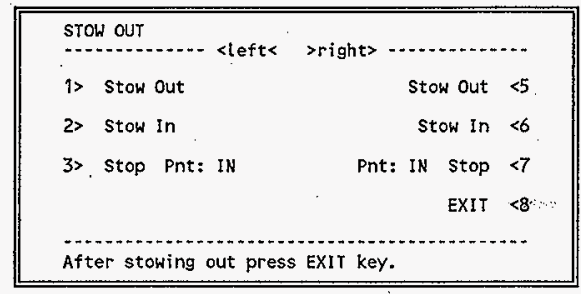

f. PRESS "1> STOW OUT" (left arm).

g. VERIFY left arm display (PNT), indicates OUT.

h. PRESS " $5>$ STOW OUT" (right arm).

i. VERIFY right arm display (PNT), indicates OUT.

j. PRESS "8> EXIT" (to MAIN menu).

k. GO TO Section VII.B, NORMAL SYSTEM OPERATION.

8. IF STARTUP WITH ERRORS screen displays, THEN, GO TO Section VII.C, STARTUP WITH SYSTEM ERRORS.

ERRORS WERE DETECTED:

LF slave not detected

RT arm not near stow point

Keys error $(00000000000100000)$

Diagnose errors before starting hydrauljos. Press the DOWN key to view the next menu. 
B. NORMAL SYSTEM OPERATION

NOTE - This section assumes dual-arm system operation. If one slave arm controller is disabled, menus and choices for only the active arm will be available.

Normal operation of the manipulator, with exception of setting different system characteristics is gained primarily from on-the-job training and experience.

The user manipulates the system via master arms on the master contro7 panel, and arm carriages, and selects or changes desired operating characteristics as needed.

This section describes basic master arm operating functions, carriage movement, and OPERATE menu selections. The user is referenced to applicable sections for setting specific characteristics.

The master control panel display is norma77y left on the OPERATE menu, which provides easy access to the most common functions.

1. VERIFY MAIN menu displayed.

$\begin{array}{ll}\text { NOTE - } & \text { User returns to MAIN menu as needed by pressing } \\ \text { EXIT from other menus unti7 MAIN menu displays. }\end{array}$

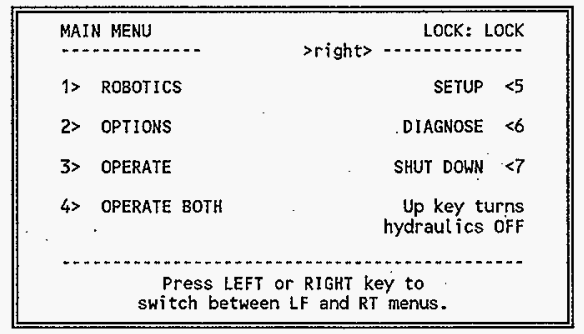


NOTE - OPERATE selects left/right OPERATE menu depending on flag when selection is made. To select opposite OPERATE menu, operator must return to MAIN menu. OPERATE BOTH a7lows operator to toggle left/right OPERATE menus using LF/RT function keys, without returning to MAIN menu.

2. IF access to only one OPERATE menu is desired, THEN:

a. PRESS LF (left) OR RT (right) function key until desired choice is flagged on MAIN menu.

b. PRESS "3> OPERATE".

3. IF access to both LEFT and RIGHT OPERATE menus is desired, THEN, PRESS "4> OPERATE BOTH".

4. VERIFY OPERATE menu displayed.

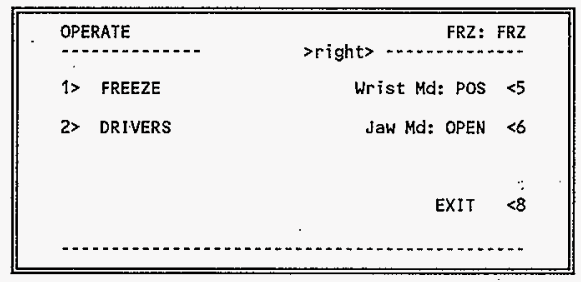

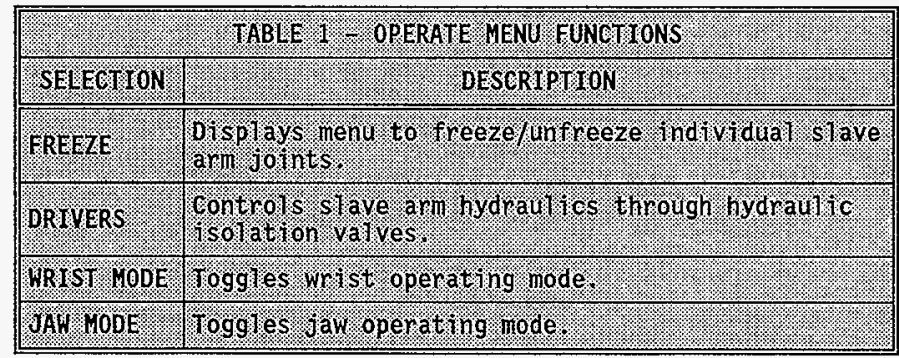

\begin{tabular}{|c|c|c|c|}
\hline $\begin{array}{l}\text { oocunent } \\
\text { WRP }\end{array}$ & pront pare $07 \%$. 97 & rev/lod & rage $7 / 2$ of 32 \\
\hline
\end{tabular}




\section{WRAP 1 PLANT OPERATING PROCEDURE}

5. Master Arm Operation

NOTE - $\quad$ Master arms (Figure 3) are miniature replicas of left and right slave arms, with the same relative range of motion. Each slave arm joint or function has a corresponding master arm action.

a. ACTUATE master arm action(s) (TABLE 2), as required, to manipulate corresponding slave arm:

\begin{tabular}{|c|c|}
\hline TABIE $2=1 \mathrm{NAS}$ & 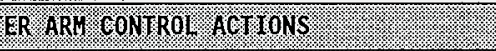 \\
\hline DESIRED SIAVH ARU FUNCIONI & OPERHOOR ACION ON YASTER ARH \\
\hline Move. SMAVE. ARHV & 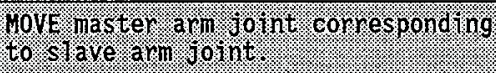 \\
\hline 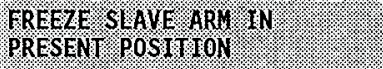 & 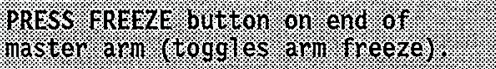 \\
\hline ROIATE GRIST & 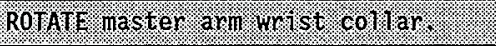 \\
\hline OPEN/GOSE JAH. & 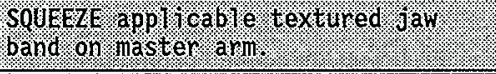 \\
\hline \multicolumn{2}{|c|}{ 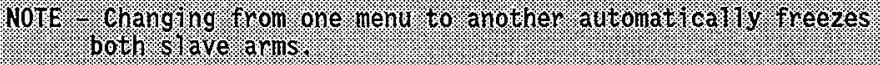 } \\
\hline
\end{tabular}

6. Changing STave Arm To Master Arm Correspondence

NOTE - When a slave arm is frozen, its master arm can be repositioned to a more comfortable operating position without moving the slave arm. This is usefuT when a task results in an awkward position for the master arm.

a. FREEZE slave arm.

b. REPOSITION master arm as desired.

c. UNFREEZE slave arm.

d. RESUME normal operation.

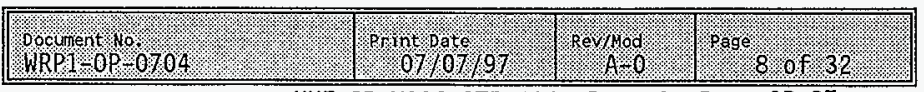




\section{WRAP 1 PLANT OPERATING PROCEDURE}

7. Jaw Operation

NOTE - STave arm must be unfrozen to change jaw mode.

a. SET jaws to desired mode as follows:

(1) PRESS "3> OPERATE", from MAIN menu.

(2) VERIFY OPERATE menu displayed.

\begin{tabular}{|c|c|c|c|}
\hline \multicolumn{2}{|c|}{ OPERATE } & \multicolumn{2}{|c|}{ >rights $\quad$ FRZ: FRZ } \\
\hline & FREEZE & Wrist Md: POS & $<5$ \\
\hline 2> & DRIVERS & JaW Md: OPEN & $<6$ \\
\hline & . & EXIT & $<8$ \\
\hline
\end{tabular}

(3) PRESS " $6>$ JAW MD" to toggle modes (TABLE 3).

\begin{tabular}{|c|c|}
\hline & . \\
\hline VNODE & 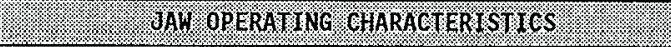 \\
\hline 6PEN & 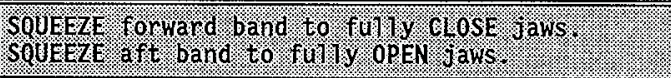 \\
\hline 70GGl & 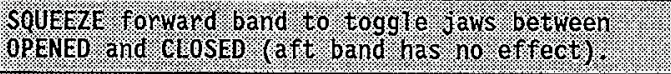 \\
\hline Lock & 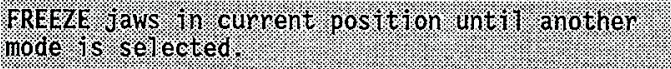 \\
\hline POS TIION & 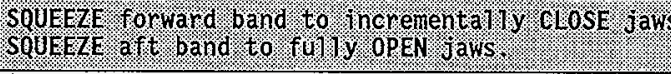 \\
\hline
\end{tabular}

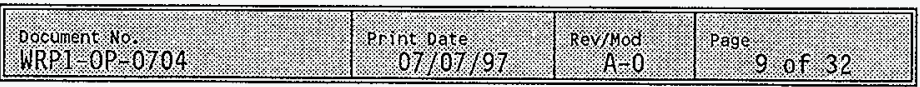


8. Controlling Wrist Rotation

NOTE - The wrist has two modes of operation: In POSITION mode the slave arm wrist follows (direction and speed) rotation of the master arm wrist collar. Rotation is limited by master arm wrist collar to $270^{\circ}$.

In RATE mode, rotating the master arm wrist collar in either direction from null position causes the slave arm wrist to rotate continuously in the corresponding direction. Rate of rotation is proportional to degree of rotation of the master collar from nu77 position. Freezing the slave arm or rotating the wrist slightly in the opposite direction will stop rotation.

a. CHANGE wrist mode as follows:

(1) ENSURE OPERATE menu displayed.

(2) ENSURE slave arm unfrozen.

(3) PRESS " $5>$ WRS MD" (toggles setting).

NOTE -

Nu7l position applies to RATE mode on7y. Nu7l position is the position of the master arm wrist collar at the point where either: (1) wrist mode was changed from POSITION to RATE, or (2) slave arm was unfrozen. This means the nuT7 position could be close to one of the wrist collar mechanical stops.

b. ESTABLISH new wrist null position as follows:

(1) FREEZE siave arm.

NOTE - NULL is norma77y set close to center of wrist collar mechanical range.

(2) MOVE wrist collar to desired null position.

(3) UNFREEZE slave arm. 


\section{WRAP 1 PLANT OPERATING PROCEDURE}

\section{CAUTION}

CARRIAGES MAY RUN INTO OBSTRUCTIONS (CABLES, HOSES, PROCESS ITEMS OR PROCESS EQUIPMENT) INSIDE GLOVEBOX.

CARRIAGE WILL STOP MOVING WHEN MECHANICAL STOP IS REACHED. DO NOT HOLD MOVEMENT BUTTONS AFTER CARRIAGE REACHES MECHANICAL STOP OR EQUIPMENT MAY BE DAMAGED.

CARRIAGE MUST COME TO A COMPLETE STOP BEFORE REVERSING DIRECTIONS.

OPERATING HORIZONTAL CARRIAGE WITH LOW HYDRAULIC PRESSURE CAN DAMAGE EQUIPMENT.

9. Moving Carriages

a. PRESS and HOLD movement button(s) on carriage controller until carriage reaches desired position,

THEN, RELEASE.

10. Vertical Carriage Obstructions

a. If vertical carriage encounters an obstruction too heavy to push aside,

THEN:

(1) RELEASE activated movement button.

(2) CHECK for obstruction(s).

(3) MOVE obstructions as necessary.

(4) RESUME normal operations. 
11. Horizontal Carriage Obstructions

NOTE - Horizontal carriage is equipped with a torque limiter which may be tripped by any of the following; horizontal carriage hits obstacle too heavy to push aside, foreign obstacle in carriage pulley or sprocket, slave arm grasping fixed object during carriage movement, carriage at end of trave7, carriage rail is bent or damaged.

HORIZ SLIP 7ight is ON only when torque limiter trips and movement button is pressed.

a. IF HORIZ SLIP 1ight illuminates, THEN:

(1) RELEASE activated movement button.

(2) CHECK for obstructions or other cause.

NOTE - Torque limiter may self reset when fault is cleared.

(3) CORRECT obstructịons or fautt as necessary.

(4) RESUME normal operations.

b. If cause of fautt is not readily apparent,

$\underline{\mathrm{OR}}$

If torque 1 imiter remains tripped after fault is cleared, THEN, RESET torque limiter as follows:

NOTE - HORIZ SLIP light will be ON when moving carriage.

(1) MOVE carriage in opposite direction of fault unti1 mechanical stop is encountered.

(2) J0G (repeatedly press and reTease) button used to move carriage to mechanical stop, until torque 1imiter resets (1isten for reset).

(3) VERIFY HORIZ SLIP light remains OFF when carriage is moved in either direction.

(4) REPEAT actions until reset.

(5) RESUME normal operations. 


\section{WRAP 1 PLANT OPERATING PROCEDURE}

12. Changing Individual System Operating Characteristics

a. CHANGE characteristics as follows:

(1) GO TO Section indicated in TABLE 4.

(2) MAKE changes as required.

(3) RESUME normal operation.

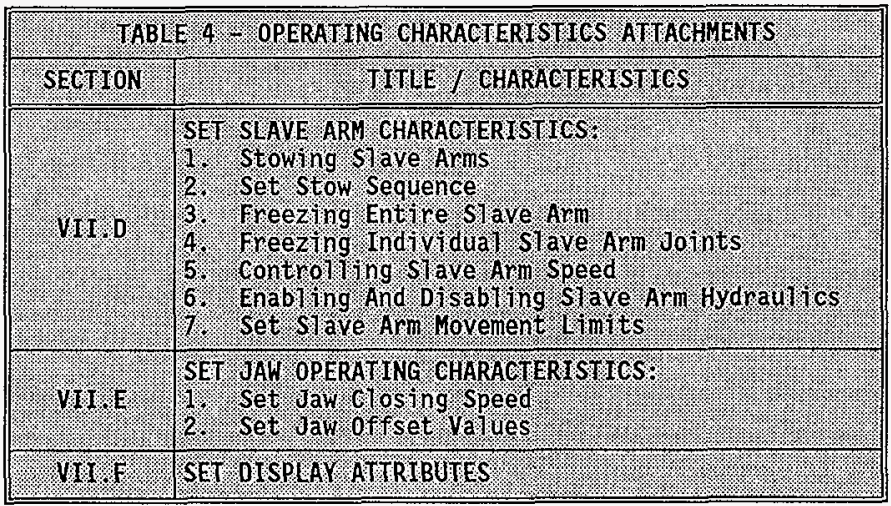

\section{c. STARTUP WITH SYSTEM ERRORS}

1. DETERMINE cause of error(s) (TABLE 5).

2. TAKE applicable corrective action per TABLE 5.

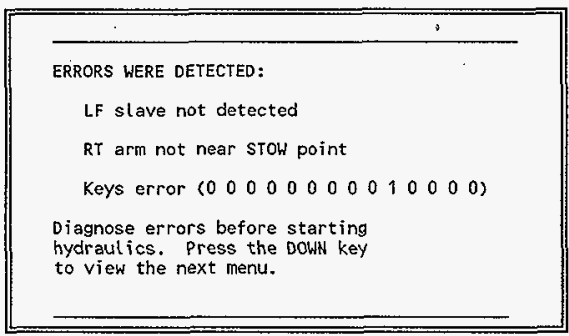

\begin{tabular}{|c|c|c|c|}
\hline $\begin{array}{l}\text { Document } \\
\text { HRPJ }-0 \mathrm{PP}\end{array}$ & $\begin{array}{l}\text { mptpate } \\
07107 \% 9\end{array}$ & \%evodod & 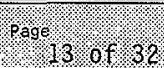 \\
\hline
\end{tabular}




\begin{tabular}{|c|c|c|}
\hline TIAB & 5 - STARTUP WTH ER & \\
\hline ERROR WESSAGE & GAUSE & 4c190 \\
\hline 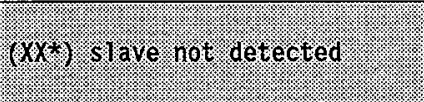 & 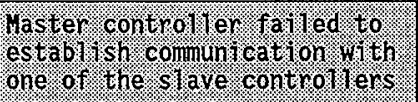 & Hottify superviston. \\
\hline$(x)(t)$ arm not near stont pos int & $\begin{array}{l}\text { Left or right stave arm not } \\
\text { in stowed position. }\end{array}$ & Go 10 step V11.0.4. \\
\hline 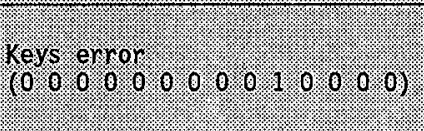 & 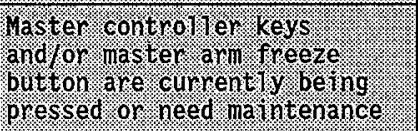 & 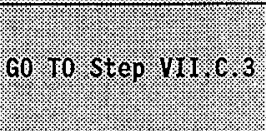 \\
\hline te to di indicates fallied c & el: If ( Teft ) or RT & \\
\hline
\end{tabular}

3. IF a key error message is displayed, THEN, PROCEED as follows:

a. IF a key or FREEZE button is actual7y being pressed, THEN :

(1) REMOVE pressure from key or REPOSITION master arm so freeze button is released.

(2) SKIP to Step VII.C.4.

b. IF a key or freeze button is not being pressed, THEN:

(I) DO NOT enable hydraulics or deploy slave arms.

(2) PRESS DN function key on master control panel.

(3) VERIFY MAIN menu displayed.

(4) NOTIFY DOS.

4. PRESS DN function key on master control pane1.

5. VERIFY MAIN menu for right slave arm displayed.

6. PRESS " $3>$ OPERATE".

\begin{tabular}{|c|c|c|c|}
\hline 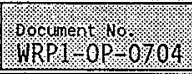 & Print oate & 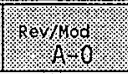 & Proc $14.0 \%$. 32 \\
\hline
\end{tabular}




\section{WRAP 1 PLANT OPERATING PROCEDURE}

7. VERIFY OPERATE menu displayed.

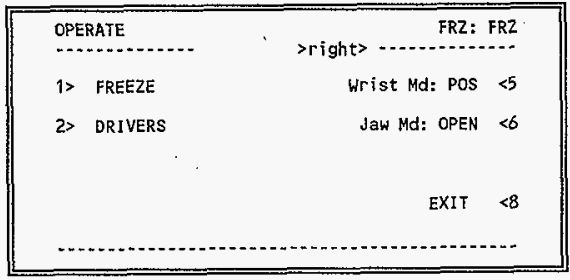

8. PRESS "2> DRIVERS".

9. VERIFY DRIVERS menu displayed.

\begin{tabular}{|lr|}
\hline DRIVERS & LOCK: LOCK \\
1> \#1: OFF & \$5: OFF $<5$ \\
2> \#2: OFF & Hydr: ON $<6$ \\
3> \#3: OFF & \\
4> \#4: OFF & EXIT $<8$ \\
\hline
\end{tabular}

10. PRESS "6> HYDR" (enable right slave arm hydraulics).

11. EXIT to MAIN menu, THEN, DISPLAY OPERATE menu for left stave arm.

12. REPEAT Steps VII.C.8 through VII.C.10 (enable left slave arm hydraulics).

13. GO TO Section VII.B, NORMAL SYSTEM OPERATION.

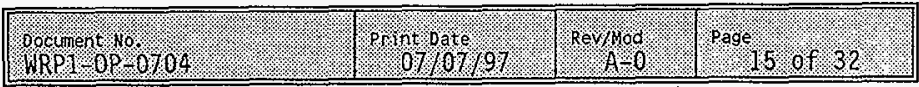




\section{WRAP 1 PLANT OPERATING PROCEDURE}

D. SET SLAVE ARM CHARACTERISTICS

1. Stowing STave Arms

NOTE - S7ave arms are moved to their STOWED IN, (protected) position for transport by carriages inside glovebox and to their STOWED OUT (deployed) position for working.

\section{CAUTION}

IF A SLAVE ARM IS NOT STOWED OUT WHEN A STOW COMMAND IS ISSUED, THE MESSAGE "ARM NOT NEAR STOW POINT" DISPLAYS. THE ARM THEN MOVES FIRST, TO THE STOW OUT POSITION, THEN TO THE COMMANDED POINT. BE PREPARED FOR ARM TO MOVE TO STOW OUT POSITION BEFORE IT MOVES THROUGH THE STOW/DEPLOY SEQUENCE.

ONCE STOW IN IS SELECTED, ARM MUST BE ALLOWED TO MOVE TO FULL STOW IN POSITION BEFORE EXITING APPLICABLE MENU.

a. ENSURE stow paths are clear of obstructions which could damage or be damaged by slave arms.

b. PRESS function keys (TABLE 6), as required, to STOW slave $\operatorname{arm}(s)$ to desired position:

(1) PRESS STOP to halt slave arm at next point in sequence.

(2) IF slave arm is being STOWED IN, THEN, PRESS STOW OUT at any point to reverse sequence.

(3) IF slave arm is being STOWED OUT, THEN, PRESS STOW IN at any point to reverse sequence.

\begin{tabular}{|c|c|c|c|}
\hline \multicolumn{4}{|c|}{ 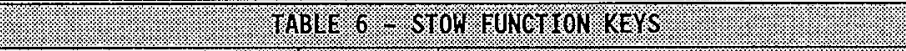 } \\
\hline EUNCT & OH KEY & \multirow{2}{*}{ IUNC T ION } & \multirow{2}{*}{ ACTION } \\
\hline YET\%ARM. & RGHU $A R H$ & & \\
\hline 新 & 5. & 5104 our & Deploys arn to stow out pos J Won. \\
\hline 2 & $1 \%$ & STOH $11 \%$ & Retracts arn tro strow hn pos r fron. \\
\hline (3) & $2.1 \%+2.1 \%$ & $(2,0 \mathrm{P}$ & $\begin{array}{l}\text { Stops ar } \\
\text { stow out hr sequence }\end{array}$ \\
\hline pNT & tes each & ow seque & ce point as arn passes through $/$ t \\
\hline
\end{tabular}

\begin{tabular}{|c|c|c|c|}
\hline Wocuent 10.6 & print oate $07 \%$ & \% & pago $16.0 \mathrm{f} / 32$ \\
\hline
\end{tabular}

HNF-SD-W026-OTR-014, Rev. 0, Page OP-95 
2. Set Stow Sequence

NOTE - $\quad$ Stow sequence is a series of arm movements that positions the slave arm between the protected (STOW IN) position and the deployed (STOW OUT) position. The sequence may consist of 2 to 16 slave arm positions (points).

a. PRESS "5> SETUP" from MAIN menu.

b. VERIFY SETUP menu displayed.

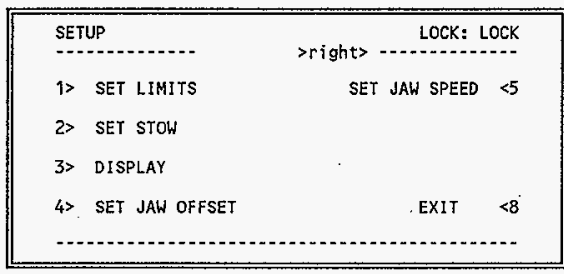

c. PRESS "2> SET STOW".

d. VERIFY SET STOW menu displayed.

\begin{tabular}{|c|c|c|c|}
\hline SET STOW & $>$ right $>$ & LOCK: & FRZ \\
\hline Point: 01 & & Last & $<5$ \\
\hline & & Next & $<6$ \\
\hline & & Teach & $<7$ \\
\hline $\begin{array}{l}\text { LAST STOW } \\
<4 \text { Point: } 16\end{array}$ & & EXIT & $<8$ \\
\hline
\end{tabular}

e. DETERMINE desired number of points in stow path sequence.

f. PRESS " $4>$ POINT", repeatediy, until determined number is displayed.

g. UNFREEZE slave arm. 


\section{WRAP 1 PLANT OPERATING PROCEDURE}

(Step VII.D.2 Cont.)

NOTE - Point 01 is first point in stow path, (STOW IN position).

h. VERIFY point counter indicates 01 .

i. MOVE arm to desired position (point).

\section{CAUTION}

WHEN THE LAST POINT SPECIFIED IN SEQUENCE IS TAUGHT, POINT COUNTER DOES NOT INCREMENT. IF "7> TEACH" IS NOT PRESSED THE LAST POINT ACTUALLY TAUGHT PRIOR TO PRESSING "B> EXIT" BECOMES THE STOW OUT POINT. IT IS IMPORTANT TO TEACH DESIRED STOW OUT POINT BEFORE EXITING SET STOW MENU.

ALL POINTS MUST BE CLEAR OF GLOVEBOX WALLS \& EQUIPMENT OR DAMAGE MAY OCCUR.

NOTE - Pressing "5> LAST", instead of "7>TEACH" returns to previous point. Pressing "6>NEXT", instead of "7> TEACH" skips point \& increments point counter.

j. PRESS "7> TEACH".

k. VERIFY point counter increments to next number.

1. REPEAT Steps VII.D.2.i through VII.D.2.k until ali desired points have been programmed.

m. PRESS "8> EXIT" (to SETUP menu).

3. Freezing Entire Slave Arm

NOTE - Changing from one menu to another automatically freezes both slave arms.

a. CHECK slave arm status in upper right corner of menu:

- REIN - normal unfrozen operation.

- $\quad$ FRZ - arm frozen (can be unfrozen with master arm freeze button).

- LOCK - arm frozen (cannot be unfrozen with freeze button from current menu).

b. PRESS freeze button (toggles FRZ/REIN) on master arm.

\begin{tabular}{|c|c|c|c|}
\hline 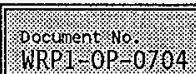 & 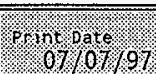 & Rey/Mod & (2) $18.0 \mathrm{f} .32$ \\
\hline
\end{tabular}


4. Freezing Individual Slave Arm Joints

NOTE - Freezing individual joints not required for a given task, provides a more stable platform for the task.

a. FREEZE stave arm.

b. PRESS "3> OPERATE" from MAIN menu.

c. VERIFY OPERATE menu displayed.

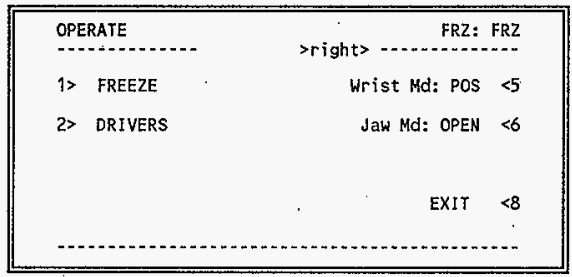

d. PRESS " $1>$ FREEZE".

e. VERIFY FREEZE menu dispiayed.

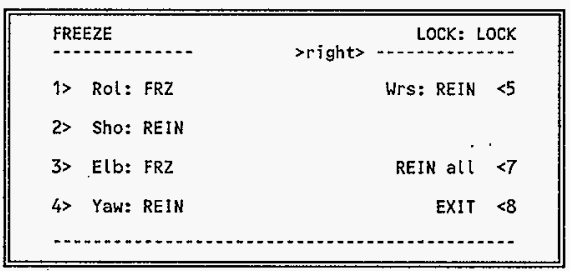

f. CHECK status of individual joints on menu.

g. PRESS indicated function to toggle status (FRZ/REIN) of each joint as desired ("7> REIN ALL", unfreezes a17).

h. PRESS "8> EXIT" (to OPERATE menu). 


\section{WRAP 1 PLANT OPERATING PROCEDURE}

5. Controlling Slave Arm Speed

NOTE - In genera7, speed of master arm movement controls speed of s7ave arm movement. System starts in FAST mode, however SLOW mode may be selected when more precise control is needed.

a. PRESS "2> OPTIONS", from MAIN menu.

b. VERIFY OPTIONS menu displayed.

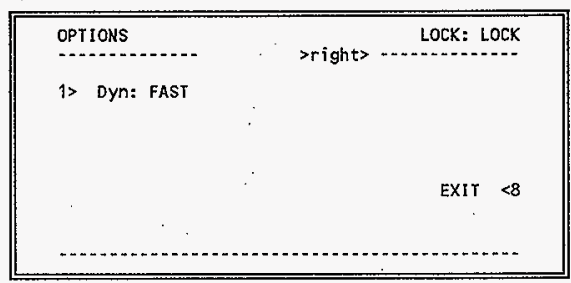

c. PRESS "I> DYNAMICS" (toggles FAST/SLOW modes).

d. PRESS "8> EXIT" (to MAIN menu). 


\section{WRAP 1 PLANT OPERATING PROCEDURE}

6. Enabling And Disabling Slave Arm Hydraulics

\section{CAUTION}

IF COMMANDED SLAVE ARM POSITION CHANGES WHILE HYDRAULICS ARE DISABLED, SLAVE. ARM MAY ABRUPTLY MOVE TO THE NEW COMMANDED POSITION WHEN HYDRAULICS ARE RE-ENABLED. (THIS CAN HAPPEN IF OPERATOR DISABLES HYDRAULICS, FREEZES A SLAVE ARM IS FROZEN, MOVES THE MASTER ARM, UNFREEZES THE SLAVE ARM, AND THEN RE-ENABLES HYDRAULICS). IT IS IMPERATIVE THAT SLAVE ARM BE FROZEN BEFORE DISABLING SLAVE ARM HYDRAULICS. HYDRAULICS MUST BE RE-ENABLED AGAIN BEFORE UNFREEZING SLAVE ARM.

NOTE - Each s7ave arm has a hydraulic isolation valve that is either OPEN (enable hydraulic flow to slave arm actuators), or CLOSED (disable arm movement). Hydraulics are enabled as part of the startup process, but may be toggled for operation as desired.

a. IF hydrautics are to be immediately disabled, THEN:

(1) FREEZE s lave arm.

(2) PRESS UP function key on master control panel.

b. If hydrautics are to be re-enabled or toggled, THEN:

(1) PRESS "2> DRIVERS" from OPERATE menu.

(2.) VERIFY DRIVERS menu displayed.

\begin{tabular}{|c|c|c|c|}
\hline DRIVERS & $>$ right $>$ & LOCK: LO & \\
\hline 1> \#1: OFF & & $\# 5:$ OFF & $<5$ \\
\hline 2> \#2: OFF & & Hydr: ON & $<6$ \\
\hline 3> \#3: OFF & & · & \\
\hline 4> \#4: OFF & & EXIT & $<8$ \\
\hline
\end{tabular}

(3) CHECK "6> HYDR" display (ON = hyd enabled, OFF $=$ disabled).

(4) PRESS "6> HYDR", to toggle hydraulics as desired.

(5) PRESS "8> EXIT" (to OPERATE menu).

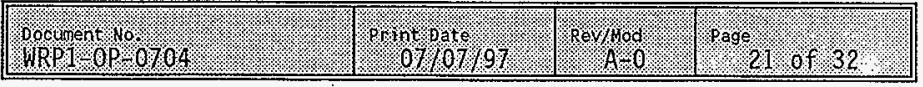




\section{WRAP 1 PLANT OPERATING PROCEDURE}

7. Set S1ave Arm Movement Limits

NOTE - Range of motion of each slave arm joint (except wrist) can be 7imited by specifying left/right or up/down position limit.

a. PRESS "5> SETUP" from MAIN menu.

b. VERIFY SETUP menu displayed.

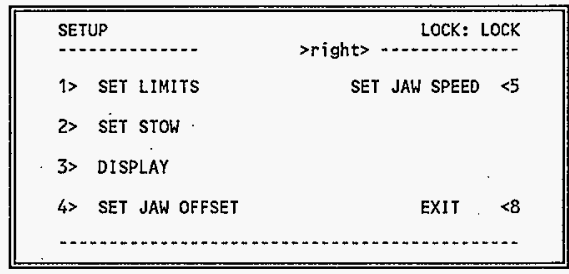

c. PRESS " $1>$ SET LIMITS".

NOTE - Limits are inactive when menu is disp7ayed.

d. VERIFY SET LIMITS menu displayed.

\begin{tabular}{|lr|}
\hline SEI LIMITS & LOCK: FRZ \\
/Count: 01 & >right $>$ \\
Limit: & Last $<5$ \\
ROL REIRACI & Next $<6$ \\
& Teach $<7$ \\
& EXIT $<8$ \\
\hline
\end{tabular}

e. UNFREEZE slave arm.

f. VERIFY count indicates 01 . 


\section{WRAP 1 PLANT OPERATING PROCEDURE}

(Step VII.D.7 Cont.)

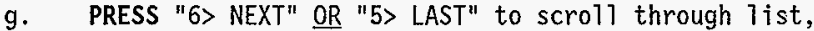
until desired limit (TABLE 7), indicates on display.

\begin{tabular}{|c|c|c|}
\hline \multicolumn{3}{|c|}{ 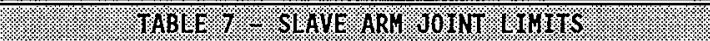 } \\
\hline YII NUABER & $001 \mathrm{~N}$ & ACTUATOR OLREOTION \\
\hline . 01 & 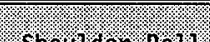 & $\mathrm{REIRACH}$ \\
\hline 02 & 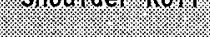 & EXTEND \\
\hline 03. & (5: & RETRAOT \\
\hline 04 & 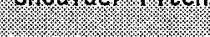 & EXTEND \\
\hline 05 & Tor & RERAQI \\
\hline 06 & Finons & EXTEND \\
\hline 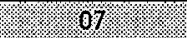 & $\sqrt{1}$ & RETBAC: \\
\hline 08 & & EXTEND \\
\hline
\end{tabular}

h. MOVE joint indicated on display to desired limit.

i. PRESS "7> TEACH".

j. VERIFY counter increments to next position number.

k. REPEAT Steps VII.D.7.g through VII.D.7.j until all desired limits have been set.

NOTE - Any joints remaining outside new limits wi7l move to position inside limits when arm is unfrozen.

1. MOVE arm inside new set limits.

m. PRESS "8> EXIT" (to SETUP menu).

\begin{tabular}{|c|c|c|c|}
\hline 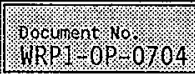 & 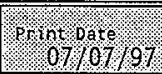 & ReV/Mod & page, $23 \%$ of 32 \\
\hline
\end{tabular}




\section{WRAP 1 PLANT OPERATING PROCEDURE}

\section{E. SET JAW OPERATING CHARACTERISTICS}

1. Set Jaw Closing Speed

NOTE - Jaw speed controls speed at which jaws

incremental7y OPEN with JAW MODE set to POSITION. closing speed is not affected.

a. SET jaw mode to POSITION.

b. PRESS "8> EXIT" (to MAIN menu).

c. PRESS "5> SETUP", from MAIN menu.

d. VERIFY SETUP menu displayed.

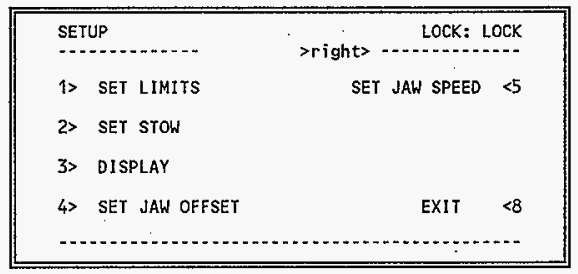

e. PRESS "5> SET JAW SPEED".

f. VERIFY SET JAW SPEED menu disp]ayed.

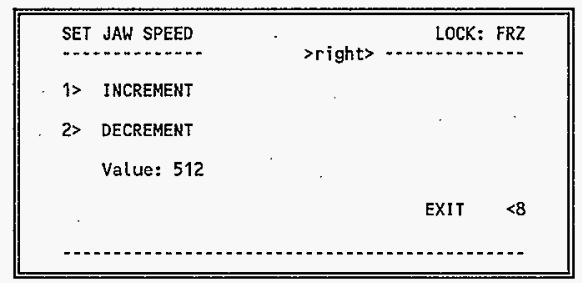

NOTE -

Jaw speed is incremented in arbitrary units (counts), ranging from 128 to 2048. Higher count setting equals faster jaw closure. Each switch press changes setting by 128.

g. PRESS " $1>$ INCREMENT" $\underline{O R}$ " $2>$ DECREMENT", untiT desired setting is displayed. 


\section{WRAP 1 PLANT OPERATING PROCEDURE}

2. Set Jaw offset Values

NOTE - Jaw off set compensates for differences in servo valves to prevent jaws from drifting from a command position during operation. Once set, jaw offset should not need to be changed, unless jaw servo valve is replaced.

a. PRESS "5> SETUP", from MAIN menu.

b. VERIFY SETUP menu displayed.

\begin{tabular}{|c|c|c|c|}
\hline \multicolumn{2}{|c|}{ SETUP } & \multicolumn{2}{|c|}{ LOCK: LOCK } \\
\hline 1> & SET LIMITS & SET JAW SPEED & $<5$ \\
\hline 2> & SET STOW & & \\
\hline 3) & DISPLAY & & \\
\hline 4> & SET JAW OFFSET & EXIT & $<8$ \\
\hline
\end{tabular}

c. PRESS " $4>$ SET JAW OFFSET".

d. VERIFY SET JAW OFFSET menu displayed.

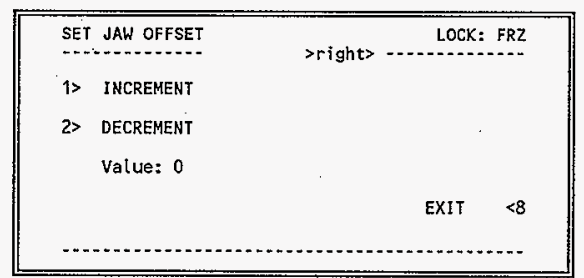

NOTE -

Jaw offset is incremented in arbitrary units

(counts), ranging from -512 to +512 . Each switch press changes setting by 32 .

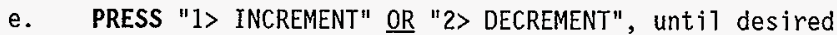
setting is displayed.

f. PRESS "8> EXIT" (to OPERATE menu).

g. RESUME normal operation. 


\section{WRAP 1 PLANT OPERATING PROCEDURE}

F. SET DISPLAY ATTRIBUTES

NOTE - Allows for change of screen viewing angle and/or reversing dark and light portions of the screen.

1. PRESS "5> SETUP", from MAIN menu.

2. VERIFY SETUP menu displayed.

\begin{tabular}{|lr|}
\hline SETUP & LOCK: LOCK \\
1> SET LIMITS & SET JAW SPEED $<5$ \\
2> SET STOW & \\
3> DISPLAY & \\
4> SET JAW OFFSET & EXIT $<8$ \\
\hline
\end{tabular}

\section{PRESS "3> DISPLAY".}

4. VERIFY DISPLAY menu displayed.

\begin{tabular}{|c|c|}
\hline DISPLAY & LOCK: LOCK \\
\hline 1) View Angle - UP & Save to Default $<5$ \\
\hline 2> View Angle - DOWN & \\
\hline 3) Invert background & \\
\hline 4> Default & EXIT $<8$ \\
\hline
\end{tabular}




\section{WRAP 1 PLANT OPERATING PROCEDURE}

5. PRESS function key(s) (TABLE 8), for desired screen attributes.

\begin{tabular}{|c|c|}
\hline 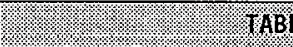 & E $8 \%$ SGRE N ATSRIBUTES \\
\hline EUACTION & SQREEN ESFECT \\
\hline VVIFANGL UP. & 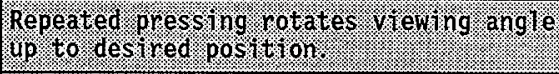 \\
\hline 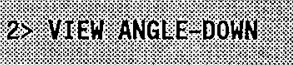 & 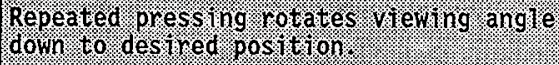 \\
\hline 3\% WUVRT BAGKGROIND & Reverses (togg (e) screen contrast. \\
\hline 4> DEAAUIT- & Revert t to start up screen contry gurat 10 r. \\
\hline $5 \%$ SAVF 10 OEFAUI) & 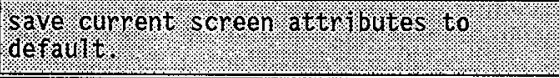 \\
\hline
\end{tabular}

6. PRESS "8> EXIT" (to MAIN menu).

G. SYSTEM SHUTDOWN

\section{CAUTION}

SHUTTING DOWN MANIPULATOR WILL CAUSE SLAVE ARMS TO SAG UNTIL MECHANICAL STOPS ARE REACHED. HYDRAULIC OR CONTROL POWER SHOULD NOT BE TURNED OFF UNTIL ARMS ARE POSITIONED IN GLOVEBOX SO THEY WILL NOT HARM, OR BE HARMED BY OTHER OBJECTS WHEN THEY SAG.

1. PRESS "7> SHUTDOWN" from MAIN menu.

2. VERIFY SHUTDOWN menu displayed.

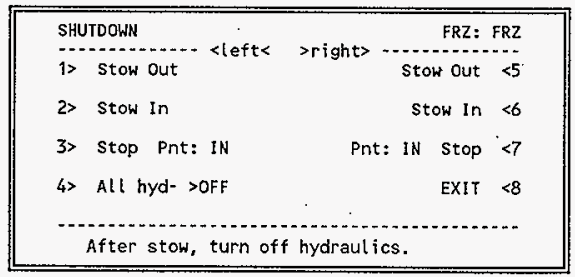

\begin{tabular}{|c|c|c|c|}
\hline 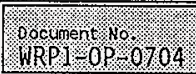 & prone oars & $\%$ \% 1000 & bagk \\
\hline
\end{tabular}




\section{WRAP 1 PLANT OPERATING PROCEDURE}

3. MOVE each slave arm to a position near its STOW OUT point.

4. PRESS "2> STOW IN" (left slave arm).

5. VERIFY left slave arm STOWS IN.

6. PRESS "5> STOW IN" (right slave arm).

7. VERIFY right slave arm STOWS IN.

8. PRESS " $4>$ ALL HYDR OFF".

9. TURN master control power switch OFF.

10. PUSH EMERGENCY STOP button on either side of carriage controller IN.

11. IF manipulator is to be electrically isolated, THEN, SET disconnect switch on C\&I enclosure to ZERO.

12. IF HPU is to be SHUT DOWN, THEN:

a. REPEAT Steps VII.G.1 through VII.G.11 for each manipulator.

\section{CAUTION}

ALL MANIPULATORS MUST BE NEAR STOW OUT POINT BEFORE SHUTTING OFF HPU. SHUTTING OFF HPU WILL DISABLE ALL MANIPULATORS.

NOTE - HPU 107-HU-07-302 is common to al7 manipulators. HPU Control Panel is located at HPU on overhead walkway.

b. PRESS "STOP" button on HPU Control Pane1.

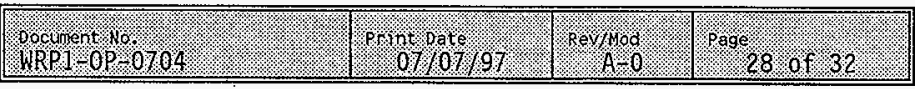




\section{WRAP 1 PLANT OPERATING PROCEDURE}

\section{H. EMERGENCY SHUTDOWN}

NOTE - $\quad$ Emergency Stop immediately shuts down manipulator by turning off all manipulator components that receive power from C\&I enclosure (master controller, carriage controller, slave controllers, slave arms, and horiz and vertical carriages).

Hydraulic power unit (HPU) remains oN but hydraulically powered manipu7ator components cannot be used without electrical power as hydraulic control valves are disabled.

\section{CAUTION}

SHUTTING DOWN MANIPULATOR WILL CAUSE SLAVE ARMS TO SAG UNTIL MECHANICAL STOPS ARE REACHED. HYDRAULIC OR CONTROL POWER SHOULD NOT BE TURNED OFF UNTIL ARMS ARE POSITIONED IN GLOVEBOX SO THEY WILL NOT HARM, OR BE HARMED BY OTHER OBJECTS WHEN THEY SAG.

1. PUSH EMERGENCY STOP button on either side of carriage controller IN.

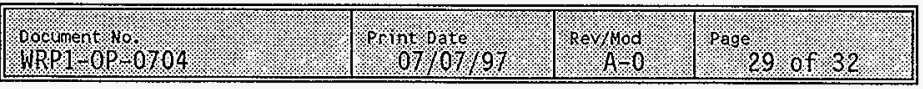




\section{WRAP 1 PLANT OPERATING PROCEDURE}

FIGURE 1 - CARRIAGE CONTROLLER

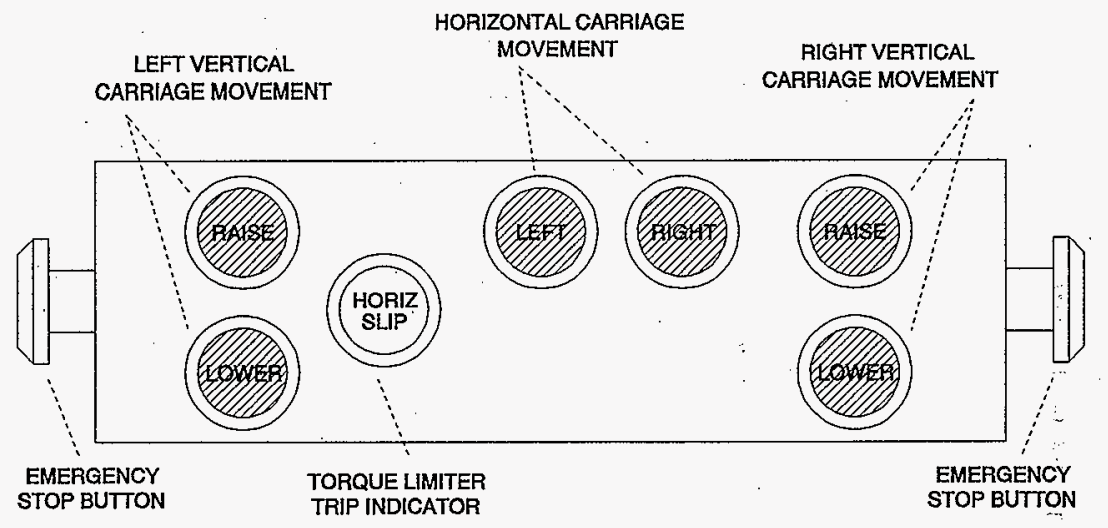

\begin{tabular}{|c|c|c|c|}
\hline 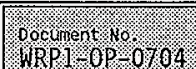 & 90.101079 & reypodi & Page \\
\hline
\end{tabular}

HNF-SD-W026-OTR-014, Rev, 0, Page OP-109 


\section{WRAP 1 PLANT OPERATING PROCEDURE}

FIGURE 2 - MASTER CONTROL PANEL.

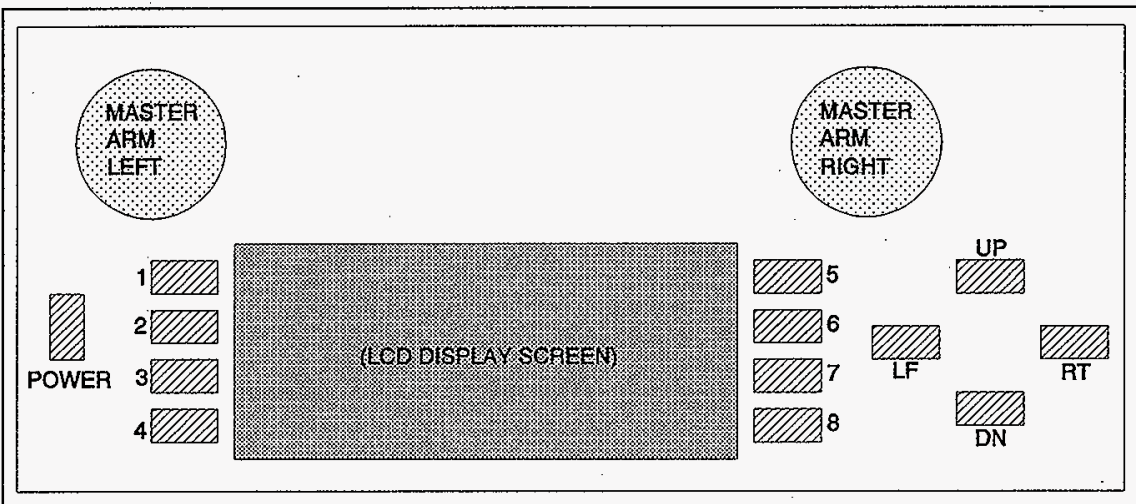

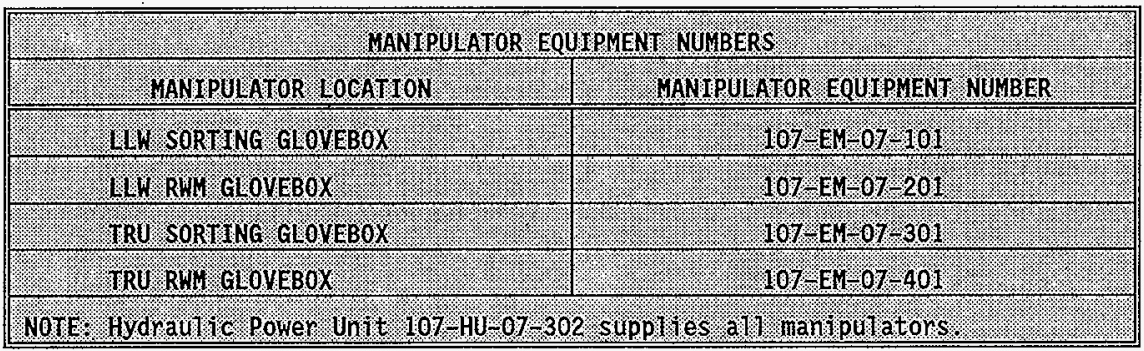

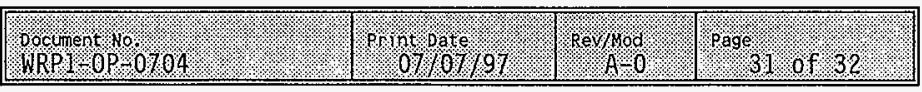


FIGURE 3 - MASTER ARM

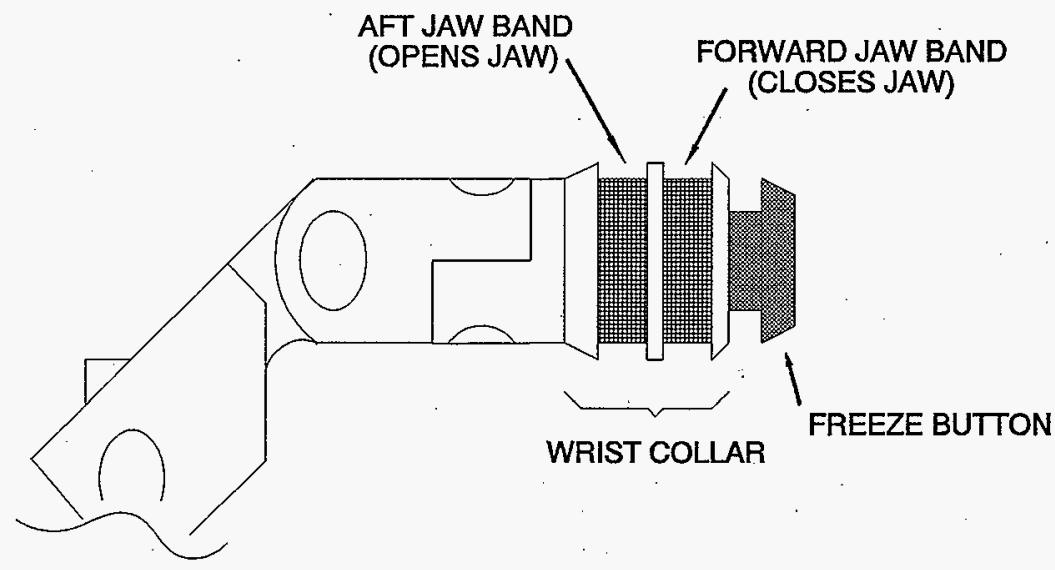

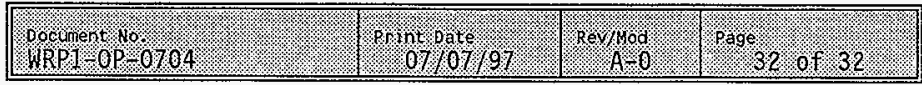




\title{
WRAP 1 PLANT OPERATING PROCEDURE
}

\author{
SYSTEW * PROCESS
}

\section{BAGLESS TRANSFER SYSTEM MANUAL OPERATION}

\section{SYSTEM DESCRIPTION}

NOTE - The bagless transfer system may also be referred to as the Drath. \& Schrader system in some plant documentation.

This procedure provides instructions for manuat operation of the Deutsche Babcock, Bagless Transfer System (200/300 Titer Drum Loading Ports).

The drum loading port is normally operated in automatic mode. However, if automatic operation is interrupted (inadvertently or otherwise), it may be necessary to enter manual mode to perform drum transfers or to return unit to proper sequencing. The drum loading port should be operated in manual only if absolutely necessary because the PLC ignores all limit switch signals. Major equipment damage is possible if improper operating sequence is used.

\section{REFERENCE DOCUMENTS}

ICF Kaiser Hanford Submittal No. 0676.A, Deutsche Babcock Technical and Operational Description, Double Lid Ports DF 674/560 and DF 742/660.

HSRCM-1, Hanford Site Radiological Control Manual.

\section{PRESTART CONDITIONS}

A11 personnel performing this procedure shall be qualified in accordance with WHC-CM-5-34, Section 1.8, "Training and Qualification", and on-the-job training.

Al1 personnel performing this procedure shatl have reviewed the applicable Radiation Work Permits (RWPs) and work under cognizance of Operations Management.

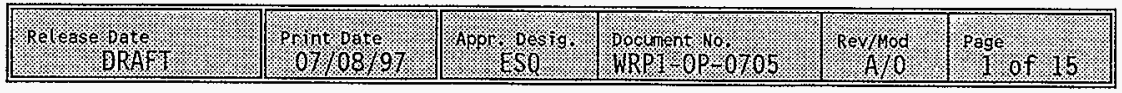




\section{SAFETY}

All potential hazards, such as lifting heavy containers or handling sharp objects present in waste containers, must be mitigated by protective equipment, procedures, and administrative controls to ensure acceptablerisk operating conditions.

\section{Radiological}

Failure to follow proper port sequencing as described in this procedure may result in contamination of drum port and surrounding area.

\section{v. TOOLS AND SUPPLIES}

None. 


\section{WRAP 1 PLANT OPERATING PROCEDURE}

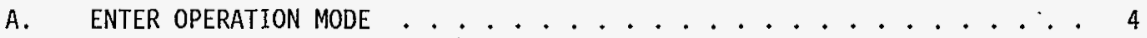

B. DETERMINE PORT STATUS .................. 6

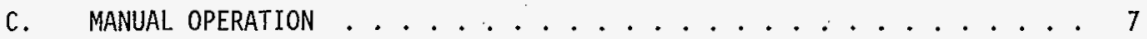

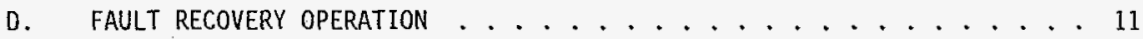

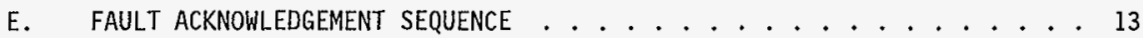

F. RESET PORT CONTROL PANEL EMERGENCY STOP . . . . . . . . . . . 13

\section{ATTACHMENTS}

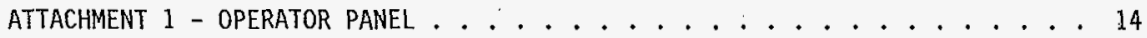




\section{PROCEDURE}

\section{CAUTION}

THE DRUM LOADING PORT SHOULD BE OPERATED IN MANUAL, ONLY IF ABSOLUTELY NECESSARY, BECAUSE THE PLC IGNORES ALL LIMIT SWITCH SIGNALS. MAJOR EQUIPMENT DAMAGE OR CONTAMINATION IS POSSIBLE IF IMPROPER OPERATING SEQUENCE IS USED.

NOTE - $\quad$ Administrative hold steps are identified by letters in parentheses at the left margin of the procedure step. The direction given in the procedure step must be satisfied before work continues.

(M) - Operations management shall approve continued operation

(R) - Radiological Control (RC) shall complete surveys or agree to permit continued operation.

\section{A. ENTER OPERATION MODE}

1. OBTAIN Operations Supervisor permission to enter MANUAL mode.

2. VERIFY Hoist chain is RAISED and will not interfere with port operation.

3. VERIFY manipulator arms will not interfere with port operation.

4. VERIFY other items wi11 not interfere with port operation.

5. ACCESS, on operator panel keyboard (Attachment 1), MANUAL mode, as follows:

a. PRESS STOP button (not EMERGENCY STOP).

b. VERIFY K5 key pilot light i11uminated.

c. PRESS K5 key.

d. VERIFY MAIN menu displayed.

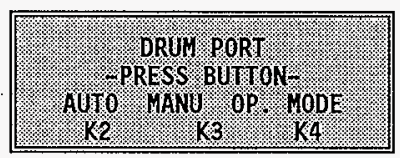

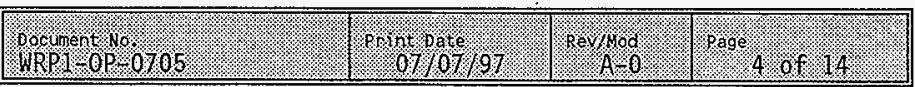




\section{WRAP 1 PLANT OPERATING PROCEDURE}

e. PRESS K3 key.

f. VERIFY disp.lay.

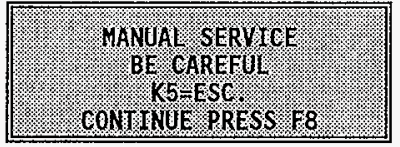

g. PRESS F8 key.

h. VERIFY display.

NOTE - Pressing ESC key at this point wi7l cancel entry to manua7.

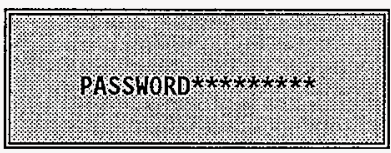

(M)

i. KEY IN password (Operations Supervisor).

j. PRESS ENTER key.

k. PRESS ESC key.

1. PRESS F8 key.

m. VERIFY display.

\begin{tabular}{|c|c|}
\hline . & 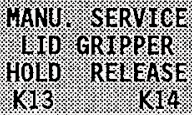 \\
\hline
\end{tabular}




\section{WRAP 1 PLANT OPERATING PROCEDURE}

\section{B. DETERMINE PORT STATUS}

1. DETERMINE port status as follows:

a. PRESS UP/DOWN arrow keys, as required, to select each function listed in Table 1 .

NOTE - Either K13 or K14 wil7 be STEADY ON, whi7e the other wi77 be FLASHING. Option 7isted above FLASHING indicator is present position/status of selected function.

b. OBSERVE K13 / K14 key at each function to determine position of corresponding actuator.

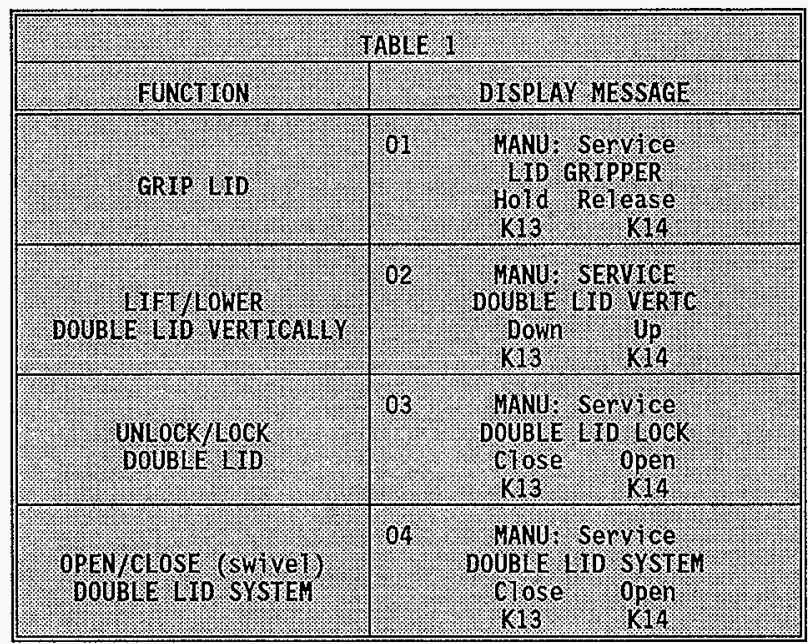

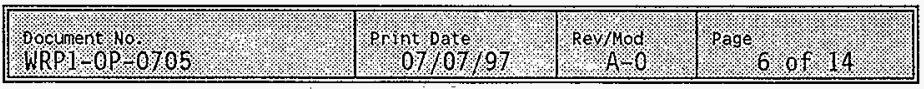




\section{WRAP 1 PLANT OPERATING PROCEDURE}

C. MANUAL OPERATION

\section{CAUTION}

FAILURE TO FOLLOW PROPER SEQUENCE MAY RESULT IN MAJOR EQUIPMENT DAMAGE OR CONTAMINATION OF EQUIPMENT AND SURROUNDING AREA.

1. ENSURE port in manual per Section VII.A.

2. VERIFY port trave? path clear of obstructions.

3. VERIFY port closed and locked as per port close sequence below.

4. VERIFY Lid Gripper in "ReTease" position.

5. ENSURE drum properly positioned under port.

6. OPEN port as follows:

a. GRIP Tid:

(1) IF the following LID GRIPPER menu with title is displayed,

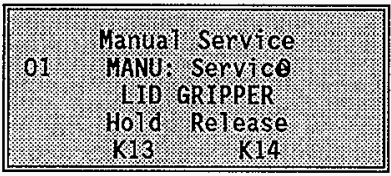

PRESS the DOWN arrow key to display the LID GRIPPER Menu without title.

(2) PRESS UP/DOWN arrow keys, as required, to access LID GRIPPER menu:

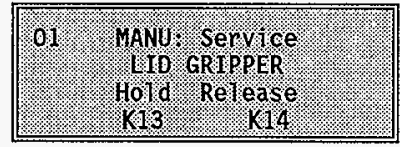

(3) PRESS K13 key.

(4) VERIFY K13 FLASHING, KI4 STEADY ON.

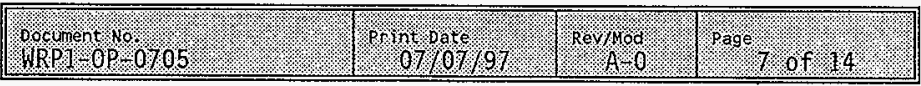




\section{WRAP 1 PLANT OPERATING PROCEDURE}

b. LIFT double lid:

(1) PRESS DOWN arrow key.

(2) VERIFY DOUBLE LID VERTC menu dispiayed.

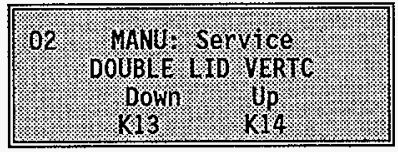

(3) PRESS K14 key.

(4) VERIFY K14 FLASHING, K13 STEADY ON.

(5) VERIFY lid retained against raised port door.

c. OPEN double lid lock:

(1) PRESS DOWN arrow key.

(2) VERIFY DOUBLE LID LOCK menu displayed.

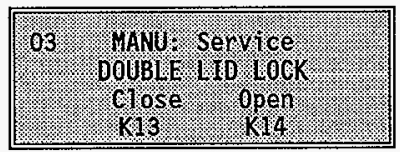

(3) PRESS K14 key.

(4) VERIFY K14 FLASHING, K13 STEADY ON.

NOTE - Upon completion of the following step, port will be rotated open and drum completely exposed to glovebox.

d. OPEN doubie lid system:

(1) PRESS DOWN arrow key.

(2) VERIFY DOUBLE LID SYSTEM menu displayed.

\begin{tabular}{|c|c|}
\hline . & 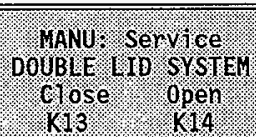 \\
\hline
\end{tabular}


(3) PRESS and HOLD K14 key.

NOTE - K14 key must be held until door reaches full OPEN pOsition.

(4) VERIFY K14 FLASHING, K13 STEADY ON.

(5). RELEASE K14 key.

7. CLOSE port as follows:

a. VERIFY no debris present on port.

b. CLOSE double lid system:

(1) PRESS UP/DOWN arrow keys, as required, to access DOUBLE LID SYSTEM menu.

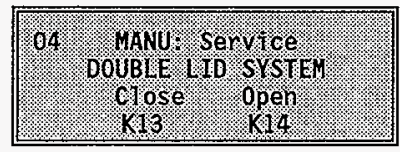

(2) PRESS and HOLD K13 key.

NOTE - K13 key must be held until door reaches full CLOSED position.

(3) VERIFY K13 FLASHING, K14 STEADY ON.

(4) RELEASE K13 key.

c. CLOSE double lid Tock:

(1) PRESS UP arrow key.

(2) VERIFY DOUBLE LID LOCK menu displayed.

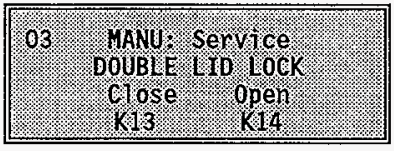

(3) PRESS K13 key.

(4) VERIFY K13 FLASHING, KI4 STEADY ON. 
d. LOWER double lid:

(1) PRESS UP arrow key.

(2) VERIFY DOUBLE LID VERTC menu displayed.

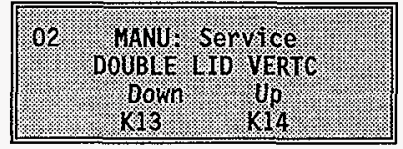

(3) PRESS K13 key.

(4) VERIFY K13 FLASHING, K14 STEADY ON.

e. RELEASE lid:

(1) PRESS UP arrow key.

(2) VERIFY LID GRIPPER menu disp 1 ayed.

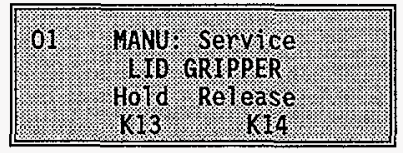

(3) PRESS K14 key.

(4) VERIFY K14 FLASHING, K13 STEADY ON.

8. WHEN desired,

THEN, RETURN to automatic operation as follows:

a. ENSURE port CLOSED per Step VII.C.7.

b. PRESS K5 key.

c. VERIFY MAIN menu displayed.

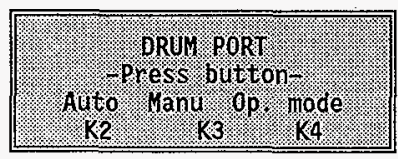

d. PRESS k2 key.

\begin{tabular}{|c|c|c|c|}
\hline 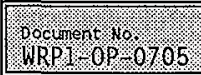 & \%) & Ko\% & 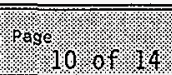 \\
\hline
\end{tabular}




\section{WRAP 1 PLANT OPERATING PROCEDURE}

NOTE - $\quad$ Port will not go into Automatic with low pressure or with emergency stop depressed.

9. IF AUTOMATIC mode cannot be accessed, THEN

a. VERIFY Bagless Transfer Port reguiator adjusted for 7 ( 6 to 8 ) bar.

b. VERIFY glovebox emergency stops NOT ACTIVATED.

\section{FAULT RECOVERY OPERATION}

NOTE - The typical fault would involve an emergency stop, a loss of power, or a loss of air pressure, stopping the port during the OPENING/CLOSING sequence. In MANUAL mode, the operator determines at what stage in the sequence the port stopped, manually operates the actuators to return to proper sequence, then CLOSES the port and returns to AUTOMATIC mode.

1. PLACE port in MANUAL mode, per Section VII.A.

2. DETERMINE port status per Section VII.B.

3. EVALUATE port for fault condition relative to proper OPENING/CLOSING sequence per Tables 2 and 3.

NOTE - Manual operating sequence must follow automatic sequences shown in Tables 2 and 3 (sequences assume design drum properly positioned).

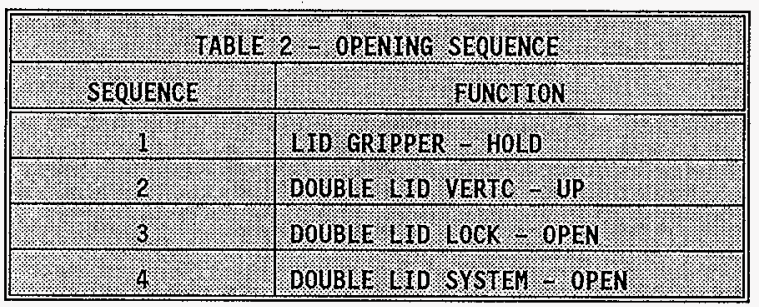

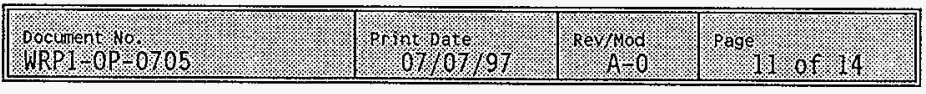




\section{WRAP 1 PLANT OPERATING PROCEDURE}

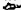

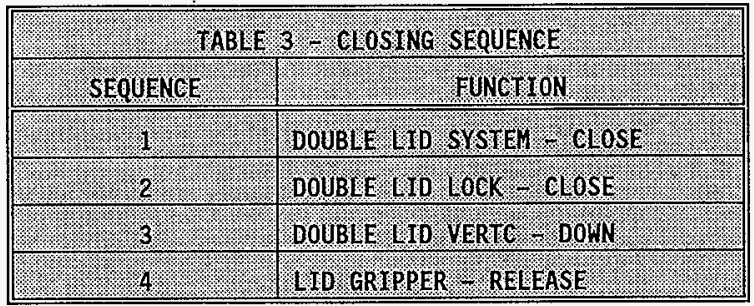

4. DETERMINE, based on observations, corrections required to return port to normal sequencing.

a. IF in doubt as to proper corrective actions, THEN:

(1) STOP work immediately.

(2) CONTACT Duty Operations Supervisor and COG Engineer for assistance.

5. CORRECT port sequencing fault using Section VII.C, as follows:

a. PRESS UP/DOWN arrow key, as applicable, to select desired function.

b. PRESS K13 / K14 key as applicable, to return corresponding actuator to proper position.

C. REPEAT Steps VII.D.5.a and VII.D.5.b, as required, to properly position each port actuator.

NOTE - Once port sequence has been interrupted, the port must be completely CLOSED before it can be returned to automatic.

6. WHEN port has been returned to correct sequencing, THEN:

a. DETERMINE port condition relative to CLOSING sequence per Step VII.C.7. AND continue sequence to full CLOSED position.

b. RETURN to automatic mode per step VII.C.8.

\begin{tabular}{|c|c|c|c|}
\hline 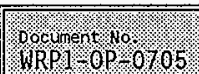 & 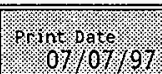 & 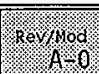 & page \\
\hline
\end{tabular}


E. FAULT ACKNOHLEDGEMENT SEQUENCE

NOTE - $\quad$ A77 faults must be acknowledged and cleared before operation can commence.

NOTE - An unacknowledged fault exists when either the "ACK" button pilot light is illuminated, or the Control Panel screen is flashing.

1. READ Fault message.

2. RECORD FauTt message.

3. PRESS "ACK" button.

4. IF "ACK" button pilot light is i1luminated AND screen is flashing, REPEAT steps VII.E.1 through VII.E.3.

5. VERIFY "ACK" button pilot light is NOT illuminated.

F. RESET PORT CONTROL PANEL EMERGENCY STOP

1. TWIST emergency stop button clockwise.

2. ACKNOWLEDGE a11 faults.

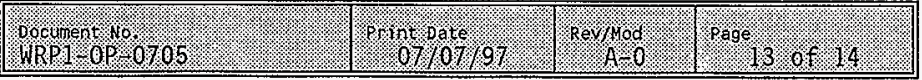


ATTACHMENT 1 - OPERATOR PANEL
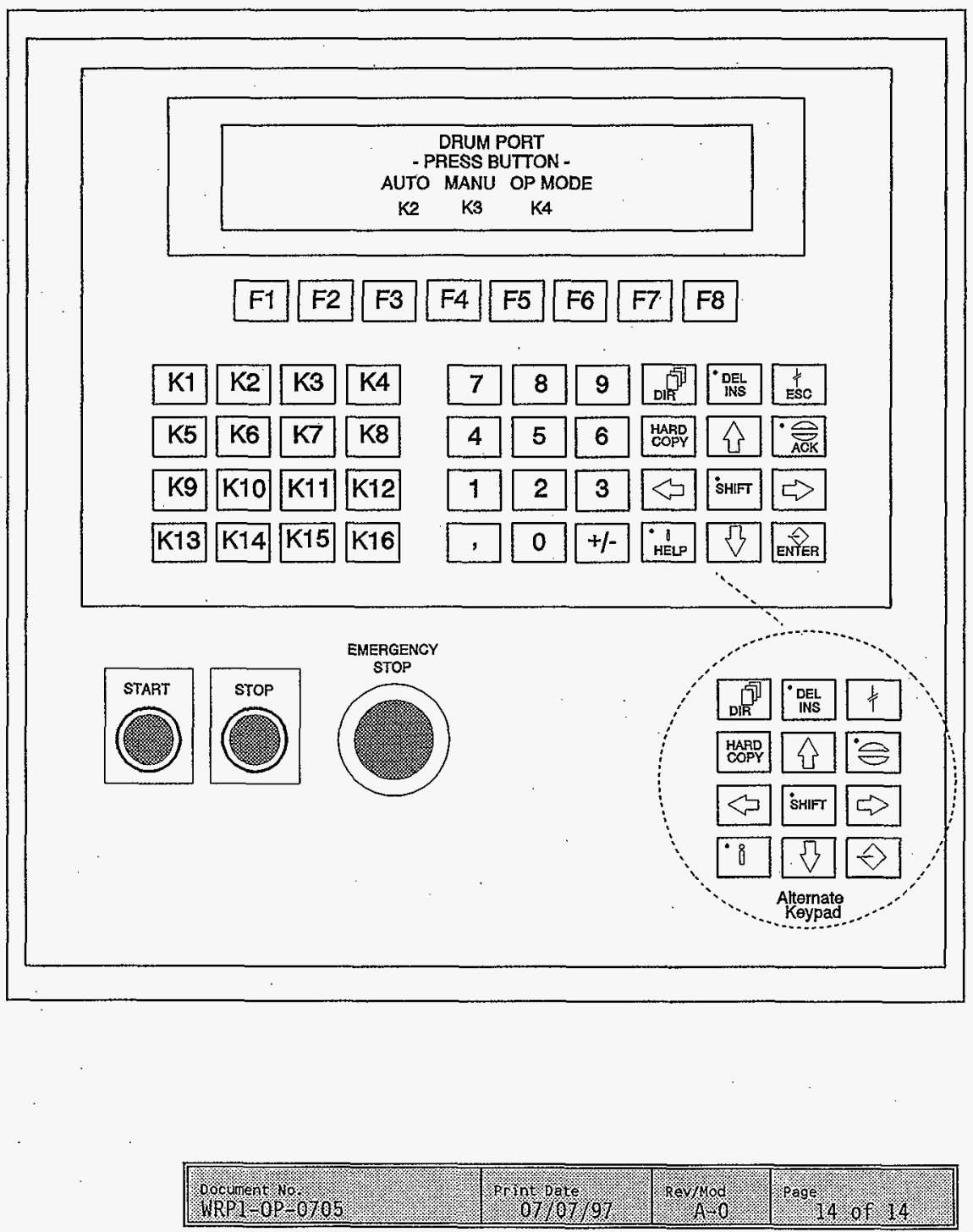


\section{WRAP 1 PLANT OPERATING PROCEDURE}

SYSTELU ENVTRONHENTAL

\section{VENT AND DRAIN AEROSOL CANS}

\section{SYSTEM DESCRIPTION}

This procedure details the steps required to properly vent and drain aerosol cans within each of the Restricted Waste Management (RWM) gloveboxes.

The device consists of a punch assembly, a coalescing filter, an activated carbon filter, one and two liter poly bottles, a pressure relief valve, and a frame bolted to the floor of the glovebox.

The punch assembly holds the can in an inverted position, locks it in place with a lock knob, punctures the can with a puncture pin, and drains the liquid contents into a one liter poly bottle.

Gasses leaving the can pass through coalescing and activated carbon filters and any foam or overflow liquids are collected in the two liter poly bottle. The coalescing filter collects $99.9 \%$ of 1 iquid particulate from the propellant released when an aerosol can is vented. Once through the coalescing filter, the gasses pass through the activated carbon filter where absorption of organic compounds takes place.

The relief valve is set at 10 psi to limit the pressure generated during the venting process.

Aerosol can contents will be treated using one of two processing methods. If incoming waste streams contain several cans with similar known contents, they will be batched, punctured, and packaged as a compatible waste stream. Collection containers containing Dry Zorb absorbent will be staged inside the glovebox for this purpose. After treatment completion of the batch, the collected compatible Tiquids will be packaged according to WRPl-0P-0717, LLW/RWM Process Glovebox Operation. Punctured cans and collected Tiquid bottles will only be staged long enough to complete processing of the batch. For unique, unknown, or incompatible aerosol can contents, the can will be punctured and packaged on a case-by-case basis in order to prevent reaction between incompatible wastes. These wastes wi1l be packaged immediately or returned to the storage carousel until more similar wastes can be collected and efficiently packaged or until sample results are available.

\begin{tabular}{|c|c|c|c|c|c|}
\hline recase pare & 00707.9 & 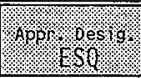 & 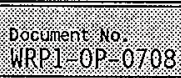 & Rexpod & prage $10 \%$ \\
\hline
\end{tabular}




\section{WRAP 1 PLANT OPERATING PROCEDURE}

\section{REFERENCE DOCUMENTS}

WRP1-0P-0710, Waste Sampling

HSRCM-1, Hanford Site Radiological Control Manua?

HNF-CM-5-34, Solid Waste Disposal Operations Administration

HNF-CM-5-36, SWD Internal Requirements

HNF-EP-0063, Hanford Site Solid Waste Acceptance Criteria

\section{PRESTART CONDITIONS}

Personnel performing this procedure shall be qualified in accordance with WHC-CM-5-34, Section 1.8, Training Plan, and on-the-job training.

Prior to operation, a pre-job safety meeting will be conducted to discuss safety issues.

\section{SAFETY}

Consult with Operations Management, Industrial Hygiene, and refer to Job Hazard Analysis (JHA) and Radiological Work Permit (RWP) to determine required PPE for handling hazardous wastes and the hazards of the can being vented and drained.

\section{TOOLS AND SUPPLIES}

Aerosol Container Puncture Unit

1 Liter, Narrow Mouth, Poly Bottles with 38-430 Threads

2 Liter, Narrow Mouth, Poly Bottles with 38-430 Threads

Dry Zorb Absorbent in convenient container(s)

V. TABLE OF CONTENTS

PAGE

A. STAGING COLLECTION CONTAINERS AND ABSORBENT . . . . . . . . 3

B. VENT AND DRAIN SINGLE AEROSOL CAN ............ 3

C. VENT AND DRAIN BATCHED, COMPATIBLE AEROSOL CANS . . . . . 6

\begin{tabular}{|c|c|c|c|}
\hline oocument & 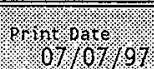 & Re\%pod & 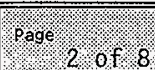 \\
\hline
\end{tabular}




\section{PROCEDURE}

NOTE - $\quad(P C S)$ in the left margin indicates an action performed by the Plant Control System.

\section{A. STAGING COLLECTION CONTAINERS AND ABSORBENT}

1. STAGE 1 liter poly bottles inside the RWM glovebox as directed by the Sample/Treatment Director.

a. STAGE empty 1 liter poly botties.

b. STAGE 1 liter poly bottles, each containing 150 grams Dry Zorb absorbent.

2. STAGE convenient container(s) of bulk Dry Zorb absorbent inside the RWM glovebox as directed by the Sample/Treatment Director.

\section{B. VENT AND DRAIN SINGLE AEROSOL CAN}

1. ENSURE Aerosol Container Puncture Unit is securely bolted to the glovebox floor.

2. ENSURE an empty two liter poly overflow bottle is securely attached to the bottom of the filter assembly.

NOTE - The \#2 in parentheses, (\#2), refers to the second Collection Container which becomes necessary if Collection Container \#1 is of insufficient volume to hold the contents of the two liter overflow bott7e.

6 3. INSERT Collection Container \#1 (\#2) into glovebox according to instructions in WRPI-0P-0710, Waste Sampling, OR

RETRIEVE a staged I liter poly bottle from inside the RWM glovebox to serve as collection Container \#1 (\#2). This retrieved container may be either of the following:

a. Empty 1 Titer poly bottle if the contents of the can are:

(1) Unknown and require sampling $\mathrm{OR}$,

(2) Not compatible with Dry Zorb OR,

(3) In need of some other treatment prior to absorption.

b. 1 liter poly bottle containing 150 grams Dry Zorb absorbent if:

(1) The contents are known to be compatible with Dry Zorb absorbent AND

(2) No other treatment is required prior to absorption. 


\section{WRAP 1 PLANT OPERATING PROCEDURE}

4. ATTACH a pre-printed barcode label to collection Container \#1 (\#2).

5. IF the contents of the aerosol can and the type and amount of absorbent material required are known to be other than Dry Zorb,

THEN PLACE the appropriate amount and type of absorbent material into Collection Container \#1 (\#2), as determined by the Sample/Treatment Director.

6. IF the contents of the aerosol can are NOT known,

THEN the contents of Collection Container \#1 (\#2), after collection of the contents of the aerosol can, will have to be sampled per WRP1-0P-0710, GLOVEBOX WASTE SAMPLING.

7. SCAN to associate Collection Container \#1 (\#2) with the packet that the aerosol can came from (refer to Table 1 below) as follows:

a. Location bar code on glovebox wall.

b. Bar code of packet that aerosol can came from.

c. Collection Container \#1 (\#2) bar code.

d. Termination bar code on glovebox wall.

Table 1

\begin{tabular}{|c|c|c|}
\hline . & PORTABUE SOANHER & 100AToN \\
\hline TRU RWM & $12-N E-303$ & TRURWSAMPL \\
\hline LLW RWM & $12-N E-313$ & LL.WRWSAMPL \\
\hline
\end{tabular}

PCS PCS sends message to DMS associating the original packet with Collection Container \#1 (\#2).

8. THREAD Collection Container \#1 onto bottom of aerosol can punch assembly until tight.

9. LOOSEN lock knob and LIFT sliding top plate to open housing sleeve.

10. REMOVE cap and from top of aerosol can.

NOTE - Large diameter cans may require removal of the plastic housing sleeve from inside the Puncture Unit housing.

11. INSERT aerosol can, NOZZLE END DOWN, into housing sleeve (or housing in the case of a large diameter can) so that shoulder of can rests on gasket.

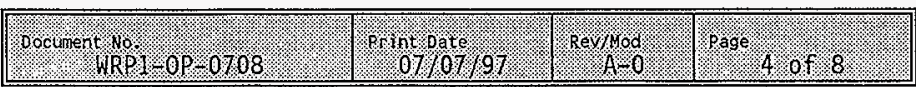




\section{CAUTION}

FAILURE TO ENGAGE SLIDING TOP PLATE AGAINST CAN BEING PUNCTURED AND SECURELY TIGHTEN LOCK KNOB, MAY CAUSE BREAKAGE OF THE PUNCTURE PIN.

12. LOWER sliding top plate and FIRMLY engage against can.

13. TIGHTEN Jock knob.

NOTE - When puncturing full and half-full cans, best results are obtained by pumping the handle several times when puncturing, to moderate evacuation pressure.

14. PUSH handle down firmiy and release.

15. WAIT 20 seconds before removing punctured aerosol can to allow residual liquids to drain.

\section{CAUTION}

FAILURE TO LIMIT LIQUID VOLUME OF A COLLECTION CONTAINER TO ONE HALF TO TWO THIRDS FULL; MAY LEAVE INSUFFICIENT SPACE IN THE COLLECTION CONTAINER FOR LATER TREATMENT (ABSORPTION, NEUTRALIZATION, ETC.) OF THE CONTENTS.

16. IF the volume of liquid drained from the aerosol can exceeds one half to two thirds the capacity of Collection Container \#1, THEN REPEAT Steps VII.B.3 through VII.B.7 to prepare Collection Container \#2 to accept the excessive fluid.

17. REMOVE empty can from punch assembiy and LOADOUT in accordance with Procedure WRP1-0P-0717, LLW/RWM Glovebox Operation.

18. REMOVE Collection Container \#1 from bottom of aerosol can punch assembly.

19. IF there was any overflow of foam or liquid into the two liter bottTe,

THEN PERFORM the following:

a. REMOVE the two liter bottle from the bottom of the filter assembly.

b. POUR the liquid from the two liter bottle back into ColTection Container \#I if there is room for it without exceeding one half to two thirds level, OR into a Collection Container \#2 if there is not.

c. RETURN the two liter bottle to its normal position under the bottom of the filter assembly.

\begin{tabular}{|c|c|c|c|}
\hline 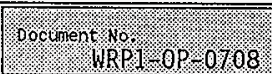 & \%proter & ReV/od & page $/ 40 \% 18$ \\
\hline
\end{tabular}


20. PLACE cap(s) on Collection Container \#1 and any other one liter bottle used to contain the overflow.

21. SEAt containe Tid(s) with tapex f Nerero.

22. ENSURE barcode 1 abe 1 is intact and PLACE any other required labels on container(s).

23. PLACE Collection Container \#1 (and Collection Container \#2, if used) on Transfer Stand and LOABOUT in accordance with Procedure WRP1-0P-0717, LLW/RWM Glovebox 0peration.

C. VENT AND DRAIN BATCHED, COMPATIBLE AEROSOL CANS

1. ENSURE Aerosol Container Puncture Unit is securely bolted to the glovebox floor.

2. ENSURE an empty two liter poly overflow bottle is securely attached to the bottom of the filter assembly.

3. INSERT Collection Container \#1 (\#2) into glovebox according to instructions in WRP1-OP-0710, Waste Sampling, OR

RETRIEVE a staged 1 1iter poly bottle from inside the RWM glovebox to serve as Collection Container \#1 (\#2). This retrieved container may be either of the following:

a. Empty I liter poly bottle if the contents of the can are:

(1) Not compatible with Dry Zorb 0R,

(2) In need of some other treatment prior to absorption.

b. 1 liter poly bottle containing 150 grams Dry Zorb absorbent if:

(1) The contents are known to be compatible with Dry Zorb absorbent AND

(2) No other treatment is required prior to absorption.

4. ATTACH a pre-printed barcode label to Collection Container \#1 (\#2).

5. IF the contents of the aerosol. cans require absorbent material other than Dry Zorb, THEN PLACE the appropriate amount and type of absorbent material into collection Container \#1 (\#2), as determined by the Sample/Treatment Director. 


\section{WRAP 1 PLANT OPERATING PROCEDURE}

6. SCAN to associate Collection Container \#1 (\#2) with the packet that EACH aerosol can came from (refer to Table 1 below) as follows:

a. Location bar code on glovebox wall.

b. Bar code of packet that aerosol can came from.

c. Collection Container \#1 (\#2) bar code.

d. Termination bar code on glovebox wal1.

Table 1

\begin{tabular}{|c|c|c|}
\hline GLOV EBOA & PORTABVY SOANER & GOCATION \\
\hline TRU RWM & $12-N E-303$ & TRURWSAMPL \\
\hline LLW RHM & 12-NE-313 & LLWRWSAMPL \\
\hline
\end{tabular}

PCS PCS sends message to DMS associating each original packet with Collection Container \#1.

7. LOOSEN lock knob and LIFT sliding top plate to open housing sleeve.

8. REMOVE cap from top of aerosol can.

NOTE - Large diameter cans may require removal of the plastic housing sleeve from inside the Puncture Unit housing.

9. INSERT aerosol can, NOZZLE END DOWN, into housing sleeve (or housing in the case of a large diameter can) so that shoulder of can rests on gasket.

\section{CAUTION}

FAILURE TO ENGAGE SLIDING TOP PLATE AGAINST CAN BEING PUNCTURED AND SECURELY TIGHTEN LOCK KNOB, MAY CAUSE BREAKAGE OF THE PUNCTURE PIN.

10. LOWER sliding top plate and FIRMLY engage against end of can.

11. TIGHTEN lock knob.

NOTE - When puncturing full and half-full cans, best results are obtained by pumping the handle several times when puncturing, to moderate evacuation pressure.

12. PUSH handle down firmly and release.

13. WAIT 20 seconds before removing punctured aerosol can to allow residual 7 iquids to drain.

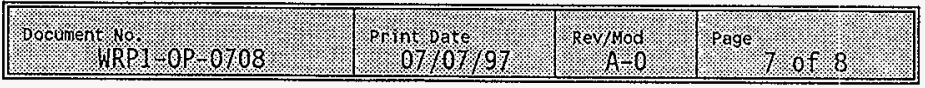

HNF-SD-W026-0TR-014, Rev. 0, Page OP-132 


\section{WRAP 1 PLANT OPERATING PROCEDURE}

\section{CAUTION}

FAILURE TO LIMIT LIQUID VOLUME OF A COLLECTION CONTAINER TO ONE HALF TO TWO THIRDS FULL, MAY LEAVE INSUFFICIENT SPACE IN THE COLLECTION CONTAINER FOR LATER TREATMENT (ABSORPTION, NEUTRALIZATION, ETC.) OF THE CONTENTS.

14. IF the volume of liquid drained from the aerosol can exceeds one half to two thirds the capacity of Collection Container \#1, THEN REPEAT Steps VII.C.3 through VII.C.6 to prepare Collection Container \#2 to accept the excessive fluid.

15. REMOVE empty can from punch assembly and STAGE in glovebox.

16. REPEAT steps VII.C.7 through VII.C.15 for each remaining batched, compatible aerosol can until collection Container \#1 is one half to two thirds full.

17. IF Collection Container \#1 is one half to two thirds full, and there are more batched, compatible aerosol cans to drain, THEN REPLACE Collection Container \#1 with Collection Container $\# 2$, and so on until all aerosol cans are vented and drained.

18. REMOVE Collection Container \#1 from bottom of aerosol can punch assembly.

19. IF there was any overflow of foam or Tiquid into the two liter bottle,

THEN PERFORM the following:

a. REMOVE the two liter bottle from the bottom of the filter assembly.

b. POUR the liquid from the two liter bottle back into Collection Container \#1 if there is room for it without exceeding two thirds level, oR into a Collection Container \#2 (or \#3, \#4, etc.) if there is not.

c. RETURN the two liter bottle to its normal position under the bottom of the filter assembly.

20. ENSURE a11 Collection Containers have their lids tightly in place.

21. SEAL all Collection Container lids with tape.

22. ENSURE barcode labels are intact and PLACE any other required labels on Collection Containers.

23. LOADOUT empty cans in accordance with WRPI-OP-0717, LLW/RWM Glovebox Operation.

24. LOADOUT all Collection Containers in accordance with WRP1-0P-0717, LLW/RWM Glovebox Operation. 
HNF-SD-WO26-OTR-O14 REV.O

WRAP I PLANT OPERATINE PROEEDURE

SYSIEH: PROGESSS

\section{GLOVEBOX WASTE SAMPLING}

\section{SYSTEM DESCRIPTION}

This procedure defines the approach that is used to sample WRAP 1 process area glovebox waste under RCRA protocol sampling requirements.

WRAP Operation's responsibility is to sample for Iaboratory analysis in accordance with this procedure. Sample data shall be recorded using either the Data Management System, DMS Screen DMSS050I, which is the primary method of recording data, or on Attachment 2, Lab Sample Acquisition which is available as a backup method.

The Sample/Treatment Director shall provide recommendations on the specific method to be used, primarily via Attachment 1, Laboratory Sample Anaiysis Request, as appropriate. The Sample/Treatment Director shall also be responsible for ensuring that the data collected by Operations personnel either in DMS or on Attachment 2, Lab Sample Acquisition, is transferred to the Facility Sample $\log$ as required.

Additionally, this procedure may also be used to collect samples that may undergo field screening within the glovebox prior to sampling for laboratory analys is.

This procedure will also provide guidance for transferring samples and other various items in and out of the gloveboxes through the use of the Sample Transfer Container (STC). This will include mounting and unmounting the container, item transfer, and a] 7 associated DMS interface requirements.

\section{REFERENCE DOCUMENTS}

HSRCM-1, Hanford Site Radiological Control Manual

WHC-CM-5-34, Solid Waste Division Operations Administration

WHC-CM-5-36, SWD Internal Requirements, Chapter 7-5, Environmental Compliance 
III. PRESTART CONDITIONS

Personnel performing this procedure shall be qualified in accordance with WHC-CM-5-34, Section 1.8, "Training Plan", and on-the-job training.

\section{SAFETY}

Appropriate protective clothing will be worn depending on the sampling activity involved. The clothing required will be identified on the RWP and/or during the pre-job evaluation by the operations personnel, operations Management, or Industrial Safety and Hygiene, as appropriate.

Anticipated radiological conditions shall be evaluated prior to starting work through the RWP and ALARA Management Worksheet (AMW), if applicable.

\section{TOOLS AND SUPPLIES (As Needed)}

Sampling equipment (i.e., glass tube or pipet sampler, scoop, hand auger, etc.)

Sample container(s)

Evidence tape

Tape

Cooler

Plastic sheets

Plastic bags

Camera

Other tools, supplies, or protective clothing as defined by the pre-job evaluation.

\begin{tabular}{|c|c|c|c|}
\hline 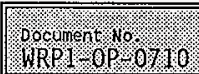 & provt pate & weyod & page 2 \\
\hline
\end{tabular}




\section{TABLE OF CONTENTS}

I. SYSTEM DESCRIPTION

II. REFERENCE DOCUMENTS

III. PRESTART CONDITIONS

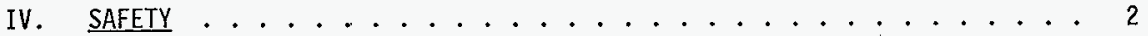

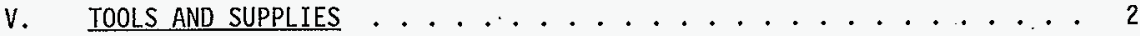

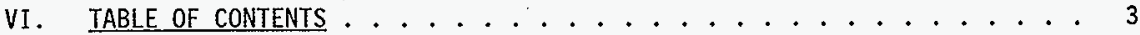

VII. PROCEDURE . . . . . . . . . . . . . . . . 4

A. FIELD SCREENING IN RWM GLOVEBOXES ............ 4

B. PREPARING TO SAMPLE ............ . . . 6

c. LOAD EMPTY SAMPLE BOTTLES INTO GLOVEBOX ........... 8

D. STC OPERATIONS ................ . . 8

1. Attach STC to Glovebox ............. 8

2: $\quad$ open STC .................. . . 9

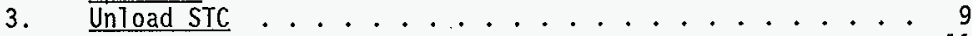

E. SAMPLING LIQUIDS . . . . . . . . . . 11

F. SAMPLING SEMI-SOLID/SOLID WASTE MATRICES ......... 12

a. Scoop Sampler . . . . . . . . . . . . . . . 13

b. Hand Auger Sampler . . . . . . . . . . . . . 14

c. Snip and Wipe Method ............. . 15

G. FINAL SAMPLE PREPARATION AND LOAD OUT ............ 15

H. SAMPLE ACTIVITY LOGBOOKS AND DMS ............ 19

I. SAMPLING ACTIVITIES PHOTO ALBUM ............. 19

ATTACHMENT 1 - LABORATORY SAMPLE ANALYSIS REQUEST . . . . . . . . . . 20

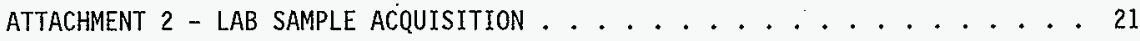

ATTACHMENT 3 - SAMPLE AND TREATMENT LOOKUP TABLES . . . . . . . . . 22

\begin{tabular}{|c|c|c|c|}
\hline 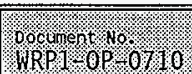 & 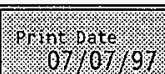 & kerorod & 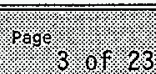 \\
\hline
\end{tabular}




\section{PROCEDURE}

NOTE - Administrative hold steps are identified by initials in parenthesis at the left margin of the procedure step. The direction given in the procedure step must be satisfied before work continues.

(M) - Operations management shati approve continued operation.

(R) - Radiological Control shal7 complete surveys or agree to permit continued operation.

NOTE - The Sample/Treatment Director has the authority to decide whether any given quantity of waste should be Field Screened or Sampled.

\section{A. FIELD SCREENING IN RWM GLOVEBOXES}

NOTE - A supply of bottles, dishes, and other supplies to be used in field screening will be kept in, or loaded into the RWM GTovebox as needed.

NOTE -

DMS Screen DMSS0508 (Samp7e Labe7s) is accessed from Screen DMSS0504 (Laboratory Sample Analysis Request) which can be accessed from DMSS0503 (Field Screening).

NOTE - DMS records the sample/packet relationship.

NOTE - This Section is used when material from a packet or collection container located at an RWM Glovebox sorting location requires field screening.

1. 1. DISPLAY screen DMSS0503, Field Screening, by selecting the "Field Screening" button on the RWM Waste Repackaging DMS Screen (DMSSO322 for LLW RWM and DMSS0342 for TRU RWM).

NOTE - Screen DMSSO503 displays a 7ist of a77 packets and collection containers located at the RWM Sorting Location.

2. SELECT the PIN of the item to be sampled from the 'list.

3. SELECT "Next Sample".

NOTE - DMS, at this point assigns a Screening ID.

4. CONDUCT field screening in accordance with the information provided on DMS Screen DMSS0503 and any additional guidance provided by the Sample Treatment Director.

\begin{tabular}{|c|c|c|c|}
\hline 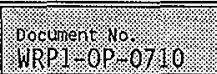 & $907 \% 10 \% 197$ & Rev/1od & page $4.4 \%$ \\
\hline
\end{tabular}


5. ENTER the results of the field screening on screen DMSSO503, including the following parameters as a minimum:

- Sampling Method

- Sample Volume

- General Comments

- Ana?ysis performed

- Results

6. ENTER a signature password and commit the data.

7. IF the Sample/Treatment Director determines a sample is required based on the results of the field screening, THEN the Sampling/Treatment Director shall perform the following:

a. PREPARE a sample analysis request using screen DMSS0504, Laboratory Sample AnaTys is Request, or Attachment 1, Laboratory Sample Analysis Request.

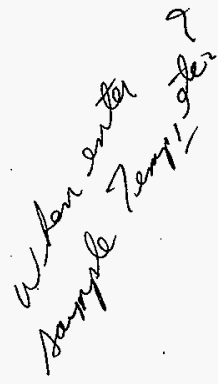

b. PRINT (in the Sample Management Office) the sample bottle labels using screen DMSS0508, Sample Bottle Labels.

8. IF another screening sample is required, THEN SELECT the NEXT SAMPLE button on the DMSS0503 screen to re-display the list of packets and collection containers at the RWM sampling location.

9. IF no other screening sample is required, THEN return to screen DMSS0322.

10. WHEN the field screening analyses are complete, PACKAGE the field screened material with the original material if possible.

NOTE: If it is necessary to package a samp7e separately, a new packet will be created and 7 abeled.

11. IF sample cannot be combined with original material, THEN perform the following:

a. SCAN the barcode for the location.

b. SCAN the barcode for the original packet.

c. LABEL the new packet.

d. SCAN the barcode for the new packet(s).

e. SCAN the end barcode.

f. SCAN the yes barcode.

g. ENTER descriptions of the new packets on the DMSS0322 Screen.

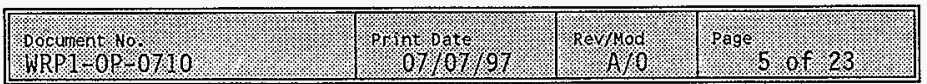




\section{WRAP 1 PLANT OPERATING PROCEDURE}

B. PREPARING TO SAMPLE

\section{WARNING}

APPROPRIATE EYE, HAND, AND PROTECTION CLOTHING AS SPECIFIED BY THE RWP, JHA OR PRE-JOB EVALUATION SHALL BE WORN DURING SAMPLING.

NOTE 1 - The appropriate methods for sample collection, required volumes, and sample containers are determined prior to sampling and recorded in DMS or on Attachment 1, Laboratory Sample Analysis Request. Sampling is performed on the following waste matrices:

- $\quad$ s7udges

- Soils, sand, silt, other particulates

- Free liquids

- Sorbents (stained or saturated only).

Other matrices can be sampled if they fa7l under matrices identified in SW-846, specific sampling and ana7ysis plan, waste analysis plan, or work package.

If discrepant articles or conditions are encountered that cannot be identified and resolved with resources available, contact the Operations Management for assistance.

NOTE 2 - Sampling documentation (including photographs) is collected throughout the sampling activity, as appropriate.

1. ENSURE the Sample/Treatment Director has performed one of the following:

- PREPARE Attachment 1, Laboratory Sample Analysis Request. - UPDATE DMS Screen DMSS0504 Laboratory Sample AnaTysis Request.

2. OBTAIN appropriate sampling equipment, sample container(s), camera, etc.

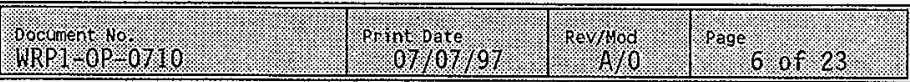




\section{CAUTION}

SAMPLE CONTAINERS MAY NOT BE OPENED PRIOR TO THEIR INTENDED USE. DOING SO MAY NEGATE THE PEDIGREE OF THE CONTAINER. DO NOT USE PREVIOUSLY OPENED CONTAINERS FOR RCRA PROTOCOL SAMPLING ACTIVITIES.

3. ENSURE empty sample containers have been maintained in a compliant manner as follows:

- Kept under lock and key (e.g., Tocked storage cabinet, locked storeroom, etc.), with limited access. A custodian and alternate(s) shall be designated by Operations.

- The outside of each case must be legibly initialed and dated by the individual opening that case. Pedigree papers for each case should be checked against the contents and any anomalies noted at that time in the logbook. Pedigree papers are kept on file.

4. ENSURE the following information is recorded in the Empty Sample Container Logbook for each sample container:

- Custodian of container(s) being removed from secured area

- Date removed

- Lot number

- Container ID number

- Size and Type

- Sampling location

- Used/Not used

- Comments (as required)

5. PRINT the sample bottle barcode labels using screen DMSS0508.

6. LABEL the empty sample bottle(s) with the barcode label(s). 
c. LOAD EMPTY SAMPLE BOTTLES INTO GLOVEBOX

1. ENSURE the sample bottle is properly labeled.

2. OPEN the door on the Consumable Materials Port.

3. INSERT a properly sized bottle into the Consumable Materials Port, pushing the bottle already in the Port all the way through to the interior of the glovebox.

4. INSERT another properly sized bottle into the Consumable Materials Port, pushing the desired sample bottle(s) into the interior of the glovebox.

D. STC OPERATIONS

1. Attach STC to GTovebox

a. REMOVE STC from transfer pig (if applicable).

b. ENSURE port door is CLOSED and LOCKED:

(1) Door latch CLOSED (down).

(2) Port door latch locking pin dropped into locking hole.

NOTE - During rotation, the STC is 7ocked and sealed to the port flange, and the STC cover is unlocked from the STC and locked and sealed to the cell port.

c. MATE STC flange to cell flange, with locking bayonets aligned to cell flange openings and ROTATE STC clockwise, $30^{\circ}$.

d. ENSURE STC fully engages port as follows:

(1) Visually CHECK mounting flanges are fully engaged.

(2) TIGHTEN STC with STC Tool as necessary.

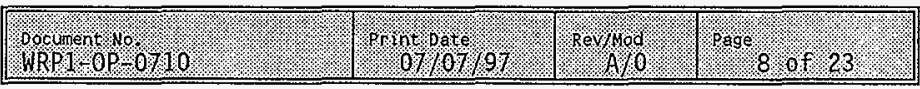




\section{WRAP 1 PLANT OPERATING PROCEDURE}

e. SCAN STC to glovebox location with the appropriate portable scanner (refer to Table 1 below) as follows:

(1) Location barcode on glovebox wall.

(2) STC barcode.

(3) End barcode on glovebox wall.

(4) Yes barcode on glovebox wall.

Table 1

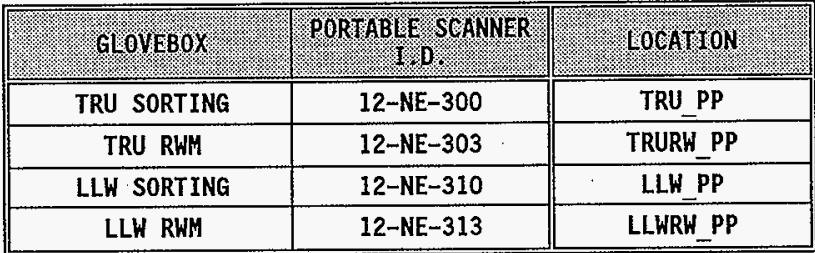

f. VERIFY OIU display on Sample Menu, "Purge Port Canister Present".

2. Open STC

NOTE - This Section Assumes that the STC is already properly mated to the Glovebox per Section VII.D.

a. PULL port door latch locking pin and TURN counter-clockwise, $30^{\circ}$.

b. MOVE port door latch to OPEN (UP).

c. OPEN port door.

3. Unload STC

a. INSPECT glovebox sampling area for objects or conditions that could puncture, rip, cut, or could otherwise injure or harm sampling personnel.

b. REQUEST Radiological Control ( $R C$ ) coverage to the extent indicated on Attachment 1, Laboratory Sample Analysis Request. 


\section{WRAP 1 PLANT OPERATING PROCEDURE}

c. UNLOAD sample container, or applicable item, into the glovebox, using gloves.

(1) If sample containers needing treatment are being un Toaded,

THEN REFER to WRP1-0P-0717, LLW/RWM GLOVEBOX

OPERATION, Section titled, "REMOVE SAMPLE BOTTLES FROM STC FOR TREATMENT".

4. Load Sample in STC

a. SELECT "Loadout Samples" on OIU.

b. SCAN barcodes (refer to Table 2 below) as follows:

(1) Location barcode on glovebox wall.

(2) Sample Bottle barcode.

(3) End barcode on glovebox watl.

(4) Yes barcode on glovebox wall.

Table 2

\begin{tabular}{|c|c|c|}
\hline . G. & PORTABIE SEANNER & WOGT ON \\
\hline TRU SORTING & $12-\mathrm{NE}-300$ & TRU_SORT \\
\hline TRU RWM & 12-NE-303 & TRURWSAMPL \\
\hline LLW SORTING & $12-N E-310$ & LLW SORT \\
\hline LLH RWM & 12-NE-313 & LLWRHSAMPL \\
\hline
\end{tabular}


$m$

E. SAMPLING LIQUIDS

NOTE 1 - Liquids may be sampled using several methods - pipet, glass tube, etc. The determination of which method and sample containers to use is made prior to sampling and is defined on Attachment 2, Laboratory Sample Analysis Request $O R$ in the DMS.

NOTE 2 - The sample container should be placed over the waste container to minimize potential contamination spread as much as practical.

1. SCAN barcodes (refer to Table 2 below) as follows:

a. Location barcode on glovebox wall.

b. Sample Bottle barcode.

c. IF and only if, sampling in an RWM Glovebox; THEN SCAN the packet or collection container being sampled.

d. End barcode on glovebox wall.

e. Yes barcode on glovebox wall.

Table 2

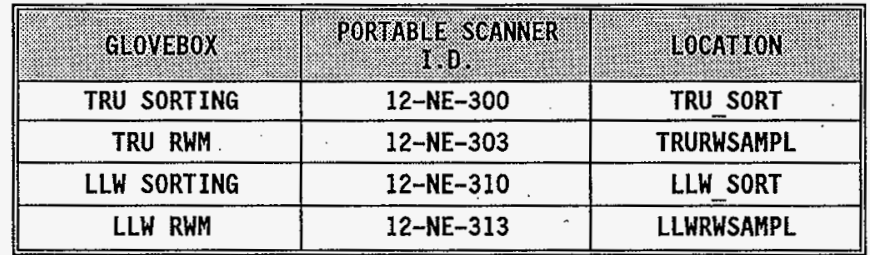

2. OBTAIN sampling information from DMS Screen DMSS0501 or from Attachment 2, Lab Sample Acquisition.

3. ENTER Room Temperature, Sample Matrix, and Comments as appropriate per Attachment 2, Lab Sample Acquisition.

4. ENTER Signature Password.

5. SELECT Commit.

6. LOWER the designated sampler into the selected waste container and obtain the sample.

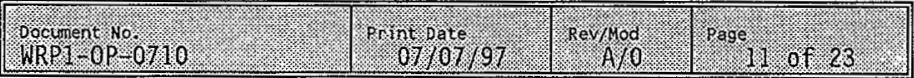




\section{WRAP 1 PLANT OPERATING PROCEDURE}

7. CAREFULLY WITHDRAW the waste sample materiat and FILL the sample container(s), as appropriate. If there are phases, FILL a sample container(s) for each phase.

a. NOTE the order of each phase and NUMBER the vials appropriately, (e.g., 1 of 3,2 of 3,3 of 3 ).

8. SECURE cap on each container(s)

9. SEAL around cap/container with tape.

10. RETURN unused waste sample material to original container.

i1. CLOSE and SECURE the lid on the waste container that was sampled.

12. G0 T0 Section VII.G, for instructions on completing sample preparation.

\section{F. SAMPLING SEMI-SOLID/SOLID WASTE MATRICES}

1. SCAN barcodes (refer to Table 2 below) as follows:

a. Location barcode on glovebox wall.

b. Sample Bottle barcode.

c. IF and only if, sampling in an RWM Glovebox, THEN SCAN the packet or collection container being sampled.

d. End barcode on glovebox wall.

e. Yes barcode on glovebox wall.

Table 2

\begin{tabular}{|c|c|c|}
\hline GYoYEBox & PORTABLG SCANNAR & GOCATIOH \\
\hline TRU SORTING & 12-NE-300 & TRU_SORT \\
\hline TRU RWM & 12-NE-303 & TRURWSAMPL \\
\hline LLW SORTING & $12-N E-310$ & LLW_SORT \\
\hline LLW RHM & $12-N E-313$ & LLWRWSAHPL \\
\hline
\end{tabular}

2. OBTAIN sampling information from DMS Screen DMSS0501 or from Attachment 2, Lab Sample Acquisition. 
3. ENTER Room Temperature, Sample Matrix, and Comments as appropriate per Attachment 2, Lab Sample Acquisition.

4. ENTER Signature Password.

5. SELECT Commit.

6. DIVIDE the waste surface into a sampling grid, as appropriate.

7. SAMPLE each distinguishable matrix using one of the following methods:

\section{a. Scoop Sampler}

NOTE - $\quad$ This sampling technique should only be used when the material is homogenous throughout the container and a representative sample can be taken from a. portion of the container.

(1). Using a scoop or shove1, thoroughty MIX the material to be sampled then Scoop enough material for a representative sample.

(2) FILL the sample container(s).

(3). SECURE cap on each container(s).

(4) SEAL around cap/container with tape.

(5) RETURN unused sample material to original waste container, if appropriate.

(6) CLOSE and SECURE the lid on sampled waste container, if applicable.

(7) GO TO Section VII.G for instruction on completing sample preparation.

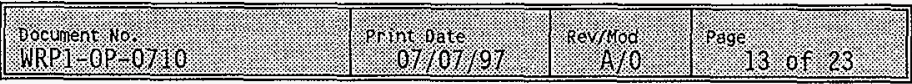


sin

b. Hand Auger. Sampler

NOTE - There are a wide variety of hand augers available in varying sizes for different materials. If the material will not stay in the auger, or if the sample hole will not stay open for reentry, an alternate sampling method should be chosen.

(1) PLACE the auger end at the selected sampling location.

(2) AUGER into the sample material.

(3) When the auger becomes full of material, REMOVE the auger by PULLING and TURNING the auger counterclockwise. Power equipment may be used as appropriate.

(4) PLACE a bowl underneath the auger. Using a spoon or other suitable tool PUSH the material from either the top or bottom of the auger. into the bow] and TAP the auger with a hammer or other suitable tool, as needed to encourage material flow.

(5) REPEAT Steps VII.F.7.b. (1) through VII.F.7.b. (4) above, the appropriate number of times in the same sampling hole to obtain sample material from the entire depth of the matrix.

(6) If more than one bowT is needed, SUB-SAMPLE from each bow? into a single composite bow7 and MIX thoroughty.

(7) Using a scoop or shove7, thoroughly MIX the material to be sampled then Scoop enough material for a representative sample.

(8) FILl: the sample container(s).

(9) SECURE cap on each container(s).

(10) SEAL around cap/container with tape.

(11) RETURN unused sample material to original waste container, if appropriate.

(12) CLOSE and SECURE the lid on sampled waste container, if applicable.

(13) GO T0 Section VII.G for instruction on completing sample preparation.

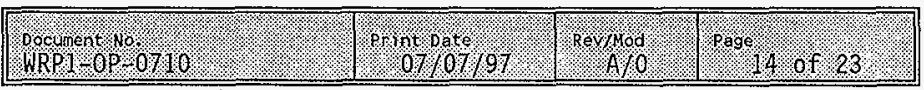


$\sin$

c. Snip and Wipe Method

(1) IF the solid item being sampled is hard, USE an approved filter paper or other media and WIPE item in such a way as to obtain a representative sample of the whole.

(2) If the solid item being sampled is soft, USE scissors and SNIP sections of the soft item in such a way as to obtain a representative sample of the whole.

(3) Prepare the sample per instructions obtained from the laboratory that will be performing the analysis and identified on Attachment 1, Laboratory Sample Analys is Request or DMS Screen DMSS0504.

(4) FILL the container as appropriate.

(5) SECURE cap on each container(s).

(6) SEAL around cap/container with tape.

(7) RETURN unused sample material to original waste container, if appropriate.

(8) CLOSE and SECURE the Iid on sampled waste container, if applicable.

(9) GO TO Section VII.G for information on completion of sample preparation.

\section{G. FINAL SAMPLE PREPARATION AND LOAD OUT}

1. Sample custodian must ENSURE sample.containers are sealed with evidence tape and appropriately labeled as follows:

- Sample number

- Date and Time

- Requested analyses

- Container type

- Container number (ie. \#1 of 4), as appropriate

- Collector's initials

\begin{tabular}{|c|c|c|c|}
\hline 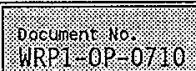 & provorat & Bryom & pase ex. \\
\hline
\end{tabular}


2. LOAD the sample containers into the STC for transfer.

NOTE - If "LOADOUT SAMPLES" is selected without a sample canister in $p 7$ ace, OIU responds with "NO PURGE PORT PRESENT".

a. SELECT "LOADOUT SAMPLES" on Operator Interface Unit (OIU) (refer to Table 3 below).

Table 3

\begin{tabular}{|c|c|}
\hline GLOVEBOX & $0 \mathrm{IU} / \mathrm{I}$ O \\
\hline TRU SORTING & $0 \mathrm{IU}-12-104 \mathrm{~B}$ \\
\hline TRU RWM & $0 \mathrm{IU}-12-106 \mathrm{~B}$ \\
\hline LLW SORTING & $0 \mathrm{IU}-12-103 \mathrm{~B}$ \\
\hline LLW RWM & $0 \mathrm{IU}-12-105 \mathrm{~B}$ \\
\hline
\end{tabular}

(PCS)

b. DISPLAY "SAMPLE LOADOUT IN PROGRESS" at 0IU-12-105B.

c. SCAN sample container barcode with portable scanner as follows (refer to Table 2 below):

(1) Location barcode on glovebox wall.

(2) Sample Bottle barcode.

(3) End barcode on glovebox wall.

(4) Yes barcode on glovebox wal1.

Table 2

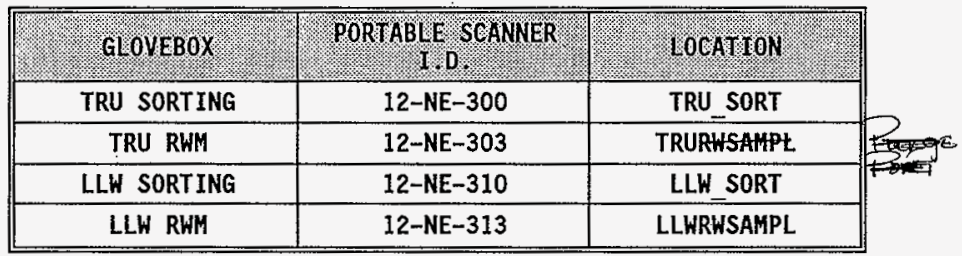

d. LOAD sample container into port canister.

e. REPEAT Steps VII.G.2.C and VII.G.2.d for each sample container to be transferred.

f. ENSURE all sample bottles in the Sample Transfer Container are displayed on DMSS0501. 
g. SELECT "END SAMPLE LOADOUT" on OIU.

h. CONFIRM "SAMPLE LOADOUT COMPLETE" is displayed at. OIU-12-105B.

3. CLOSE and DETACH the STC from the glovebox.

a. CLOSE port door.

b. MOVE port door latch to CLOSED position (down).

c. TURN port door latch locking pin clockwise, $30^{\circ}$ and RELEASE.

d. ENSURE port door latch locking pin drops into locking hole.

NOTE - During rotation, the STC cover is locked and sealed to the STC.

\section{CAUTION}

FAILURE TO SURVEY STC AND CELL FLANGE MAY RESULT IN EQUIPMENT AND/OR PERSONNEL CONTAMINATION.

e. REQUEST RCT coverage for STC removal.

f. ROTATE STC counter-clockwise, $60^{\circ}$ and REMOVE from port flange. 


\section{WRAP 1 PLINTT OPERATING PROGEDURE}

$\infty$

g. SCAN STC out of glovebox location with portable scanner (refer to Table 1 below), as follows:

(1) Location barcode on glovebox wa11.

(2) STC barcode.

(3) Transport PIG (if used).

(4) End barcode on glovebox wall.

(5) Yes barcode on giovebox wal1.

Table 1

\begin{tabular}{|c|c|}
\hline GLOVBBX & PORMABLE SCANNER I.O. \\
\hline TRU SORTING & $12-N E-300$ \\
\hline TRU RWM & $12-N E-303$ \\
\hline LLW SORTING & $12-N E-310$ \\
\hline LLW RWM & $12-N E-313$ \\
\hline
\end{tabular}

4. VERIFY OIU displays, "NO PURGE PORT CANISTER PRESENT".

5. REQUEST RCT to survey and appropriately release the sealed STC.

6. IF surface contamination is found, DECONTAMINATE the container.

7. IF/WHEN no surface contamination is detected, PLACE the STC in transport PIG or cooler as required.

8. ENSURE the COC/SAR information is entered into screen DMSSO502 or the hard copy is attached.

9. IF the COC of the STC is transferred between operations personnel prior to removal from the Process Area, ENSURE the COC/SAR, DMSS502 "Re] inquished By" and the "Relinquished To" information is entered into screen DMSSO502 on the DMS or on the hard copy.

10. TRANSFER the STC to the Sample Management Area, OR

a. SECURE in a locked cabinet until such time that the containers will be transported to the Sample Management Area.

b. RECORD time, date, and person placing the sample into storage in the cabinet $\log$ sheet.

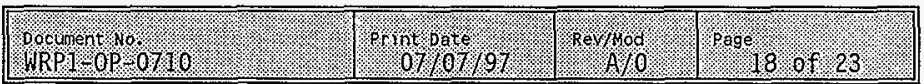


sor

11. RELINQUISH sample(s) to the sample custodian

12. ENSURE the COC/SAR "ReTinquished By" and the "ReTinquished To" information is entered into Screen DMSS0502 or on the hard copy form.

H. SAMPLE ACTIVITY LOGBOOKS AND DMS

NOTE - DMS or Attachment 2, Lab Sample Acquisition, shall be used for data collection. The Sample/Treatment Director shall transfer the information to the Sample Logbook, in a timely manner as required for compliant record keeping.

1. Sample/Treatment Director ENSURE and/or RECORD the following information applicable to the samples taken, in the Sample Log book and in the DMS:

- Container Identification Number (CIN)

- Manifest Number or other tracking number (e.g.,

Radioactive Shipping Record)

- Sampling Location

- Date

- Time

- Volume

- Container size and type

- Sampler's name

- Recorder's signature

- Physical Characteristics (Color, Texture, Viscosity)

- Anomalies and prohibited items

- Description of overall sampling event/Comments

- Requested Analysis

2. NOTE any discrepancies in the Comments Section of the Sample Log Book.

\section{SAMPLING ACTIVITIES PHOTO ALBUM}

1. RECORD the following information for each photo taken and ENTER in the Samping Activities Photo Album:

- Title of activity

- Roll number

- Picture number

- Negative number

- CIN of waste container sampled

- Date taken

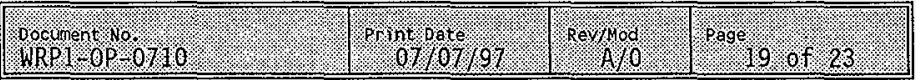




\section{ATTACHMENT 1 - LABORATORY SAMPLE ANALYSIS REQUEST}

\section{NOTE}

The data recorded in this attachment corresponds to the data recorded on DMSS0504 - Laboratory Sample Analys is Request.

Requested by:

Date:

(Corresponds to Signature Password on DMSS0504)

Drum/Packet PIN: Sample Number: Sample Location:

Analyses Selected: (Using codes from Attachment 3, Sample and Treatment Lookup Tables, each selected Laboratory Analysis will be associated with the following predetermined parameters: Description, Preservative, Container Type, No. of Containers, and required volume. Enter codes below for each requested analysis.)

Description of each Analysis:

Total number, type, and volume of sample containers required:

Preservative required:

Special Instructions/Graphics:

Additional sheet(s) attached: __ YES _ N0

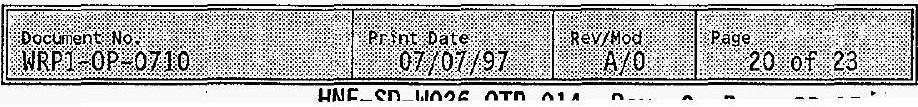




\section{ATTACHMENT 2 - LAB SAMPLE ACQUISITION}

\section{NOTE}

The data recorded in this attachment corresponds to the data recorded on DMSS0501 - Lab Sample Acquisition.

Requested by:

Date:

(Corresponds to Signature Password on. DMSS050I)

Drum/Packet PIN:

Room Temperature:

$\left({ }^{\circ} \mathrm{C}\right)$
STC PIN:

Sample Matrix: (from Attachment 3)

Bottles in STC by Bottie PIN and Anaiysis Description Code for each from Attachment 3, Sample and Treatment Lookup Tables:

Bottle PIN Analysis Code

$\frac{1}{1}$

Bottle PIN

Analysis Code

Comments:

Additional sheet(s) attached: _ YES _ NO 


\section{ATTACHMENT 3 - SAMPLE AND TREATMENT LOOKUP TABLES (Page 1 of 2)}

\begin{tabular}{|c|c|c|c|c|c|}
\hline \multicolumn{6}{|c|}{ Laboratory Analysis Table (LABANAL) } \\
\hline $\begin{array}{l}* \text { LABANAL CD } \\
\text { VCHAR2 }(8)\end{array}$ & $\begin{array}{l}\text { LABANAL_CNTR_COUNT } \\
\text { NUMBER }(\overline{1})\end{array}$ & $\begin{array}{l}\text { - LABANAL CNTYP_CD } \\
\text { VCHAR2 (3) }\end{array}$ & $\begin{array}{l}\text { LABANAL DESCR } \\
\operatorname{VCHAR2}(\overline{2} 0) \\
\end{array}$ & $\begin{array}{l}\text { LABANAL PRESERV } \\
\text { VCHAR2(12) }\end{array}$ & $\begin{array}{l}\text { LABANAL REQD_VOL } \\
\text { NUMBER }(\overline{4})\end{array}$ \\
\hline$A Q L I Q$ & 1 & $\mathrm{P}$ & Aqueous liquid & None & 250 \\
\hline OILS & 1 & $p$ & $0 \mathrm{ils}$ & None & 250 \\
\hline SOLVENTS & 1 & G & Solvents & None & 250 \\
\hline TCLP & 1 & P & TCLP & None & 250 \\
\hline SOLIDS & 1 & MC & Solids & None & 250 \\
\hline COND & 1 & $\mathrm{p}$ & Conductivity & None & 250 \\
\hline
\end{tabular}

\begin{tabular}{|l|l|}
\hline \multicolumn{2}{|l|}{ Sample Container Type (SAMPCONTYP) DMSSO463 } \\
\hline \hline $\begin{array}{l}\text { *SAMP_CNTYP_CD } \\
\text { VCHAR2 (3) }\end{array}$ & $\begin{array}{l}\text { SAMP_CNTR DESCR } \\
\text { VCHAR2 (30) }\end{array}$ \\
\hline \hline$P$ & Polyethylene Bottle \\
\hline$G$ & Glass \\
\hline$A G$ & Amber glass \\
\hline$A G S$ & Amber glass septa \\
\hline$G S$ & Glass septa \\
\hline$M C$ & Metal can \\
\hline
\end{tabular}


WRAP 1 PLANT OPLEATING PROGEDURE

ATTACHMENT 3 - SAMPLE AND TREATMENT LOOKUP TABLES

(Page 2 of 2)

\begin{tabular}{|c|c|}
\hline Sample Matrix Tabl & (SAMPMATX) DMSS0464 \\
\hline $\begin{array}{l}\text { *SAMPMATX CD } \\
\text { VCHAR2 (3)- }\end{array}$ & $\begin{array}{l}\text { SAMPMATX DESCR } \\
\operatorname{VCHAR2}(1 \overline{2})\end{array}$ \\
\hline $\mathrm{s}$ & Soil \\
\hline SE & Sediment \\
\hline so & Solid \\
\hline$S G$ & Solid - Gran \\
\hline SM & Solid - Metal \\
\hline SS & Solid - Sludge \\
\hline SL & Sludge \\
\hline$w$ & Water \\
\hline 0 & $0 i 1$ \\
\hline A & Air \\
\hline DS & Drum Sotids \\
\hline $\mathrm{DL}$ & Drum Liquids \\
\hline$T$ & Tissue \\
\hline WI & Wipe \\
\hline$L$ & Liquid \\
\hline$x$ & Other \\
\hline
\end{tabular}

\begin{tabular}{|l|l|}
\hline \multicolumn{2}{|c|}{ Sampling Hethod (SAMPMETH) DMSS0465 } \\
\hline $\begin{array}{l}\text { *SAMPLING METHOD_CD } \\
\text { VCHAR2(4) }\end{array}$ & $\begin{array}{l}\text { SAMPLING METHOD_DESC } \\
\text { R VCHAR2(30) }\end{array}$ \\
\hline \hline PIPE & Pipet sampler \\
\hline SCOP & Scoop sampler \\
\hline TRIR & Trier sampler \\
\hline AUGR & Hand auger sampler \\
\hline SNIP & Solid snip sample \\
\hline SWIP & Swipe sample \\
\hline CLWS & Colowasa sampler \\
\hline INS & Insitu \\
\hline
\end{tabular}

\begin{tabular}{|c|c|c|}
\hline \multicolumn{3}{|c|}{ Field Analysis Type Table (FLDANALTYP) DMSS0459 } \\
\hline $\begin{array}{l}\text { *FAT CD } \\
\text { VCHAR } 2(4) \\
\end{array}$ & $\begin{array}{l}\text { FAT - DESCR } \\
\text { VCHAR2 }(70)\end{array}$ & $\begin{array}{l}\text { FAT UNITS } \\
\text { VCHAR2 }(6) \\
\end{array}$ \\
\hline LEAD & Lead stick test & $\mathrm{red} / \mathrm{no}$ \\
\hline PHDP & pH dip strip & $\mathrm{pH}$ \\
\hline PHMR & $\mathrm{pH}$ meter & $\mathrm{pH}$ \\
\hline PCBO & CLOR-N-OIL & ppm \\
\hline PCBS & CLOR-N-SOIL & $\mathrm{ppm}$ \\
\hline $\mathrm{CLOR}$ & CIOR-D-TECT & npm \\
\hline
\end{tabular}




\section{WASTE TREATMENT}

\section{SYSTEM DESCRIPTION}

This procedure provides instructions for the treatment of hazardous components of the WRAP 1 Mixed Waste stream or waste that is not in compliance with disposal criteria for the Waste Isolation Pilot Plant (WIPP) or the Hanford Site. The treatment processes will take place in the Restricted Waste Management (RWM) gloveboxes and include:

- Deactivation (neutralization, cementing, absorption, and controlled reaction with water)

- $\quad$ Stabilization (cementing, absorption, and sealing)

- Amalgamation (1iquid, elemental mercury is treated with inorganic reagent such as copper, zinc, nickel, gold, or sulfur, to produce a non-Tiquid semi-solid amalgam)

The waste to be treated can be grouped into four general categories:

- Liquid Organic Solutions with a variety of dissolved organic constituents, oils, solvents, fuel

- Solid Organic Soil, resins, paper, rags, wood

- Liquid Inorganic Acid, base, and salt solutions, solutions containing heavy metals

- Solid Inorganic pipe, fluorescent tubes (elemental mercury), machinery, shielding lead, sheet metal

\section{REFERENCE DOCUMENTS}

HSRCM-1, Hanford Site Radiological Control Manual

WHC-CM-5-34, Sol id Waste Disposal Operations Administration

WHC-CM-5-36, SWD Interna7 Requirements

WHC-EP-0063, Hanford Site Solid Waste Acceptance Criteria

WRP1-0P-0710, Glovebox Waste Sampling

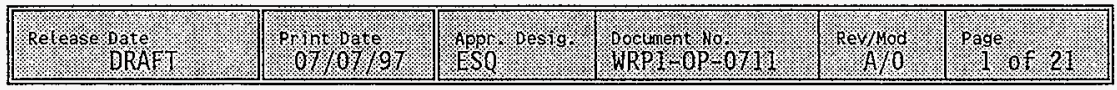




\section{WRAP 1 PLANT OPERATING PROCEDURE}

\section{PRESTART CONDITIONS}

A pre-job briefing will be held to review the completed Treatment Work Sheet (Attachment 1) and/or DMS Screen DMSS0325, LLW RWM Processing Instructions, provided by the Sampling/Treatment Director, to ensure a11 information to complete the job is provided.

An ALARA Management Work Sheet, Job Hazards Analysis (JHA), and a Radiological Work Permit (RWP) will be reviewed in the pre-job briefing as required.

Prior to treatment operation, ensure the fire protection system is operable.

OBTAIN completed copy of Attachment 1, Treatment Work Sheet 0R, ENSURE the worksheet data has been entered in the DMS on Screen DMSS0506.

OBTAIN tools and materials specified on Attachment 1, Treatment Work Sheet or DMS Screen DMSS0325, LLW RWM Processing Instructions.

\section{SAFETY}

Potential hazards, such as lifting heavy containers or handling sharp objects, must be mitigated by protective equipment, procedures, and administrative controls to ensure acceptable-risk operating conditions.

Consult with Operations Management and Industrial Hygiene, and refer to the Job Hazards Analysis (JHA), Radiological Work Permit (RWP) and Material Safety Data Sheets (MSDS) to determine required Personal Protective Equipment (PPE).

Check glovebox contents prior to startup/restart for any materials which might require special handling precautions (i.e. flammable, reactive, etc.). If problems exist, or if unsure that glovebox operation may be safely conducted, then notify Duty Operations Supervisor (DOS).

This procedure requires accessing gloveboxes through gloveports on a regular basis. Operators sha17 perform self survey upon exiting gloveport after each use. Radiological Control Technician (RCT) shall be contacted anytime self survey limits are exceeded.

Spills inside the glovebox shall be cleaned up per WRP1-0P-0701, Glovebox Housekeeping. 


\section{WRAP 1 PLANT OPERATING PROCEDURE}

$\infty$

Warning - Use care to prevent injury. Possible safety hazards include:

- Tripping/S1ipping

- Injuries to the hands at g]ovebox ports

- Radiological Contamination

- Interference with the Automatic Guided Vehicle (AGV)

If during performance of this procedure, any of the following conditions are found, immediately STOP WORK, place equipment in. a safe condition, and notify Operations management:

- Any equipment malfunction which could prevent fulfillment of its functional requirements

- Personnel error

When treating reactive material, on $7 y$ one container of reactive material sized up to 1 gallon will be located in the glovebox at any one time unless reviewed and covered under a separate Job Hazards Analysis.

Containers of $f$ lammable or reactive materials shall not be stored in the gloveboxes for extended periods of time or overnight. Open containers of flammable or reactive material shall not be left unattended in the gloveboxes.

\section{WARNING:}

Caution must be used to avoid spills when handling acids or caustics.

Extreme caution must be used when handling reactive materials due to potential flammability or explosion.

Do not handle bottles with bulging or other signs of pressurization.

Do not handle any containers with crystalline material formation until it is determined they are not shock sensitive.

\section{TOOLS AND SUPPLIES}

As specified on specific Attachment 1, Waste Treatment Work Sheet or DMS Screen DMSS0325, LLW RWM Processing Instructions, for each job.

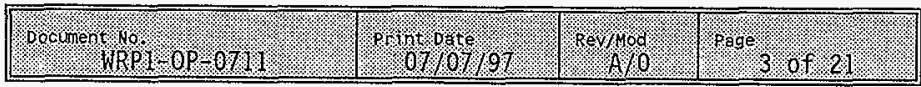




\section{TABLE OF CONTENTS}

PAGE

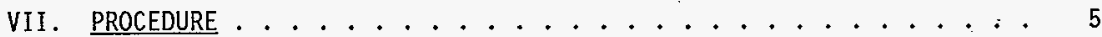

ATTACHMENT 1 - TREATMENT WORK SHEET . . . . . . . . . . 9

ATTACHMENT 2 - NEUTRALIZATION ................ 10

ATTACHMENT 3 - ABSORPTION . . . . . . . . . . . . 13

ATTACHMENT 4 - CONTROLLED REACTION WITH WATER . . . . . . . . 15

ATTACHMENT 5 - GROUTING ....................... 17

ATTACHMENT 6 - AMALGAMATION ................. 20

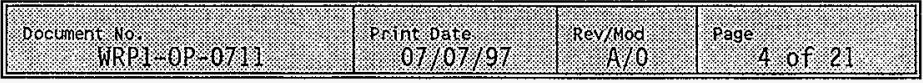




\section{PROCEDURE}

\section{WARNING}

CAUTION MUST BE USED TO AVOID SPILLS WHEN HANDLING ACIDS OR CAUSTICS.

EXTREME CAUTION MUST BE USED WHEN HANDLING REACTIVE MATERIALS DUE TO POTENTIAL FLAMMABILITY OR EXPLOSION.

NOTE 1 - Administrative hold steps are identified by letters in parentheses at the left margin. Direction given in the procedure step must be satisfied before work continues.

(M) - Operations management shall approve operation.

(R) - Radiological Control (RC) shaj7 complete surveys or agree to permit continued operation.

NOTE 2 -. UNLESS OTHERWISE SPECIFIED, se7ections and displays throughout this procedure are on applicable Glovebox operator Interface Unit (OIU, RWM GTovebox):

- OIU-12-105B, LLW/RWM glovebox

- OTU-12-106B, TRU/RWM gTovebox

NOTE 3 DMS Screen DMSS0506, Worksheet Data Entry, is used to select items for treatment and prepare the worksheet on DMS.

NOTE 4 - $\quad$ AT7 Data Management System (DMS) data entry, is performed on DMS Termina7 (107-TE-12-103).

NOTE 5 - Treatment information is displayed inside glovebox on applicab7e OTU:

- OTU-12-105A, LLW/RWM glovebox

- OTU-12-106A, TRU/RWM glovebox

NOTE 6 - Al7 scanning is performed with applicable portable scanner:

- 12-NE-313, LLW/RWM gTovebox

- 12-NE-303, TRU/RWM glovebox

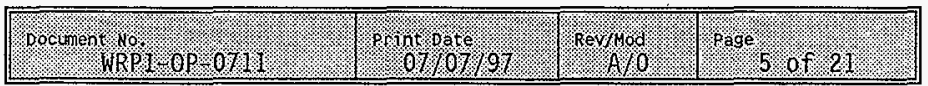

HNF-SD-W026-0TR-014, Rev. 0, Page 0P-161 


\section{WRAP: 1 PLANT OPERATING PROCEDURE}

(M) A. If at any time during performance of this procedure, any unique or unusual everit occurs,

THEN:

1. STOP work immediately.

2. NOTIFY Duty Operations Supervisor (DOS).

3. PROCEED when and as directed by DOS.

4. RECORD description of event and actions taken in Treatment Logbook and in appropriate comment space of DMSSO326 screen.

\section{WARNING}

DO NOT HANDLE BOTTLES HITH BULGING OR OTHER SIGNS OF PRESSURIZATION.

DO NOT HANDLE ANY CONTAINERS WITH CRYSTALLINE MATERIAL FORMATION UNTIL IT IS DETERMINED THEY ARE NOT SHOCK SENSITIVE.

(M) B. IF at any time during performance of this procedure, any bottles or other waste/sample containers show either of the following:

- Signs of pressurization (bulging, etc.)

- Crystalline material formation

THEN:

1. STOP work immediately.

2. NOTIFY Duty Operations Supervisor (DOS).

3. PROCEED when and as directed by DOS.

4. RECORD description of event and actions taken in Treatment Logbook and in appropriate comment space of DMSS0326 screen.

C. OBTAIN the completed copy of Attachment 1, Treatment Worksheet, OR SELECT Worksheet from List of Values on DMS Screen DMSSO3/4/34\%

D. VERIFY all items on the worksheet are at the treatment station by performing one of the following:

1. CHECK the items against the worksheet.

2. CHECK Screen DMSS03,24, LLW RWM Tréatment Item Assembly, shows zero "Item Containers to be Retrieved" for both packets and samples.

\begin{tabular}{|c|c|c|c|}
\hline 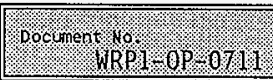 & Proporto & Kovod & \% \\
\hline
\end{tabular}


NOTE - $\quad$ The PCS Barcode System does not support splitting of packets at the RWM Treatment Location. Splitting of packets must be done at the sorting location.

NOTE - When a collection container bar code is scanned, any packets scanned afterwards are associated with this collection container.

NOTE - For DMS item tracking, all treated items must be associated with a treatment container ID. If a separate treatment container is not used, a treatment container barcode label must be attached to the container used for treatment and the barcode $7 a b e 7 s$ scanned to associate the original container with new treatment container ID.

E. SCAN the following bar codes for EACH item to be treated:

1. Location bar code.

2. Treatment container bar code.

3. Bar code Tabel of each item to be treated.

4. "YEND" bar code.

5. "YYES" bar code.

F. TREAT item(s) per the Processing Instructions found either on Attachment 1, Treatment Work Sheet, or DMS Screen DMSS0325, Processing Instructions.

1. USE Attachments 2 through 6 for conducting specific treatment procedures as follows:

a. Attachment 2, Neutralization

b. Attachment 3, Absorption

c. Attachment 4, Controlled Reaction With Water

d. Attachment 5, Grouting

e. Attachment 6, Amalgamation

2. On DMS Screen DMSS0326, LLW RWM Treatment, ENTER the following information as appropriate:

a. Material group code.

b. Waste description.

c. Treatment comments for the treatment container.

3. If compliant waste from an item is added to the compliant waste loadout drum:

a. CHECK the COMPLIANT box for the item.

b. UPDATE the waste description for the Compliant Loadout Drum. 


\section{WRAP 1 PLANT OPERATING PROGEDURE}

$\infty$

G. ENSURE Attachment 1, Treatment Work Sheet has been completed, signed and dated $O R$ the Operator's signature password has been entered on Screen DMSSO326 and the data has been committed.

H. RETURN completed Attachment 1, Treatment Work Sheet to DOS OR ENSURE DMS Screen DMSS0506 is updated.

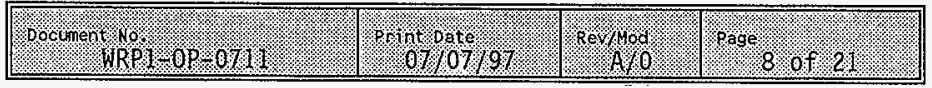




\section{ATTACHMENT 1 - TREATMENT WORK SHEET}

\begin{tabular}{|l|l|l|l|l|}
\hline WORK SHEET NUMBER: & RWP \#: & AMW \#: \\
\hline TREATMENT TYPE: & CHEMIST SIGNATURE: & \\
\hline & WASTE/SAMPLE PIN TO BE TREATED & \\
\hline & & & & \\
\hline & & & & \\
\hline & & & & \\
\hline
\end{tabular}

TOOLS AND MATERIALS:

PROCESSING INSTRUCTIONS:

NEW TREATED WASTE CONTAINER PINS:

\begin{tabular}{|r|r|r|r|}
\hline & & & \\
\hline & & & \\
\hline & & & \\
\hline & & & \\
\hline
\end{tabular}

WASTE PACKAGING AND HANDLING INSTRUCTIONS:

JOB COMPLETION COMMENTS:

OPERATOR SIGNATURE:

DATE:

OPERATION MANAGEMENT SIGNATURE:

DATE: 
ATTACHNENT 2 - NEUTRALIZATION

Page 1 of 3

\section{WARNING}

FAILURE TO EXERCISE EXTREME CAUTION AND ATTENTION TO DETAIL WHEN WORKING AROUND STRONG ACIDS AND BASES CAN CAUSE SEVERE INJURY DUE TO CHEMICAL BURNS.

\section{GENERAL NEUTRALIZATION INFORMATION}

NOTE - Corrosive waste with a pH $\leq 2.0$ and $\geq 12.5$ will be deactivated by neutralization.

NOTE - The target as an endpoint to treatment shall be a neutral pH with a range between 5.0 and 9.0 considered acceptab7e.

A. Generally, if waste has a $\mathrm{pH} \leq 2.0$ or $\geq 12.5$, neutraljzation witl be the first step in the overall treatment. Once neutraijized, the waste may require other treatment such as absorption or stabilization.

B. It shall be the responsibility of the Sample/Treatment Director to:

Decide, based on chemical properties of the waste and desired chemical end products, which reagent(s) to use in the neutralization process.

Perform any and al1 necessary stoichiometric calculations and/or produce titration curves to determine accurate quantities of neutralizing agent to be introduced to the waste ensuring minimal end waste volume.

- Perform al1 necessary and required safety analyses related to the actual chemical processes needed for neutralization (e.g., generation of poisonous gasses, heat production, potential for explosion, etc.) and make personnel safety recommendations as needed.

\begin{tabular}{|c|c|c|c|}
\hline Wocrontrko & ront 0 ate 0197 & Revroo & Poge 10001 \\
\hline
\end{tabular}




\section{WRAP 1 PLANT OPERATING PROCEDURE}

\section{ATTACHMENT 2 - NEUTRALIZATION}

Page 2 of 3

\section{GENERAL NEUTRALIZATION INFORMATION (continued)}

C. Typical bases used to neutralize acids include:

- Calcium Carbonate, $\left(\mathrm{CaCO}_{3}\right)$ - Generally desirable neutralizing agent due to its tendency to produce nonsoluble salts. Has the advantage of producing many insoluble carbonate metal salts and the fact that these carbonates are compatible with portland cement. It should be anticipated when neutralizing with calcium carbonate, that the reaction may produce frothing due to the evolution of carbon dioxide.

- $\quad$ Calcium Hydroxide, $(\mathrm{CaOH})$ - Generally desirable neutralizing agent due to its tendency to produce non-soluble saits.

- $\quad$ Sodium Hydroxide ( $\mathrm{NaOH})$ - Less desirable neutralizing agent due to its tendency to produce soluble salts.

D. Typical acids used as to neutralize bases include:

- Hydrochloric Acid (Hcl) - Appropriate caution must be taken to avoid injury due to potential generation of chlorine gas.

- Sulfuric Acid $\left(\mathrm{H}_{2} \underline{\mathrm{SO}}_{4}\right)$

- $\quad$ Nitric Acid $\left(\mathrm{HNO}_{3}\right)$

\section{NEUTRALIZATION PROCEDURE}

\section{WARNING}

FAILURE TO NOTE ANY SPECIAL SAFETY PRECAUTIONS ON THE FOLLOWING DMS SCREENS, PRIOR TO INITIATING ANY TREATMENT ACTIVITY COULD CAUSE PERSONNEL INJURY AND/OR EQUIPMENT DAMAGE:

- DMSS0326, LLW RHM TREATMENT

- DMSSO325, LLW RHM PROCESSING INSTRUCTIONS

A. ENSURE the PIN of the item to be treated matches the PIN of the selected DMS Screen DMSS0326, LLW RWM Treatment.

|

HNF-SD-W026-OTR-014, Rev. 0, Page 0P-167 


\section{WRAP 1 PLANT OPERATING PROCEDURE}

$m-$

\section{ATTACHMENT 2 - NEUTRALIZATION}

Page 3 of 3

\section{NEUTRALIZATION PROCEDURE (Continued)}

B. TEST the contents of the selected Treatment Container. for $\mathrm{pH}$, using a pH dip test.

C. IF the $\mathrm{pH}$ is between 2.0 and 12.5 , THEN EXIT this Attachment.

D. If the reading is lower than 2.0 or greater than 12.5 , THEN CONTINUE with this pH neutralization process.

E. IF the pH cannot be accurately determined using a dip strip, THEN use a pH meter according to WRP1-0P-0707, Operation and Setpoint Check of the Orion pH Meter.

F. ADD the designated amount of the neutralizing agent as indicated on DMS Screen DMSS0325, LLW RWM Processing Instructions.

G. SECURE the lid on the treatment container.

H. PLACE the treatment container on the jar mill for the amount of time indicated on DMS Screen DMSS0325, LLW RWM Processing Instructions.

I. WHEN mixing is complete,

THEN REMOVE the treatment container from the jar mill.

J. TEST the treated solution with $\mathrm{pH}$ paper.

K. If pH is at, or between 5.0 and 9.0 ,

THEN PROCEED with the remainder of this Attachment.

L: IF $\mathrm{pH}$ is NOT at, or between 5.0 and 9.0 , THEN CONSULT the Sample/Treatment Director for further neutralization instructions.

M. RECORD the results of the pH dip test on DMS Screen DMSS0326, LLW RWM Treatment.

N. PROCEED with other treatment activities or treated item load out procedures as required.

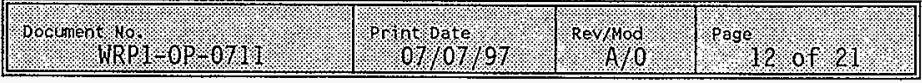




\section{ATTACHMENT 3 - ABSORPTION}

Page 1 of 2

\section{GENERAL ABSORPTION PROCESS INFORMATION}

A. The absorption of Tiquid waste material is conducted to render the waste non-fluid.

B. Sufficient absorbent must be added to the waste to ensure there is no free liquid remaining after the addition of the absorbent material, while not adding so much absorbent that it unnecessarily increases the volume of waste.

C. Absorbent material approved for use on the Hanford Site is 1isted in the Hanford Site Solid Waste Acceptance Criteria, WHC-EP-0063, and in the TRU Waste Acceptance Criteria for the Waste Isolation. Pilot Plant, WIPP-DOE-069, and includes but is not 7 imited to:

- Polyacrylates

- Polypropytene

- Superabsorbent Polymer

- Cellutose

D. The absorption process is judged effective by conducting a visual inspection.

E. If the overail treatment process is completed after the addition of the absorbent and is ready for disposal, a paint filter test may be performed to verify that the waste no longer contains free Tiquids.

\section{ABSORPTION PROCEDURE}

\section{WARNING}

FAILURE TO NOTE ANY SPECIAL SAFETY PRECAUTIONS ON THE FOLLOWING DMS SCREENS, PRIOR TO INITIATING ANY TREATMENT ACTIVITY COULD CAUSE PERSONNEL INJURY AND/OR EQUIPMENT DAMAGE:

\section{DMSS0326, LLW RWM TREATMENT}

DMSSO325, LLW RWM PROCESSING INSTRUCTIONS

A. ENSURE the PIN of the item to be treated matches the PIN of the selected DMS Screen DMSS0326, LLW RWM Treatment.

\begin{tabular}{|c|c|c|c|}
\hline 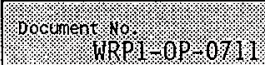 & 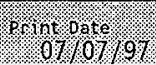 & revardo & Poge, 6.1 \\
\hline
\end{tabular}




\section{WRAP 1 PLANT OPERATING PROCEDURE}

\section{ATTACHMENT 3 - ABSORPTION \\ Page 2 of 2}

B. TEST the contents of the selected Treatment Container for pH, using a $\mathrm{pH}$ dip test.

C. IF the $\mathrm{pH}$ cannot be accurately determined using a dip strip, THEN use a pH meter according to WRP1-0P-0707, Operation and Setpoint Check of the Orion $\mathrm{pH}$ Meter.

D. IF the $\mathrm{pH}$ is at, or between 2.0 and 12.5,

THEN PROCEED with the remainder of this Attachment.

E. IF the reading is lower than 2.0 or greater than 12.5, THEN NEUTRALIZE the liquid prior to absorption per Attachment 2, NEUTRALIZATION.

F. DETERMINE the correct type and amount of absorbent material to use from DMS Screen DMSS0325, LLW RWM Processing Instructions.

G. SECURE the lid on the treatment container.

H. PLACE the treatment container on the jar mill for the amount of time indicated on DMS Screen DMSS0325, LLW RWM Processing Instructions.

I. WHEN mixing is complete,

THEN REMOVE the treatment container from the jar mill.

J. TEST the treated solution by visual inspection:

1. If there is any visible free liquid in the Treatment Container,

THEN add $25 \%$ of the original amount of absorbent to the container and repeat Steps II.G through II.J.

2. IF there is no visible free liquid in the container, THEN RECORD the test resuits on DMS Screen DMSS0326, LLW RWM Treatment.

K. PROCEED with other treatment activities or treated item load out procedures as required.

\begin{tabular}{|c|c|c|c|}
\hline hocrinert ror & . & revod & Proge. $14.0 \mathrm{of} / 2$ \\
\hline
\end{tabular}

HNF-SD-W026-0TR-014, Rev. 0, Page OP-170 


\title{
WRAP 1 PLANT OPERATING PROCEBURE
}

\author{
ATTACHMENT 4 - CONTROLLED REACTION WITH WATER
}

Page 1 of 2

I. CONTROLLED REACTION WITH WATER - GENERAL INFORMATION

\section{WARNING}

FAILURE TO EXERCISE EXTREME CAUTION IN HANDLING METALLIC SODIUM AROUND WATER, CAN CAUSE A VIOLENT EXOTHERMIC REACTION RESULTING IN A RAPID HYDROGEN BURN RESEMBLING AN EXPLOSION.

A. A very slow, controlled reaction with water (usually vapor or mist) is used to deactivate some reactive material such as sodium metal.

B. To ensure complete deactivation, the geometry of the metal object may have to be altered (cut or shaved into small thin particles).

C. Upon completion of the controlled reaction, a visual inspection of the reactive material is necessary.

D. The treated metal should no longer appear shiny and silvery gray. It should appear dul1 and dark or completely dissolved.

\section{PROCEDURE FOR CONTROLLED REACTION WITH WATER}

\section{WARNING}

FAILURE TO NOTE ANY SPECIAL SAFETY PRECAUTIONS ON THE FOLLOWING DMS SCREENS, PRIOR TO INITIATING ANY TREATMENT ACTIVITY COULD CAUSE PERSONNEL INJURY AND/OR EQUIPMENT DAMAGE:

- DMSS0326, LLW RWM TREATMENT

- DMSSO325, LLW RHM PROCESSING INSTRUCTIONS

A. ENSURE the PIN of the item to be treated matches the PIN of the selected DMS Screen DMSSO326, LLW RWM Treatment.

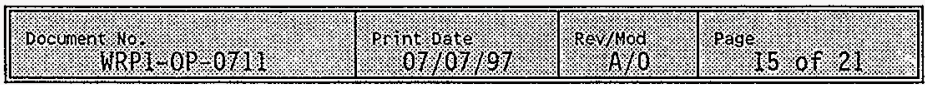

HNF-SD-H026-OTR-014, Rev. 0, Page OP-171 


\section{ATTACHMENT 4 - CONTROLLED REACTION WITH WATER}

Page 2 of 2

B. DETERMINE the following from DMS Screen DMMS0325, LLW RWM Processing Instructions:

1. Geometric configuration of waste (e.g., shaved, granulated, chipped, etc.).

2. Quantity of water to be introduced.

3. Method by which the water is to introduced.

4. Rate at which the water is to introduced.

C. ENSURE the geometric configuration of the material to be deactivated by reaction with water is as specified on DMS Screen DMSS0325, LLW RWM Processing Instructions.

D. ADD specified quantity of water, at the specified rate, using the specified method, to the Treatment Container.

E. WHEN the entire amount of water specified on DMS Screen DMSSO325 has been added to the Treatment Container, THEN VERIFY the following:

1. There is no free liquid in the Treatment Container.

2. The metal being treated no longer displays a shiny, silver-grey appearance.

3. The metal being treated displays a dark, dult appearance.

F. IF there is any free liquid,

THEN CHECK pH and neutralize as necessary per Attachment 2, Neutralization.

G. RECORD the results of the reaction with water on DMS Screen DSS0326, LLW RWM Treatment.

H. PROCEED with other treatment activities or treated item load out procedures as required.

\begin{tabular}{|c|c|c|c|}
\hline 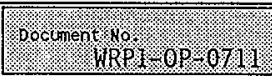 & 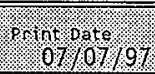 & R.\% $10 \%$ & pag $10 \%$ of \\
\hline
\end{tabular}




\section{ATTACHMENT 5 - GROUTING}

Page 1 of 3

\section{GENERAL GROUTING PROCESS INFORMATION}

A. Grouting is used to deactivate reactive ignitables consisting of metal fines and radioactive or corrosive material.

B. The mechanism of this deactivation technique is to mix and bind the active elements of the waste with an jnert material. This inert material is typically portland cement.

c. The optimum water to cement ratio for portland cement is 0.7 meaning 7 parts water to ten parts cement, with a $3 \%$ by weight bentonite content.

D. The waste is thoroughly mixed in with the cement as soon as the water is added:

E. The resultant matrix is allowed to cure for a minimum of 24 hours before handling.

NOTE: Stability is defined as the resistance of waste to degrade into smaller particles as a function of time. Durability is defined as the resistance of waste particles to abrade into smaller particles during anticipated handling and transportation.

F. Following curing, the matrix is visually inspected for signs of free liquid, stability, and durability.

G. Since grouting is a treatment technology and there are no other hazardous constituents, no additional testing is required to meet RCRA Requirements. 


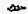

\section{GROUTING PROCEDURE}

\section{ATTACHMENT 5. - GROUTING}

Page 2 of 3

\section{WARNING}

FAILURE TO NOTE ANY SPECIAL SAFETY PRECAUTIONS ON THE FOLLOWING DMS SCREENS, PRIOR TO INITIATING ANY TREATMENT ACTIVITY COULD CAUSE PERSONNEL INJURY AND/OR EQUIPMENT DAMAGE:

- DMSSO326, LLW RHM TREATMENT

- DMSSO325, LLH RHM PROCESSING INSTRUCTIONS

A. ENSURE the PIN of the item to be treated matches the PIN of the selected DMS Screen DMSS0326, LLW RWM Treatment.

B. DETERMINE the following from DMS Screen DMMS0325, LLW RWM Processing Instructions:

1. Type of grout (cement) to use.

2. Quantity of grout to use.

3. Amount of water to be mixed with the grout.

4. Any special additives to be mixed. with the grout.

\section{CAUTION}

FAILURE TO ACT QUICKLY AFTER THE DRY GROUT HAS BEEN ADDED TO THE LIQUID WASTE, CAN CAUSE INCOMPLETE STABILIZATION.

C. ADD the designated amount of dry grout to the Treatment Container.

D. IMMEDIATELY ADD any water, or additives, as required by DMS Screen DMMS0325, LLW RWM Processing Instructions.

E. IMMEDIATELY SECURE the 7 id on the treatment container.

F. IMMEDIATELY PLACE the treatment container on the jar mill for the amount of time indicated on DMS Screen DMSS0325, LLW RWM Processing Instructions.

G. WHEN mixing is complete,

THEN REMOVE the treatment container from the jar mill.

H. PLACE the treatment container in a location where it can remain undisturbed for a minimum of twenty four hours.

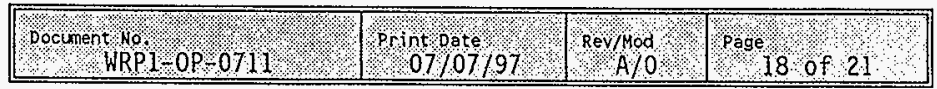


ATTACHMENT 5 - GROUTING

Page 3 of 3

I. INSPECT the contents of the Treatment Container after the twenty four hour curing time to determine if there is any free liquid remaining:

1. IF there is any detectable free liquid in the Treatment Container, THEN CONSULT the Sample/Treatment Director for further instructions.

2. If there is no visibie free liquid in the container, THEN RECORD the test results on DMS Screen DMSS0326, LLW RWM Treatment.

J. PROCEED with treated item load out procedures as required. 


\section{ATTACHMENT 6 - AMALGAMATION}

Page 1 of 2

\section{GENERAL AMALGAMATION PROCESS INFORMATION}

A. Amalgamation is a technique used to treat liquid, elemental mercury, that results in a non-7iquid semi-solid amalgam, thereby reducing potential emissions of elemental mercury vapors to the air.

B. Amalgamation treatment uses inorganic reagents including the following:

$\begin{array}{ll}: & \text { Copper } \\ : & \text { Zinc } \\ \text { Nickel } \\ \text { Gold } \\ \text { Sulphur }\end{array}$

C. The Sampling/Treatment Director is responsible for determining which reagents, what amount, and method of introduction, based on the chemical constituents of the waste other than the mercury.

D. The resultant matrix is a non-liquid, semi-solid amalgam.

E. The amalgam is visually inspected to verify compliance.

F. Since Amalgamation is a technology based treatment standard, additional testing or laboratory analysis to show compliance to RCRA requirements is not required.

\section{AMALGAMATION PROCEDURE}

\section{WARNING}

FAILURE TO NOTE ANY SPECIAL SAFETY PRECAUTIONS ON THE FOLLOWING DMS SCREENS, PRIOR TO INITIATING ANY TREATMENT ACTIVITY COULD CAUSE PERSONNEL INJURY AND/OR EQUIPMENT DAMAGE:

\section{DMSSO326, LLW RWM TREATMENT \\ DMSSO325, LLW RWM PROCESSING INSTRUCTIONS}

A. ENSURE the PIN of the item to be treated matches the PIN of the selected DMS Screen DMSS0326, LLW RWM Treatment.

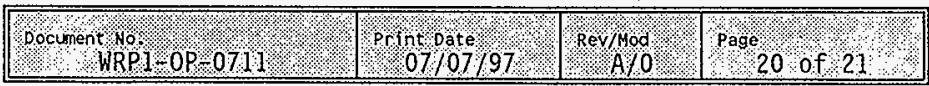




\section{WRAP 1 PLANT OPERATING PROCEDURE}

\section{ATTACHMENT 6 - AMALGAMATION \\ Page 2 of 2}

II. AMALGAMATION PROCEDURE (Continued)

B. DETERMINE the following from DMS Screen DMMS0325, LLW RWM Processing Instructions:

1. Which reagent to use.

2. Proper concentration for the reagent.

3. Quantity of reagent to be introduced.

4. Method of reagent introduction.

C. ADD the designated amount of amalgamation reagent to the Treatment Container, using the method described on DMS Screen DMMS0325, LLW RWM Processing Instructions.

D. SECURE the lid on the Treatment Container.

E. PLACE the Treatment Container on the jar mitT for the amount of time indicated on DMS Screen DMSS0325, LLW RWM Processing Instructions.

F. WHEN mixing is complete,

THEN REMOVE the treatment container from the jar mil1.

G. VISUALLY INSPECT the contents of the Treatment Container to ENSURE no Tiquid, elemental mercury remains:

1. IF there is any liquid, elemental, mercury visible, CONSULT the Sample/Treatment Director for further treatment instructions.

2. IF there is no visible liquid, elemental mercury visible in the container,

THEN RECORD the test results on DMS Screen DMSS0326, LLW RWM Treatment.

H. PROCEED with treated item load out procedures as required.

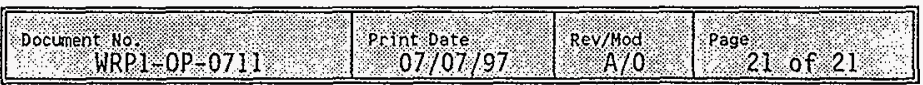

HNF-SD-W026-0TR-014, Rev. 0, Page 0P-175 


\title{
WASTE RECEIVING AND PROCESSING (WRAP) PLANT OPERATING PROCEDURE
}

\author{
SYSTEH FROOESS
}

\section{RWM GLOVEBOXES MANUAL OPERATION}

\section{SYSTEM DESCRIPTION}

This procedure provides instructions for manual (Operator Interface Unit (OIU) Maintenance Menu) operation of the Transuranic (TRU) and Low Level Waste (LLW), Restricted Waste Management (RWM) Gloveboxes.

In Manual (Maintenance) mode automatic operations normally performed by the Plant Control System (PCS) may be individually actuated. This allows correction of interlock conflicts, time-outs or other out-of-sequence conditions which prevent the normal sequence of events from proceeding. once prohibiting conditions are corrected, automatic operation is resumed.

Maintenance Menus of the OIU are password protected and Duty Operation Supervisor (DOS) permission is required for activation. Depending on the situation, the DOS may simply correct a single prohibiting condition and return the system to normal, or in cases of numerous problems such as might occur after an Emergency Stop or power loss, the DOS may activate the maintenance mode and request additional assistance for the Operator (i.e., Cognizant Engineer).

This procedure addresses each OIU function individually and is not intended to govern sequence of operations. Sequence of operations, hold points and non-0IU related activities are governed by applicable glovebox operating procedures. Attachment 2, Tables 1 through 2, define interlock and permissive signals in each glovebox and reference procedure sections or other procedures which manipulate the indicated source switch for the interlock or permissive. When a function will not actuate, applicable interlocks should be verified to the proper state, then the function rechecked. If all interlocks are properly verified and a function will still not actuate, maintenance is required.

If there is a need to review initiation times and/or sequences of specific OIU messages, they are aI1 recorded on the RTAP Printer.

\section{REFERENCE DOCUMENTATION/DOCUMENTED BASIS}

HSRCM-1, Hanford Site Radiological Control Manual. WRP1-0P-0703, Glovebox Lift Table Operation.

WRP1-0P-0701, GTovebox Housekeeping.

WRP1-0P-0704, Waste Manipulator Operation.

WRP1-0P-0705, Bagless Transfer Manual Operation.

WRP1-0P-0717, LLW/RWM Process Glovebox Operation.

WRP1-0P-0728, IRU/RWM Process Glovebox Operation.

\begin{tabular}{|c|c|c|c|c|c|}
\hline Releas morte & priptores & Approsig. & 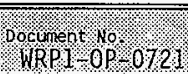 & sevmod & page.6. \\
\hline
\end{tabular}




\section{PRESTART CONDITIONS}

All personnel performing this procedure shall be qualified in accordance with WHC-CM-5-34, Section 1.8, "Training Plan," and on-the-job training.

Process area Heating, Ventilation, Air Conditioning (HVAC) in OPERATION.

\section{SAFETY}

Potential hazards, such as lifting heavy containers or handling sharp objects present in waste containers, must be mitigated by protective equipment, procedures, and administrative controls to ensure acceptable-risk operating conditions.

Consult with Operations Management and Industrial Hygiene, and refer to the Job Hazards Analysis (JHA), Radiological Work Permit (RWP) and Material Safety Data Sheets (MSDS) to determine required Personal Protective Equipment (PPE).

Check glovebox contents prior to startup/restart for any materials which might require special handling precautions (i.e. flammable, reactive, etc.). If problems exist, or if unsure that glovebox operation may be safely conducted, then notify Duty Operations Supervisor (DOS).

This procedure requires accessing gloveboxes through gloveports on a regular basis. Operators shall perform self survey upon exiting gloveport after each use. Radiological Control Technician (RCT) shall be contacted anytime self survey limits are exceeded.

Spilis inside gloveboxes shall be cleaned up per WRP1-0P-0701, Glovebox Housekeeping.

CAUTION - When an OIU is in maintenance mode, most interlocks and safety features are bypassed. It is possible to damage equipment, and/or bypass safety restrictions such as glovebox containment. Caution must be exercised to prevent unwanted violation of operational safety requirements or equipment damage.

WARNING - Use care to prevent injury. Possible safety hazards include:

- Tripping/STipping

- Radiological Contamination

- Interference with Automatic Guided Vehicle (AGV)

WARNING - Improper operation of glovebox equipment including hoist, manipulators, etc. may cause injury. Caution must be exercised if reaching through gloveports while equipment is in operation.

\section{TOOLS AND SUPPLIES}

None.

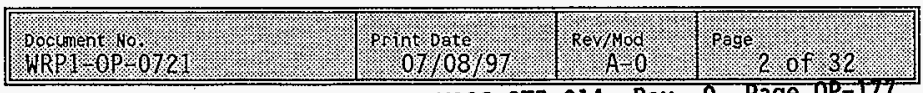




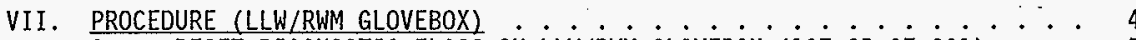

A. RESET DIAGNOSTIC FLAGS ON LLW/RWM GLOVEBOX (107-GB-07-201) . . 5

B. ACCESS LLW/RWM GLOVEBOX MAINTENANCE MENUS .......... 7

C. LLW/RWM GLOVEBOX HOIST (107-CR-07-201) MANUAL OPERATIONS $\ldots .7$

D. BAGLESS TRANSFER PORT (107-D0-07-201) MANUAL OPERATIONS . . . 7

1. MATE DRUM TO BAGLESS TRANSFER PORT $(107-D 0-07-201) \ldots . .7$

2. OPEN/CLOSE BAGLESS TRANSFER PORT (107-D0-07-201) .... 9

3. REMOVE DRUM FROM BAGLESS TRANSFER PORT (107-D0-07-201) . $: 9$

E. COMPLIANT WASTE PORT (107-D0-07-202) MANUAL OPERATIONS . . . 10

1. MATE DRUM TO COMPLIANT WASTE PORT $(107-D 0-07-202) \ldots \ldots$

2. OPEN COMPLIANT WASTE PORT $(107-D 0-07-202) \ldots \ldots . . . . .11$

3. CLOSE COMPLIANT WASTE PORT (107-D0-07-202) . . . . 12

4. REMOVE DRUM FROM COMPLIANT WASTE PORT (107-D0-07-202) . . 13

F. NON-COMPLIANT WASTE PORT (107-D0-07-203) MANUAL OPERATIONS . . . 13

1. MATE DRUM TO NON-COMPLIANT WASTE PORT $(107-D 0-07-203) \ldots 13$

2. OPEN NON-COMPLIANT WASTE PORT $(107-D 0-07-203) \ldots \ldots$

3. CLOSE NON-COMPLIANT WASTE PORT (107-D0-07-203) … 16

4. REMOVE DRUM FROM NON-COMPLIANT WASTE PORT

$(107-D 0-07-203) \ldots \ldots \ldots . \ldots 17$

VIII. PROCEDURE (TRU/RUM GLOVEBOX) $\ldots \ldots \ldots \ldots$

A. RESET DIAGNOSTIC FLAGS ON TRU/RWM GLOVEBOX (107-GB-07-401) . . 19

B. ACCESS TRU/RWM GLOVEBOX MAINTENANCE MENUS ......... 21

C. . TRU/RWM GLOVEBOX HOIST (107-CR-07-401) MANUAL OPERATION .... 21

D. BAGLESS TRANSFER PORT (107-D0-07-401) MANUAL OPERATION . . . 21

1. MATE DRUM TO BAGLESS TRANSFER PORT (107-D0-07-401) . . . 21

2. OPEN/CLOSE BAGLESS TRANSFER PORT (107-D0-07-401) .... 23

3. REMOVE DRUM FROM BAGLESS TRANSFER PORT (107-D0-07-401) . . 23

E. COMPLIANT WASTE PORT (107-D0-07-402A) MANUAL OPERATIONS $\ldots 23$

1. MATE DRUM TO COMPLIANT WASTE PORT $(107-D 0-07-402 A) \ldots .23$

2. OPEN COMPLIANT WASTE PORT $(107-D 0-07-402 A) \ldots \ldots 24$

3. CLOSE COMPLIANT WASTE PORT (107-D0-07-402A) ..... 24

4. REMOVE DRUM FROM COMPLIANT WASTE PORT (107-D0-07-402A) * . 25

F. NON-COMPLIANT WASTE PORT (107-D0-07-402B) MANUAL OPERATIONS . . 25

1. MATE DRUM TO NON-COMPLIANT WASTE PORT (107-D0-07-402B) . . 25

2. OPEN NON-COMPLIANT WASTE PORT (107-D0-07-402B) .... 25

3. CLOSE NON-COMPLIANT WASTE PORT $(107-D 0-07-402 B) \ldots 26$

4. REMOVE DRUM FROM NON-COMPLIANT WASTE PORT $(107-D 0-07-402 B) \ldots \ldots . . . \ldots 26$

ATTACHMENTS

ATTACHMENT 1: RWM GLOVEBOX HOIST CONTROL FUNCTIONS . . . . . . . . . . 27

ATTACHMENT 2: GLOVEBOX INTERLOCK SIGNALS ............. 28

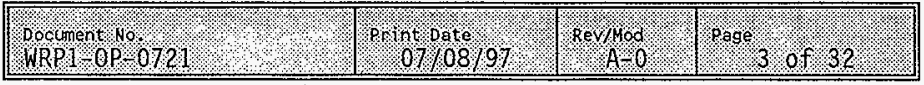




\section{WRAP 1 PLANT OPERATING PROCEDURE}

\section{PROCEDURE (LLW/RWM GLOVEBOX)}

\section{WARNING}

IMPROPER OPERATION OF GLOVEBOX EQUIPMENT INCLUDING HOIST, MANIPULATORS, ETC. MAY CAUSE INJURY. CAUTION MUST BE EXERCISED IF REACHING THROUGH GLOVEPORTS WHILE EQUIPMENT IS IN OPERATION.

\section{CAUTION}

WHEN AN OIU IS IN MAINTENANCE MODE, MOST INTERLOCKS AND SAFETY FEATURES ARE BYPASSED. IT IS POSSIBLE TO DAMAGE EQUIPMENT, AND/OR BYPASS SAFETY

RESTRICTIONS SUCH AS GLOVEBOX CONTAINMENT. CAUTION MUST BE EXERCISED TO PREVENT VIOLATION OF OPERATIONAL SAFETY REQUIREMENTS OR EQUIPMENT DAMAGE.

NOTE 1 - Administrative hold steps are identified by letters in parentheses at the left margin of the procedure step. The direction given in the procedure step must be satisfied before work continues.

(M) - Operations management shal7 approve operation.

(R) - Radiological Control (RC) shall complete surveys or agree to permit continued operation.

NOTE 2 - Actions performed by the P7ant Control System (PCS) or Data Management System (DMS) are identified by (PCS) or (DMS) in the left margin.

NOTE 3 - In Maintenance mode, OIU selections must be selected (screen touched) until the actuated device is visually verified to have reached the desired state.

NOTE 4 - Gloveports and manipulators are used as needed to perform tasks throughout this procedure. Manipulator Operation is performed per WRP1-0P-0704, G7ovebox Manipulator Operation.

NOTE 5 - Manual operation of Bag7ess Transfer Ports 107-D0-07-201 and 107-D0-07-401 is performed per WRP1-0P-0705, Bagless Transfer Manua7 Operation.

NOTE 6 - Data Management System (DMS) actions in Section VII are performed on DMS Monitor (107-0IU-12-105A).

NOTE 7 - UNLESS OTHERWISE SPECIFIED, selections and disp7ays in Section VII are on LLW/RWM Operator Interface Unit (OIU-12-105B).

\begin{tabular}{|c|c|c|c|}
\hline Bocumern vo 0.72 . & $.6 \% 1008.9 \%$ & 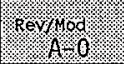 & 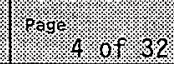 \\
\hline
\end{tabular}




\section{WRAP 1 PLANT OPERATING PROCEDURE}

NOTE 8 - Lift Table controls in Section VII are located as follows:

$$
\text { Lift Table Lift Table Control Pedestal }
$$

$$
\begin{array}{ll}
107-L T-09-201 D & 107-P C-09-201 D \\
107-L T-09-201 E & 107-P C-09-201 E \\
107-L T-09-201 F & 107-P C-09-201 F
\end{array}
$$

Manua7 lift tab7e operation is performed per WRP1-0P-0703, Glovebox Lift Table Operation.

NOTE 9 - Hoist activities in Section VII are performed on Hoist Control (107-PC-07-009A \& 009B). Functions must be activated until associated indicator illuminates. Reference Attachment 1.

A. RESET DIAGNOSTIC FLAGS ON LLW/RWM GLOVEBOX (107-GB-07-201)

1. SELECT "DIAGNOSTICS MENU" from "LLW RWM GLOVEBOX MAIN MENU".

NOTE - Parameters flagged with red displays on the DIAGNOSTICS MENU indicate that associated equipment is in other than the most stable default mode, indicated in green.

(PCS) Displays flagged parameters in red, with negative messages and default parameters in green with positive messages.

2. OBSERVE diagnostic menu messages (Reference Table VII-1 on the following page).

3. ACCESS MAINTENANCE MENU screens per applicable sections of this procedure, to reset diagnostic flags as required.

4. VERIFY desired diagnostic flags are reset by performing the following:

a. RETURN to "MAINTENANCE MAIN MENU" .

b. SELECT "DIAGNOSTICS MENU".

c. ENSURE messages assocjated with the particutar equipment in question are displayed in green.

5. WHEN all required red flags have been reset (to green):

\begin{tabular}{|c|c|c|c|}
\hline $\begin{array}{l}\text { oocunent } \\
\text { HRP }\end{array}$ & 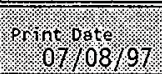 & Kro & pagk \\
\hline
\end{tabular}

a. RETURN to "MAINTENANCE MAIN MENU".

b. SELECT "MAIN MENU".

c. GO TO desired section(s). 


\section{WRAP 1 PLANT OPERATING PROCEDURE}

TABLE VII-1: LLW/RWM GLOVEBOX DIAGNOSTICS MENU MESSAGES

\begin{tabular}{|c|c|}
\hline DIAGNOSTIC IESSAGE (negative state) & STGNAL SOUREE \\
\hline LT-09-201F M/0/N NOT IN NORMAL & O9-HS-231A \\
\hline LT-09-201F LIFT TABLE NOT AT AGV HEIGHT & $09-Z S-231 G$ \\
\hline DO-07-202 DOOR NOT CLOSED AND LOCKED & $\begin{array}{l}07-Z S-900 \mathrm{~A} \\
07-Z S C-908 \mathrm{~B} \\
\end{array}$ \\
\hline D0-07-202 VACUUM NOT RELEASED & $.07-P S-900$ \\
\hline D0-07-202 CENTERING CLAMPS NOT RETRACTED & $\begin{array}{l}07-Z S 0-914 A \\
07-Z S 0-914 B \\
\end{array}$ \\
\hline D0-07-202 HVAC SEAL NOT DEFLATED & $07-P S-916$ \\
\hline LT-09-201E M/O/N NOT IN NORMAL & $09-\mathrm{HS}-229 \mathrm{~A}$ \\
\hline LT-09-201E LIFT TABLE NOT AT AGV HEIGHT & $09-Z S-229 G$ \\
\hline DO-07-203 DOOR NOT CLOSED AND LOCKED & $\begin{array}{l}\text { 07-ZS-912A } \\
07-Z S C-910 B \\
\end{array}$ \\
\hline D0-07-203 VACUUM NOT RELEASED & 07-PS-901 \\
\hline D0-07-203 CENTERING CLAMPS NOT RETRACTED & $\begin{array}{l}07-Z S 0-915 A \\
07-Z S 0-915 B \\
\end{array}$ \\
\hline D0-07-203 HYAC SEAL NOT DEFLATED & $07-P S-917$ \\
\hline LT-09-20ID M/O/N NOT IN NORMAL & $09-H S-227 A$ \\
\hline LT-09-201D LIFT TABLE NOT AT AGV HEIGHT & $09-Z S-227 G$ \\
\hline EM-07-201 MANIPULATOR NOT PARKED & 07-ZS-920A \\
\hline EM-07-20I LEFT ARM NOT PARKED & $07-Z S-920 C$ \\
\hline EM-07-201 RIGHT ARM N0T PARKED & $07-Z S-920 D$ \\
\hline \multicolumn{2}{|c|}{ 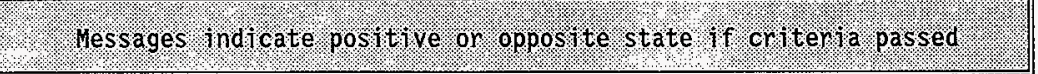 } \\
\hline
\end{tabular}

\begin{tabular}{|c|c|c|c|}
\hline opockent vol & . & revorod & (e. \\
\hline
\end{tabular}




\section{WRAP 1 PLANT OPERATING PROCEDURE}

NOTE - - Sections VII.C through VII.F assume Maintenance Main Menu has been accessed per Section VII.B.

B. ACCESS LLW/RWM GLOVEBOX MAINTENANCE MENUS

1. SELECT "MAINT ACCESS MENU" from 0IU-12-105B, LLW RWM GLOVEBOX MAIN MENU.

2. VERIFY display "ENTER ACCESS CODE".

3. KEY in majntenance password,

THEN, SELECT "ENTER".

4. VERIFY display "MAINTENANCE MAIN MENU".

5. SELECT desired maintenance submenu(s).

6. WHEN desired,

RETURN to Operating Menus as follows:

a. IF at any maintenance submenu,

b. SHEN, SELECT "MAINT

C. LLW/RWM GLOVEBOX HOIST (107-CR-07-201) MANUAL OPERATIONS

NOTE - Manual hoist operation is performed the same as in Automatic Mode, except hoist control is continuously enabled.

1. OPERATE hoist as required for glovebox tasks or maintenance.

D. BAGLESS TRANSFER PORT (107-D0-07-201) MANUAL OPERATIONS

NOTE - Lift Table controls and indicators referred to in this Section are located on Lift Table Control Pedestal 107-PC-09-201F.

1. MATE DRUM TO BAGLESS TRANSFER. PORT (107-D0-07-201)

a. ENSURE GTovebox Manipulator is PARKED (Reference WRP1-0P-0704, Glovebox ManipuTator Operation).

b. ENSURE. lift table MAINT/OFF/NORMAL switch (09-HS-231A) set to "MAINT".

c. SELECT "PORT D0-07-201 MENU" from MAIN MENU.

d. VERIFY display "DRUM AT LIFT TABLE" on 0IU-12-105B.

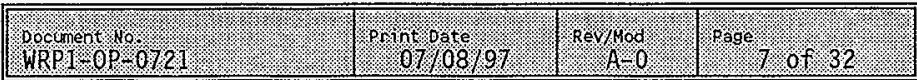


e. SCAN in order:

(1) Location (2Y) barcode on side of glovebox.

(2) Drum (S) barcode.

(PCS) Verifies drum PIN and location match that input by AGV delivery. Halts process if mismatched.

(M)

f. IF scanner displays "PIN MIS-MATCH",

THEN:

(1) RECORD PIN displayed on barcode scanner on PIN Change Form.

(2) SCAN Cancellation barcode.

(PCS) Clears pin mis-match message.

(3) NOTIFY DOS.

(4) CORRECT PIN information in PCS.

g. SCAN End (Y) barcode.

h. SCAN Yes (Y) barcode.

(PCS)

PCS transmits drum location update message to the DMS.

(DMS)

DMS Screen DMSS0321 displays Transfer Drum PIN and associated packets.

NOTE - Operator cannot control the 7ift table with AGV/PANEL switch in "AGV" position.

i. PLACE lift table AGV/PANEL switch (09-HS-2310) to "PANEL".

j. IF drum is fitted with outer lid,

THEN, REMOVE outer lid as follows:

(1) LOWER $1 \mathrm{ift}$ table as required.

(2) REMOVE outer lid bolts.

(3) REMOVE outer Tid.

(4) POSITION lift table to "AGV LOAD HEIGHT".

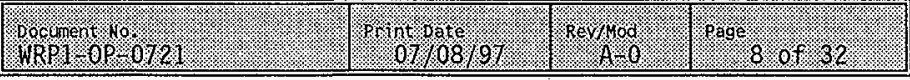




\section{WRAP 1 PLANT OPERATING PROCEDURE}

k. FORHARD lift table to "DRUM UNDER PORT" position, using FORWARD push button at Control Pedestal PC-07-201F until drum is under port.

1. ENSURE drum centered under port D0-07-201.

m. VERIFY "DRUM UNDER PORT" Tight iTluminated.

NOTE - Lift table cannot be raised unless the drum is located under the port. Drum under port is indicated by sensor illumination.

n. PRESS AND HOLD "UP" push button at control pedestal PC-07-201F until drum is approximately 2 inches below port.

\section{CAUTION}

DO NOT EXCEED WEIGHT SETPOINT BY MORE THAN 50 KILOGRAMS. FAILURE TO CAREFULLY JOG "UP" BUTTON TO REACH WEIGHT SETPOINT WILL CAUSE OVERLOADING LIFT TABLE.

0. BRIEFLY PRESS AND RELEASE "UP" button unti] weight setpoint reached.

NOTE - $\quad$ Setpoint must be initially met or exceeded for "DRUM IN POSITION" Tight" to i7luminate. Weight may later fall below setpoint due to gasket set.

p. VERIFY "DRUM IN POSITION" 7ight iTluminated.

2. OPEN/CLOSE BAGLESS TRANSFER PORT (107-D0-07-201)

a. ENSURE desired drum is mated to port per Section VII.D.1.

b. ENSURE hoist chain (107-CR-07-201) is at "RAISED LIMIT".

c. ENSURE hoist TROLLEY is at "LEFT".

d. ENSURE Manipulator (EM-07-201) is PARKED.

e. OPERATE port per WRP1-0P-0705, Bagiess Transfer Manua1 Operation.

3. REMOVE DRUM FROM BAGLESS TRANSFER PORT (107-D0-07-201)

a. ENSURE port is CLOSED and LOCKED.

b. LOWER 1 ift table to (107-LT-09-201F) "AGV LOAD HEIGHT".

- REQUEST RCT SURVEY DRUM for CONTAMIMATION. I. IF DRUM is CONTAMINATEP,

C. REVERSE lift table to "DRUM AT AGV END". THEN DECOMTAMINATE ASDIRECTED. 2. RE SURVEY DRUM.

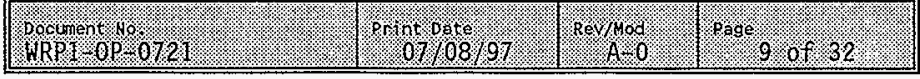


E. COMPLIANT WASTE PORT (107-D0-07-202) MANUAL OPERATIONS

1. MATE DRUN TO COMPLIANT WASTE PORT (107-D0-07-202)

a. ENSURE desired drum is on lift table (107-LT-09-201E).

b. ENSURE drum clamp band is removed.

c. SCAN "LLW RWM COMPLIANT" location barcode with seaner12-NF-313 to fix drum-7ocation.

d. VERIFY scanner displays correct location.

e. SCAN drum barcode with-seanner $12-1 F-313$.

f. VERIFY scanner displays correct drum pin.

g. SCAN "YEND" barcode to complete sequence.

h. SCAN "YYES" barcode to confirm sequence.

i. PLACE Lift Table 202E Switch 09-HS-229D in PANEL.

j. PLACE Lift Table 202E Switch 09-HS-229A in MAINTENANCE.

k. FORWARD lift table conveyor to. "DRUM UNDER PORT" position.

1. VERIFY "DRUM UNDER PORT" light iliuminated.

m. SELECT "PORT D0-07-202 MAINTENANCE MENU" at 0IU-12-105B.

n. VERIFY "PORT D0-07-202 MAINTENANCE MENU" displayed.

o. SELECT "RETRACT LID DETACH".

p. VERIFY display "RETRACT SOLENOID ACTIVATED".

q. ENSURE Manipulator (EM-07-201) is PARKED.

$r$ ENSURE Manipulator Arms are RAISED.

s. ENSURE display "CENTERING CLAMPS RETRACTED".

t. ENSURE display "HVAC SEAL DEFLATED".

u. RAISE 1ift table to "DRUM CENTERING HEIGHT" as indicated by the local arrow labels.

v. SELECT "EXTEND CENTERING CLAMPS". 


\section{WRAP 1 PLANT OPERATING PROCEDURE}

W. VERIFY display "CENTERING CLAMPS EXTENDED".

$x$. SELECT "TURN ON VACUUM".

y. VERIFY display "VACUUM GENERATED".

z. RAISE lift tab]e to "LID IN POSITION HEIGHT".

aa. VERIFY display "LID IN POSITION"

ab. SELECT "INFLATE HVAC SEAL".

ac. VERIFY display "HVAC SEAL INFLATED".

2. OPEN COMPLIANT WASTE PORT (107-D0-07-202)

a. ENSURE desired drum is mated to Compliant Waste Port 107-D0-07-202 per Section VII.E.1.

b. SELECT "MAINT MAIN MENU" at 0IU-12-105B.

c. VERIFY display, "MAINTENANCE MAIN MENU".

d. ENSURE Manipulator (EM-07-201) is PARKED.

e. ENSURE Manipulator (EM-07-201): Arms are RAISED.

f. SELECT "PORT D0-07-202 MAINTENANCE MENU".

g. VERIFY display "D0-07-202 MAINTENANCE MENU".

h. SELECT "UNLOCK PORT".

i. VERIFY display "PORT UNLOCKED".

NOTE - Steps VII.E.2.j, VII.E.2.k, and VII.E.2.7 are performed concurrent7y.

j. SELECT and HOLD "OPEN VENTS".

k. VERIFY display "VENTS OPEN".

7. RAISE 1ift table (107-LT-09-201E) until port door lifts $\sim 1 / 2$ inch.

m. VERIFY display "DOOR IN POSITION" .

n. RELEASE "OPEN VENTS".

o. SELECT "RAISE DOOR".

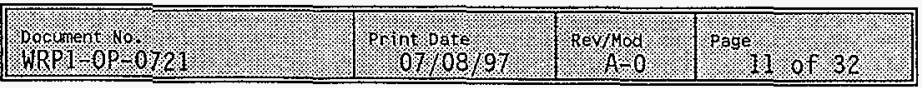


p. VERIFY dispTay "DOOR RAISED".

q. SELECT "ROTATE PORT OPEN".

$r$ VERIFY display "PORT ROTATED OPEN".

S. VERIFY port OPEN.

t. SELECT "MAINT MAIN MENU".

3. CLOSE COMPLIANT WASTE PORT (107-D0-07-202)

a. ENSURE Manipulator (EM-07-201) is PARKED.

b. ENSURE Manipulator Arms (EM-07-201) are RAISED.

c. SELECT "PORT D0-07-202 MAINTENANCE MENU".

d. VERIFY display "D0-07-202 MAINTENANCE MENU"

e. SELECT "ROTATE PORT CLOSED".

f. VERIFY display "PORT ROTATED CLOSED".

g. SELECT "LOWER DOOR".

h. VERIFY display "DOOR IN POSITION".

i. LOWER lift table to "LID IN POSITION HEIGHT".

j. VERIFY display "DOOR LOWERED".

k. SELECT "LOCK PORT".

1. VERIFY display "PORT LOCKED"

m. SELECT "TURN OFF VACUUM".

n. SELECT "EXTEND LID DETACH".

o. VERIFY display "EXTEND SOLENOID ACTIVATED".

p. LOWER lift table (107-LT-09-201E) to "DRUM CENTERING HEIGHT" as indicated by local arrow 7 abels.

q. SELECT "RETRACT LID DETACH".

$r$. VERIFY display "RETRACT SOLENOID ACTIVATED".

s. SELECT "DEFLATE HVAC SEAL". 


\section{WRAP 1 PLANT OPERATING PROCEDURE}

t. VERIFY display "DEFLATE SOLENOID ACTIVATED".

u. VERIFY display "HVAC SEAL DEFLATED".

v. SELECT "RETRACT CENTERING CLAMPS".

w. VERIFY display "CENTERING CLAMPS RETRACTED".

x. SELECT "MAINT MAIN MENU".

4. REMOVE DRUM FROM COMPLIANT WASTE PORT (107-D0-07-202)

a. ENSURE port is CLOSED and LOCKED.

b. VERIFY Centering $\mathrm{Cl}$ amps are retracted.

c. LOWER lift table (107-LT-09-201E) to "AGV LOAD HEIGHT".

d. REVERSE lift table to "DRUM AT AGV END".

F. NON-COMPLIANT WASTE PORT (107-D0-07-203) MANUAL OPERATIONS

1. MATE DRUM TO NON-COMPLIANT WASTE PORT (107-D0-07-203)

a. ENSURE desired drum is on lift table (107-LT-09-201D).

b. ENSURE drum clamp band is removed.

c. SCAN "LLW RWM NON-COMPLIANT" location barcode with scanner 12-NT-313 to fix drum location.

d. VERIFY scanner displays correct location.

e. SCAN drum barcode with scanner 12-NT-313.

f. VERIFY scanner displays correct drum pin.

g. SCAN "YEND" barcode to complete sequence.

h. SCAN "YYES" barcode to confirm sequence.

i. PLACE Lift Table 202D Switch 09-HS-227D in PANEL.

j. PLACE lift Table 202D Switch 09-HS-227A in MAINTENANCE.

k. FORWARD lift table conveyor to position drum to "DRUM UNDER PORT POSITION".

1. VERIFY "DRUM UNDER PORT" light j11uminated. 
m. SELECT "PORT DO-07-203 MAINTENANCE MENU" at OIU-12-105B.

n. VERIFY "PORT D0-07-203 MAINTENANCE MENU" dispiayed.

o. SELECT "RETRACT LID DETACH".

p. VERIFY display "RETRACT SOLENOID ACTIVATED".

q. ENSURE Manipulator (EM-07-201) is PARKED.

$r$ ENSURE Manipulator Arms are RAISED.

s. ENSURE display "CENTERING CLAMPS RETRACTED".

t. ENSURE display "HVAC SEAL DEFLATED".

u. RAISE lift table to "DRUM CENTERING HEIGHT" as indicated by the local arrow labels.

v. SELECT "EXTEND CENTERING CLAMPS".

w. VERIFY display "CENTERING CLAMPS EXTENDED".

$x$. SELECT "TURN ON VACUUM",

y. VERIFY display "VACUUM GENERATED".

z. RAISE lift table to "LID IN POSITION HEIGHT".

aa. VERIFY dispTay "LID IN POSITION"

ab. SELECT "INFLATE HVAC SEAL".

ac. VERIFY display "HVAC SEAL INFLATED". 
2. OPEN NON-COMPLIANT WASTE PORT (107-D0-07-203)

a. ENSURE desired drum is mated to Non-Compliant Waste Port 107-D0-07-203 per Section VII.F.1.

b. SELECT "MAINT MAIN MENU" at OIU-12-105B.

c. VERIFY display, "MAINTENANCE MAIN MENU".

d. ENSURE Manipulator (EM-07-201) is PARKED.

e. ENSURE Manipulator (EM-07-201) Arms are RAISED.

f. SELECT "PORT D0-07-203 MAINTENANCE MENU".

g. VERIFY display "D0-07-203 MAINTENANCE MENU".

h. SELECT "UNLOCK PORT".

i. VERIFY display "PORT UNLOCKED".

NOTE - Steps VII.F.2.j, VII.F.2.k, and VII.F.2.1 are performed concurrentiy.

j. SELECT and HOLD "OPEN VENTS".

k. VERIFY display "VENTS OPEN".

1. RAISE lift table (107-LT-09-201D) until port door 1ifts "1/2 inch.

m. VERIFY dispTay "DOOR IN POSITION".

n. RELEASE "OPEN VENTS".

o. SELECT "RAISE DOOR".

p. VERIFY display "DOOR RAISED".

q. SELECT "ROTATE PORT OPEN".

$r$. VERIFY display. "PORT ROTATED OPEN".

s. VERIFY port OPEN.

t. SELECT "MAINT MAIN MENU". 


\section{WRAP 1 PLANT OPERATING PROCEDURE}

3. CLOSE NON-COMPLIANT WASTE PORT (107-D0-07-203)

a. ENSURE Manipulator (EM-07-201) is PARKED.

b. ENSURE Manipulator Arms (EM-07-201) are RAISED.

c. SELECT "PORT D0-07-203 MAINTENANCE MENU".

d. VERIFY display "D0-07-203 MAINTENANCE MENU"

e. SELECT "ROTATE PORT CLOSED".

f. VERIFY display "PORT ROTATED CLOSED".

g. SELECT "LOWER DOOR",

h. VERIFY door lowered on top of drum.

i. LOWER lift table to "DOOR IN POSITION" height.

j. VERIFY dispTay "DOOR IN POSITION".

k. LOWER lift table to "LID IN POSITION" height.

1. VERIFY display "DOOR LOWERED".

m. SELECT "LOCK PORT",

n. VERIFY display "PORT LOCKED"

. SELECT "TURN OFF VACUUM".

p. SELECT "EXTEND LID DETACH".

q. VERIFY display "EXTEND SOLENOID ACTIVATED".

$r$. VERIFY display "HVAC SEAL DEFLATED" not displayed.

s. LOWER lift table (107-LT-09-201D) to "DRUM CENTERING HEIGHT" as indicated by local arrow labels.

t. SELECT "RETRACT LID DETACH" .

u. VERIFY display "RETRACT SOLENOID ACTIVATED".

v. SELECT "DEFLATE hVAC SEAL".

w. SELECT "RETRACT CENTERING CLAMPS".

x. VERIFY display "CENTERING CLAMPS RETRACTED". 
y. VERIFY Centering $\mathrm{Cl}$ lamps are actualyy retracted.

$z$. SELECT "MAINT MAIN MENU".

4. REMOVE DRUM FROM NON-COMPLIANT WASTE PORT (107-D0-07-203)
a. ENSURE port is CLOSED and LOCKED.
b. LOWER 7 ift table (107-LT-09-201D) to "DOWN OVERTRAVEL".
c. REVERSE lift table to "DRUM AT AGV END". 


\section{PROCEDURE (TRU/RWM GLOVEBOX)}

\section{HARNING}

IMPROPER OPERATION OF GLOVEBOX EQUIPMENT INCLUDING HOIST, SORTING TABLE, MANIPULATORS, TRANSFER CAR, ETC. MAY CAUSE INJURY. CAUTION MUST BE EXERCISED IF REACHING THROUGH GLOVEPORTS WHILE EQUIPMENT IS IN OPERATION.

\section{CAUTION}

WHEN AN OIU IS IN MAINTENANCE MODE, MOST INTERLOCKS AND SAFETY FEATURES ARE BYPASSED. IT IS POSSIBLE TO DAMAGE EQUIPMENT, AND/OR BYPASS SAFETY RESTRICTIONS SUCH AS GLOVEBOX CONTAINMENT. CAUTION MUST BE EXERCISED TO PREVENT VIOLATION OF OPERATIONAL SAFETY REQUIREMENTS OR EQUIPMENT DAMAGE.

NOTE 1 - Administrative hold steps are identified by letters in parentheses at the left margin of the procedure step. The direction given in the procedure step must be satisfied before work continues.

(M) - Operations management sha77 approve operation.

(R) - Radiological Control (RC) shall complete surveys or agree to permit continued operation.

NOTE 2 - Actions performed by the Plant Control System (PCS) or Data Management System (DMS) are identified by (PCS) or (DMS) in the left margin.

NOTE 3 - In Maintenance mode, OIU selections must be selected (screen touched) until the actuated device is visually verified to have reached it's desired state.

NOTE 4 - Manual operation of Bag7ess Transfer Port 107-00-07-401 is performed per WRP1-0P-0705, Bag7ess Transfer Manual Operation.

NOTE 5 - Data Management System (DMS) actions in Section VIII are performed on DMS Monitor (107-0IU-12-106A).

NOTE 6 - $\quad$ UNLESS OTHERWISE SPECIFIED, selections and displays in Section VIII are on TRU/RWM Operator Interface Unit (OIU-12-106B).

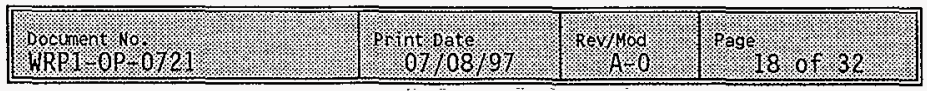


NOTE 7 - Lift Table controls in Section VIII are located as follows: Lift Tab7e Lift Table Control Pedestal

$\begin{array}{ll}107-L T-09-201 A & 107-P C-09-201 A \\ 107-L T-09-201 B & 107-P C-09-201 B \\ 107-L T-09-201 C & 107-P C-09-201 C\end{array}$

Manual lift table operation is performed per WRP1-0P-0703, Glovebox Lift Table Operation.

NOTE 8. - Hoist activities in Section VIII are performed on Hoist Control (107-PC-07-010A \& 010B). Functions must be activated unt $i 7$ associated indicator illuminates. Reference Attachment 1.

\section{A. RESET DIAGNOSTIC FLAGS ON TRU/RWM GLOVEBOX (107-GB-07-401)}

NOTE - Parameters flagged with red displays on the DIAGNOSTICS MENU indicate that associated equipment is in other than the most stable default mode, indicated in green.

1. SELECT "DIAGNOSTICS MENU" from "TRU RWM GLOVEBOX. MAIN MENU" on OIU-12-106B.

Displays flagged parameters in red, with negative messages and default parameters in green with positive messages.

2. OBSERVE diagnostic menu messages (Reference Table VIII-1 on the following page).

3. ACCESS MAINTENANCE MENU screens per applicable sections of this procedure, to reset diagnostic flags as required.

4. VERIFY desired diagnostic flags are reset by performing the following:
a. RETURN to "MAINTENANCE MAIN MENU".
b. SELECT "DIAGNOSTICS MENU".
c. ENSURE messages associated with the particular equipment in question are displayed in green.

5. WHEN a 71 required red flags have been reset (to green):
a. RETURN to "MAINTENANCE MAIN MENU".
b. SELECT "DIAGNOSTICS MENU".
c. GO TO desired section $(s)$.

6. VERIFY PORT 401 is CLOSED AND IN ANTO MONE USING PORT 401 OFERATOR PAIEL. REFERENCE WRAIDPOTOS FOR MANUAL OPER ATONS IF REQUIRED.

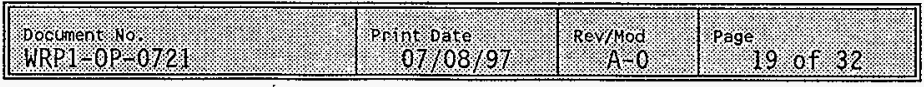




\section{WRAP 1 PLANT OPERATING PROCEDURE}

TABLE VIII-1: TRU/RWM GLOVEBOX DIAGNOSTICS MENU MESSAGES

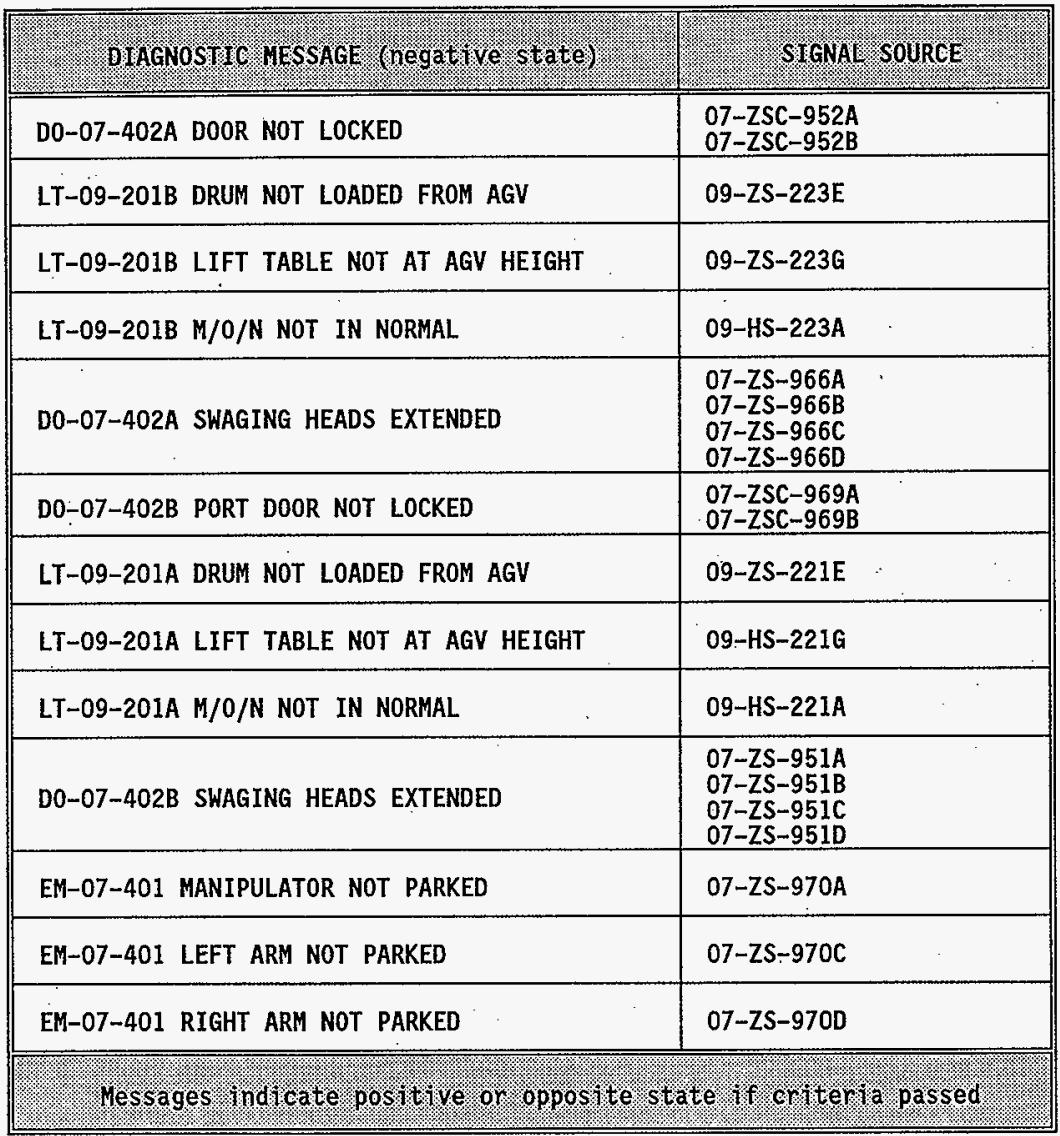

\begin{tabular}{|c|c|c|c|}
\hline pocumeroro & 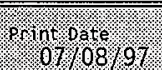 & Rev/rod & 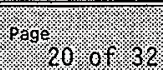 \\
\hline
\end{tabular}




\section{WRAP 1 PLANT OPERATING PROCEDURE}

NOTE - Sections VIII.C through VIII.F.4 assume Maintenance Main Menu has been accessed per Section VIII.B.

B. ACCESS TRU/RWM GLOVEBOX MAINTENANCE MENUS

1. SELECT "MAINT ACCESS MENU" from OIU, TRU RWM GLOVEBOX MAIN MENU.

2. VERIFY display "ENTER ACCESS CODE".

3. KEY in majntenance password, THEN, SELECT "ENTER".

4. VERIFY display "MAINTENANCE MAIN MENU".

5. SELECT desired maintenance submenu(s).

6. WHEN desired, RETURN to Operating Menus as follows:
a. IF at any maintenance submenu, THEN, SELECT "MAINT MAIN MENU"
b. SELECT "MAIN MENU".

C. TRU/RWM GLOVEBOX HOIST (107-CR-07-401) MANUAL OPERATION

NOTE 1 - Manual hoist operation is performed the same as in Automatic Mode, except hoist control is continuously enabled.

1. OPERATE hoist as required for glovebox tasks or maintenance.

D. BAGLESS TRANSFER PORT (107-D0-07-401) MANUAL OPERATION

NOTE - Lift Table controls and indicators referred to in this Section are located on Lift Table Control Pedestal 107-PC-09-201C.

1. MATE DRUM TO BAGLESS TRANSFER PORT (107-D0-07-401)

a. ENSURE 1 ift table MAINT/OFF/NORMAL switch (09-HS-225A) set to "NORMAL". MAINT.

b. SELECT "PORT D0-07-401 MENU" from MAIN MENU.

c. VERIFY dispTay "DRUM AT LIFT TABLE".

\begin{tabular}{|c|c|c|c|}
\hline 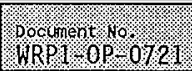 & popre & Kard & hage \\
\hline
\end{tabular}




\section{WRAP 1 PLANT OPERATING PROCEDURE}

d. SCAN in order:

(1) Location (2Y) barcode on side of giovebox. -

(2) Drum (S) barcode.

(PCS)

(M)

Verifies drum PIN and location match that input by AGV delivery. Halts process if mismatched.

e. IF scanner displays "PIN MIS-MATCH",

THEN:

(1) RECORD PIN displayed on barcode scanner on PIN Change Form.

(2) SCAN Cancellation barcode.

(PCS), Clears pin mis-match message.

(3) NOTIFY DOS.

(4) CORRECT PIN information in PCS.

f. SCAN End ( $Y$ ) barcode.

g. SCAN Yes (Y) barcode.

(PCS)

PCS transmits drum location update message to the DMS.

(DMS) DISPLAY "DRUM LOCATED AT TRU-RWM ENTRY PORT."

NOTE - . Operator cannot control the lift table with AGV/PANEL switch in "AGV" position.

h. PLACE Tift table AGV/PANEL switch (09-HS-225D) to "PANEL".

i. IF drum is fitted with outer lid, THEN, REMOVE outer 1id as follows:

(1) LOWER 1 ift table as required.

(2) REMOVE outer lid bolts.

(3) REMOVE outer lid.

(4) POSITION lift table to "AGV LOAD HEIGHT".

j. FORWARD jift table to "DRUM UNDER PORT" position, using FORWARD push button at Control Pedestal PC-09-201C unt il drum is under port.

k. ENSURE drum centered under port D0-07-401.

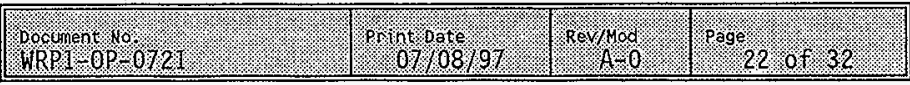




\section{WRAP 1 PLANT OPERATING PROCEDURE}

1. VERIFY "DRUM UNDER PORT" light illuminated.

NOTE - Lift table cannot be raised unless the drum is located under the lift table. Drum under port is indicated by sensor illumination.

m. PRESS "UP" push button at control pedestal PC-09-201C until drum is approximately 2 inches below port.

\section{CAUTION}

DO NOT EXCEED WEIGHT SETPOINT BY MORE THAN 50 KILOGRAMS. FAILURE TO CAREFULLY JOG "UP" BUTTON TO REACH WEIGHT SETPOINT WILL CAUSE OVERLOADING LIFT TABLE.

n. BRIEFLY PRESS AND RELEASE "UP" button unti1 weight

setpoint reached. PER PORT MATNG PRESSURE TAB LE IN OP-0703.

NOTE - Setpoint must be initial7y met or exceeded for

"DRUM IN POSITION" 7ight to i7luminate. Weight may

later fall below setpoint due to gasket set.

o. VERIFY "DRUM IN POSITION" light i 17uminated.

2. OPEN/CLOSE BAGLESS TRANSFER PORT (107-D0-07-401)

a. ENSURE desired drum is mated to port per Section VIII.D.I.

b. ENSURE hoist chain (107-CR-07-401) is at "RAISED LIMIT".

c. ENSURE hoist TROLLEY is at "LEFT".

d. ENSURE Manipulator (EM-07-401) is PARKED.

e. OPERATE port per WRP1-0P-0705, Bagless Transfer Manua1 Operation.

3. REMOVE DRUM FROM BAGLESS TRANSFER PORT (107-D0-07-401)

a. ENSURE port is CLOSED and LOCKED.

b. LOWER lift table to (107-LT-09-401F) "AGV LOAD HEIGHT".

c. REVERSE lift table to "DRUM AT AGV END".

E. COMPLIANT WASTE PORT (107-D0-07-402A) MANUAL OPERATIONS

1. MATE DRUM TO COMPLIANT WASTE PORT (107-D0-07-402A)

a. ENSURE desired drum is on lift table (107-LT-09-201B).

b. ENSURE drum clamp bandA t's removed.

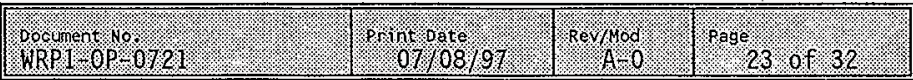




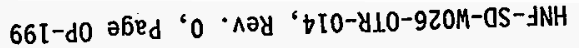

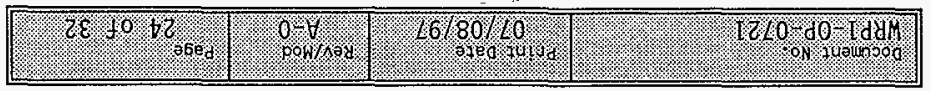

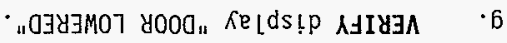

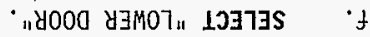

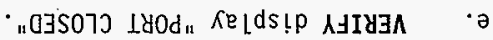

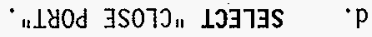

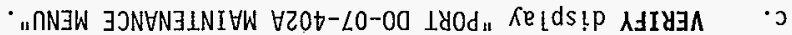

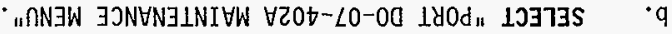

- äXYYd S! ([0t-LO-W]) soze [nd!̣ew JynSN

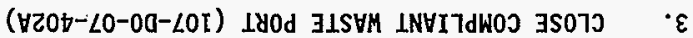

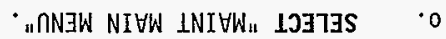

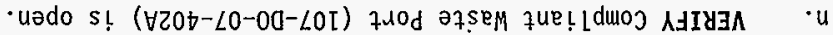

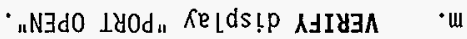

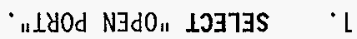

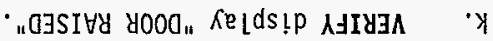

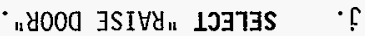

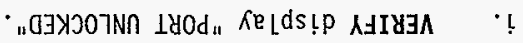

" "LYOd XJOTNR" LOJTIS 4

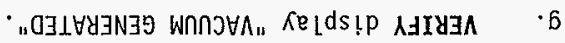

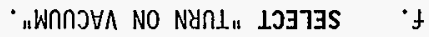

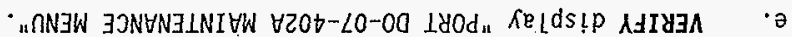

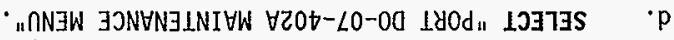

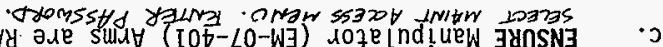

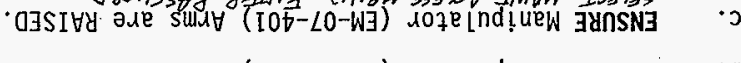

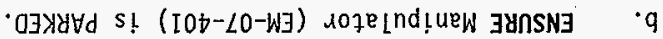

i. I. IIII

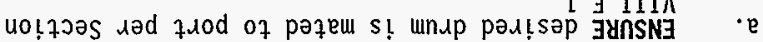

(YZOD-LO-OO-LOI) IYOd JLSHM INYITdWOJ NJdO 'Z

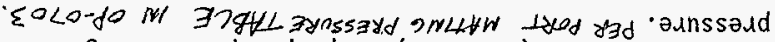

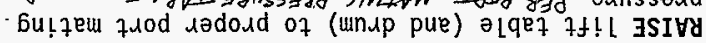

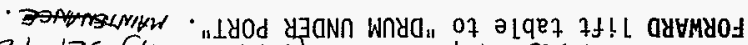

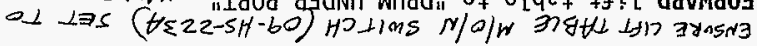




\section{WRAP 1 PLANT OPERATING PROCEDURE}

h. SELECT "EXTEND SWAGING HEADS".

i. VERIFY disp1ay "SWAGING HEADS EXTENDED".

j. SELECT "TURN OFF VACUUM".

k. VERIFY display "TURN ON VACUUM" (E.G., "VACUUM GENERATED" not displayed.)

1. SELECT "LOCK PORT".

m. VERIFY display "PORT LOCKED".

$n$. SELECT "MAINT MAIN MENU".

o. VERIFY disp1ay "MAINTENANCE MAIN MENU".

4. REMOVE DRUM FROM COMPLIANT WASTE PORT (107-D0-07-402A)

a. ENSURE port is CLOSED and LOCKED per Section VIII.E.3.

b. LOWER lift table (107-LT-09-201B) to "AGV LOAD HEIGHT".

c. REVERSE lift table to "DRUM AT AGV END".

F. NON-COMPLIANT WASTE PORT (107-D0-07-402B) MANUAL OPERATIONS

1. MATE DRUM TO NON-COMPLIANT WASTE PORT (107-D0-07-402B)

a. ENSURE desired drum is on lift table (107-LT-09-201A).

b. AND OUTER LIS ARE

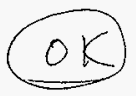

b. ENSURE drum $\mathrm{cl}$ amp band $\mathrm{A}+\mathrm{s}$ removed.

c. ENSURE UFT TABLE M/ON SWUTCH CO 9- HS-221A

d. RAISE lift table to proper port mating pressure. PeR PORT MATNC

2. OPEN NON-COMPLIANT WASTE PORT (107-D0-07-402B)

a. ENSURE desired drum is mated to port.

b. ENSURE Manipulator (EM-07-401) is PARKED.

c. ENSURE Manipulator (EM-07-401) Arms are RAISED.

SELECT MAINT. ACCESS MENO. ENTER PASSIWORD.

d. SELECT "PORT D0-07-402B MAINTENANCE MENU".

e. VERIFY display "PORT D0-07-402B MAINTENANCE MENU".

f. SELECT "TURN ON VACUUM".

g. VERIFY display "VACUUM GENERATED".

h. SELECT "UNLOCK PORT".

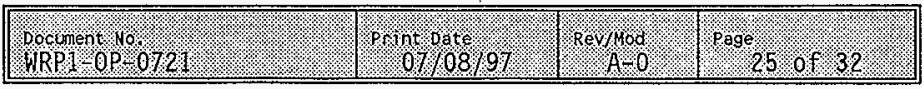


i. VERIFY display "PORT UNLOCKED".

$j . \quad$ SELECT "RAISE DOOR".

k. VERIFY display "DOOR RAISED":

1. SELECT "OPEN PORT".

m. VERIFY dispTay "PORT OPEN".

n. VERIFY Compliant Waste Port (107-D0-07-402B) is open.

o. SELECT "MAINT MAIN MENU".

3. CLOSE NON-COMPLIANT WASTE PORT (107-D0-07-402B)

a. ENSURE Manipulator (EM-07-401) is PARKED.

b. SELECT "PORT D0-07-402B MAINTENANCE MENU" .

c. VERIFY display "PORT DO-07-402B MAINTENANCE MENU".

d. SELECT "CLOSE PORT".

e. VERIFY dispTay "PORT CLOSED".

f. SELECT "LOWER DOOR".

g. VERIFY display "DOOR LOWERED".

h. SELECT "EXTEND SWAGING HEADS".

- i. VERIFY display "SWAGING HEADS EXTENDED".

j. SELECT "TURN OFF VACUUM".

k. VERIFY display "TURN ON VACUUM" (E.G., "VACUUM GENERATED" not displayed.)

T. SELECT "LOCK PORT".

m. VERIFY display "PORT LOCKED".

n. SELECT "MAINT MAIN MENU".

o. VERIFY display "MAINTENANCE MAIN MENU".

4. REMOVE DRUM FROM NON-COMPLIANT WASTE PORT (107-D0-07-402B)

a. ENSURE port is CLOSED and LOCKED per Section VIII.F.3.

b. LOWER 1 ift tab7e (107-LT-09-201A) to "AGV LOAD HEIGHT".

c. REVERSE lift table to "DRUM AT AGV END".

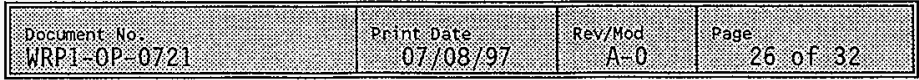




\section{WRAP 1 PLANT OPERATING PROCEDURE}

\section{ATTACHMENT 1: RWM GLOVEBOX HOIST CONTROL FUNCTIONS}

NOTE - Controllers for $L L W / R W M$ and TRU/RWM gloveboxes are the same except for equipment number.

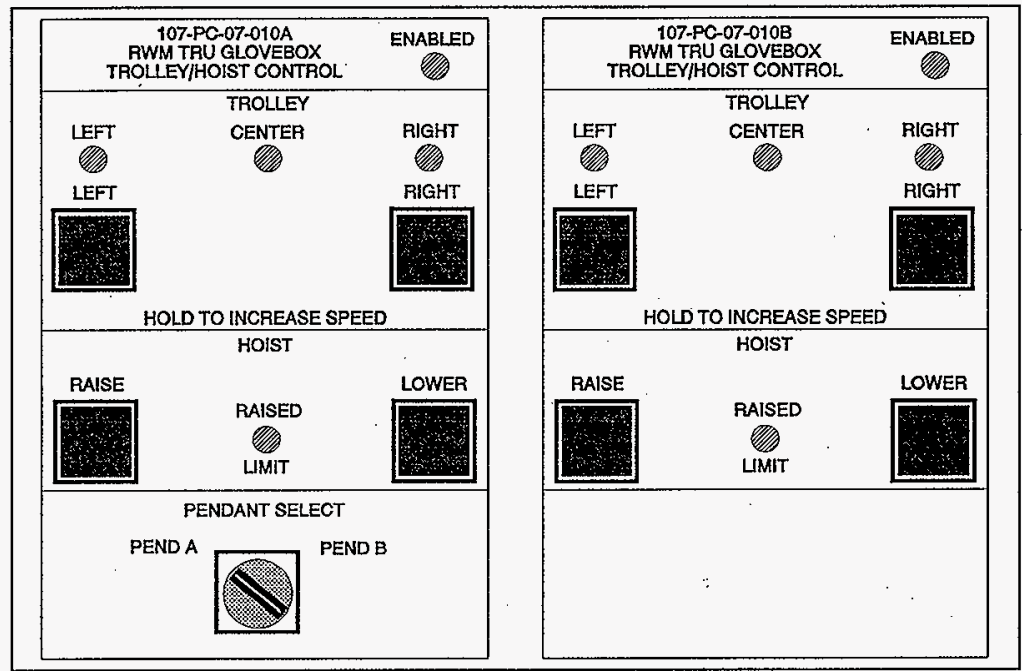

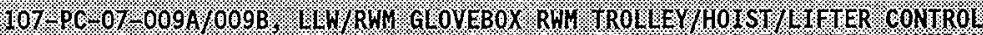

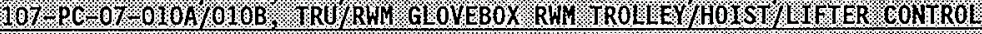
GONTROL SHITCHES

\begin{tabular}{|c|l}
\hline SMITCH & FUNCTION \\
\hline LEFT / RIGHT & MOVE HOIST TROLLEY LEFT / RIGHT \\
\hline RAISE / LOWER & RAISE / LOWER HOIST \\
\hline
\end{tabular}

PENDANT SELECT $\left(^{*}\right)$ SELECTS ACTIVE CONTROL 107-PC-07-010A or 010B

NOTE (6). Actrve control is selected at operator discrettion.

\section{INDICATIORS}

\begin{tabular}{|c|l|}
\hline LEFT & HOIST LOCATED ABOVE RWM TRANSFER PORT 107-D0-07-402A \\
\hline CENTER & HOIST LOCATED ABOVE RHM TRANSFER PORT 107-D0-07-402B \\
\hline RIGHT & HOIST LOCATED ABOVE RWM TRANSFER PORT 107-D0-07-401 \\
\hline RAISED LIMIT & HOIST RAISED TO UPPER HEIGHT LIMIT \\
\hline
\end{tabular}

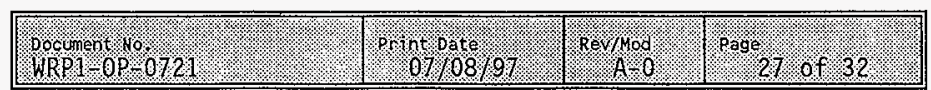




\section{WRAP 1 PLANT OPERATING PROCEDURE}

\section{ATTACHMENT 2: GLOVEBOX INTERLOCK SIGNALS}

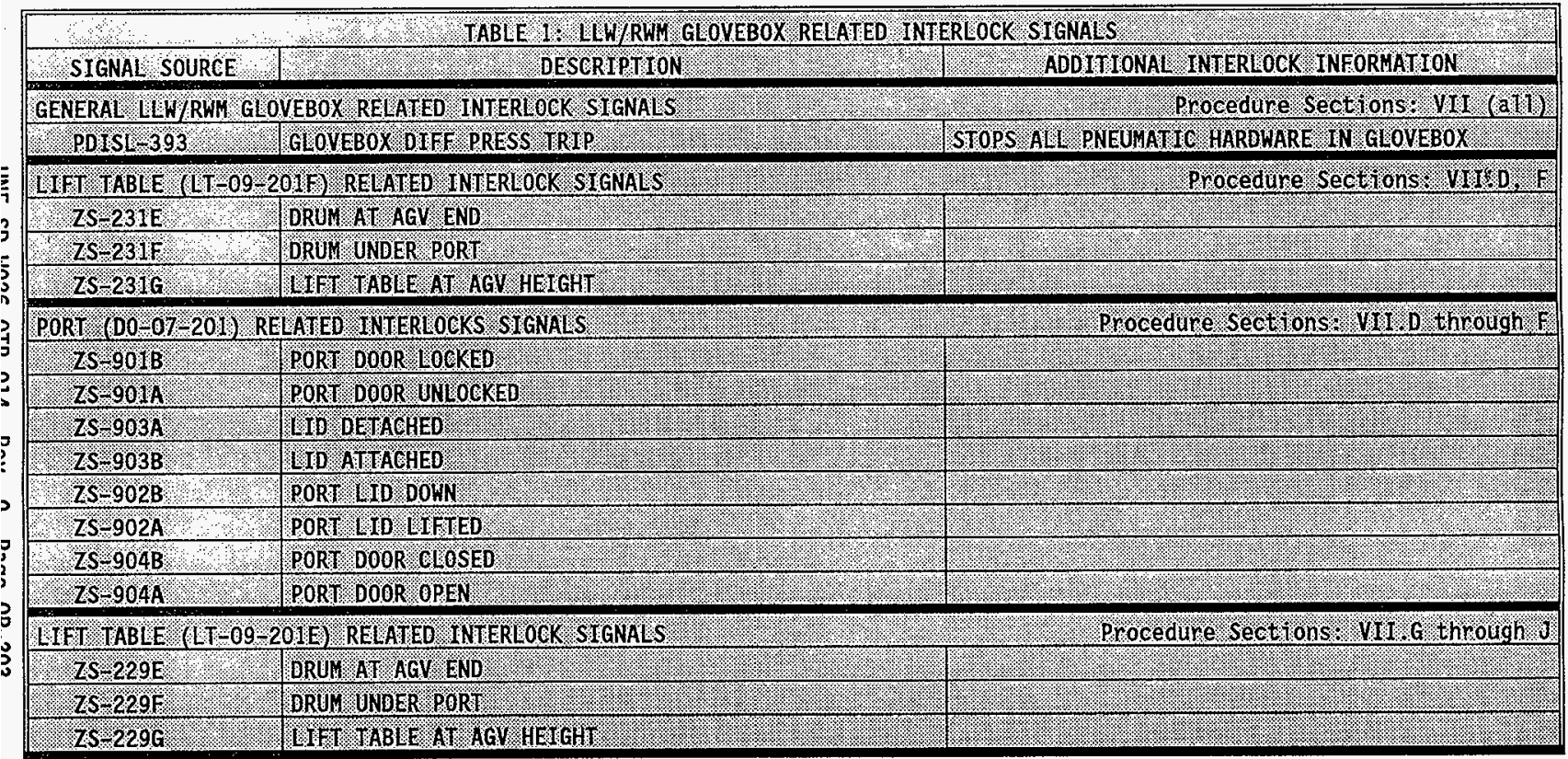

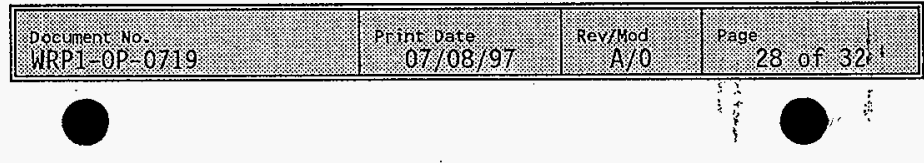




\section{WRAP 1 PLANT OPERATING PROCEDURE}

TABLE 1. LW/RWH GLOVEBOX RELATED INIERLOGK SIGNALS

STGNAL SOURCE DESCRIPTIION

ADUITIONAL INIERLOCK INEORMATLON

PORT (DO-07-202) RELATED INTERLOCR SIGNAES ZSC 908A \& B 7 DORT CLOSED ANO LOCKED

$2 S-907.7$ PORT DRUM L TD IN ROSITION

ZS-900A 2 P PORT DRUH DOOR CLOSED

ZSS $900 \mathrm{~B}$ ? RORT DRUH IN POSIMTON

\begin{tabular}{l|l}
$2 \mathrm{~S}=900 \mathrm{O}$ & PORI DRUS DODR OPEN
\end{tabular}

\begin{tabular}{l|ll}
$2 \mathrm{SC} .909$ & PORT DOOR DOWN \\
\hline
\end{tabular}

250-909

PS -900

PORT DOOR ROTATED

PORT LID VACUUM

LIFT TABLE (LT-0.9-2O1D) RELAIED INTERLOEK SIGNALS

Procedure Sectilons: NM. A through N

ZS-22,7E DRUN AT AGV END

ZS $227 \mathrm{~F}$ DRUI UNDER PORT

2S: $227 \mathrm{G}$ WITT TABLE AT AGV HETGHI

PORT (DO-07-203) RELATED INIERLOCK ST GNALS Procedure Sectilons: VII.G through J 2SC 910 A \& B 1 PORT CLOSED AND LOCKED

ZS -913 ४ 1 PORT DRUM IID IN POSITL ION

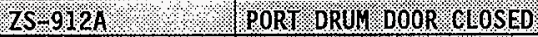

ZS-912B 4 PORT DRUM W ROSITION

ZS-912C \थ PORT DRUM DOOOR OPEN

ZSC. 911

ZSO -911

CORT DOOR DOWN

BS: 901

TORT D.0R RORATED

RORT IID YACUUH

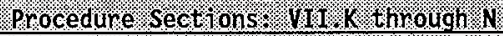

IRP $10.0 \mathrm{P}-0.1 \%$

Poto

$07 \% 089$

80.100.

80 

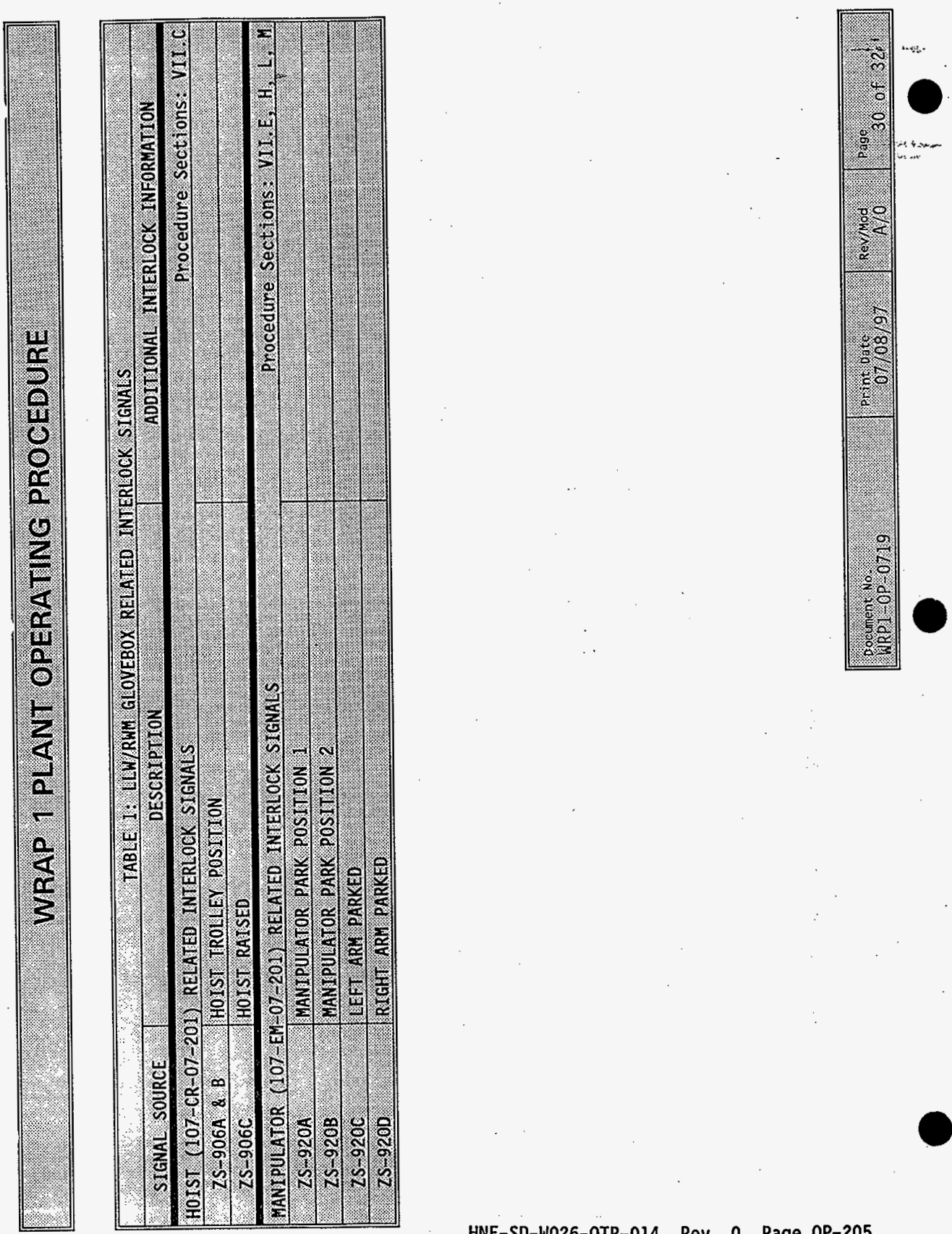

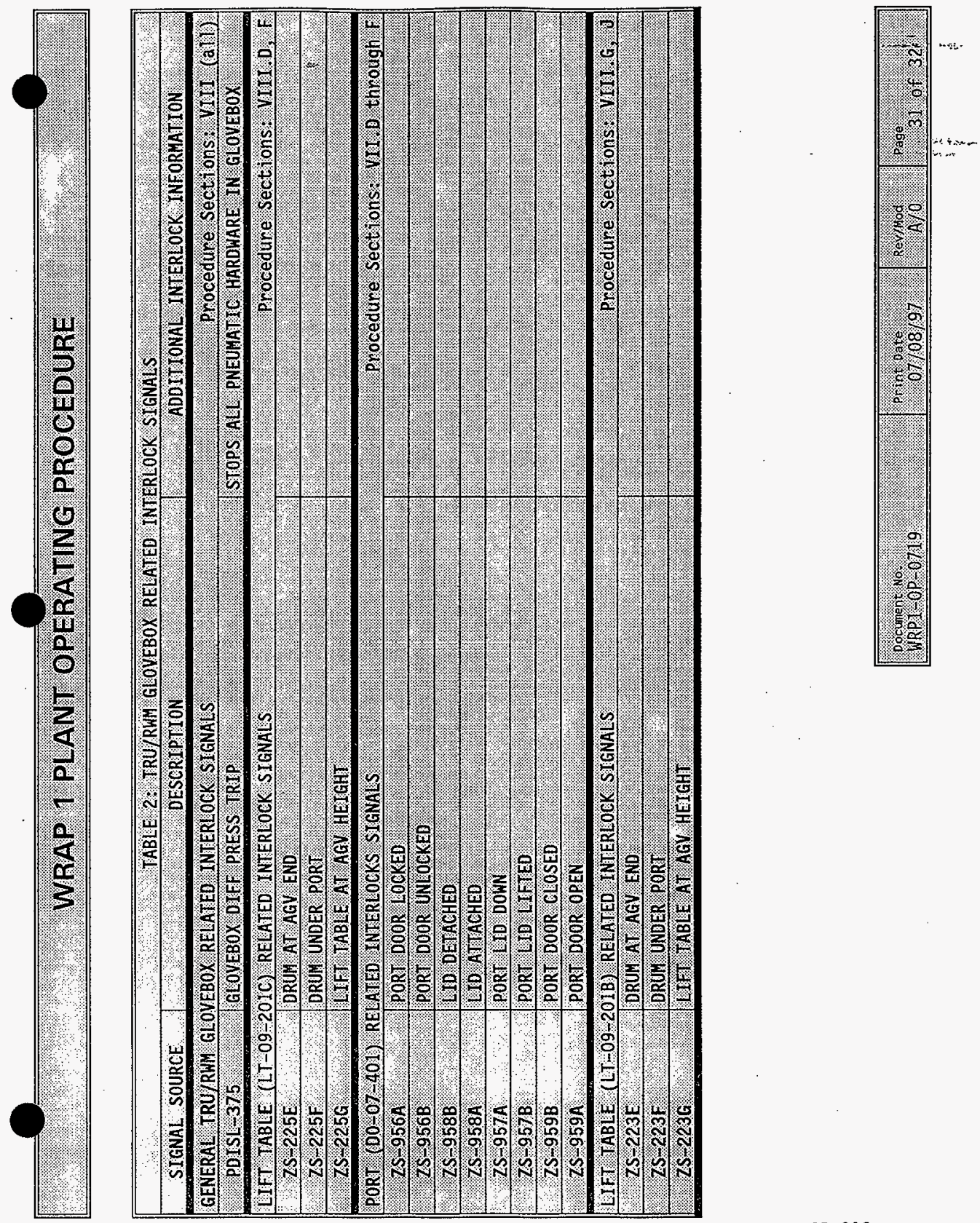


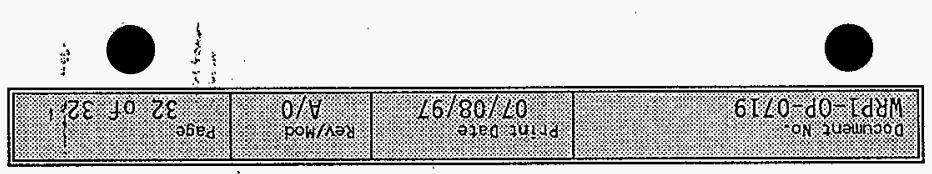

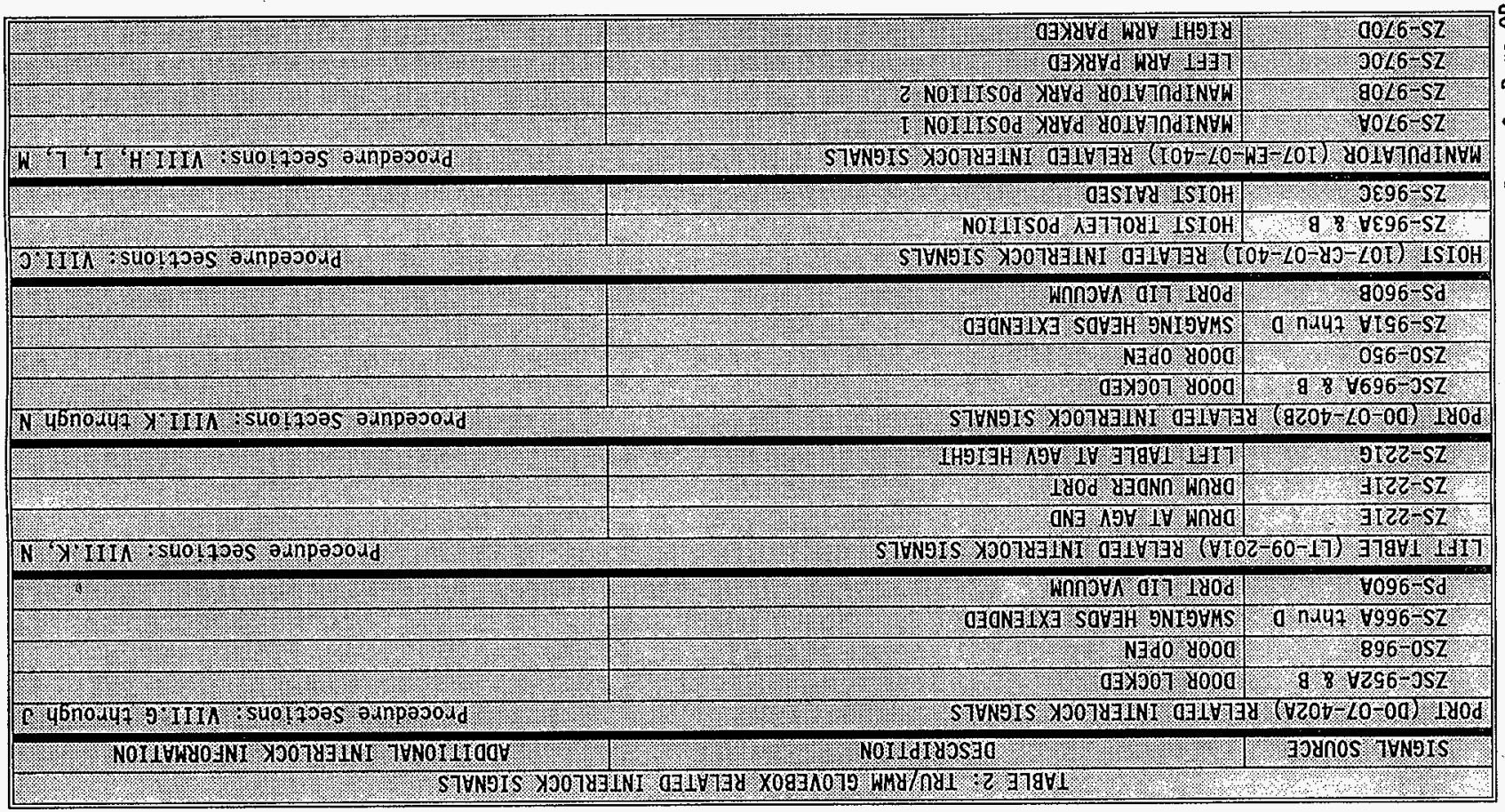

\section{ayn@}




\section{WASTE RECEIVING AND PROCESSING (WRAP) PLANT OPERATING PROCEDURE}

STSTEMT: PROCESS

\section{RESTART RWM GLOVEBOX OPERATION}

\section{SYSTEM DESCRIPTION}

This procedure provides instructions for restart of the Low Level Waste (LLW) and Transuranic (TRU), Restricted Waste Management (RWM) Gloveboxes after Emergency Stop, Fire Alarm, Power Outage, Equipment Failure, Low Glovebox Pressure or other abnormal shutdown.

Emergency Stop or other abnorma? shutdown of the gloveboxes may Teave various glovebox equipment including entry/exit ports, hoists, lift tables etc. in an abnormal state (i.e., partially open/closed, seals deflated or inflated at the wrong time, etc.). Prior to restart, the condition of all glovebox equipment must be analyzed, and as required, returned to normal initialization status. Because of numerous interlocks and other safety features, return of most equipment to the proper position must be accomplished manually through use of the Maintenance Mode menus on the glovebox Operator Interface Units (OIUs), Lift Table Controllers and other operator control stations.

\section{REFERENCE DOCUMENTS}

HSRCM-1, Hanford Site Radiological Control Manual. WRP1-0P-0721, RWM Gloveboxes Manual Operation. WRP1-0P-0717, LLW/RWM Glovebox Operation. WRPI-0P-0728, IRU/RWM Process Glovebox Operation.

\section{PRESTART CONDITIONS}

All personnel performing this procedure shall be qualified in accordance with WHC-CM-5-34, Section 1.8, "Training Plan," and on-the-job training.

Process area Heating, Ventilation, Air Conditioning (HVAC) in OPERATION. Applicable portions of plant electrical system in OPERATION.

Compressed air system in OPERATION.

\begin{tabular}{|c|c|c|c|c|c|}
\hline 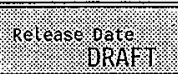 & print orter & apror pessol & $\begin{array}{l}\text { orocurert } \\
\text { HRP }\end{array}$ & Revinod & 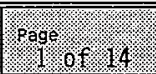 \\
\hline
\end{tabular}




\section{WRAP 1 PLANT OPERATING PROCEDURE}

\section{SAFETY}

Potential hazards, such as lifting heavy containers or handling sharp objects present in waste containers, must be mitigated by protective equipment, procedures, and administrative controls to ensure acceptable-risk operating conditions.

Consult with Operations Management and Industrial Hygiene, and refer to the Job Hazards Analysis (JHA), Radiological Work Permit (RWP) and Material Safety Data Sheets (MSDS) to determine required Personal Protective Equipment (PPE).

This procedure requires accessing gloveboxes through gloveports on a regular basis. Operators shall perform self survey upon exiting gloveport after each use. Radiological control Technician (RCT) shall be contacted anytime self survey limits are exceeded.

Warning - Use care to prevent injury. Possible safety hazards include:

- Tripping/Slipping

- Injuries to the hands at glovebox ports

- Radiological Contamination

- Interference with the Automatic Guided Vehicle (AGV)

When an OIU is in maintenance mode, most interlocks and safety features are bypassed. This allows equipment to be manipulated to a desired state to satisfy operational needs. It is also possible to damage equipment, and/or. bypass safety restrictions such as glovebox containment. Caution must be exercised to prevent unwanted violation of operational safety requirements or personnel injury.

\section{TOOLS AND SUPPLIES}

None.

\begin{tabular}{|c|c|c|c|}
\hline 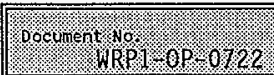 & 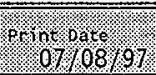 & \%oryog & \% \\
\hline
\end{tabular}




\section{TABLE OF CONTENTS}

PAGE

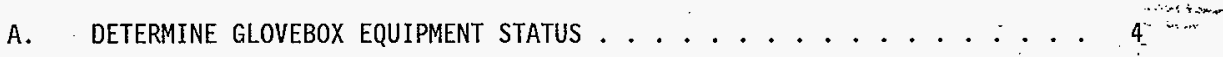

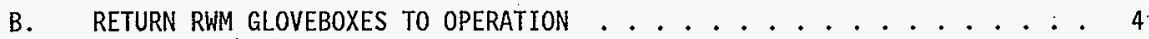
ATTACHMENTS

ATTACHMENT 1 : LLW/RWM GLOVEBOX EQUIPMENT STATUS . . . . . . . . . 5

ATTACHMENT 2 : TRU/RUM GLOVEBOX EQUIPMENT STATUS $\ldots \ldots \ldots \ldots \ldots \ldots$

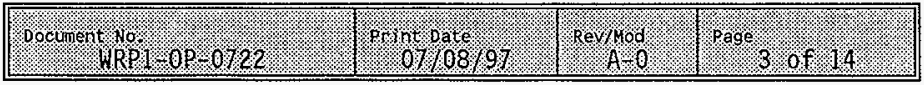




\section{WRAP 1 PLANT OPERATING PROCEDURE}

\section{PROCEDURE}

NOTE - Equipment status is determined by visual observation of equipment and status indicating lights as applicable.

\section{A. DETERMINE GLOVEBOX EQUIPMENT STATUS}

1. If restarting $L L W / R W M$ Glovebox,

PERFORM Attachment 1 LLW/RWM Glovebox Equipment Status.

2. IF restarting TRU/RWM Glovebox,

PERFORM Attachment 2, TRU/RWM Glovebox Equipment Status.

\section{B. RETURN RWM GLOVEBOXES TO OPERATION}

1. SELECT attachment for glovebox to be returned to service:

a. ATTACHMENT 1: LLW/RWM GLOVEBOX EQUIPMENT STATUS

b. ATTACHMENT 2: TRU/RWM GLOVEBOX EQUIPMENT STATUS

2. COMPARE AS FOUND status on selected attachment to the following:

a. INITIALIZE POSITION listed on selected attachment:

b. Interlock information in applicable section of WRP1-OP-0721, RWM Gloveboxes Manual Operation.

c. Operational sequencing in applicable operating procedure:

- WRP1-OP-0717, LLW/RWM Glovebox 0peration.

- WRP1-OP-0728, TRU/RWM Process Glovebox Operation

3. RECORD required equipment corrections in COMMENTS section of selected attachment.

4. GO TO appiicable section(s) of WRP1-OP-0721 and PERFORM necessary actions to return equipment to desired status.

5. RETURN glovebox 0IU to automatic operation per WRP1-OP-0721.

6. REPEAT Steps VII.B.1 through VII.B.5 as required for additional gTovebox. 


\section{WRAP 1 PLANT OPERATING PROCEDURE}

\section{ATTACHMENT 1: LLW/RWM GLOVEBOX EQUIPMENT STATUS}

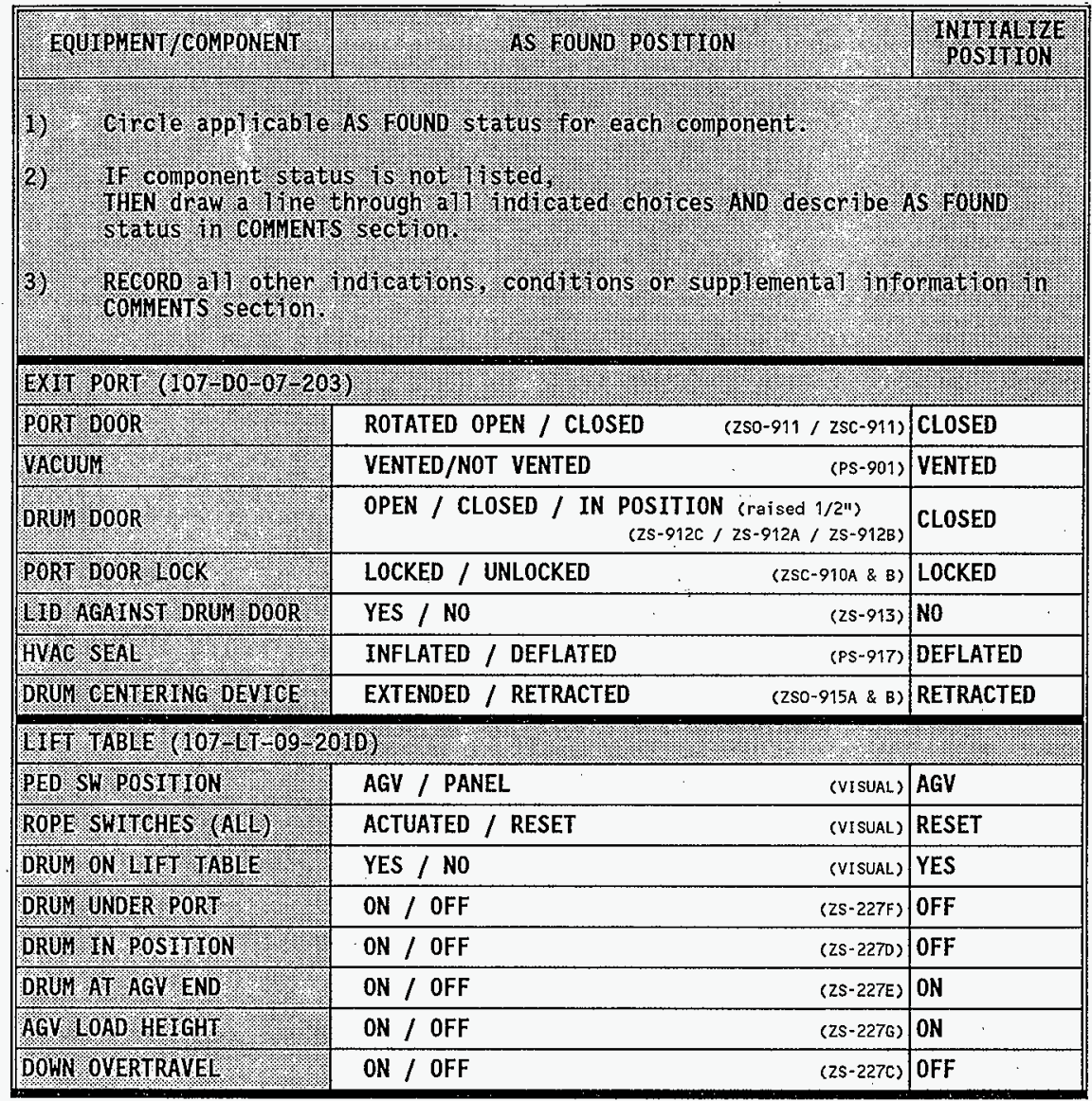

\begin{tabular}{|c|c|c|c|}
\hline 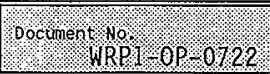 & 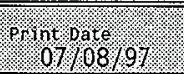 & Revolo & Page $/ 5.6 \%$ of 14 \\
\hline
\end{tabular}

HNF-SD-W026-0TR-014, Rev, 0, Page OP-212 


\section{WRAP 1 PLANT OPERATING PROCEDURE}

\section{ATTACHMENT 1: LLW/RWM GLOVEBOX EQUIPMENT STATUS}

\begin{tabular}{|c|c|c|}
\hline EQUTPMEN//COMPONENT: & AS ROUNO POST $110 \mathrm{~N}$ & $\begin{array}{l}\text { INITHALER } \\
\text { Position }\end{array}$ \\
\hline \multicolumn{3}{|c|}{ 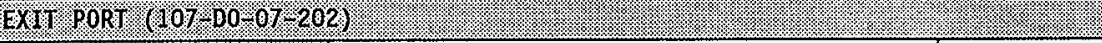 } \\
\hline PORT DOOR & ROTATED OPEN / CLOSED (2s0-909 / 2SC-909) & CLOSED \\
\hline VAcouM : & VENTED / NOT VENTED & VENTED \\
\hline DRUH DOOR : : : & $\begin{array}{l}\text { OPEN / CLOSED / IN POSITION (raised 1/2") } \\
\text { (ZS-900C/ZS-900A / ZS-900B) }\end{array}$ & CLOSED \\
\hline PORI DOOR LOCK : : & LOCKED / UNLOCKED & LOCKED \\
\hline 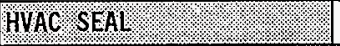 & INFLATED / DEFLATED & DEFLATED \\
\hline YD AGAINST DRUH DOOR & YES / NO & N0 \\
\hline DRUM CENTERING DEYICE & EXTENDED / RETRACTED & RETRACTED \\
\hline \multicolumn{3}{|c|}{ 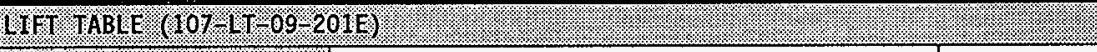 } \\
\hline PED SH POSTITON : & AGV / PANEL & AGV \\
\hline ROPE SHITCHES (ALI) & ACTUATED / RESET & RESET \\
\hline DRUY ON $M$ IFT TABLE: & YES / NO & YES \\
\hline DRUM UNDER PORT: & ON / OFF & OFF \\
\hline DRUM IN POSTITION: & ON / OFF & OFF \\
\hline DRUH AT AGY ENB & ON / OFF & ON \\
\hline AGV LOAD HEIGH & ON / OFF & ON \\
\hline DOWN OVERTRAVEL: : & ON / OFF & OFF \\
\hline \multicolumn{3}{|c|}{ RWM IRANSFER PORT $(107-00=07-201)$ : } \\
\hline DOUBLE ID : & (zS-904A / zs-904B) & CLOSE \\
\hline DOUBEE I TD LOCK & OPEN / CLOSE & CLOSE \\
\hline DOUBLE I ID VERT MCAY : & $(Z S-902 A / Z S-902 B)$ & DOWN \\
\hline WD GRIPPER & HOLD / RELEASE & RELEASE \\
\hline
\end{tabular}

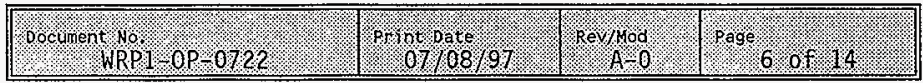

HNF-SD-W026-0TR-014, Rev. 0, Page OP-213 


\section{WRAP 1 PLANT OPERATING PROCEDURE}

\section{ATTACHMENT 1: LLW/RWM GLOVEBOX EQUIPMENT STATUS}

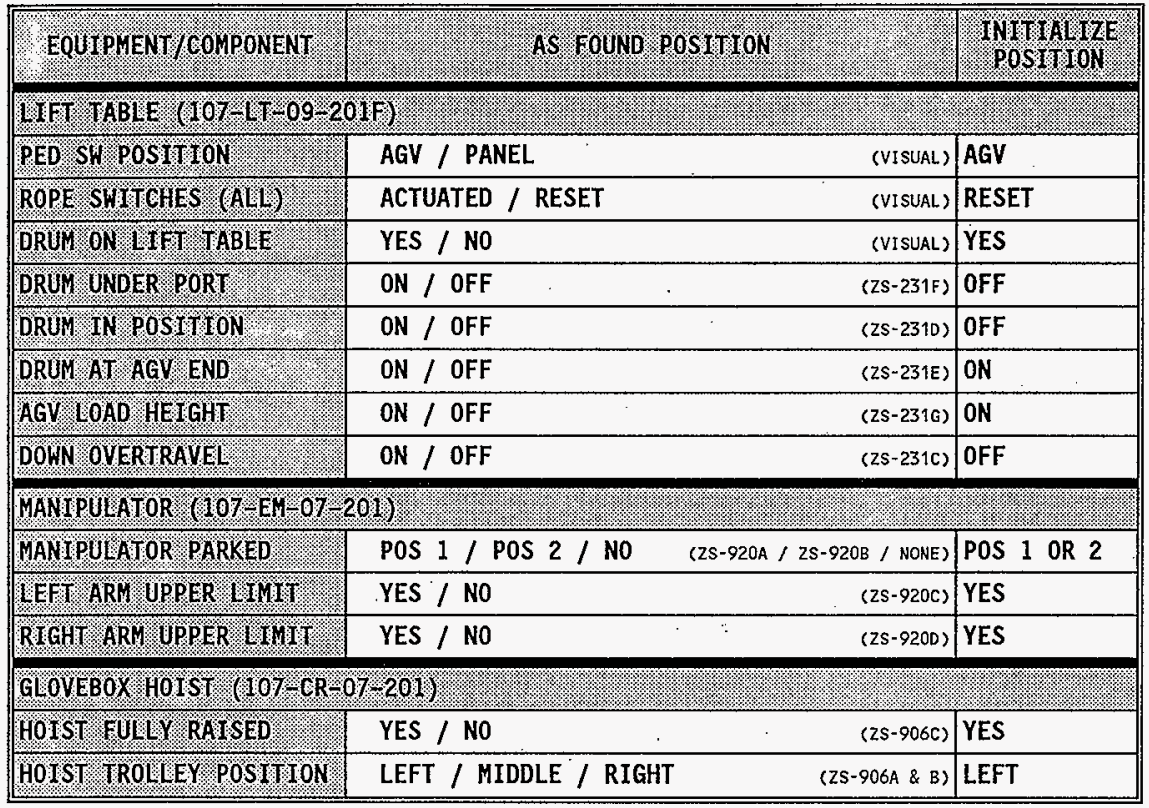

\begin{tabular}{|c|c|c|c|}
\hline 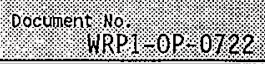 & pron pate 6 & 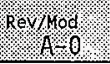 & 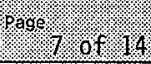 \\
\hline
\end{tabular}




\section{WRAP 1 PLANT OPERATING PROCEDURE}

\section{ATTACHMENT 1: LLW/RWM GLOVEBOX EQUIPMENT STATUS}

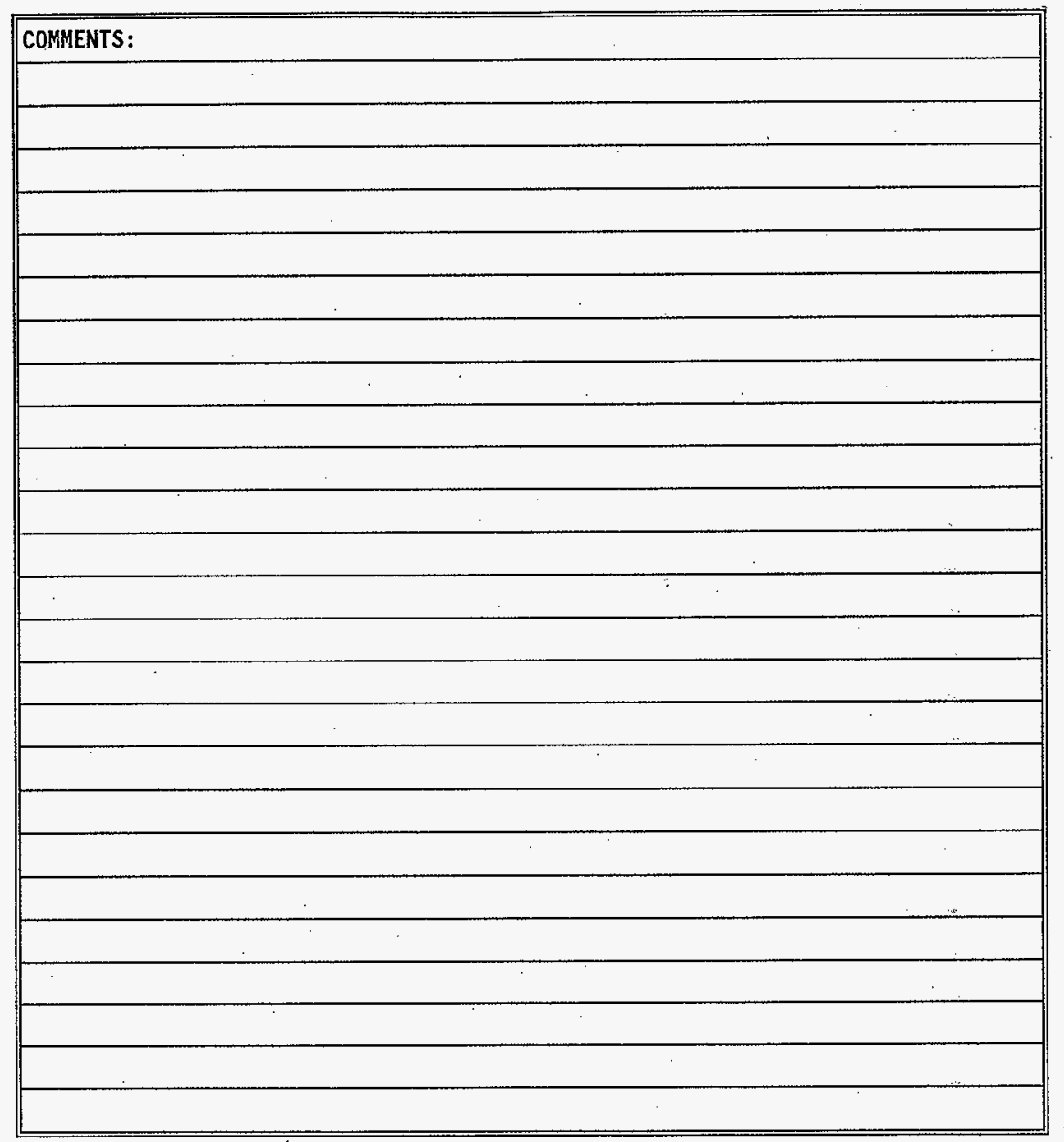

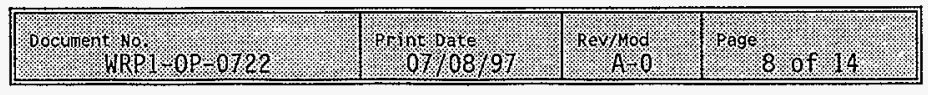




\section{ATTACHMENT 1: LLW/RWM GLOVEBOX EQUIPMENT STATUS}

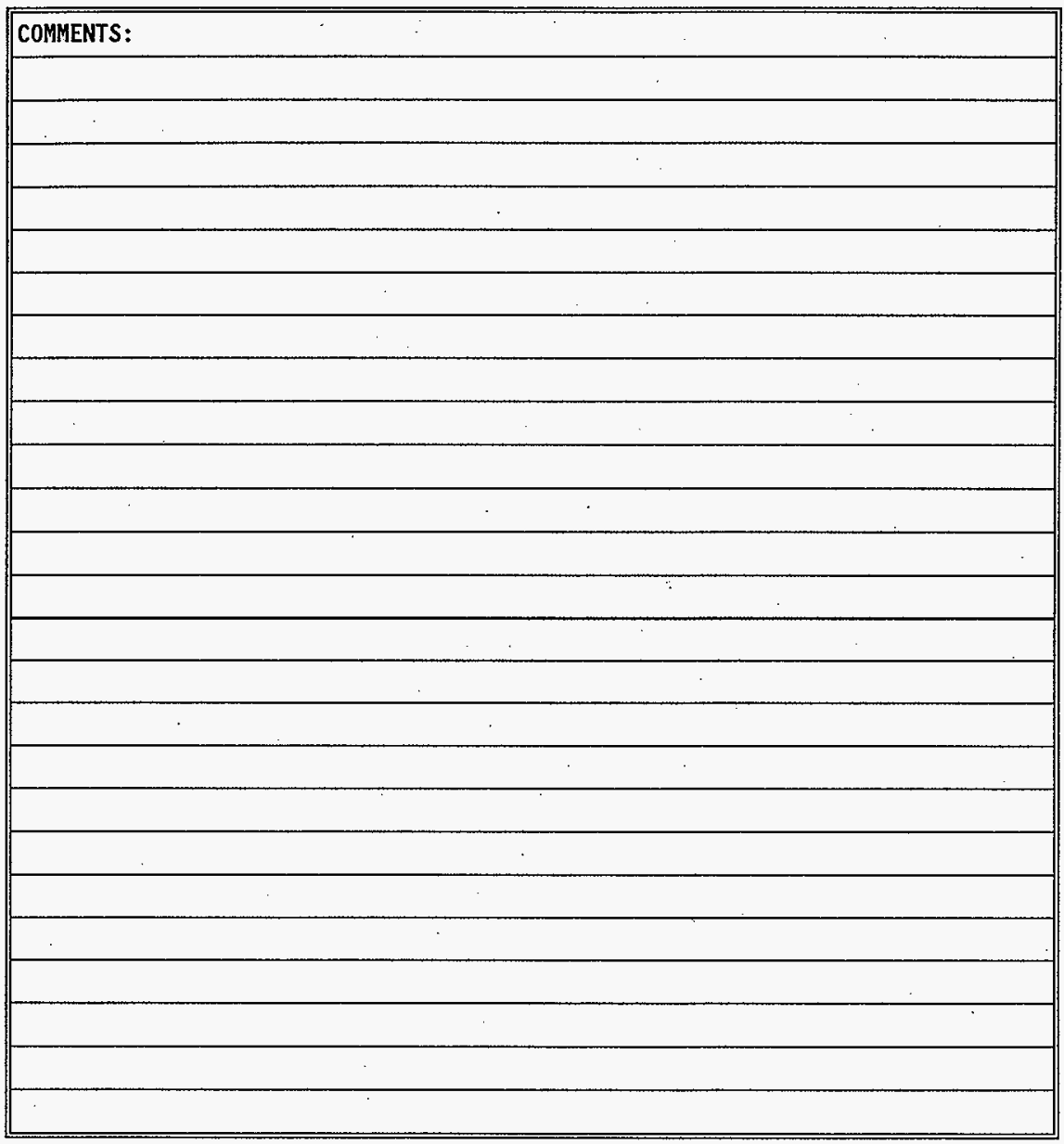




\section{WRAP 1 PLANT OPERATING PROCEDURE}

\section{ATTACHMENT 2: TRU/RWM GLOVEBOX EQUIPMENT STATUS}

\begin{tabular}{|c|c|c|}
\hline EQUIPUENT COMPONENI: & AS FOOND POSTITION & \\
\hline \multicolumn{3}{|c|}{ 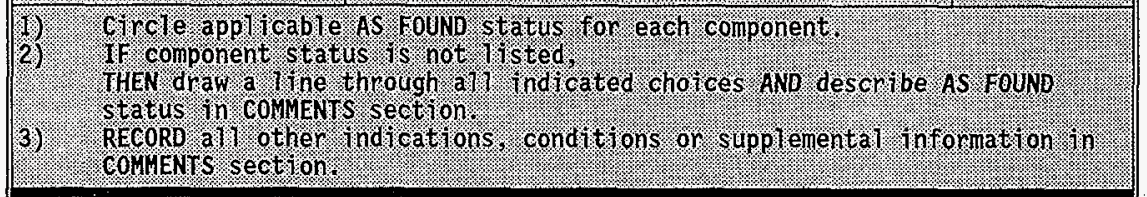 } \\
\hline \multicolumn{3}{|c|}{ EXIT PORT ( $107-00-07.402 \mathrm{~B})$} \\
\hline PORT DOOR: & $\begin{array}{l}\text { TRANSLATED OPEN / TRANSLATED (CLOSED) } \\
(250-950 \& 2 \mathrm{zC}-950)\end{array}$ & $\begin{array}{l}\text { TRAN } \\
\text { CLOS }\end{array}$ \\
\hline PORT $000 \mathrm{R}:=2:=0$ & RAISED KLOWERED & LOWE \\
\hline PORT DOOR LOCK : : & LOCKED / UNLOCKED & LOCK \\
\hline WHAGING HEADS : & EXTENDED KRETRACTED & RETh \\
\hline IO VACOUM : & OFF \& VENTED/ VACUUM ON & OFF \& VENTE \\
\hline \multicolumn{3}{|c|}{ 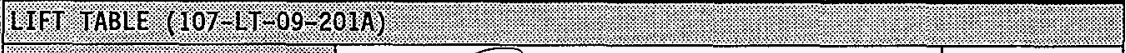 } \\
\hline PED SH. POSTITON : & AGV / PANEL (VISUAL) & AGV \\
\hline Rope SHIMH & ACTUATED / RESET & RESET: \\
\hline DRUM ON IIF & GES / NO & YES \\
\hline DRUM UNOER PORT & ON $/$ OFE & OFF \\
\hline DRUM IN POSTION & ON / OFF & OFF \\
\hline DRUM AT AGV END : & (ON)/ OFF & ON \\
\hline AGV LOAD HETGHT & (ON) OFF & ON \\
\hline DOWN OVERTRAVL : & ON / OED & OFF \\
\hline \multicolumn{3}{|c|}{ EXIT PORT $(107-00-07-4024)$} \\
\hline PORI DOOR. & $\begin{array}{l}\text { TRANSLATED OPEN / TRANSLATED CLLSED) } \\
\text { (2S0-968 \& 2SC-968) }\end{array}$ & \\
\hline PORT DOOR. & RAISED') / LOWERED & \\
\hline PORT DOOR LOCK & LOCKED / (NLOCKED & \\
\hline SWAGING HEADS : & EXTENDED / RETRACTED & RETRACTED \\
\hline ID VACUUM & OFF \& VENTED / VACUUM ON & OFF \& VENTED \\
\hline
\end{tabular}




\section{WRAP 1 PLANT OPERATING PROCEDURE}

\section{ATTACHMENT 2: TRU/RWM GLOVEBOX EQUIPMENT STATUS}

\begin{tabular}{|c|c|c|}
\hline EQUIPNENTYOONPONENI & MS. FoUNO POSI ION & W WOS \\
\hline \multicolumn{3}{|c|}{ 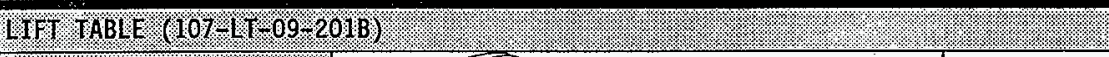 } \\
\hline PED SH POSTTON & AGV / PANEL & AGV \\
\hline ROPE SHITHES (A) & ACTUATED / RESET & RESET \\
\hline DRUM ON KTF TABLE & YES / NO & YES \\
\hline DRUM YNDER PORT & (0N OFF & OFF \\
\hline DRUM IN POSITION & ON / OFF & OFF \\
\hline DRUM $/ \mathrm{AG}$ AG END & ON / OFB & ON \\
\hline AGV YOAD Y WGHT & ON / OFF & ON \\
\hline DOWN OVERTRAVEL & ON /OFE & OFF \\
\hline \multicolumn{3}{|c|}{ RWM TRANSFER PORT $(10 \%$ Do-07 401$)$} \\
\hline DOUBLE U1D . & CPEN / CLOSE & CLOSE \\
\hline DOUBLE LID YOOK. & (OPEN / CLOSE & CLOSE \\
\hline DOUBLE WIO VERI CAL. & (UP) / DOWN & DOWN \\
\hline MOV GRPPER & HOLD / RELEASE & RELEASE \\
\hline \multicolumn{3}{|c|}{ 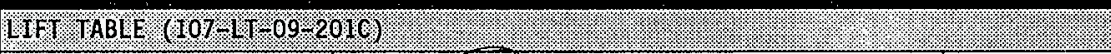 } \\
\hline PED SW POSTITON & AGV / PANED & AGV \\
\hline ROPE SWITCHES (AL) & ACTUATED / RESET & RESET \\
\hline DROM ON III I TABLE & (YES) / NO & YES \\
\hline QRUM UNDER PORT & ON $/ \mathrm{FF}$ & OFF \\
\hline DRUM IN POSTI TON. & ON OFF & OFF \\
\hline DRUM AT AGV END, & ON $/$ OFF & ON \\
\hline AGV $10 A D$ HEIGHT & ON / OFE & ON \\
\hline DOWN OVERTRAVEL & ON $/$ OFE & OFF \\
\hline \multicolumn{3}{|c|}{ 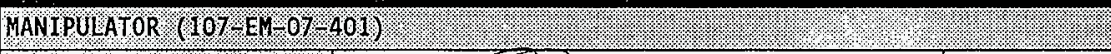 } \\
\hline MANIPULATOR PARKED & POS $1 / \operatorname{POS} 2 /$ NO & POS 1 OR 2 \\
\hline LEFT ARM UPPER YIMIT & XES / NO & YES \\
\hline RIGHT ARM UPPER I IMIT & (TES)/ NO & YES \\
\hline
\end{tabular}

\begin{tabular}{|c|c|c|c|}
\hline bocunent Wo & Proptote 0708 & $\begin{array}{l}\text { Rev/rod } \\
\text { A }\end{array}$ & rage 1 of $/ 4$ \\
\hline
\end{tabular}

HNF-SD-W026-0TR-014, Rev. O, Page OP-218. 
ATTACHMENT 2: TRU/RWM GLOVEBOX EQUIPMENT STATUS

\begin{tabular}{|c|c|c|c|}
\hline EQUTPHENT/COMPONENI & As r Founo Postriton & & 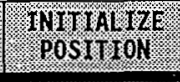 \\
\hline \multicolumn{4}{|c|}{ 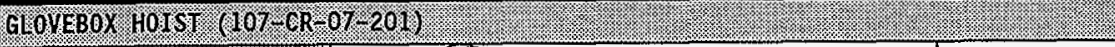 } \\
\hline HOIST EUYY RATSED & YES $K$ NO & $(z s-963 c)$ & YES \\
\hline HOLST TROLLEY pOSITION & LEFT / NIDDLE / RIGHT & (ZS-963A \& B) & LEFT \\
\hline
\end{tabular}




\section{WRAP 1 PLANT OPERATING PROCEDURE}

\section{ATTACHMENT 2: TRU/RWM GLOVEBOX EQUIPMENT STATUS}

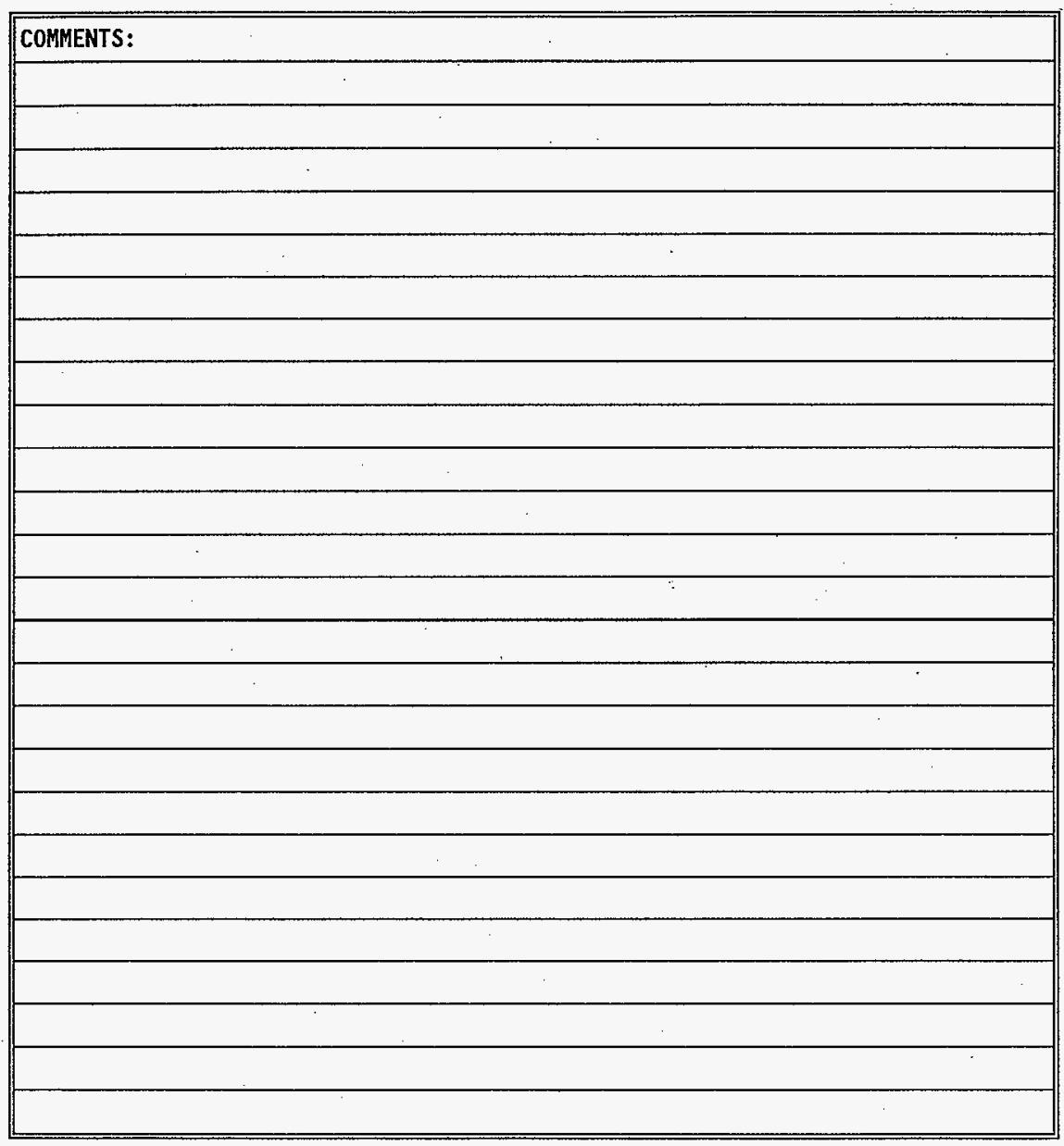




\section{ATTACHMENT 2: TRU/RWM GLOVEBOX EOUIPMENT STATUS}

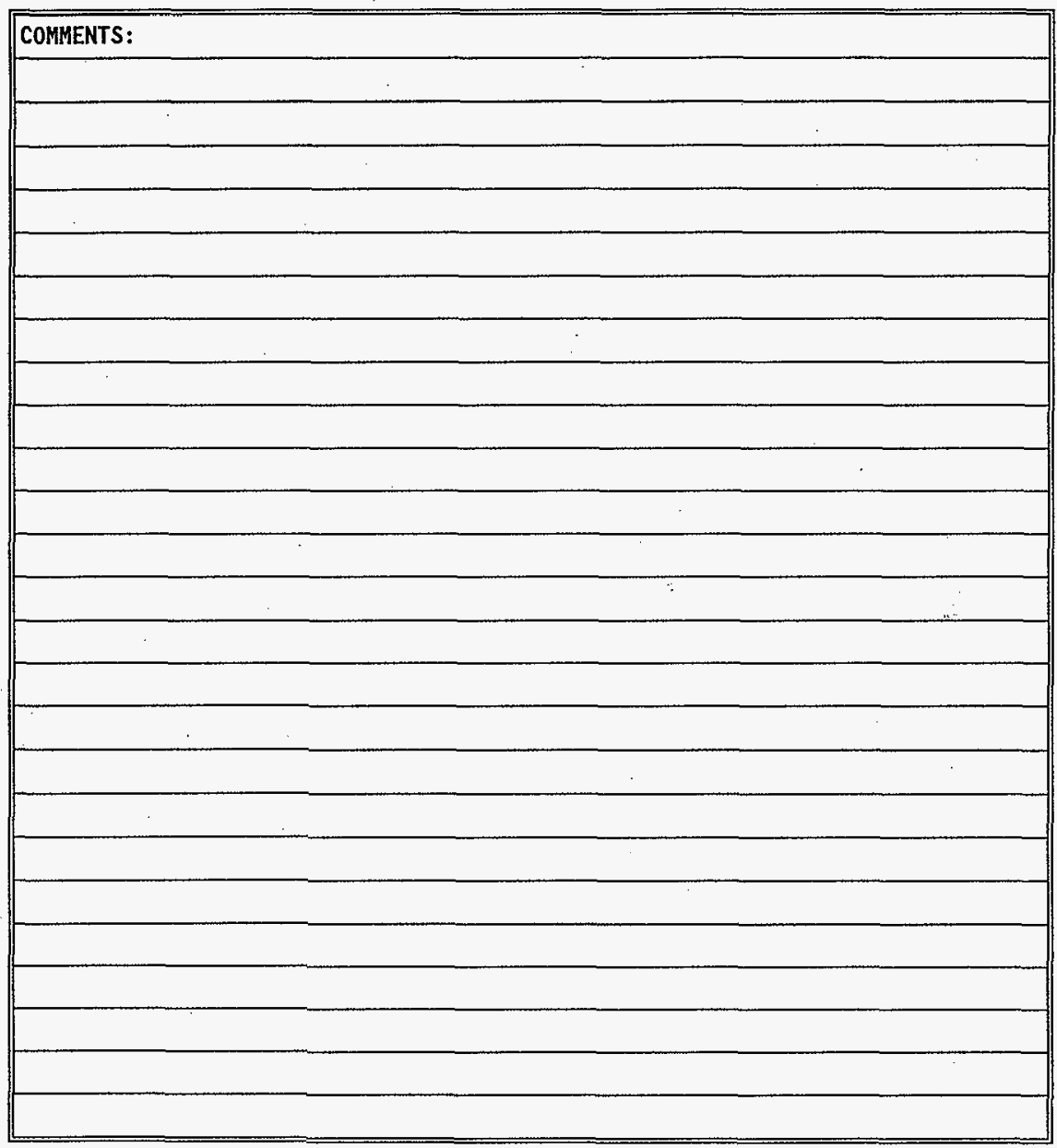




\section{WASTE RECEIVING AND PROCESSING (WRAPI PLANT OPERATING PROCEDURE \\ SYSTEH\% PROOESS}

\section{TRU/RWM GLOVEBOX OPERATION}

\section{SYSTEM DESCRIPTION}

This procedure provides instructions for operation of the Transuranic Waste, Restricted Waste Management (TRU/RWM) Glovebox (107-GB-07-401).

\section{REFERENCE DOCUMENTS}

HSRCM-1, Hanford Site Radiological Control Manual.

WRP1-0P-0703, Glovebox Lift Table Operation.

WRP1-0P-0704, GTovebox Manipulator Operation.

WRP1-0P-0708, Vent Aerosol Cans.

WRP1-0P-0710, Waste Sampling.

WRP1-0P-0711, Waste Treatment.

WRP1-0P-1708, Packaging Low-Level Waste.

WRP1-0P-1709, Packaging Mixed Waste.

IiI. PRESTART CONDITIONS

All personnel performing this procedure shall be qualified per WHC-CM-5-34, Section 1.8, "Training Plan," and on-the-job training.

Process area Heating, Ventilation, Air Conditioning (HVAC) in OPERATION. Applicable light curtains in OPERATION.

Applicable portions of plant electrical system in OPERATION.

Compressed air system in OPERATION.

Waste has been sorted in the TRU Processing glovebox and/or otherwise has been determined to be restricted. Restricted items are on a Non-Compliant Item Transfer Stand inside of a Drath and Schrader drum, 1ocated on the AGV end of Lift Table 107-LT-09-201C.

\section{SAFETY}

Potential hazards, such as lifting heavy containers or handling sharp objects present in waste containers, must be mitigated by protective equipment, procedures, and administrative controls to ensure acceptable-risk operating conditions. 
IV. SAFETY (Cont.)

Consult with Operations Management and Industrial Hygiene, and refer to the Job Hazards Analysis (JHA), Radiological Work Permit (RWP) and Material Safety Data Sheets (MSDS) to determine required Personal Protective Equjpment (PPE).

Check glovebox contents prior to startup/restart for any materials which might require special handling precautions (i.e. flammable, reactive, etc.). If problems exist, or if unsure that glovebox operation may be safely conducted, then notify Duty Operations Supervisor (DOS).

This procedure requires accessing gloveboxes through gloveports on a regular basis. Operators shall perform self survey upon exiting gloveport after each use. Radiological Control Technician (RCT) shall be contacted anytime self survey limits are exceeded.

Warning - Use care to prevent injury. Possible safety hazards inciude:

- Tripping/Slipping

- Injuries to the hands at glavebox ports

- Radiological Contamination

- Interference with the Automatic Guided VehicTe (AGV)

Waste must be segregated by hazard class to avoid dangerous reactions or explosions. If reactive, pyrophoric, or explosive materials are identified upon opening of waste packets, immediately STOP WORK and contact Duty operations Supervisor (DOS).

Aerosol cans which have contained acutely hazardous waste are placed in RWM drums only if the cans have been punctured and emptied. Contents must be captured and managed as waste. Contact Environmental Compliance Group (ECG) if questions arise concerning hazard class of waste items contained in aerosol cans.

Ensure that sharp objects and large dense objects are wrapped and packaged to minimize potential for puncturing the container and to prevent shifting during drum handling.

\section{TOOLS AND SUPPLIES}

None.

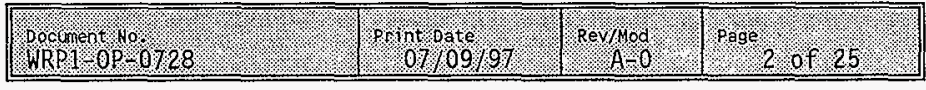




\section{WRAP 1 PLANT OPERATING PROCEDURE}

VI. TABLE OF CONTENTS

PAGE

A. INITIALIZE SYSTEM .................. 5

B. OPEN BAGLESS TRANSFER PORT $(107-D 0-07-401) \ldots \ldots \ldots$

C. REMOVE NON-COMPLIANT ITEM TRANSFER STAND FROM RWM DRUM . . . . . . . 7

D. REMOVE WASTE ITEMS FROM TRANSFER STAND FOR SORTING/SAMPLING . . . . . 7

E. REMOVE WASTE ITEMS FROM TRANSFER STAND FOR TREATMENT ....... 8

F. REMOVE SAMPLE BOTTLES FROM STC FOR TREATMENT .......... 9

G. OPEN COMPLIANT WASTE PORT $(107-D 0-07-402 A) \ldots \ldots 10$

H. OPEN NON-COMPLIANT WASTE PORT $(107-D 0-07-402 B) \ldots \ldots$ Il

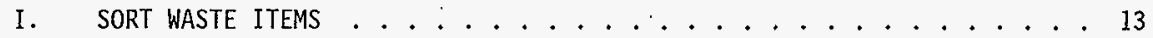

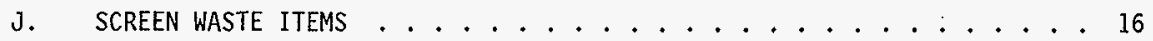

L. TREATED WASTE ITEM LOADOUT ...................... 17

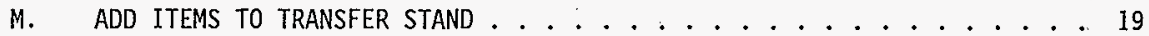

N. LOAD TRANSFER STAND INTO RWM DRUM ............... 20

0. CLOSE BAGLESS TRANSFER PORT $(107-D 0-07-401) \ldots \ldots \ldots$

P. CLOSE COMPLIANT WASTE PORT $(107-D 0-07-402 A) \ldots \ldots \ldots \ldots$

Q. CLOSE NON-COMPLIANT WASTE PORT $(107-D 0-07-402 B) \ldots \ldots . \ldots 23$ ATTACHMENTS:

ATTACHMENT 1: SORTING GLOVEBOX HOIST CONTROL FUNCTIONS . . . . . . . 25

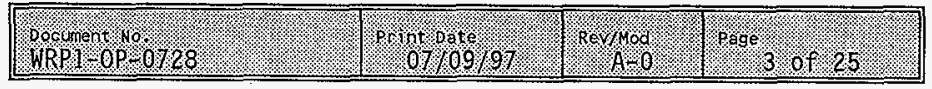




\section{WRAP 1 PLANT OPERATING PROCEDURE}

VII. PROCEDURE

NOTE 1 - Administrative hold steps are identified by letters in parentheses at the left margin of the procedure step. The direction given in the procedure step must be satisfied before work continues.

(M) - Operations management shall approve operation.

(R) - Radiological Control (RC) shall complete surveys or agree to permit continued operation.

NOTE 2 - UNLESS OTHERWISE SPECIFIED, selections and displays throughout this procedure are on TRU/RWM Glovebox Operator Interface Unit (0IU-12-106B).

NOTE 3 - A77 Data Management System (DMS) data entry, is performed on DMS Termina7 (107-TE-12-103). DMS sample treatment information is displayed on DMS display (0IU-12-106A).

NOTE 4 - A77 hoist control activities in this procedure are performed on Hoist Control (107-PC-07-010A or 010B) at operator discretion. Active controller is selected by switch on 107-PC-07-010A. Functions must be activated until associated indicator illuminates. Reference Attachment 1.

NOTE 5 - Manipulators are used throughout this procedure as required, to move and sort items in the gloveboxes. Manipulator operation is per WRP1-OP-0704, Glovebox Manipulator Operation.

NOTE 6 - Manual Lift table operation when specified throughout this procedure is performed per WRP1-0P-0703, G7ovebox Lift Table operation.

NOTE 7 - A77 scanning is performed with Portable Scanner 12-NE-303 or assigned spare. 


\section{A. INITIALIZE SYSTEM}

(PCS) When powered up, performs system initialization check and displays results on OIU Main Menu.

1. REVIEW OIU "MAIN MENU".

2. SELECT "DIAGNOSTICS MENU".

(PCS) Displays failed parameters in red, with negative messages. Passed parameters are displayed in green with positive message.
a. VERIFY display "DIAGNOSTICS MENU".
b. NOTIFY DOS.
c. SELECT "MAINT. ACCESS" on OIU.
d. ENTER MAINTENANCE access password on OIU and SELECT ENTER.
e. ACCESS MAINTENANCE MENU screens as necessary to correct indicated initialization faults.
f. WHEN all faults have been corrected, SELECT "MAIN MENU".

B. OPEN BAGLESS TRANSFER PORT (107-D0-07-401)
NOTE - Lift Table 107-LT-09-201C controls and indicators in Section VII.B are 7ocated on Lift Table Control Pedestal 107-PC-09-201C.

1. ENSURE $1 \mathrm{ift}$ table MAINT/OFF/NORMAL switch (09-HS-225A)

(PCS) Verifies drum PIN and location match that input by AGV delivery. Halts process if mismatched. 


\section{WRAP 1 PLANT OPERATING PROCEDURE}

(M)

5. IF scanner displays "PIN MIS-MATCH", THEN:

a. RECORD PIN displayed on barcode scanner on PIN Change Form.

b. SCAN Cancellation barcode.

(PCS) Clears pin mis-match message.

c. NOTIFY DOS,

d. CORRECT PIN information in PCS.

6. SCAN End (Y) barcode.

7. SCAN Yes (Y) barcode.

(DMS) Updates drum location.

8. PLACE ifft tabie AGV/PANEL switch (09-HS-225D) to "PANEL".

9. IF drum is fitted with outer 1 id,

THEN, REMOVE outer Tid as follows:

a. LOWER lift table as required.

b. REMOVE outer lid bolts.

c. REMOVE outer Tid.

d. POSITION lift table to "AGV LOAD HEIGHT".

10. FORWARD lift table to "DRUM UNDER PORT" position.

(PCS) Moves drum under port.

11. RAISE 1ift table to "DRUM IN POSITION" height.

12. ENSURE manipulator (107-EM-07-401) is PARKED.

13. SELECT "OPEN PORT".

14. VERIFY display "PORT OPENING..."

15. WAIT for display "PORT OPEN".

(PCS) Writes OIU displays, completes port opening sequence.

\begin{tabular}{|c|c|c|c|c|}
\hline $\begin{array}{l}\text { oocument } \\
\text { HRP }\end{array}$ & poxtros & Revpod & page/ 6 & \%8\% \\
\hline
\end{tabular}




\section{WRAP 1 PLANT OPERATING PROCEDURE}

c. REMOVE NON-COMPLIANT ITEM TRANSFER STAND FROM RWM DRUM

1. ENSURE manipulator (107-EM-07-401) is PARKED.

2. ENSURE RWM Transfer Port (107-D0-07-401) is OPEN.

3. PERFORM with hoist control, as required:

a. POSITION TROLLEY (hoist hook) above Port (107-D0-07-401).

b. LOWER HOIST to transfer stand.

c. ATrACH hoist hook to transfer stand.

d. RAISE HOIST until transfer stand clears glovebox floor.

e. POSITION TROLLEY to glovebox sorting or treatment area, as desired.

NOTE - $\quad$ At operators discretion, hoist can remain attached to transfer stand and transfer stand may be set on glovebox floor or left suspended, as desired for packet remova7.

f. LOWER HOIST unti1 transfer stand is at desired height.

D. REMOVE WASTE ITEMS FROM TRANSFER STAND FOR. SORTING/SAMPLING

1. SELECT in order, on DMS terminal, as required:

a. "PROCESS OPS".

b. "TRU RWM GLOVEBOX".

c. "RWM WASTE SORTING".

2. VERIFY DMS screen "DMSS0341 TRU RWM Waste Sorting" is displayed.

3. VERIFY correct drum and packet IDs displayed on DMS screen.

4. SELECT in order on OIU, as required:

a. "MAIN MENU".

b. "TRANSFER STAND MENU".

c. "REMOVE ITEMS".

5. VERIFY display "REMOVAL OF ITEMS IN PROGRESS...". 


\section{WRAP 1 PLANT OPERATING PROCEDURE}

6. SCAN Sample Sorting Location (2Y) barcode on glovebox.

7. REMOVE packet from transfer stand.

8. SCAN Packet identification (20T) barcode.

9. PLACE scanned packet in glovebox sorting area.

10. SCAN End ( $Y$ ) barcode.

11. SCAN Yes $(Y)$ barcode.

(DMS) Removes packet ID from RWM transfer drum inventory.

12. REPEAT Steps VII.D.6 through VII.D.11 until all items have been removed from transfer stand.

\section{CAUTION}

ALL ITEMS MUST BE REMOVED FROM TRANSFER STAND AND SCANNED PRIOR TO SELECTING "END REMOVE" OR PCS WILL NOT CORRECTLY UPDATE PACKET LOCATIONS IN DMS.

13. SELECT "END REMOVE" on OIU screen.

14. VERIFY display "REMOVAL OF ITEMS COMPLETE".

15. SELECT "REFRESH" on DMS screen.

16. VERIFY PINs of packets removed from transfer stand are displayed in DMS "Sample Station Packets" column.

\section{E. REMOVE WASTE ITEMS FROM TRANSFER STAND FOR TREATMENT}

1. SELECT in order, on DMS terminal:
a. "PROCESS OPS".
b. "TRU RWM GLOVEBOX".
c. "RWM WASTE TREATMENT".

2. VERIFY DMS screen "DMSS0344 - TRU RWM Treatment Item Assemb7y".

3. SELECT proper worksheet from. "LIST OF VALUES".

4. VERIFY correct drum and packet IDs displayed on DMS screen.

5. SCAN Treatment. Location (2Y) barcode on glovebox.

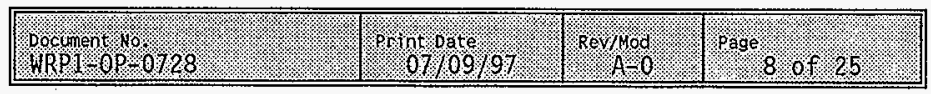




\section{WRAP 1 PLANT OPERATING PROCEDURE}

6. REMOVE packet from STE. TAASFCR 5 TAND

7. SCAN Packet identification (20T) barcode

8. PLACE scanned packet in glovebox treatment area.

9. SCAN End $(Y)$ barcode.

10. SCAN Yes $(Y)$ barcode.

(DMS) Removes packet ID from RWM transfer drum inventory.

11. REPEAT Steps VII.E.5 through VII.E.10 until all items have been removed from transfer stand.

\section{F. REMOVE SAMPLE BOTTLES FROM STC FOR TREATMENT}

1. SELECT in order, on DMS terminal:
a. "PROCESS OPS".
b. "TRU RWM GLOVEBOX".
c. "RWM WASTE TREATMENT".

2. VERIFY DMS screen "DMSS0344 - TRU RWM Treatment Item Assembly".

3. SELECT proper worksheet from "LIST OF VALUES".

4. SCAN Treatment Location (2Y) barcode on glovebox.

5. REMOVE sample bottle from tanser-stand. $S T C$

6. SCAN sample bottle identification (1S) barcode.

7. PLACE scanned sample bottle in glovebox treatment area.

8. SCAN End $(Y)$ barcode.

9. SCAN Yes $(Y)$ barcode.

(DMS) Removes sample bottle from STC inventory.

10. REPEAT Steps VII.F.4 through VII.F.9 until all sample bottles on Treatment Work Sheet or DMS Screen DMSS0345, Processing Instructions have been removed from STC. 


\section{WRAP 1 PLANT OPERATING PROCEDURE}

G. OPEN COMPLIANT HASTE PORT (107-D0-07-402A)

NOTE - Lift Table controls and indicators in Section VII:G are located on Lift Table Control Pedestal 107 PC-09-201B.

1. PREPARE TRU Waste or Mixed Waste, 55 gallon drum per WRP1-0P-1709, Packaging Mixed Waste.

2. SELECT in order, as required:

a. "MAIN MENU".

b. "PORT D0-07-402A".

3. VERIFY dispTay "DRUM AT LIFT TABLE".

4. SCAN in order:

a. Location (2Y) barcode on side of glovebox.

b. Drum (S) barcode.

c. End (Y) barcode.

d. Yes (Y) barcode.

(PCS) Verifies drum PIN and location match that input by AGV delivery. Halts process if mismatched.

(DMS) Updates drum Tocation.

(M) 5. IF scanner displays "PIN MIS-MATCH", THEN:

a. RECORD PIN displayed on barcode scanner on PIN Change Form.

b. SCAN Cancellation barcode.

(PCS) Clears pin mis-match message.

c. NOTIFY DOS.

d. CORRECT PIN information in PCS.

6. PLACE lift tabTe AGV/PANEL switch (09-HS-225D) to "PANEL".

\begin{tabular}{|c|c|c|c|}
\hline $\begin{array}{l}\text { oocument } \\
\text { 10\% }\end{array}$ & $\begin{array}{l}\text { Print pats } \\
\text { \%ol } 09997\end{array}$ & ReV Mod & pagr $10 \% 0 \%$ \\
\hline
\end{tabular}


7. REMOVE clamp band and outer lid from drum as follows:
a. LOWER lift table as required.
b. REMOVE clamp band.
c. REMOVE outer lid.
d. POSITION lift table to "AGV LOAD HEIGHT".

8. FORWARD lift table to "DRUM UNDER PORT".

(PCS) Moves drum under port.

9. RAISE 1ift table to "DRUM IN POSITION" height.

10. SELECT "FURH ON VACUUM".

10. SELECT WURT ON VACUUM" HECD

11. VERIFY display "VACUUM EENERATED".

12. SELECT "UNLOCK PORT".

13. VERIFY disp?AY "PORT UALOCKEQ"-

14. SELECT "OPEN PORT" POR ONING D. DISPLAY \&

15. VERIFY display "PORT OPEN".

(PCS) Writes OIU displays, completes port opening sequence.

H. OPEN NON-COMPLIANT WASTE PORT (107-D0-07-402B)

NOTE - Lift Tab7e 107-LT-09-201A contro7s and indicators in Section VII.H are located on Lift Table Control Pedestal. 107-PC-09-201A.

1. PREPARE Mixed Waste 85 gallon drum per WRP1-0P-1709, Packaging Mixed Waste.

2. SELECT "MAIN MENU" on OIU-12-106B.

3. VERIFY display "TRU RWM GLOVEBOX MAIN MENU".

4. SELECT "PORT DO-07-402B MENU".

5. VERIFY display "PORT D0-07-402B MENU".

6. VERIFY dispTay "LIFT TABLE WEIGHT" SETPOINT". ARE DISPCAYED. 
7. SCAN the following:

a. Location (2Y) barcode on side of glovebox.

b. $\operatorname{Drum}(S)$ barcode.

c. End $(Y)$ barcode.

d. Yes $(Y)$ barcode.

(PCS) Verifies drum PIN and Tocation match that input by AGV delivery. Halts process if mismatched.

(M)

8. IF scanner displays "PIN MIS-MATCH", THEN:

a. RECORD PIN displayed on barcode scanner on PIN Change Form.

b. SCAN Cancellation barcode.

(PCS) Clears pin mis-match message.

c. NOTIFY DOS.

d. CORRECT PIN information in PCS.

9. PLACE lift table AGV/PANEL switch (09-HS-221D) to "PANEL".

10. REMOVE clamp band and outer lid from drum as follows:

a. LOWER $1 \mathrm{ift}$ table as required.

b. REMOVE clamp band.

c. REMOVE outer lid.

d. POSITION 7 ift table to "AGV LOAD HEIGHT".

11. FORWARD 1 ift table to "DRUM UNDER PORT" position.

(PCS) Moves drum under port.

12. RAISE lift table to "DRUM IN POSITION" height.

13. SELECT "GENERATE VACUUM", THEN, WAIT for display "VACUUM HELD".

\begin{tabular}{|c|c|c|c|}
\hline 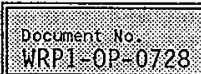 & Provt pate & Rev/110d & Page: $12.6 \%$ \% 25 \\
\hline
\end{tabular}


f. SELECT "COMMIT."

7. PLACE packet in compliant loadout drum.

8. If item requires field screening to determine comp $7 i$ ant/non-comp 7 iant status, THEN, PERFORM field screening per section VII.J.

9. PROCESS non-compliant items from the packet as follows:

a. IF the non-compliant item is an aerosol container, THEN perform the following:

(1) PROCESS per WRP1-0P-0708, Vent and Drain Aerosol

(2) PROCEED to Step VII.I.10.

b. IF non-compliant item requires containment/packaging, THEN, PLACE item in proper container/packing.

c. ATTACH new barcode to non-compliant item/package.

NOTE - Each scan of original packet barcode must be followed by scan of a new barcode, or an error message occurs. For multiple items in a single packet, original packet barcode must be scanned prior to each new barcode.

d. SCAN in order:

(1) Sorting location (2Y) barcode.

(2) Original packet identification (20T) barcode.

e. VERIFY barcode reader displays operation prompt "NEW PACKET" and original packet barcode.

f. SCAN new packet identification (20T) barcode.

g. VERIFY new packet barcode displayed on barcode reader.

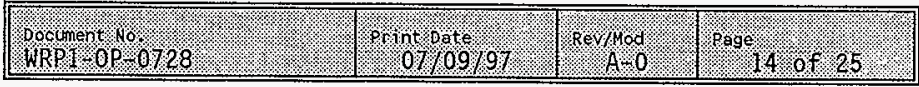




\section{WRAP 1 PLANT OPERATING PROCEDURE}

h. IF OIU displays "Barcode ERROR",

THEN:

(1) SELECT "ACKNOWLEDGE ERROR"

(2) RE-SCAN original packet identification (20T) barcode.

(3) RE-SCAN new packet identification (20T) barcode.

i. SCAN End $(Y)$ barcode.

j. SCAN Yes $(Y)$ barcode.

10. ENTER data for new container as follows:

a. SELECT "NEXT NEW CONTAINER" from screen "DMSSO342 TRURWM WASTE REPACKAGING".

b. VERIFY DMS displays data for original packet and ID for new packet.

c. ENTER Container Type, Material Group Code, and Waste Description for new container.

11. ENTER data for compliant portion of packet (includes wrapping and compliant material found in the packet) as follows:

a. CHECK the Compliant Waste block if wrapping or other compliant waste has been separated from original packet.

b. MODIFY compliant loadout drum waste description.

12. SAVE data to the DMS database as follows:
a. ENTER signature password
b. SELECT "COMMIT".

(DMS) Stores entered data in DMS database.

13. LOAD compliant material into compliant loadout drum.

14. REPEAT Steps VII.I.9 through VII.I.13 for each non-compliant item in packet.

15. REPEAT StepS VII.I.3 through VII.I.13 for a11 remaining packets. 


\section{WRAP 1 PLANT OPERATING PROCEDURE}

16. SELECT "RETURN TO SORTING" on DMS terminaT.

17. VERIFY DMS display "DMSS0341 - TRU RWM Waste Sorting".

18. SELECT "COMPLIANT LOADOUT" on DMS Terminat.

19. VERIFY DMS display, "DMSSO343 - TRU RWM Compliant Waste Loadout.

20. SELECT "PHYSCOMP" on DMS Terminat.

21. ENTER the following for items added to the drum:
a. "Description"
b. "Vol \%"
c. . "Weight"

22. ENTER signature password.

23. SELECT "COMMIT".

24. IF "Storage Category" has been modified,

a. ENTER signature password.

b. SELECT "COMMIT."

\section{J. SCREEN WASTE ITEMS}

1. If item has not been completely characterized, THEN, PERFORM field screening per WRP1-OP-0710, Glovebox Waste Sampling.

2. SAMPLE remaining non-compliant items per applicable procedure:

- WRPI-OP-0710, Waste Sampling

- WRP1-0P-0708, Vent and Drain Aerosol Cans

K. TREAT NON-COMPLIANT WASTE ITEMS

1. TREAT characterized waste per WRP1-OP-0711, Waste Treatment.

2. LOADOUT treated/non-compliant waste per Section VII.L.

\begin{tabular}{|c|c|c|c|}
\hline 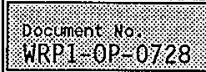 & erint polo & Rev/god & fogg. $16.0 \mathrm{of}: 25$ \\
\hline
\end{tabular}




\section{TREATED HASTE ITEM LOADOUT}

1. SELECT in order on OIU as required:
a. "MAIN MENU".
b. "TREATED ITEM MENU".
c. "LOADOUT TREATED ITEMS".

2. VERIFY display "TREATED ITEM LOADOUT IN PROGRESS..."

3. SCAN treatment location barcode.

4. VERIFY barcode reader displays operation prompt "TREATED ITEM LOADOUT".

5. SCAN treated item barcode.

6. LOAD item into drum at port 107-D0-07-402B.

7. SCAN End $(Y)$ barcode.

8. SCAN Yes (Y) barcode.

9. IF more items require loadout, THEN, REPEAT steps VII.L.3 to VII.L.8.

10. SELECT "END TR. ITEM LDOUT".

11. VERIFY display "TREATED ITEM LOADOUT COMPLETE".

12. WHEN drum is ful1, THEN, PERFORM the following steps:

a. SELECT in order, on DMS termina7, as required:

(1) "PROCESS OPS".

(2) "TRU RWM GLOVEBOX",

(3) "TREATED WASTE LOADOUT".

b. VERIFY DMS display "DMSS0348 - TRU RWM TREATED WASTE LOADOUT" shows correct "TREATED WASTE LOADOUT DRUM".

c. SELECT "PHYSCOMP". 
d. ENTER Physical Component Description and corresponding volume percents and weights. AND COMMIT DATH.

e. SELECT "RETURN" EX/T.

f. SELECT "CHEMCOMP".

g. ENTER I.D. and weight of any hazardous components, tayD comn $D A 7 \not$.

h. SELECT "RETURNH. EXIT:

i. ENTER the following:

(1) Primary Waste Type.

(2) Hazardous Waste Storage Category.

(3) Assay Profite ID.

(4) Assay Default Flag.

(5) Liner Venting Method.

(6) Drum Filter Model \# (if installed).

(7) Void filler type and weight (if used) ( $(\omega)$ only)

j. ENTER signature password.

k. SELECT "COMMIT". 


\section{ADD ITEMS TO TRANSFER STAND}

1. SELECT in order on OIU, as required:

a. "MAIN MENU".

b. "TRANSFER STAND MENU".

c. "ADD ITEMS".

2. VERIFY display "ADD ITEMS IN PROGRESS".

3. SELECT packet to be added to transfer stand.

4. SCAN Sample Location (2Y) barcode on inside of glovebox.

5: VERIFY barcode reader dispTays operation prompt "ADD ITEMS ".

6. SCAN packet identification (20T) barcode.

7. PLACE scanned packet on transfer stand.

8. SCAN End (Y) barcode.

9. SCAN Yes (Y) barcode.

(DMS) Removes packet ID from glove box inventory.

10. REPEAT Steps VII.M.3 through VII.M.9 until all items have been added to transfer stand.

11. SELECT "END ADD".

12. VERIFY display "ADD ITEMS COMPLETE".

13. SELECT in order, on DMS terminal, as required:
a. "PROCESS OPS".
b. "TRU. RWM GLOVEBOX".
c. "RWM WASTE SORTING".

14. VERIFY DMS screen "DMSS0341 - TRU RWM Waste Sorting".
a. SELECT "REFRESH".

15. VERIFY PINs for packets added to transfer stand are dispiayed in DMS "Transfer Drum Packets" column.

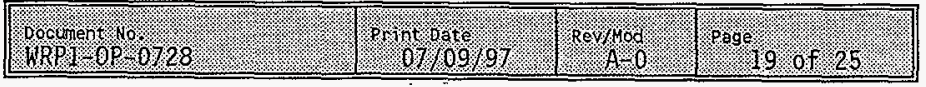




\section{WRAP 1 PLANT OPERATING PROCEDURE}

N. LOAD TRANSFER STAND INTO RHM DRUM

1. ENSURE manipuTator (107-EM-07-401) is PARKED.

2. ENSURE RWM Transfer Port (107-D0-07-401) is OPEN.

3. LOAD transfer stand into transfer drum using Glovebox Hoist.

0. CLOSE BAGLESS TRANSFER PORT (107-D0-07-401)

NOTE - Lift Table controls and indicators in Section VII.O are located on Lift Table Contro7 Pedestal 107-PC-09-201C.

1. ENSURE $1 \mathrm{ift}$ table MAINT/OFF/NORMAL switch (09-HS-225A) set to "NORMAL".

ENSURE HOIST IS RAISED CLEAR OF BTP LID.

2. SELECT on OIU-12-106B, in order:
a. "MAIN MENU".
b. "PORT DO-07-401 MENU".
c. "CLOSE PORT".

3. VERIFY display "PORT CLOSING...".

4. WAIT for display "PORT CLOSED".

(PCS) Writes OIU displays, completes port closing sequence.

5. PLACE 7 ift table AGV/PANEL switch (09-HS-225D) to "PANEL".

6. POSITION lift table to "AGV LOAD HEIGHT".

(R) 7. REQUEST RCT survey drum for contamination.
a. IF drum is contaminated,
THEN DECONTAMINATE as directed.
b. RESURVEY drum.

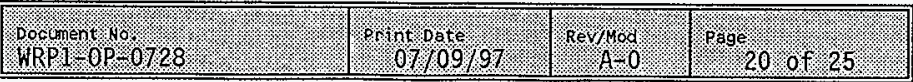




\section{WRAP 1 PLANT OPERATING PROCEDURE}

8. IF drum was fitted with outer $1 j \mathrm{~d}$,

THEN, REINSTALL outer Tid as follows:

a. LOWER lift table as required.

b. REINSTALL outer 1id.

c. REINSTALL outer Tid bolts.

9. REVERSE lift table to "DRUM AT. AGV END" position.

(PCS)

Moves drum to AGV end of lift table.

10. PLACE lift table AGV/PANEL switch to "AGV".

P. CLOSE COMPLIANT WASTE PORT (107-D0-07-402A)

NOTE - Lift Table controls and indicators in Section VII.P are located on Lift Table Contro7 Pedestal 107-PC-09-201B.

1. ENSURE drum is filled to desired level.

2. SELECT in order, on DMS terminal, as required:

a. "PROCESS OPS".

b. "TRU RWM GLOVEBOX".

c. "RWM WASTE SORTING".

d. "RWM COMPLIANT LOADOUT".

3. VERIFY DMS screen "DMSS0343 - TRU RWM Compliant Waste Loadout".

4. ENTER the following information on waste in the compliant waste loadout drum:

a. Primary waste type.

b. Hazardous waste storage category for drum.

c. Assay profile and default profile flag.

5. SELECT "PHYSCOMP" on DMS termina1.

6. UPDATE compliant loadout drum waste Description, if required. SELECT "RETURN-TO-SORTIAG TABLE-SCREEN".

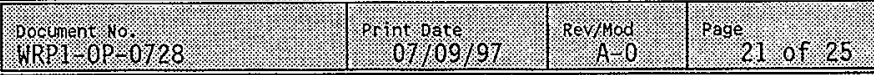




\section{WRAP 1 PLANT OPERATING PROCEDURE}

8. VERIFY DMS screen "DMSS0343 - TRU RWM Compliant Waste Loadout".

9. ENTER signature password

10. SELECT "COMMIT".

(DMS) Store data in database.

11. SELECT in order, as required:
a. "MAIN MENU".
b. "PORT D0-07-402A".
c. "CLOSE PORT".

12. VERIFY display "PORT CLOSING...".

13. WAIT for OIU display "PORT CLOSED".

Writes OIU displays, completes port closing sequence.

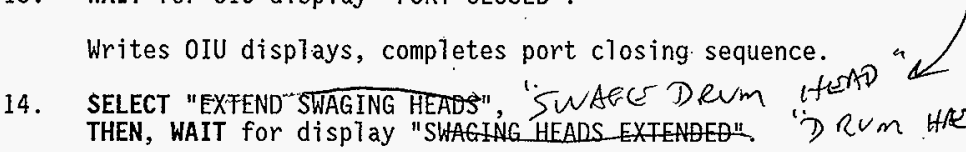

"D) RUM HAETS TWAGO

15. SELECT "FURN OFF WACHUH". ReECSASE MACUVM

16. VERIFY display "THRNONACUUA" (e.g., VACUUM GENERATED display changes state.) VACVUM RELEA EEO

17. PLACE lift tabTe AGV/PANEL switch (09-HS-229D) to "PANEL".

18. LOWER lift table to "AGV HEIGHT".

$(\mathrm{R})$

19. REQUEST RCT survey drum for contamination.

a. IF drum is contaminated,

THEN DECONTAMINATE as directed.

b. RESURVEY drum.

20. REVERSE lift table to "DRUM AT AGV END" position.

(PCS) Moves drum to AGV end of lift table. 


\section{WRAP 1 PLANT OPERATING PROCEDURE}

21. SELECT in order, on DMS terminal, as required:
a. "PROCESS OPS".
b. "TRU RWM GLOVEBOX".
c. "RWM WASTE TREATMENT".
d. "COMPLIANT LOADOUT".

22. VERIFY DMS screen "DMSS0343 - TRU RWM Compliant Waste Loadout".
a. ENTER "Beta/Gamma Dose Rate" and "Seal No.: for drum.
b. ENTER signature password.
c. SELECT "COMMIT".

23. SELECT "RETURN".

24. PLACE lift table AGV/PANEL switch to "AGV".

Q. CLOSE NON-COMPLIANT WASTE PORT (107-D0-07-402B)

NOTE - Lift Table controls and indicators in Section VII.Q are located on Lift Table Control Pedestal 107-PC-09-201A.

1. ENSURE drum is fu71 and contents properly documented.

2. SELECT on OIU in order, as required:

a. "MAIN MENU".

b. "PORT DO-07-402B MENU".

c. "CLOSE PORT".

3. VERIFY display "PORT CLOSING...".

4. WAIT for display "PORT CLOSED".

(PCS) Writes OIU displays, completes port closing sequence. "SWAGE DRUM IFSAD" DRUM HEATS SWAGE IN

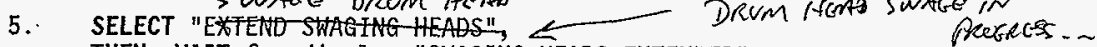
THEN, WAIT for display "SWAGING HEADS EXTENDED".

6. ' SELECT "TURN OFF VACUUM".

\begin{tabular}{|c|c|c|c|c|c|}
\hline 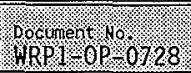 & 3rot date & revaro & page & 09 & 25 \\
\hline
\end{tabular}




\section{WRAP 1 PLANT OPERATING PROCEDURE}

7. VERIFY display "TURN ON VACUUM" (e.g., VACUUM GENERATED display changes state.)

8. PLACE lift table AGV/PANEL switch (09-HS-221D) to "PANEL".

9. LOWER lift table to "AGV HEIGHT"

10. REVERSE 7ift table to "DRUM AT AGV END" position.

(PCS)

(R)

(PCS)

14. SELECT in order, on DMS terminal, as required:
a. "PROCESS OPS".
b. "TRU RWM GLOVEBOX".
c. "RWM WASTE TREATMENT".
d. "TREATED WASTE LOADOUT".

15. VERIFY DMS screen "DMSSO348 - TRU RWM Treated Waste Loadout".
a. ENTER "Beta/Gamma Dose Rate" and "Seal No.: for drum.
b. ENTER signature password
C. SELECT "COMMIT".

16. SELECT "RETURN".

17. PLACE lift table AGV/PANEL switch to "AGV". 


\section{WRAP 1 PLANT OPERATING PROCEDURE}

\section{ATTACHMENT 1: SORTING GLOVEBOX HOIST CONTROL FUNCTIONS}

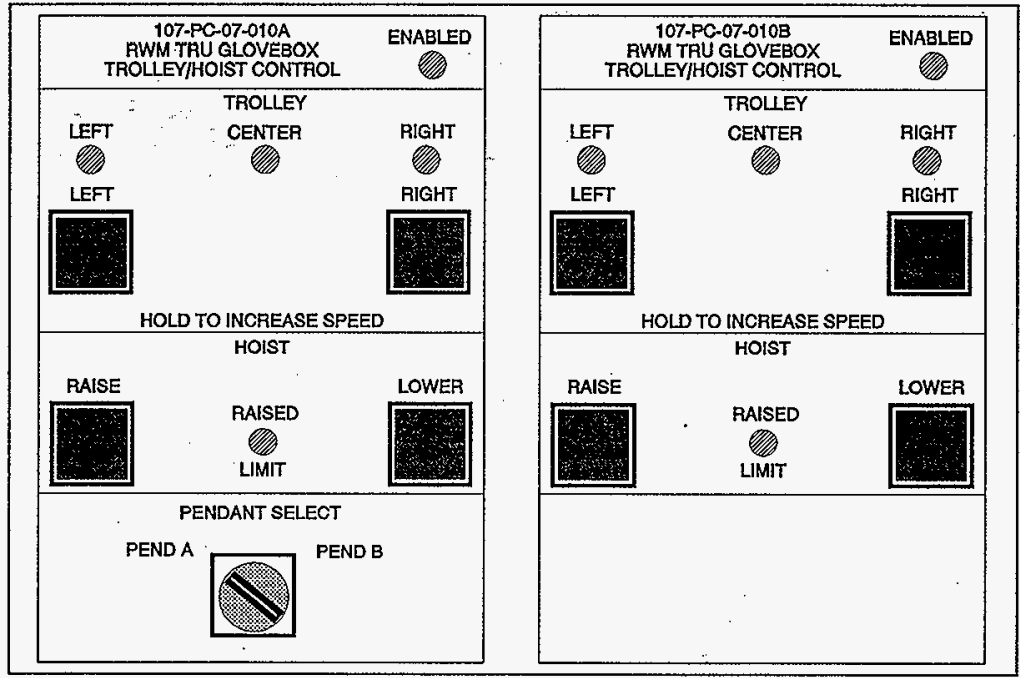

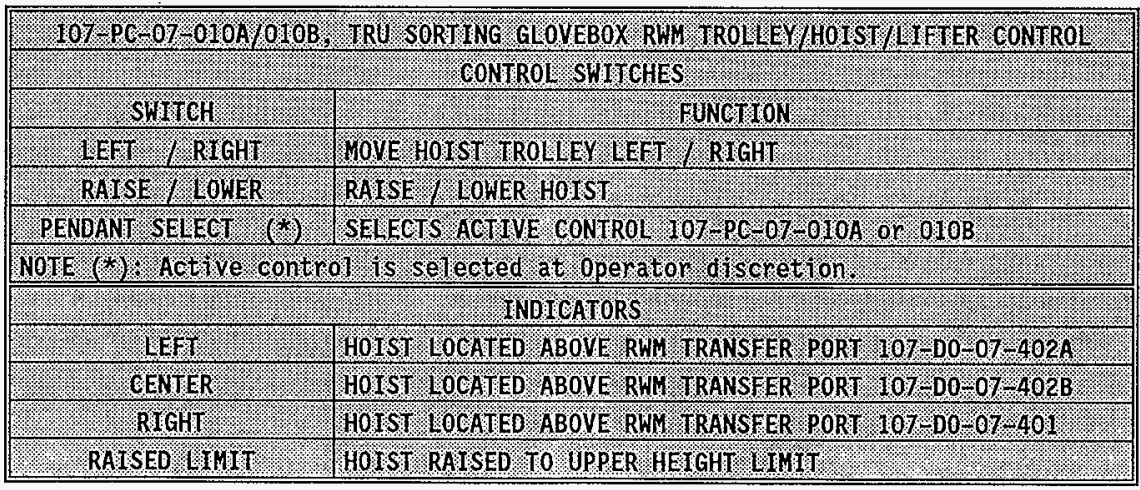

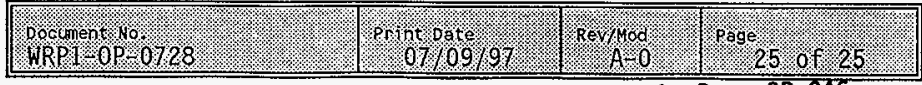




\section{DISTRIBUTION SHEET}

\begin{tabular}{|c|c|c|c|c|c|}
\hline \multirow{2}{*}{$\begin{array}{l}\text { To } \\
\text { Distribution }\end{array}$} & \multirow{2}{*}{\multicolumn{3}{|c|}{$\begin{array}{l}\text { From } \\
\text { WRAP Engineering }\end{array}$}} & \multicolumn{2}{|l|}{ Page 1 of 1} \\
\hline & & & & \multicolumn{2}{|c|}{ Date $02 / 03 / 98$} \\
\hline \multirow{2}{*}{\multicolumn{4}{|c|}{$\begin{array}{l}\text { Project Title/Work Order } \\
\text { HNF-SD-W026-0TR-014, Rev. 0, TRU RWM OPERATIONAL TEST REPORT }\end{array}$}} & \multicolumn{2}{|c|}{ EDT No. 621918} \\
\hline & & & & \multicolumn{2}{|c|}{ ECN No. N/A } \\
\hline Name & MSIN & $\begin{array}{l}\text { Text } \\
\text { With All } \\
\text { Attach. }\end{array}$ & Text Only & $\begin{array}{l}\text { Attach./ } \\
\text { Appendix } \\
\text { Only }\end{array}$ & $\begin{array}{l}\text { EDT/ECN } \\
\text { Only }\end{array}$ \\
\hline
\end{tabular}

RJ Bottenus

T4-51

$x$

WG Jasen

T4-52

X

JK Kersten

T4-52

$x$

RJ Kol1

T4-51

$x$

KJ Leist

T4-52

$x$

WR Thackaberry

T4-52

$x$

JR Weidert

T4-52

$x$

Engineering Files

A3-88

$x$ 